\title{
Dusty Star-Forming Galaxies at High Redshift
}

\author{
Caitlin M. Casey ${ }^{1,2}$, Desika Narayanan ${ }^{3}$, Asantha Cooray ${ }^{1}$ \\ ${ }^{1}$ Department of Physics and Astronomy, University of California, Irvine, CA 92697 \\ ${ }^{2}$ Institute for Astronomy, University of Hawai' $i, 2680$ Woodlawn Dr, Honolulu, HI 96822 \\ ${ }^{3}$ Department of Physics and Astronomy, Haverford College, 370 Lancaster Avenue, Haverford, PA 19041
}

\begin{abstract}
Far-infrared and submillimeter wavelength surveys have now established the important role of dusty, star-forming galaxies (DSFGs) in the assembly of stellar mass and the evolution of massive galaxies in the Universe. The brightest of these galaxies have infrared luminosities in excess of $10^{13} \mathrm{~L}_{\odot}$ with implied star-formation rates of thousands of solar masses per year. They represent the most intense starbursts in the Universe, yet many are completely optically obscured. Their easy detection at submm wavelengths is due to dust heated by ultraviolet radiation of newly forming stars. When summed up, all of the dusty, star-forming galaxies in the Universe produce an infrared radiation field that has an equal energy density as the direct starlight emission from all galaxies visible at ultraviolet and optical wavelengths. The bulk of this infrared extragalactic background light emanates from galaxies as diverse as gas-rich disks to mergers of intense starbursting galaxies. Major advances in far-infrared instrumentation in recent years, both spacebased and ground-based, has led to the detection of nearly a million DSFGs, yet our understanding of the underlying astrophysics that govern the start and end of the dusty starburst phase is still in nascent stage. This review is aimed at summarizing the current status of DSFG studies, focusing especially on the detailed characterization of the bestunderstood subset (submillimeter galaxies, who were summarized in the last review of this field over a decade ago, Blain et al. 2002), but also the selection and characterization of more recently discovered DSFG populations. We review DSFG population statistics, their physical properties including dust, gas and stellar contents, their environments, and current theoretical models related to the formation and evolution of these galaxies.
\end{abstract}

Keywords: Galaxies, Cosmology, Galaxy evolution, Galaxy formation, Infrared galaxies

\section{Contents}

1 Introduction

2 Selection of Distant Infrared-Luminous Galaxies

2.1 Local Infrared-Luminous Galaxies . . . . . . . . . . . . . . . . . . . . . . . . . . . 7

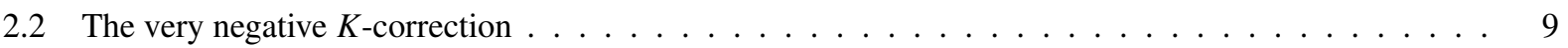

2.3 Dusty Galaxy Selection from $\sim 8-2000 \mu \mathrm{m} \ldots \ldots \ldots$. . . . . . . . . . . . . . . . 11

2.3.1 Facilities and Instruments Discovering high- $z$ DSFGs . . . . . . . . . . . . . . . 11

2.3.2 Notable surveys focused on DSFG Discovery . . . . . . . . . . . . . . . . 17

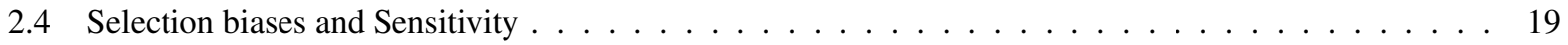

2.4 .1 Intrinsic Variation in SEDs . . . . . . . . . . . . . . . . . 20

2.4.2 Identifying Multi-wavelength Counterparts $\ldots \ldots \ldots \ldots \ldots \ldots \ldots \ldots \ldots$

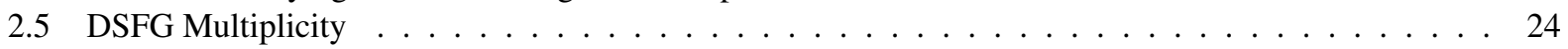

3 Submillimeter Number Counts 25

3.1 Confusion Noise . . . . . . . . . . . . . . . . . . . . . . 25

3.2 Using Monte Carlo Simulations in Number Counts Analysis . . . . . . . . . . . . . . . . . 25

3.2.1 Estimating Deboosted Flux Densities . . . . . . . . . . . . . . . . . . . 27

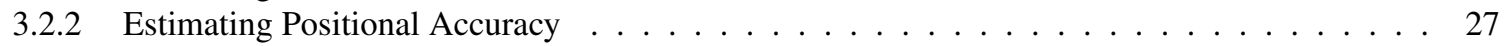


3.2.3 Estimating Sample Contamination \& Completeness . . . . . . . . . . . . . . . . 29

3.3 Number Counts . . . . . . . . . . . . . . . . . . . . . . . . . . 29

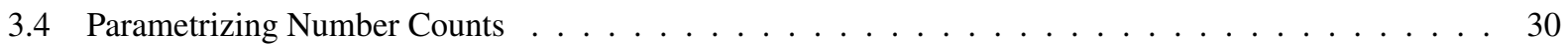

3.5 Bright-End Counts: Gravitationally Lensed DSFGs . . . . . . . . . . . . . . . . . . . . . 33

3.6 The Cosmic Infrared Background and $\mathrm{P}(\mathrm{D})$ Analysis $\ldots \ldots \ldots \ldots$

4 Redshifts and Spectral Energy Distributions of Infrared-Luminous Galaxies 3

4.1 Acquiring Spectroscopic or Photometric Redshifts . . . . . . . . . . . . . . . . . . 36

4.1.1 Millimetric Spectroscopic Redshifts . . . . . . . . . . . . . . . . . . . . . 37

4.1.2 Millimetric Photometric Redshifts . . . . . . . . . . . . . . . . . . . . . 38

4.1.3 Redshift Distributions of $24 \mu \mathrm{m}$ selected DSFG populations . . . . . . . . . . . . 38

4.1.4 Redshift Distributions of $850 \mu \mathrm{m}-1.4 \mathrm{~mm}$-selected DSFG populations . . . . . . . . . . . 38

4.1.5 Redshift Distributions of $250 \mu \mathrm{m}-500 \mathrm{~mm}$-selected DSFG populations . . . . . . . . . . . 41

4.2 Infrared SED Fitting for DSFGs . . . . . . . . . . . . . . . . . . . . . . 43

4.2.1 Employing dust radiative transfer models and empirical templates . . . . . . . . . . 44

4.2 .2 Direct modified blackbody SED modeling . . . . . . . . . . . . . . . . 46

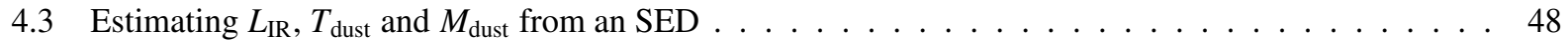

4.4 Luminosity Functions . . . . . . . . . . . . . . . . . . . . . . 50

4.5 Contribution to Cosmic Star Formation Rate Density $\ldots \ldots \ldots \ldots \ldots \ldots \ldots$

5 Physical Characterization 55

5.1 Star Formation History \& Dynamical Time $\ldots \ldots \ldots \ldots \ldots \ldots \ldots \ldots \ldots$

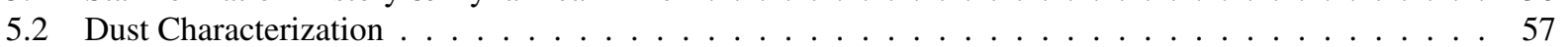

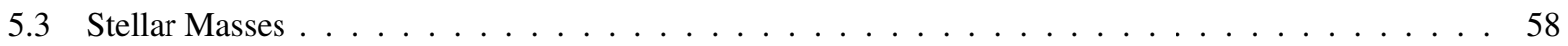

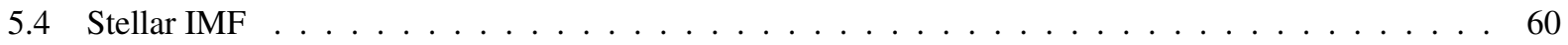

5.5 Rest-frame Ultraviolet \& Optical Spectral Characterization . . . . . . . . . . . . . . . . 61

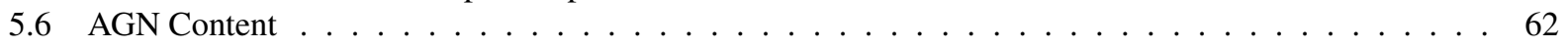

5.7 Mid-Infrared Diagnostics . . . . . . . . . . . . . . . . . . . . . . . . . . . . . . . . 64

5.8 Mid-Infrared Spitzer-selected Populations ～. . . . . . . . . . . . . . . . . . . . . 65

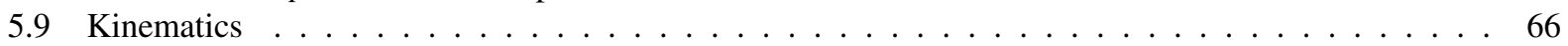

5.10 Physical Size and Morphology . . . . . . . . . . . . . . . . . . . . . 66

5.11 Relationship to Normal Galaxies: the Infrared Main Sequence $\ldots \ldots \ldots \ldots$. . . . . . . . 69

5.12 The FIR/Radio Correlation . . . . . . . . . . . . . . . . . . . . . . 70

6 Detailed Studies of Individual Dusty Star-Forming Galaxies 73

6.1 SMM J02399-0136 . . . . . . . . . . . . . . . . . . . . . . . . . . . . 73

6.2 SMM J2135-0102: the Cosmic Eyelash … . . . . . . . . . . . . . . . . . . . . . . . 74

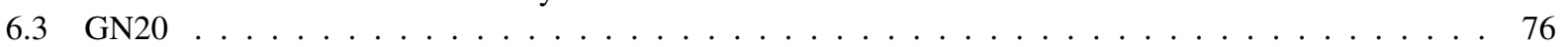

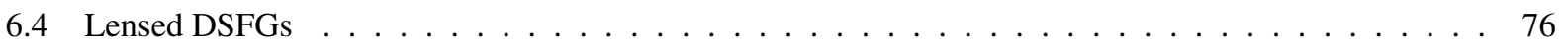

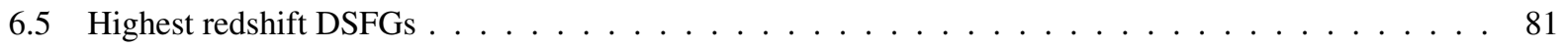

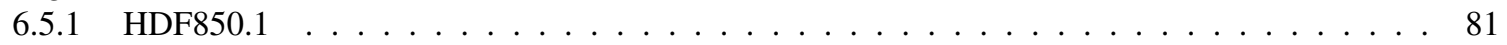

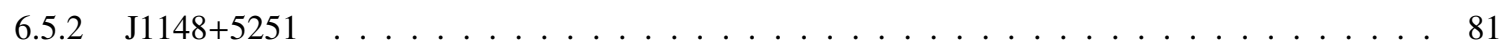

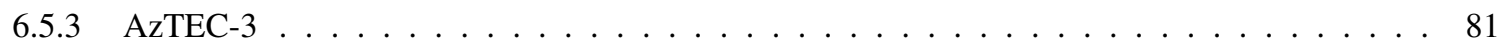

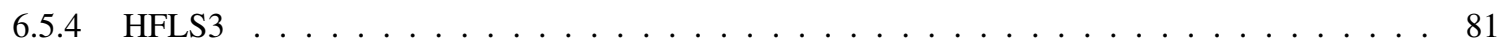

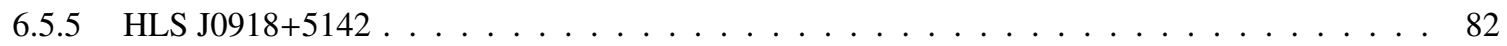

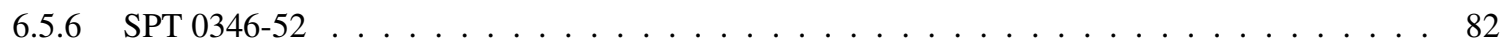

7 Clustering and Environment $\quad 83$

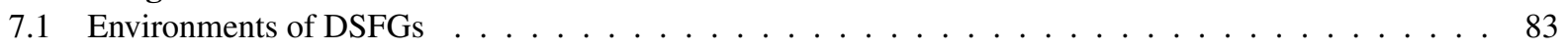

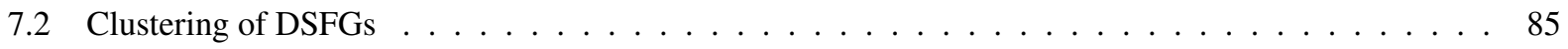

7.3 Clustering of Faint, Unresolved DSFGs through the CIB Anisotropy Power Spectrum . . . . . . . . 87

7.4 Cosmic Magnification of Submm Sources . . . . . . . . . . . . . . . . . . . 89 


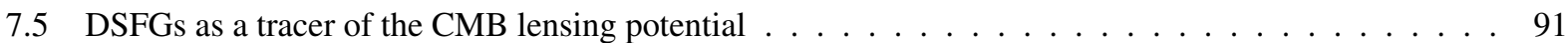

8 Molecular Gas and Star Formation 92

8.1 Basic Definitions . . . . . . . . . . . . . . . . . . . . . . . . . 92

8.2 Deriving $\mathrm{H}_{2}$ Gas Masses from High-Redshift Galaxies ～. . . . . . . . . . . . . . . . 92

8.3 Star Formation Laws and Efficiencies $\ldots \ldots \ldots \ldots \ldots \ldots \ldots$

8.4 The Role of Dense Molecular Gas . . . . . . . . . . . . . . . . . . . . . . . . . . 100

8.4.1 Physics Learned from the Milky Way and Local Galaxies . . . . . . . . . . . . . . . . . . . 100

8.4.2 Dense Gas at High-Redshift . . . . . . . . . . . . . . . . . . . . . . . . . . . . 101

8.5 CO Excitation and Spectral Line Energy Distributions . . . . . . . . . . . . . . . . . . . . 102

8.6 Molecular Gas Fractions . . . . . . . . . . . . . . . . . . . . . . . . . . . . . . . . 104

8.7 Molecular Gas Morphology and Dynamics . . . . . . . . . . . . . . . . . . . . . 105

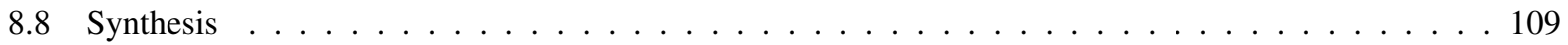

9 Atomic Lines 110

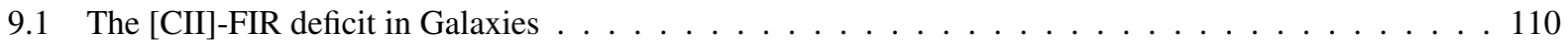

$9.2[\mathrm{CII}]$ as a Star Formation Rate Indicator . . . . . . . . . . . . . . . . . . . . . . 111

9.3 [CII] Morphologies and Dynamics . . . . . . . . . . . . . . . . . . . . . . . . . . 111

10 The Theory of forming Dusty Galaxies 114

10.1 Overview of Dusty Galaxy Modeling Methods . . . . . . . . . . . . . . . . . . . . . . . 114

10.1.1 Semi-Analytic Models . . . . . . . . . . . . . . . . . . . . . . . . . . 114

10.1.2 Cosmological Hydrodynamic Simulations . . . . . . . . . . . . . . . . . . 115

10.1.3 Idealized and Hybrid Models . . . . . . . . . . . . . . . . . . . . . . 116

10.1.4 Empirical Methods . . . . . . . . . . . . . . . . . . . . . . . . 116

10.2 Main Results from Theories of Dusty Galaxies ～. . . . . . . . . . . . . . . . . . . . . . 117

10.2.1 Semi-Analytic Methods . . . . . . . . . . . . . . . . . . . . . . . . . 117

10.2.2 Cosmological Hydrodynamic Simulations . . . . . . . . . . . . . . . . . 120

10.2 .3 Idealized and Hybrid Simulations . . . . . . . . . . . . . . . . . . . 121

10.3 Testable Predictions and Key Differences between Models . . . . . . . . . . . . . . . . . . 123

11 Future directions

12 Glossary of Dusty Star-Forming Galaxy Acronyms 128 


\section{Introduction}

Understanding the origins of the cosmic background radiation, from the X-ray to the radio, has led to detailed analysis of distant galaxy populations and their formation and evolution. While astronomers have made significant progress in studying the stellar mass content of galaxies, the growth and assembly of stellar populations, the physics surrounding supermassive black holes in galactic centers, and the large number of luminous galaxies in the early Universe-all from optical, $\mathrm{X}$-ray and radio observations-it has become increasingly evident that a significant portion of galaxies' light is emitted at far-infrared and submillimeter wavelengths.

In the early 1990s, the Far-InfraRed Absolute Spectrophotometer (FIRAS) aboard the space-based Cosmic Background Explorer $(C O B E)$ measured the absolute energy spectrum of the Universe at far-infrared and sub-millimeter wavelengths above $150 \mu \mathrm{m}$. These measurements, along with prior observations of nearby galaxies with IRAS in the 1980 s, showed for the first time that the Universe emits a comparable energy density at infrared and sub-millimeter wavelengths as it does in the more traditionally studied optical and ultraviolet. The implications of this are significant: optical and ultraviolet observations alone will miss roughly half of the star formation activity in the Universe. More troublesome is that this problem is exacerbated at high-redshift, where the bulk of the cosmic star formation activity takes place. The original COBE measurements of the cosmic infrared background (CIB), combined with galaxy surveys at optical wavelengths, showed that there must be either a population of galaxies that are enshrouded in dust and/or numerous dust-enshrouded regions within optically-detected galaxies where newly born massive stars are likely born. The radiation coming from stars in dusty regions heat the dust and the thermal emission from the dust at far-infrared and submm wavelengths (from roughly $10-1000 \mu \mathrm{m}$ ) leaves the signature of their presence.

At these long wavelengths, one encounters multiple observational limitations: the water vapor in the atmosphere forces sensitive observations to be made from either high and dry locations on Earth or from space. Another challenge has been the hitherto poor sensitivity of detectors operating at these long infrared wavelengths. Still, since the original measurements of the IR background by COBE, the field has seen an explosion of interest. In the late 1990s, deep blank field pointings with the Submillimeter Common-User Bolometer Array at $850 \mu \mathrm{m}$ on the James Clerk Maxwell Telescope (JCMT) directly detected, for the first time, populations of high-redshift galaxies that are extremely bright at far-IR/submm wavelengths but are nearly invisible in the optical. These ground-breaking studies revolutionized the field of galaxy formation, and turned the study of high- $z$ dusty galaxies into one of the fastest growing areas of extragalactic astronomy. Following surveys by the Spitzer Space Telescope at mid- and far-IR wavelengths, as well as by both balloon and ground-based submillimeter (submm) and millimeter $(\mathrm{mm})$ single dish radio facilities, have since detected galaxies with a wide range of inferred star formation rates, stellar masses, and black hole luminosities. Some of the most significant and recent developments have come from the Herschel Space Observatory which, during its 4 year operations between 2009 and 2013, mapped more than $1300 \mathrm{deg}^{2}$ of the sky between 100 and $500 \mu \mathrm{m}$ and detected more than a million galaxies bright at far-infrared and submm wavelengths (Figure 1), and the South Pole Telescope, which has mapped $\sim 2500 \mathrm{deg}^{2}$ at $1.4-2.0 \mathrm{~mm}$, detecting hundreds of brightly lensed dusty galaxies. The increases afforded by these facilities have allowed for large statistical studies of a cosmologically crucial populations of galaxies which is a marked improvement over the original samples of $\sim 200$ galaxies discovered in the original submm surveys with SCUBA in the late 1990s. With the recent commissioning of ScUBA-2 on the JCMT, as well as the Atacama Large Millimeter Array (ALMA) and soon-to-be construction of the Cerro Chajnantor Atacama Telescope (CCAT) in Chile, unprecedented statistics and detailed physical characterization of precisely-identified submm bright galaxies at high- $z$ are imminent.

Since their initial discovery, dusty star-forming galaxies (DSFG 1 ) at high- $z$ have become a critical player in our understanding of cosmic galaxy formation and evolution. The most luminous of these systems are the brightest galaxies in the Universe, and are seen back to just $\sim 800 \mathrm{Myr}$ after the Big Bang. These DSFGs are the most intense stellar nurseries in the Universe, and have inferred star formation rates (SFRs) of as much as a few thousand solar

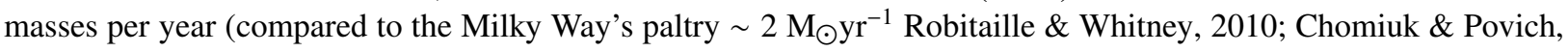
2011). Much of this is happening in a spatial extent so compact, that the star formation rate surface densities of these galaxies are among the largest known. These DSFGs provide a unique laboratory for investigating the physics of star

\footnotetext{
${ }^{1}$ Hereafter, we generalize all galaxies at high- $z$ that have been originally selected at infrared or sub-millimeter wavelengths as dusty star-forming galaxies (DSFGs). This encompasses a diverse zoo of galaxies, that we discuss in greater detail in $\$ 2$
} 


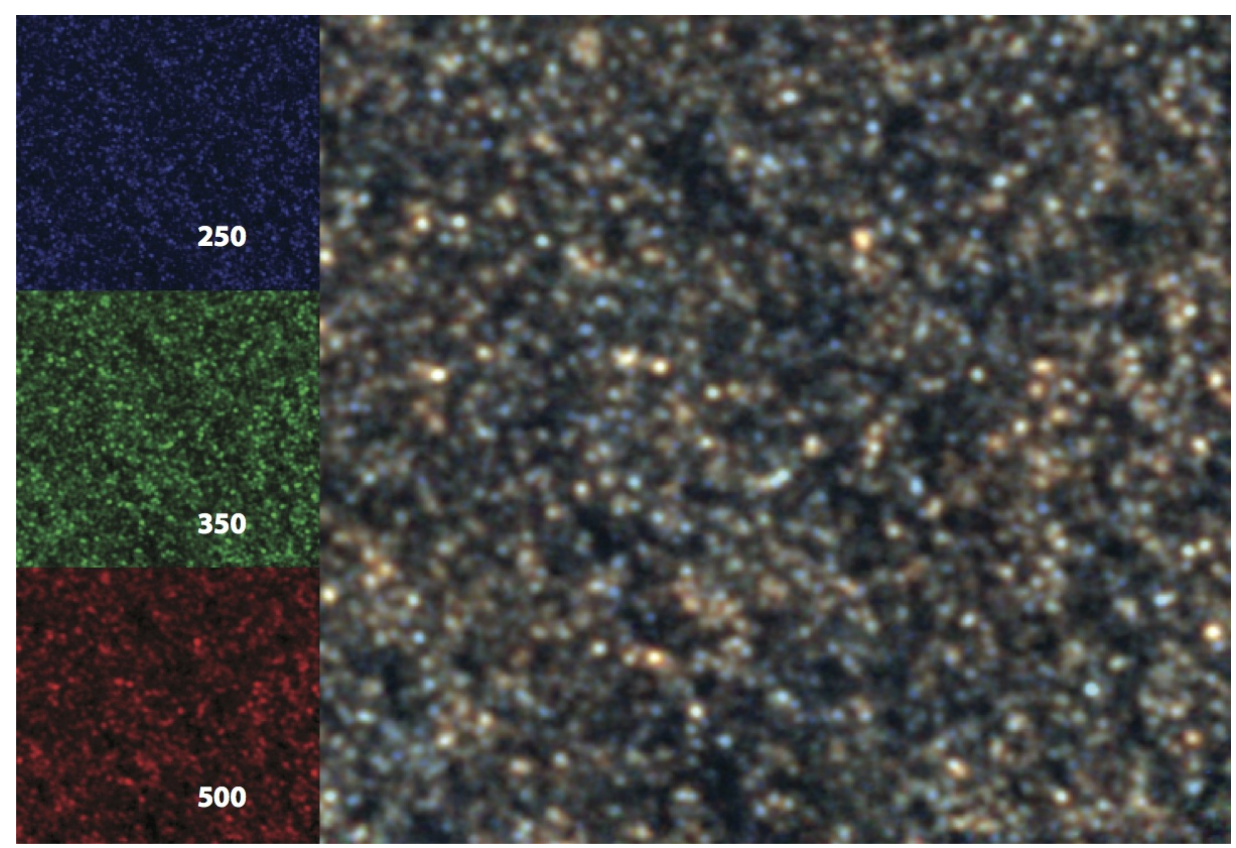

Figure 1: The false-color image of Herschel-SPIRE instrument map of the GOODS-N region of the sky. The three panels to the left show the sky at 250,350 and $500 \mu \mathrm{m}$ in blue, green, and red, respectively. At right, the combined false-color image shows galaxies that are brighter at 250 $\mu \mathrm{m}$ (bluer) vs those that are brighter at $500 \mu \mathrm{m}$ (redder). This color change could come from either differences in the thermal dust temperature or due to differences associated with the redshifting of the thermal spectrum as a function of the redshift. The red color then indicates galaxies that contain colder dust or are at higher redshifts. As we discuss later, follow-up observations have shown the latter case to be the primary reason for the color changes. The image spanning $30 \times 30 \mathrm{arcmin}^{2}$ contains close to a few hundred individually detected galaxies that are brighter at these submm wavelengths and makes up less than a thousandth of the area surveyed by Herschel. This image was done with 30 orthogonal scans of the Herschel-SPIRE instrument. Most of the extragalactic sky area covered by Herschel-SPIRE involves two orthogonal scans which effectively reaches the same confusion-limited depth as this data but is less useful for advanced statistical test (for an example of a map with two scans see Fig. 34 .

formation in environments far more extreme than can be found in our own galaxy ${ }_{2}^{2}$

A sub-sample of DSFGs are known to harbor heavily dust-enshrouded supermassive black holes. A significant fraction of these galaxies' infrared luminosity can actually be dominated by AGN heating mechanisms, rather than star formation processes, as is the case for the recently characterized WISE-selected galaxies (e.g. Blain et al., 2013). In fact, a variety of lines of evidence suggest that these galaxy may serve as precursors to luminous quasars, and serve as the site of a rapid growth phase of central black holes, as they approach nearly a billion solar masses. At the other extreme, fainter galaxies just barely detected in the far-IR/submm form stars at rates of tens to hundreds of solar masses per year, and contribute principally to a cosmic infrared background that is comparable to the energy density of all direct starlight from all galaxies in the ultraviolet and optical wavelength regimes.

The discovery of copious numbers of submm and infrared emitting galaxies at high- $z$ has proven to be a significant challenge for theoretical models of galaxy formation. As we will discuss later, cosmological models of structure formation and galaxy evolution have had a difficult time understanding the origin and evolutionary destiny of these heavily star-forming systems utilizing simulations, especially those designed ab initio. Whether or not they are simply scaled up versions of local extreme galaxies (such as UltraLuminous Infrared Galaxies, ULIRGs), or different beasts all together is still a heavily debated topic today.

Extragalactic infrared-based astronomy is at a period of great growth. With the development of the James Webb Space Telescope (JWST; space-based near and mid-IR), continued development and operations of the Atacama Large Millimeter Array (ALMA; ground-based far-IR and submm interferometer) and the planning of CCAT, the community is investing heavily in facilities that will enable the detection of and physical characterization of dusty systems out to

\footnotetext{
${ }^{2}$ While certain regions of the Milky Way have star formation surface densities as high, distant DSFGs are unique in that the high density environment encompasses the entire ISM (Wu et al. 2009).
} 
the Universe's earliest epochs.

In this review, we summarize what has been learned about high- $z$ dusty star-forming galaxies over the past decade, from the characterization of original submm sources detected by ScuBA, to newer DSFGs found by AzTEC, Herschel, ScuBA-2, SPT and others. We will summarize both the population statistics-number counts, redshift distribution, luminosity functions - as well as detailed physical properties of these dusty, star-forming galaxies. We will review the contribution of these galaxies to the cosmic star-formation rate density, the stellar mass build-up of the Universe, the formation of massive early-type galaxies, and properties of DSFGs' star-forming regions. We will also present results related to source clustering and the anisotropies of the background. Finally, we review theoretical attempts over the last decade to understand the origin and evolution of DSFGs in a cosmological context.

In this review, we summarize what has been learned about high- $z$ dusty star-forming galaxies over the past decade, from the original days of ScuBA, the the flourishing, diverse datasets and simulations we have today. This review is organized as follows. In $\S 2$ we summarize basic properties of various galaxy populations that are characterized as DSFGs and the observational programs at submm and far-IR wavelengths. In $\S 3$ we review existing measurements related to the number counts of DSFGs at a variety of wavelengths, including methods for analyzing counts in submm maps to gravitationally lensed counts at the bright-end of submm $/ \mathrm{mm}$ flux densities. In $\S 4$ we review the redshift distributions of DSFGs, how to fit spectral energy distributions to their far-infrared data, and the implied luminosity functions and measurements of the cosmic star formation rate density. The internal physical characterization of DSFGs, including multi-wavelength properties, morphologies, and dynamics are reviewed in $\S 5$. Although $\S 5$ discusses the role of AGN in DSFGs, we note here this review focuses primarily on extreme star-forming galaxies and does not explicitly focus on dusty luminous galaxies for which luminous black holes are thought to dominate the bolometric luminosity. In $\S 6$ we review some of the basic physical properties of individual DSFGs that are studied in detail in the literature. The spatial distribution of DSFGs and galaxy clustering and environmental effects are in $\S 7$. In $\S 8$ and $\S 9$ we review the molecular gas, mainly $\mathrm{CO}$ and dense gas tracers such as $\mathrm{HCN}$, and ionized gas, such as [CII], properties of star-forming galaxies, respectively. § 10 presents a review of theoretical models related to DSFG formation and evolution, from numerical and hydrodynamical simulations to semi-analytical recipes in the literature. We conclude our review with a summary of outstanding scientific questions for future research programs in $\S 11$. When quoting results, as needed, we assume a general cosmological model consistent with Planck data (Planck Collaboration et al. 2013b). We state our initial mass function (IMF) assumptions when appropriate, and throughout, address how changes in the IMF will alter select results. Similarly, when appropriate, we discuss the issue of AGN dust heating and how that impacts estimated star formation rates and the physical interpretation of certain DSFGs. 


\section{Selection of Distant Infrared-Luminous Galaxies}

This section discusses the selection of dusty star-forming galaxies at far-infrared and submillimeter wavelengths. Given past and current survey detection limits, these galaxies are typically prolific star formers that exhibit infraredbased star formation rates as much as a few orders of magnitude above a normal $L_{\star}$ galaxy. Although they sometimes only represent the tip of the iceberg in terms of galaxy mass halos and star formation rates, they shed light on how galaxy formation processes in the early Universe might differ from the Universe today. The detection and selection of these sources is the first step to studying them.

\subsection{Local Infrared-Luminous Galaxies}

Any discussion of the high-redshift infrared-bright galaxy population requires a brief overview of the local Luminous InfraRed Galaxy (LIRG; $\mathrm{L}_{\mathrm{IR}}>10^{11} \mathrm{~L} \odot$ ) population, even though it's not clear whether or not high- $z$ DSFGs closely relate or not. The InfraRed Astronomy Satellite (IRAS; Neugebauer et al., 1984) was responsible for the discovery of these extremely bright extragalactic sources during its short lifetime in 1983 . While close to 250,000 extragalactic sources were logged by IRAS over the entire sky, the subset of 'bright galaxies' became the most wellstudied infrared sources (629 of which make up the 'Revised Bright Galaxy Sample,' or RBGS; Sanders et al., 2003). Further populations of local infrared-bright galaxies were discovered by ISOPHOT aboard the ISO satellite in the mid-1990s (Lemke et al. 1996). The majority of local IRAS-selected and ISO-selected galaxies were infrared galaxies with infrared luminosities within $10^{11}<L_{\mathrm{IR}}<10^{12} \mathrm{~L}_{\odot}$, though a small subset ( $\sim 12$ galaxies) were ultraluminous infrared galaxies, or ULIRGs, with $10^{12}<L_{\mathrm{IR}}<10^{13} \mathrm{~L} \odot$. Careful analysis of these galaxies, from studying their morphological structure in the optical, near-infrared, molecular gas and dust emission, indicated that the majority of systems above $\sim 10^{11.5} \mathrm{~L}_{\odot}$-or above star formation rates $\sim 50 \quad \mathrm{M}_{\odot} \mathrm{yr}^{-1}$-were major mergers of two or more equalmass galaxies (de Jong et al. 1984; Soifer et al. 1984; Lonsdale et al. 1984, Joseph \& Wright 1985, Veilleux et al. 2002, for a review, see Sanders \& Mirabel, (1996).

The observation that high infrared luminosity correlated with high star formation rates and major mergers led to the widely accepted evolutionary picture for extreme luminosity systems first proposed by Sanders et al. (1988). A schematic diagram of this evolutionary picture is shown in Figure 2, here reproduced from Hopkins et al. (2008). This formulation places the LIRG or ULIRG phenomenon at a fixed stage in a larger evolutionary sequence whereby two gas-rich disk galaxies collide and ignite an intense phase of star formation by the rapid compression and cooling of gas. This collision and subsequent star formation triggers the prolific formation of dust particles which, in turn, absorb rest-frame optical and ultraviolet emission from young, blue stars and re-radiates that light at infrared/submillimeter wavelengths during the (U)LIRG phase. At the moment when the two galaxy cores and supermassive black holes (SMBHs) merge during final coalescence, an active galactic nucleus (AGN) is formed and is fed by an accretion disk of material, further fueled by the infall from the outer realms of the galaxy merger (Hopkins et al., 2012ba). The (U)LIRG phase is proposed to be short-lived due to limited gas supply and high star formation rates, and the possible feedback winds generated from the AGN. The resulting galaxy might shine brightly after the ULIRG phase as an obscured or unobscured AGN (or quasar), but eventually, the system lacks gas to form new stars. The galaxy could then be categorized as an 'elliptical galaxy' as it approaches a virialized state from the merger and is characterized by an old stellar population. Significant theoretical progress toward this picture has been made by Hopkins et al. (2005a b. 2006); Kim et al. (2009); Younger et al. (2009a); Teyssier et al. (2010); Hayward et al. (2013c) and others.

While this merger-driven picture of local infrared galaxies seems quite elegant and meaningful, particularly to the formation of the most massive elliptical galaxies in the Universe, local infrared galaxies are very rare relative to 'normal' Milky Way type galaxies and are not thought to contribute substantially to the $z=0$ cosmic star formation rate density. If high- $z$ (U)LIRGs are more common than local (U)LIRGs, does this imply that major mergers dominate cosmic star formation at early times? Or could they be dominated by a different physical evolutionary sequence not yet known? As the wealth of information on high- $z$ infrared galaxies mounts, we are inching closer to answers, but it is critical to recognize that our current high- $z$ studies would be lost without the large body of fundamental work done on local (U)LIRG samples in the decades leading up to the submillimeter galaxy (SMG) era. 
(c) Interaction/"Merger"

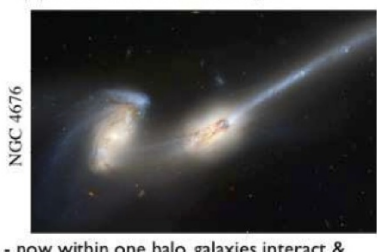

ow within one halo, galaxies interact \& lose angular momentur

SFR starts to increase

- stellar winds dominate feedback - rarely excite QSOs (only special orbits)

(b) "Small Group"

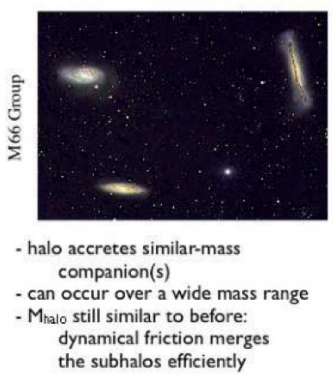

(a) Isolated Disk

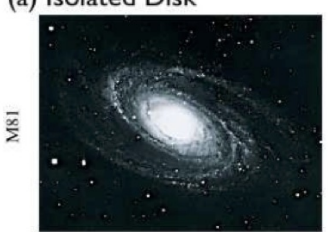

- halo \& disk grow, most stars formed - secular growth builds bars \& pseudobulges - "Seyfert" fueling (AGN with MB $>-23$ ) - cannot redden to the red sequence (d) Coalescence/(U)LIRG

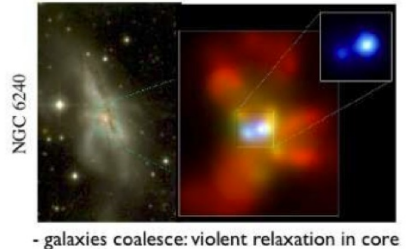

- galaxies coalesce: viol
- gas inflows to center:

starburst \& buried ( $X$-ray) AGN

-starburst dominates luminosity/feedback

but, total stellar mass formed is small (e) "Blowout"

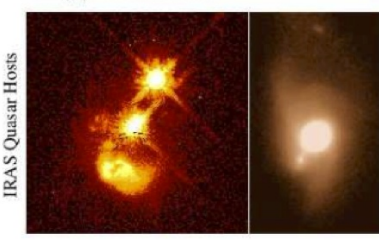

- $\mathrm{BH}$ grows rapidly: briefly dominates luminosity/feedback - remaining dust/gas expelled get reddened (but not Type II) QSO: recent/ongoing SF in high Eddington ratios (f) Quasar

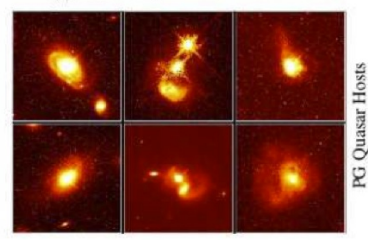

- dust removed: now a "traditional" QSO host morphology difficult to observe: tidal features fade rapidly

(g) Decay/K+A
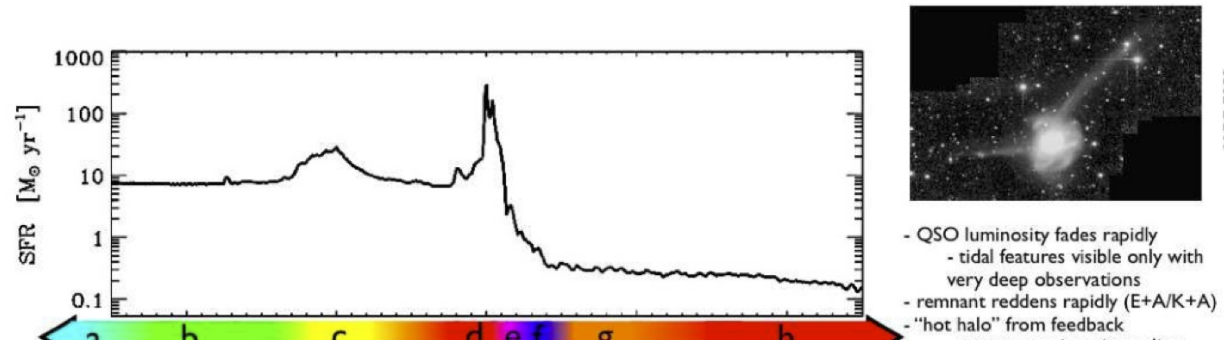

QSO luminosity fades rapidly - tidal features visible only with very deep observations remnant reddens rapidly $(\mathrm{E}+\mathrm{A} / \mathrm{K}+\mathrm{A})$ hot halo" from feedback

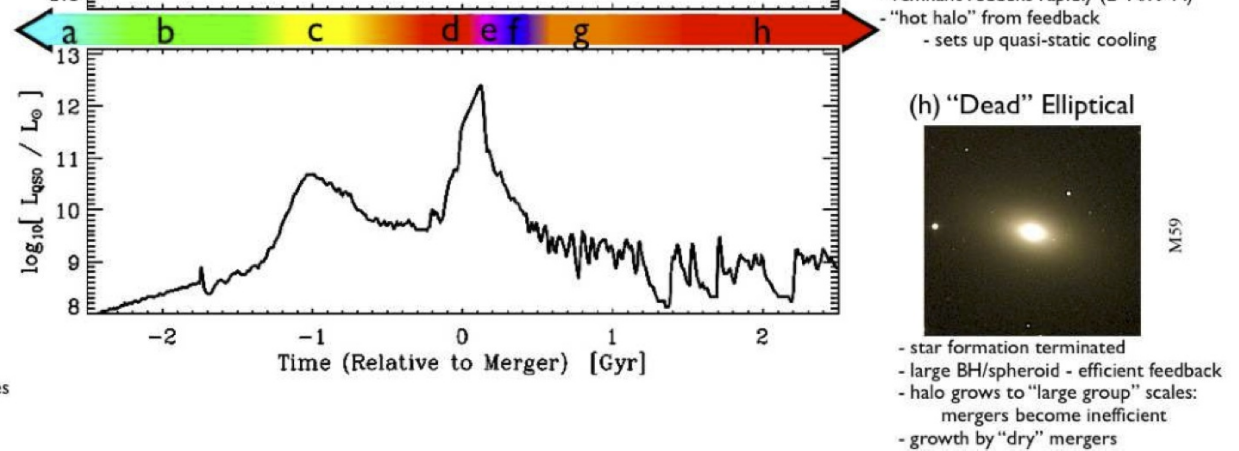

(h) "Dead" Elliptical

- growth by "dry" mergers

Figure 2: A schematic diagram of the evolution of a galaxy undergoing a major merger of gas-rich disks during its lifetime. This figure is reproduced from Hopkins et al. (2008) with permission from the authors and AAS. Image credits: (a) NOAO/AURA/NSF; (b) REU program/NOAO/AURA/NSF; (c) NASA/STScI/ACS Science Team; (d) Optical (left): NASA/STScI/R. P. van der Marel \& J. Gerssen; X-ray (right): NASA/CXC/MPE/S. Komossa et al.; (e) Left: J. Bahcall/M. Disney/NASA; Right: Gemini Observatory/NSF/University of Hawaii Institute for Astronomy; (f) J. Bahcall/M. Disney/NASA; (g) F. Schweizer (CIW/DTM); (h) NOAO/AURA/NSF. 


\subsection{The very negative $K$-correction}

Before the advent of the Submillimeter Common User Bolometric Array (ScuBA) in 1997 the first of several high-sensitivity submillimeter arrays, the number of distant $(z \gtrsim 0.3)$ infrared-luminous galaxies known amounted to only a handful. This included the spectacularly luminous IRAS F10214+4724 with apparent $L_{\mathrm{IR}}=3 \times 10^{14} \mathrm{~L}_{\odot}$ at $z=2.3$ (Rowan-Robinson et al. 1991) and APM 08279+5255 with $L_{\mathrm{IR}}=5 \times 10^{15} \mathrm{~L}_{\odot}$ at $z=3.9$ (Irwin et al. 1998 ), both gravitationally lensed by factors $\gtrsim 10$. They were both so unique and rare that little was known about the population of unlensed dusty galaxies to which they might relate. With submillimeter sensitivities an order of magnitude improved over previous generations of instruments, Scuba ushered in a new era of discovery in the high- $z$ Universe by revealing the unlensed high- $z$ infrared-luminous galaxy population.

Tens to hundreds of luminous sources were detected with Scuba in the first deep-field maps (e.g. Smail et al. 1997, Hughes et al., 1998, Barger et al. 1998), although their properties were hard to study (Ivison et al., 1998) due to the large beamsize of ScuBA's observations ( $\sim 15^{\prime \prime}$ at $850 \mu \mathrm{m}$; at cosmological redshifts, roughly $\left.120 \mathrm{kpc}\right)$ and the difficulty in identifying multi-wavelength counterparts. However, at the time it was already thought that these submillimeter sources would be predominantly located at high-redshift. This is because submillimeter observations of extragalactic sources, particularly those conducted at $\sim 1 \mathrm{~mm}$, benefit from a special trait relating to having a negative $K$-correction.

A $K$-correction is applied to a redshifted object's absolute magnitude (or its flux) to convert from observed-frame to rest-frame. The K-correction depends only on the inferred shape of the galaxy's spectral energy distribution, and is independent of the correction between apparent and absolute magnitudes. The $K$-correction is typically called 'positive' if the flux density decreases with increasing redshift and 'negative' if it increases with redshift (this terminology was built around magnitudes, hence the reversal of positive and negative).

Galaxies' submillimeter emission has a negative $K$-correctior ${ }^{4}$. This is because dust emission in ULIRGs resembles a modified blackbody which peaks at rest-frame wavelengths $\sim 100 \mu \mathrm{m}$, and the long-wavelength portion of the spectrum is the Rayleigh-Jeans regime where $S_{v} \propto v^{2+\beta}$, where $S_{v}$ is the measured flux density (given in units $\propto \mathrm{Jy}$ ) and $\beta$ is the dust emissivity spectral index, discussed more in $\S 5$. Beyond $\sim 3 \mathrm{~mm}$, galaxies' emission is no longer dominated by dust emission, but by a mix of synchrotron and free-free emission, where the $K$-correction is positive.

However, it is not the negative $K$-correction alone which makes extragalactic submillimeter observing special. It's the fact that submillimeter observations have a very negative $K$-correction such that high- $z$ galaxies have roughly constant brightness at submillimeter wavelengths from $z=1-8$. If a galaxy of fixed luminosity $L$ is pushed back in redshift, the observed flux density at a given frequency $v$ diminishes as the luminosity distance increases approximately $\propto(1+z)^{4}$ (since $S_{v}=L_{v} / 4 \pi D_{\mathrm{L}}^{2}$ and $D_{\mathrm{L}}$ can be approximated as $D_{\mathrm{L}} \propto(1+z)^{2}$ at $0.5<z<3$ ). The SED also shifts towards shorter rest-frame wavelengths. In the Rayleigh-Jeans regime, the flux density will behav $\varepsilon^{5}$ like $S_{\nu}(z) \propto$ $v^{2+\beta} / 4 \pi D_{\mathrm{L}}^{2} \propto v_{\text {rest }}^{2+\beta}(1+z)^{2+\beta} /(1+z)^{4} \propto(1+z)^{\beta-2}$. Later we will verify that $\beta=1.5-2.0$ is a reasonable assumption for dusty galaxies, which then leads us to $S_{v}(z)$ is roughly constant. In other words, across the wide range of redshifts for which the Rayleigh-Jeans approximation is applicable ( $z \approx 1-8$ for $850 \mu$ m observations), the observed flux density is roughly constant or might even increase slightly. This is what makes the $K$-correction in the submillimeter very negative.

Figure 3 makes it clear that the submillimeter regime is unique in making the high-redshift Universe readily accessible. This figure highlights the expected change in observed flux density with redshift for a template DSFG SED of fixed luminosity (Pope et al. 2008b) across many observed-frame wavelengths, from the optical ( $i$-band, $0.8 \mu \mathrm{m})$ through the near-IR, mid-IR, far-IR, millimeter, and the radio $(1.4 \mathrm{GHz})$. The $K$-correction at $250<\lambda<500 \mu \mathrm{m}$ is still negative, though is far less dramatic than the very negative $K$-correction at $\sim 1 \mathrm{~mm}$ and the very positive $K$-correction at optical/near-infrared and radio wavelengths which causes a dramatic drop in redshift flux density. Promisingly, the negative $K$-correction at $850 \mu \mathrm{m}-2 \mathrm{~mm}$ implies that higher redshift galaxies will actually be easier to detect than their low-redshift counterparts, making the motivation for the original $850 \mu \mathrm{m}$ ScuBa surveys quite clear.

\footnotetext{
${ }^{3}$ A single-element bolometer named UKT14 actually predated ScuBA on the JCMT, and managed to detect several high- $z$ radio quasars to $\sim 4$ mJy sensitivity (Hughes et al. 1997).

${ }^{4}$ Note that heavily-absorbed soft X-ray sources and self-absorbed radio sources also have negative $K$-corrections, although not as steep as the submillimeter.

${ }^{5}$ Note that at slightly higher redshifts $(z>3)$, where $D_{\mathrm{L}} \propto(1+z)^{1.5}$, this becomes $S_{v}(z) \propto(1+z)^{\beta-1.5}$
} 


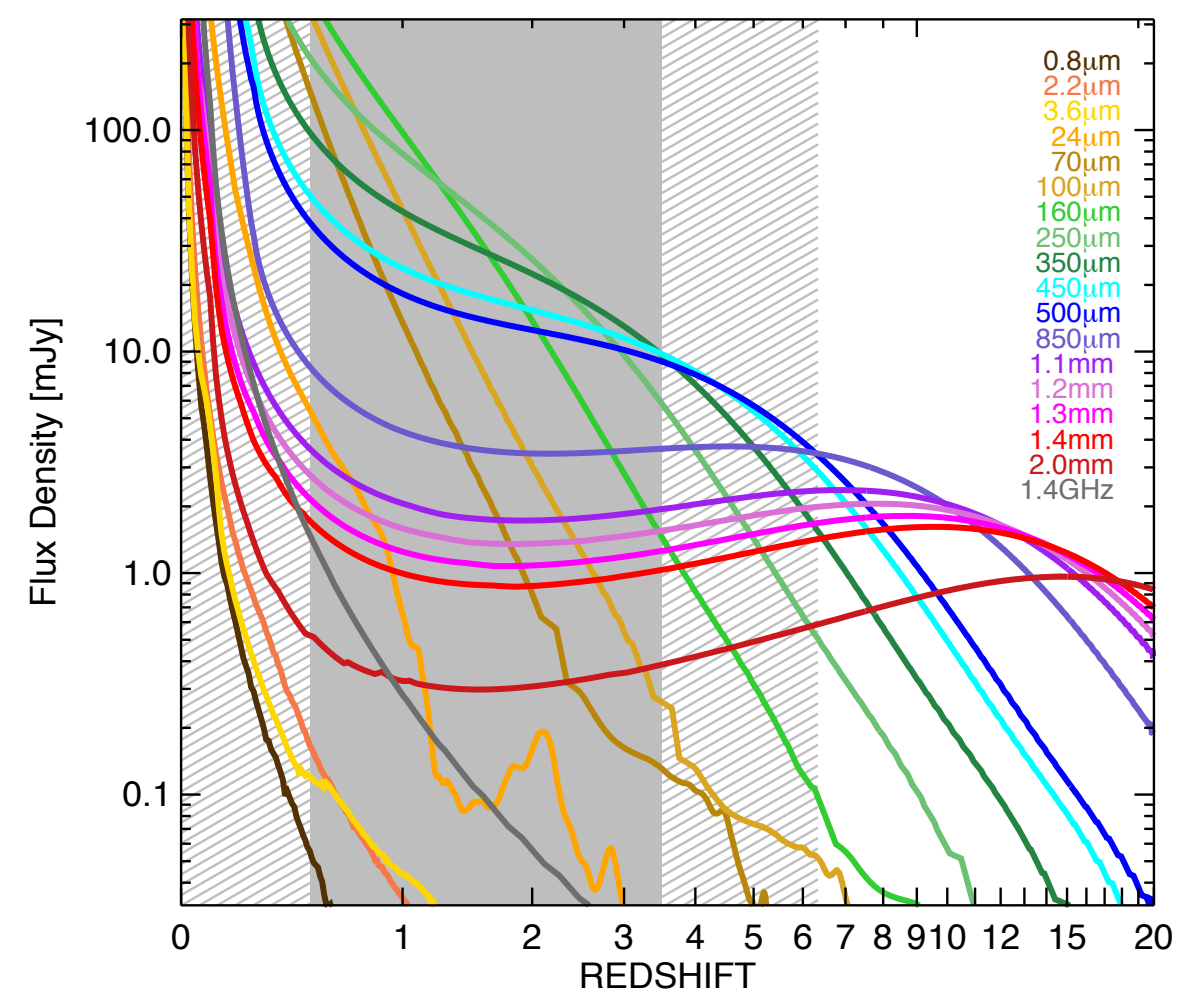

Figure 3: The observed flux densities for a typical $10^{12.5} \mathrm{~L} \odot$ infrared-luminous galaxy as a function of redshift. This illustrates the nearly-unchanged flux densities which DSFGs have in the $\sim 1 \mathrm{~mm}$ bands across a wide range of epochs $1 \lesssim z \lesssim 10$. The Pope et al. (2008b) composite SMG SED is used to generate the flux evolution, or $K$ - correction, at wavelengths $24 \mu \mathrm{m}-2 \mathrm{~mm}$, and at $1.4 \mathrm{GHz}$. An Arp $220 \mathrm{SED}$, adjusted to have a luminosity of $10^{12.5} \mathrm{~L}_{\odot}$, is used to generate optical and near-IR $K$-corrections. The peak epoch of DSFG discovery is highlighted in solid gray, from $0.5<z<3.5$, where redshift follow-up and characterization has been efficient, particularly for $850 \mu \mathrm{m}$-selected SMGs. The shaded areas, at $0<z<0.5$ and $3.5<z<6.4$ highlight redshift space where $\log \left(L_{\mathrm{IR}}\right) \approx 12.5$ galaxies are perceived to be rare. At $0<z<0.5$ this is because they have a very low volume density, attributable to cosmic downsizing (Cowie et al. 1996), whereas at high-redshift $(3.5<z<6.4)$ it is unknown whether or not the volume density of DSFGs is much lower since galaxies are much more difficult to spectroscopically confirm. At present, no purely star-forming DSFG at redshifts above $z \approx 6.4$ has been discovered. 


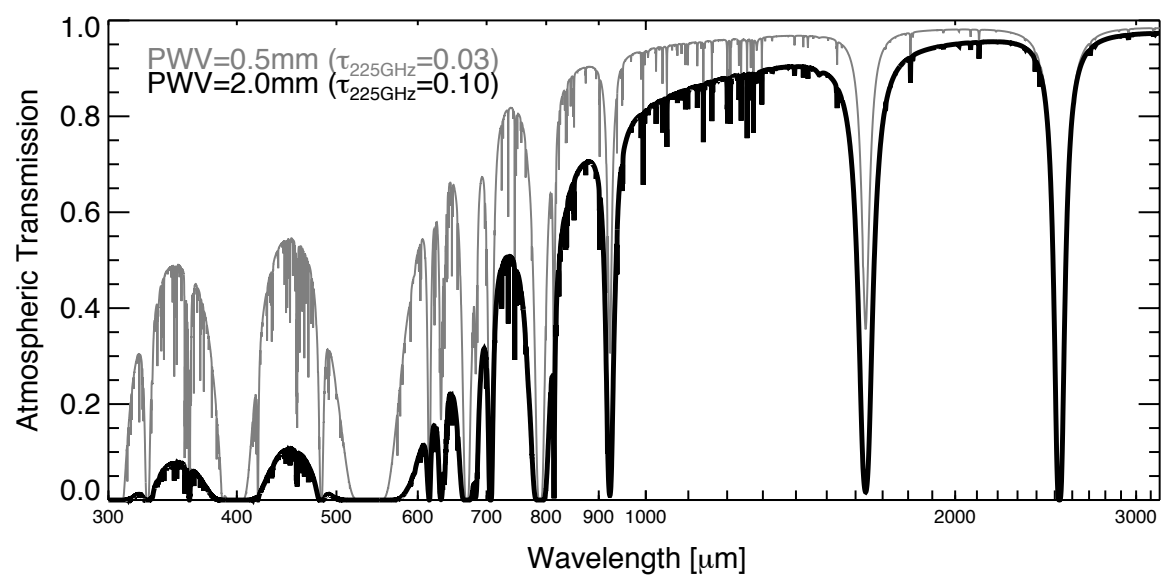

Figure 4: The atmospheric transmission as seen from Mauna Kea, Hawai'i under two weather conditions with precipitable water vapor (PWV) levels of $0.5 \mathrm{~mm}$ and $2.0 \mathrm{~mm}$. The PWV is the amount of water vapor in the atmosphere integrated from the top of the atmosphere down to the telescope. In the submillimeter regime $(\lambda<1 \mathrm{~mm})$ the atmosphere is very opaque, even under the driest weather conditions. Some natural atmospheric windows occur at $350 \mu \mathrm{m}(860 \mathrm{GHz}), 450 \mu \mathrm{m}(670 \mathrm{GHz}), 770 \mu \mathrm{m}(390 \mathrm{GHz}), 870 \mu \mathrm{m}(345 \mathrm{GHz}), 1.2 \mathrm{~mm}(250 \mathrm{GHz})$ and $2 \mathrm{~mm}(150 \mathrm{GHz})$. An optical depth of $\tau \leq 0.10$ only occurs $\sim 10 \%$ of nights on Mauna Kea, and the truly exceptional $\tau \leq 0.03$ conditions are extremely rare, only happening a few days out of the year. Note that a few more bands occur $\sim 200 \mu \mathrm{m}$ but require even drier conditions to observe from the ground.

\subsection{Dusty Galaxy Selection from $\sim 8-2000 \mu m$}

Infrared galaxy selection has largely been limited by the opacity of the Earth's atmosphere or, alternatively, the limited instrumentation which we are able to send to space. Beyond the local samples discovered by IRASand ISO, high-redshift far infrared galaxy searches have primarily focused on the $345 \mathrm{GHz}$ and $230 \mathrm{GHz}$ transmission 'windows' with recent expansions to other wavelengths due to instrumental improvements. The atmosphere's transmission at different infrared-to-radio wavelengths is shown in Figure 4 under two different water column densities, illustrating all of the naturally occurring submm and $\mathrm{mm}$ atmospheric windows.

Understanding the atmospheric windows and the initial limitations of infrared bolometer array technology is critical to understanding the selection of the first high- $z$ dusty starbursts. Below, in $\$ 2.3 .1$, we describe the facilities used to discover distant infrared galaxy populations; the order is roughly chronological, as the populations were first observed and described. Table 1 lists some basic properties of the facilities in the same chronological order.

While reviewing the facilities by which high- $z$ DSFGs were discovered, the reader should keep in mind the underlying shape of a dusty starburst's spectral energy distribution (SED) and how that behaves with redshift and wavelength, as shown in Figure 3 Figure 5 illustrates a characteristic SED of a $z=2 L_{\mathrm{IR}}=3 \times 10^{12} \mathrm{~L}_{\odot}$ galaxy (Pope et al., 2008b) in relation to some well known local ULIRGs (Arp 220, Mrk 231, templates provided by Polletta et al. 2007). Over-plotted on this SED are characteristic detection limits for the deepest mid-IR, submillimeter and millimeter facilities available. When reviewing the respective facilities, it is important to keep in mind the impact of the selection wavelength on the various DSFG populations.

\subsubsection{Facilities and Instruments Discovering high-z DSFGs}

Submillimeter Common User Bolometric Array [ScUBA], (1997-2005)

Scuba was commissioned on the James Clerk Maxwell Telescope (JCMT) atop Mauna Kea in Hawai'i in 1997 and operated simultaneously at $450 \mu \mathrm{m}$ and $850 \mu \mathrm{m}$ (in the $670 \mathrm{GHz}$ and $345 \mathrm{GHz}$ atmospheric windows). Although Scuba was not the first bolometer-array in use, it had unrivaled sensitivity at the time with a fairly substantial field of view $\left(\sim 5 \operatorname{arcmin}^{2}\right)$. While the $450 \mu \mathrm{m}$ sensitivity was significantly worse than the $850 \mu \mathrm{m}$ sensitivity $\left(\sigma_{450} \approx 30 \times \sigma_{850}\right)$, so not as constraining, the $850 \mu \mathrm{m}$ arrays could reach $\sim 2 \mathrm{mJy}$ sensitivity with 6 hours of integration, easily detecting $10^{12.5} \mathrm{~L}_{\odot}$ galaxies out to $z \approx 8$. The first few submillimeter deep-field maps which were published (e.g. Smail et al., 1997; Barger et al., 1998; Hughes et al., 1998) detected several galaxies at $850 \mu \mathrm{m}$ within several square arcminutes. A 


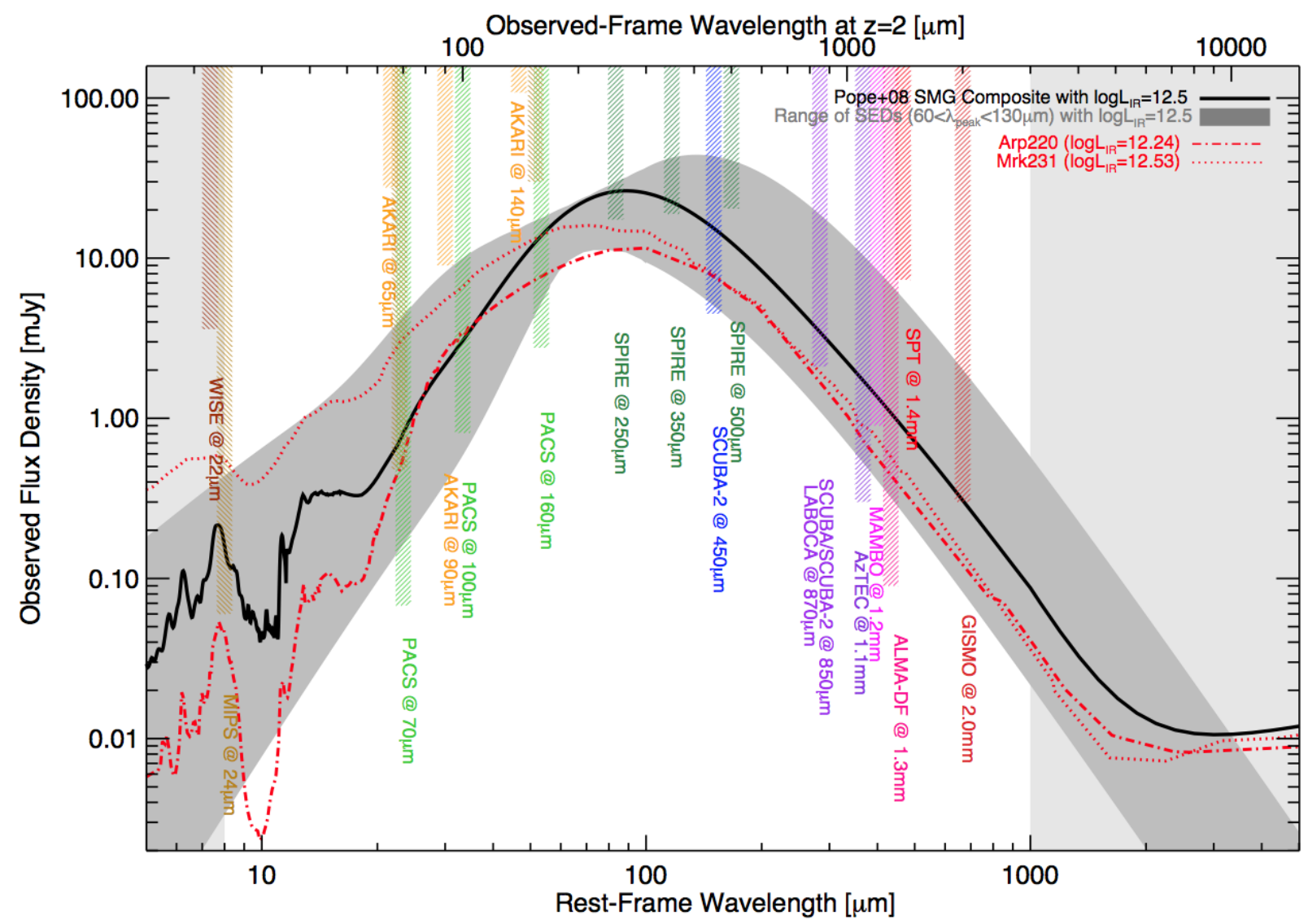

Figure 5: A schematic spectral energy distribution for a dusty star forming galaxy at $z=2$. A representative SED for a $\sim 500 \mathrm{M}_{\odot} \mathrm{yr}^{-1} \mathrm{SMG}$ (black; Pope et al. 2008b) is overplotted on a gray band representing the plausible range of SED types for a galaxy of fixed infrared luminosity $10^{12.5} \mathrm{~L} \odot$ with peak SED wavelengths ranging from $70-130 \mu \mathrm{m}$ (corresponding to temperatures $\sim 30-58 \mathrm{~K}$ ). Model SEDs for local galaxies Arp 220 and Mrk 231 are also overplotted in red dot-dashed and dotted lines. Vertical shaded bands represent the sensitivities of different far-infrared observatories; the minimum flux density value of each colored band represents the $3 \sigma$ detection limit for the given instrument. From short wavelengths to long, we include WISE at $22 \mu \mathrm{m}$, MIPS at $24 \mu \mathrm{m}, 70 \mu \mathrm{m}$, and $160 \mu \mathrm{m}$, AKARI at $65 \mu \mathrm{m}, 90 \mu \mathrm{m}$, and $140 \mu \mathrm{m}$, PACs at $70 \mu \mathrm{m}, 100 \mu \mathrm{m}$, and $160 \mu \mathrm{m}$, Sire at $250 \mu \mathrm{m}, 350 \mu \mathrm{m}, 500 \mu \mathrm{m}$, ScuBa- 2 at $450 \mu \mathrm{m}$ and $850 \mu \mathrm{m}$, ScuBa at $850 \mu \mathrm{m}$ and LaBoca at $870 \mu \mathrm{m}$, AzTEC at $1.1 \mathrm{~mm}$, MaMBo at $1.2 \mathrm{~mm}$, the ALMA Deep Field pointing at $1.3 \mathrm{~mm}$, the South Pole Telescope at $1.4 \mathrm{~mm}$, and GISMO at $2.0 \mathrm{~mm}$. References given in the text and Table 1 
Table 1: Single-Dish Facilities used for DSFG discovery

\begin{tabular}{|c|c|c|c|c|c|c|}
\hline Instrument & Telescope & $\begin{array}{c}\text { Years } \\
\text { Active }\end{array}$ & $\begin{array}{c}\text { Beam- } \\
\text { size }\end{array}$ & $\begin{array}{l}\text { Wave- } \\
\text { bands }\end{array}$ & $\begin{array}{c}\text { Deepest } \\
\text { Sensitivity }\end{array}$ & $\begin{array}{l}\text { Instrument } \\
\text { Reference }\end{array}$ \\
\hline IRAS & IRAS & 1983 & $\begin{array}{l}0.5^{\prime} \dagger \\
0.5^{\prime} \dagger \\
1.0^{\prime} \dagger \\
2.0^{\prime} \dagger\end{array}$ & $\begin{array}{c}12 \mu \mathrm{m} \\
25 \mu \mathrm{m} \\
60 \mu \mathrm{m} \\
100 \mu \mathrm{m}\end{array}$ & $\begin{array}{l}0.4 \mathrm{Jy} \\
0.5 \mathrm{Jy} \\
0.6 \mathrm{Jy} \\
1.0 \mathrm{Jy}\end{array}$ & Neugebauer et al. (1984) \\
\hline ISOPHOT & ISO & 1995-1998 & $\begin{array}{l}7^{\prime \prime} \\
11^{\prime \prime} \\
44^{\prime \prime} \\
79^{\prime \prime}\end{array}$ & $\begin{array}{c}15 \mu \mathrm{m} \\
25 \mu \mathrm{m} \\
100 \mu \mathrm{m} \\
180 \mu \mathrm{m}\end{array}$ & $\begin{array}{c}14 \mathrm{mJy} \\
90 \mathrm{mJy} \\
250 \mathrm{mJy} \\
800 \mathrm{mJy}\end{array}$ & Lemke et al. (1996) \\
\hline ScUBA & JCMT & $1997-2005$ & $\begin{array}{c}15^{\prime \prime} \\
7^{\prime \prime}\end{array}$ & $\begin{array}{l}850 \mu \mathrm{m} \\
450 \mu \mathrm{m}\end{array}$ & $\begin{array}{c}1 \mathrm{mJy} \\
30 \mathrm{mJy}\end{array}$ & Holland et al. (1999) \\
\hline Мамво-1 & IRAM $30 \mathrm{~m}$ & 1998-2002 & $11^{\prime \prime}$ & $1.2 \mathrm{~mm}$ & $0.8 \mathrm{mJy}$ & Kreysa et al. (1999) \\
\hline Мамво-2 & IRAM $30 \mathrm{~m}$ & $2002-2011$ & $11^{\prime \prime}$ & $1.2 \mathrm{~mm}$ & $0.8 \mathrm{mJy}$ & \\
\hline SHARC-II & $\mathrm{CSO}$ & $2002-2013$ & $9^{\prime \prime}$ & $350 \mu \mathrm{m}$ & $5 \mathrm{mJy}$ & Dowell et al. (2003) \\
\hline BOLOCAM & $\mathrm{CSO}$ & $2002-2013$ & $30^{\prime \prime}$ & $1.1 \mathrm{~mm}$ & $1.9 \mathrm{mJy}$ & Laurent et al. $(\overline{2005})$ \\
\hline MIPS & Spitzer & $2003-2009$ & $\begin{array}{c}6^{\prime \prime} \\
17^{\prime \prime} \\
38^{\prime \prime}\end{array}$ & $\begin{array}{c}24 \mu \mathrm{m} \\
70 \mu \mathrm{m} \\
160 \mu \mathrm{m}\end{array}$ & $\begin{array}{c}7 \mu \mathrm{Jy} \\
0.8 \mathrm{mJy} \\
9.4 \mathrm{mJy}\end{array}$ & Rieke et al. $(\overline{2004})$ \\
\hline FIS & AKARI & $2006-2011$ & $\begin{array}{l}26^{\prime \prime} \\
36^{\prime \prime} \\
56^{\prime \prime} \\
64^{\prime \prime}\end{array}$ & $\begin{array}{c}65 \mu \mathrm{m} \\
90 \mu \mathrm{m} \\
140 \mu \mathrm{m} \\
160 \mu \mathrm{m}\end{array}$ & $\begin{array}{c}9.2 \mathrm{mJy} \\
3 \mathrm{mJy} \\
36 \mathrm{mJy} \\
120 \mathrm{mJy}\end{array}$ & Murakami et al. 2007) \\
\hline BLAST & BLAST & 2008 & $\begin{array}{l}33^{\prime \prime} \\
46^{\prime \prime} \\
66^{\prime \prime}\end{array}$ & $\begin{array}{l}250 \mu \mathrm{m} \\
350 \mu \mathrm{m} \\
500 \mu \mathrm{m}\end{array}$ & $\begin{array}{l}18 \mathrm{mJy} \\
13 \mathrm{mJy} \\
12 \mathrm{mJy}\end{array}$ & Devlin et al. (2009) \\
\hline SPIRE & Herschel & $2009-2013$ & $\begin{array}{l}18^{\prime \prime} \\
26^{\prime \prime} \\
36^{\prime \prime}\end{array}$ & $\begin{array}{l}250 \mu \mathrm{m} \\
350 \mu \mathrm{m} \\
500 \mu \mathrm{m}\end{array}$ & $\begin{array}{l}5.8 \mathrm{mJy} \\
6.3 \mathrm{mJy} \\
6.8 \mathrm{mJy}\end{array}$ & Griffin et al. (2010) \\
\hline Pacs & Herschel & $2009-2013$ & $\begin{array}{l}12^{\prime \prime} \\
7^{\prime \prime} \\
5^{\prime \prime}\end{array}$ & $\begin{array}{c}160 \mu \mathrm{m} \\
100 \mu \mathrm{m} \\
70 \mu \mathrm{m}\end{array}$ & $\begin{array}{l}0.9 \mathrm{mJy} \\
0.4 \mathrm{mJy} \\
0.4 \mathrm{mJy}\end{array}$ & Poglitsch et al. (2010) \\
\hline WISE & WISE & 2009-2011 & $\begin{array}{c}7^{\prime \prime} \\
12^{\prime \prime}\end{array}$ & $\begin{array}{l}12 \mu \mathrm{m} \\
22 \mu \mathrm{m}\end{array}$ & $\begin{array}{l}0.2 \mathrm{mJy} \\
1.2 \mathrm{mJy}\end{array}$ & Wright et al. (2010) \\
\hline AzTEC & $\begin{array}{c}\text { JCMT } \\
\text { ASTE } \\
\text { LMT }(32 \mathrm{~m}) \\
\text { LMT }(50 \mathrm{~m})\end{array}$ & $\begin{array}{c}2005-2006 \\
2007-2008 \\
2011-2015 \\
2015-\end{array}$ & $\begin{array}{c}19^{\prime \prime} \\
29^{\prime \prime} \\
9^{\prime \prime} \\
6^{\prime \prime}\end{array}$ & $\begin{array}{l}1.1 \mathrm{~mm} \\
1.1 \mathrm{~mm} \\
1.1 \mathrm{~mm} \\
1.1 \mathrm{~mm}\end{array}$ & $\begin{array}{c}1.5 \mathrm{mJy} \\
1.2 \mathrm{mJy} \\
\ldots \\
\ldots\end{array}$ & Wilson et al. (2008) \\
\hline LABOCA & APEX & $2006-$ & $19^{\prime \prime}$ & $870 \mu \mathrm{m}$ & $1.2 \mathrm{mJy}$ & Siringo et al. (2009) \\
\hline ACT & ACT & $2007-$ & $\begin{array}{l}54^{\prime \prime} \\
69^{\prime \prime} \\
98^{\prime \prime}\end{array}$ & $\begin{array}{l}1.1 \mathrm{~mm} \\
1.4 \mathrm{~mm} \\
2.0 \mathrm{~mm}\end{array}$ & $\begin{array}{l}6.0 \mathrm{mJy} \\
3.7 \mathrm{mJy} \\
2.3 \mathrm{mJy}\end{array}$ & Swetz et al. $(\overline{2011)}$ \\
\hline SPT & SPT & $2008-$ & $\begin{array}{l}69^{\prime \prime} \\
63^{\prime \prime}\end{array}$ & $\begin{array}{l}2.0 \mathrm{~mm} \\
1.4 \mathrm{~mm}\end{array}$ & $\begin{array}{l}1.3 \mathrm{mJy} \\
3.4 \mathrm{mJy}\end{array}$ & $\begin{array}{c}\text { Mocanu et al. }(2013) ; \\
\text { Vieira et al. }(2010)\end{array}$ \\
\hline SABOCA & APEX & $2009-$ & $8^{\prime \prime}$ & $350 \mu \mathrm{m}$ & $30 \mathrm{mJy}$ & Siringo et al. $(\overline{2010})$ \\
\hline GISMO & IRAM 30m & $2011-$ & $24^{\prime \prime}$ & $2.0 \mathrm{~mm}$ & $0.1 \mathrm{mJy}$ & Staguhn et al. $(\overline{2012})$ \\
\hline ScubA-2 & JCMT & $2011-$ & $\begin{array}{c}15^{\prime \prime} \\
7^{\prime \prime}\end{array}$ & $\begin{array}{l}850 \mu \mathrm{m} \\
450 \mu \mathrm{m}\end{array}$ & $\begin{array}{l}0.7 \mathrm{mJy} \\
1.7 \mathrm{mJy}\end{array}$ & $\overline{\text { Holland et al. }}(\overline{2013})$ \\
\hline
\end{tabular}

$\lceil$ The IRAS detector's pixels were much larger than the beamsize. 
was soon shown (e.g. Ivison et al., 1998), these galaxies sit predominantly at high- $z$ (due to the anticipated benefit of the negative $K$-correction), the detection of these galaxies was enough to confirm that there had to be strong evolution in the cosmic star formation rate density, or infrared luminosity density, out to high-z. Put another way, if the density of (U)LIRGs at $z \sim 1-2$ mirrored the local density, then the limited volumes probed by the original ScuBA surveys would not have been large enough to detect a single source.

While the detection of Scuba galaxies provided an exciting confirmation of an evolving Universe, follow-up on individual galaxies was arduous given the large $15^{\prime \prime}$ beamsize. At first, attempts to use deep optical data provided lengthy lists of multiple candidate counterparts for every submillimeter source (Smail et al., 1998). Although efforts to follow-up these galaxies with spectroscopy were able to obtain redshifts (Barger et al. 1999), it remained uncertain whether or not these redshifts corresponded directly to the source of submillimeter emission. Later, the realization that these submillimeter sources should also be faint $\mu$ Jy radio galaxies (Ivison et al., 1998, 2000, Smail et al., 2000) by virtue of the FIR/radio correlation seen in local starburst galaxies (see $\$ 5.12$ ), lead to a breakthrough in SMG counterpart identification. Deep $\mu \mathrm{Jy}$ radio data were obtained with the Very Large Array (VLA) at $1.4 \mathrm{GHz}$ (e.g. Ivison et al. 2002$)$ at substantially higher resolution $\left(\sim 1^{\prime \prime}\right)$ than the submillimeter maps. Since radio sources are far more rare on the sky than optical sources, and there would typically only be one radio galaxy within the searchable beamsize of SCUBA, the precise positions of single $\mu \mathrm{Jy}$ radio sources provided the missing link necessary to characterize SMGs. The process of SMGs' redshift follow-up and characterization is discussed again in more detail in $\S 4$

The results of the redshift follow-up effort on $850 \mu \mathrm{m}$ SMGs revealed a population that, indeed, sat at high redshifts. The median radio-identified SMG had a redshift of $z \approx 2.2$ (Chapman et al., 2004a, 2005) with far-infrared luminosities $>10^{12.5} \mathrm{~L}_{\odot}$ and star formation rates $>500 \mathrm{M}_{\odot} \mathrm{yr}^{-1}$. By 2006, about 75 SMGs had confirmed spectroscopic redshifts (Swinbank et al., 2004, Chapman et al., 2005) and many were being followed up at other wavelengths to understand their comprehensive energy budget and evolutionary mechanisms. We describe the follow-up physical characterization of ScuBA-selected SMGs more in $\S 5$. We highly recommend the Blain et al. (2002) review for a thorough summary of SMG science in the earlier days of Scuba. Scuba was decommissioned in 2005 to make way for the second generation instrument for the JCMT, ScUBA-2.

MAx-planck Millimeter BOlometer [MАмво], (2002-2011)

Мамво represents a family of bolometer arrays designed and built at the Max-Planck-Institut für Radioastronomie and installed on the Institut de Radioastronomie Millimétrique (IRAM) $30 \mathrm{~m}$ Telescope at Pico Veleta in southern Spain (Kreysa et al., 1999). After a few prototypes, the first generation of Maмво (known as "Mамво-1") was a 37 channel array used from 1998-2002 until the development of a second generation 117 channel array ("MAмво-2") installed on the $30 \mathrm{~m}$ in early 2002. The latter was far more sensitive and enabled the deep mapping of blank fields over $\sim 4 \operatorname{arcmin}^{2}$ similar to ScuBA.

Some of the deeper MАмво blank-field pointings cover $150 \operatorname{arcmin}^{2}$ both in the Elais-N2 and Lockman Hole North fields to $0.8 \mathrm{mJy}$ RMS (Greve et al., 2004), $400 \mathrm{arcmin}^{2}$ to $1 \mathrm{mJy}$ RMS in COSMOS (Bertoldi et al. 2007), and $287 \mathrm{arcmin}^{2}$ to $0.7 \mathrm{mJy}$ in the GOODS-N field (Greve et al., 2008). An updated an expanded version of the Lockman Hole North map has a $0.75 \mathrm{mJy}$ RMS over $566 \operatorname{arcmin}^{2}$ (Lindner et al., 2011).

Submillimeter High Angular Resolution Camera-II [SHARc-II], 2002-2013

The SHARC-II camera operated at $350 \mu \mathrm{m}$ and at $450 \mu \mathrm{m}$ at the Caltech Submillimeter Observatory (Dowell et al., 2003) on Mauna Kea and was used extensively for far-infrared follow-up of Scuba submillimeter-selected galaxies at $350 \mu \mathrm{m}$ with a $2.3 \mathrm{arcmin}^{2}$ field of view and $9^{\prime \prime}$ beamsize. Since the atmospheric opacity is quite high at $350 \mu \mathrm{m}$ making observations only accessible in the driest weather conditions, SHARc-II was not used readily for blank-field mapping. Kovács et al. (2006) and Coppin et al. (2008a) used SHARc-II to follow-up 850 $\mu$ m-detected SMGs to further constrain their SEDs near the peak of their modified blackbody emission.

BOLOmeter CAMera [Bolocam], 2002-2013

The Bolocam instrument is designed for observations at $1.1 \mathrm{~mm}$ and $2.1 \mathrm{~mm}$ at the Caltech Submillimeter Observatory. Observations were not done simultaneously at both wavelengths, and the only deep field survey work done with Bolocam was done at $1.1 \mathrm{~mm}$ with a 30" FWHM beam (Laurent et al. 2005). Observations at $2.1 \mathrm{~mm}$ were motivated by searches for clusters via the Sunyaev-Zeldovich effect. A deep $1.1 \mathrm{~mm}$ BoLocam map of the COSMOS field exists and overlaps significantly with the AzTEC $1.1 \mathrm{~mm}$ COSMOS pointings.

Multiband Imaging Photometer for Spitzer [MIPS], 2003-2009 
MIPS aboard the Spitzer Space Telescope (formerly known as the Space Infrared Telescope Facility, SIRTF) has been fundamental in the detection of distant galaxies at mid-infrared wavelengths (Rieke et al., 2004). MIPS had detector arrays operating at $24 \mu \mathrm{m}, 70 \mu \mathrm{m}$, and $160 \mu \mathrm{m}$ with beamsizes $6^{\prime \prime}, 18^{\prime \prime}$ and $41^{\prime \prime}$, respectively. The $24 \mu \mathrm{m}$ channel was the most sensitive and widely used; $70 \mu \mathrm{m}$ was also used for some deep field pointings, while the $160 \mu \mathrm{m}$ channel was the least sensitive. MIPS completed a series of large legacy mapping programs over several square degrees at $24 \mu \mathrm{m}$ in deep extragalactic fields (Dickinson et al., 2003, Lonsdale et al., 2003; Egami et al., 2004, Le Floc'h et al., 2004; Dunlop et al., 2007, Sanders et al., 2007, Dickinson et al., in preparation, Chary et al., in preparation).

MIPS provided the first look at the population of mid-infrared $(\sim 20-70 \mu \mathrm{m})$ luminous galaxies in the distant Universe; the work complemented the submillimeter mapping work done at $\sim 850 \mu \mathrm{m}$ and was well suited for characterizing galaxies at $z<2$. Not only was the MIPS $24 \mu \mathrm{m}$ beamsize significantly smaller than the bolometer arrays' beamsize, but the MIPS maps covered much larger areas on the sky, providing statistically significant populations of dusty galaxies.

Selection of galaxies at $24 \mu \mathrm{m}$ is a bit more complex than selection in the submillimeter on the Rayleigh-Jeans portion of the dusty modified blackbody. The rest-frame $24 \mu \mathrm{m}$ emission of a dusty starburst is dominated by hot dust emission $(\sim 100-200 \mathrm{~K})$ and not the canonical cold $(\sim 30-50 \mathrm{~K})$, diffuse dust dominating the bulk of infrared emission at longer wavelengths. This hot dust could emanate from more compact star-forming regions, or 'clumps' within galaxies or it could emanate from hot, dusty tori surrounding black hole accretion disks at the galaxies' center. At redshifts $z \sim 1-2$, the observed $24 \mu \mathrm{m}$ band could be dominated by emission features generated by heavier dust grains, in particular Polycyclic Aromatic Hydrocarbons (PAHs; Lagache et al. 2004) which are associated with starforming regions (see more in $\S 5.7$ ). Although the physical mechanisms driving observed $24 \mu \mathrm{m}$ emission are varied and complex, there is no doubt that the emission is dust-generated.

A key population identified with Spitzer MIPS are $24 \mu \mathrm{m}$ sources (Yan et al., 2004a b;, Sajina et al., 2008, Donley et al., 2010, Zamojski et al., 2011; Sajina et al., 2012), and the subset of this population, Dust Obscured Galaxies (DOGs Dey et al. 2008). DOG selection requires $24 \mu \mathrm{m}$ emission and a very red color between optical $R$-band and $24 \mu \mathrm{m}$. The DOG population (defined formally in the glossary, $\S 12$ ), is representative of the family of mid-infrared bright dusty galaxies, ranging from pure starbursts, to obscured AGN-dominated sources, to very luminous PAH emitters.

\section{Far-Infrared Surveyor [FIS], 2006-2011}

The Far-Infrared Surveyor on board the $68.5 \mathrm{~cm}$ AKARI telescope (formerly known as the ASTRO-F satellite; Murakami et al., 2007, Kawada et al., 2007) imaged the sky at $65 \mu \mathrm{m}, 90 \mu \mathrm{m}, 140 \mu \mathrm{m}$, and $160 \mu \mathrm{m}$. Similar to the original IRAS survey, FIS conducted an all-sky far-infrared survey (Yamamura et al., 2010) at relatively shallow depths (i.e. insufficient to detect high- $z$ unlensed galaxies) but conducted a few deep field pointings, including the $A K A R I$ deep field South (ADF-S) covering $\sim 12 \mathrm{deg}^{2}$ (Clements et al., 2011). The ADF-S hosts several tens of bright $90 \mu \mathrm{m}$ galaxies (the $90 \mu \mathrm{m}$ channel being the most sensitive) yet to be characterized in detail; a number of spectroscopic redshifts for the galaxies have been compiled in Sedgwick et al. (2011). The AKARI observatory also hosted the InfraRed Camera (IRC; Onaka et al., 2007) operated at shorter wavelengths, from 2.4-24 $\mu$ m, yet was not focused on deep-field extragalactic work as Spitzer IRAC and MIPS fulfilled that role.

\section{Balloon-borne Large Aperture Submillimeter Telescope [BLAST], 2006-2007}

BLAST provided a unique look into the 250-500 $\mu \mathrm{m}$ sky (Devlin et al.,2009) in advance of SPIRE on the Herschel Space Observatory. Since the sky is virtually opaque at these wavelengths, the BLAST detector was launched on a balloon to rise above most of the Earth's atmosphere on a number of scheduled flights in the Arctic and Antarctic circles. BLAST's scientific flights took place in 2005 (launched from Esrange, Sweden) and 2006-2007 (launched from McMurdo, Antarctica). The latter flight resulted in near-destruction of the telescope but total recovery of the data. In that flight, BLAST conducted the first deep extragalactic surveys at 250-500 $\mu \mathrm{m}$, one covering $9 \mathrm{deg}^{2}$ encompassing $^{2}$ the Extended Chandra Deep Field South (ECDF-S Devlin et al. 2009) and another $8 \mathrm{deg}^{2}$ near the south ecliptic pole. While BLAST discovered many individual FIR-luminous galaxies not yet previously discovered (Dunlop et al. 2010, Chapin et al., 2010), its large beamsize and large area coverage were best suited for measurements of the CIB. More details on BLAST's CIB results and clustering measurements are given in $\S 7$ A documentary film entitled BLAST! recounts the drama of the BLAST launches.

Spectral and Photometric Imaging Receiver [SPIRE], 2009-2013 
The SPIRE instrument was launched aboard the Herschel Space Observatory in May of 2009 and operated until April 2013. SPIRE consisted of both a spectrometer and an imaging photometer operating in three wavebands simultaneously at $250 \mu \mathrm{m}, 350 \mu \mathrm{m}$, and $500 \mu \mathrm{m}$. The photometer was used primarily for mapping large areas of sky under the Herschel Multitiered Extragalactic Survey (HerMES; Oliver et al. 2012) and the Herschel-ATLAS Survey (Eales et al. 2010) to varying depths. The beamsize of $250 \mu \mathrm{m}, 350 \mu \mathrm{m}$, and $500 \mu \mathrm{m}$ observations was $18^{\prime \prime}, 26^{\prime \prime}$ and $36^{\prime \prime}$, respectively with a confusion limit of $5.8 \mathrm{mJy}, 6.3 \mathrm{mJy}$ and $6.8 \mathrm{mJy}$, respectively. SPIRE has been very useful for constraining measurements of the CIB and discovering rare, isolated bright far-IR sources which have sometimes been found to be very distant lensed submillimeter galaxies, many of which are described throughout this review.

Photodetector Array Camera \& Spectrometer [Pacs], 2009-2013

The PACs instrument, launched aboard the Herschel Space Observatory along with SPIRE, consisted of both an integral field spectrometer and imaging photometer. Although less commonly used for high- $z$ submillimeter sources, the spectrometer provided spectral coverage from $57-210 \mu \mathrm{m}$ and was very valuable for identifying rarer species of gas emission in nearby ULIRGs (e.g. van der Werf et al., 2010). The imaging photometer provided simultaneous two-band imaging at $70 \mu \mathrm{m}, 100 \mu \mathrm{m}$, and $160 \mu \mathrm{m}$ and was used primarily by the PAcs Evolutionary Probe team (PEP; Lutz et al., 2011), and the GOODS-Herschel team (Elbaz et al., 2011). Although PAcs had a much smaller beamsize than SPIRE due to the lower wavelengths probed $\left(5^{\prime \prime}, 7^{\prime \prime}\right.$ and $12^{\prime \prime}$ at 70,100 , and $160 \mu \mathrm{m}$, respectively) and the confusion limit was much lower at these wavelengths, PACs mapping was not as efficient as SPIRE mapping, making it more difficult to cover large areas of sky. Between the two major extragalactic deep field surveys PEP and GOODS-Herschel, about $3 \mathrm{deg}^{2}$ were imaged at $100 \mu \mathrm{m}$ and $160 \mu \mathrm{m}$, with $<1 \mathrm{deg}^{2}$ at $70 \mu \mathrm{m}$.

Wide-field Infrared Survey Explorer [WISE], 2009-2011

The WISE satellite conducted an all-sky survey at 3.4, 4.6, 12 and $22 \mu \mathrm{m}$ with a $40 \mathrm{~cm}$ diameter telescope and led to important discoveries of near earth objects and Y-dwarf stars; most relevant to the detection of DSFGs is the all-sky $22 \mu \mathrm{m}$ coverage (Wright et al., 2010). Although the detection limit at $22 \mu \mathrm{m}$ was quite shallow compared to, e.g., Spitzer MIPS at $24 \mu \mathrm{m}$ (see Figure 5), the huge increase in sky area meant that WISE detected many ultraluminous and hyper-luminous infrared galaxies, many at high-z. The DSFGs detected by WISE are notably warm, since they were selected at much shorter wavelengths than traditional SMGs or even local IRAS galaxies (Bridge et al., 2013, Blain et al., 2013, Tsai et al. 2013).

AzTEC, 2005-present

AzTEC, which is not an acronym, is a 144 element bolometer array camera operating at $1.1 \mathrm{~mm}$ currently mounted on the Large Millimeter Telescope (LMT) on the Sierra Negras outside of Puebla, Mexico (Wilson et al., 2008). AzTEC was commissioned in 2005 first on the JCMT after ScuBA was taken down, and over a short two month period, AzTEC surveyed $\approx 1 \mathrm{deg}^{2}$ to $1 \mathrm{mJy}$ RMS, roughly the same sky area covered by the many previous surveys of Scuba. While on the JCMT, AzTEC's imaging beamsize was 19". AzTEC was later taken to the $10 \mathrm{~m}$ Atacama Submillimeter Telescope Experiment (ASTE) in Chile where it mapped sky to similar depths over large areas, but with a 29" beamsize, until 2008. AzTEC was transported to the LMT in 2009 and has since been undergoing tests and commissioning, awaiting the completion of the $50 \mathrm{~m}$ telescope (which currently is only complete out to a $32 \mathrm{~m}$ diameter). The beamsize at the LMT is significantly improved over the large beamsizes at JCMT and ASTE, currently $9^{\prime \prime}$ with a $32 \mathrm{~m}$ dish which will improve to $6^{\prime \prime}$ with a $50 \mathrm{~m}$ dish.

AzTEC has performed some of the deepest and widest ground-based extragalactic field pointings, including several pointings in the COSMOS field (e.g. Scott et al. 2008, Aretxaga et al. 2011) and has been responsible for the discovery of some of the highest redshift SMGs known around $z \approx 5$ (e.g. Capak et al., 2011).

Large Apex BOlometer CAmera [LABOCA], 2006-present

LABOCA was developed by the bolometer development group at the Max-Planck-Institute für Radioastronomie as a multi-channel bolometer array for $870 \mu \mathrm{m}$ continuum mapping installed at the Atacama Pathfinder EXperiment (APEX) telescope in Chile (Siringo et al., 2009). It was first brought to APEX for science observations in 2006/2007 and started full science operations in 2008. The LABOca beamsize is 19", similar to that of ScuBA and AzTEC on the JCMT, although LABOCA's field of view is $11.4 \mathrm{arcmin}^{2}$. The first deep extragalactic pointing carried out with LABocA, and still the most uniform large coverage area from the instrument, is in the Chandra Deep Field South (Weiß et al. 2009a). The DSFGs discovered by LABOca in this LESS survey were some of the first to be followed up with continuum observations at ALMA interferometrically (Karim et al., 2013, Hodge et al., 2013b). 


\section{Atacama Cosmology Telescope [ACT], 2007-present}

The ACT experiment (Swetz et al. 2011) is a $6 \mathrm{~m}$ millimeter telescope which was installed on Cerro Toco in Chile in 2007. With a beamsize about an arcminute across, observations are conducted $1.1 \mathrm{~mm}, 1.4 \mathrm{~mm}$ and $2.0 \mathrm{~mm}$ with the primary goal understanding the CMB through the Sunyaev-Zeldovich (SZ) effect and measuring temperature variations of the CMB down to arcminute scales. In surveying $455 \mathrm{deg}^{2}$ in the 2008 ACT Southern Survey to $\sim 0.03 \mathrm{Jy}$, it has also contributed somewhat to the study of DSFGs (e.g. Marsden et al. 2013), although perhaps not as much as its later counterpart, South Pole Telescope.

South Pole Telescope [SPT], 2008-present

The South Pole Telescope is a $10 \mathrm{~m}$ millimeter wave telescope located at the geographic south pole in Antarctica designed to detect low-contrast signals like anisotropies in the cosmic microwave background (Carlstrom et al., 2011). The bolometer array on the SPT completed a $87 \mathrm{deg}^{2}$ survey of the sky looking for the detection of galaxy clusters via the Sunyaev-Zel'dovich effect (Staniszewski et al., 2009; Vieira et al., 2010), then later some larger surveys to $770 \mathrm{deg}^{2}$ (Mocanu et al. 2013); the full survey area completed in 2011 was $2500 \mathrm{deg}^{2}$ with $\sim 100$ bright DSFG detections. While its purpose was to detect galaxy clusters, the SPT instrument was also ideal for detecting some of the brightest galaxies emitting at long wavelengths (Vieira et al. 2010). Due to their extreme perceived luminosities, galaxies detected by SPT have been shown to be gravitationally lensed (e.g. Vieira et al., 2013; Bothwell et al., 2013b). We describe the follow-up of some of the more interesting SPT discoveries in $\$ 6$

Submillimetre Apex BOlometer CAmera [sABOCA], 2009-present

The SABOCA instrument is a 39 channel bolometer array operating at $350 \mu \mathrm{m}$ at APEX on Cerro Chajnantor in Chile. Although SABOCA has a relatively small beamsize for submillimeter bolometer observations, it is not sufficiently sensitive to detect unlensed DSFGs at high- $z$. In the best weather conditions (PWV $=0.2 \mathrm{~mm}$ ) the RMS at $350 \mu \mathrm{m}$ would reach $10.3 \mathrm{mJy} /$ beam after 13 hours, or $17.5 \mathrm{mJy} /$ beam in average weather conditions. With large overheads, sABOCA has not been as widely used for submm mapping as other submillimeter bolometers, nor has it been used extensively for dedicated source follow-up at $350 \mu \mathrm{m}$ as SHARc-II.

Goddard-Iram Superconducting 2-Millimeter Observer [GISMO], 2011-present

GISMO is a $8 \times 16$ pixel bolometer camera operating at $2 \mathrm{~mm}$ built at the Goddard Space Flight Center and is installed on the IRAM $30 \mathrm{~m}$ telescope at Pico Veleta in Spain (Staguhn et al. 2012). GISMO (actually named GISMO2 after the earlier prototype, GISMO; Staguhn et al. 2006) is designed to detect the highest-redshift DSFGs by taking advantage of the dramatic $K$-correction at $2 \mathrm{~mm}$ (see Figure 3). The field of view is $1.8 \times 3.7 \mathrm{arcmin}$ and the beamsize 16.7". Some small deep fields have already been obtained, particularly in a $5 \times 5$ arcmin portion of GOODS-N to $120 \mu \mathrm{Jy} /$ beam RMS (Staguhn et al. 2013) with more, wider-field pointings being planned in fields like COSMOS.

Submillimeter Common User Bolometer Array-2 [ScuBA-2], 2011-present

Scuba-2 (Holland et al., 2013) is the second generation bolometer array for the James Clerk Maxwell Telescope and finished commissioning in 2011. Scuba-2, a 10000 pixel camera with 100-150 times the mapping speed of Scuba, operates simultaneously at $450 \mu \mathrm{m}$ and $850 \mu \mathrm{m}$. SCUBA-2 is the first ground-based instrument to map blank field sky efficiently in the $450 \mu \mathrm{m}$ window. Since the wavelength is half that of $850 \mu \mathrm{m}$ mapping, the resolution is also improved by a factor of 2 , with a 7 " beamsize.

At $850 \mu \mathrm{m}$, ScuBa-2 produces very similar science results to the previous Scuba instrument, although it is a much more efficient mapper, by a factor of 5-10 (the best increase in mapping speed is seen at $450 \mu \mathrm{m}$ ). At $450 \mu \mathrm{m}, \mathrm{ScUBA}-2$ is much more efficient than Scuba. The Scuba-2 Cosmology Legacy Survey (S2CLS) is the Guaranteed Time project on ScuBA-2 which is dedicating the most observing time to blank-field extragalactic mapping at both $450 \mu \mathrm{m}$ and $850 \mu \mathrm{m}$. Several initial works (Chen et al. 2013a; Geach et al., 2013, Casey et al., 2013, Chen et al., 2013b; Roseboom et al., 2013) describe galaxies selected at $450 \mu \mathrm{m}$ and compare and contrast them to galaxies selected at longer wavelengths.

\subsubsection{Notable surveys focused on DSFG Discovery}

While the instruments above have contributed a great deal to the collective knowledge and census of DSFG activity in the high-redshift Universe, their contributions have been radically varied in scope and limitations. Figure 6 illustrates some of the more prominent 'legacy' surveys conducted in the submillimeter to date by survey area and depth, given here in units of solar luminosities. Luminosity is used as the method of quoting survey depth so that different surveys conducted at different wavelengths can be compared. Unfortunately, the conversion from a flux 

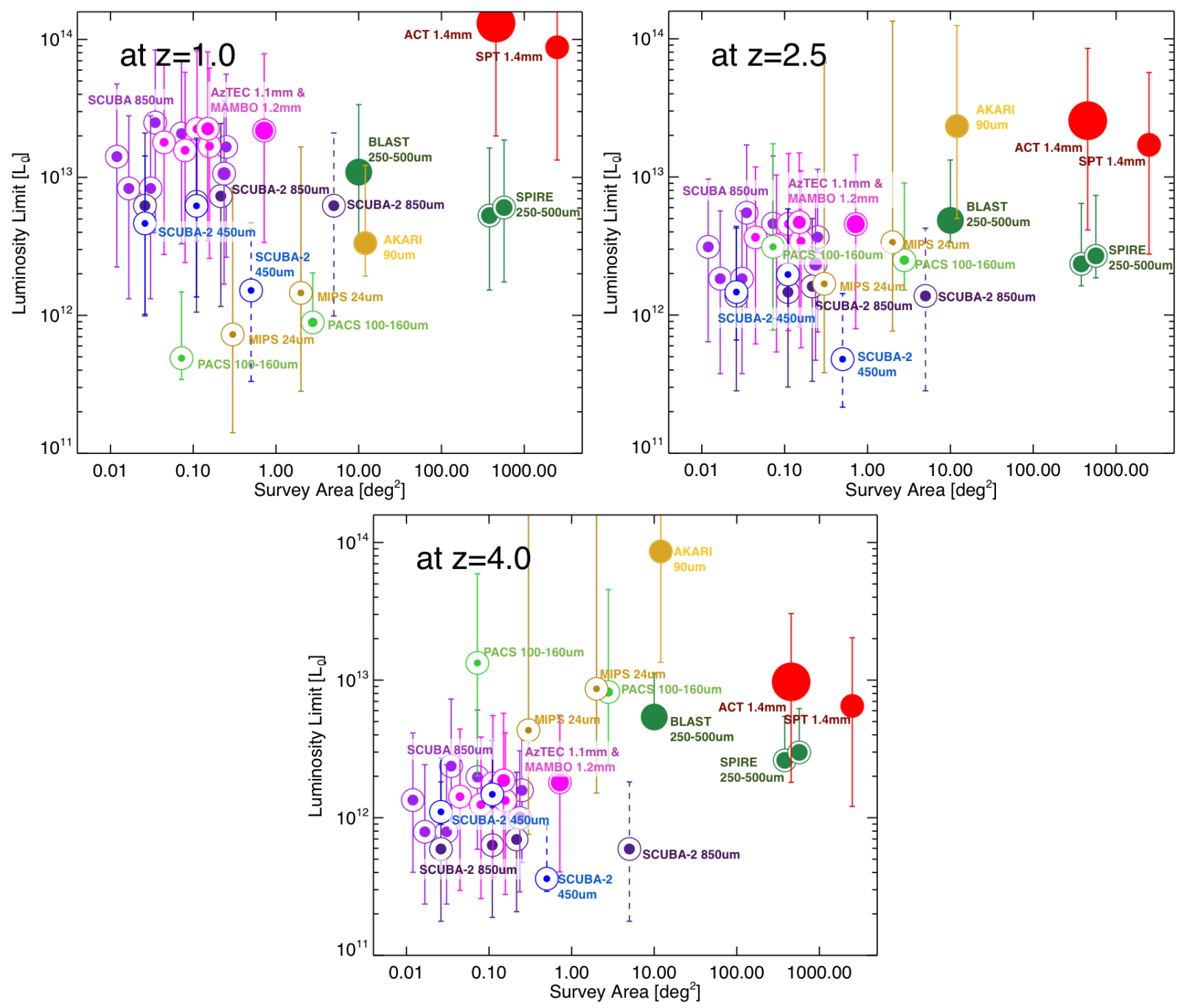

Figure 6: Survey Area against sensitivity, in solar luminosities, for several far-infrared surveys in the literature. Survey depth in solar luminosities is determined from the range of plausible SEDs (taken from Figure 5 which could have a given flux density, $S_{v}$, at a given selection wavelength, $\lambda$, at the given redshift. The survey depths at $z=1$ are given on the upper left panel, at $z=2.5$ are given in the upper right panel, and survey depths at $z=4$ are shown at bottom. The symbol size corresponds linearly with the survey's beamsize, ranging from 7-90". Note that this plot omits all-sky far-infrared surveys from Planck, WISE, and AKARI, although their sensitivities are not deep enough to detect unlensed, non-AGN dominated DSFGs. The Scuba $850 \mu$ m surveys included on this plot (purple) are the Hawai'i Hubble map (Barger et al. [1999), the 8 mJy survey (Scott et al. 2002 |Fox et al. 2002), the HDF Scan map (Borys et al. 2003. Pope et al. 2005), CUDSS (Webb et al.| 2003), the Scuba Lens survey (Smail et al. 2002), and the SHADES survey (Coppin et al. 2005). At 870 $\mu \mathrm{m}$ (also purple), we include the LESS LABoca survey (Weiß et al. 2009a). From Scuba-2 at $850 \mu \mathrm{m}$ (dark purple) and 450 $\mu \mathrm{m}$ (blue), we include recent surveys from Chen et al. (2013a) and Casey et al. (2013). We also include future survey estimates from the ScuBA-2 Cosmology Legacy Survey (Smail, private communication). At $1.1 \mathrm{~mm}$ and $1.2 \mathrm{~mm}$ (magenta) we include the MAMBO surveys of GOODS-N, Elais N2, and Lockman Hole North (Greve et al. 2004 2008 Lindner et al. 2011, and COSMOS (Bertoldi et al. 2007) and the AzTEC surveys of COSMOS (Scott et al. 2008, Aretxaga et al.|2011). At 250-500 $\mu$ m (dark green), we show the BLAST ECDFS survey (Devlin et al. 2009) and Herschel-SPIRE legacy programs HerMES (Oliver et al. 2012) and H-ATLAS (Eales et al. 2010). At 100 $\mu \mathrm{m}$ (light green), we show the results of the PAcs PEP and GOODS-Herschel surveys (Lutz et al. 2011. Elbaz et al. 2011). The AKARI $90 \mu \mathrm{m}$ deep field (Clements et al. 2011) is also shown (gold) as are the Spitzer MIPS coverages of GOODS-N and COSMOS (Sanders et al. 2007. Dickinson et al., in prep). At $1.4 \mathrm{~mm} \mathrm{(red),} \mathrm{we} \mathrm{also} \mathrm{include} \mathrm{the} \mathrm{ACT} \mathrm{survey} \mathrm{(Marsden} \mathrm{et} \mathrm{al.} \mathrm{2013)} \mathrm{and} \mathrm{the} \mathrm{SPT} \mathrm{survey} \mathrm{(Vieira} \mathrm{et} \mathrm{al.}$ 2013). 
density limit to a luminosity limit is intrinsically uncertain and dependent on the SED shape of any given galaxy (which is impacted mostly by its characteristic dust temperature). The survey depths in Figure 6 therefore have an characteristic uncertainty which is dominated by the intrinsic variation of SED shapes, a topic which is addressed later in $\$ 2.4 .1$ Also critical to note is that survey depth is redshift dependent. Shorter selection wavelengths will be most sensitive to detecting low luminosity systems at low redshift, whereas long wavelengths will be efficient at a range of redshifts, thanks to the varying $K$-correction (see Figure 3). What constitutes a deep survey at $100 \mu \mathrm{m}$ at $z=1$, is not that deep at $z=2.5$; Figure 6 shows various survey limits at both of these epochs.

The largest survey conducted with ScuBa was the SHADES survey (Coppin et al., 2005) covering $0.25 \mathrm{deg}^{2}$ to $2 \mathrm{mJy}$ RMS, though several prior surveys at $850 \mu \mathrm{m}$ opened up the discovery space for far-infrared galaxies: the Hawai'i Hubble map with 0.2-4 mJy RMS over $110 \operatorname{arcmin}^{2}$ (Barger et al. 1999), the $260 \operatorname{arcmin}^{2} 2.5 \mathrm{mJy}$ RMS 8 mJy survey (Scott et al., 2002, Fox et al., 2002; Lutz et al., 2001; Almaini et al., 2003; Ivison et al., 2002), the HDF Scan map with $125 \mathrm{arcmin}^{2}$ to $3 \mathrm{mJy}$ (Borys et al. 2003), CUDSS with $1 \mathrm{mJy}$ RMS over $60 \mathrm{arcmin}^{2}$ (Webb et al., 2003), and the lens survey with $1.7 \mathrm{mJy}$ RMS over $45 \mathrm{arcmin}^{2}$ (Smail et al., 2002). In the mid-2000's, MaMbo began producing similarly fruitful results in Elais N2 and Lockman Hole, with $0.8 \mathrm{mJy}$ RMS over $160 \operatorname{arcmin}^{2}$ (Greve et al., 2004), 1 mJy RMS over $400 \operatorname{arcmin}^{2}$ in COSMOS (Bertoldi et al., 2007), 0.7 mJy RMS over $287 \operatorname{arcmin}^{2}$ in GOODS$\mathrm{N}$ (Greve et al., 2008), and $0.75 \mathrm{mJy}$ RMS over $566 \mathrm{arcmin}^{2}$ in Lockman Hole North (Lindner et al. 2011). AzTEC soon followed with even larger sky areas at $1.1 \mathrm{~mm}$ with deep maps in COSMOS, to $1.3 \mathrm{mJy}$ RMS over $0.15 \mathrm{deg}^{2}$ (Scott et al., 2008) and $1.25 \mathrm{mJy}$ RMS over $0.72 \mathrm{deg}^{2}$ (Aretxaga et al., 2011). The $870 \mu \mathrm{m}$ LABOCA coverage of CDFS (Weiß et al., 2009a) is also comparably large, and provided the first uniform SMG sample followed up with ALMA interferometrically (Karim et al. 2013; Hodge et al., 2013b).

Also in the mid-2000's were some surveys at shorter wavelengths; notably the Spitzer MIPS large sky coverage at $24 \mu \mathrm{m}$. Although not probing the peak of the modified blackbody emission directly, the $24 \mu \mathrm{m}$ surveys covered much larger areas than prior submm mapping. Some of the deepest maps were in GOODS-N (Dickinson et al., in preparation) and COSMOS (Sanders et al. 2007) with SWIRE covering larger areas $50 \mathrm{deg}^{2}$ to shallower depths of $280 \mu \mathrm{Jy}$ (Lonsdale et al. 2003). The deepest map from AKARI, the $10 \mathrm{deg}^{2}$ AKARI deep field (Clements et al., 2011), places it in a regime where it can detect unlensed high- $z$ DSFGs.

Immediately prior to the launch of the Herschel Space Observatory, the BLAST and SPT experiments (Carlstrom et al. 2011) conducted their work at 250-500 $\mu \mathrm{m}$ and 1.4-2.0 mm respectively. The former completed a $10 \mathrm{deg}^{2} \mathrm{deep}^{-}$ map pointing around the ECDFS to $\sim 15 \mathrm{mJy}$ RMS (Devlin et al., 2009) while the latter completed a much larger and shallower $87 \mathrm{deg}^{2}$ survey to $11 \mathrm{mJy}$ RMS at $1.4 \mathrm{~mm}$ (Vieira et al., 2010).

By the end of the 2000's, the Herschel Space Observatory was launched and large scale $\sim 100 \mathrm{deg}^{2}$ sensitive submillimeter surveys became reality. The legacy programs of the SPIRE instruments immediately set out to survey vast areas of sky at $250-500 \mu \mathrm{m}$. The H-ATLAS survey (Eales et al., 2010) covers $\sim 570 \mathrm{deg}^{2}$ to $35-45 \mathrm{mJy}$ RMS while the HerMES survey (Oliver et al., 2012) covered $\sim 380 \mathrm{deg}^{2}$ in a wedding cake style; HerMES data were largely confusion limited with sensitivities 5-10 mJy. By the end of SPIRE's life in 2013, it had surveyed about $1300 \mathrm{deg}^{2}$ to varying depths. Unfortunately the PACs instrument did not have the efficient mapping capabilities of SPIRE; nevertheless, the depth achieved by PAcs PEP (Lutz et al., 2011) and GOODS-Herschel (Elbaz et al., 2011) legacy surveys-particularly at $z=1-$ is unrivaled.

\subsection{Selection biases and Sensitivity}

A discussion on the DSFGs selection methods would not be complete without an analysis of their selection biases and relative sensitivities. Galaxies' flux densities at any given wavelength depend not only on their intrinsic luminosities, but also their SED characteristics. Furthermore, the physical characteristics of DSFGs which we infer from these surveys depends on successful counterpart and redshift identification of the systems. How are our conclusions regarding DSFG evolution impacted by potential SED variation? How are they impacted by the process and success rates of individual source follow-up? What have we missed? The two subsections below address these two problems in detail. First, the impact of intrinsic variation in SED types in $\$ 2.4 .1$, and second, the impact of multi-wavelength counterpart matching in $\S 2.4 .2$ 

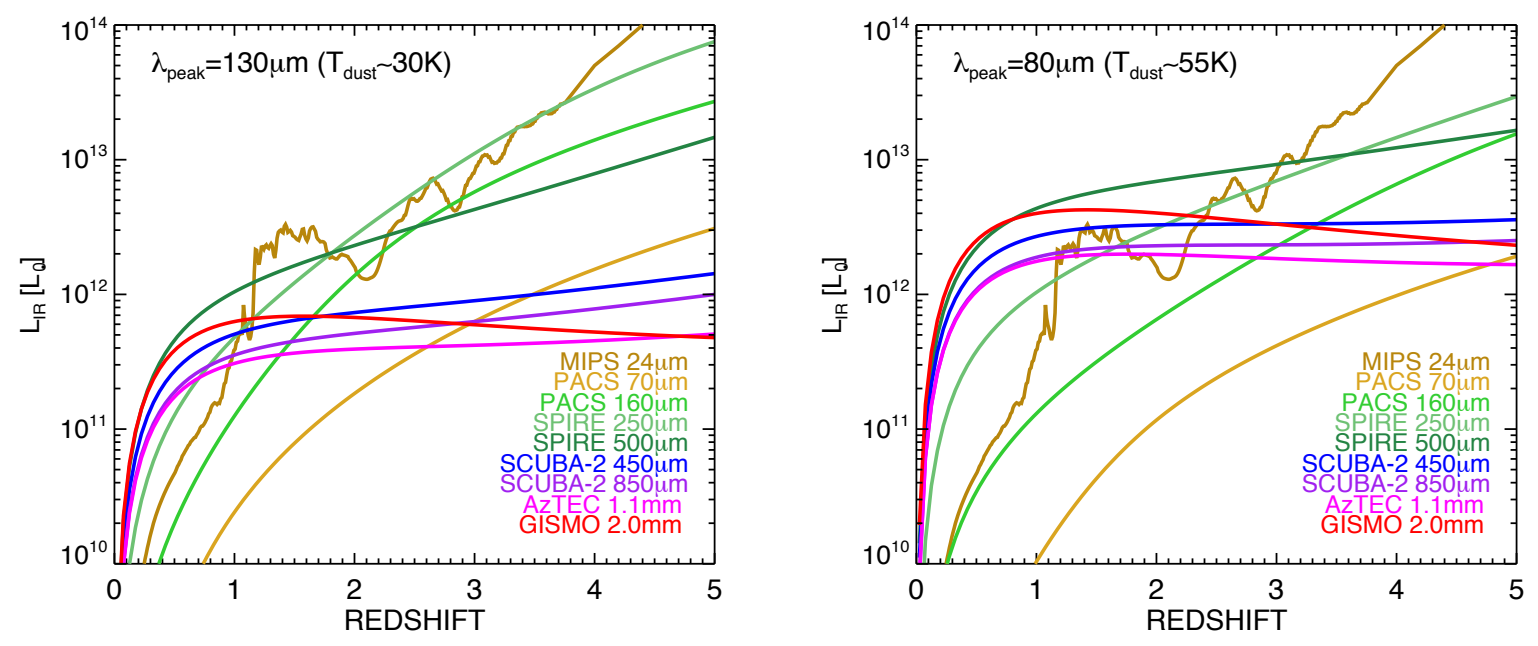

Figure 7: The luminosity limits of different submm bands as a function of redshift for two DSFG SEDs, used to highlight the strong dependence of survey depth and completeness on assumed dust temperature. On the left, we use a DSFG SED (modified blackbody of the type given in Eq77 which peaks in $\mathrm{S}_{v}$ at $130 \mu \mathrm{m}$ and can be characterized with a temperature of $30 \mathrm{~K}$. On the right, we use an SED peaking at $80 \mu \mathrm{m}$ and has a temperature of $55 \mathrm{~K}$. Both SED types are within the range of typical expected ULIRG temperatures (20-60 K). This illustrates the dramatic impact that SED shape can have on the sensitivity limits of submillimeter observations at certain redshifts. The wavelengths most impacted by dust temperature are those $>500 \mu \mathrm{m}$, while those at 70-250 $\mathrm{m}$ are minimally affected (but might be affected by the presence of AGN emission, not accounted for here).

\subsubsection{Intrinsic Variation in SEDs}

The average measured dust temperature of DSFGs is $\sim 30-40 \mathrm{~K}^{6}$ This temperature is characteristic of dust heated by ambient star formation activity in molecular clouds scattered throughout the galaxy. The volume of dust surrounding these star-forming regions might be diffuse, thus difficult to heat in bulk substantially above $\sim 50 \mathrm{~K}$ without a very bright nuclear source. From studies of local galaxies, particularly the Revised Bright Galaxy Sample (RBGS; Sanders \& Mirabel, 1996), dust temperatures for star-formation dominated DSFGs range between 20-60 K. This dust temperature range represents the intrinsic variation of SED shapes in local ULIRGs, not the uncertainty by which the temperatures are constrained. How does intrinsic DSFG SED variation impact the detection and selection of distant dusty starbursts in the submillimeter? How does it impact the perceived completeness of a population of DSFGs selected in a single band?

Figure 7 illustrates the evolving luminosity detection limits from $0<z<5$ at different detection wavelengths for a $30 \mathrm{~K}$ dust SED and a $55 \mathrm{~K}$ dust SED which we use to illustrate the significant dependence of survey depth on assumed dust temperature. While some limits remain stable within a factor of $\sim 2$ between the panels (e.g. $24 \mu \mathrm{m}$, $70 \mu \mathrm{m}, 160 \mu \mathrm{m}, 250 \mu \mathrm{m}$ ) other luminosity thresholds change dramatically (e.g. $850 \mu \mathrm{m}, 1.1 \mathrm{~mm}, 2.0 \mathrm{~mm}$ ) where much fainter cold-dust sources are detectable than warm-dust. This is known as the submillimeter dust-temperature selection effect (Blain, 1996; Eales et al., 2000; Blain et al., 2004a). This effect implies that, luminosities being equal, even minor differences in SED shape can impact the measured flux densities at any given submillimeter flux and select against galaxies of certain SED types. At $850 \mu \mathrm{m}$, this effect was described by Blain et al. (2004a) and observationally verified by Chapman et al. (2004a) and Casey et al.(2009c), where at and $z \sim 2$, a galaxy's 850 $\mu$ m flux density goes as $S_{850} \propto L_{\mathrm{IR}} T_{\text {dust }}^{-3.5}$. Galaxies with fixed luminosity $L_{\mathrm{IR}}$ and warmer dust $(\sim 50 \mathrm{~K})$ will have dramatically lower $S_{850}$ and a much lower likelihood of being selected as a submillimeter galaxy, even though it might have an infrared luminosity typical of other submillimeter galaxies.

The first inclination that the canonical ScuBA-selected SMG population was incomplete and biased against warmdust SEDs (see Blain et al. 2004a, for the first detailed discussion of the topic) lead to the investigation of possible warm-dust SMG cousins. Given the procedure for identifying SMGs' counterparts relied on identification in the radio, where emission is dominated by synchrotron emission mixed with free-free emission (a topic we discuss in the next

${ }^{6}$ This represents a weighted average for dust distributed throughout the galaxy and should not be taken literally. 


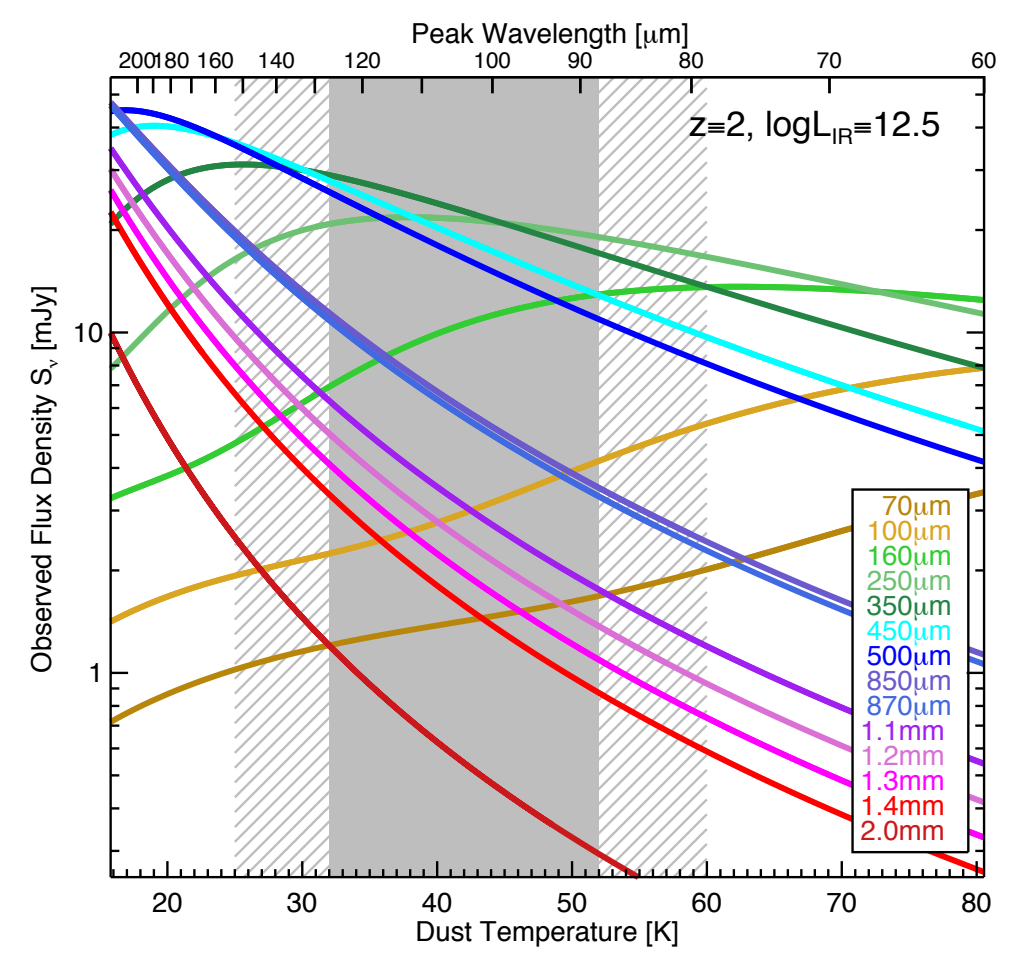

Figure 8: For a galaxy of fixed redshift $(z=2)$ and luminosity $\left(\log \left(L_{\mathrm{IR}}\right)=12.5\right)$, the relationship between SED shape or dust temperature and measured flux density at a variety of wavelengths from the mid-infrared through the millimeter. The solid gray stripe in the background represents the typical characteristic dust temperature range of local ULIRGs; the hashed region represents the dust temperature range of all local infraredbright galaxies (i.e. from RBGS Sanders \& Mirabel 1996). Within the peak dust temperature range, $\sim 32-52 \mathrm{~K}, 850 \mu \mathrm{m}-1.2 \mathrm{~mm}$ flux densities can vary up to 1 dex. Observed wavelengths that are nearer the peak in the SED show a shallower dependence on temperature.

subsection), a natural place to search for warm-dust SMG cousins is the temperature-independent radio waveband. Chapman et al. (2004a) summarizes the results of the first search of the warm-dust analogue of SMGs as Optically Faint Radio Galaxies (OFRGs). OFRGs later became known as Submillimeter-Faint Radio Galaxies (SFRGs) in later work (Casey et al., 2009c a; Magnelli et al., 2010; Casey et al., 2011b) which confirmed that they were truly warm-dust analogues of SMGs with similar physical properties.

Figure 8 illustrates the submillimeter dust-temperature selection effect another way. For a galaxy of fixed luminosity and redshift, the observed flux density in a given submillimeter band will vary with dust temperature, or SED peak wavelength. The flux density dependencies on dust temperature are critical to keep in mind when considering 'completeness' of populations. Particularly in the context of completing an accurate census of all infrared-luminous activity, this type of prominent selection bias needs to be considered.

\subsubsection{Identifying Multi-wavelength Counterparts}

Although long-wavelength submillimeter observations benefit substantially from the negative $K$-correction (Figure 3, enabling detection of sources out to very high- $z$, the difficulty in following up those sources and characterizing them at other wavelengths can bias the interpretation of the population. The large beamsize of single-dish submillimeter observations is the key limiting factor. Bright submillimeter sources will have positional uncertainties of order several to tens of arcseconds and the number of possible counterpart galaxies corresponding to that source is in the tens. Although direct far-infrared/submillimeter interferometric follow-up is the most certain way of narrowing down the position of the submillimeter source to $\sim 1^{\prime \prime}$, interferometric follow-up for submillimeter sources has often been observationally expensive. Other methods can be employed to identify the multiwavelength counterpart to 
infrared-luminous sources.

Radio counterparts:

Traditionally, this was done by identifying counterparts at radio wavelengths. Radio interferometric observations are far easier to make than far-infrared interferometric observations due to the atmospheric transmission (see Figure 4), and most of the deep extragalactic legacy fields containing submillimeter data already have been surveyed by radio arrays at $1.4 \mathrm{GHz}$ like the Very Large Array, which is now known as the Jansky Very Large Array (VLA) after being largely rebuilt. Searching for infrared-bright galaxies at radio wavelengths exploits the locally observed correlation between radio emission and far-infrared emission in starburst galaxies, described first in Helou et al. (1985) and Condon (1992). Although the physics of this correlation is debated, it is clear that radio synchrotron emission arising from supernova remnants trace the obscured star formation quite well, and that there seems to be little to no evolution in this relationship out to high-redshifts (Murphy, 2009; Ivison et al., 2010a b).

The advantage of matching submillimeter sources to radio counterparts is that radio sources are much more rare than optically-bright galaxies (Ivison et al., 2007). Although tens of optically-bright galaxies might be visible within one submillimeter beam, it is rare to have more than one radio-bright galaxy (here, radio-bright simply means radiodetected at $S_{1.4} \gtrsim 50 \mu \mathrm{Jy}$ ). Once a radio position is in hand, the source can quickly be identified at other wavelengths and even followed-up using traditional spectroscopic methods in the optical and near-infrared (which was done most famously by Chapman et al. 2005, for a set of $\sim 75$ Scuba-selected SMGs). With accurate positions and redshifts in hand, follow-up physical characterization can be done.

Of course, the disadvantage of matching to radio counterparts is that a large fraction of submillimeter sources $d o$ not have radio counterparts. Chapman et al. (2003b) and Barger et al. (2007) study the $\mu$ Jy radio galaxy population in detail and how they relate to the $850 \mu \mathrm{m}$-selected Scuba population. In one of the deepest radio continuum maps, only $66 \%$ of $S_{850}>5 \mathrm{mJy}$ SMGs were radio detected. In slightly shallower coverage areas, the fraction is more like 40-50\%. If so many submillimeter sources lack radio counterparts, how will our interpretation of the submillimeter galaxy population be impacted if we only consider those that do?

Unlike submillimeter observations, radio $1.4 \mathrm{GHz}$ observations do not benefit from a negative $K$-correction at high-redshift (see Figure 3); galaxies at $z \gtrsim 3.5$ are very difficult to detect at radio wavelengths. This could imply that the submillimeter sources without radio counterparts sit at high redshifts, but that is difficult to constrain as some other factors could also lead to a radio-faint submillimeter source (like submillimeter multiplicity discussed in $\S 2.5$ or variation in the far-infrared/radio correlation discussed more in $\$ 5.12$. Nevertheless, our ability to deduce intrinsic properties for the DSFG population, like the peak epoch of formation, is hampered by our inability to identify roughly half of the submillimeter population.

\section{$\mathbf{2 4} \mu$ m cross-identifications:}

Recognizing the low fraction of submillimeter sources identified at radio wavelengths, work has been done to match submillimeter emission to detection in other bands. This has primarily been done with Spitzer MIPS $24 \mu \mathrm{m}$ maps, as they cover large areas of sky to sufficient depths (e.g. Pope et al., 2006; Dye et al., 2008). While mid-infrared emission does roughly correlate with far-infrared emission, a few aspects of mid-infrared emission complicate the relation. From $z=0$ to $z=4$, emission and absorption features from Polycyclic Aromatic Hydrocarbons (PAHs; discussed further in $\S 5.7$ and silicates alter the underlying warm-dust continuum. This manifests in an irregular detection boundary with redshift; as seen in Figure 7, a $10^{12} \mathrm{~L}_{\odot}$ galaxy is detectable at $24 \mu \mathrm{m}$ at $z<1$ and in the narrow range $2<z<2.2$. Furthermore, as we will see later, the variety of mid-infrared spectral types in DSFGs is large and does not always map directly to the integrated far-infrared luminosity. One further disadvantage of matching far-infrared emission to $24 \mu \mathrm{m}$ is that the sky density of $24 \mu \mathrm{m}$ sources is significantly higher than those in the radio, although still much lower than in the optical. Despite these drawbacks, deep $24 \mu \mathrm{m}$ imaging from Spitzer is far more abundant in extragalactic legacy fields than sufficiently deep radio maps, making it a natural second choice for counterpart matching.

More recent data from Herschel have made use of the multi-wavelength counterpart technique to constrain the positions of infrared-luminous sources. Roseboom et al. (2010) introduced a cross-matching technique, dubbed 'XID,' which uses $24 \mu \mathrm{m}$ and radio positional priors to determine both accurate positions as well as deboosted flux densities for Herschel-selected galaxies (HSGs). The technique (updated in Roseboom et al., 2012) identifies significant Herschel detections and investigates nearby $24 \mu \mathrm{m}$ or radio sources as possible counterparts using a likelihood estimator. Since Herschel maps are confusion limited, this multi-wavelength counterpart matching must be done iteratively across the entire map, and not individually source by source. While the disadvantages of the XID method are the same 

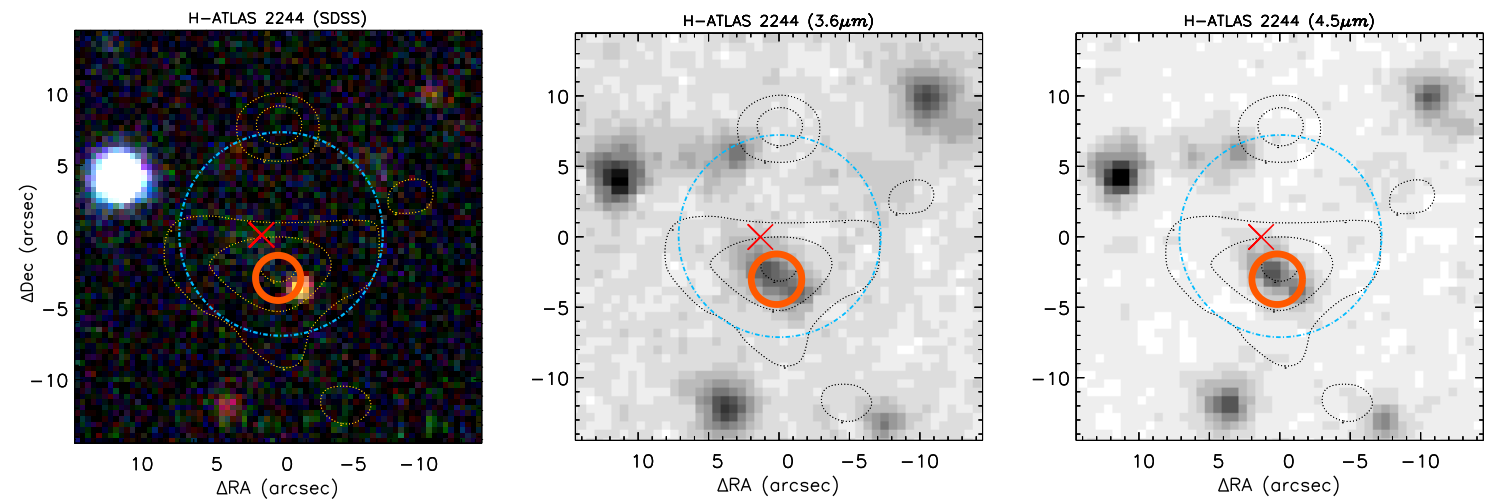

Figure 9: An example of a Herschel source that was identified using the likelihood ratio (LR) method with SDSS and IRAC. From left-to-right we show a three-color SDSS image ( $g, r$, and $i$ bands), the $3.6 \mu \mathrm{m}$, and the $4.5 \mu \mathrm{m}$ data. The galaxies identified as the SDSS and IRAC counterparts to the SPIRE source are marked with an ' $\mathrm{X}$ ' and a small circle, respectively. The LR method prefers the IRAC identification marked with a circle over the SDSS-based identification as the counterpart to the SPIRE source. The large circle has $7.2^{\prime \prime}$ radius and encompasses the SPIRE $3 \sigma$ pos area in which counterparts are identified. Contours show the SPIRE $250 \mu \mathrm{m}$ emission at 5, 7, 9, $11 \sigma$ levels. In the absence of high-resolution sub-mm imaging we cannot determine whether this SPIRE source is the results of blended emission from the two identified galaxies, or whether one of those counterparts is a chance association. The figure is reproduced from Kim et al. (2012) with permission from the authors and AAS.

as prior attempts as using radio or $24 \mu \mathrm{m}$ counterparts (a potentially high fraction of high- $z$ galaxies will be missing at $24 \mu \mathrm{m}$ or $1.4 \mathrm{GHz}$ ), this technique can be used to assess statistically large populations of $z \lesssim 2$ far-infrared selected galaxies.

\section{Optical and near-IR counterparts using likelihood ratio:}

Although matching a large-beamsize submillimeter position directly to an optical/near-infrared source can have high failure rates, there is a technique which assesses the quality of such a match. Performing a likelihood analysis that accounts for both the relative positions and other observable properties of optical sources within a beamsize gives a somewhat reliable estimate as to cross-identification purity. The likelihood ratio can be written as the probability that an optical or near-IR source is the correct counterpart to the longer wavelength submm galaxy with an equivalent probability for an unassociated background source, written as

$$
L=\frac{q(m) f(r)}{n(m)}
$$

where $q(m)$ and $n(m)$ are the normalized magnitude distributions of counterparts and background sources, respectively. The radial probability distribution of the separation between submm source and the shorter wavelength galaxies is denoted by $f(r)$. This likelihood ratio (LR) method was developed in Sutherland \& Saunders (1992) with additional improvements in Ciliegi et al. (2003), Brusa et al. (2007) and-in the context of submillimeter data-in Chapin et al. (2011). This likelihood ratio estimator is similar to the calculation of " $p$-values," or the corrected-Poissonian probability. This is the probability of counterpart alignment for a member of a certain population (e.g. $24 \mu \mathrm{m}$-selected galaxies, optical $i$-band selected galaxies, etc) having a given space density. The $p$-value is calculated via the following as described in Downes et al. (1986):

$$
p=1-\exp \left(-\pi n \theta^{2}\right)
$$

where $n$ is the source density of the given counterpart type, $\theta$ is the angular offset between original source and counterpart. The $p$-value itself represents the probability or random coincidence, and generally, a match is considered reliable if $p<0.05$ (e.g. Ivison et al., 2002, Pope et al., 2006, Chapin et al., 2009, Yun et al., 2012; Hodge et al., 2013b, Alberts et al., 2013). The $p$-value is more appropriate for catalogs in which the surface density is low (e.g., ra- 
dio identifications) and favors counterparts that are brighter than the background population. Since the surface density of optical and near-infrared sources is high, the $L R$ method is favorable when rarer counterpart types are not available.

Both likelihood matching methods have been implemented to match submillimeter counterparts to optical and near-IR counterparts (e.g. Smith et al., 2011, Fleuren et al., 2012, Bond et al., 2012, Kim et al. 2012), and both methods have been naturally extended to account for other properties of galaxies beyond positional and flux information, such as near-infrared luminosity and color (Wang et al., 2006, Serjeant et al., 2008, Kim et al., 2012, Alberts et al., 2013). This type of matching is especially necessary when the availability of $24 \mu \mathrm{m}$ or radio ancillary data is lacking, e.g. over areas spanning many tens to hundreds of square degrees, as recently done with Herschel in the H-ATLAS collaboration. Over these wide areas, near-IR and optical coverage has and will be much more plentifully available and can be used as a direct way of identifying counterparts (see example in Figure 9) The primary drawback of this method is that it assumes one counterpart to each submm source and has no mechanism to account for source multiplicity; however, as discussed in $\$ 2.5$, that can be difficult to surmise from radio and $24 \mu \mathrm{m}$ counterpart identifications as well.

One can also use Bayesian techniques (Budavári \& Szalay, 2008) that uses a priori knowledge of the counterpart population from one area to guide the identification process of another sky area. This technique has been frequently used to cross-identify X-ray sources in mid and near-IR data (Brand et al., 2006; Gorjian et al., 2008). Bayesian methods are not currently pursued to cross-identify far-IR and submm sources due to the need for a priori in describing the cross-identifications, perhaps with another method. The $L R$ analysis is advantageous in that you can make use of prior information if you have it, unlike the $p$-statistic (see again Chapin et al., 2011, Alberts et al., 2013, for examples of intelligent multi-dimensional priors).

\subsection{DSFG Multiplicity}

One of the more recent advances in DSFG work has come from high-resolution far-IR maps providing the initial constraints on the multiplicity of SMGs: in other words, the number of galaxies that contributes to a given submm source's flux density. For confusion-limited surveys (see $\$ 3.1$ where sources' flux density would not evolve substantially with redshift (due to the negative $K$-correction), the probability that two less luminous galaxies masquerade as a single, line-of-sight submm source is not low (it is much higher in the submm than in the optical, where sources at different redshifts are unlikely to have comparable flux densities). For observers studying the environments of heavy star formation at high- $z$, the initial worry was that some of the most extreme galaxies contributing to the bright-end tail of the flux density distribution may actually resolve into multiple counterparts when examined at high spatial resolution. Indeed, most theoretical models that have aimed to understand the origin of SMGs and other high- $z$ dusty sources have found great difficulty in reproducing the numbers of the brightest sources as individual galaxies (c.f. $\S[10$ ). In fact some observational works argue with likelihood estimates that submillimeter sources are probably mostly multiples (Chapin et al. 2011). Wang et al. (2011c) used the SMA to map the morphologies of two SMGs, and found that both resolved into multiple counterparts. This study was expanded upon by Barger et al. (2012), who found nearly $\sim 1 / 4$ of their sample of 16 SMGs observed with the SMA resolved into multiple counterparts as well.

With the advent of ALMA (even in Cycle 0), this field has been revolutionized. Hodge et al. (2013b) and Karim et al. (2013) found that potentially between $30-50 \%$ of a sample of $\gtrsim 100$ high- $z$ SMGs break up into multiple counterparts, and all sources above $S_{870}>10 \mathrm{mJy}$ are intrinsic multiples. Their initial detections as a single source by single dish telescopes was confused by the poor resolution of these facilities (e.g. the JCMT) compared to ALMA. To some degree, this phenomena was expected from early radio studies (e.g. Pope et al., 2006; Ivison et al., 2007), clustering measurements (Blain et al., 2004a; Hickox et al., 2012), and numerical models (Hayward et al., 2012). We note that not all high-resolution studies of SMGs have revealed multiplicity to the same extent as the Hodge et al. (2013b) and Karim et al. (2013) which could be biased due to the known underdensity of bright SMGs in the Chandra Deep Field South. For example, Hezaveh et al. (2013) and Chen et al. (2013a) find fewer multiples, the latter work estimating a multiple fraction of SMGs of $10 \%$ with several examples of intrinsic $>10 \mathrm{mJy}$ single-source SMGs. In all studies, sample sizes are still relatively small (although the ALESS sample is $\sim 90$ galaxies strong, they are biased fainter than most SMG populations), and we can only expect that much larger, statistically significant samples will become available with ScUBA-2 and expanded ALMA operations target this burgeoning field. 


\section{Submillimeter Number Counts}

This section describes the basic attributes of submillimeter/far-infrared maps and the scientific conclusions we can reach from direct measurement of those maps. While optical and near-infrared maps are fairly straightforward to interpret since there is a clear division between source and background, submillimeter maps from single-dish observatories are often dominated by confusion noise, where the beamsize is larger than the space between neighboring sources and it becomes difficult to pinpoint individual galaxies. Figure 10 illustrates the changing resolution over a single patch of sky in the COSMOS field from optical $i$-band through to $1.4 \mathrm{GHz}$ radio continuum.

The community of submillimeter astronomers analyzing submillimeter maps have developed strategies to unravel the confusion brought on by large beamsizes; these techniques-including estimating positional accuracy, deboosted flux densities, sample completeness, etc-are described below in $\$ 3.2$. These techniques are essential to the analysis of large-beamsize submillimeter observations until more direct constraints (via interferometric observations) can be made. This review not only touches on the now-standard techniques for analyzing submillimeter maps, but also briefly describes different yet complimentary techniques, including stacking and a technique called $\mathrm{P}(\mathrm{D})$ analysis.

While the eventual goal of characterizing the sources in a submillimeter map is understanding the galaxies and their physical processes, this section describes a more basic measurement: submillimeter number counts. Number counts are simply the number of sources above a given flux density per unit area, often denoted $N(>S)\left[\mathrm{deg}^{-2}\right]$ in cumulative form or $d N / d S\left[\mathrm{mJy}^{-1} \mathrm{deg}^{-2}\right]$ in differential form. Although the number counts might seem like a simple quantity to measure, inferring the number counts in the submillimeter can be challenging. The challenge is worth pursuing since number counts can provide key constraints on the cosmic infrared background (CIB), as well as galaxy formation theory (see $\S 10$. Unlike studies of sources' redshifts, luminosities, SEDs, etc., the number counts measurement is not as plagued by sample incompleteness. Even without information on the physical characteristics of individual sources, number counts shed much needed light on the dominating sources of the extragalactic background light, EBL.

\subsection{Confusion Noise}

Confusion noise arises when the density of sources on the sky is quite high and the beamsize of observations is large; it is present when more than one source is present in a telescope beam. Optical observations are rarely confusion limited except in the case of very crowded star cluster fields, where the density of stars per resolution element is greater than one. However, confusion noise is far more common in the infrared and submillimeter given the large beamsizes of single-dish telescopes (see Table 1 in the previous chapter). Since fainter sources are far more numerous than bright sources, there will be some threshold in flux density beyond which a survey will become confusion limited. For a beamsize $\Omega_{\text {beam }}$ (an angular area), the confusion limit flux density $S_{\text {conf }}$ is the limit at which the spatial density of sources at or above that flux density multiplied by the beamsize is unity. In terms of the cumulative number counts $N(S)$-the number of sources above flux density $S$-this confusion limit can be expressed where the following is fulfilled: $\Omega_{\text {beam }} N\left(S_{\text {conf }}\right)=1$. Even though an instrument or survey might be able to integrate longer to beat down instrumental noise, there will be minimal gain in field depth below the confusion limit.

\subsection{Using Monte Carlo Simulations in Number Counts Analysis}

The identification of individual point sources in a submillimeter map requires Monte Carlo simulations to characterize completeness, bias, and false positive rates. This technique was first outlined by Eales et al. (2000), Scott et al. (2002) and Cowie et al. (2002) and used in Borys et al. (2003) for the Scuba HDF survey and in Coppin et al. (2006) for the ScuBA SHADES survey. It has since been updated for point source identification in Herschel extragalactic surveys such as HerMES (Smith et al. 2012), but probably the most elegant updates to the technique have come out of work done by the AzTEC team (see Perera et al., 2008; Scott et al., 2008; Austermann et al., 2010; Scott et al. 2012). These simulations go beyond the simple identification of sources; they provide a more accurate estimate of sources' intrinsic emission. By using a Markov Chain Monte Carlo statistical technique of analyzing submillimeter maps, sources' positional accuracy, intrinsic flux densities can be constrained, thus number counts, as well as sample contamination and completeness. The only major shortcoming of MCMC analysis is the lack of consideration of clustered sources, which might or might not be a significant effect (see $\$ 7$ ).

This technique is based on the injection of fake sources into noise maps. The distribution of sources in spatial density and flux densities, $S$, is an input assumption and the more the resulting source-injected map resembles the real 


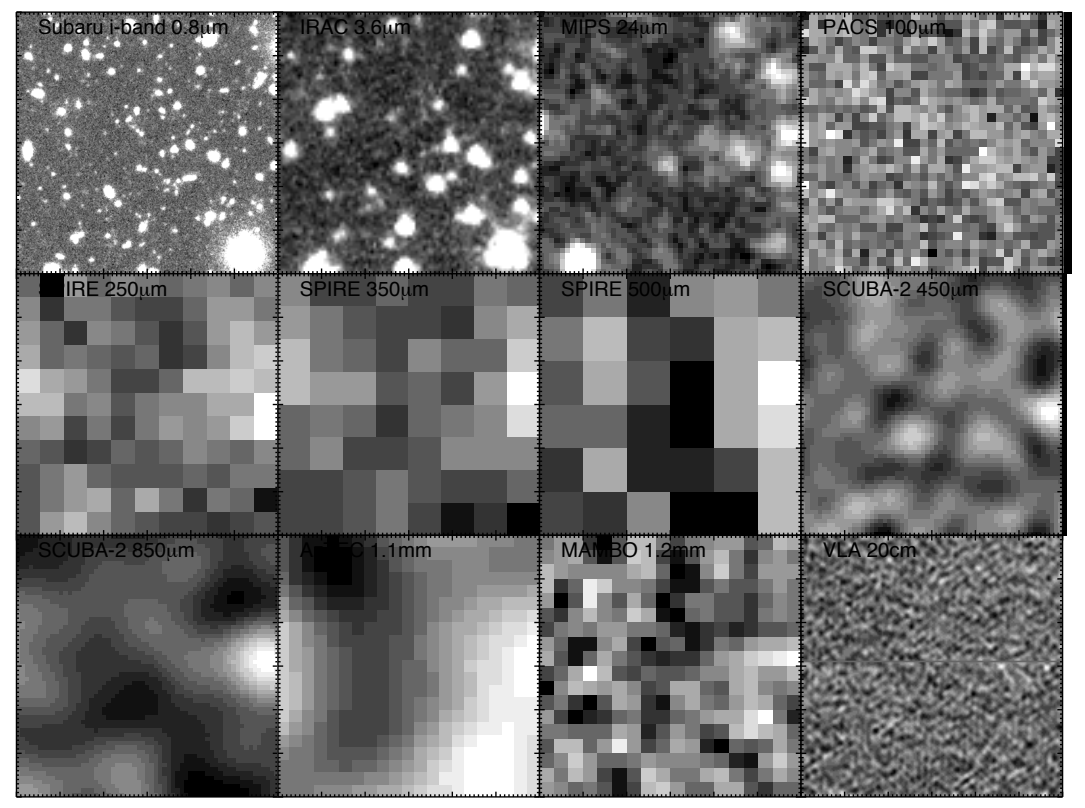

Figure 10: Twelve $1 \times 1$ arcmin cutouts from the COSMOS field imaged at different wavelengths with different facilities. From shortest wavelengths to long (top left to bottom right): Subaru $i$-band $(0.8 \mu \mathrm{m})$, IRAC $3.6 \mu \mathrm{m}$, MIPS $24 \mu \mathrm{m}$, PACS $100 \mu \mathrm{m}$, SPIRE $250 \mu \mathrm{m}$, SPIRE $350 \mu \mathrm{m}$, SPIRE $500 \mu \mathrm{m}$, ScuBA-2 $450 \mu \mathrm{m}$, ScuBa-2 $850 \mu \mathrm{m}$, AzTEC $1.1 \mathrm{~mm}$, MAMBO $1.2 \mathrm{~mm}$, and VLA $20 \mathrm{~cm}(1.4 \mathrm{GHz})$. The resolutions vary substantially, from $\sim 0.5^{\prime \prime}$ ( $i$-band) to $36^{\prime \prime}(500 \mu \mathrm{m})$. This illustrates the challenge which most submillimeter mapping facilities in distinguishing individual galaxies which have a high spatial density. 
data, the more accurate the input assumptions. The noise map used in these tests is often referred to as a 'jackknife' map and is constructed by taking two halves of the given submm data set and subtracting one from the other; then the noise is scaled down to represent the noise for the total integration time $T$ (as a simple subtraction only represents an integration time of $T / 2$ ). Since real sources should appear in each half of the data, they should not be present in the jackknife map, even at substantially low signal-to-noise. In that sense, the jackknife map represents pure noise.

With some assumption about the underlying distribution of sources in the field, individual delta function sources are convolved with the beam and injected into the jackknife map at random positions (assuming little to no influence from clustering) and, when finished injecting, the entire map is analyzed for source detections. If the distribution in individual point sources extracted from the resulting map mirrors the distribution of real data, then we have learned that the input assumptions might well be representative of the underlying parent population.

Determining an appropriate functional form of the input population (sources per unit flux density per unit sky area, $d N / d S$ ) is an iterative process, taking into account the functional form observed from the raw data map and the observed distributions at other submillimeter wavelengths. $\S 3.3$ addresses the functional approximations of the differential number counts $d N / d S$. Whatever functional form is assumed, the free parameters of $d N / d S$ are adjusted via a Markov Chain Monte Carlo until the differences between real and simulated map number counts are minimized.

Besides measuring agreement between real and simulated map number counts, a few other parameters can be estimated from the simulated maps. These include the positional accuracy of submillimeter sources, the difference between intrinsic flux density and measured flux density, and population contamination and completeness. Figure 11 illustrates an example flow chart of the simulations technique and the output estimates for the given $d N / d S$ formulation.

\subsubsection{Estimating Deboosted Flux Densities}

One of the most critical output estimates from this technique is the correction for flux boosting. Sources' flux densities are boosted in two different ways. The first is the statistical variation around sources' true flux densities. More sources are intrinsically faint than bright, and therefore, more of those faint sources scatter towards higher flux densities than bright sources scatter down towards lower flux densities. This Eddington boosting (first described by Eddington. 1913) assumes that fainter sources are more numerous than brighter sources. The second form of boosting comes from confusion noise, as discussed in $\$ 3.1$, which is caused by sources below the detection threshold contributing flux to sources above the detection threshold. These two boosting factors are independent although their effect is the same so they are measured together as a function of signal-to-noise and flux density (both dependencies are important in the case where maps do not have uniform noise, see further discussion in Crawford et al., 2010). In simulations, the boosting is measured as an average multiplicative factor between input flux density and measured output flux density as a function of output signal-to-noise ratio. Boosting-or inversely, the deboosting factor-is estimated as a function of signal-to-noise because very high signal-to-noise sources are likely to only have a negligible contribution from statistical or confusion boosting.

Note that galaxies who benefit from gravitational lensing either will be detected at a substantially high signal-tonoise or substantially high flux density and thus, only be minimally affected by flux boosting. As the signal-to-noise of such sources becomes high, the fractional contribution of faint sources to measured flux density goes towards zero. Even in the case where the given submm survey is not substantially deep, sources with high flux densities well above the confusion noise threshold are unlikely to be substantially boosted as sources with comparable flux densities are exceedingly rare, and the only sources contributing to boosting will be negligible when compared to the instrumental noise uncertainty.

\subsubsection{Estimating Positional Accuracy}

Along with flux deboosting, the positional accuracy of submillimeter sources is estimated by contrasting the input 'injected' sources with the measured output sources. The output positions might be different from input positions due to confusion from sources nearby or instrumental noise. Like flux deboosting, positional accuracy is measured as a function of source signal-to-noise, as the sources of higher significance will only be marginally impacted by confusion. The average offset between input and output position is roughly indicative of the positional accuracy of submillimeter sources at the given signal-to-noise and has provided the initial search-area for corresponding counterparts at nearIR, mid-IR, and radio wavelengths (e.g. Weiß et al., 2009a; Biggs et al., 2011). Note, however, that Hodge et al. 


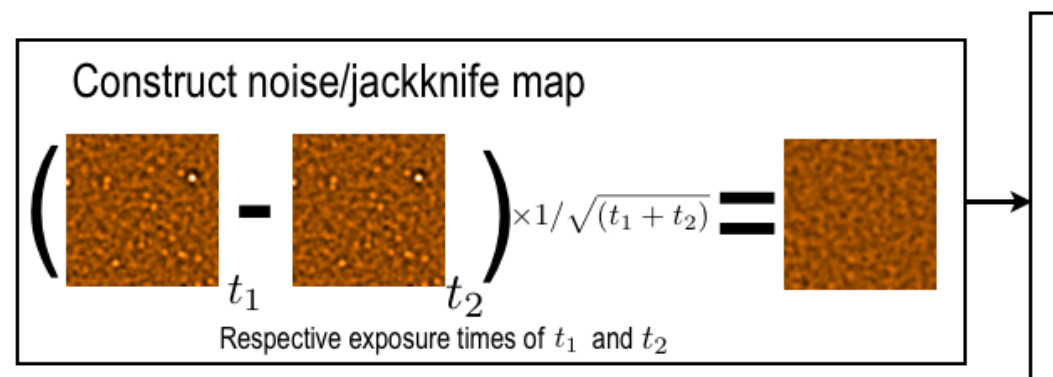

Inject sources drawn from
dN/dS into jackknife map, for example:

$\frac{d N}{d S}= \begin{cases}\frac{N_{0}}{S_{0}}\left(\frac{S}{S_{0}}\right)^{-\alpha} & : S \leq S_{0} \\ \frac{N_{0}}{S_{0}}\left(\frac{S}{S_{0}}\right)^{-\beta} & : S>S_{0}\end{cases}$

Choose best-guess $N_{0}, S_{0}, \alpha, \beta$

Randomize source positions, convolve with beam.

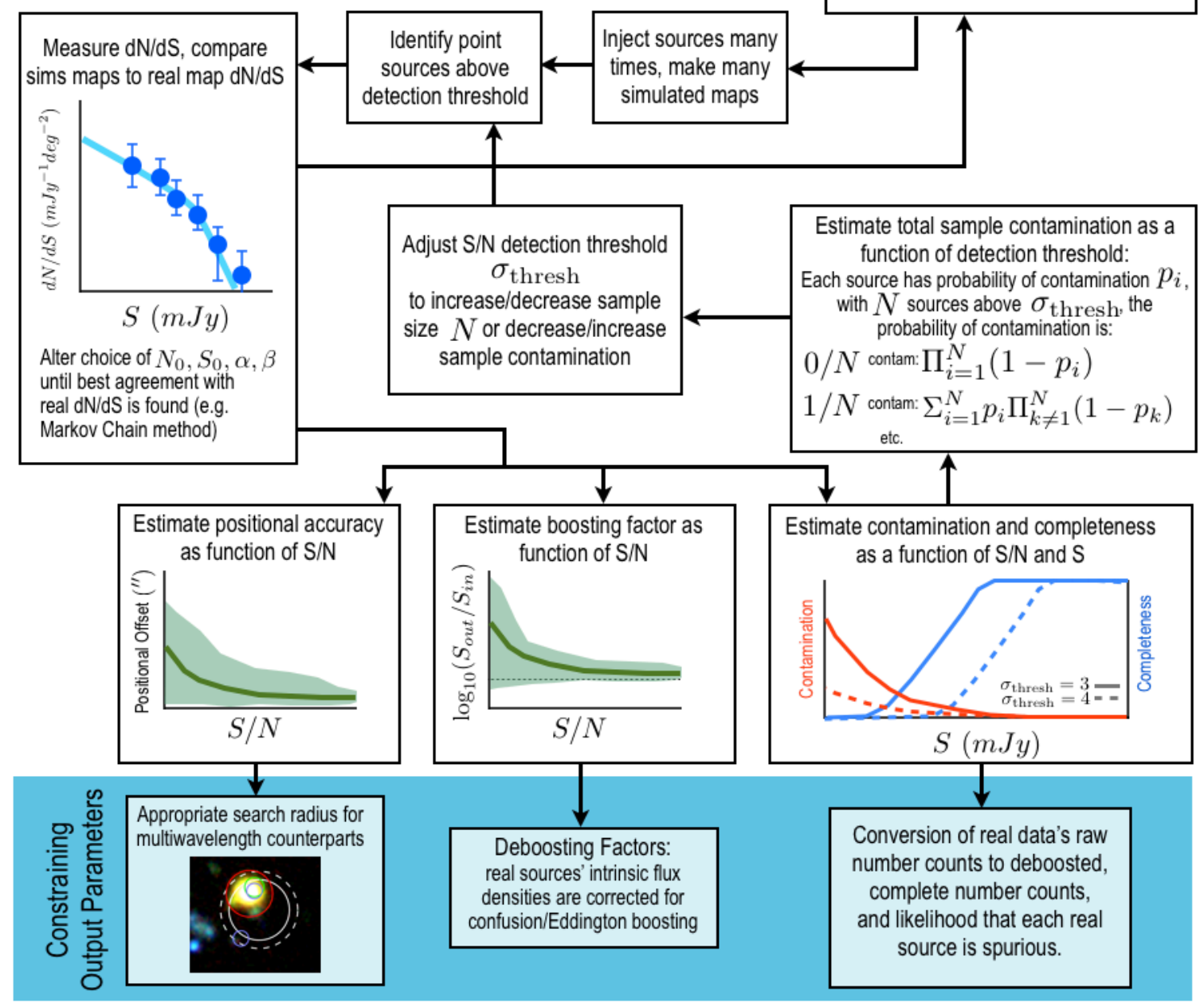

Figure 11: Flow chart illustrating the use of Monte Carlo simulations for analysis of submillimeter maps. The process begins with the construction of a jackknife or pure noise map in which real sources have been removed. Fake sources are then injected into the jackknife map using a number counts model assumption over several iterations. The parameters of the number counts can be optimized using a Markov Chain Monte Carlo technique. Once ideal parameters are determined, the positional accuracy, boosting factor of individual sources can be estimated, along with sample completeness and contamination. Analyzing the contaminating fraction within the whole sample can be used to determine the optimum detection threshold, $\sigma_{\text {thresh }}$. The final constraining output parameters of the Monte Carlo Simulations are: (a) the appropriate search radius to use when looking for multi-wavelength counterparts, (b) the deboosting factor to use when correcting a galaxy's raw measured flux density to intrinsic, and (c) how to convert raw number counts to incompleteness-corrected, deboosted number counts. 
(2013b) use ALMA interferometric follow-up to determine that this method of determining positional accuracy is not completely reliable.

\subsubsection{Estimating Sample Contamination $\mathcal{E}$ Completeness}

Simulations also provide estimates for sample contamination and completeness and can guide a best-choice signalto-noise $(\mathrm{S} / \mathrm{N})$ detection threshold. If a detection threshold is too conservative, contamination will be very low and completeness high, but the given submm source population risks being too small for substantial population analysis or more heavily biased against certain galaxy types, e.g. as $850 \mu \mathrm{m}$ mapping biases against warm-dust systems, discussed in $\$ 2.4 .1$ Conversely, a low detection threshold that is too liberal risks having a high contaminating fraction.

Sample completeness at a given flux density $S$ can be estimated by considering the number of injected sources with $S$ which are recovered in the simulated maps as real detections. The user can adjust the detection threshold to see increases or decreases in completeness. Note that nearly all submillimeter maps have non-uniform noise and that completeness is not only a function of flux density $S$ but also detection signal-to-noise. Sample contamination is also measured as a function of output flux density and signal-to-noise as the fraction of detections which are not expected to be detected based on the input catalog. In other words, a source is considered a contaminant or spurious if the input flux density of sources within a beamsize is substantially lower than the nominal deboosted flux density limit. This can happen if the density of input sources with low flux densities is high, so the conglomerate of flux from multiple sources leads to a single 'spurious' source of much higher flux density. It can also happen when a single source of intrinsic flux density $S$ is boosted significantly above the expected boosting factor at its signal-to-noise.

Together, the sample completeness and contamination can provide a good idea of what the strengths and weaknesses are of a given sample. These quantities can motivate the choice of a certain signal-to-noise threshold if there is a certain target contamination rate in mind. Often a target contamination rate of $\lesssim 5 \%$ in a submillimeter map will result in a detection threshold between $3<\sigma<4$. The contamination rate for an entire sample may be estimated using the probability that each individual source, of a given $\mathrm{S} / \mathrm{N}$, is real or a contaminant.

\subsection{Number Counts}

The measured number counts of a submillimeter map can be given in raw units-measured directly from the map-or deboosted and corrected units-after the sources' flux densities have been deboosted and the sample has been corrected for contamination and incompleteness. The latter is largely what has been published in the literature, albeit using slight variants on the method above used to correct the counts. Depending on the scale of the given survey (small, targeted deep field versus large sky area, shallow survey), the units of number counts are quoted direct or Euclidean-normalized units7 given as galaxies per unit flux density per unit sky area (e.g. $\mathrm{mJy}^{-1} \mathrm{deg}^{-2} \mathrm{or} \mathrm{Jy}^{-1} \mathrm{str}^{-1}$ ) and galaxies times flux density to the 1.5 power per unit sky area (e.g. $\mathrm{mJy}^{1.5} \mathrm{deg}^{-2}$ or $\mathrm{Jy}^{1.5} \mathrm{str}^{-1}$, calculated as $d N / d S \times S^{2.5}$ ) respectively.

The $850 \mu \mathrm{m}$ number counts are the best-studied amongst submillimeter maps with over 15 literature sources reporting independent measurements spanning four orders of magnitude in flux density; another regime where the number counts are fairly well constrained, although only recently, are at 450-500 $\mu \mathrm{m}$, from recent Herschel and ScuBA-2 measurements over three orders of magnitude. Figure 12 illustrates both $850-870 \mu$ m number counts (from Scuba, Laboca, Scuba-2, and ALMA Blain et al. 1999: Scott et al., 2002, Chapman et al. 2002, Cowie et al., 2002, Borys et al. 2003, Webb et al., 2003, Barnard et al., 2004, Coppin et al., 2006, Scott et al., 2006; Knudsen et al., 2008, Beelen et al., 2008; Weiß et al. 2009a Karim et al. 2013, Casey et al. 2013, Chen et al. 2013a) and 450-500 $\mu$ m (from Scuba, Herschel and Scuba-2 Smail et al., 2002, Oliver et al., 2010, Clements et al., 2010, Béthermin et al., 2012b, Geach et al., 2013: Casey et al., 2013, Chen et al., 2013a).

Figure 13 illustrates other infrared and submillimeter number counts in the literature in direct units, from $70 \mu \mathrm{m}$ (Dole et al., 2004; Béthermin et al. 2010a, Berta et al., 2011), 100 $\mu \mathrm{m}$ (Héraudeau et al., 2004; Rodighiero \& Franceschini. 2004; Kawara et al. 2004; Berta et al., 2011; Magnelli et al. 2013b), 160 $\mu \mathrm{m}$ (Dole et al., 2004: Kawara et al., 2004 Béthermin et al. 2010a: Berta et al. 2011; Magnelli et al. 2013b), 250 $\mu \mathrm{m}$ and 350 $\mu \mathrm{m}$ (Patanchon et al., 2009.

\footnotetext{
${ }^{7}$ Euclidean-normalized units in this context are normal number counts multiplied by flux density to the 2.5 power. They are useful for converting an observed function which varies over several orders of magnitude to something relatively 'flat' where functional fits to data are performed simply and the extreme ends of the datasets do not dominate fit solutions.
} 

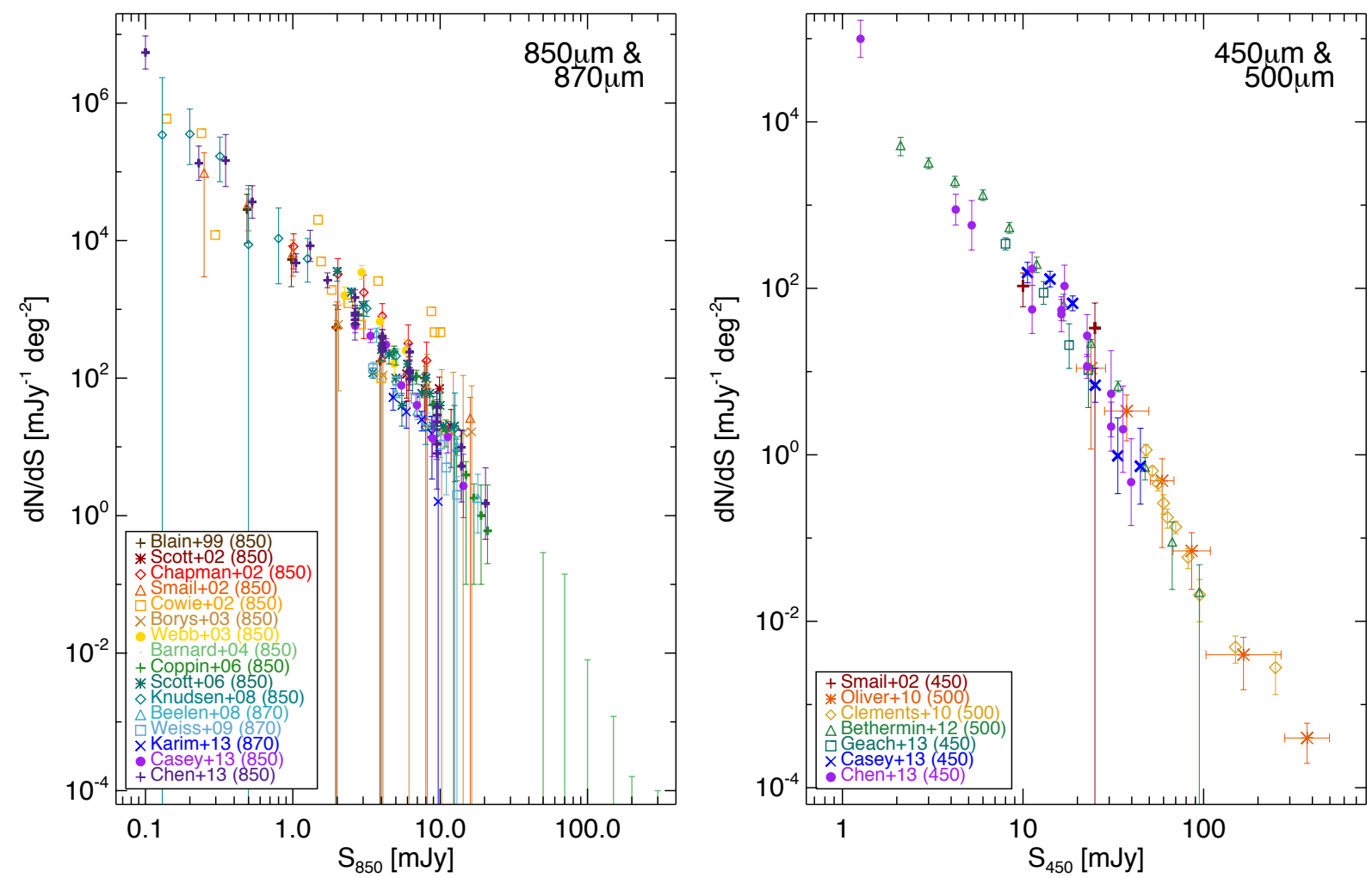

Figure 12: Differential submillimeter number counts at $850 \mu \mathrm{m} / 870 \mu \mathrm{m}$ (left) and $450-500 \mu \mathrm{m}$ (right). The $850 \mu \mathrm{m}$ and $870 \mu \mathrm{m}$ number counts come the initial Scuba surveys (Blain et al. 1999, Scott et al. 2002, Chapman et al. 2002, Cowie et al. 2002, Borys et al. 2003, Webb et al. 2003 , Barnard et al. 2004. Coppin et al. |2006. Scott et al. |2006.|Knudsen et al. |2008 , shown in brown, dark red, red, dark orange, orange, dark gold yellow, light green, green, dark teal, and teal respectively). Data from Beelen et al. (2008), Weiß et al. (2009a), and Karim et al. (2013) are taken at $870 \mu \mathrm{m}$ rather than $850 \mu \mathrm{m}$, the two former from LABOCA and the latter from interferometric ALMA data. ScuBa-2 $850 \mu \mathrm{m}$ data from Casey et al. (2013) and Chen et al. (2013b) are also plotted, the latter including the lens field work of Chen et al. (2013a). The Cowie et al. (2002) results do not quote uncertainties and the Barnard et al. (2004) results represent upper limits on number counts at very high flux densities, covering larger areas than the nominal Scuba survey. The work of Blain et al. (1999), Smail et al. (2002), Cowie et al. (2002), Knudsen et al. (2008) and Chen et al. (2013a) used gravitational lensing in cluster fields to survey flux densities $<1 \mathrm{mJy}$.

Oliver et al. 2010; Clements et al., 2010; Béthermin et al. 2010b, 2012b), and $1.1 \mathrm{~mm}$ (Perera et al., 2008; Austermann et al. 2010; Scott et al. 2010; Hatsukade et al., 2011; Aretxaga et al. 2011; Scott et al. 2012).

Figure 14 gathers all of these results together and plots all submillimeter number counts together in Euclideannormalized units, allowing for a more clear view of how the slope, normalization, and intrinsic variance vary between selection wavelengths and what relative dynamic range is probed at each wavelength. $\$ 3.6$ goes into greater detail of what these measurements imply for resolving the cosmic infrared background.

\subsection{Parametrizing Number Counts}

Observed number counts are often fit to functional forms which assume a certain shape for the underlying distribution. This parametrization often is given as a Schechter function

$$
\frac{d N}{d S}=\frac{N_{0}}{S_{0}}\left(\frac{S}{S_{0}}\right)^{-\alpha} e^{-\left(\frac{S}{S_{0}}\right)}
$$



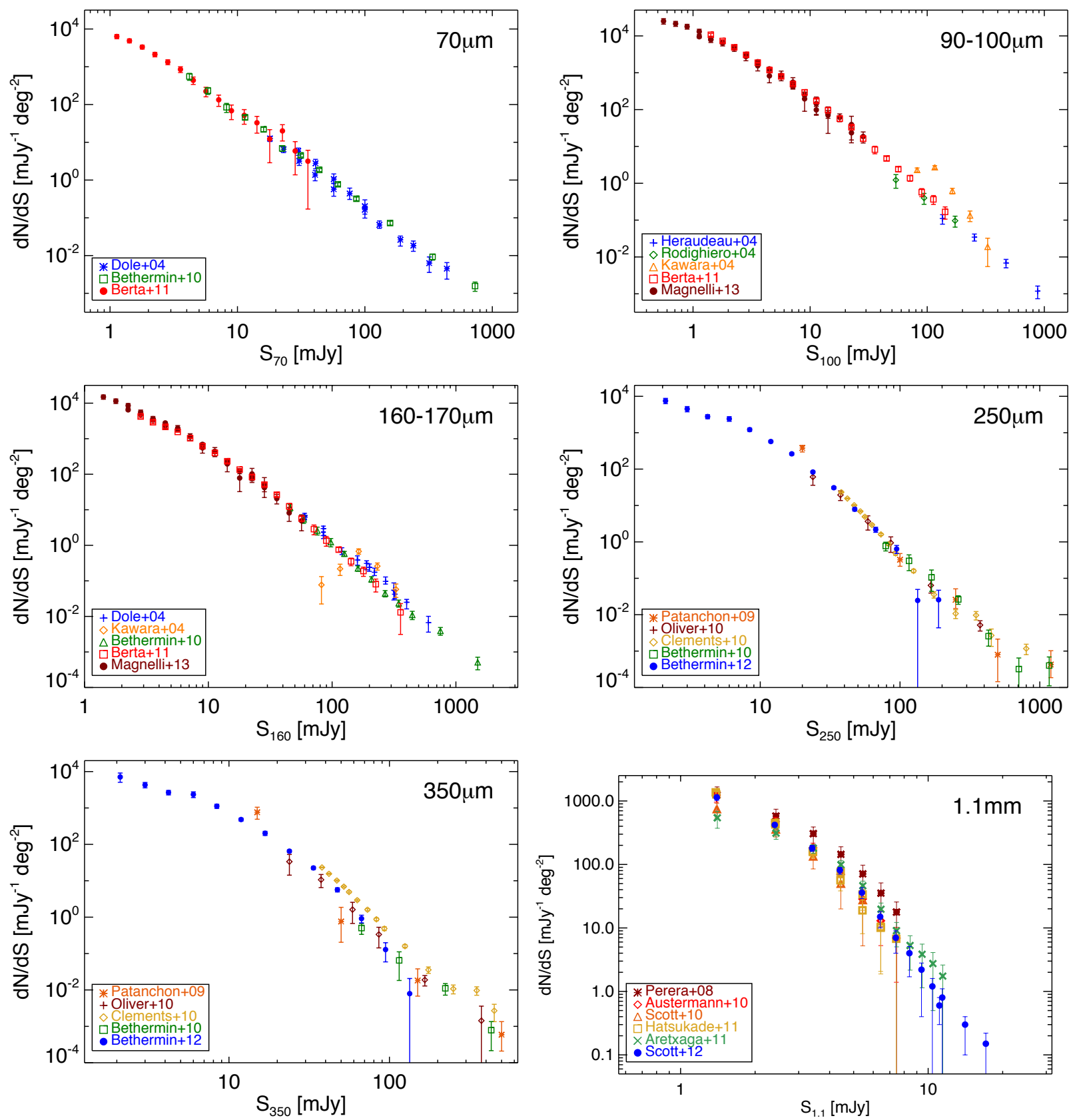

Figure 13: Differential submillimeter number counts at $70 \mu \mathrm{m}, 100 \mu \mathrm{m}, 160 \mu \mathrm{m}, 250 \mu \mathrm{m}, 350 \mu \mathrm{m}$, and $1.1 \mathrm{~mm}$. The $70 \mu \mathrm{m}$ data is collated from Spitzer MIPS (Dole et al. 2004 Béthermin et al. 2010a) and Herschel PACS (Berta et al. 2011). At 100 $\mu \mathrm{m}$, data are from the ISOPHOT instrument aboard ISO (Héraudeau et al. 2004 Rodighiero \& Franceschini |2004, Kawara et al.| 2004) and Herschel PACS (Berta et al. |2011, Magnelli et al. 2013b). At $160 \mu \mathrm{m}-170 \mu \mathrm{m}$, data are from Spitzer MIPS (Dole et al. |2004 |Béthermin et al. 2010a), ISO ISOPHOT (Kawara et al. |2004), and Herschel PACS (Berta et al. 2011. Magnelli et al. 2013b). At both $250 \mu \mathrm{m}$ and 350 $\mu \mathrm{m}$, data come from BLAST (Patanchon et al. 2009 |Béthermin et al. 2010b) and Herschel SPIRE (Oliver et al. 2010. Clements et al. 2010, Béthermin et al. 2012b). All $1.1 \mathrm{~mm}$ number counts studies have been undertaken with the AzTEC instrument on JCMT and ASTE and summarized in Scott et al. (2012), including prior datasets described in Perera et al. (2008), Austermann et al. (2010), Scott et al. (2010), Hatsukade et al. (2011), and Aretxaga et al. (2011). 


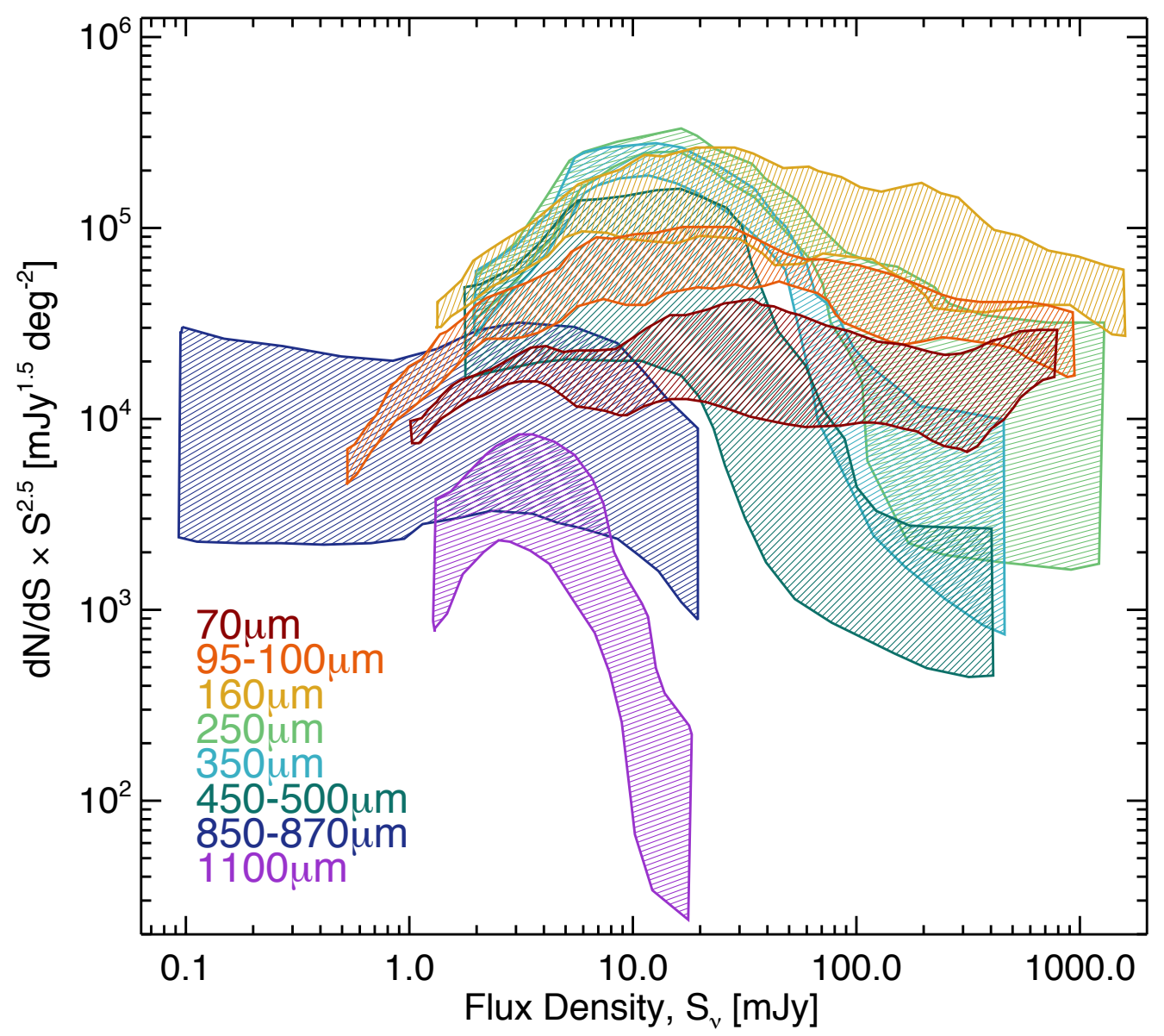

Figure 14: All differential submillimeter number counts replotted from Figures 12 and 13 in Euclidean units. For clarity in plotting, the individual points have been removed and replaced with a polygon representing the median $1 \sigma$ spread in number counts measurements. 


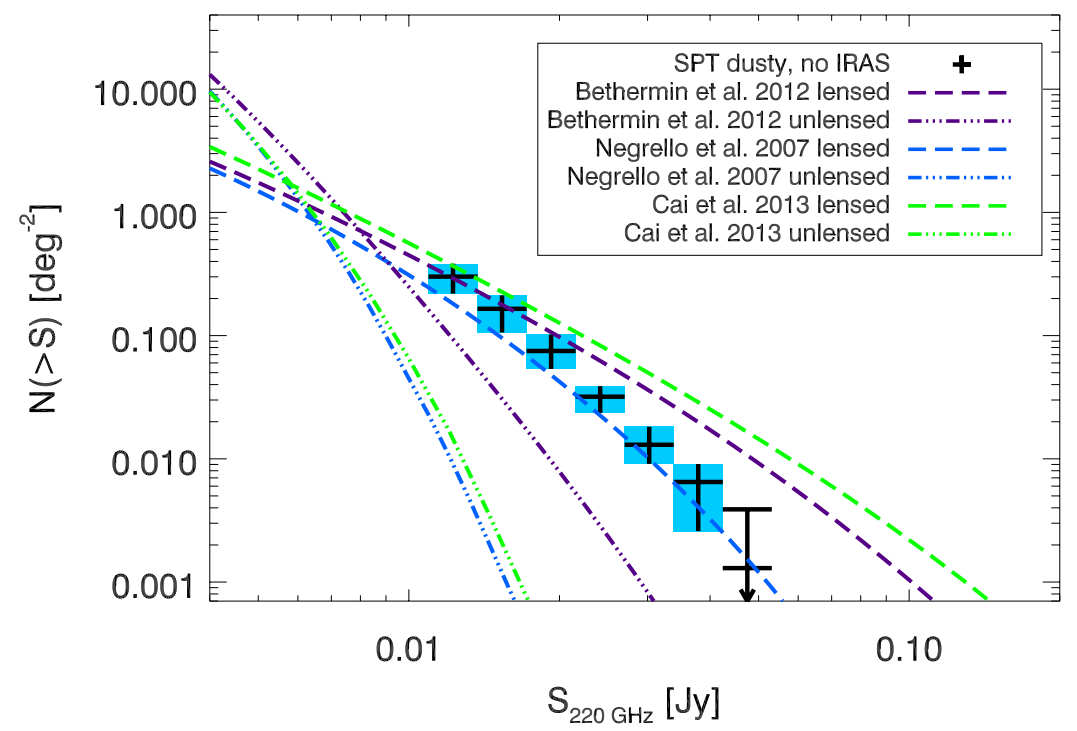

Figure 15: The $1.4 \mathrm{~mm}$ number counts of gravitationally lensed dusty sources from the South Pole Telescope from their $770 \mathrm{deg}^{2}$ survey, after removal for nearby non-lensed IRAS-luminous galaxies. Three predictive models for unlensed and lensed galaxies are compared: Negrello et al. (2007), Béthermin et al. (2012b), and Cai et al. (2013). The Negrello et al. (2007) model combines a physical model from Granato et al. (2004) on the evolution of spheroidal galaxies with phenomenological models on starburst, radio and spiral galaxy populations. The Béthermin et al. [2012b] model is an empirical model based on two star formation modes-main sequence and starburst. The Cai et al. (2013) model is a physically forward model evolving spheroidal galaxies and AGN with a backwards evolution model for spiral type galaxies. This figure is reproduced from Mocanu et al. (2013) with permission from the authors and AAS.

or a double power law

$$
\frac{d N}{d S}= \begin{cases}\frac{N_{0}}{S_{0}}\left(\frac{S}{S_{0}}\right)^{-\alpha} & : S \leq S_{0} \\ \frac{N_{0}}{S_{0}}\left(\frac{S}{S_{0}}\right)^{-\beta} & : S>S_{0}\end{cases}
$$

While both typically provide reasonable fits to any given dataset over a narrow dynamic range, it is important to recognize that the shape of the number counts is probably intrinsically much more complex. Number counts represent flux density, not luminosity, so the conversion from a physically-motivated luminosity function to an observationallyderived number count function might not be straightforward. For example, if a Schechter function is the assumed shape to a luminosity function and that luminosity function evolves gradually with redshift, then the resulting number counts function will be non-Schechter; local galaxies influence the high flux density end, along with lensed galaxies, while the bump at lower flux densities is dominated by moderate to high-redshift galaxies $(0.5<z<2.5$, depending on the selection wavelength). An excellent paper summarizing power-law distributions in empirical data, their applicability and some common flaws in their overuse, is given by Clauset et al. (2007). The Clauset et al. discussion would be readily applicable to submillimeter number counts.

\subsection{Bright-End Counts: Gravitationally Lensed DSFGs}

Gravitationally lensed DSFGs constitute a significant fraction of the bright-end number counts at submm wavelengths at or above $500 \mu \mathrm{m}$. This is for two reasons. First, submm galaxy number counts are steep with a sharp intrinsic cut-off, thus flux magnification by gravitational lensing moves sources from the the steep faint end to the bright end and produce counts that are flatter at the bright flux densities (this was first discussed as an interesting sub-population in Blain 1996). Second, due to negative $K$-correction, longer wavelength observations probe higher redshift galaxies where the optical depth to lensing is rapidly increasing (see Figure 6 of Weiß et al. 2013). At wavelengths shorter than $500 \mu \mathrm{m}$, while lensed DSFGs do exist, the bright-end counts are dominated by star-forming galaxies at low red- 
shifts where the lensed counts do not make up an appreciable fraction (Negrello et al., 2007; Bussmann et al., 2013, Wardlow et al. 2013).

At all submm wavelengths the bright-end number counts can be reconstructed from local galaxies, active galactic nuclei such as radio blazars, and distant lensed infrared galaxies. The lensed sources can be identified relatively easily when submm maps are combined with multi-wavelength data. Local galaxies are easy to cross-identify through wide area shallow optical surveys such as SDSS while radio blazars are easily identifiable with shallow radio surveys such as NVSS. Theoretical predictions are such that at $500 \mu \mathrm{m}$, once accounting for local galaxies and radio blazars, lensed DSFGs should make up all of the remaining counts at $S_{500}>80 \mathrm{mJy}$ (Negrello et al., 2007). This implies that there are no DSFGs at high redshifts with intrinsic $500 \mu \mathrm{m}$ flux densities above $80 \mathrm{mJy}$. Observationally, this has yet to be properly tested but there are tentative results indicating that the lensed fraction is below $100 \%$ due to rare sources such as DSFG-DSFG mergers (Fu et al., 2013; Ivison et al., 2013). Existing followup results show that the sources that are neither associated with local galaxies nor radio blazars are gravitationally lensed with an efficiency better than $90 \%$ at $500 \mu \mathrm{m}$ (Negrello et al., 2010, Wardlow et al. 2013). At $1.4 \mathrm{~mm}$ a similar (or better) success rate at identifying lensed DSFGs is clear with bright sources $\left(S_{1.4}>60 \mathrm{mJy}\right)$ in the arcminute-scale cosmic microwave background (CMB) anisotropy maps made with the South Pole Telescope (SPT) (Vieira et al. 2010, 2013; Mocanu et al., 2013). In Figure 15 at $1.4 \mathrm{~mm}$ from SPT in contrast to several models. In $\$ 6$ we will return to lensed DSFGs and review the key results that have been obtained with multi-wavelength followup programs. As the sources magnified, the improvement in both the flux density and the spatial resolution facilitates the followup of lensed galaxies over the intrinsic population.

\subsection{The Cosmic Infrared Background and $P(D)$ Analysis}

The total intensity of the cosmic infrared background (CIB) at submm wavelengths is now known from absolute photometry measurements (Puget et al., 1996; Fixsen et al., 1998; Dwek et al. 1998). While the integrated intensity is known, albeit highly uncertain, we still do not have a complete understanding of the sources responsible for the background, the key reason being that existing surveys are limited by large beamsizes and confusion noise. For example, recent deep surveys with Herschel and ground-based sub-mm and mm-wave instruments have only managed to directly resolve about $5 \%$ to $15 \%$ of the background to individual galaxies at wavelengths longer than $250 \mu \mathrm{m}$ (Coppin et al. 2006; Scott et al. 2010; Oliver et al. 2010; Clements et al., 2010). At far-infrared wavelengths of 100 and $160 \mu \mathrm{m}$, deep surveys with Herschel/PACS have now resolved $\sim 60 \%$ and $75 \%$ of the COBE/DIRBE CIB intensity (Berta et al. 2011). Additional aid from gravitational lensing in cluster fields and higher resolution $450 \mu \mathrm{m}$ mapping with ScuBa-2 mean that $\sim 50 \%$ of the $450 \mu \mathrm{m}$ background has been resolved (Chen et al. 2013a). The notable exception in this realm of direct detection of the faintest sources contributing to the CIB is the recent work of Hatsukade et al. (2013) who summarize the faint end of the $1.3 \mathrm{~mm}$ number counts from serendipitous detections within targeted ALMA follow-up observations. Assuming no correlation from clustering or lensing, they claim to resolve $80 \%$ of the CIB at $1.3 \mathrm{~mm}$.

Note that Béthermin et al. (2012b) use a stacking analysis of $24 \mu \mathrm{m}$-emittors (similar to the methodology outlined by Marsden et al., 2009, Pascale et al. 2009, used for analysis of BLAST data) using Herschel data to recover the FIRAS CIB and estimate the underlying redshift distribution of $250 \mu \mathrm{m}, 350 \mu \mathrm{m}$, and $500 \mu \mathrm{m}$ sources. The resulting estimates to number counts (extrapolated well beyond the nominal Herschel confusion limit) held up to more recent results from Scuba-2 (Chen et al., 2013a; Geach et al. 2013; Casey et al., 2013). Furthermore, Viero et al. (2013b) use a stacking analysis on $\sim 80,000 K$-band selected sources based on optical color selection, which is largely independent of mid-IR or far-IR emission. Viero et al. claim to resolve $\sim 70 \%$ of the CIB at $24 \mu \mathrm{m}, 80 \%$ at $70 \mu \mathrm{m}, 60 \%$ at $100 \mu \mathrm{m}$, $80 \%$ at $160 \mu \mathrm{m}$ and $250 \mu \mathrm{m}, \sim 70 \%$ at $350 \mu \mathrm{m}$ and $500 \mu \mathrm{m}$ and $45 \%$ at $1100 \mu \mathrm{m}$. Of those resolved sources, $95 \%$ of sources are star-forming galaxies and 5\% are quiescent. They go on to suggest that the galaxies which dominate the CIB have stellar masses $\sim 10^{9.5-11} \mathrm{M}_{\odot}$, and that the $\lambda<200 \mu \mathrm{m}$ CIB is generated at $z<1$ while the $\lambda>200 \mu \mathrm{m} \mathrm{CIB}$ is generated at $1<z<2$.

While individual detections are confused by fainter sources, in maps where the confusion noise dominates over the instrumental noise, important statistical information on the fainter sources that make up the confusion can be extracted from the maps. Probability of deflection statistics, $P(D)$ analysis, focuses on the pixel intensity histogram after masking out the extended (and sometimes bright) detected sources. The measured histogram in the data is then either compared to histograms made with mock simulation maps populated with fainter sources below the confusion with varying levels of number counts, both in terms of the number count slope and the overall normalization, or 
they are analyzed with the FFT formalism. With an accounting of the noise and noise correlations across the map, the simulations can be used to constrain the faint-end slope and count normalization that gives the best matching histogram to the data. Again, a major caveat of $P(D)$ analysis is the lack of understanding for population clustering and its impact on residual flux in a map.

These $P(D)$ statistics capture primarily the variance of the intensity in the map at the beam scale and, to a lesser extent, higher order cumulants of the intensity variation, again at the beam scale. $P(D)$ method allows a constraint on the faint-end counts below confusion and has been used widely in sub-mm maps since the SCUBA surveys (Hughes et al. 1998). Patanchon et al. (2009) expanded the technique with a parameterized functional form for faint-end counts with knots and slopes. The number count model parameters extracted through the $P(D)$ histogram data under such a model are correlated and these correlations need to be taken into account either when comparing faint-end $P(D)$ counts to number count models or when using $P(D)$ counts to distinguish cosmological models of the DSFG population. Extending below the nominal confusion limit of about 5-6 mJy of Herschel-SpIRE at 250-500 $\mu \mathrm{m}, P(D)$ studies have allowed the counts to be constrained down to the $1 \mathrm{mJy}$ level. The parameterized model-fits to the $P(D)$ counts resolve $\sim 60 \%$ and $45 \%$ of the CIB intensity at $250 \mu \mathrm{m}$ and $500 \mu \mathrm{m}$, respectively (Glenn et al., 2010). This is a significant improvement over the CIB fraction resolved by point sources counts alone that are at $15 \%$ and $6 \%$ at $250 \mu \mathrm{m}$ and $500 \mu \mathrm{m}$ respectively.

An alternative method, outlined by Refregier \& Loeb (1997) involves the lensed intensity pattern through a massive galaxy cluster when compared to the intensity away from the cluster. The technique can be understood as follows: when viewed through the galaxy cluster, faint distant DSFGs will be magnified by a factor $\mu$. But this resulting increase in the flux density is compensated by a decrease in the volume probed such that the total number counts seen through the cluster changes to $N / \mu$. If the faint-end count slope scales as $d N / d S \propto S^{-\alpha}$, then through the cluster is modified to $d N^{\prime} / d S \propto \mu^{\alpha-2} S^{-\alpha}$. There is either an enhancement or a decrement of fainter sources towards the galaxy cluster, relative to the background population, depending on whether $\alpha<2$ or $>2$. Averaged over the whole cluster, the effect will result in no signal as the total surface brightness is conserved under gravitational lensing. A measurement of the decrement has been reported with Herschel-SpIRE maps of four galaxy clusters in Zemcov et al. (2013). Instead of constraining the faint-end number counts below the confusion level authors used the lensing profile to obtain an independent measurement of the CIB level at SPIRE wavelengths, subject to uncertainties in the absolute flux calibration of SPIRE maps. They report a CIB intensity of $0.69_{-007}^{+0.12} \mathrm{MJy} \mathrm{sr}^{-1}$ at $250 \mu \mathrm{m}$. The $C O B E$ CIB measurement at $240 \mu \mathrm{mis}$ at the level of $0.9 \pm 0.2 \mathrm{MJy} \mathrm{sr}^{-1}$ (Puget et al., 1996, Fixsen et al., 1998; Dwek et al., 1998). While the two agree within one $\sigma$ uncertainties, the lensing-based measurement is likely an underestimate as it only focuses on the sources behind the cluster and a separate accounting of the sources in the foreground needs to be made to obtain the total background intensity. In any case the demonstration of Zemcov et al. (2013) shows that more detailed statistical measurements on the fainter source can be obtained through galaxy cluster lensing and the expectation is that future instruments, including ALMA, will exploit this avenue for further studies. 


\section{Redshifts and Spectral Energy Distributions of Infrared-Luminous Galaxies}

This section describes some of the basic bulk characteristics of distant dusty infrared star-forming galaxies. Included for discussion is the redshift distributions of DSFGs-how they have been measured in the past and how they will likely be measured en masse in the future-along with best estimates of luminosity functions and the total contribution of some DSFG populations to the cosmic infrared luminosity density or star formation rate density. Once redshift is in hand, we also discuss how one estimates the basic physical characteristics from a far-IR SED fit, whether using direct analytic fits or templates.

\subsection{Acquiring Spectroscopic or Photometric Redshifts}

Before the physical nature of individual DSFGs or the bulk nature of their population can be understood, redshifts are needed. As described in the first section of this review, $\S 2$, redshift acquisition is unfortunately not straightforward for dusty, infrared-selected samples. Efforts to secure redshifts are hampered by significant extinction in the rest-frame ultraviolet and optical, where most of the classic emission-line redshift indicators lie, and the large beamsize of infrared/submillimeter observations which makes multi-wavelength counterpart identification ambiguous (see $\S 2.4 .2$.

The ambiguity of multi-wavelength counterpart matching has historically led to a dependence on intermediate bands for counterpart identification. $\$ 2.4 .2$ describes how radio wavelengths and mid-infrared $24 \mu \mathrm{m}$ imaging has often been used as such an intermediate band by which counterpart galaxies can be identified on 1-2" scales, providing the precision needed to acquire redshifts.

In general, high-redshift galaxies may be studied in detail with either photometric or spectroscopic redshift identifications. Although the former is less precise and less reliable, the increase of available multi-wavelength coverage in deep extragalactic fields (e.g. as in the COSMOS field; Scoville et al., 2007) has made photometric redshifts increasingly reliable (e.g. Ilbert et al. 2009) and much less observationally expensive than obtaining spectroscopic redshifts. Spectroscopic redshifts for faint $(i \sim 23-25)$ galaxies might require a few hours of integration on an $>8 \mathrm{~m}$ class telescope. One might conclude that, if no further analysis of the galaxies' optical/near-IR spectra are needed, photometric redshifts are preferable particularly when counterparts are ambiguous. However, there are a few important details which must be kept in mind when considering redshifts for DSFGs in contrast to more 'normal' high-redshift galaxies.

First, dusty, infrared-selected galaxies are subject to significant optical extinction. More than making counterpart identification difficult, this could impact the quality and reliability of photometric redshift estimates. Spectroscopic campaigns of $\sim 500 \mathrm{M}_{\odot} \mathrm{yr}^{-1} 850 \mu \mathrm{m}$-selected SMGs have often found bright emission lines with no detected continuum (Chapman et al., 2003b, 2005), with emission-line-to-continuum ratios far higher than observed in, e.g. 10 $\mathrm{M}_{\odot} \mathrm{yr}^{-1}$ Lyman-Break Galaxies (Shapley et al. 2003). The quality of photometric redshifts for SMGs, or any similar dusty, high-SFR DSFG, relies on the input stellar population model assumptions accounting for bursty star formation episodes, very significant extinction factors, and very high star formation rates. While a broad range of stellar population models have been successfully used to measure accurate photometric redshifts of optically-identified galaxies, no systematic study of their reliability for dustier, starbursts galaxies has been carried out, and often, photometric redshifts have been found to fail catastrophically for DSFGs (e.g. Casey et al., 2012b).

Second, in the era when submillimeter surveys had a smaller angular footprint on the sky, the preferred method of redshift acquisition was different. Pre-2009, the sky coverage of submillimeter maps was limited to a few square degrees scattered about the sky in a few legacy fields and around several galaxy clusters. The latter tended to lack sufficiently deep optical ancillary data needed to compute reliable photometric redshifts. Given individual areas only several-to-tens of square arcminutes in size, spectroscopic follow-up with instruments of a similar field-of-view was often more efficient and precise. Furthermore, spectroscopic identifications have the added benefit of facilitating follow-up kinematic and dynamical studies, like resolved $\mathrm{H} \alpha$ observations from adaptive optics IFUs or CO molecular gas observations 8

Although more recent infrared observatories have dramatically increased the angular footprint of submm mapping, and thus dramatically increased the number of detected DSFGs, the same limitations in ancillary data apply, with most

\footnotetext{
${ }^{8}$ Throughout the 2000 s, the bandwidth for most millimeter line observations was narrow, so spectroscopic redshifts needed to be very secure to observe CO lines.
} 


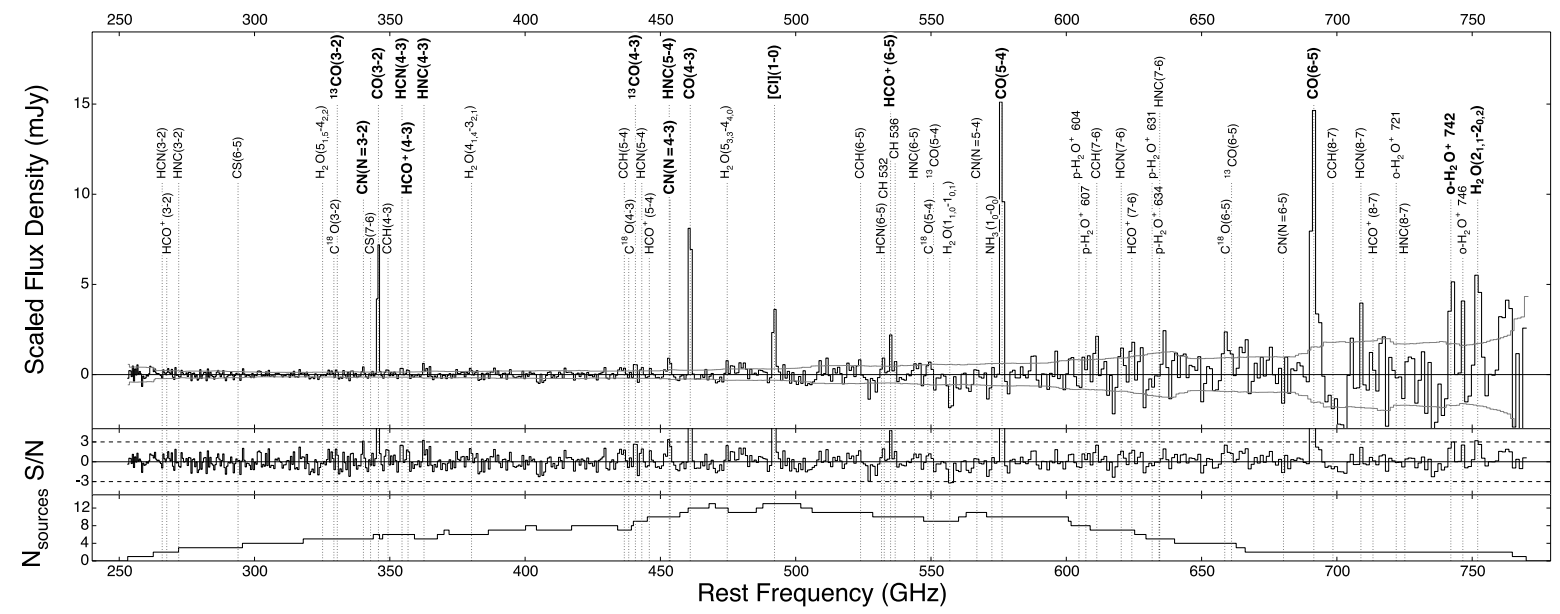

Figure 16: A composite, continuum-subtracted millimeter spectrum of 22 South Pole Telescope-detected brightly lensed DSFGs. These DSFGs constitute the most complete, unambiguously identified DSFG sample with spectroscopic redshifts. This figure is reproduced with permission from Spilker et al., submitted.

of the mapped sky not having sufficiently deep multi-wavelength data to analyze samples by photometric redshift or even secure reliable radio or mid-infrared counterparts for optical/near-infrared spectroscopic follow-up. For the most rare and scattered submillimeter sources on the sky, millimetric photometric redshifts might be the only initial handle we have on their redshifts, and perhaps millimetric spectroscopic redshifts are the most efficient follow-up technique. When considering different methods of redshift acquisition, the scale of survey, availability of ancillary data, and maximum science gain should all be considered.

\subsubsection{Millimetric Spectroscopic Redshifts}

While millimetric spectroscopic redshifts-the ability to spectroscopically confirm a DSFG directly in the millimeter via mm-wavelength molecular gas emission lines-were a pipe dream for much of the 2000s, the past few years have seen significant advances in the area. The primarily limitation in prior years was receiver bandwidth. Existing correlators' bandwidths were always too narrow to serve as an efficient method of searching for galaxies' redshifts. One of the first improvements in widening millimetric receivers' bandwidths came with WIDEX on the Plateau de Bure Interferometer (PdBI). WIDEX is a 3.6 GHz bandwidth dual polarization correlator, a factor of four improved over its predecessor correlators. In a few short years with a variety of widened receivers, this direct method of spectroscopic confirmation was proven, e.g. using the EMIR receivers at the IRAM $30 \mathrm{~m}$ (Weiß et al., 2009a), the Z-SPEC instrument at the Caltech Submillimeter Observatory (Bradford et al. 2009), Zeus also at the CSO (Nikola et al. , 2003), the Zpectrometer on the Green Bank Telescope (Harris et al., 2007, 2010, 2012), the redshift search receiver for the the Large Millimeter Telescope (UMASS; Five College Radio Astronomy Observatory) or the upgraded receivers on the Combined Array for Research in Millimeter-wave Astronomy (CARMA, e.g. Riechers et al., $2010 \mathrm{~b})$. The benefits of the millimetric spectroscopic technique are huge: there is no uncertainty with respect to multi-wavelength counterpart identification and these galaxies are already detected in the millimeter and expected to have luminous mm emission lines facilitating spectroscopic identification. ALMA is optimally designed to follow-up DSFGs in the submillimeter and identify redshifts via, e.g., CO molecular gas or the CII cooling line. This type of follow up has been done for brightly lensed DSFGs detected by the South Pole Telescope (Vieira et al., 2013). Figure 16 illustrates a composite millimeter spectrum from these blindly CO-identified DSFGs (Spilker et al., in preparation). Unfortunately this technique is not yet sufficiently efficient to obtain redshifts for substantially large populations of unlensed DSFGs (current limitations are to $\leq 100$ for lensed DSFGs, and $\leq 10$ for unlensed DSFGs), but once ALMA enters full-science observations, DSFG follow-up will become an efficient way of confirming source redshifts. 


\subsubsection{Millimetric Photometric Redshifts}

An alternate form of redshift determination which has gained some traction in the past few years is millimetric photometric redshifts (e.g. see Carilli \& Yun, 1999, Hughes et al., 2002, Aretxaga et al., 2007, Chapin et al., 2009, Yun et al. 2012; Roseboom et al., 2012; Barger et al., 2012; Chen et al. 2013a). These 'millimetric redshifts' are determined from the shape of the far-infrared or submillimeter SED or its colors rather than any properties indicated by its stellar emission characteristics in the optical/near-IR or emission line signatures. This method assumes that the far-infrared SED of DSFGs is roughly fixed (e.g. to an Arp 220 SED, or an SED with some adopted representative temperature) and the FIR colors can be used to estimate the galaxy's redshift. This technique can be useful to roughly estimate sources' redshifts if they are impossible to probe using other methods.

An important observation to make about millimetric redshifts is that the precision can be quite poor and its accuracy is dependent on the intrinsic variation in SED types for the given population. As will be addressed in $\S 5.2$. temperature, manifested as SED peak wavelength correlates with infrared luminosity and both can be degenerate with redshift. An SED which has a dust temperature between 30-50 K9 the rest-frame peak wavelength can vary between $72-125 \mu \mathrm{m}$. If a $30 \mathrm{~K} \mathrm{SED}$ is used to estimate that galaxy which peaks in the observed-frame at $400 \mu \mathrm{m}$, the millimetric photometric redshift will be $z=2.2$. If the $50 \mathrm{~K} \mathrm{SED} \mathrm{(approaching} \mathrm{the} \mathrm{temperature} \mathrm{of} \mathrm{some} \mathrm{typical} \mathrm{local}$ ULIRGs, e.g. Arp 220 and Mrk 231) is assumed then the millimetric redshift will be $z=4.6$. So while millimetric photometric redshifts can be quite useful to gauge the overall redshift regime of DSFGs (e.g. is it at $z \sim 1$ or $z \sim 5$ ?) and the bulk redshift distribution of a population, they should not be regarded as precise on a case-by-case basis. In contrast, several works use millimetric redshifts to study redshift distributions in a statistical sense, as in e.g. Greve et al. (2012), which can be useful to understand aggregate populations.

\subsubsection{Redshift Distributions of $24 \mu \mathrm{m}$ selected DSFG populations}

Desai et al. (2008) and Fiolet et al. (2010) addresses the redshift distribution of $24 \mu \mathrm{m}$-selected sources by confirming a set of $>400$ sources spectroscopically out of $\sim 600$ targeted. They find that the redshift distribution of $24 \mu \mathrm{m}$ galaxies brighter than $300 \mu \mathrm{Jy}$ peaks at $z \sim 0.3$ with a possible second peak at $z \sim 0.9$ and a tail of galaxies detected out to $z \sim 4.5$. This confirmed earlier photometric work on $24 \mu \mathrm{m}$ sources in COSMOS (Le Floc'h et al., 2005) which finds the vast majority of sources at $z<1$, with a clear link between $24 \mu \mathrm{m}$ and $K$-band populations. Given the $24 \mu \mathrm{m}$ observed selection function (shown in Figure 7), this is along the lines of what might be expected given the enhanced sensitivity of observed $24 \mu \mathrm{m}$ at very low redshift and in certain redshift ranges that align the $24 \mu \mathrm{m}$ band with bright PAH emission features (see more in $\$ 5.7$ ). Desai et al. (2008) also find a population of extremely infrared luminous DSFGs at $z \sim 2$ that, although difficult to spectroscopically confirm due to wavelength restrictions of optical spectrographs, were thought to be largely dominated by AGN-driven emission $(\sim 55 \%)$.

This population of $z \sim 224 \mu \mathrm{m}$-selected DSFGs was studied in detail by Dey et al.(2008) who outline the selection of Dust Obscured Galaxies (DOGs) to especially target this extremely luminous subset of the $24 \mu \mathrm{m}$-population at $\langle z\rangle=1.99 \pm 0.45$. The AGN contribution to these DOGs' luminosity is estimated at $\sim 50 \%$, although this fraction is a function of observed $24 \mu \mathrm{m}$ flux density, $S_{24}$, with fainter targets less dominated by AGN-driven emission. Fiore et al. (2008) present a similarly selected population of galaxies as DOGs, although they approached the analysis using the fact that all of their targets were also AGN candidates. By stacking X-ray emission in the CDFS, Fiore et al. find that many of these DOG-like galaxies could harbor Compton-thick AGN. Read more about AGN content in DSFGs in $\$ 5.6$

\subsubsection{Redshift Distributions of $850 \mu \mathrm{m}-1.4 \mathrm{~mm}$-selected DSFG populations}

The $850 \mu \mathrm{m}$-selected SMG population is the best-studied subset of DSFGs and their redshift distribution is illustrated in Figure 17. The initial assessment of the SMG redshift distribution came from Chapman et al. (2005), now one of the most well known compilations of DSFGs in the literature. Chapman et al. describe a population of $850 \mu \mathrm{m}-$ selected SMGs which have radio counterparts; these radio counterparts have astrometric precision $<1^{\prime \prime}$ and provide excellent multi-slit spectroscopic targets. The Chapman et al. sample of 73 galaxies was spectroscopically observed at Keck Observatory using the LRIS instrument (Oke et al., 1995) and has a median redshift of $z=2.2$ (some of these redshifts had been reported in earlier works, e.g. Barger et al., 2000, Chapman et al., 2003b).

\footnotetext{
${ }^{9}$ Assuming a modified blackbody fit where the optical depth is unity at $\sim 100 \mu \mathrm{m}$.
} 


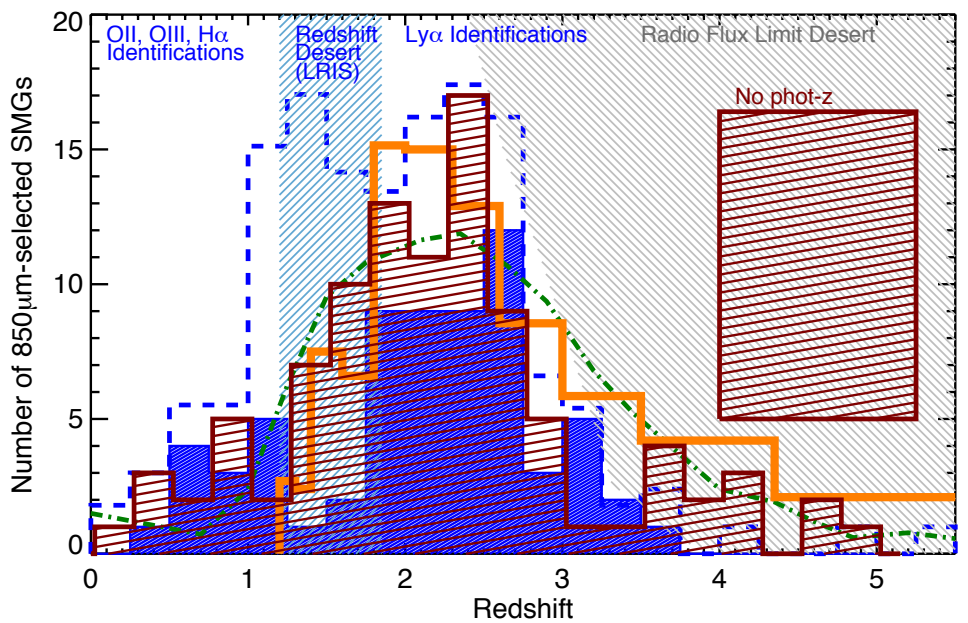

Figure 17: The redshift distribution of $850 \mu \mathrm{m}$ and $870 \mu \mathrm{m}$-selected SMGs from the literature. The Chapman et al. (2005) redshift distribution (background solid blue) is a collation of $73850 \mu \mathrm{m}$-selected radio galaxy redshifts obtained spectroscopically with the LRIS instrument on Keck 1 (also including data from Chapman et al. 2003b). This distribution suffers from a radio flux limit at high redshifts (gray striped area) and a spectroscopic desert between $1.2<z<1.9$ (this particular range is specific to the LRIS instrument on Keck 1). Sources with $z \gtrsim 2$ are primarily identified via $\operatorname{Ly} \alpha$ emission and sources at $z \lesssim 1.3$ are identified via OII, OIII or $\mathrm{H} \alpha$. An updated version of the Chapman et al. redshift distribution is shown as a dashed-blue line, including DEIMOS-observed radio SMGs from Banerji et al. (2011) specifically targeted to fill the redshift desert gap of LRIS, and a handful of high- $z$ 850 $\mu \mathrm{m}$-selected SMGs (Coppin et al. 2009. Daddi et al. 2009. Walter et al. 2012). Also over-plotted is the Lewis et al. (2005) and Chapman et al. (2003b) phenomenological model 850 $\mu \mathrm{m}$ SMG redshift distribution without radio flux selection (dark green dot dashed line). We also over-plot the Wardlow et al. (2011) redshift distribution for the LESS $870 \mu \mathrm{m}$ sample in CDFS (red line filled histogram) and the update to that sample-courtesy of ALMA follow-up establishing unambiguous counterparts- from Simpson et al. (2013) in orange. The latter is plotted in equal-time redshift bins to account for duty cycle correction. These redshifts are photometric and do not require radio sub-selection as a prerequisite, so they do not suffer from redshift deserts at intermediate or high redshifts, although a significant fraction (57/126) do not have any redshift indicators, primarily because they are too optically faint to characterize. Their relative contribution to this plot is given by the large line-filled red square on the right.
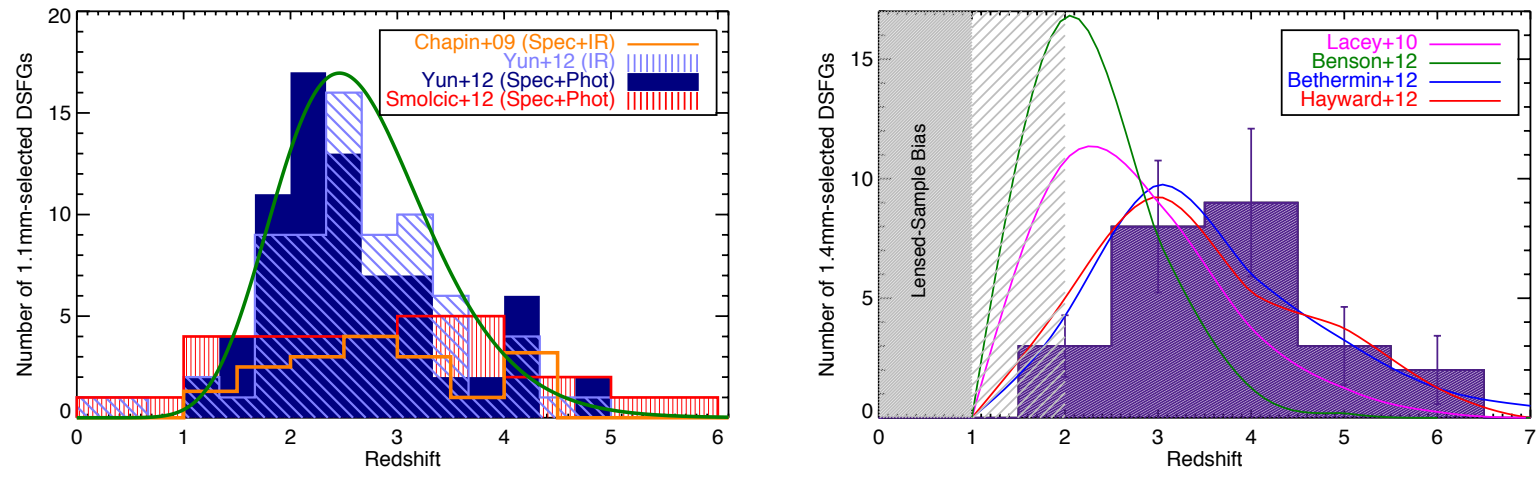

Figure 18: (Left:) The redshift distribution of $1.1 \mathrm{~mm}$-selected DSFGs in the literature, all detected with the AzTEC instrument. The orange histogram represent secure redshifts (spectroscopic and millimeter photometric) from AzTEC sources in GOODS-N (Chapin et al. 2009) and the red is from AzTEC sources in COSMOS (Smolčić et al. 2012). The solid dark blue histogram represents both spectroscopic and optical photometric redshifts for AzTEC sources in GOODS-S (Yun et al. |2012) who suggest the distribution is a log-normal in nature with median value $z=2.6$ (solid green line). The distribution in infrared photometric redshifts for the same GOODS-S sample is illustrated in hashed light blue to demonstrate its overall consistency with the optical spectroscopic/photometric sample. (Right:) The redshift distribution of $1.4 \mathrm{~mm}$-selected DSFGs detected with the South Pole Telescope (Vieira et al. 2013. Weiß et al. 2013). The redshifts are all spectroscopically confirmed in the millimeter. The sample is known to be gravitationally lensed due to observing depth of observations and confirmed Einstein rings around many sources (Vieira et al. 2013), and since they are lensed, no sources are detected at $z<1.5$. The mean redshift of the sample is $z=3.5$, seemingly much higher than the mean redshift for $850 \mu \mathrm{m}$-selected or $1.1 \mathrm{~mm}$-selected samples. 
Presented as is, this distribution (Figure 17) has two significant selection effects: (1) a required sub-selection in radio $1.4 \mathrm{GHz}$ continuum, and (2) a dearth of sources between $1.2<z<1.9$, which corresponds to the LRIS spectroscopic redshift desert. The requirement of radio detection in the Chapman et al. sample selects against galaxies at the highest redshifts since those galaxies are very unlikely to be $1.4 \mathrm{GHz}$-detected, as can been surmised from radio's positive K-correction as seen in Figure 3 . Thus, radio sub-selection has cut off the high- $z$ tail of the $850 \mu$ m distribution (as indicated by the hashed gray area on Figure 17). This is confirmed by the phenomenological models of Chapman et al. (2003b) and Lewis et al. (2005) who surmise that there should be a missing population of SMGs at high- $z$ not detected in the radio. Chapman et al. also describe how radio sub-selection can also select against very cold-dust SMGs, as they'll have much higher $\mathrm{S}_{850} / \mathrm{S}_{1.4}$ ratios than typical SMGs.

The second selection effect in the Chapman et al. sample is the LRIS redshift desert which spans $1.2<z<1.9$. This is the redshift range for which no bright emission lines would be present in a galaxy's LRIS spectrum. At lower redshifts, the rest-frame optical features of OII $3727 \AA$, H $\beta 4861 \AA$, OIII $5007 \AA$, and H $\alpha 6563 \AA$ would be readily available. At higher redshifts, $\operatorname{Ly} \alpha 1216 \AA$ would be accessible. In the desert, OII has redshifted too far out of LRIS' spectroscopic coverage and Ly $\alpha$ is not redshifted enough to be detected; LRIS' coverage at the time spanned $\approx 3000-8000 \AA$. It is important to note that different optical multi-object spectrographs have different redshift deserts if they have different spectroscopic coverage. For example, the DEIMOS spectrograph on Keck (Faber et al., 2003) has approximate maximum coverage 4500-9500 $\AA$ in low resolution mode, which corresponds to a redshift desert of $1.6<z<3.2$, making it not as ideal an instrument for follow-up of a population which peaks at $z \sim 2$. Note that since the Chapman et al. (2005) spectroscopic survey has been conducted, the red arm of the LRIS instrument has been upgraded so the coverage now runs $\approx 3000-10000 \AA$, with only a small desert around $z \approx 1.7$.

Various efforts to remedy the biases of the Chapman et al. sample have been made. Several high- $z(z>4)$ $850 \mu \mathrm{m}$-selected SMGs have now been spectroscopically confirmed, including GN20 at $z=4.055$ (Daddi et al., 2009), LESS J033229.4-275619 at $z=4.76$ (Coppin et al., 2009) and HDF 850.01 at $z=5.17$ (Walter et al., 2012), confirming that SMGs exist at the earliest epochs in the Universe's history, 1 Gyr after the Big Bang. Banerji et al. (2011) also work to fill in the LRIS spectroscopic desert with spectroscopic confirmations from DEIMOS in the neighborhood of $z \sim 1.5$. These have lead to a more contiguous picture of the $850 \mu \mathrm{m}$ redshift distribution.

Wardlow et al. (2011) presented an optical/near-infrared photometric analysis to the $870 \mu \mathrm{m}$ LABocA-selected population; the advantage of the Wardlow et al. sample is the LABOca map of the ECDFS is remarkably uniform in noise properties and fairly large. The $126 \mathrm{SMGs}$ analyzed in that work represent one of the first complete and relatively unbiased samples of $850 / 870 \mu \mathrm{m}$-selected SMG populations. Furthermore, radio (or $24 \mu \mathrm{m}$ ) detection was not a necessary requirement for analysis as the paper goes to great efforts to estimate the 'missing' sources' redshifts through statistical groupings of near-infrared samples. The Wardlow et al. redshift distribution, perhaps not surprisingly, lacks some of the clear sample biases of the Chapman et al. sample and happens to resemble the Lewis et al. model redshift distribution peaking at $z \sim 2.5$. Although more recent follow-up of the Wardlow et al. sample indicate that some of the LABOCA sources split into multiples in ALMA imaging (Karim et al., 2013; Hodge et al., 2013b), the re-analysis of the population's photometric redshifts with the high-resolution data (Simpson et al., 2013) find largely the same results. This sample remains the only representative sample of the $850 / 870 \mu \mathrm{m}$ population which has been unequivocally identified interferometrically and analyzed without substantial follow-up bias. Note that most differences between perceived median redshifts of $850 \mu \mathrm{m}$ samples is due to depth and area limits of the given surveys. Brighter sources tend to sit at higher redshifts (Pope et al., 2006; Koprowski et al., 2013).

At slightly longer wavelengths, the AzTEC $1.1 \mathrm{~mm}$-selected DSFG population has been studied in a little less detail than $850 \mu \mathrm{m}$-selected SMGs but provide equally large samples of $\sim 100$ galaxies with a good grasp on sample completeness. Figure 18 (left) illustrates the redshift distribution studies carried out at $1.1 \mathrm{~mm}$. Beyond the millimeter photometric redshifts work by Aretxaga et al. (2007), Chapin et al. (2009) compile 28 secure redshift identifications for $1.1 \mathrm{~mm}$-selected DSFGs in GOODS-N and measure a median redshift of $z=2.7$ which they claim is statistically distinct from the $z=2.2$ mean measured in the Chapman et al. sample and $z=2.5$ in the Wardlow et al. sample. Yun et al. (2012) look at the $1.1 \mathrm{~mm}$-detected DSFGs in GOODS-S and exploit the deep multi-wavelength ancillary data to measure redshifts as best as possible in the optical and near-infrared if spectroscopic redshifts do not already exist. Yun et al. also show that the millimetric redshift distribution of the sample is nearly identical to the optical/nearinfrared redshift distribution, both of which have a median redshift of $z=2.6 \pm 0.1$, similar to the median redshift of $z=2.7 \pm 0.2$ found by Chapin et al. (2009). Yun et al. demonstrate that the $1.1 \mathrm{~mm}$ DSFG redshift distribution can be 
well-modeled by a log-normal distribution of the form

$$
f(z)=\frac{1}{(1+z) \sigma \sqrt{2 \pi}} \exp \left(-\frac{\left[\ln (1+z)-\ln \left(1+z_{\mu}\right)\right]^{2}}{2 \sigma^{2}}\right)
$$

with $z_{\mu}=2.6$ and $\sigma=0.2$ in $\ln (1+z)$. Although DSFG populations selected at other wavelengths do not often analyze the population with this parametrized fit, the shapes of most populations could largely be generalized in this form, with different mean redshifts and dispersions for populations selected at different rest-frame wavelengths.

One more notable analysis of the $1.1 \mathrm{~mm}$-selected population is summarized by Smolčić et al. (2012) who describe interferometric follow-up observations of DSFGs in the COSMOS field. Like the interferometric work on the LABOCA CDFS sample from ALMA (summarized in Karim et al., 2013, Hodge et al., 2013b), the Smolčić et al. work measures the accuracy of previous counterpart identifications to bolometer-detected sources and identifies submillimeter multiples (see more in $\$ 2.5$. Smolčić et al. suggest a revised strategy for assessing the optical/near-IR photometric redshifts of DSFGs by considering multiple minima in the $\chi^{2}$ photometric redshift fitting. They find a median redshift of $z=3.1 \pm 0.3$ for the $1.1 \mathrm{~mm}$ sample which is offset from the Chapin et al. and Yun et al. findings, but is also a small sample, consisting of only 17 galaxies. The discrepancy is most certainly due to the different strategy in analyzing photometric redshifts (including lower limits and choosing higher-redshift phot- $z$ solutions) but it could also be due in part to cosmic variance, where the COSMOS field is known to have several notable, very distant DSFGs at $z>4.5$ (e.g. Capak et al. 2008, Riechers et al. 2010b). At the moment, it is unclear to what extent the method of fitting photometric redshifts to DSFGs needs revision, as larger statistical samples are necessary to really determine the relative shortcomings of any redshift-distribution measurement technique.

Figure 18 (right) shows the observed FIR-spectroscopically-confirmed redshift distribution of $1.4 \mathrm{~mm}$-selected DSFGs from Weiß et al. (2013). This sample is unique in that it consists of galaxies that are most certainly gravitationally lensed (Vieira et al. 2013) because the $1.4 \mathrm{~mm}$ imaging from the South Pole Telescope is too shallow to detect distant DSFGs unlensed. Lensing does impact the observed redshift distribution since lower redshift galaxies $(z<2)$ are less likely to be gravitationally lensed by a foreground object than higher redshift galaxies. Weiß et al. (2013) argue that this effect is strong at $z<2$, especially at $z<1$, but it has little impact on lensing of higher redshift galaxies. In Figure 18 this lensing-bias is indicated by hashed gray areas at $z<2$. The $1.4 \mathrm{~mm}$ redshift distribution is compared to several literature models which employ a phenomenological approach (Béthermin et al., 2012b), hybrid cosmological hydrodynamic approach (Hayward et al., 2013a), and semi-analytic approach (Lacey et al., 2010. Benson, 2012). It is important to stress that, although the SPT sample is small, these galaxies are the most spectroscopically complete DSFG sample of any; 26 targets were blindly chosen, for which 23 millimetric spectroscopic redshifts were measured from ALMA (their composite millimeter spectrum is shown in Figure 16. The Weiß et al. (2013) work also includes an interesting discussion of size evolution and its potential impact on the observed redshift distribution. If the mean physical size of DSFGs evolves with redshift (i.e. they are more compact or extended at high-redshift) then this behavior can impact the redshift distribution due to the influence of size evolution on lensing (Hezaveh \& Holder, 2011). The more compact the higher-redshift DSFGs, the more likely it is for them to be detected in the $1.4 \mathrm{~mm}$ survey of lensed DSFGs. If the intrinsic redshift distribution of $1.4 \mathrm{~mm}$-selected DSFGs mirrors the intrinsic redshift distribution of the $850 \mu \mathrm{m}$-selected SMGs, then a fairly extreme size evolution is needed to reconcile observations.

\subsubsection{Redshift Distributions of $250 \mu \mathrm{m}-500 \mathrm{~mm}$-selected DSFG populations}

Moving from long wavelengths to shorter wavelengths, here we address the redshift distribution of galaxies selected at $250-500 \mu \mathrm{m}$. Unlike surveys conducted at $850 \mu \mathrm{m}-1.1 \mathrm{~mm}$, BLAST, H-ATLAS and HerMES (see $\S 2.3 .2$ for details) have imaged several hundred square degrees of sky in large surveys. The nature of the galaxies detected in these large surveys is going to be more varied due to the large dynamic range in luminosities than those found in smaller surveys.

On small angular scales $\left(<1 \mathrm{deg}^{2}\right)$, the population of DSFGs which are luminous at $250-500 \mu \mathrm{m}$ (at $\left.\gtrsim 20 \mathrm{mJy}\right)$ might be thought of as very similar to $850 \mu \mathrm{m}$ or $1.1 \mathrm{~mm}$ galaxies. In one sense, this is a correct assumption, since most galaxies which are $\sim 5 \mathrm{mJy}$ at $850 \mu \mathrm{m}$ will be $\sim 30 \mathrm{mJy}$ at $\sim 500 \mu \mathrm{m}$ if they sit at intermediate redshifts $z<4$. However, some important details are lost in this interpretation. Although most $850 \mu \mathrm{m}$-selected galaxies might be detectable at $250-500 \mu \mathrm{m}$, not all $250-500 \mu \mathrm{m}$ galaxies will be $850 \mu \mathrm{m}$ detected either because they sit at different redshifts or have 
different SED characteristics (see the discussion of the temperature bias of SMGs in $\$ 2.4$, also particularly Figure 20 of Casey et al.2012a); more importantly, a population's selection wavelength (within 250-500 $\mu$ m) matters quite a bit to their implied redshift distribution (as pointed out by Béthermin et al. 2012b) as $250 \mu \mathrm{m}$ populations will be very different from $500 \mu \mathrm{m}$ populations, where the latter more closely resemble the canonical $850 \mu \mathrm{m}$-selected SMGs.

By observing the increasing median redshift of samples with longer wavelengths in the previous section, one also might assume that longer wavelength populations correspond to higher redshifts, and shorter wavelengths lower redshifts. Thus, the $250-500 \mu \mathrm{m}$ redshift distribution would peak below $z \sim 2$. This interpretation is partially correct since the peak of the dust blackbody emission does shift towards longer wavelengths at higher redshifts. However, this assumption can be too simplistic when not accounting for sample biases, intrinsic SED variation, and survey detection limits in the different bands. For example, a wide-and-shallow survey at $500 \mu \mathrm{m}$ might overlap completely with a wide-and-shallow survey at $1.4 \mathrm{~mm}$, in that they might both be efficient at picking up the same lensed dusty galaxies at high- $z$, but perhaps the equivalent deep pencil-beam surveys at the same wavelengths, $500 \mu \mathrm{m}$ and $1.4 \mathrm{~mm}$, reveal two completely different, non-overlapping populations. Therefore, it becomes important to clearly define population selection before comparing and contrasting distributions.

Figure 19 shows some of these contrasting populations selected at $250-500 \mu \mathrm{m}$, for both deep pencil-beam surveys and wide-field shallow surveys for lensed DSFGs. Negrello et al. (2007) present some of the first predictive measurements for 250-500 $\mu \mathrm{m}$-selected lensed populations by combining physical and phenomenological models. Negrello et al. predict a redshift distribution for $S_{350}>100 \mathrm{mJy}$ galaxies peaking at $z \approx 2$ (red line on Figure 19). In contrast, earlier predictive work by Lagache et al. (2005) addresses the overall redshift distribution for the underlying $350 \mu$ m population, which peaks at substantially lower redshift, $z \approx 1$, with a long tail out to high redshifts. Another phenomenological approach described in Béthermin et al. (2011), working backwards from constrained luminosity functions to redshift distributions and number counts predicts a peak at very low redshifts with a secondary peak ${ }^{10}$ at $z \sim 2$. Béthermin et al. (2012b) present yet another new approach which is strictly empirical using the observed evolution in the stellar mass function of star-forming galaxies and the observed infrared main sequence of galaxies (Rodighiero et al., 2011, Sargent et al. 2012); they use empirical templates from Magdis et al. (2012b) as a function of main-sequence status to predict number counts and also source redshift distributions.

The first data results of redshift distributions in this regime came from Amblard et al. (2010) who use SPIRE colors of H-ATLAS sources to constrain redshift based on a millimetric redshift identification technique. Amblard et al. generate SEDs at a variety of redshifts and SpIRE colors and then identify the color-color space where most SPIRE sources are located and what redshifts they are most likely associated with. They estimate a median redshift of $z=2.2 \pm 0.6$.

Two more recent samples use both spectroscopic and optical/near-IR photometric redshifts to determine the 250 $500 \mu \mathrm{m}$ redshift distribution. Chapin et al. (2011) characterize the 69 BLAST-detected ECDFS sources, finding a flat distribution between $0.5<z<3$ with a strong peak at $z \sim 0.3$. Casey et al. (2012a b) summarize the efforts of a large spectroscopic follow-up campaign for $\approx 1600$ HerMES SPIRE-selected galaxies in multiple fields covering $\sim 1 \mathrm{deg}^{2}$, nearly 800 of which are spectroscopically confirmed in the optical. They find a median redshift of $z=1.1$ with peak around $z=0.8$ with a long tail out to $z \approx 5$. The Chapin et al. and Casey samples are statistically consistent when removing the $z \sim 0.3$ peak in the BLAST sample which is thought to be an over-density in ECDFS caused by cosmic variance. Both of these datasets agree with the Lagache et al. (2005) model distribution, potentially the Béthermin et al. (2012b) model distribution if some different SED assumptions are used, but are statistically distinct from the Negrello et al. (2007) model prediction for lensed galaxies.

The disagreement between the Amblard et al. (2010) and Chapin et al. and Casey results is likely due to (a) the assumptions about SED shape of SPIRE galaxies made by Amblard et al. (whereby a different assumed temperatureluminosity relation could have given a lower redshift peak) or (b) biases in the optical redshift samples which exclude higher redshift galaxies for lack of $1.4 \mathrm{GHz}$ or $24 \mu \mathrm{m}$ counterparts. Whatever the case, it is clear that work on the redshift distributions of Herschel-selected galaxies has not yet reached maturity.

In contrast to the large scale work done with Herschel, some further strides have been made at $450 \mu \mathrm{m}$ using the ScuBA-2 instrument on smaller scales. This population has not been probed until very recently since the $450 \mu \mathrm{m}$ bolometers on ScuBa were very difficult to use due to difficult sky subtraction. Although SHARC-II on the CSO probed

\footnotetext{
${ }^{10}$ This shape perhaps seems a bit counter-intuitive, but depends significantly on the input SED shape assumed for the population.
} 

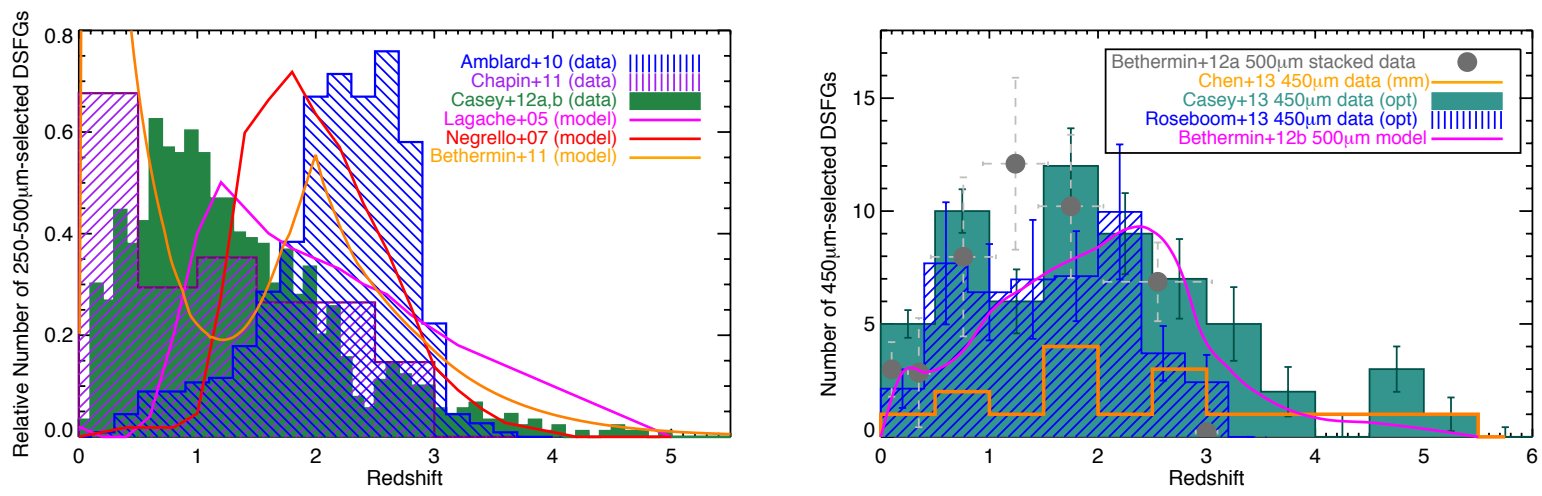

Figure 19: (Left:) Redshift distributions of 250-500 $\mu \mathrm{m}$-selected DSFGs in the literature and comparisons to model 250-500 $\mu \mathrm{m}$ predictions. Histograms have been renormalized since sample sizes vary from $\sim 70$ to $\sim 2000$ galaxies. The Amblard et al. (2010) distribution (blue hashed region) is generated through statistical means by fitting millimetric redshifts to $\sim 2000 \mathrm{H}$-ATLAS Herschel-SPIRE $350 \mu$ m-selected DSFGs. The Chapin et al. (2011) distribution (purple hashed region) includes various efforts to characterize the 69 BLAST 250-500 $\mu$ m-selected DSFGs in ECDFS (including spectroscopic and photometric redshifts collated from Dunlop et al. 2010, Casey et al. 2011a). The Casey et al. (2012a) distribution (solid green region) (also including data from Casey et al. 2012b) is a collection of $\sim 1600$ Herschel-SpIRE 250-500 $\mu$ m-selected DSFGs, half photometric, half spectroscopically confirmed. The model distributions for this population are phenomenological and are described in Lagache et al. (2005) (magenta line), Negrello et al. 2007) (red line), and Béthermin et al. (2011) (orange line). (Right:) Redshift distributions of $450 \mu \mathrm{m}$-selected galaxies in the literature, observed with the ScuBA-2 instrument, and contrasted to predictions and measurements of similar $500 \mu \mathrm{m}$ galaxies observed by Herschel. The $500 \mu \mathrm{m}$ Herschel sample (gray circles) is from an analysis of stacked data based on $24 \mu \mathrm{m}$ priors and is suggested to peak at $z \sim 1.5$ (Béthermin et al. 2012b). At $450 \mu \mathrm{m}$, there are three datasets summarized in Chen et al. (2013a) (orange line histogram), Casey et al. (2013) (teal filled histogram) and Roseboom et al. (2013) (blue hashed histogram). The Chen et al. redshifts are millimetric photometric redshifts while the Casey et al. and Roseboom et al. are optical/near-IR photometric redshifts from COSMOS. The latter two samples overlap although are drawn from two different datasets; the Casey et al. dataset is more shallow and wide while the Roseboom et al. dataset is deeper and smaller. Casey et al. measure a median redshift of $z=1.95 \pm 0.19$ while Roseboom et al. measure $z=1.4$.

this wavelength regime at $350 \mu \mathrm{m}$, SHARc-II was never used for mapping blank-field sky to detect its own population. The unique advantage of $450 \mu \mathrm{m}$ mapping with ScUBA-2 is its superb spatial resolution, $\sim 7^{\prime \prime}$, which is significantly better than the spatial resolution of Herschel at $500 \mu \mathrm{m}, \sim 36^{\prime \prime}$. Figure 19 (right panel) plots the redshift distribution for galaxies selected at $450 \mu \mathrm{m}$ from data described by Chen et al. (2013a), Casey et al. (2013), Geach et al. (2013) and Roseboom et al. (2013) and compares it to some statistical determinations of the redshift distribution for $500 \mu \mathrm{m}$ selected galaxies from Herschel (Béthermin et al., 2012b a). The 12-galaxy Chen et al. sample redshifts are derived from the millimeter $\left(\mathrm{S}_{450} / \mathrm{S}_{850}\right.$ colors) and span $0<z<6$ with a median of $z=2.3$. The Casey et al. sample and Roseboom et al. sample (the latter of which is the same dataset analyzed in Geach et al., 2013) sit in an overlapping region of the COSMOS fields. The Roseboom et al. map is $\sim 1 / 4$ the size of the Casey et al. map although it is also 4 times deeper (an RMS of $1.2 \mathrm{mJy}$ versus $4.1 \mathrm{mJy}$ ). The respective redshift distributions could be drawn from the same parent population despite the fact that the quoted median redshifts for the samples are dissimilar $(z=1.4$ in Roseboom et al. and $z=1.95 \pm 0.19$ in Casey et al.). The differences could be caused by the different field depths, where the Casey work extends over larger areas and is able to detect rare high- $z$ systems while the Roseboom work is more sensitive to fainter galaxies at $z \sim 1-2$.

\subsection{Infrared SED Fitting for DSFGs}

Spectral energy distribution (SED) fitting in the far-infrared is needed to extract basic properties of galaxies' dust emission: its infrared luminosity, thus obscured star formation rate, dust temperature and dust mass. Far-IR SED fitting varies from the use of template libraries, the use of scaling relations, to direct data fitting to parametrized fits. Unlike data in the optical or near-infrared (Bolzonella et al., 2000; Bruzual \& Charlot, 2003, Maraston, 2005), far-infrared data often suffers from a dearth of photometric data with far fewer bands available for comparison with models. At most, individual galaxies will have $\sim 10$ photometric data points in the far-infrared, and more like 3-5 on average, versus $30+$ bands in the optical. Unfortunately, the parameters needed to describe the far-infrared emission are no less complex than those in the optical/near-IR, including dust distribution, composition, dust grain type, orientation, galaxy structure, AGN heating, emissivity and optical depth. Below we describe the techniques commonly used in 
the literature to measure far-infrared SEDs' basic characteristics and summarize them in Table 2 . As a sidenote to all infrared SED fitting for DSFGs, the effect of far-infrared emission lines from CO or CII should be considered, as emission lines can contaminate broadband submm flux densities by up to 20-40\% (Smail et al., 2011).

SED fitting techniques can be broken down into categories: (1) direct comparison to models (radiative transfer or empirical) using, for example, $\chi^{2}$ techniques, (2) comparison with model templates using Bayesian techniques, or (3) direct FIR fitting methods to simple modified blackbody-like functions. The first two are described in $\S 4.2 .1$ and the last in $\S 4.2 .2$ Different applications of data call for different types of SED modeling. While the last set of methods is the most computationally straightforward to apply to galaxies with fewer data points, it might be useful for the user to fit the stellar emission and dust emission simultaneously, therefore use some of the more sophisticated models based on stellar synthesis modeling, accurate attenuation curves and dust grain analysis.

\subsubsection{Employing dust radiative transfer models and empirical templates}

Despite the relative lack of detailed data, detailed radiative transfer models and empirical template libraries have modeled the complex dust infrared emission from stars, molecular clouds and dust grains over a wide range of galaxy geometries and luminosities (Silva et al., 1998; Chary \& Elbaz, 2001; Dale et al., 2001; Dale \& Helou, 2002; Abel \& Wandelt, 2002; Siebenmorgen \& Krügel, 2007; Draine \& Li, 2007). When discussing these models, it's important to keep in mind that the accuracy or applicability of these models cannot be tested with data since it simply does not exist in enough detail to disentangle effects of geometry, distribution, optical depth, etc. It's also important to note that many have done work in this area, particularly modeling radiative transfer in local starburst populations to generate SEDs (Efstathiou \& Rowan-Robinson, 1995; Efstathiou et al., 2000; Efstathiou \& Rowan-Robinson, 2003; Efstathiou \& Siebenmorgen, 2009, Nenkova et al., 2002, Dullemond \& van Bemmel, 2005, Piovan et al., 2006, Nenkova et al., 2008; Takagi et al., 2003; Fritz et al., 2006; Hönig et al., 2006, Schartmann et al., 2008), but here we try to focus on the techniques which have been most commonly employed for SED fitting of high- $z$ dusty starburst ${ }^{11}$

Silva et al. (1998) developed the GrasiL code to model galaxy emission by explicitly accounting for dust absorption and emission from the ultraviolet through to the far-infrared. They use stellar population synthesis models and a chemical evolution code to generate integrated spectra for simple stellar populations of different ages, metallicities, star formation rates, gas fractions, relative gas trapped in molecular clouds versus diffuse ISM, dust geometry and dust grain size distribution (small grains versus PAHs). Chary \& Elbaz (2001) use GrasIL and the analysis of Silva et al. (1998) to generate template SEDs for infrared-luminous galaxies out to moderately high redshifts $(z \sim 1)$ when measuring contributions of different galaxy populations to the CIB. Chary \& Elbaz generate four SEDs with GRASIL to fit data from nearby galaxies which each represent a different decade in infrared luminosity-Arp 220, NGC 6090, M 82, and M 51. They discard the mid-IR portion of the model spectrum and replace it with data from ISOCAM (Smith et al., 1989; Charmandaris et al., 1997; Laurent et al., 2000, Förster Schreiber et al., 2001; Roussel et al., 2001). They then interpolated between the four galaxies' SEDs to span intermediate luminosities. The templates were then split at $20 \mu \mathrm{m}$ and additional FIR templates $(20-1000 \mu \mathrm{m})$ were taken from Dale et al. (2001) to span a wider range of spectral shapes, i.e. dust temperatures.

In contrast to the Silva et al. (1998) and Chary \& Elbaz (2001) work, Dale et al. (2001) present a different model for SEDs in the far-infrared. SEDs are constructed from various dust emission curves and the assumption that there is a power-law distributions of dust masses (thus dust temperatures) over a wide range of radiation fields. Small, large and PAH grains are all taken into account and the models are compared to data (from IRAS, ISOCAM and ISOPHOT) of 69 nearby normal galaxies for constraints. Dale et al. find that normal galaxy SEDs can be described solely by a range of FIR colors defined by the IRAS bands, i.e. $S_{60} / S_{100}$. Dale \& Helou (2002) builds on this phenomenological approach by extending calibration at wavelengths $>120 \mu \mathrm{m}$ and correcting the Dale et al. (2001) model assumptions regarding dust emissivity and radiation field intensity.

Dopita et al. (2005) present another modeling technique which reproduces SEDs from the ultraviolet through the far-infrared, and out through the radio by combining stellar synthesis output from STARBURST99 (Leitherer \& Heckman, 1995), nebular line emission modeling, a dynamic evolution model of Hir regions and a simplified synchrotron emissivity model to construct self-consistent SEDs for solar-metallicity starbursts with durations $\sim 100 \mathrm{Myr}$. They

\footnotetext{
${ }^{11}$ While these models have been widely used to model the SEDs of high- $z$ dusty starbursts, we do not recommend their blind use on large DSFG datasets without an intimate understanding of the governing parameters.
} 


\begin{tabular}{|c|c|}
\hline $\begin{array}{l}\text { GrasiL; Silva et al. } \\
\text { (1998) }\end{array}$ & $\begin{array}{l}\text { Stellar population synthesis model which accounts for dust obscuration from UV through the } \\
\text { FIR; incorporates chemical evolution, gas fraction, metallicity, ages, relative fraction of star- } \\
\text { forming molecular gas to diffuse gas, the effect of small grains and PAHs and compares to } \\
\text { observations from ISO. Can be used to investigate SFR, IMF, and supernova rate in nearby } \\
\text { starbursts and normal galaxies. }\end{array}$ \\
\hline Dopita et al. 2005) & $\begin{array}{l}\text { Stellar population synthesis from STARBURST } 99 \text { combined with nebular line emission mod- } \\
\text { eling, a dynamic evolution model of HII regions and simplified synchrotron emissivity model. } \\
\text { Constructed for solar-metallicity starbursts with duration } \sim 100 \text { Myr and checked for consistency } \\
\text { using local starbursts. }\end{array}$ \\
\hline $\begin{array}{l}\text { Siebenmorgen \& } \\
\text { Krügel (2007) }\end{array}$ & $\begin{array}{l}7000 \text { templates; spherically symmetric radiative transfer model accounting for a variety of star } \\
\text { formation rates, gas fractions, sizes or dust masses. No accounting for dust clumpiness or } \\
\text { asymmetry, although they argue it is insignificant. }\end{array}$ \\
\hline \multicolumn{2}{|r|}{ EMPIRICAL TEMPLATES } \\
\hline \begin{tabular}{|l|} 
Dale et al. $(2001) ;$ \\
Dale \& Helou (2002)
\end{tabular} & $\begin{array}{l}69 \text { FIR phenomenological models } \text { (Dale et al. } 2001 \text { ) supplemented by FIR and submm data } \\
\text { presented in Dale \& Helou (2002). SEDs are generated assuming power-law distribution of } \\
\text { dust mass over a range of ISM radiation fields and constrained with IRAS and ISOPHOT data } \\
\text { for } 69 \text { normal nearby galaxies. Mid-IR spectral features taken from ISO observations. }\end{array}$ \\
\hline $\begin{array}{l}\text { Chary \& Elbaz } \\
(2001)\end{array}$ & $\begin{array}{l}105 \text { templates; used basic Silva et al. (1998) models to reproduce SEDs for four local galax- } \\
\text { ies (Arp 220, NGC 6090, M } 82, \text { M I , representing ULIRGs, LIRGs, starbursts and normal } \\
\text { galaxies) and ISOCAM CVF 3-18 } 18 \text { m observations to determine mid-IR spectra and continuum } \\
\text { strength. Interpolated between four SEDs and used Dale et al. (2001) templates to create a } \\
\text { larger range of FIR spectral shapes. }\end{array}$ \\
\hline Draine \& Li (2007) & $\begin{array}{l}69 \text { templates, } 25 \text { consistent with the most IR-luminous; model focused on mid-IR emission } \\
\text { by balancing size distribution of PAHs with small grains, starlight intensities, and the relative } \\
\text { fraction of dust which is heated by starlight. Checked for consistency against Spitzer data. }\end{array}$ \\
\hline Rieke et al. (2009) & $\begin{array}{l}14 \text { templates, based on IRS and ISO spectra of eleven local LIRGs and ULIRGs connected to } \\
\text { modified blackbodies with temperatures } 38-64 \mathrm{~K} \text { (where temperature scales with luminosity). } \\
\text { Resulting templates span } 5 \times 10^{9}-10^{13} \mathrm{~L}_{\odot} \text {, where most luminous sources have strongest silicate } \\
\text { absorption. }\end{array}$ \\
\hline \multicolumn{2}{|r|}{ ENERgy Balance TechNiques } \\
\hline $\begin{array}{l}\text { MagPhys; da Cunha } \\
\text { et al. (2008) }\end{array}$ & $\begin{array}{l}\text { Constrains UV-FIR SED empirically using an energy balance argument. Accounts for wide } \\
\text { variety of star formation histories and adjustable input stellar synthesis template library (which } \\
\text { by default uses those from Bruzual \& Charlot 2003); attenuation determined from mix of hot } \\
\text { and cool dust grains and PA } \overline{\text { Hs. }}\end{array}$ \\
\hline $\begin{array}{l}\text { Cigale; Burgarella } \\
\begin{array}{l}\text { et al. (2005); Noll } \\
\text { et al. }(2009)\end{array}\end{array}$ & $\begin{array}{l}\text { Combines stellar population models from Maraston }(2005) \text { with Calzetti et al. dust attenuation } \\
\text { curves and far-infrared SEDs from Dale \& Helou }(2 \overline{002}) \text { to fit UV-FIR SEDs for a variety of } \\
\text { galaxies. }\end{array}$ \\
\hline \multicolumn{2}{|r|}{ DIRECT FIR-ONLY METHODS } \\
\hline Modified Blackbody & $\begin{array}{l}S(\nu, T)=\frac{\left(1-e^{-\tau(v)}\right) v^{3}}{e^{h / / k T}-1}, \text { where the optical depth is given by } \tau(v)=\left(v / v^{0}\right)^{\beta} \cdot \beta=1.5 \text { is a common } \\
\text { assumption, although some work find its value varies from } \beta \approx 1-2 . \text { Most data indicates } \\
v_{0} \approx 1.5 \mathrm{THz} \text {. The optically thin assumption reduces the }\left(1-e^{-\tau(v)}\right) \text { term to } \nu^{\beta} \text {. }\end{array}$ \\
\hline $\begin{array}{l}\text { Two-temperature } \\
\text { Modified Blackbody } \\
\text { (e.g. Dunne \& Eales }\end{array}$ & $\begin{array}{l}S\left(\nu, T_{\text {cold }}\right)+S\left(v, T_{\text {warm }}\right) \text {,i.e. the sum of two modified blackbodies as given above. } T_{\text {cold }} \text { is } \\
\text { here intended to dominate the FIR SED longward of } \sim 100 \mu \mathrm{m} \text {, while the } T_{\text {warm }} \text { component helps } \\
\text { reconstruct the emission at mid-infrared wavelengths. This procedure has more free parameters } \\
\text { than the fitting methods below, so use with caution. }\end{array}$ \\
\hline $\begin{array}{l}\text { Piecewise Modified } \\
\text { Blackbody+ } \\
\text { Powerlaw (e.g. } \\
\text { Younger et al., 2007) }\end{array}$ & $\begin{array}{l}S(v, T)=\left\{\begin{array}{cc}\frac{\left(1-e^{-\tau(v)}\right) v^{3}}{e^{h \nu / k T}-1} & : v \leq v_{c} \\
v^{-\alpha} & : v>v_{c}\end{array} \text { where }\left.\frac{d S}{d v}\right|_{v_{c}}=-\alpha . \text { Procedurally, this fitting method is }\right. \\
\text { identical to the modified blackbody fit, where the Wien side is removed and replaced by mid- } \\
\text { infrared power-law consistent with any mid-IR data available; in the absence of mid-IR data, } \\
\text { a powerlaw slope of } \alpha=2 \text { is consistent with starbursts and slightly more shallow, } \sim 1.5, \text { for } \\
\text { starbursts with AGN (Blain et al. 2003, Casey, 2012, Koss et al. 2013). }\end{array}$ \\
\hline $\begin{array}{l}\text { Power-law of dust } \\
\text { temperatures (e.g. } \\
\text { Dale et al. 2001) }\end{array}$ & $\begin{array}{l}S\left(v, T_{c}\right)=(\gamma-1) T_{c}^{\gamma-1} \int_{T_{c}}^{\infty}\left(1-e^{-\tau(v)}\right) B_{v}(T) T^{-\gamma} d T \text {, where } B_{v}(T) \text { is the Planck function and } \\
\gamma \text { is a parameter determining the slope of the mid-IR power-law (note } \gamma \neq \alpha) \text {. See Kovács et al. } \\
\text { (2010) for fitting procedure. }\end{array}$ \\
\hline $\begin{array}{l}\text { Analytic Approx. } \\
\text { Mod. Blackbody+ } \\
\text { Powerlaw (Casey, } \\
\text { 2012) }\end{array}$ & $\begin{array}{l}S(v, T)=N_{\mathrm{bb}} \frac{\left(1-e^{-\tau(\nu)}\right) v^{3}}{e^{h \nu / k T}-1}+N_{\mathrm{pl}} v^{-\alpha} e^{-\left(v_{c} / v\right)^{2}} \text {; an analytic approximation to the power-law of dust } \\
\text { temperatures fit which is computationally straightforward to fit to data. } N_{\mathrm{pl}} \text { is a fixed function } \\
\text { of } N_{\mathrm{bb}} \text {, the normalization factors, and } v_{c} \text { is where } d S / d v=-\alpha \text {, as is the case for the piecewise } \\
\text { modified blackbody+ powerlaw fits. }\end{array}$ \\
\hline
\end{tabular}


describe the dependence of the far-infrared emission on the ambient pressure of the starburst. Although the Dopita et al. (2005) models are not often widely used amongst the high- $z$ dusty galaxy community, their application could be appropriate, especially for solar-metallicity systems.

The Chary \& Elbaz (2001) and Dale \& Helou (2002) have garnered significant traction in the dusty galaxy community for SED fitting, particularly for galaxies detected in the mid-IR for which estimates of far-IR emission are necessary, but newer models have recently become available which make use of more sophisticated, later datasets and modeling techniques specifically tweaked for extreme starbursts.

Siebenmorgen \& Krügel (2007) describe a spherically symmetric radiative transfer model for dusty starburst nuclei and ULIRGs and argue that the symmetry assumption, and lack of accounting for dust clumpiness, does not significantly change a dusty galaxy's SED. They present a library of 7000 SEDs which can be fit to galaxies either locally or at high- $z$ and accurately be used to measure luminosity, size, dust or gas mass. Their SEDs have been applied in several more recent studies, e.g. Symeonidis et al. (2013), which study the bulk characteristics of FIR SEDs at high- $z$.

Draine \& Li (2007) present another modeling method, focused instead on the emission from dust in the midinfrared portion of the spectrum, but following through to the far-infrared. The models balance the size distribution of PAH grains and starlight intensities and the relative fraction of dust which is heated by starlight above a certain intensity. The models are constrained with data from Spitzer.

Another set of templates used extensively by the DSFG community are those described in Rieke et al. (2009). Rieke et al. use detailed Spitzer observations of eleven local LIRGs and ULIRGs to construct a library of templates spanning $0.4 \mu \mathrm{m}-30 \mathrm{~cm}$ and luminosities $5 \times 10^{9}-10^{13} \mathrm{~L} \odot$. The spectral characteristics of the templates at rest-frame wavelengths $\lesssim 35 \mu \mathrm{m}$ are comprised of IRS and ISO spectra, consistently matched to $0.4 \mu \mathrm{m}-5 \mu \mathrm{m}$ stellar photospheric templates with a simple reddening law. The far-infrared portion of the spectrum is a modified blackbody with fitted temperatures ranging $38-64 \mathrm{~K}\left(70<\lambda_{\text {peak }}<125 \mu \mathrm{m}\right)$ and emissivity $0.7<\beta<1$.

Contemporaneous to the works describing the latest empirical templates, Bayesian fitting codes have come about which are explicitly designed to fit observed data to template SEDs from the ultraviolet through the far-infrared. The Code Investigating GALaxy Emission (CigALE) was developed from an algorithm described in Burgarella et al. (2005) and formally presented in Noll et al. (2009). CigALE is based on model spectra generated in the optical/near-infrared by Maraston (2005) which account for thermally pulsating asymptotic giant branch (TP-AGB) stars, synthetic dust attenuation curves based on the modified laws presented in Calzetti et al. (1994) and Calzetti (2001) and far-infrared SED templates from Dale \& Helou (2002). In contrast, the Multi-wavelength Analysis of Galaxy Physical Properties, or MAGPHYs code, is a modeling package described in da Cunha et al. (2008) which empirically constrains the output of an SED from the ultraviolet through far-infrared using an energy balance argument. The infrared portion of SEDs are generated by modeling emission from hot grains (mid-infrared continuum; temperatures 130-250 K), PAHs (midinfrared spectral lines), and grains in thermal equilibrium (far-infrared continuum; temperatures $\sim 30-60 \mathrm{~K}$ ). The stellar component of SEDs is generated from Bruzual \& Charlot (2003) stellar population synthesis, and the spectrum is attenuated using the angle-averaged model of Charlot \& Fall (2000), and then the starlight which is attenuated in the optical is accounted for in the re-radiated infrared emission. The da Cunha et al. (2008) model technique is particularly versatile for constraining SEDs for galaxies of a wide range of star formation histories since any number of input stellar emission templates can be adjusted accordingly.

\subsubsection{Direct modified blackbody SED modeling}

In contrast to detailed models, many works instead approximate the far-infrared portion of the spectrum as a modified blackbody ${ }^{12}$. Before the launch of Herschel, when DSFGs commonly only had one photometric point in the far-infrared-the photometric point corresponding to its detection band, e.g. $24 \mu \mathrm{m}$ or $850 \mu \mathrm{m}$-even more simplistic interpretations of the modified blackbody were made simply because more sophisticated models would have been unconstrained. For example, in $850 \mu \mathrm{m}$-selected SMG samples, the $850 \mu \mathrm{m}$ flux density was commonly converted directly to a far-infrared luminosity then star formation rate by assuming a modified blackbody of roughly fixed temperature, e.g. between 30-40 K, or an SED template of a local ULIRG like Arp 220 (Barger et al., 2012). The

\footnotetext{
${ }^{12}$ Note that the term greybody is often used interchangeably with modified blackbody, however the former refers to the condition where the optical depth not wavelength dependent.
} 
disadvantage of this method is that it does not account for variation in dust temperature which can impact the measured $850 \mu \mathrm{m}$ flux density significantly for fixed infrared luminosity or star formation rate (see Figure 8 in $\$ 2.4 .1$.

An alternate approach when only one far-infrared measurement is at hand is to use the FIR/radio scaling relation for starbursts (Helou et al. 1985, Condon, 1992, also see this review $\S 5.12$. This empirical relation relates the rest-frame radio continuum luminosity of synchrotron radiation scattering off supernovae remnants to galaxies' restframe far-infrared dust modified blackbody emission and is shown to hold out to high redshifts in starbursts with either shallow or no evolution (Ivison et al., 2010a b). With one far-infrared measurement and one radio continuum measurement, the integrated IR luminosity, thus star formation rate, is determined from the radio while the dust temperature of the modified blackbody is determined by the SED which best fits the given far-infrared data constraint. This was the procedure largely adopted for $850 \mu \mathrm{m}$-selected SMGs (e.g. Smail et al., 2002; Blain et al., 2002; Chapman et al., 2004a, 2005) and lead to the first measurements of SMGs' dust temperatures. The dust temperatures implied from the FIR/radio correlation were, as expected (Eales et al., 2000, Blain et al., 2004a; Chapman et al., 2004a), statistically colder on average than local ULIRGs by $\sim 9 \mathrm{~K}$, a difference which has been attributed to the dust temperature selection effect of $850 \mu \mathrm{m}$ samples (Casey et al., 2009a; Chapman et al., 2010; Magdis et al., 2010).

DSFGs which do have more photometric constraints in the far-infrared, e.g. from Herschel PAcs and SPIRE bands (possibly in addition to other submillimeter data), a more sophisticated direct SED fitting can be done which does not rely on the radio luminosity or an assumed dust temperature. The most simplistic direct far-infrared SED fit is the blackbody fit, or Planck function, $B_{v}(T)$ which is a function of temperature, $T$. However, given that galaxies' temperature is not uniform and inevitably variant, along with the fact that galaxies' dust is not perfectly non-reflective (source emissivity) and there is variation in opacity (i.e. non-uniform screen of dust), galaxies' flux density should be modeled as a modified blackbody of the form $S_{v} \propto\left(1-e^{-\tau(v)}\right) B_{v}(T)$, or

$$
S(v, T) \propto \frac{\left(1-e^{-\tau(v)}\right) v^{3}}{e^{h v / k T}-1}
$$

where $S(v, T)$ is the flux density at $v$ for a given temperature $T$ in units of $\mathrm{erg} \mathrm{s}^{-1} \mathrm{~cm}^{-2} \mathrm{~Hz}^{-1}$ or Jy. The optical depth is $\tau(v)$ is defined by $\tau(v)=\kappa_{v} \Sigma_{\text {dust }}$ and is commonly represented as $\tau(v)=\left(v / v_{0}\right)^{\beta}$, where $\beta$ is the spectral emissivity index and $v_{0}$ is the frequency where optical depth equals unity (Draine, 2006), often assumed as $=3 \mathrm{THz}$ from laboratory experiments $(\approx 100 \mu \mathrm{m})$ although the measured value from a number of galaxies tends more towards $1.5 \mathrm{THz}(\approx 200 \mu \mathrm{m}$ Conley et al., 2011, Rangwala et al., 2011). The dust mass absorption coefficient has an identical frequency dependence, $\kappa_{v}=\kappa_{0}\left(v / v_{0}\right)^{\beta}$, since $\tau \equiv \kappa \Sigma_{\text {dust }}$. The spectral emissivity index, $\beta$, is often assumed to be 1.5 (and is found to usually range between 1-2 in starburst galaxies Hildebrand, 1983, Dunne \& Eales, 2001; Chapin et al., 2011). Note that several works on nearby molecular clouds and dusty regions in nearby galaxies debate whether or not $\beta$ also has temperature dependence, with laboratory experiments suggesting an anti-correlation (Lisenfeld et al., 2000, Dupac et al., 2003, Paradis et al. 2009, Shetty et al., 2009a b; Veneziani et al., 2010, Bracco et al., 2011; Tabatabaei et al., 2013), although that has little impact on the implied SED fit for unresolved distant DSFGs where the effective temperature is only representative of the aggrigate dust temperatures contained within. Note that some works assume that DSFGs galaxies can be approximated as optically thin modified blackbodies, such that the $\left(1-e^{-\tau(v)}\right)$ term reduces to $\nu^{\beta}$. This assumption is perfectly valid at long rest-frame wavelengths $\gtrsim 450 \mu \mathrm{m}$ where $\tau \ll 1$.

While the modeling of a single-temperature component modified blackbody goes a long way in accurately describing a galaxy's far-infrared emission, especially on the Rayleigh-Jeans tail of the distribution (where $850 \mu \mathrm{m}-1.2 \mathrm{~mm}$ observations will sit), the short wavelength regime rarely provides a suitable solution. Most galaxies will exhibit a noticeable flux density excess at mid-infrared wavelengths above what is expected from the Wien tail, between $\sim 8-$ $50 \mu \mathrm{m}$ rest-frame. This mid-infrared excess is generated from smaller clumps of hotter dust within the galaxy. Areas with more compact dust, particularly around a galaxy's nucleus, are more easily heated (either by star forming regions or AGN); higher energy radiation will more easily escape from an optically thin medium (see the thorough discussion of radial density distributions, dust clouds' opacity and dust mass coefficients in Scoville \& Kwan, 1976).

There have been a few methods used in the literature to address the mid-infrared excess when fitting modified blackbodies. The first is to fit the SED to two modified blackbodies of different temperatures simultaneously. The cold component dominates the long-wavelength portion (corresponding to the vast cold dust reservoir), and a warmer component is used to make up the flux deficit at mid-infrared wavelengths. In fact, a number of works over the last decade have suggested using this two-component SED fitting technique (e.g. Dunne \& Eales, 2001, Farrah et al., 2003, 
Galametz et al., 2012). (Kirkpatrick et al., 2012) have argued that for a sample of high- $z$ galaxies, a two component model may fare much better than a single component model. This said, this technique comes at the expense of introducing extra unconstrained parameters to fit (both dust temperatures, both normalizations, both emissivities).

Alternatively, other methods have dealt with this mid-infrared excess differently. First, several works fit the longwavelength data ( $\gtrsim 50 \mu \mathrm{m}$ ) to a single temperature modified blackbody and then cut-off the SED at short wavelengths and attach a power-law SED such that $S(v) \propto v^{-\alpha}$ where $d S / d v \lesssim-\alpha$ (Blain et al., 2003, Younger et al., 2007, 2009b, Roseboom et al., 2013). In other words,

$$
S(v, T)=\left\{\begin{array}{ll}
\frac{\left(1-e^{-\tau(v)}\right) v^{3}}{e^{h / k T}-1} & : v \leq v_{c} \\
v^{-\alpha} & : v>v_{c}
\end{array} \text { where }\left.\frac{d S}{d v}\right|_{v_{c}}=-\alpha\right.
$$

This is a very straightforward method which accounts for the mid-infrared excess, but has the disadvantage of not being easy to directly optimize simultaneously to a set of data which spans the divide at $v_{c}$ without first fitting the long-wavelength blackbody without the powerlaw. Another method is to assume instead that the aggregate SED is the composite of many SEDs of different temperatures, and the distribution of temperatures within a galaxy follows a powerlaw, such that

$$
S\left(v, T_{c}\right)=(\gamma-1) T_{c}^{\gamma-1} \int_{T_{c}}^{\infty}\left(1-e^{-\tau(v)}\right) B_{v}(T) T^{-\gamma} d T
$$

where the integrand is the modified blackbody from Equation 6 multiplied by $T^{-\gamma}, T_{c}$ is the critical or 'minimum' temperature which corresponds to the temperature of the most massive dust reservoir, and $\gamma$ is a parameter of the fit which represents the slope of the powerlaw distribution in dust temperatures (see Kovács et al. 2010, for a detailed description of this method). This formulation is the most physically motivated method of fitting the mid-infrared portion of the SED through direct methods, but can be challenging to constrain as the integral must be computed numerically or can be alternatively written in closed form as an incomplete Riemann zeta function, $Z\left(\gamma-1, h v / k T_{c}\right)$ and $\Gamma(\gamma-1)$ (see Kovács et al. for more). A fourth method for dealing with this mid-infrared spectral component seeks an analytical approximation to the power-law temperature distribution with

$$
S(v, T)=N_{\mathrm{bb}} \frac{(1-\exp [-\tau(v)]) v^{3}}{\exp [h v / k T]-1}+N_{\mathrm{pl}} v^{-\alpha} \exp \left[-\left(v_{c} / v\right)^{2}\right]
$$

where the first component represents the long-wavelength modified blackbody and the second component represents the mid-infrared powerlaw (Casey, 2012). Here $v_{0}$ is the frequency where opacity is unity, $\alpha$ is the mid-infrared powerlaw slope, $v_{c}$ is the frequency at which $d S / d v=-\alpha$ and $N_{\mathrm{bb}}$ and $N_{\mathrm{pl}}$ are the relative normalizations of the two components; $N_{\mathrm{bb}}$ is a free parameter and $N_{\mathrm{pl}}$ is a fixed function ${ }^{13}$ of $T$ and $\beta$.

While much of the earlier work (2009 and prior) in direct SED fitting, particularly for $850 \mu \mathrm{m}$-selected SMGs, was based on the single temperature modified blackbody fit, more recent datasets, with coverage on both sides of the SED peak have lead to the latter fits, incorporating both cold-dust modified blackbody and mid-infrared powerlaw. All three methods will produce very similar fits with indistinguishable luminosities and peak SED wavelengths, although the choice of fitting method (e.g. least squares fit, generate templates and perform a $\chi^{2}$ test, etc.) can impact the subtleties of the fits (Kelly et al., 2012).

\subsection{Estimating $L_{\mathrm{IR}}, T_{\text {dust }}$ and $M_{\text {dust }}$ from an $S E D$}

With an SED in hand, the measurement of infrared luminosity, $L_{\mathrm{IR}}$, is simply the integral under the curve in the infrared. The upper and lower limits of this integral are not completely standard in the literature, although most often are taken from $8-1000 \mu \mathrm{m}$ (e.g. Kennicutt 1998a). While $8-1000 \mu \mathrm{m}$ is inclusive of all dusty emission, the drawback is that such a wide range captures multiple types of dust emission processes, from cold diffuse dust which dominates the long-wavelength portion of the SED to hot-dust and PAH emission in the mid-infrared (described more in $\S 5.7$ ) to non-star formation driven heating, like AGN heating ( $\$ 5.6$. Non-star forming processes can come close to dominating the $8-1000 \mu \mathrm{m}$ infrared luminosity, particularly for optically or X-ray identified AGN at rest-frame wavelengths

\footnotetext{
${ }^{13}$ When expressed in wavelength units, $N_{\mathrm{pl}}=N_{\mathrm{bb}} \frac{\left(1-\exp \left[-\left(\lambda_{0} / \lambda_{c}\right)^{\beta}\right]\right) \lambda_{c}^{-3}}{\exp \left[h c / \lambda_{c} k T\right]-1}$; see Casey (2012) for details.
} 
$\lesssim 40 \mu \mathrm{m}$ (Sanders et al., 1988; Koss et al., 2013). As a result, some works have pushed a narrower range of integration limits to restrict the computation of $L_{\mathrm{IR}}$ to star-formation-driven emission only. This range is sometimes taken as 40-120 $\mu \mathrm{m}$ (from the IRAS-era, e.g. Helou et al., 1988) or a slightly broader range, 40-1000 $\mu \mathrm{m}$. Despite the fact that

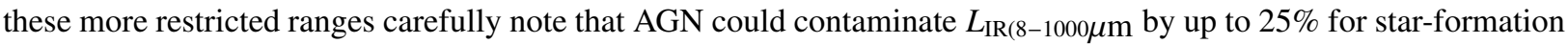
dominated DSFGs (and more for obvious AGN or quasars), the literature largely still uses the 8-1000 $\mu$ m integration limits for historical reasons.

The conversion from $L_{\mathrm{IR}}$ to star formation rate is not straightforward and relies on an understanding of the dust composition and initial mass function (IMF; see the review of Bastian et al., 2010). Most work on DSFGs assume the conversion given in Kennicutt (1998a) of

$$
\operatorname{SFR}\left(M_{\odot} y r^{-1}\right)=4.5 \times 10^{-44} L_{\mathrm{IR}}\left(\operatorname{erg~s}^{-1}\right)=1.71 \times 10^{-10} L_{\mathrm{IR}}\left(L_{\odot}\right)
$$

which takes the radiative transfer models of Leitherer \& Heckman (1995) for continuous starbursts ranging in age from 10-100 Myr and a Salpeter IMF (Salpeter, 1955). Note importantly that this conversion does not account for AGN heating of dust in this wavelength regime, even though AGN fractional contribution is known to be non-negligible (10$30 \%$ ). Here $L_{\mathrm{IR}}$ corresponds to the full infrared 8-1000 $\mu \mathrm{m}$; Kennicutt (1998a) find that most other published work on calibrating this relation lies within $\pm 30 \%$, notwithstanding differences based on IMF assumptions. Some more recent work (e.g. Swinbank et al., 2008) have assumed a Chabrier IMF (Chabrier, 2003) which alters the $L_{\mathrm{IR}}$ to SFR calibration by a factor of $\sim 1.8$ or 0.23 dex, in other words, assuming a Chabrier IMF will produce star formation rates a factor of 1.8 lower than a Salpeter IMF. It is important to note that the conversion from $L_{\mathrm{IR}}$ to SFR is only reliably calibrated locally for moderate-luminosity star forming galaxies. While high-redshift work in the literature have freely applied this scaling for lack of a better solution, it is not yet clear whether or not the conditions of this relation should change under different environments like more extreme luminosity systems at high redshift, and whether or not other dust-heating sources contribute significantly to infrared output, thus contaminating estimated star formation.

Besides $L_{\mathrm{IR}}$ and SFR, a few other properties can be constrained from an SED fit including dust temperature, dust mass and emissivity index. As discussed in $\$ 4.2 .2$ dust temperature scales with the inverse of the far-infrared SED peak, in other words, the wavelength at which the SED peaks in $S_{v}$, dubbed $\lambda_{\text {peak }}$. However, critical to the interpretation of dust temperature, is the understanding that only $\lambda_{\text {peak }}$ is constrainable by current data and the conversion from $\lambda_{\text {peak }}$, what is measured, to dust temperature requires a model assumption. Different assumptions in model dust opacity and emissivity can have dramatically different outcome dust temperatures, as shown in Figure 20 . The differences generated by opacity assumptions (i.e. optically thin or $v_{0}$ value) dominate over emissivity assumptions $(\beta$ value), although both can impact temperature measurements. Any work which hopes to compare temperatures between galaxies in the literature should have a firm understanding of the input model assumptions before interpreting differences, or rather, the comparison can be carried out in the observed quantity, $\lambda_{\text {peak }}$.

Both dust mass and emissivity spectral index are measured from the Rayleigh-Jeans regime of the infrared SED fit. In the optically thin regime (where $S_{v} \approx \tau B_{v}(T)$ ) dust mass is related to dust temperature and flux density via

$$
S_{v}=\kappa_{v} B_{v}(T) M_{\mathrm{dust}} D_{\mathrm{L}}^{-2}
$$

where $\kappa_{v}$ is the dust mass absorption coefficient at frequency $v, B_{v}(T)$ is the Planck function at temperature $T, M_{\text {dust }}$ is the total dust mass of the emitting body and $D_{\mathrm{L}}$ is the luminosity distance. The modified blackbody is effectively represented by the product $\kappa_{v} \Sigma_{\text {dust }} B_{v}(T)$, the product of a perfect blackbody, the dust mass coefficient and dust density. In this optical thin approximation, the galaxy's luminosity thus scales as $L_{v} \propto S_{v} / B_{v}(T) \propto v^{-2}$. Note that at wavelengths shorter than rest-frame $350 \mu \mathrm{m}$, where the optically thin approximation breaks down, dust temperature has a profound effect on the dust mass measurement, since $B_{v}(T)$ is highly dependent on $T$ (Draine \& Li, 2007); for example, a $4 \mathrm{~K}$ difference between $18 \mathrm{~K}$ and $22 \mathrm{~K}$ results in a $150 \%$ increase in measured $M_{\text {dust }}$. This dependence on dust temperature originates from the thermal emission per unit dust mass at a given $\lambda$ being proportional to $\propto\left(e^{h c / \lambda k T}-1\right)^{-1}$. Unfortunately the dust absorption coefficients are poorly constrained, particularly at short wavelengths, so the vast majority of dust mass measurements are taken at long wavelengths $\sim 1 \mathrm{~mm}$. Weingartner \& Draine (2001) and Dunne et al. (2003) measure the dust absorption coefficient at rest-frame $850 \mu \mathrm{m}$ to be $\kappa_{850}=0.15 \mathrm{~m}^{2} \mathrm{~kg}^{-1}$. A few corollaries from the dust mass calculation are the following proportionalities between dust mass, measured flux density, dust temperature, 


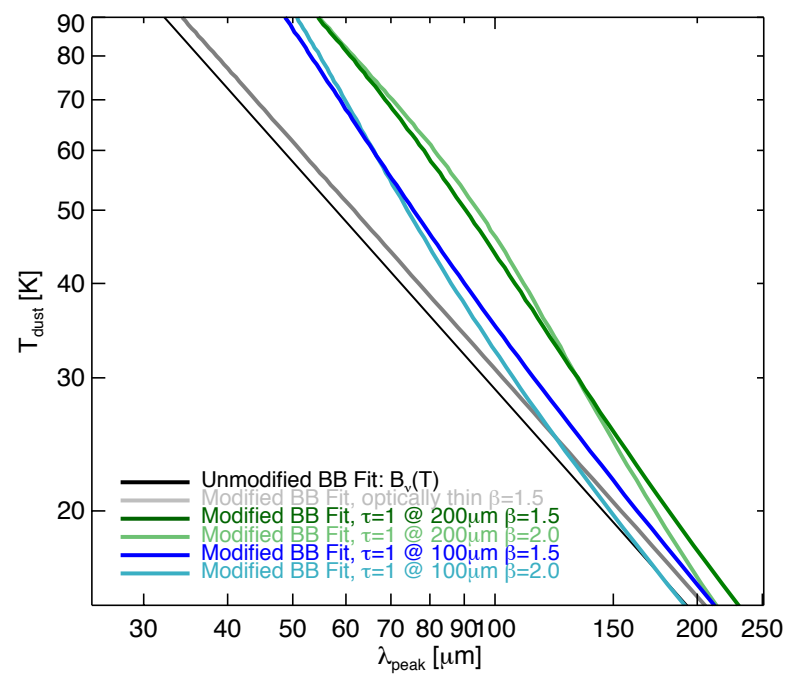

Figure 20: The relationship between SED peak wavelength, $\lambda_{\text {peak }}$, and measured dust temperature, $T_{\text {dust }}$, for six different type of direct SED model assumptions. An unmodified blackbody SED, represented by the Planck function $B_{v}(T)$ is shown in black and represents Wien's displacement law, i.e. $\lambda_{\text {peak }} T=b$, where $b \approx 2.898 \times 10^{-3} \mathrm{~m} \mathrm{~K}$. The gray line is the relation for an optically thin modified blackbody as in Equation 6 Different assumptions regarding opacity, and under what wavelength regime it is equal to unity, are also shown, in green for the $\tau=1$ at $200 \mu \mathrm{m}$ assumption and in blue for the $\tau=1$ at $100 \mu \mathrm{m}$ assumption, with the darker shades denoting an assumed emissivity spectral index of $\beta=1.5$ and lighter shades denoting $\beta=2.0$.

and infrared luminosity:

$$
M_{\text {dust }} \propto S_{v} T_{\text {dust }}^{-1} \quad \text { and } \quad M_{\text {dust }} \propto L_{\mathrm{IR}} T_{\text {dust }}^{-(4+\beta)} .
$$

This highlights the fact that submillimeter flux density does not map simply to dust mass and that knowledge of the dust temperature, or $\lambda_{\text {peak }}$, should be somewhat constrained. Note also that due to the lack of straightforward mapping of $S_{v}$ to $L_{\mathrm{IR}}$, dust mass's relation to luminosity depends steeply on dust temperature. Dust mass can sometimes be used to derive gas mass via an assumed constant gas-to-dust ratio (e.g. Scoville, 2012; Eales et al., 2012; Scoville et al. 2013). Note however that this gas-to-dust ratio is only constrained in Milky Way molecular clouds and some local (U)LIRGs where molecular gas masses measured from millimeter lines can be directly compared to reliable dust masses measured from thermal continuum. A few high-z SMGs which have CO(1-0) observations (Ivison et al., 2011) have also been used to calibrate galaxies' gas-to-dust ratio $\sim 100$, which has been subsequently used to estimate some high-z DSFGs' gas masses (e.g. Swinbank et al., 2013).

It should be noted that there is a degeneracy between dust temperature and the emissivity spectral index $\beta$ similar, yet not as pronounced as the degeneracy between dust temperature and assumed opacity model. Figure 20 illustrates this with different modified blackbodies using the same opacity models but with varying assumptions of $\beta$.

\subsection{Luminosity Functions}

Coupling results from SED fitting and redshift acquisitions-giving you flux and distance-luminosity functions can be constructed. Unlike redshift distributions which extend out to $z \sim 6$ for DSFGs, infrared luminosity functions require additional understanding of sample selection and completeness so, as of yet, only extend to $z \approx 2-3$. Luminosity functions studied beyond that redshift regime are very limited by sample selection effects, potential biasing and incompleteness. This subsection summarizes integrated luminosity function measurements made in the infrared for galaxies beyond the local samples detected by IRAS. We note that many works have also addressed the luminosity function in specific bands, e.g. $12 \mu \mathrm{m}, 24 \mu \mathrm{m}, 35 \mu \mathrm{m}$, etc., however these are not discussed here since they are less physical in nature than a discussion of the total integrated infrared luminosity, $L_{\mathrm{IR}}(8-1000 \mu \mathrm{m})$ which should scale directly with infrared-based star formation rate.

The first measurements of the integrated infrared luminosity function out to $z \sim 1$ were carried out using Spitzer $24 \mu \mathrm{m}$ data (Le Floc'h et al. 2005), extrapolating $24 \mu \mathrm{m}$ flux densities to integrated infrared using local scalings and 
existing knowledge of mid-infrared spectral features and their impact on $24 \mu \mathrm{m}$ flux with redshift. The $1 / V_{\max }$ method of calculating a luminosity function uses the following methodology. The integrated luminosity function is given as

$$
\int_{L_{1}}^{L_{2}} \phi(L) d L=\sum_{i=1}^{n_{\mathrm{obs}}} 1 / V_{\max }(i) \quad \text { where } \quad V_{\max }(i) \equiv \int_{\Omega} \int_{z_{\min }(i)}^{z_{\max }(i)} \frac{d^{2} V}{d \Omega d z} d z d \Omega
$$

where the the redshifts $z_{\min }(i)$ and $z_{\max }(i)$ define the maximum and minimum redshifts that source $i$ would still have been accessible or detected in the given survey. While it presents the simpliest method of arriving at a volume density of sources, the $1 / V_{\max }$ method might not be well suited for sources with increasing flux density at high-redshift (e.g. 1-2 mm detected sources Wall et al. 2008); however, alternative methods rely on model SED and redshift distribution assumptions. Further Spitzer work was presented in Caputi et al. (2007) and Magnelli et al. (2011), with similar conclusions as Le Floc'h et al. with the first extensions out to $z \sim 2$. Within uncertainty, results from the $A K A R I$ satellite out to $z \sim 1.6$ agree with Spitzer results (Goto et al., 2010).

Herschel-based luminosity functions have a clear advantage over previous Spitzer work since Herschel probes the peak of infrared emission directly rather than indirectly; work from Magnelli et al. (2009), Casey et al. (2012a b), Magnelli et al. (2013b), and Gruppioni et al. (2013) have all published integrated luminosity functions all the way out to $z \sim 3.6$ using SED fits to PACS and SPIRE data. These Spitzer and Herschel luminosity functions are plotted together-segregated by redshift regime-in Figure 21

Most literature presentations of the integrated luminosity function also present a analytic approximation, sometimes given as a Schechter function and sometimes as a double power law (Saunders et al., 1990). The double power law is represented as a function of four parameters $\left(L_{\star}, \Phi_{\star}, \alpha\right.$, and $\left.\sigma\right)$ such that:

$$
\Phi(L)=\Phi_{\star}\left(\frac{L}{L_{\star}}\right)^{1-\alpha} \exp \left(\frac{-1}{2 \sigma^{2}} \log ^{2}\left(1+\frac{L}{L_{\star}}\right)\right)
$$

or, alternatively:

$$
\Phi(L)= \begin{cases}\Phi_{\star}\left(\frac{L}{L_{\star}}\right)^{a_{1}} & : L<L_{\star} \\ \Phi_{\star}\left(\frac{L}{L_{\star}}\right)^{a_{2}} & : L<L_{\star}\end{cases}
$$

While all four parameters in Equations 14 and 15 are perhaps constrainable locally, no more than two can be constrained beyond $z \approx 0$, so most works attempt to measure $\Phi_{\star}$ and $L_{\star}$ by fixing $\alpha$ and $\sigma$ (or in Equation 15 , by fixing $a_{1}$ and $a_{2}$ ). Unfortunately, the uncertainties in the luminosity function itself mean that even 'fixed' parameters $-\alpha$ and $\sigma$ or $a_{1}$ and $a_{2}$-are themselves unconstrained, and fixing them arbitrarily results in different absolute calibrations of $L_{\star}$ and $\Phi_{\star}$, as shown in Figure 22. Any physical interpretation of a measured $L_{\star}$ or $\Phi_{\star}$ value should therefore be cautious. While the literature seems to uniformly observe an increase in $L_{\star}$ with redshift no matter which $\alpha$ and $\sigma$ are assumed, despite absolute calibration off by $\sim 1 \mathrm{dex}$, a concrete trend is not observed in the measured $\Phi_{\star}$ values. It is critical to also recognize the interdependence of the two measurements; no abrupt 'break' or 'knee' is seen in the data, so a fit with high- $L_{\star}$ and low- $\Phi_{\star}$ might be as equally adequate as a fit with low $-L_{\star}$ and high- $\Phi_{\star}$. In the future, as more significant samples become available, Monte Carlo Markov Chains should be used to hone in on the key parameter values.

\subsection{Contribution to Cosmic Star Formation Rate Density}

The star formation rate density (SFRD) represents the total star formation occurring per unit time and volume at a given epoch; determining the contribution of infrared galaxies to the Universe's SFRD has been an important focus of DSFG surveys, particularly in understanding the role of dust obscuration at high redshifts. The SFRD is better constrained than infrared luminosity functions as the former is the integral of the latter. Like the calculation of the luminosity function, the $1 / V_{\max }$ method is most commonly used to form an understanding of a galaxy's accessible volume; in other words, given the limits of the survey (e.g. $S_{850}>2 \mathrm{mJy}$ covering, e.g., $0.5 \mathrm{deg}^{2}$ ), the maximum volume is found by shifting the given galaxy to higher redshift until that galaxy would no longer be detectable at its measured luminosity. The volume is then calculated based on that maximum redshift, and star formation rate is determined from infrared luminosity assuming a scaling like that of Equation 10 The infrared luminosity density (IRLD) may be calculated as an alternate to the SFRD, removing the uncertainty of the $L_{\mathrm{IR}}-S F R$ calibration. As 

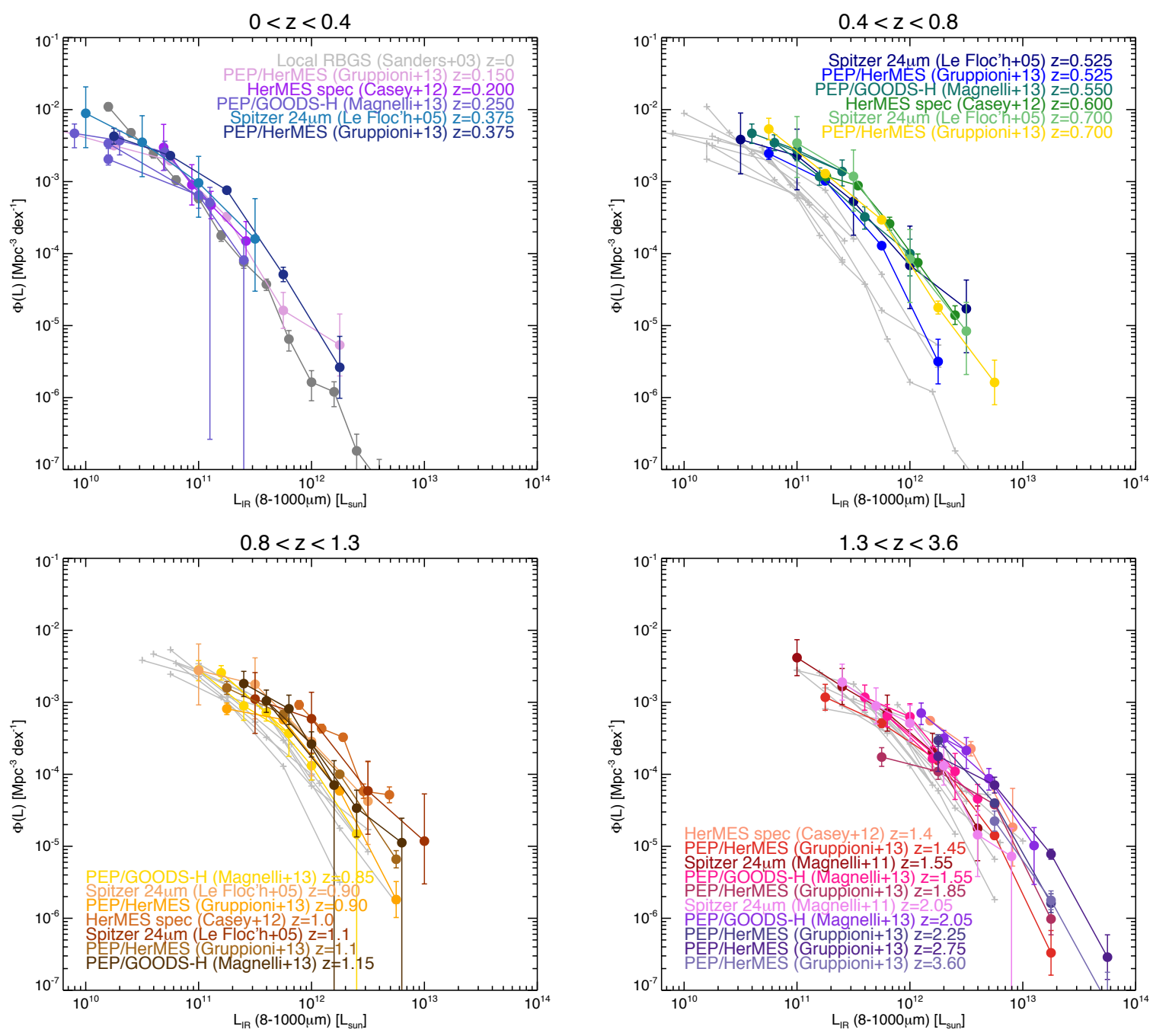

Figure 21: Measured integrated infrared luminosity functions from the literature, including work on the local RBGS sample (Sanders et al. 2003), Spitzer 24 $\mu$ m-selected samples (Le Floc'h et al. 2005. Magnelli et al. 2009. 2011), and from Herschel via the PEP and HerMES surveys (Casey et al. 2012a Magnelli et al. 2013b Gruppioni et al. 2013). Here we only include data points themselves and not analytic approximations to the luminosity function, which is often given as a double power law. The four redshift bins are plotted with the same dynamic range, with the previous (lower) redshift bin illustrated in gray underneath to demonstrate evolution. 

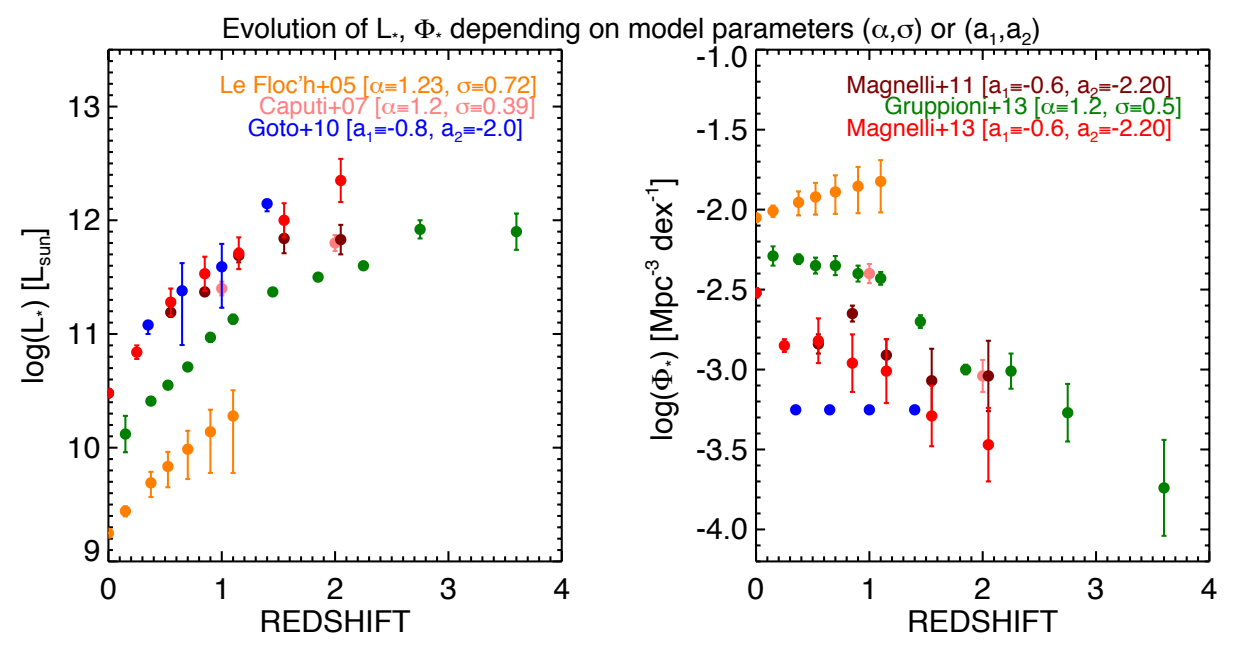

Figure 22: The evolution of $L_{\star}$ and $\Phi_{\star}$ for the integrated infrared luminosity function from the literature. One of two models is adopted in these works. Le Floc'h et al. (2005), Caputi et al. (2007) and Gruppioni et al. (2013) all use the model described in Equation 14 while Goto et al. (2010), Magnelli et al. (2011) and Magnelli et al. (2013b) use the model from Equation 15 While $L_{\star}$ shows clear signs of downsizing in all models, irrespective of absolute calibration, the evolution in $\Phi_{\star}$ is more model dependent.

long as there is a clear understanding of a survey's depth and sky area coverage, the IRLD or SFRD is easily calculated as the total IR luminosity or star formation rate of the sample divided by its volume. Splitting the measurement into redshift bins then gives more detailed information on sample evolution. Plots of SFRD against redshift or look-back time are often referred to as a Lilly-Madau diagram, first discussed in Lilly et al. (1995) and Madau et al. (1996).

Figure 23 illustrates infrared-based estimates to the SFRD contribution from a variety of surveys and Figure 24 shows their contributions broken down by luminosity class. Both of these SFRD figures provide essential illustrations to the interpretation of cosmic star formation. While the first (Figure 23) includes samples known to suffer from incompleteness and biases, it is a useful tool for visualizing the net contribution from any one sample. For example, even though the $850 \mu \mathrm{m}$-selected SMG sample from Chapman et al. (2005) is known to be biased against warm-dust and radio-quiet galaxies, one can still determine that their SFRD contribution is $\sim 10 \%$ of the total at $z \sim 2$. Knowing it is incomplete, one then knows that the real contribution is $\gtrsim 10 \%$. On the other hand, if the goal is to measure the total contribution of a population to cosmic star formation, then Figure 24 is more useful, as samples have been corrected for incompleteness and grouped in equal luminosity bins so as to compare like-with-like.

As shown previously in, e.g., Le Floc'h et al. (2005), the contribution from ultraluminous infrared galaxies evolves strongly. ULIRGs have an insignificant role in cosmic star formation in the present-day Universe, but contributes $\sim 10 \%$ at $z \sim 1$ and $\sim 50 \%$ at $z \approx 2$ (see Figure 24). The contribution from slightly less luminous galaxies-LIRGs-appears to peak at $z \sim 1$, as verified by multiple literature sources, amounting to $\sim 50 \%$ of all star formation at that epoch. A key goal of the next several decades will be to improve the estimates of infrared samples' SFRD contributions out to higher redshifts with as much precision as the current optical and rest-frame ultraviolet estimates from pencil-beam surveys (e.g. Hopkins \& Beacom, 2006) currently provide. Despite some limitations in infrared samples, the last decade's work on DSFGs has made it clear that dust-obscured star formation is non-negligible in the high-redshift Universe and should be studied carefully if one wishes to understand star formation processes at early times. 


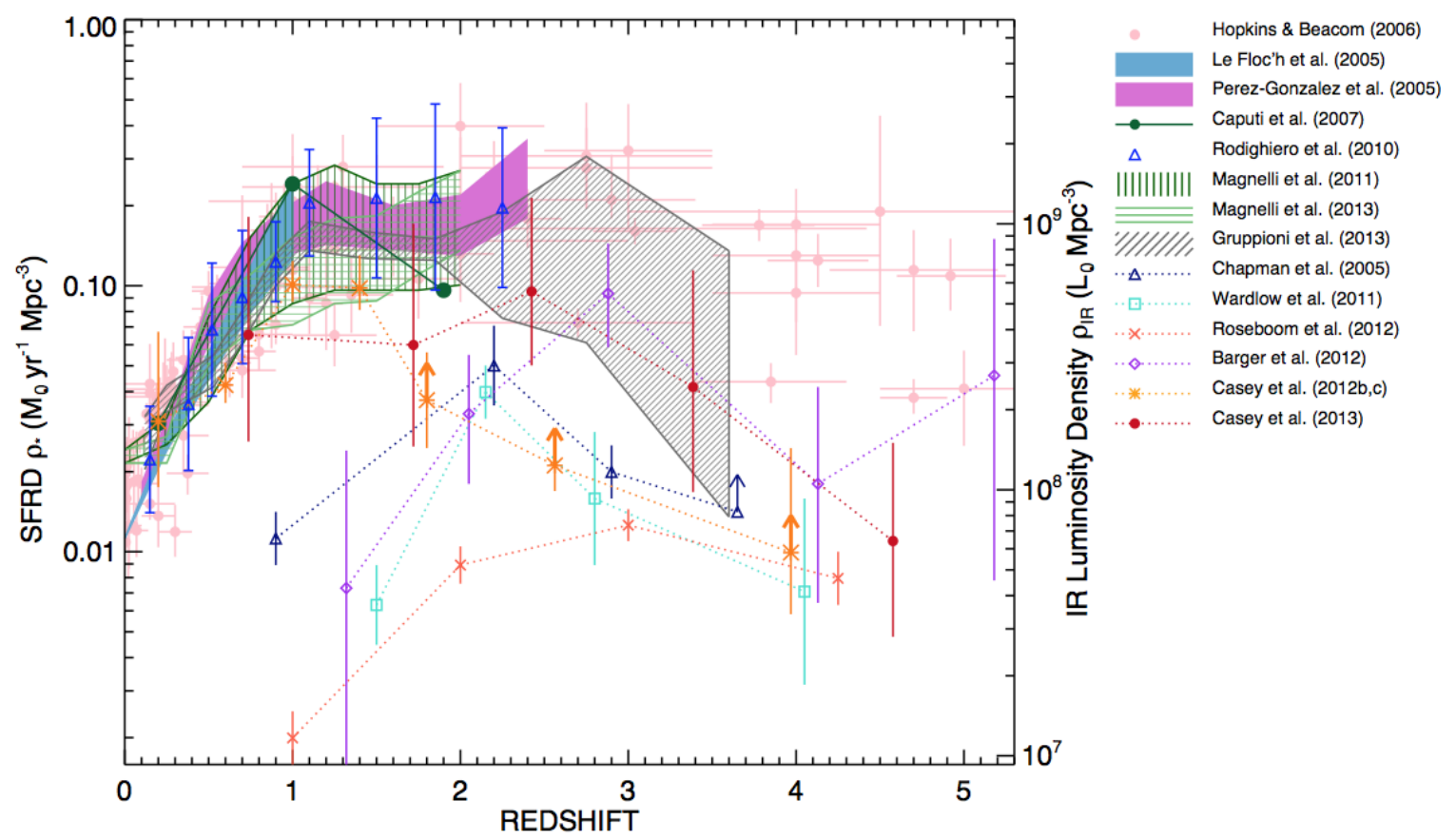

Figure 23: The contribution of various galaxy populations to the cosmic star formation rate density (left y-axis) or infrared luminosity density (right y-axis). The Kennicutt (1998a) scaling for $L_{\mathrm{IR}}$ to SFR in Equation 10 is assumed. This SFRD plot shows the contributions from total surveyed infrared populations, whereas Figure 24 shows the break-down of contributions by luminosity bins between $0<z<2.5$. All infraredbased estimates are compared to the optical/rest-frame UV estimates compiled in Hopkins \& Beacom (2006) which have been corrected for dust extinction, and therefore represent an approximation to the total star formation rate density in the Universe at a given epoch. The total infrared estimates from the literature (integrated over $\sim 10^{8}-10^{13.5} \mathrm{~L}_{\odot}$ ) come from Spitzer samples (Le Floc'h et al. 2005. Pérez-González et al. 2005. Caputi et al. 2007. Rodighiero et al. 2010|. Magnelli et al. 2011) and Herschel samples (Gruppioni et al.||2013). In contrast, several samples from the submm and $\mathrm{mm}$ are also included; although they are known to be incomplete, they provide a benchmark lower limits for the true contributions from their respective populations. These estimates include 850/870 $\mu \mathrm{m}$-selected SMGs (Chapman et al. |2005. Wardlow et al. |2011||Barger et al. 2012 Casey et al. 2013), $1.2 \mathrm{~mm}$-selected MMGs (Roseboom et al. 2012), 250-500 $\mu \mathrm{m}$-selected Herschel DSFGs (Casey et al. 2012a b) and $450 \mu \mathrm{m}$ Scuba-2-selected DSFGs (Casey et al. 2013). See legend for color and symbol details. 


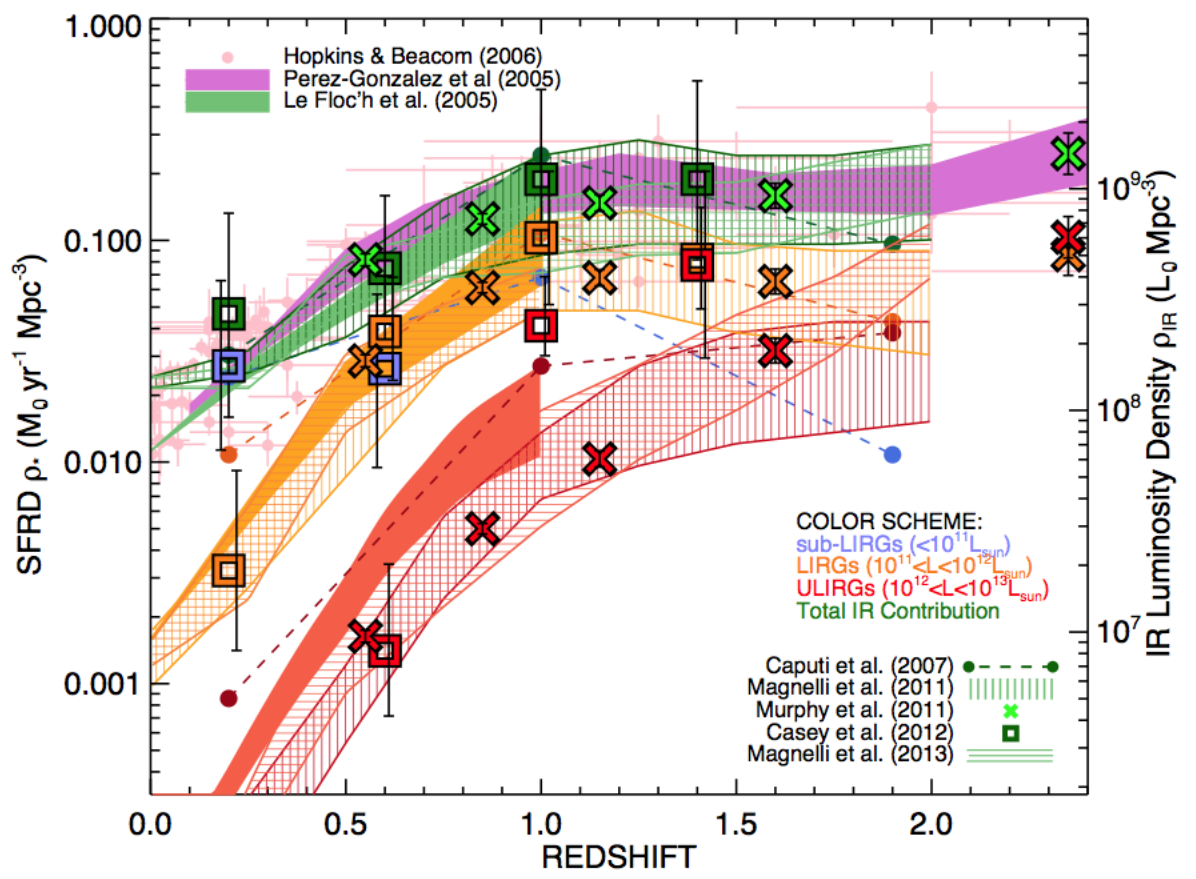

Figure 24: Estimates of DSFGs' contributions to cosmic star formation and infrared luminosity densities given by decade in luminosity. The overall color scheme adopted is that the total integrated infrared contribution is shown in green, the sub-LIRG population (i.e. $L<10^{11} \mathrm{~L}_{\odot}$ ) in blue, the LIRG population $\left(10^{11}<L<10^{12} \mathrm{~L} \odot\right)$ in yellow-orange, and the ULIRG population $\left(10^{12}<L<10^{13} \mathrm{~L}_{\odot}\right)$ in red. The literature sources of each estimate are given by different symbols or line-types as indicated (Le Floc'h et al. 2005 . Pérez-González et al. 2005 . Hopkins \& Beacom 2006. Caputi et al. 2007. Magnelli et al. 2011 2013b. Murphy et al. 2011. Casey. 2012). Note that not all literature derivations of different luminosity bins are computationally equivalent since some luminosities are derived directly from the far-infrared bands while others are extrapolations from mid-infrared bands. Most estimates agree within uncertainty in all luminosity bins, except the ULIRG estimates at $z \sim 1$, where Caputi et al. (2007) and Casey et al. (2012a) estimates are $\sim 0.5$ dex higher than those from Magnelli et al. (2011) 2013b) and Murphy et al. (2011). Overall, all datasets find the nearly insignificant role of ultraluminous galaxies at $z \sim 0$ while a dominating role of ultra-bright galaxies at high redshifts $z \gtrsim 1.5$. As more direct-FIR measurements become commonplace, the SFRD contributions from different luminosity types will be better constrained. 


\section{Physical Characterization}

While the previous sections focus on the surveys which find dusty star forming galaxies, their observational strengths and limitations, this section addresses DSFGs' physical characterization. Basic information like redshifts and luminosities are needed before carrying out a more detailed characterization, but it is the characterization which tells us the physical setup driving DSFGs' intense infrared luminosities, shedding light on the entire process of galaxy formation in a cosmological framework. Furthermore, physical characterization provides essential clues to the interplay between active Active Galactic Nuclei (AGN) and starburst regions, the kinematic history of gas and stars in galaxies' disks, the total stellar, dynamical, dust and gas masses, the physical extent of galaxies, and whether or not those galaxies have recently undergone interactions with other galaxies.

This detailed information on individual galaxies is what we would hope to obtain for all DSFGs, but unfortunately, the observations it requires are expensive. Nearly all observations described in this section have constituted major time investments on behalf of cutting-edge telescopes world-wide, and sometimes, only provide insight for a small handful of galaxies (3-20 SMGs for example). Although our capabilities are improving with the next generation of facilities (e.g. ALMA, CCAT, SPICA, GMT, TMT, and E-ELT), physical characterization will always be available for only a subset of galaxies detected in our large surveys. As such, the selection function determining which galaxies are characterized with follow-up observations is always critical to keep in mind when interpreting these data. For example, most of the initial follow-up of $850 \mu \mathrm{m}$-selected SMGs was carried out on the most luminous subset of SMGs at $L_{\mathrm{IR}}>10^{12.5} \mathrm{~L}_{\odot}$; those systems were determined at the time to be extreme, scaled-up analogues of local ULIRGs with star formation rates SFR $>1000 \mathrm{M}_{\odot} \mathrm{yr}^{-1}{ }^{14}$. Although true, that characterization is not appropriate for the whole population. Since many of the observations below are only representative of DSFG subset populations, it is essential that the reader keep this type of bias in mind when interpreting results.

From an observer's perspective, this section addresses most types of detailed physical characterization from the radio through the X-ray except molecular gas characterization. The latter has been such a substantial piece of the puzzle in interpreting DSFG evolution that it deserved its own section $(\$ 8)$ which follows this section. Included below are discussions of DSFGs' star formation history and dynamical time, dust characterization, stellar masses, stellar IMF, AGN content, kinematics, and physical size.

\subsection{Star Formation History \& Dynamical Time}

The dynamical time of DSFGs is constrained observationally from a small sample of galaxies at high- $z$ and, more frequently, from inference of local ULIRG IRAS samples. The timescale for evolution in starbursting galaxies can be represented as the timescale of the burst, observationally probed as the depletion timescale $\left(\tau_{\text {depl }}\right)$ for molecular gas, or molecular gas mass over current star formation rate. Even with a large potential of star forming fuel, the high star formation rates seen in DSFGs often implies short depletion timescales.

Solomon \& Sage (1988) provides a succinct summary of the depletion time, or rather $L_{\mathrm{FIR}} / L_{\mathrm{CO}}$, for local ULIRGs divided by morphological merger classes, given prior suggestions that mergers and interactions are responsible for elevated $L_{\mathrm{FIR}} / L_{\mathrm{CO}}$ or lower $\tau_{\text {depl }}$ over normal star-forming galaxies (Sanders \& Mirabel, 1985; Sanders et al., 1986 , Young et al. 1986 ). While they find non-interacting LIRGs have gas depletion timescales of $\approx 150_{-30}^{+20} \mathrm{Myr}$, merging or merged galaxies have $\tau_{\text {depl }}=60_{-20}^{+30} \mathrm{Myr}$ and galaxies with tidal tails and bridges (e.g. evidence of the initial stages of a merger) have $\tau_{\text {depl }}=16_{-4}^{+6}$ Myr. The difference between merging or interacting galaxies and non-merging systems is striking.

At higher redshifts, the best measurements of DSFG timescales come from $850 \mu$ m-selected SMGs with CO measurements (Neri et al. 2003: Greve et al., 2005; Tacconi et al. 2006, 2008, Coppin et al., 2008b; Bothwell et al. 2010, Casey et al., 2011a) Bothwell et al. 2013a). Bothwell et al. (2013a) provides a summary of all CO surveys directed at $850 \mu \mathrm{m}$-selected sources, and more recently Carilli \& Walter (2013) summarizes molecular gas surveys at high- $z$. As described later in $\S 8$, these surveys measure typical depletion timescales of $\sim 100-200 \mathrm{Myr}$ for SMGs versus much longer $\sim 1$ Gyr timescales for normal galaxies (e.g. Tacconi et al., 2010). Although these timescales are longer than seen in local ULIRGs, this is primarily due to elevated gas fractions in high-redshift galaxies. In other words, for a fixed SFR, the gas masses at high- $z$ are higher by factors of $2-3$.

\footnotetext{
${ }^{14}$ The evidence for this characterization comes in this section and $\S 8$ although we note that this description has come into question in recent years.
} 

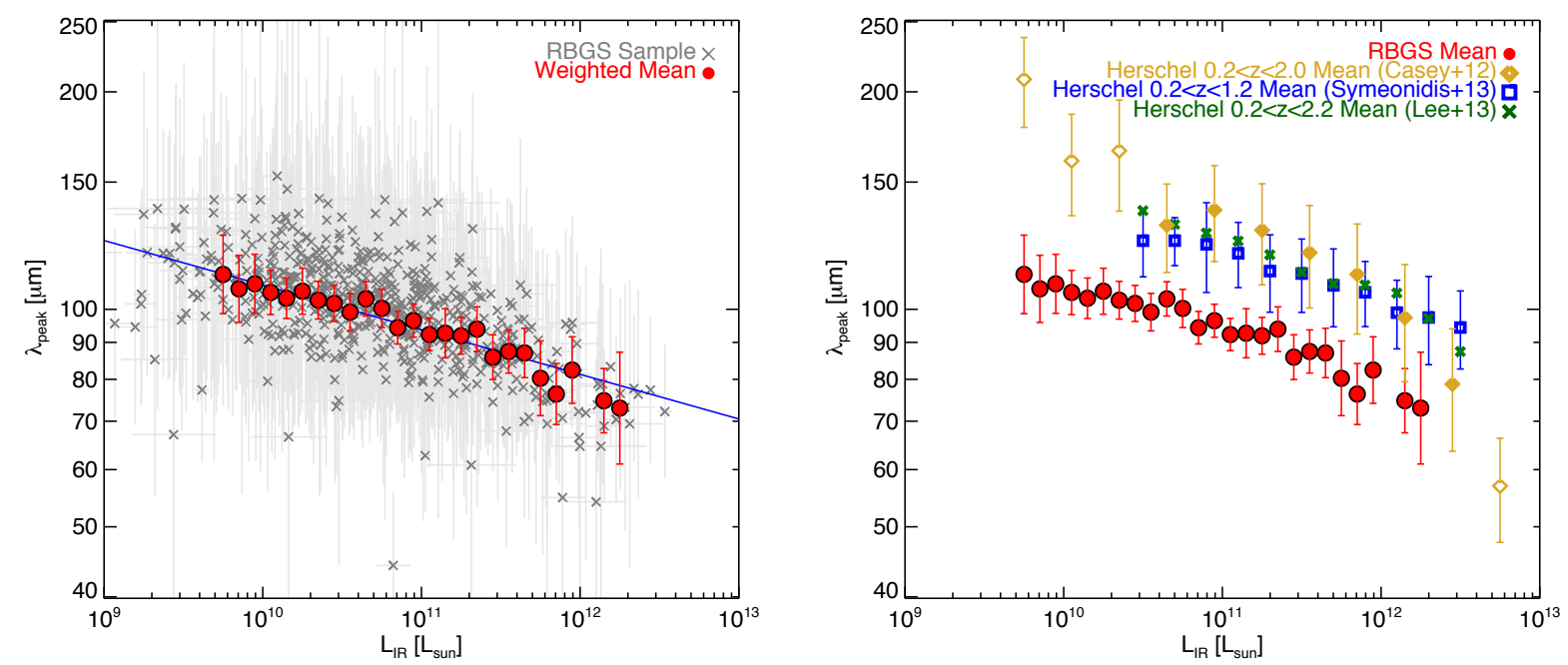

Figure 25: The relationship between luminosity and dust temperature plotted in $L_{\mathrm{IR}}-\lambda_{\text {peak }}$ space. $\lambda_{\text {peak }}$ is inversely proportional to dust temperature, the exact conversion being dependent on the assumed opacity model. At right, the relation is plotted for the local and unbiased Revised Bright Galaxy Sample (Sanders et al. 2003 Chapman et al. 2003c). Higher-luminosity sources appear to be warmer, following a power-law type relation with $\lambda_{\text {peak }} \propto L_{\mathrm{IR}}^{-0.06}$. At left, we illustrate the same relation measured for limited samples of high- $z$ sources detected with Herschel-SpIRE(Casey et al. 2012a, Symeonidis et al. 2013. Lee et al. 2013, who find a similar slope but offset relation. The offset implies that galaxies of equal luminosity at higher redshift have colder dust. Colder dust could be caused by different dust composition or geometries.

\subsection{Dust Characterization}

Figure 25 contrasts the measured dust temperatures of DSFGs in the local and high- $z$ Universe; the local samples are collated from the Revised Bright Galaxy Sample (Chapman et al.2003c most recently with re-analyzed SEDs in U et al. 2012) and high- $z$ samples are all Herschel-SpIRE selected (Casey, 2012; Symeonidis et al., 2013; Lee et al., 2013). Chapman et al. (2003c) fit the $L_{\mathrm{IR}}$ dust temperature relation using the $100 \mu \mathrm{m}$ to $60 \mu \mathrm{m}$ color of the local sample in lieu of temperature. Here we convert that value to $\lambda_{\text {peak }}$ (a conversion which is by-and-large independent of SED fitting method). Unfortunately, most measurements of dust temperature in the high- $z$ Universe pre-Herschel were limited to a handful of objects, mostly SMGs, which had more than one photometric constraint in the $\sim 50-300 \mu \mathrm{m}$ rest-frame wavelength range. Most SMGs (e.g. Chapman et al., 2005) lacked FIR SED measurements to constrain dust temperature directly. In addition, because SMGs have been selected primarily on the Rayleigh-Jeans tail, they carry a known bias against warmer dust temperature systems. Since Herschel-SpIRE's selection straddles the FIR emission peak out to $z \sim 2$ it's an excellent tool for constraining high- $z$ temperatures, as has been done by Casey et al. (2012a) for spectroscopic samples, and Symeonidis et al. (2013) and Lee et al. (2013) for much larger photometric samples. Notably, DSFGs at higher redshifts have cooler SEDs than those locally, even when correction for selection bias is taken into account. This is thought to be due to more extended dust distributions (e.g. Swinbank et al., 2013) in high- $z$ DSFGs on scales $\gtrsim 2 \mathrm{kpc}$, versus the more compact ISM seen in local ULIRGs $\approx 1 \mathrm{kpc}$ (see more on DSFGs' sizes in $\S 5.10$.

Dust masses for DSFGs have not been straightforward to measure precisely given the lack of FIR photometry on most galaxies, however, as noted in $\S 4.3$, even if dust temperature is not well constrained, a flux density measurement on the Rayleigh-Jeans tail (i.e. optically-thin portion) of the black body provides a decent dust mass estimator. For example, the nominal $850 \mu \mathrm{m}$-selected SMGs, with $S_{850} \approx 5-10 \mathrm{mJy}$ (Smail et al., 2002 ), $\langle z\rangle \sim 2.2$ (Chapman et al., 2005), and dust temperatures $T_{\text {dust }} \approx 20-40 \mathrm{~K}$ (Kovács et al., 2006) imply dust masses of $\approx 5-20 \times 10^{8} \mathrm{M}_{\odot}$. In contrast, the dust masses of more recently observed Herschel-selected galaxies, with flux densities of $S_{250-500} \approx 20$ $60 \mathrm{mJy},\langle z\rangle \sim 1$, and $T_{\text {dust }} \approx 30-50 \mathrm{~K}$ (Casey et al., 2012a) are much lower at $M_{\text {dust }} \approx 1-20 \times 10^{8} \mathrm{M}_{\odot}$, due in part to the fact that $850 \mu \mathrm{m}$ preferentially selected colder, more massive dusty galaxies than Herschel (from Equation 12 ) and Herschel-selected galaxies sit at lower redshifts. At high- $z$, the $850 \mu \mathrm{m}$-selected SMG sample described by Ivison et al. (2011) summarize $\mathrm{CO}(1-0)$ observations, enabling a direct comparison of SMGs' dust-to-gas ratios to those of 
local galaxies through the measured quantity $\left\langle L_{C O}^{\prime} / L_{850}\right\rangle$. By and large, the gas-to-dust ratio measured is consistent with local values, $\approx 100$, enabling a direct scaling between dust mass and gas mass, a far more direct and simple observational investment than conversions from $\mathrm{CO}$ molecular gas transitions (see more about gas mass measurements in $\$ 8$.

Note that recent work on Herschel samples of modest star formers at $0.2<z<2$ have found tight correlations between dust masses and stellar mass (e.g. Dunne et al., 2011; Buat et al., 2012, Magdis et al., 2012b) or specific starformation rate, $s S F R$ (Santini et al., 2013). These works assume that the gas-to-dust mass ratio varies with metallicity and can be used to constrain gas fractions on and off the star forming main sequence.

\subsection{Stellar Masses}

Determining stellar masses for high- $z$ dusty star-forming galaxies has been a task that has proven to be highly uncertain and the subject of heavy debate. For example, for the exact same $850 \mu \mathrm{m}$-selected submillimeter galaxies, Hainline et al. (2011) and Michałowski et al. (2012) find values that show up to an order of magnitude difference from one another. The uncertainties that plague stellar mass measurements of high- $z$ dusty galaxies are both those common to all stellar mass measurements from galaxies, as well as some that are unique to dusty galaxies at highredshift. These encompass both our theoretical understanding of stellar population modeling and the varying quality of observational constraints.

The first component in determining the stellar mass of DSFGs that introduces some uncertainty is the assumed star formation history (SFH). Typical assumptions involve an exponentially declining SFH, a constant SFH, a single burst of star formation, or a multiple-component SFH. As noted by Dunlop (2011), a multiple component SFH can lead to higher inferred stellar masses than a single component model. In a single burst, the entire stellar population must be young in order to match the observed UV emission. This typically results in estimated stellar masses that are somewhat low. For a continuous star formation history, the current SFR is set by the current UV flux, and the length of time over which the galaxy has been forming stars is set by the longer wavelength optical and NIR emission. The multi-component fits allow, in principle, both possibilities. The burst can drive the observed UV emission, while the continuous SFH may contribute to the optical emission with ages that can be somewhat older than when solely assuming a single $\mathrm{SFH}$.

In principle, the SFHs of massive galaxies that form stars in a quiescent mode (that is, not undergoing a burst that would drive them significantly off of the SFR- $M_{*}$ relation) are reasonably well constrained. Thomas et al. (2005), McDermid et al. (2012) and Pacifici et al. (2013) find older stellar populations for galaxies of increasing mass. Davé et al. (2012) and others show that a lognormal SFH for galaxies that peaks at increasing amplitude and earlier times for more massive galaxies provides a good fit to the typical SFH for galaxies in cosmological simulations. Thus, in principle, a reasonable SFH for a DSFG would be a lognormal SFH calibrated for the halo mass of the galaxy under question, with potentially a second late burst component added on. This, of course, is predicated on some knowledge of the halo mass (we discuss halo mass determinations of DSFGs in more detail in $\S 7$ ).

The second key issue involved is in the choice of stellar population synthesis (SPS) model parameters. SPS calculations are recently reviewed by Conroy (2013), and we refer the reader to that review for a more thorough discussion of uncertainties in population synthesis calculations. Briefly, we note that Hainline et al. (2011) explored both Bruzual \& Charlot (2003) and Maraston (2005) stellar evolutionary models, and found that utilizing the Bruzual $\&$ Charlot (2003) models resulted in roughly 50\% higher masses than the Maraston (2005) models. Note, however, than the Hainline et al. (2011) stellar masses account for this systematic uncertainty.

The third main uncertainty involves the choice of a stellar initial mass function. As we discuss both in the next subsection ( $\$ 5.4$, as well as in $\S 10$, both observational constraints and theoretical models that aim to pin down the IMF in high- $z$ DSFGs result in a wide range of potential options. Most variations from a locally-calibrated IMF at high- $z$ tend to go in the direction of more massive stars and less low-mass stars; this said, observational constraints of the IMF in present day ellipticals, the supposed descendants of high- $z$ DSFGs suggest that the IMF may actually be bottom-heavy in these. Even minor differences such as the usage of a Chabrier (2003) IMF versus a Salpeter (1955) IMF can result in a factor $\sim 1.8$ difference in the calculated stellar mass (and, consequently, the star formation rate).

Borys et al. (2005) provided one of the first large samples of stellar mass measurements of high- $z$ DSFGs, focusing on the submillimeter galaxy population. Borys et al. assumed both instantaneous burst SFHs, as well as constant SFHs as two potential limiting cases. With an assumed Miller-Scalo IMF (Miller \& Scalo, 1979), this group provided 
evidence that SMGs are an extremely massive galaxy population, with derived stellar masses $\operatorname{ranging} \operatorname{from} \log \left(M_{*}\right)$ $=[11.14,12.15]$ (with one outlier at $\log \left(M_{*}\right)=10.56$ ) using the conversion $M_{*}=10^{-0.4\left(M_{K}-3.3\right)} / L K M$ where $L K M$ is the light-to-mass ratio, taken on average to be $\sim 3.2$. The median of the Borys et al. (2005) sample was $\left\langle M_{*}\right\rangle=$ $2.5 \times 10^{11} \mathrm{M}_{\odot}$. Because this study was aimed at investigating X-ray detected SMGs (to compare stellar masses to black hole masses), contamination of the stellar masses by AGN is a potential issue, particularly because rest-frame $K$-band luminosities were used in the derivation. Dye et al. (2008) determine the stellar masses for galaxies in the SHADES survey with only 8 bands of photometry utilizing the synthetic spectra of Bruzual \& Charlot (2003); while they find comparable stellar masses to the work of Borys et al. (2005), the margin of uncertainty from lack of observational constraints dominate.

In 2011, two nearly contemporaneous papers came out with starkly different results for the stellar masses of the same sample of SMGs. Hainline et al. (2011) examined 70 SMGs, and found that 10\% of their sample had substantial contributions to the SED from AGN, much like those analyzed in Borys et al. (2005). Instead of deriving stellar masses from rest-frame $K$-band $(2.2 \mu \mathrm{m})$ luminosities, which can potentially be contaminated by powerlaw emission from AGN heating, Hainline et al. (2011) make use of rest-frame $H$-band luminosities (1.6$\mu \mathrm{m})$ which probe the peak of stellar emission more directly and therefore, produce a more accurate measure of stellar mass. Hainline et al. find lower stellar masses for their sample of SMGs than most other studies, with a median $M_{*}$ of $\left\langle M_{*}\right\rangle \approx$ $7 \times 10^{10} \mathrm{M}_{\odot}$. At the same time, Michałowski et al. (2010) found a median stellar mass in the Hainline et al. (2011) sample of $76 \mathrm{SMGs}$ of $\left\langle M_{*}\right\rangle \approx 3.5 \times 10^{11} \mathrm{M}_{\odot}$. When correcting for assumed IMF effects, Hainline et al. (2011) find that their median mass comes to within roughly a factor of 3 of the Michałowski et al. (2010) results. Michałowski et al. (2012) followed up on these works, and performed a systematic analysis of the discrepancies in the Hainline et al. (2011) and Michałowski et al. (2010) stellar masses. These authors claimed that the discrepancy was not dominated by AGN contamination, but rather different choices of stellar IMFs, population synthesis models, and the star formation history.

In the absence of strong constraints on either the stellar IMF in high- $z$ SMGs, or the star formation history, it is reasonable to expect an inherent factor $\sim 2-3$ uncertainty in any stellar mass measurement of high- $z$ DSFG. One promising way forward is to utilize other mass constraints. For example, CO dynamical mass measurements (e.g. Greve et al. 2005, Tacconi et al., 2008, Bothwell et al., 2013a) in combination with an assumed dark halo profile can provide a constraint on the remaining stellar mass. This neglects any contribution to the mass by HI, and comes with the uncertainty of an assumed dynamical state of SMGs. The abundance matching methodology of Conroy \& Wechsler (2009) and Behroozi et al. (2013) can provide some constraints on the average $M_{*}$ that must exist in DSFGs (Béthermin et al., 2013), given some a priori knowledge of their typical halo masses. The abundance matching technique assumes that the most (stellar) massive galaxies at a given redshift reside in the most massive haloes, least massive galaxies in the least massive haloes, and corresponding matches at intermediate masses. By employing such a technique, Behroozi et al. (2013) constrain the average stellar mass of galaxies in haloes between $M_{\text {halo }}=10^{12}-10^{13} \mathrm{M}_{\odot}$ at $z=2$ to range from $M_{*} \approx 5 \times 10^{9}-10^{11} \mathrm{M}_{\odot}$. If we assume the Hickox et al. (2012) halo mass measurements of $850 \mu \mathrm{m}$ selected SMGs (c.f. $\S 7$ ) of $\sim 6 \times 10^{12} h^{-1} \mathrm{M}_{\odot}$, one arrives at a typical stellar mass of a $z \sim 2 \mathrm{SMG}$ of $\sim 1.1 \times 10^{11} \mathrm{M}_{\odot}$. We note that this is significantly below the empirical upper limit for stellar masses of $z \sim 2$ SMGs of $\sim 1-3 \times 10^{12} \mathrm{M}_{\odot}$ derived by Hayward (2013).

Outside of SMGs, the number of measurements of stellar masses of high- $z$ DSFGs are relatively limited, though extremely important in terms of working toward a synthesis picture of how different breeds of DSFGs in the high- $z$ zoo may or may not be related. Berta et al. (2007) and Lonsdale et al. (2009) constrained the stellar masses for some Spitzer-selected ULIRGs at $z \sim 2$ that were selected at $5.8 \mu \mathrm{m}(>25.8 \mu \mathrm{Jy})$ and $24 \mu \mathrm{m}(>400 \mu \mathrm{Jy})$, respectively. In order to investigate a potential evolutionary connection between $24 \mu \mathrm{m}$ DOGs and $850 \mu \mathrm{m}$ SMGs, Bussmann et al. (2012) examined the stellar masses for a sample of DOGs that had both bump-like and powerlaw-like mid-IR colors. While the spectral bump owes to an opacity feature in stellar spectra, the powerlaw feature likely owes either to AGN contribution, or to dust opacity in that region (Narayanan et al., 2010a; Snyder et al., 2013). Bussmann et al. (2012) utilized a fixed method of stellar mass determination for their sample of DOGs, and a sample of SMGs, and found that DOGs selected at this flux density cut tended to be roughly twice as massive as traditional SMGs.

Going forward, accurately constraining the stellar masses of DSFGs will be a critical step toward our understanding their relationship to other galaxy populations at high- $z$, and whether or not they lie on the galaxy main sequence at a given redshift. Resolved near-IR IFU work may help to disentangle any potential contribution from AGN to the luminosity. Similarly, accurate CO or [CII] dynamical masses (which will require resolved morphologies in order to 
constrain the galaxy inclination angle) may help to place stronger constraints on the stellar masses of high- $z$ DSFGs.

\subsection{Stellar IMF}

Whether or not the stellar initial mass function (IMF) varies with physical conditions in galaxies is a question of fundamental importance that impacts nearly every aspect of extragalactic astrophysics. The topic is still open, and is most recently reviewed by Bastian et al. (2010). We refer the reader to the Bastian review for a comprehensive picture of potential IMF variations in the Galaxy and other galaxies, and concentrate here on evidence for IMF variations at high- $z$. We employ the following definitions, and assume a basic IMF shape that is has a log-normal shape in $d N / d \log m-M$ space, with potentially steeper slopes at low and high-masses (c.f. Figure 1 from Bastian et al. 2010). "Bottom light" refers to a deficit in low-mass stars, and implies that the IMF slope does not vary, but rather just the mass at which the IMF turns over varies. "Bottom heavy" is the opposite. "Top heavy", in contrast, refers to a changing of the slope of the IMF. Both bottom light and top heavy IMFs have the consequence of having more massive stars per unit stellar mass formed than a typical Milky Way IMF, while bottom light has more low mass stars.

As is discussed in more detail in $\S 10$, some of the first claims for a varying IMF in high- $z$ dusty galaxies came from theoretical groups, who found difficulty in finding enough submm luminous galaxies in cosmological simulations. (Baugh et al. 2005) suggested that if the IMF varied from a traditional Kennicutt (1983) form $\left(d N / d \log m \propto m^{-x}\right.$, with $x=0.4$ for $m<1 \mathrm{M}_{\odot}$ and $m=1.5$ for $m>1 \mathrm{M}_{\odot}$ ) to a flat IMF ( $x=0$ for all masses) for star bursting galaxies. The physical motivation for this was that the simulations required more galaxies with a colder dust spectrum in order to match the then-available SCUBA counts. The top heavy IMF assumed in this case allowed both for more UV photons from massive stars, as well as a higher yield of dust (via metal enrichment from Type II supernovae). The combination of these gave rise to enough SMGs in the simulations to match observations. This said, other groups (e.g. Hayward et al. 2013b) have found that it is possible to match the observed SMG counts without varying the IMF from what is observed locally, although more work needs to be done to match more than one observational constraint simultaneously.

Other papers have argued for either top heavy or bottom light IMFs at high- $z$. Tacconi et al. (2008) simultaneously modeled the CO- $\mathrm{H}_{2}$ conversion factor, stellar masses and IMFs of $z \sim 2$ SMGs, and found that the IMF may have an excess of high-mass stars, with a best fit mass to light $(M / L)$ ratio roughly half that off a standard Kroupa IMF. This however assumes that high- $J$ CO transitions trace the same region as low- $J$ CO lines, however this often cannot be the case (Ivison et al. 2011) as the integrated SMG star formation history would exceed the local baryon density (Blain et al. 1999). Similarly, potential discrepancies in the integrated cosmic star formation history, and evolution of the stellar mass function may imply either a bottom light or top heavy IMF at high- $z$, with the idea that such an IMF would cause inferred SFRs to decrease, and bring the two values into agreement (Hopkins \& Beacom, 2006, Elsner et al. 2008; Pérez-González et al. 2008; Wilkins et al., 2008), though issues related to luminosity function integration as well as nebular line contamination in stellar mass estimates may relieve some of these tensions (Reddy \& Steidel, 2009; Stark et al., 2013). van Dokkum (2008) suggested that the color evolution of early type galaxies at $z \sim 1$, combined with their mass to light ratios may be well described by a bottom light IMF, though note in van Dokkum \& Conroy (2012) that the same observations could be consistent with a Salpeter IMF. Davé(2008) note that most cosmological simulations are unable to match the observed SFRs of main sequence galaxies at $z \sim 2$ (at a given stellar mass), and suggest that even a mildly bottom light IMF in these systems may go some distance toward reducing the inferred SFRs of $z \sim 2$ galaxies enough to bring the discrepancies into accord.

Indeed, in high star formation rate surface density environments at low- $z$, which may resemble conditions at high- $z$ (e.g. Kruijssen \& Longmore, 2013), some indications suggest bottom light or top heavy IMFs as well. Rieke et al. (1993) and Förster Schreiber et al. (2003) find potentially that the turnover mass may be a factor $2-6$ larger than a traditional Kroupa (2002) IMF in M82. Similarly, Fardal et al. (2007) examine the present day $K$-band luminosity density, cosmic background radiation, and cosmic star formation rate density, and suggest that there is an excess of intermediate mass stars. In the Galactic Center of the Milky Way, Nayakshin \& Sunyaev (2005) and Stolte et al. (2005) suggest a top-heavy IMF.

On the other hand, both dynamical methods, as well as stellar population modeling of present-epoch massive galaxies suggest that these systems may have a bottom heavy IMF. For example, observations of gravity sensitive stellar absorption lines (such as FeH, the so called "Wing-Ford" band; Ca II and $\mathrm{Na}$ I) aimed at distinguishing $\mathrm{K}$ and $\mathrm{M}$ dwarfs from $\mathrm{K}$ and $\mathrm{M}$ giants have found the IMF to be bottom heavy in $z \sim 0$ early type galaxies (van Dokkum \& Conroy, 2010, 2012, Conroy \& van Dokkum, 2012ab, Spiniello et al., 2012, Ferreras et al., 2013). Similarly, 


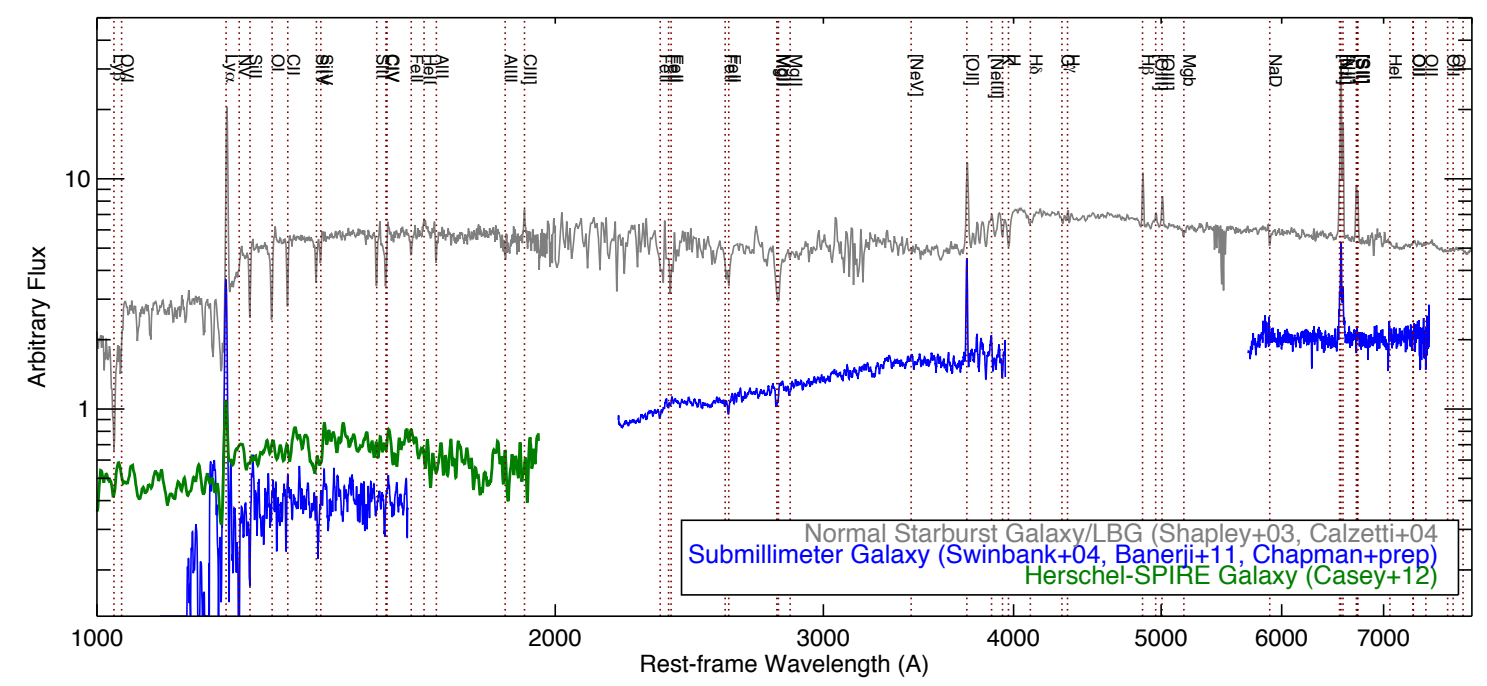

Figure 26: The piecewise composite of a Submillimeter Galaxy's rest-frame ultraviolet and optical spectrum (blue) compared to a composite spectrum for a normal, dusty star-forming Lyman Break Galaxy at $z \sim 2$ (gray; Shapley et al. 2003. Calzetti 2001). The SMG's composite spectrum is comprised of three datasets centered around the detection of three prominent emission lines: H $\alpha$ (Swinbank et al. 2004), [OII] (Banerji et al. 2011), and Ly $\alpha$ (the median stack of star-formation dominated SMGs from Chapman et al. 2005. ; Chapman et al., in preparation). Also included is the rest-frame ultraviolet spectral stack from Casey et al. (2012b) for a subset of $z>2$ Herschel-SPIRE selected galaxies (dark green; offset from SMG spectrum for clarity). Prominent emission and absorption line features are labeled. Despite assuming the dustiest template for a starburst in the rest-frame optical from Calzetti (2001) to join with the median LBG spectrum from Shapley et al. (2003), the composite spectrum from the SMG is significantly more reddened and extinguished. Detection of continuum in SMGs is rare beyond $z \sim 2$ and even difficult to detect in a composite.

constraints on the stellar mass to light ratio from kinematics have suggested a similar result (e.g. Auger et al., 2010, Treu et al., 2010; Spiniello et al., 2011, Cappellari et al., 2012, 2013; Brewer et al., 2012, Dutton et al., 2013; Tortora et al. 2013), though we note that increased mass to light ratios can result from both bottom-light and bottom-heavy IMFs (the former owing to increased numbers of low mass stars, which the latter originating in increased numbers of stellar remnants).

Considering both the indirect evidence of potential IMF variations at high- $z$, as well as observations of presentepoch massive galaxies (which are likely descendants of starbursts at high- $z$ ), it is fair to say that the form of the IMF in high- $z$ systems is still a completely open issue. A number of theoretical models have attempted to understand how the IMF may vary with physical environment. Some are able to motivate physical origins for bottom light IMFs in heavily star-forming systems (e.g. Narayanan \& Davé, 2012, 2013), while others argue for a bottom-heavy IMF in starbursts (e.g. Hennebelle \& Chabrier, 2008; Hopkins, 2012; Krumholz, 2011a). This said, to date, no model to date can accommodate a bottom light/top heavy IMF in starbursts, as well as a bottom heavy IMF in their descendants.

\subsection{Rest-frame Ultraviolet $\mathcal{E}$ Optical Spectral Characterization}

Although obscuration significantly hampers our ability to study the optical characteristics of DSFGs in detail, several works have painstakingly amassed spectral observations of DSFGs to infer redshifts and subsequently properties like AGN content, metallicity, wind outflows and extinction factors. Like the other subsections in this chapter, the literature of optical spectral characterization of DSFGs has been limited primarily to radio-detected, $850 \mu \mathrm{m}$-selected SMGs.

The first comprehensive rest-frame optical study of SMGs was done by Swinbank et al. (2004) who spectroscopically confirm 30 galaxies in $\mathrm{H} \alpha$ emission whose redshifts were originally reported via detection of Ly $\alpha$ in Chapman et al. (2003a) and Chapman et al. (2004b). Swinbank et al. measure [NII]/H $\alpha$ ratios and $\mathrm{H} \alpha$ line widths to deduce presence of AGN in at least $40 \%$ of the sample. Figure 26 illustrates the $\mathrm{H} \alpha$ composite spectrum for SMGs without AGN. Even for SMGs without AGN, a broad line component to $\mathrm{H} \alpha$ is measured with equal flux as the narrow component. The $\mathrm{H} \alpha$ line widths are significant at $400 \mathrm{~km} \mathrm{~s}^{-1}$ and spatial extent large at $\lesssim 4-8 \mathrm{kpc}$, implying large dynamical 
masses $\sim 1-2 \times 10^{11} \mathrm{M}_{\odot}$ and short dynamical times of 10-20 Myr. Along with concurrent results on CO observations of SMGs (see $\S 8$, this work concluded that these SMGs represent massive, metal-rich merging galaxies with high star-formation rates, significant dust obscuration (with H $\alpha$ SFRs suppressed 10× in comparison to far-infrared), and containing non-negligible AGN populations, thus being the likely progenitors to massive, local elliptical galaxies (e.g. Figure 2).

The rest-frame ultraviolet (UV) spectral properties of SMGs are discussed in Chapman et al. (2005) who also report their redshift distribution. Chapman et al. find that, much worse than the $10 \times$ extinction in the rest-optical, the UV luminosities of SMGs underestimate the far-infrared star formation rates by a median factor of $\sim 120$, even after nominal dust-correction via the prescriptions of Meurer et al. (1997) and Adelberger \& Steidel (2000). They measure a UV spectral index $\beta=-1.5 \pm 0.8$, corresponding to an $E(B-V)=0.14 \pm 0.15$ for a Calzetti extinction law, close to expectation for LBGs. This suggests that SMGs' UV properties do not differ significantly from those of LBGs (Adelberger \& Steidel, 2000), even though it might be thought that SMGs would have significantly redder slopes (Smail et al. 2004). However, the selection bias of the Chapman et al. is important; SMGs with detectable rest-frame UV features are likely to be bluer than the median SMG, or could even be the close, unobscured companions to SMGs.

With a UV spectral index of $\beta=-1.5 \pm 0.8$, we can surmise that the expected $L_{\mathrm{IR}} / L_{\mathrm{UV}}$ ratio for SMGs would be around $\sim 10$ if a dust attenuation relation with $\beta$ from Meurer et al. (1999) is assumed, but again, factors of $\sim 100$ are more typical for SMGs. Reddy et al. (2012) recently studied the direct dust luminosity of UV-selected galaxies, comparing the UV spectral slope directly to stacked Herschel flux densities in GOODS-Herschel and found that the attenuation and reddening of the slope agree with previous results calibrated from local starburst galaxies. While the Reddy et al. (2012) results are promising, indicating no evidence for evolving dust properties out to $z \sim 2$, the fact that these dust attenuation curves do not apply to luminous DSFGs is concerning. Future work investigating this relationship in DSFGs is urgently needed.

A composite of the rest-frame ultraviolet spectra of SMGs without AGN is over-plotted in Figure 26, taken from Chapman et al., in preparation. Although not strictly representative of the $850 \mu \mathrm{m}$ SMG population, the similarly luminous Herschel-SPIRE selected galaxies at $z>2$ discussed in Casey et al. (2012b) were all identified via rest-frame UV features; their composite is also over-plotted in Figure 26 for comparison.

After the initial redshift surveys and spectral analysis of SMGs in the rest-frame UV and $\mathrm{H} \alpha$, a $z \sim 1.5$ subset of the SMG population (along with some SFRGs, submillimeter-faint radio galaxies) were studied in Banerji et al. (2011) around the nebular [OІI] 3727 $\AA$ line emission. Banerji et al. measure line widths similar to the $z \sim 2$ SMGs from Swinbank et al. (2004), arguing that they have similar dynamical masses and evolutionary histories as the higher-redshift, higher-luminosity SMGs. Large-scale wind outflows are measured via a $-240 \pm 50 \mathrm{~km} \mathrm{~s}^{-1}$ blueshift of interstellar absorption lines (MgII and FeII) and are consistent with momentum-driven wind models and the $V \propto \mathrm{SFR}^{0.3}$ local envelope seen in low-z ULIRGs (Martin, 2005). The Banerji et al. composite around [OrI] is over-plotted on Figure 26

\subsection{AGN Content}

A major focus of galaxy evolution has been the coevolution of supermassive black holes (SMBHs) within their host galaxies and the interplay between active galactic nuclei (AGN) and starbursts (e.g. Connolly et al., 1997; Merloni, 2004; Hopkins et al. 2007). The classic evolutionary sequence-merger, to starburst, quasar, then elliptical-is not only a phase of some growth for stellar populations, but also has been shown to account for $\sim 30 \%$ of the Universe's integrated black hole growth through highly obscured accretion (Treister et al., 2009, 2010). Probing the AGN content of DSFGs provides essential limits on their evolutionary history while also shedding light on SMBH growth in extreme environments. AGN have a number of observational probes, the most common being via direct detection in the Xrays, but otherwise through radio emission, optical line diagnostics, near-infrared colors, or mid-infrared continuum spectral slope (the latter two simply probe the presence of warm dust surrounding the inner torus region exterior to the SMBHs' accretion disk).

The X-ray properties of DSFGs have been studied in detail over the past decade, in particular for $850-870 \mu \mathrm{m}-$ selected SMGs (Fabian et al., 2000; Alexander et al., 2005a b, Pope et al., 2006; Laird et al., 2010, Lutz et al., 2010, Georgantopoulos et al., 2011; Gilli et al., 2011, Hill \& Shanks, 2011, Bielby et al., 2012; Johnson et al., 2013, Wang et al., 2013b). Unfortunately, all of these studies are limited by small number statistics $\left(N_{\text {sources }}<100\right)$ cause by very time-intensive, deep X-ray observations which are required to disentangle X-ray emission dominated by AGN 

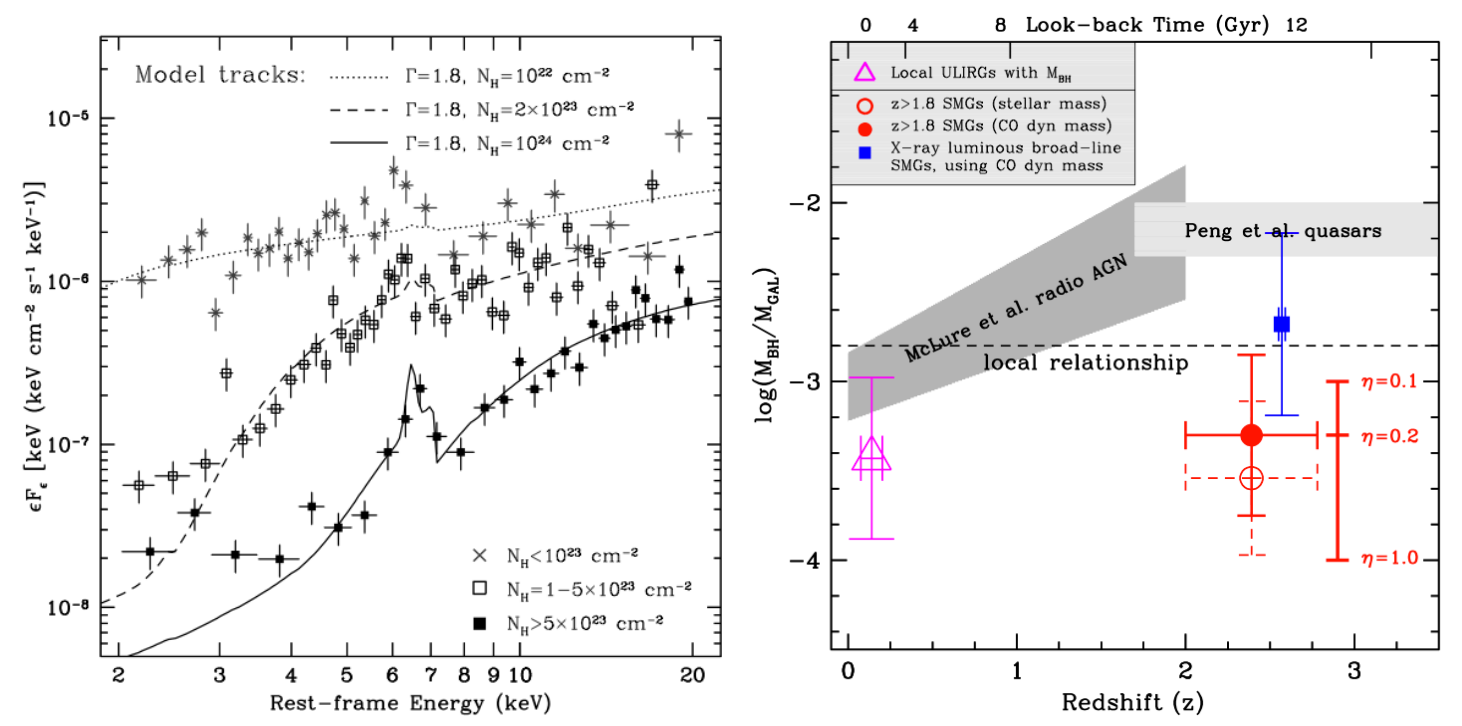

Figure 27: A summary of X-ray observations of $\sim 850 \mu$ m-selected SMGs. At left, we re-plot the composite rest-frame 2-20 keV spectra for SMGs within three obscuration classes. The $\mathrm{Fe} \mathrm{K} \alpha$ line is clearly visible-in particular for the most obscured AGN in SMGs-at $6.4 \mathrm{keV}$. This figure is reproduced from Alexander et al. (2005a) with permission from the authors and AAS. At right, the measured black-hole to host galaxy mass ratio measured in SMGs against other galaxy populations. This figure is reproduced from Alexander et al. (2008) with permission from the authors and AAS. The local relationship is given by the dashed line (Häring \& Rix 2004), whereas the measurement for radio-selected AGN between $0<z<2$ is shown in dark gray (McLure et al. 2006) and $z>2$ quasars in light gray (Peng et al. 2006). Also overplotted are measurements of local obscured ULIRGs (Veilleux et al.||1997||1999).

activity versus star formation (the latter originating from high-mass X-ray binaries, HMXBs). The results of these studies have been mixed in reporting different 'AGN fractions.' Here AGN fraction is the fraction of the population which have X-ray implied star formation rates (from HMXBs) much higher than estimates from near- to far-IR, thus the emission must originate from nuclear processes. The first measurement of AGN in SMGs (Alexander et al., 2005a) showed that $75 \%$ of radio-selected SMGs host AGN activity, with 1/3 of those constituting luminous AGN. The SMGs not hosting AGN were consistent with X-ray emission from star-formation and HMXBs. Further X-ray analysis of SMGs estimate AGN fractions of 20-29( \pm 7$) \%$ (Laird et al., 2010), <26 $49 \%$ (Georgantopoulos et al., 2011), 14-28\% (Johnson et al., 2013) and $17\left(_{-6}^{+16}\right) \%$ (Wang et al., 2013a). The Wang et al. (2013a) work is of particular note since its sample is the $\sim 100$ ALMA-confirmed $870 \mu \mathrm{m}$-selected sources in CDFS with unambiguous counterparts, enabling effectively more precise measurements in both X-ray and FIR. An important follow-up to these X-ray studies of SMGs came in Alexander et al. (2008). Alexander et al. place observational constraints on central black-hole masses for SMGs using $\mathrm{H} \alpha$ (or $\mathrm{H} \beta$ ) line analysis in addition to the X-rays. They find that SMGs have black-hole masses to galaxy mass ratios 3-5× lower than local relationship (Häring \& Rix, 2004), and much lower than other high-redshift populations of AGN (McLure et al., 2006, Peng et al., 2006). A summary of results regarding X-ray studies of SMGs and their AGN is summarized in Figure 27

While X-rays provide the most definitive signature of AGN, sometimes AGN are missed there due to high column densities of dust which can obscure soft X-rays (e.g. Daddi et al., 2007a). Therefore, it is most useful to compare quantitative measures of AGN content from a multi-wavelength perspective. Although optical spectral line diagnostics can provide a valuable probe to AGN activity in galaxies via comparison of emission line ratios (e.g. Kewley et al., 2006; Juneau et al. 2011), DSFGs, even if they have optical spectral observations, often lack the high-quality, high$\mathrm{S} / \mathrm{N}$ spectra necessary for classification.

The near- to mid-infrared portion of the SED can indicate AGN contribution, even when spectral information is not available. Presence of an infrared power-law on the Wien side of the far-infrared blackbody is an indication that a galaxy contains a significant amount of warm-dust $(100-1000 \mathrm{~K})$ which is likely to be heated by an AGN (e.g. Desai et al. 2009, Melbourne et al. 2011). For example, a normal star-forming galaxy with a $10^{8} \mathrm{M}_{\odot}$ reservoir of cold $(\sim 30 \mathrm{~K})$ dust might only have $\sim 10 \mathrm{M}_{\odot}$ of hot $500 \mathrm{~K}$ dust (heated by new stars in dense star-forming regions), 


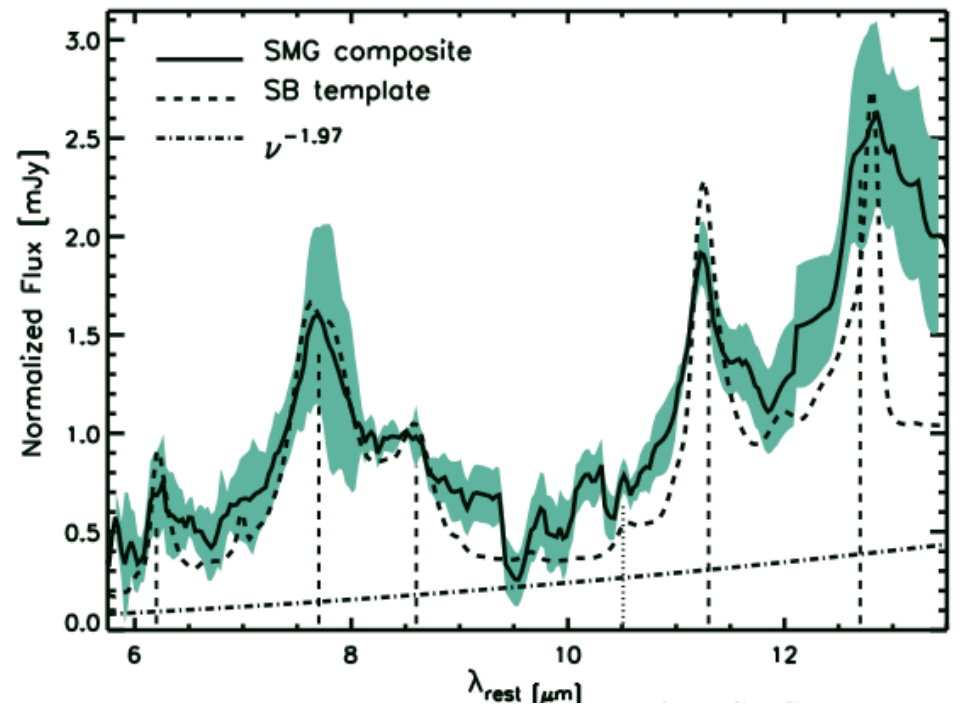

Figure 28: The composite mid-infrared spectrum of non-AGN dominated SMGs from Menéndez-Delmestre et al. (2009) compared to a starburstdominated composite spectrum of low-redshift galaxies from Brandl et al. (2006). Underlying the PAH spectral features is a power-law continuum component with spectral slope of $\alpha=1.97$, most likely originating from optically-thick emission around HII in star-forming regions. This figure is reproduced with permission from Menéndez-Delmestre et al. (2009) with permission from the authors and AAS.

while a similar galaxy with an AGN can have $10 \times$ as much warm-dust $\left(\sim 100 \mathrm{M}_{\odot}\right)$ which could be detectable and dominate the mid-infrared output, potentially contaminating estimates of stellar mass or $24 \mu \mathrm{m}$-based star formation rate. For this reason, obtaining mid-infrared spectral observations are critical to segregate contributions of emissionlines associated with star formation, star-light from old stellar populations and emission from AGN-heated warm dust, discussed in $\$ 5.7$

Motivated by the fact that X-rays can be absorbed in high column density environments, a number of groups developed mid-IR color selection techniques to identify AGN at high- $z$ utilizing the bandpasses available on the Spitzer Space Telescope Lacy et al. (2004) and Stern et al. (2005). These were recently revisited by Donley et al. (2012), under the premise that the previously developed color-selection "wedges" were contaminated by star-forming galaxies given deep enough IRAC data.

\subsection{Mid-Infrared Diagnostics}

As alluded to in $\S 4.2 .2$, the rest-frame mid-infrared portion of the spectrum is complex. Unlike the far-infrared which is dominated by smooth continuum generated through cold dust modified blackbody emission and the occasionally narrow gas emission line, the mid-infrared portion has emission and absorption features generated by heavy molecules and smaller dust grains. Polycyclic Aromatic Hydrocarbons (PAHs) are a few Å-diameter heavy molecules containing hundreds of carbon atoms which exist in cold molecular clouds (Leger \& Puget, 1984;, Allamandola et al. 1985 ) and when irradiated by young stars, emit spectral line features at discrete wavelengths from $\approx 3-19 \mu \mathrm{m}$ (Weingartner \& Draine, 2001). It follows that PAH emission strength scales with star formation rate (which relies on the assumption that stellar emission heats the photo-dissociation regions where the PAHs reside Farrah et al., 2007). Aside from PAH features, the mid-infrared can also be absorbed by dust silicates, indicative of significant obscuration in warm dust with high-column densities (e.g. Houck et al., 2005). Unfortunate for observational constraints, the PAH emission features at $\sim 8 \mu \mathrm{m}$ and $\sim 11 \mu \mathrm{m}$ almost perfectly bracket the $9.7 \mu \mathrm{m} \mathrm{Si}$ absorption, making measurements of line strengths difficult. Underlying both these emission and absorption features is the continuum which, itself, is dependent on the relative dust distribution and bolometric heating sources in a galaxy. Heating from star formation (i.e. HII regions) will only amount to hot-to-cold dust ratios of $\ll 1 / 1000$ while an AGN can skew the relative distribution of dust temperatures higher, flattening out the SED through the mid-infrared. Without a spectrum of a galaxy, which enables one to distinguish between these complex mechanisms of emission and absorption, it can be very difficult to 
ascertain a physical interpretation of mid-infrared flux densities, e.g. from observed $24 \mu \mathrm{m}$ Spitzer-MIPS work from $z \sim 1-4$.

In-depth studies of high-redshift galaxies' mid-infrared spectra was not possible before the launch of the Spitzer Space Telescope's Infrared Spectrograph (IRS) instrument. The timing was perfect for investigating the mid-infrared spectral properties of $850 \mu \mathrm{m}$-selected SMGs, now described in Menéndez-Delmestre et al. (2007), Valiante et al. (2007), Pope et al. (2008b) and Menéndez-Delmestre et al. (2009). These works find that SMGs have a majority of broad PAH emission features (80\%) with the remaining $20 \%$ of SMGs being dominated in the mid-IR by AGN. The continuum spectral slope for SMGs in the mid-IR spectral region is measured as $\alpha=2$, where $F_{v} \propto v^{-\alpha}$. This agrees with prior results of the estimated slope from non-spectral observations (Blain, Barnard, \& Chapman, 2003) which estimated $\alpha=2$ for sources without prominent AGN (and a shallower value, $\alpha \sim 1$ for luminous AGN, e.g. Koss et al., 2013). Interestingly, Menéndez-Delmestre et al. (2009) find that the ratio between $6.2 \mu \mathrm{m}$ and 7.7 $\mu \mathrm{m}$ PAH emission is higher than in local ULIRGs or nuclear starbursts, pointing to a more extended distribution of both cool and warm dust in SMGs than in compact local ULIRGs. A further set of AGN-dominated SMGs are described in Coppin et al. (2010) who demonstrate that even though AGN can dominate the mid-IR spectra of SMGs, they rarely dominate their bolometric luminosities. The Menéndez-Delmestre et al. mid-infrared SMG composite spectrum, excluding bright AGN, is shown in Figure 28 .

Elbaz et al. (2011) revisit mid-infrared analysis of DSFGs with data from Herschel-PAcs, in the context of the infrared main sequence of star forming galaxies (DSFGs in the context of the main sequence are discussed more in $\S$ 5.11). They define a parameter IR $8 \equiv L_{\mathrm{IR}} / L_{8}$, where $L_{8}$ is the rest-frame $8 \mu \mathrm{m}$ luminosity, an approximation for the $7.7 \mu \mathrm{m}$ PAH strength. They make use of observations of suppressed PAH emission in local LIRGs and ULIRGs (whereby heavy molecules are destroyed in the most dense star forming environments, e.g. Rigby et al., 2008, DíazSantos et al., 2010) to distinguish two modes of star formation where normal main sequence galaxies have IR8 values consistent with local LIRGs, whereby starbursts have elevated IR8 ratios, consistent with local ULIRGs. They find that ULIRGs at $z \sim 1-2$ have IR8 ratios consistent with normal main sequence star forming galaxies and thus conclude that most Herschel-PAcs detected galaxies are not merger dominated. As discussed further in $\S 5.11$, this supports other observational and theoretical evidence that $z \sim 2$ DSFGs have different star formation histories than $z \sim 0$ DSFGs. However, recent follow-up from Herschel-SpIRE selected galaxies in COSMOS (Lee et al., 2013) suggest that ULIRGs at $z \sim 2$ do indeed have elevated IR8 values compared to less luminous sources, after correcting for the depths of both mid-infrared and far-infrared surveys. Lee et al. (2013) also present some evidence that the infrared main sequence that appears to be quite tight at mid-infrared and optical wavelengths, dissolves when far-infrared-based star formation rates are considered. Although preliminary evidence points to IR8 being a good indicator of starbursts, the impact of certain observational assumptions and biases need to be better understood.

\subsection{Mid-Infrared Spitzer-selected Populations}

Despite the complexity of mid-infrared spectra, the deep and wide $24 \mu \mathrm{m}$ Spitzer surveys of the high- $z$ Universe provided a revolutionary look at dusty galaxies with large studies of thousands of galaxies pre-dating Herschel. The population of Dust Obscured Galaxies (DOGs; Dey et al., 2008; Pope et al., 2008a) define the population of 24 $\mu$ mselected galaxies that have extremely red colors (i.e. (R-[24]) $\geq 14$ mags [Vega]). In a multiwavelength analysis, a large fraction of these DOGs seemed to be "mid-IR excess" sources, or galaxies that have unusually strong rest-frame $8 \mu \mathrm{m}$ emission compared to the integrated infrared (Daddi et al., 2007b; Papovich et al., 2007; Magnelli et al., 2011). The physical origins of DOGs mid-infrared emission could have been AGN-heating or bright PAH emission lines. Rigby et al. (2008), Farrah et al. (2008), Murphy (2009), Fadda et al. (2010) and Takagi et al. (2010) found the latter (note however that not all of these works explicitly use the DOG selection criterion, their samples overlap substantially). Indeed, the AGN fraction of DOGs (and similar $z \sim 2 B z K s$ ) appears to be smaller than was originally anticipated $\sim 30 \%$ at $S_{24}<1 \mathrm{mJy}$ (Pope et al., 2008a, Alexander et al., 2011). Brand et al. (2006) provide a thorough analysis of the likelihood of an AGN dominating the mid-infrared spectral regime using $>20,00024 \mu \mathrm{m}$-identified sources. They determine an AGN fraction of just $9 \%$ at $S_{24}=350 \mu \mathrm{Jy}$ increasing up to $74 \pm 20 \%$ at $S_{24} \approx 3 \mathrm{mJy}$. Also see Kirkpatrick et al. (2013) for a detailed discussion of how AGN fraction changes with $24 \mu \mathrm{m}$ flux density limit.

The near-infrared SED of DOGs has been critical to placing them in an evolutionary context with SMGs. Galaxies with large stellar masses exhibit a near-infrared 'bump' originating at rest-frame $1.6 \mu \mathrm{m}$ that owes to a local minimum in the atmospheric opacity in massive stars (John, 1988; Simpson \& Eisenhardt, 1999, Farrah et al., 2008). Generally speaking, galaxies with a mid-IR bump (observed frame at $z \sim 2$ ) are associated with being star formation dominated, 
while those with a powerlaw mid-IR SED are assumed to be AGN dominated. Bussmann et al. (2009, 2011) examined the HST morphologies of both power-law and bump DOGs at $z \sim 2$, and advocated a merger-driven scenario in which bump DOGs evolved into power-law DOGs (i.e. star formation dominated galaxies evolved into AGN dominated galaxies). This was predicated on evidence that the bump-DOGs had more extended (and somewhat irregular) morphologies than the power-law DOGs, while the latter were more dynamically relaxed. This scenario was given some theoretical backing by Narayanan et al. (2010a).

\subsection{Kinematics}

Kinematic studies of DSFGs are much more observationally expensive than basic photometric or spectral constraints, and are therefore naturally limited to smaller sample sizes. Nevertheless, a substantial effort has been made to survey $850 \mu \mathrm{m}-\mathrm{SMGs}$ kinematically, through ionized gas around HII regions (typically $\mathrm{H} \alpha$ integrated field unit observations) and cold molecular gas (typically CO mm-line interferometric observations).

Key works on H $\alpha$ kinematics in SMGs is summarized by Swinbank et al. (2006), Alaghband-Zadeh et al. (2012) and Menéndez-Delmestre et al. (2013). Collectively, they observe 16 SMGs at $2.0<z<2.5$ and present strong evidence for merger-driven histories-many at an early stage first pass, where multiple components are seen separated by $\sim 8 \mathrm{kpc}$ and $200 \mathrm{~km} \mathrm{~s}^{-1}$, while others are later stage single-component systems with high-dispersion and buried AGN. Figure 29 illustrates the line-profile characteristics of these SMGs with respect to local starbursts from the SINGS sample, the $z \sim 2$ SINS sample of star-forming galaxies (Shapiro et al., 2008), some simulated SMGs Davé et al. (2010), and simulated disk and merger templates (also from Shapiro et al. 2008). The divide between mergers and disks is quite clear and narrow in this sample, with $\sim 8.5 / 10$ of SMGs lying in the unambiguous merger-driven region of the plot. Results from high-resolution CO molecular gas observations of SMGs support this merger-driven model of SMGs, albeit with limited statistics and only within the most luminous $>10^{12.5} \mathrm{~L}_{\odot}$ subset, the details of which are described more in $\S 8$.

\subsection{Physical Size and Morphology}

Measuring the physical sizes of DSFGs at high redshift requires high-resolution imaging $\ll 1^{\prime \prime}$ (beyond $z \sim 1$, $1^{\prime \prime}$ corresponds roughly to $8 \mathrm{kpc}$ ). High-resolution imaging can be gathered from either optical/near-infrared stellar continuum or from direct infrared interferometric observations in the millimeter or radio. Millimeter or radio followup is more likely for highly obscured sources like DSFGs (where the optical magnitudes can be staggeringly faint, $\left.i_{\mathrm{AB}} \sim 25-26\right)$.

When molecular gas CO observations are taken at high-resolution (e.g. Tacconi et al., 2008, Bothwell et al., 2010, Engel et al., 2010, for SMGs), a consequence of obtaining kinematics is also obtaining the simple size measurement of the cold gas reservoir. While the morphologies of the sources themselves might be disturbed, these works reached a consensus that SMGs have effective radii of $r_{e}=2 \pm 1 \mathrm{kp}{ }^{15}$ on average twice the physical size of local ULIRGs which are very compact with $r_{e} \lesssim 1 \mathrm{kpc}$. Both SMGs' sizes and molecular gas masses seemed to be twice as large as local ULIRGs, thus the population was dubbed their scaled-up analogues. Note that by these measures, both SMGs and local ULIRGs are much more compact than normal disk galaxies of comparable masses, which extend $\gtrsim 8 \mathrm{kpc}$.

One important caveat of the molecular gas size measurements is that the measured emission was made on high- $J$ transitions of $\mathrm{CO}$ and not the ground state, $\mathrm{CO}(1-0)$. In fact more recent results (Ivison et al., 2011, Riechers et al., 2011d) show that emission from $\mathrm{CO}(1-0)$ is more extended, both in line width velocity space and spatially, with a typical FWHM of $540 \mathrm{~km} \mathrm{~s}^{-1}$ (broader than the typical high- $J$ transition line width of $\sim 150 \mathrm{~km} \mathrm{~s}^{-1}$ ) and spatial FWHM of $\sim 16 \mathrm{kpc}$. While complementary work at radio wavelengths using the high-resolution MERLIN interferometer (Chapman et al. 2004b; Biggs \& Ivison, 2008, Casey et al., 2009a) corroborate the high- $J$ molecular gas size measurements with sizes of $r_{e} \approx 2 \mathrm{kpc}$ measured for radio continuum emission, we note that extended emission could either be resolved out from earlier MERLIN results or the radio continuum is only probing areas of dense star formation with higher incidents of supernovae (see $\S 5.12$ for a discussion of radio emission in DSFGs). Furthermore, these works also found that SMG sizes were diverse, from unresolved point-sources near the Eddington starburst limit, to sources

\footnotetext{
${ }^{15}$ Note that the "size" of a galaxy quoted in the literature can range from the diameter of a galaxy to the full width at half maximum (FWHM) radius to the full width at zero intensity (FWZI) radius to $r_{\mathrm{e}}$; there is no standard as to which is used so the reader of these papers should pay careful attention to the authors' methods while interpreting their results.
} 


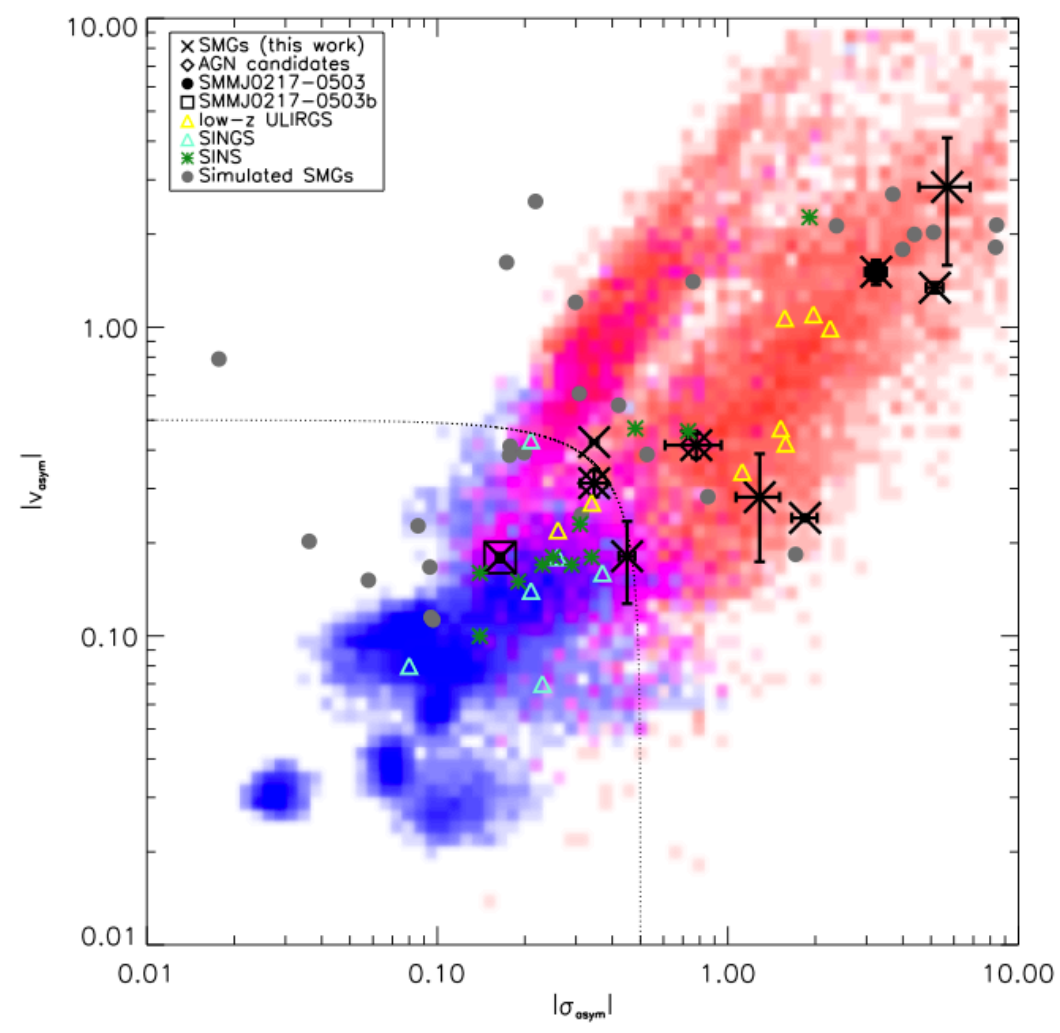

Figure 29: Line-profile characteristics of resolved $\mathrm{H} \alpha$ IFU observations of 10 SMGs [Swinbank et al. 2006, Alaghband-Zadeh et al. 2012. Menéndez-Delmestre et al. 2013). The $x$-axis plots velocity dispersion field asymmetry while the $y$-axis plots the velocity field asymmetry. This figure is reproduced with permission from Alaghband-Zadeh et al. (2012). Blue background points represent regions dominated by smoothly rotating disk galaxies while red points are expected to be dominated by merger-driven templates (Shapiro et al. 2008). The triangles represent local samples: both normal star-forming galaxies from SINGS (blue) and ULIRGs (yellow). Simulated SMGs from the SPH simulations work of Davé et al. (2010) are shown as gray circles; note that although many of the Davé et al. simulations appear in the 'merger' portion of this diagram, only $1 / 41$ is actually a merger (suggesting that perhaps the morphological and dynamical signatures of mergers at high- $z$ are not straightforwardly calibratable using low- $z$ samples). The high- $z$ star forming galaxies from SINS (Förster Schreiber et al. 2009) are small green crosses, while SMGs are large black crosses. Within this small sample, it appears that $100 \%$ of SMGs are consistent with being merger-driven (this however includes one special system which is a merger and a disk all at the same redshift), which is consistent with findings from kinematic studies of the brightest SMGs in cold molecular gas (e.g. Engel et al. 2010 Riechers et al. 2011d see more in $\$ 8$. 

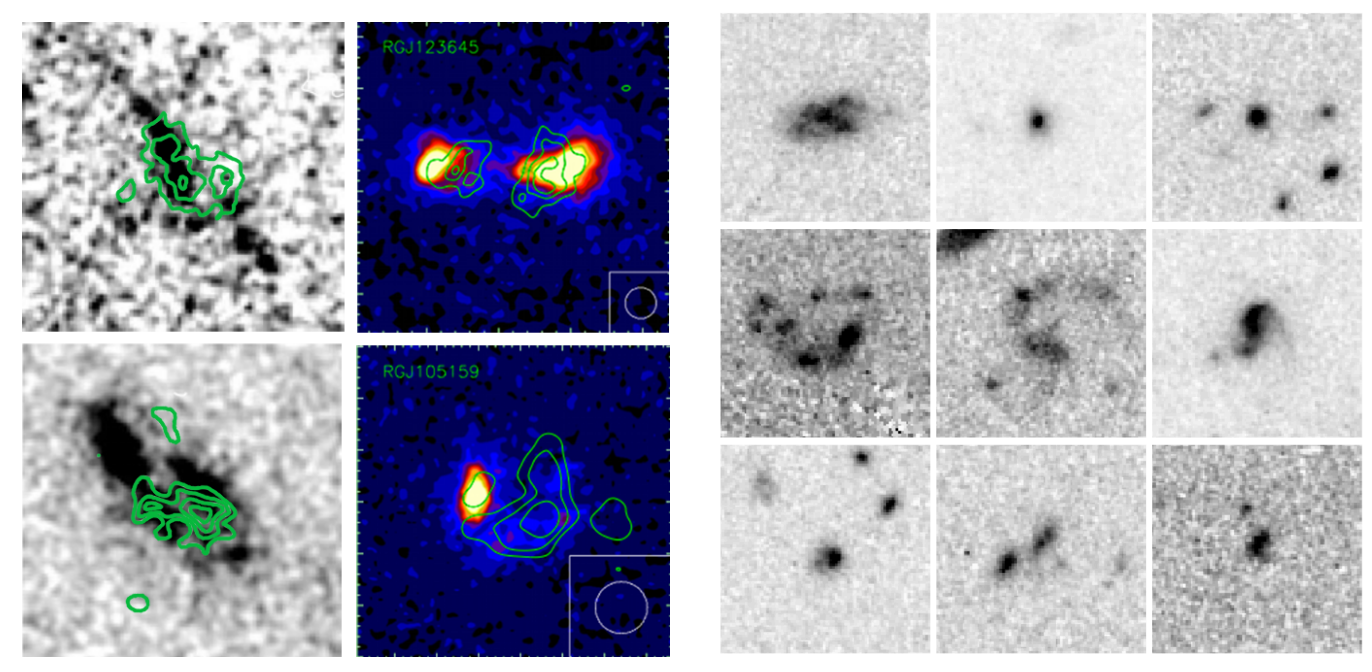

Figure 30: At left, radio $1.4 \mathrm{GHz}$ high-resolution continuum is shown in contour over $3^{\prime \prime} \times 3^{\prime \prime}$ optical $i$-band ACS images of two SMGs from Chapman et al. (2004b) and two SFRGs from Casey et al. (2009a). Morphologies in the optical alone are ambiguous, especially at $z>2$ where the K-correction is not straightforward and highly dependent on spectral type. The addition of radio continuum-which traces the FIR continuum for starbursts-sheds insight on obscured star-formation, while also sensitive to AGN, which should manifest as unresolved point sources iCasey et al. 2009c Biggs et al. 2010). Radio and optical emission are often offset from one another indicated disturb or irregular morphologies consistent with mergers. At right, example near-IR cutouts from HS T-WFC3 of Herschel-PAcs selected ULIRGs in a CANDELS field, 72\% of which exhibit interacting or merging morphologies (Kartaltepe et al. 2012). These figures are reproduced in part from Chapman et al. (2004b), Casey et al. (2009a) and Kartaltepe et al. (2012) with permissions from the authors and AAS.

extended over $\sim 8 \mathrm{kpc}$. Biggs et al. (2010) furthered investigated SMG morphologies with VLBI radio observations, showing that most SMGs do not have a compact radio core. These authors used this to argue that the radio emission in SMGs are not likely powered by nuclear AGN, but rather galaxy-wide starbursts.

As is the case with many other physically constrained parameters in this section, the measured constraints on size come from only a handful of sources. Clearly measured size depends on which observational probe is being used-whether it samples dense star-forming regions or extended, low-excitation cold gas reservoirs. Although size can be an essential stepping stone for measuring gas or star formation density (thus making density arguments), it is critical that the relative difference between observational probes is understood.

Beyond providing a simple visual characterization of the system of interest, the morphology of a galaxy at particular wavebands can reveal a host of underlying physical processes. For example, the extent of the FIR-bright region can provide constraints as to whether a starburst is Eddington-limited (e.g. Younger et al., 2008; Riechers et al., 2013b), which can be informative for models of star formation feedback in ultra-luminous galaxies (e.g. Thompson et al., 2005; Hopkins et al., 2013c a).

One of the principle reasons for obtaining radio morphologies of high- $z$ galaxies soon after their discovery was to exploit an assumed correlation between the radio and FIR flux (which was a fair assumption, given the strong local correlation; Murphy, 2009), and therefore place constraints on the size of the FIR emitting region. With advances in (sub)mm-wave interferometry during the early 2000s, direct FIR morphology measurements became available. These measurements confirmed the relatively large spatial extents of the star formation activity in high- $z$ SMGs as compared to local ULIRGs (e.g. Younger et al. 2008), and has driven the rise of models where the luminosity density of high- $z$ ULIRGs is one of the strongest indicators for the physical properties of the IR emitting environment (Rujopakarn et al., 2011).

Morphological classification of DSFGs is often too difficult to attempt in the optical or near-infrared due to extreme dust obscuration, however, out to $z \sim 1.2$, Kartaltepe et al. (2007) find high fractions of optically-luminous galaxies in pairs (the evolution of the pair fraction going as $\propto(1+z)^{n=3.1 \pm 0.1}$ ), implying almost $\sim 50 \%$ of galaxies at $z \sim 2$ should be in close pairs. When completing a similar analysis on DSFGs selected at $70 \mu \mathrm{m}$ with Spitzer, the majority of galaxies appear to be undergoing interactions, and an overall increase in merger fraction is seen from $z \sim 0$ to $z \sim 1$. With deeper high-resolution near-infrared imaging from the CANDELS survey, Kartaltepe et al. (2012) find similar results 
with visual classifications of Herschel-PAcs-selected galaxies (see right-hand side of Figure 30 of almost $\sim 73 \%$ of ULIRGs out to $z \sim 2$ undergoing potential interactions from morphological signatures.

Swinbank et al. (2010) present targeted Hubble Space Telescope ACS and NICMOS observations of 850 $\mu \mathrm{m}-$ selected SMGs measuring sizes and morphologies for 25 galaxies spanning $z \sim 0.6-3.0(\langle z\rangle=2.1)$ drawn from the Chapman et al. (2005) spectroscopically confirmed SMG sample. They measure characteristic sizes of $r_{i}=$ $2.3 \pm 0.3 \mathrm{kpc}$ and $r_{H}=2.8 \pm 0.4 \mathrm{kpc}$ at observed $i$ - and $H$-bands respectively, not statistically different than submmfaint field galaxies (like Lyman Break Galaxies). They attribute the difference in measured size between bands to structured dust obscuration, impacting the measurement of $i$-band sizes. Furthermore, by fitting Sersic indices to the $H$-band light, they find that SMGs are more analogous to spheroidal or an elliptical galaxy light distribution than disky light distribution (i.e. $n \sim 2$, where $n \sim 1$ would represent an exponential disk and $n \sim 4$ represents a spheroidal de Vaucouleurs profile). Using estimates of the same SMGs' stellar masses, Swinbank et al. determine that the stellar density of SMGs is comparable if not a bit higher than local early-type galaxies and red, dense galaxies at $z \sim 1.5$ which are proposed to be SMGs' direct descendants. Importantly, they also note that the rest-frame UV/optical morphologies of SMGs seem to be decoupled from all millimeter-determined observables.

Note that gravitationally lensed samples, some of which are discussed in the next chapter, can provide important insight into sources' gas, dust and stellar distribution and sizes by virtue of providing increased spatial resolution. However, as noted in Hezaveh et al. (2012), the size distributions measured in lensed galaxies are biased towards more compact sources.

\subsection{Relationship to Normal Galaxies: the Infrared Main Sequence}

Many recent works have framed analysis of new Herschel-detected DSFG populations in the context of the "Main Sequence" of galaxies (Elbaz et al., 2011; Rodighiero et al., 2011; Nordon et al., 2012, 2013; Sargent et al., 2012, Magnelli et al. 2013a). The main sequence was first presented in Noeske et al. (2007b a) as terminology for the perceived tight relationship between galaxies' stellar masses and their star formation rates. This correlation is seen to evolve towards high-redshift, where galaxies of a fixed stellar mass are likely to have star formation rates ten times larger at $z \sim 1$ than at $z \sim 0$. The initial sample used to measure the tightness of the relation was a set of opticallyselected galaxies for which $\mathrm{H} \alpha$ emission line star formation rates or $24 \mu \mathrm{m}$ flux densities were available. Daddi et al. (2007b) found similar results when expanding selection to dustier galaxies (i.e. $24 \mu \mathrm{m}$-selected galaxies).

A key implication of the tight correlation between stellar mass and star formation rate is that the star formation rate in galaxies is, to first order, dependent on the gas accretion rate from the intergalactic medium (IGM) (Davé et al., 2012). Star formation is supply dependent, while the gas accretion rate scales with the ever-growing stellar potential. Cosmological hydrodynamic simulations, accordingly, show a tight relationship between the star formation rate and stellar masses in galaxies 16 Galaxies undergoing a burst (owing, perhaps, to a merger) may depart the main sequence, and exhibit elevated specific star formation rates. In fact, in recent years, this has come to be a new accepted definition of the term starburst: a galaxy with elevated sSFR (specific star formation rate $\equiv S F R / M_{\star}$ ) compared to the main sequence.

From an observer's perspective, this theoretical picture makes sense. The measured tightness of the SFR-M relation implies that galaxies' SFRs must be steady over long timescales, even for $>100 \mathrm{M}_{\odot} \mathrm{yr}^{-1}$ IR-bright galaxies at $z \sim 2$. If these galaxies' SFRs were only elevated $\left(\gtrsim 100 \mathrm{M}_{\odot} \mathrm{yr}^{-1}\right)$ for a short period of time $(\ll 1 \mathrm{Gyr})$ then the $z \gtrsim 1$ observed main sequence would not be as tight as it is observed to be, or, perhaps, it would not be observed at all. Furthermore, Stark et al. (2009) and Papovich et al.(2011) show that the star formation rates and stellar masses of most galaxies at $3<z<8$ increase gradually with time. This is determined by observed rest-frame ultraviolet luminosity functions (e.g. Bouwens et al., 2006, Reddy et al., 2008; Oesch et al., 2010) at high redshift and the observed main sequence (Noeske et al., 2007b; Stark et al., 2013).

In the context of this review, three critical questions exist regarding the relationship between DSFGs and the main sequence. First, do DSFGs lie on the main sequence? This is equivalent to asking whether DSFGs are currently undergoing elevated SFRs compared to field galaxies at a similar $M_{*}$, or whether they are simply massive galaxies with corresponding elevated SFRs. Their exact location on the SFR- $M_{*}$ locus depends, of course, on precise SFR and

\footnotetext{
${ }^{16}$ We note that this is not based on an assumed relationship between the galaxy mass and accretion rate in the simulations; rather, this relationship is a direct result of the models.
} 
$M_{*}$ calibrations, both of which are at present highly uncertain in DSFGs. If we use the stellar masses of SMGs from Hainline et al. (2011), SMGs are predominantly identified as main sequence outliers whereas the masses assumed by Michałowski et al.(2012) would imply that SMGs are high-mass, high-SFR main sequence galaxies.

The second critical question we might ask is how uncertainties in $M_{*}$ and SFR affect the tightness of the main sequence relation. The tightness, or relative lack of dispersion, of the SFR-M $M_{\star}$ relation is a primary piece of evidence supporting steady state galaxy growth. This tightness is what also implies that main sequence galaxies' duty cycles are of order unity (i.e. main sequence galaxies will be observable in their current state for most of their lifetimes). Some recent works challenge the relation by pointing out that, amongst DSFG populations, the SFR- $M_{*}$ trend disappears (Lee et al., 2013). This points out that tightness of the relation relies significantly on how galaxies' star formation rates are calculated-whether it be from rest-frame UV, optical, emission line, or infrared indicators, and whether or not corrections for extinction and dust attenuation are well understood or should be re-calibrated (e.g. as Kriek \& Conroy, 2013, show is necessary for massive early-type galaxies).

Lastly, it is an open question how much stellar mass is built up in galaxies via major mergers. Observations suggest that main sequence galaxies at higher redshifts $(z \sim 2)$ undergo a higher fraction of mergers and interactions than their $z \sim 0$ counterparts (e.g. Lotz et al. 2008, Kartaltepe et al., 2010, 2012). Similarly, much of the work on SMGs indicate that most have major merger histories with short duty cycles (Engel et al., 2010, Alaghband-Zadeh et al. 2012; Bothwell et al., 2013a). These results are closely tied with whether or not a main sequence galaxy can have a merger origin. In principle, a sizable fraction of a galaxy's mass could be built from a merger. In a cartoon example, a galaxy which has undergone a $500 \mathrm{M}_{\odot} \mathrm{yr}^{-1}$ burst for $100 \mathrm{Myr}$ has built up $5 \times 10^{10} \mathrm{M}_{\odot}$ of stars during that time; comparing that to the median stellar masses of SMGs from Hainline et al. (2011) reveals that $\sim 70 \%$ of that galaxy's stellar mass is built in the burst, versus $30 \%$ pre-dating the burst (N.B. that these fractions are much lower, if the high $M_{*}$ values of Michałowski et al., 2012, are assumed). In contrast, that galaxy will only spend $\sim 2 \%$ of its time at high- $z$ observed in burst mode, while the remaining $98 \%$ percent of the time is spent on the main sequence. Of course, the picture changes if the burst duration is not as long, the star formation rates lower, or the stellar masses of SMGs larger by factors of a few, all of which could actually imply burst-built stellar mass fractions $<10 \%$.

To investigate the predominance of mergers or disks among DSFGs on and off the main sequence, Hung et al. (2013) find that merger rates are equally likely to correlate to $L_{\mathrm{IR}}$ (or star formation rate) as sSFR, the specific star formation rate. This indicates that among infrared-luminous systems (in this case Herschel-selected galaxies), merger rates do not follow a strict luminosity cutoff as they do locally (whereby nearly all galaxies at $>10^{11.5} \mathrm{~L}_{\odot}$ are mergers Sanders \& Mirabel, 1996) nor do they follow a strict sSFR cutoff limit as more recent main sequence works suggest (Rodighiero et al., 2011; Nordon et al., 2012, Sargent et al., 2012). More analyses like this, but comprising both samples of obscured and unobscured galaxies are needed to bolster the statistical analysis of calculating merger fractions and truly understanding the meaning of the galaxies main sequence.

Going forward, it will be critical to quantify at what $L_{\mathrm{IR}}$ or $L_{\mathrm{bol}}$ mergers begin to dominate the origin of DSFGs. Are (for example) typical SMGs just an extenstion of the main sequence? Or are they outliers, with more extreme SFRs and lower $M_{*}$ values? Does the main sequence itself change when viewed from a more bolometric standpoint? As we will discuss in $\S 10$, theoretical models are divided as to the exact role of mergers in driving SMG-like luminosities. Similarly, observational groups have not yet reached a consensus on the relationship of SMGs to the main sequence. While much of the formative work on the population present ample evidence of short-lived starbursts, some more recent observations hint that SMGs might only represent the most massive, luminous extention of the galaxy main sequence (Dunlop, 2011; Michałowski et al., 2012, Targett et al., 2013).

\subsection{The FIR/Radio Correlation}

The correlation between galaxies' far-infrared/submillimeter emission and their radio emission has been empirically known for several decades, first investigated by van der Kruit, P.C. (1971, 1973) who observed that $\sim 1.4 \mathrm{GHz}$ emission correlated well with $10 \mu \mathrm{m}$ emission within Seyfert galaxy nuclei spanning five orders of magnitude in luminosity. While at first both infrared and radio emission was thought to be generated by synchrotron radiation, Harwit \& Pacini (1975) suggested that thermal re-radiation from dust-enshrouded HII regions dominated the infrared while the radio originated from synchrotron radiation of relativistic electrons off of supernovae remnants. In this context, the correlation naturally falls out because the massive stars which produce supernovae are the same population which heat the surrounding gas and dust, ionizing the HII regions. The infrared emission was confirmed to be thermallydriven when IRAS data became available in the mid 1980s (Helou et al., 1985, de Jong et al., 1985). A nice review of 
radio emission in galaxies is given in Condon (1992) with substantial follow-up of the FIR/radio correlation in local galaxies given in Yun \& Carilli (2002); Bell (2003); Murphy et al. (2008); Tabatabaei et al. (2005), 2007); Murgia et al. (2005); Dumas et al. (2011). The correlation can be generalized by the parameter $q_{\mathrm{IR}}$ where

$$
q_{\mathrm{IR}}=\log \left(\frac{S_{\mathrm{IR}}}{3.75 \times 10^{12}\left[\mathrm{~W} \mathrm{~m}^{-2}\right]}\right)-\log \left(\frac{S_{1.4 \mathrm{GHz}}}{\left[\mathrm{W} \mathrm{m}^{-2} \mathrm{~Hz}^{-1}\right]}\right)
$$

and $S_{\mathrm{IR}}$ is the integrated flux density (in $\mathrm{W} \mathrm{m}^{-2}$ ) between $42.5-122.5 \mu \mathrm{m}$ and $3.75 \times 10^{12}$ is the frequency at $80 \mu \mathrm{m}$, the mid-point of that band. All quantities are rest-frame. Although the $42-120 \mu \mathrm{m}$ limits of $S_{\text {IR }}$ made sense in the IRAS era, we now have observational access to far-infrared data spanning $10-1000 \mu \mathrm{m}$, so a somewhat more applicable definition of $q_{\mathrm{IR}}$ today (Ivison et al., 2010a) is

$$
q_{\mathrm{IR}}=\log \left(\frac{1.01 \times 10^{18} L_{\mathrm{IR}}}{4 \pi D_{L}^{2}\left[L_{\odot}\right]}\right)-\log \left(\frac{10^{-32} S_{1.4 G H z}}{(1+z)^{\alpha-1}[\mu \mathrm{Jy}]}\right)
$$

where $L_{\mathrm{IR}}$ is the integrated $8-1000 \mu \mathrm{m}$ luminosity, $D_{L}^{2}$ the luminosity distance, $S_{1.4 \mathrm{GHz}}$ the observed $1.4 \mathrm{GHz}$ flux density, and $\alpha$ the radio spectral index. The radio spectral index is defined by $S_{v} \propto v^{\alpha}$, where the radio portion of the spectrum is ubiquitously well-represented by a powerlaw and the value of $\alpha$ is negative in the vast majority of sources. If radio flux density is observed in some other frequency $v$ which is not $1.4 \mathrm{GHz}$, it can be converted to observed-frame $1.4 \mathrm{GHz}$ via $S_{1.4 \mathrm{GHz}}=S_{v}(1.4 \mathrm{GHz} / v *(1+z))^{\alpha-1}$. Yun et al. (2001) show that the value of $q_{\mathrm{IR}}$ in local starburst galaxies is constrained at $q_{\mathrm{IR}}=2.34 \pm 0.72$ with only a handful of outliers.

Several works have addressed whether or not the correlation evolves at high-redshift. Magnelli et al. (2010) measure $q_{\mathrm{IR}}=2.17 \pm 0.19$ in a sample of SMGs and OFRGs-a value which is lower than seen in local starbursts. This could suggest that DSFGs either have a FIR excess or that there is evolution in $q_{\mathrm{IR}}$. Ivison et al. (2010a b) visit this issue in large samples of BLAST and Herschel-selected starbursts and suggest a shallow redshift evolution of $q_{\mathrm{IR}} \propto(1+z)^{-0.26 \pm 0.07}$ and $\propto(1+z)^{-0.15 \pm 0.03}$ respectively. Casey et al. (2012a) measure a slightly steeper evolution, $\propto(1+z)^{-0.30}$ out to $z \sim 2$ for spectroscopically confirmed Herschel-selected DSFGs. However, these works all deal with IR-selected samples and thus is intrinsically biased as pointed out by Ivison et al. (2010b); the underlying unbiased evolution in $q_{\mathrm{IR}}$ can only be measured with very large samples of both radio- and IR-selected galaxies using stacking. Note that Sargent et al. (2010) present a detailed discussion of the possible evolution of the correlation and claim, after taking the many selection effects into account, that the relationship does not evolve.

The value of the radio spectral index is often assumed to be $\alpha=-0.8$ (Condon, 1992) based on measurements from nearby star forming galaxies, however more recent studies have found evidence for shallower values for fainter radio sources $\left(S_{1.4}<1 \mathrm{mJy}\right.$ Bondi et al., 2007; Garn \& Alexander, 2008) between -0.6 and -0.7 (Ibar et al., 2009); Ivison et al. (2010a b) adopt $\alpha=-0.75 \pm 0.06$ with little evidence of evolution from $0<z<3$ (i.e. the redshift dependence they measure is $\left.\alpha \propto(1+z)^{0.14 \pm 0.20}\right)$. Indeed, the uncertainty in the radio spectral index can add scatter to the measured value of $q_{\mathrm{IR}}$ for high-redshift sources.

When applied to high-redshift DSFGs, the FIR/radio correlation can be used to estimate $L_{\mathrm{IR}}$ in the absence of infrared data. It can also be used to estimate SED characteristics like dust temperature if only 1-2 far-infrared flux densities are in hand and not enough to constrain the far-infrared peak wavelength independently (this is what was done for Scuba galaxies when only $S_{850}$ was available in the far-infrared; Ivison et al., 2002, Chapman et al., 2003c). The FIR/radio correlation can likewise be used to learn more about obscured star formation at radio wavelengths since $\mathrm{SFR} \propto L_{\mathrm{IR}} \propto L_{1.4 \mathrm{GHz}}$. This is particularly useful considering radio interferometric maps have much better resolution than submillimeter single-dish maps (both deep blank-field intermediate-resolution $\sim 1^{\prime \prime}$ maps, e.g. from the Jansky VLA, and high-resolution $\sim 0.1^{\prime \prime}$ maps from, e.g. VLBA or $e$-MERLIN). This enables precise identification of multi-wavelength counterparts and infer characteristics of the distribution of obscured star formation (e.g. as seen in Figure 30). Note that one caveat of the FIR/radio correlation is that, although it holds well on galaxy-scales, often radio and FIR morphologies are substantially distinct; more detailed follow-up at the highest resolutions with ALMA and the VLA are needed to shed additional light on the matter.

Despite the usefulness of the FIR/radio correlation in DSFGs, the underlying physical mechanisms leading to it are not well understood. One explanation offered up by Volk (1989) and Lisenfeld et al. (1996) is the "electron calorimeter" theory by which the synchrotron cooling timescale is much shorter than the escape time for electrons, 
and only a few percent of energy input from supernovae is sufficient enough to recover a relation of $v L_{v} \approx 2 \times 10^{-6} L_{\mathrm{IR}}$. However, calorimetry predicts a radio spectrum which is too steep $(\alpha \sim-(1-1.3)$, not $-(0.6-0.7))$. Bremsstrahlung radiation and ionization can flatten the spectrum, but would meanwhile break the FIR/radio correlation, so cosmic ray proton cooling is needed to make up for radio emission lost due to Bremsstrahlung and ionization (Thompson et al. 2007; Lacki et al., 2010, Lacki \& Thompson, 2010). Very recently, a new theoretical approach explains both the FIR/radio correlation and its evolution towards high-redshift using recent advancements in understanding magneticfield amplification in galaxies (Schleicher \& Beck, 2013, Zweibel, 2013); the physical quantities which underly this formulation will be testable with the next generation of radio telescopes. 

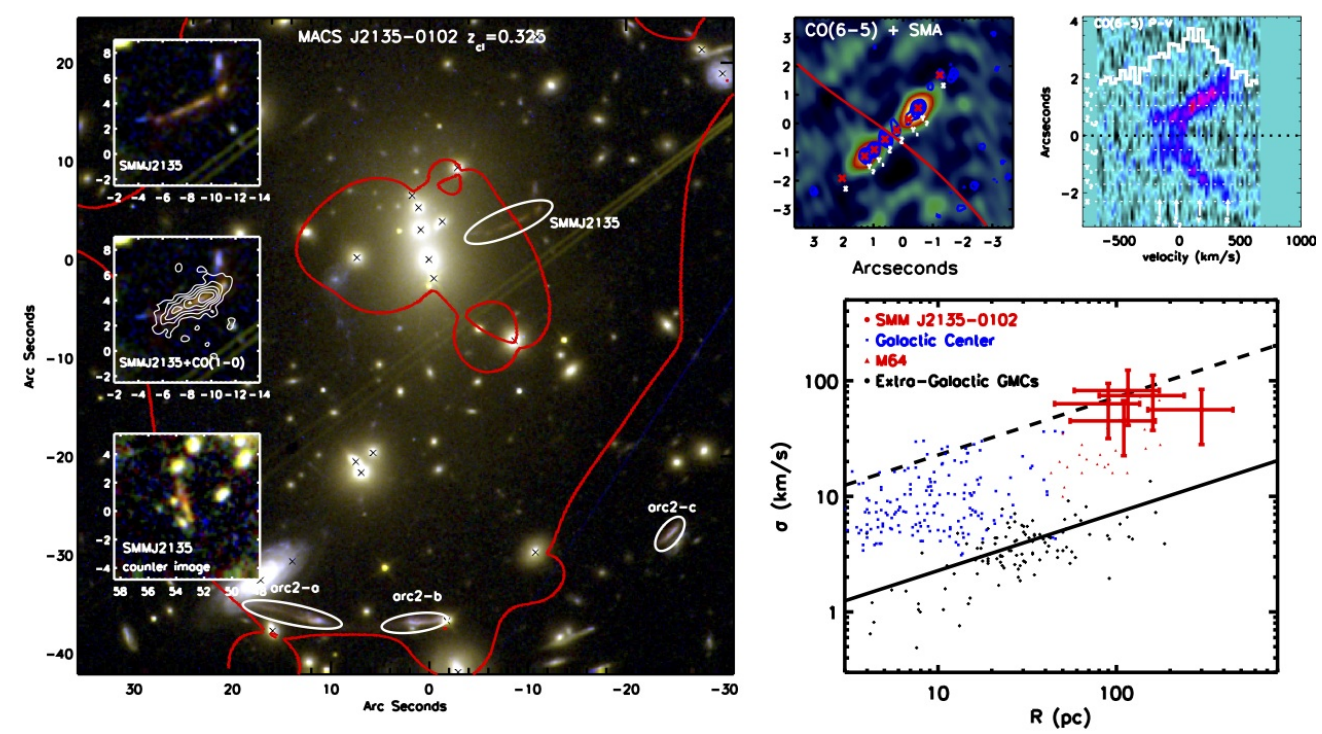

Figure 31: Left: HST image of the cluster MACSJ2135-010217. The red line is the $z=2.3$ critical curve. The insets show the images of the lensed DSFG SMMJ2135-0102 and another triple-imaged galaxy at $z=2.3$. The middle inset shows the CO1-0 map VLA. Right: The top two panels show the IRAM/PdBI CO(6-5) map with SMA $870 \mu \mathrm{m}$ contours while the second panel shows the image-plane position-velocity diagram of the $\mathrm{CO}(6-5)$ line emission extracted along a position angle of 45 degrees east or north across the lensed galaxy in the long direction. The PV diagram shows a velocity gradient of $500 \mathrm{~km} \mathrm{~s}^{-1}$ across an angular extent of 6 arcseconds. The lower panel shows the scaling relation of velocity dispersion versus cloud radius. The local relation shown by the solid line Larson (1981) is consistent with extragalactic GMCs in quiescent environments. The GMCs in gas-rich, high-turbulent-pressure environments, such as the Galactic Center, are systematically offset from this relation. The line width data for star-forming regions in SMMJ2135-0102 are compatible with a higher normalization than the Milky Way. These figures are reproduced from Swinbank et al. (2011) with permission from the authors and AAS.

\section{Detailed Studies of Individual Dusty Star-Forming Galaxies}

In this chapter we summarize properties of DSFGs as obtained from detailed individual galaxy studies. In this context, gravitational lensing has played a critical role because the spatial enhancement associated with magnification provides a way to investigate the internal structure of distant, faint galaxies to levels unattainable with the current generation of instrumentation for typical DSFGs. A second important fact here is that large samples of gravitationally lensed galaxies can be efficiently selected by searching for bright sources in wide area submillimeter surveys (Blain, 1996; Perrotta et al., 2002; Negrello et al., 2007, 2010; Hezaveh \& Holder, 2011, Wardlow et al., 2013). While the early submm surveys were limited to smaller sky areas, the advent of wide-field surveys, especially with Herschel and SPT, at sub/millimeter wavelengths have allowed detections of large samples of lensed DSFGs that can be followed up with a host of radio and submm/mm-wave interferometers. Similarly, and pre-dating the large area survey work, important targets of lensed DSFGs have come from submm/mm-wave imaging of known massive lensing galaxy clusters Smail et al. (1997).

\subsection{SMM J02399-0136}

The DSFG SMM J02399-0136, with $S_{850}=26 \pm 3 \mathrm{mJy}$, was the first SCUBA SMG to be unambiguously identified with a optical/near-IR counterpart (Ivison et al., 1998). The SMG is located towards the massive galaxy cluster A370 leading to a lensing magnification factor of 2.5 (Ivison et al., 1998). The optical identification allowed the redshift of 2.80 to be determined with optical spectroscopy. This was later confirmed with the first detection of $\mathrm{CO}$ molecular gas in a SMG, $\mathrm{CO}(3-2)$ line in this case, leading to a molecular gas mass estimate of $8 \times 10^{10} \mathrm{M}_{\odot}($ Frayer et al., 1998) extending over at least $25 \mathrm{kpc}$. The system is made up of at least three components within this large reservoir of gas. This includes a X-ray detected broad absorption line (BAL) quasar (Bautz et al., 2000, Genzel et al., 2003; Valiante et al. . 2007), a dusty starburst galaxy coincident with an extended Ly-alpha cloud (Vernet \& Cimatti, 2001), and a faint third component (Ivison et al., 2010c). The $L_{I R} \sim 3 \times 10^{13} \mathrm{~L}_{\odot}$ resulted in a first detection of the [NII] 
$122 \mu \mathrm{m}$ line from a galaxy at $z>2.5$ (Ferkinhoff et al., 2010). In combination with the [OIII] $88 \mu \mathrm{m}$ line intensity, Ferkinhoff et al. (2010) find that the [OIII]/[NII] line ratio of SMM J02399-0136 is consistent with a scenario where the dominant source for the line emission is HII regions ionized by massive O9.5 stars, especially in light of the fact that the detected AGN is no longer considered to be the dominant contributor the IR luminosity of this system (Ivison et al., 2010c). Detected free-free emission from the system implies an AGN contribution to IR luminosity of at most $35 \%$ (Thomson et al., 2012).

\subsection{SMM J2135-0102: the Cosmic Eyelash}

The DSFG SMMJ2135-0102 (the "Cosmic Eyelash") was serendipitously identified by Swinbank et al. (2010) in a LABOCA $870 \mu \mathrm{m}$ observation of the galaxy cluster MACSJ2135-010217 $(z=0.325)$ with a $870 \mu \mathrm{m}$ flux density of $106 \mathrm{mJy}$. The redshift was identified to be $z=2.33$ from CO line emission and lens models of the cluster mass distribution shows it to be magnified by a factor of 32 ; the fact that it is magnified by a cluster and not a galaxy means that differential extinction is not a substantial concern (Hezaveh et al., 2012, Serjeant, 2012). Once corrected for magnification, the source has an intrinsic luminosity consistent with a ULIRG at $z \sim 2$ with a SFR of around 200 $\mathrm{M}_{\odot} /$ year. The observed flux density at $500 \mu \mathrm{m}$ of $325 \mathrm{mJy}$ (Ivison et al., 2010d) puts this source among the brightest of all the lensed DSFGs discussed in the literature with Herschel-SPIRE (Negrello et al. 2010; Wardlow et al., 2013, Harris et al. 2012). Moreover, the high magnification of 32, compared to a magnification of about 10 for most lensed DSFGs found with Herschel (Bussmann et al., 2013, which are for galaxy-galaxy lenses, also more prone to differential lensing uncertainties), allows spatial resolution down to 100 parcsec scales within the galaxy. The lensing magnification resolves the individual giant molecular clouds (GMCs) in SMM J2135-0102, which were identified to be larger and more luminous than the GMCs of local star-forming galaxies by about a factor of 100 Swinbank et al. (2010).

Ivison et al. (2010d) presented the Herschel-SPIRE FTS spectrum with a clear detection of the $158 \mu \mathrm{m}$ [CII] line. The $\mathrm{L}_{[\mathrm{CII}]} / \mathrm{L}_{\text {bol }}$ ratio was found to be significantly higher than in local ULIRGS, but consistent with the ratio of local star-forming galaxies. Combined with $\mathrm{CO}$ measurements, the photo-dissociation regions (PDRs) were found to have a characteristic gas density of $10^{3} \mathrm{~cm}^{-3}$ and a UV radiation field, $\mathrm{G}_{0}, 1000$ times stronger than that of the Milky Way. In combination, Ivison et al. (2010d) has suggested that the galaxy contains kpc-scale starburst clumps distributed over a large disk, different from nuclear starbursting local ULIRGS. Further PDR modeling using a combination of ${ }^{12} \mathrm{CO},[\mathrm{CI}]$, and HCN line intensities are presented in Danielson et al. (2011).

Using IRAM/PdBI and VLA CO(6-5) and CO(1-0) high resolution imaging data, respectively, Swinbank et al. (2011) studied the kinematics of this galaxy as traced by the CO molecular gas. The CO velocity maps showed the galaxy to be rotationally-supported disk with a rotation speed of $320 \pm 25 \mathrm{~km} \mathrm{~s}^{1}$ and with a ratio of rotational-todispersion support of $v / \sigma=3.5 \pm 0.2$. The disk has a dynamical mass of $(6.0 \pm 0.5) \times 10^{10} \mathrm{M}_{\odot}$ within a radius of $2.5 \mathrm{kpc}$. The linewidth-size scaling relation based on the highest resolution CO data was found to be significantly offset from the local Larson scaling relation for molecular clouds (Fig. 31). Swinbank et al.(2011) argues that such a high offset is evidence for the importance of supersonic turbulence on scales 100 times smaller than in the kinematically quiescent interstellar medium of the Milky Way. Given the high external hydrostatic pressure of the ISM of this galaxy, with $P_{\text {tot }} / k_{B} \sim 2 \times 10^{7} \mathrm{~K} \mathrm{~cm}^{-3}$, the subsonic star-forming regions of are expected to have densities in exces ${ }^{17}$, of $10^{8}$ $\mathrm{cm}^{-3}$.

The lensing flux density enhancement has also allowed studies, for the first time at high redshifts, of the spectral line energy distributions (SLEDs) of ${ }^{13} \mathrm{CO}$ and $\mathrm{C}^{18} \mathrm{O}$ from $J=1-0$ to $J=7-6$ transitions of this galaxy Danielson et al. (2013). The ${ }^{13} \mathrm{CO}$ emission from optically thin regions imply a total gas mass for the galaxy of $\sim 1.5 \times 10^{10}$ $\mathrm{M}_{\odot}$ and a conversion of $\mathrm{CO}$ to $\mathrm{H}_{2}$ mass $\alpha_{\mathrm{CO}} \sim 0.9$, consistent with bright SMG-like DSFGs for a ULIRG system at $z \sim 2$. The velocity-integrated flux ratio ${ }^{13} \mathrm{CO} / \mathrm{C}^{18} \mathrm{O} \sim 1$ implies an abundance ratio $\left[{ }^{13} \mathrm{CO}\right] /\left[\mathrm{C}^{18} \mathrm{O}\right]$ that is at least a factor of seven below that of the Milky Way. The enhanced $\mathrm{C}^{18} \mathrm{O}$ abundance implies star-formation is preferentially biased to high-mass stars. The ISM is best-modeled with two phases: a cold phase at $\sim 50 \mathrm{~K}$ with a density of $10^{3}$ $\mathrm{cm}^{-3}$ and a warm phase at $90 \mathrm{~K}$ with density $10^{4} \mathrm{~cm}^{-3}$. Further modeling suggests that the ISM heating contribution from cosmic-rays and UV is adequate to explain this warm phase at $90 \mathrm{~K}$. It has a cooling rate of $(1-20) \times 10^{-25} \mathrm{erg}$

\footnotetext{
${ }^{17}$ Note that upon a correction to the calculation in Swinbank et al. 2011, the actual pressure is even higher, an order of magnitude above these published values (Jacqueline Hodge, private communication).
} 

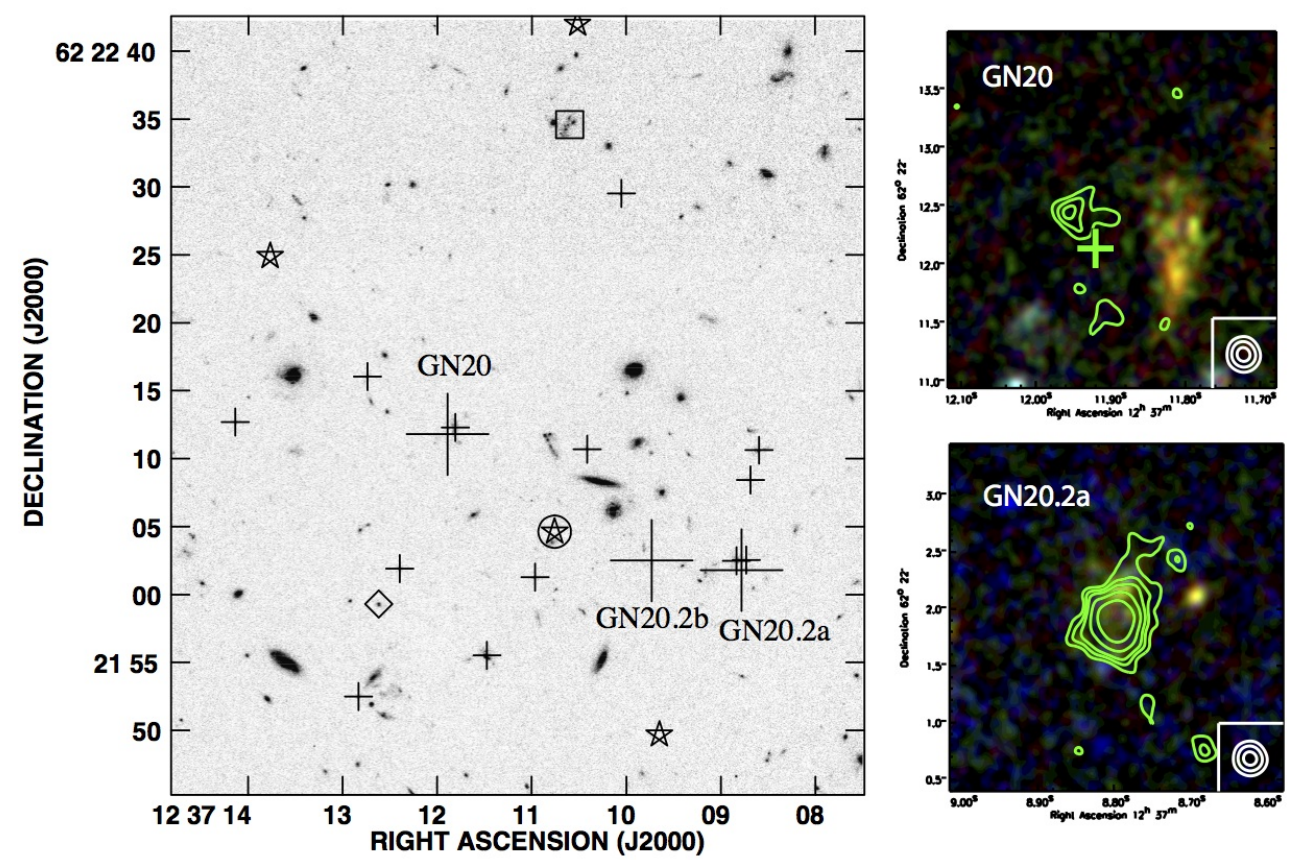

Figure 32: Left: HST/ACS F850W z-band image of the GN20 proto-cluster field. Large crosses mark the $1.4 \mathrm{GHz}$ positions of the three known SMGs in the field. Small crosses mark the positions of the LBGs within 25 arcseconds of the SMG GN20. The stars mark the positions of the new $\mathrm{CO}$ emission line sources and the diamond marks the LBG with a possible detection in $\mathrm{CO}(2-1)$ as found by Hodge et al. (2013a). The circled star is the emission line candidate with an optical counterpart within one arcsecond. The figure is reproduced from Hodge et al.(2013a) with permission from the authors and AAS. Right: Combined MERLIN and VLA radio contours overlaid on HST/ACS color images of GN20 and GN20.2a. The field sizes are $3 \times 3$ arcseconds and the MERLIN beam size is 0.3 arcseconds. The levels of the radio contours are drawn at 3, 4, 5, 7, and 10 $\sigma$. The emission centroid for GN20 in the mid-IR (Spitzer IRAC/MIPS) is consistent with the radio and submm continuum position, while GN20 shows a statistically significant offset between submm and optical emission. GN 20.2b is undetected in the radio. These panels are reproduced with permission from Casey et al. 2009b). 
$\mathrm{s}^{-1}$ per $\mathrm{H}_{2}$ molecule from the CO SLED. The high temperature of 140 to $200 \mathrm{~K}$ derived for the highest density regions of the warm phase implies that the cosmic ray heating is more important than UV heating where the star-formation is most active.

\subsection{GN20}

GN20 (Pope et al. 2005), whose redshift was serendipitously discovered by Daddi et al. (2009), is a bright SMG in a proto-cluster of multiple DSFGs. GN20 is the brightest SMG in the GOODS-N field with a $850 \mu \mathrm{m}$ flux density of $20.3 \mathrm{mJy}$, suggesting a SFR at the level of $1800 \mathrm{M}_{\odot} \mathrm{yr}^{-1}$ (Fig. 32, the environment of this source is discussed in Section 77. The individual DSFG components have been well-studied over the last few years with a variety of interferometers. At high resolution with EVLA in $\mathrm{CO}(2-1)$ the gas in GN20 appears to be resolved into at least five star-forming molecular clumps of size around $1.3 \mathrm{kpc}$ (limited by the beamsize of observations) with line widths of $\sim 100$ to $500 \mathrm{~km} \mathrm{~s}^{-1}$ (FWHM) and a mass surface densities in excess of $3000 \mathrm{M}_{\odot} \mathrm{pc}^{-2}$ (Hodge et al. 2012). The clumps are self-gravitating and they have CO-to- $\mathrm{H}_{2}$ conversion factors $\alpha_{\mathrm{CO}}$ with values between 0.2 and 0.7 . The gas reservoir extends to a diameter of $\sim 15 \mathrm{kpc}$ and also shows clumpy structure. GN20 is a rotating disk with a maximum rotational velocity of $575 \pm 100 \mathrm{~m} / \mathrm{s}$ and a dispersion of $100 \pm 30 \mathrm{~km} / \mathrm{s}$. The dynamical mass for GN20 is $5 \pm 2 \times 10^{11}$ $\mathrm{M}_{\odot}$ (Hodge et al. 2012, Carilli et al. 2011) with gas a fraction at the level of $40 \%$ (though see $\$ 8.6$ for reasons why this may be an overestimate).

The $6.2 \mu \mathrm{m}$ polycyclic aromatic hydrocarbon (PAH) line from GN20 was detected by Riechers et al.(2013a) using Spitzer/IRS. This remains the highest redshift SMG to which a PAH feature has been detected. SED modeling of the rest-frame 4 to $7 \mu \mathrm{m}$ continuum emission shows the dominant contribution to the mid-IR flux density is from a faint, dust-obscured AGN. Using the scaling relation between $6 \mu \mathrm{m}$ continuum and 2 to $10 \mathrm{keV} \mathrm{X-ray} \mathrm{luminosity,} \mathrm{Riechers}$ et al. (2013a) estimate a Eddington limit for the black hole mass of GN20 AGN to be $1.5-3 \times 10^{8} \mathrm{M}_{\odot}$ at the level of $0.03-0.06 \%$ of the dynamical mass of the galaxy, and consistent with the average ratio of $0.05 \%$ for AGN-dominated SMGs at $z \sim 2$ (Alexander et al., 2005a).

The second component GN20.2 is made up of two separate galaxies GN20.2a and GN20.2b with de-convolved sizes of $\sim 5 \times 3 \mathrm{kpc}$ and $\sim 8 \times 5 \mathrm{kpc}$ (Gaussian FWHM) in CO(2âĂŞ1) imaging data with VLA, respectively (Hodge et al. 2013a). GN20.2a is radio bright (Pope et al., 2006; Casey et al., 2009b), while GN20.2b is not. GN20.2a has the largest gas surface density of all galaxies in the proto-cluster with a surface density estimated to be at the level of $13,000 \mathrm{M}_{\odot} \mathrm{pc}^{-2}$ for the most compact components of the galaxy in the highest resolution data. The gas surface density of GN20.2b is lower at $1700 \mathrm{M}_{\odot} \mathrm{pc}^{-2}$. The difference in the gas surface densities suggest that the two galaxies are two different stages of star-formation with the possibility that GN20.2a is fueled by a major merger to reach the high surface density observed.

\subsection{Lensed DSFGs}

The wide area surveys with Herschel-SPIRE, especially H-ATLAS (Eales et al., 2010) and HerMES (Oliver et al. 2012), and the South Pole Telescope (Vieira et al. 2013) have now resulted in large samples of rare and bright lensed DSFGs. The selection is especially easy since the lensed sources are the brightest sources in the submm maps, and only basic spectral filtering for radio-bright blazars is needed to find the lensed DSFGs in millimeter maps. Negrello et al. (2010) demonstrated through multi-wavelength follow-up observations of the first five Herschel-selected DSFGs with $S_{500}>100 \mathrm{mJy}$ at $500 \mu \mathrm{m}$, after accounting for nearby spiral galaxies and radio-bright blazars, are all gravitationally lensed (Fig. 34). However due to small sample size it was not possible to conclusively establish whether all of the extragalactic sources with $S_{500}>100 \mathrm{mJy}$ are gravitationally lensed or whether there are intrinsically luminous, but possibly rare, DSFGs at such high flux densities. With 13 DSFGs with $S_{500}>100 \mathrm{mJy}$, Wardlow et al. (2013) addressed the same issue and found $>93 \%$ of the sample was confirmed to be gravitational lensed DSFGs implying $<7 \%$ of the sources would be intrinsically bright. At $z>1$, the non-lensed sources would have luminosities with $L>10^{13} \mathrm{~L}_{\odot}$ (HyLIRGs). Detailed follow-up of such luminous sources are rare, but in the two Herschel-selected cases, one from HerMES in Wardlow et al. (2013) and another from Herschel-ATLAS in Harris et al. (2012), they have been both confirmed to be DSFG-DSFG (Fu et al. 2013) or multi-DSFG (Ivison et al., 2013) merger.

With a surface density for $S_{500}>100 \mathrm{mJy}$ lensed DSFGs at the level of $0.2 \mathrm{deg}^{-2}$ (Negrello et al., 2010, Wardlow et al. 2013; Bussmann et al. 2013), and with close to $1200 \mathrm{deg}^{2}$ of SPIRE imaging data in the Herschel Science 


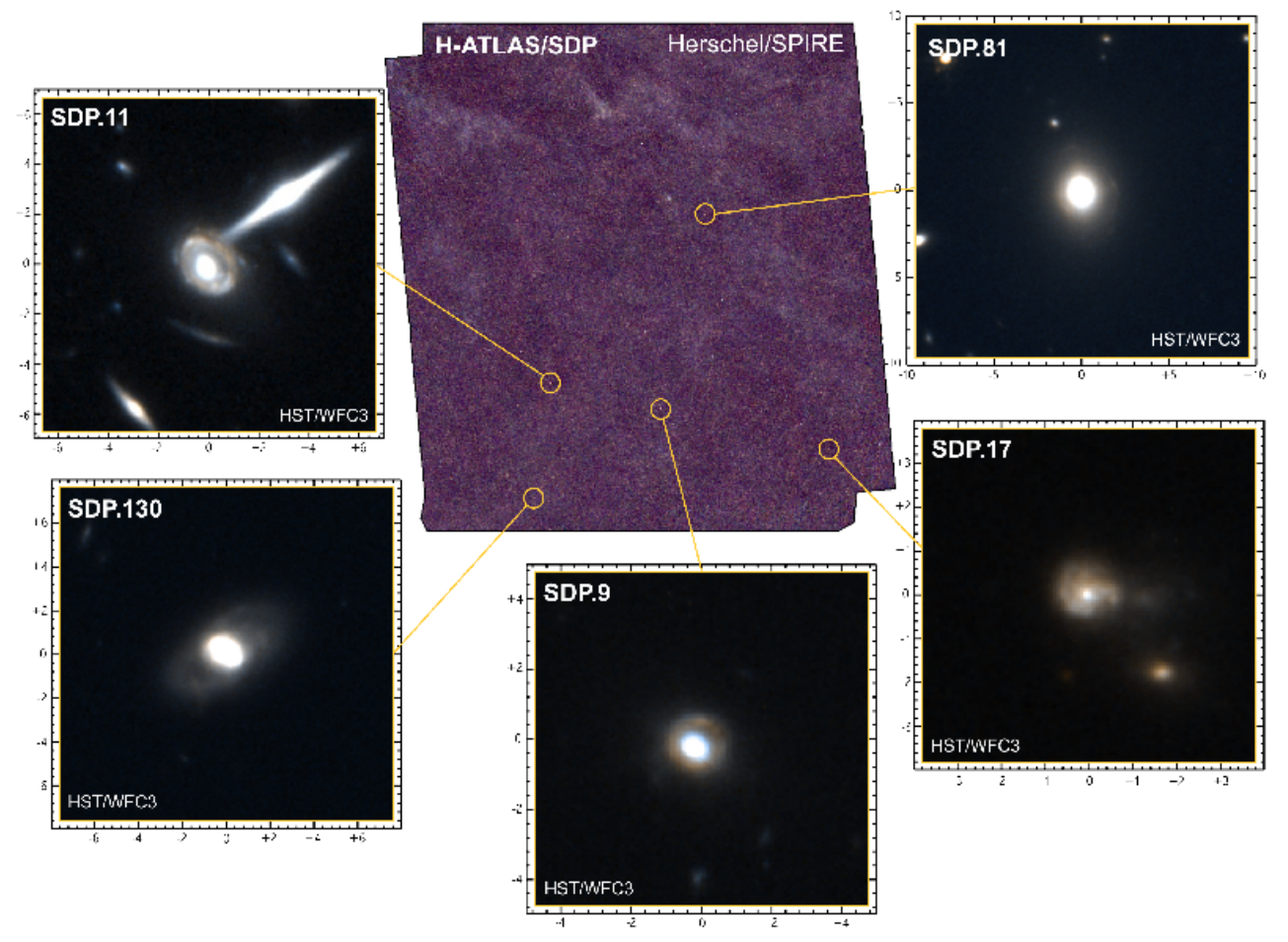

Figure 33: HST/WFC3 F110W and F160W images of the first five confirmed gravitationally lensed DSFGs from Herschel-SpiRe (Negrello et al. 2010. The center image shows the three-color $250 \mu \mathrm{m}, 350 \mu \mathrm{m}$, and $500 \mu \mathrm{m}$ SPIRE image of the H-ATLAS Science Demonstration Phase (SDP) field from which these lensed DSFGs were identified, simply based on their $500 \mu \mathrm{m}$ flux density having values with $S_{500}>100 \mathrm{mJy}$. The figure is reproduced from Negrello et al. (2013) with permission from the authors and AAS.

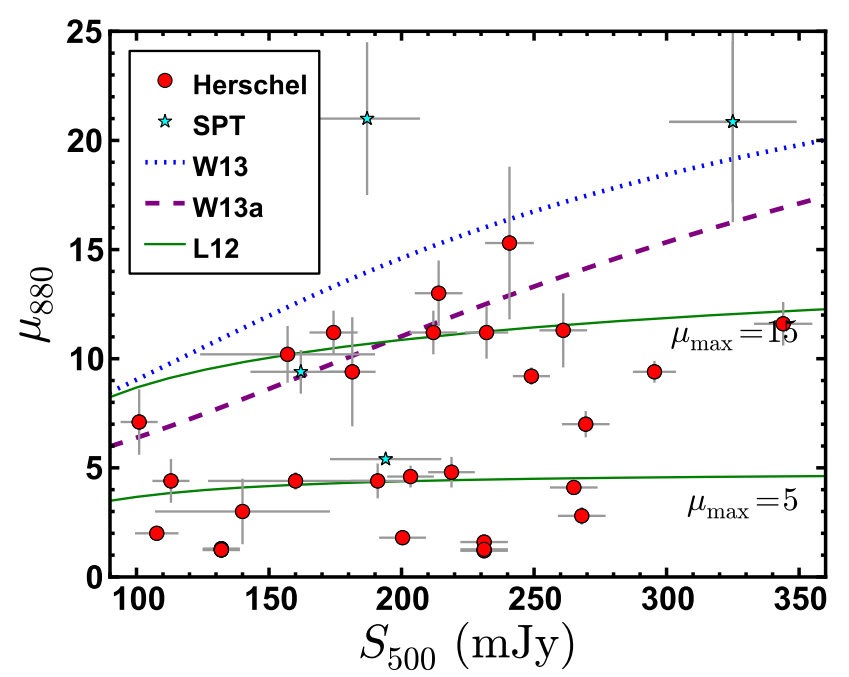

Figure 34: Magnification factors from lens modeling of high-resolution Submillimeter Array (SMA) imaging data of Herschel-selected DSFG samples with $S_{500}>100 \mathrm{mJy}$. The lines are various model predictions on the expected magnification as a function of the $500 \mu \mathrm{m}$ flux density with 'W13' and 'W13a' predictions from Wardlow et al. (2013) and 'L13' from Lapi et al. (2011). The plot also shows magnification factors of two SPT-selected lensed DSFGs from Hezaveh et al. (2013). This figure is reproduced from Bussmann et al. (2013) with permission from the authors and AAS. 
Table 3: Summary of Currently Known Highest Redshift DSFGs at $z>5$

\begin{tabular}{|c|c|c|c|}
\hline Name & Redshift & $S_{850}^{\text {obs }}(\mathrm{mJy})$ & Reference \\
\hline $\mathrm{J} 1148+5251^{\dagger}$ & 6.42 & $7.8 \pm 0.7$ & Wang et al. (2007) \\
\hline HFLS3 & 6.34 & $33 \pm 2$ & Riechers et al. (2013b) \\
\hline SPT0243-49 & 5.69 & $73 \pm 12$ & Vieira et al.(2013) \\
\hline SPT0346-52 & 5.65 & $138 \pm 24$ & Vieira et al. (2013) \\
\hline Aztec-3 & 5.30 & $8.7 \pm 1.5$ & Capak et al. (2011) \\
\hline HLS J0918+5142 & 5.24 & $125 \pm 8$ & Combes et al. (2012) \\
\hline HDF850.1 & 5.18 & $7.0 \pm 0.5$ & Walter et al. (2012) \\
\hline
\end{tabular}

$\dagger \mathrm{J} 1148+5251$ is a QSO which happens also to be a bright SMG.

Archive, there should be close to 250 lensed DSFGs. To confirm if all of these are indeed lensed rather than intrinsically bright requires time-consuming multi-wavelength follow-up programs. In addition to high resolution interferometric imaging, a lensing event can be confirmed with redshift of the background lensed DSFG and an optical image showing the presence of a foreground lensing galaxy. As the sample sizes of lensed Herschel-selected DSFGs are increasing, physical properties of those sources are now starting to appear in the literature (Hopwood et al., 2011, Negrello et al. 2013; Dye et al., 2013; Bussmann et al. 2013).

An example of a detailed study of the kind possible with Herschel-selected lensed DSFGs is HERMES J1057+5730 (Conley et al., 2011; Gavazzi et al., 2011). It has $S_{500} \sim 230 \mathrm{mJy}$ and an intrinsic luminosity that puts it among HyLIRGs with $L=1.4 \pm 0.1 \times 10^{13} \mathrm{~L}_{\odot}$, with a SFR of $2500 \mathrm{M}_{\odot} \mathrm{yr}^{-1}$ (Conley et al. 2011 ). The CO observations, especially the velocity structure traced by $\mathrm{CO}$ at high resolution, show that it is a gas-rich (a.k.a. "wet") merger (Riechers et al. 2011b) and the molecular gas properties show at least a two-phase medium with with $T_{\text {kin }} \sim 100 \mathrm{~K}$ and $n_{\text {gas }} \sim 10^{3} \mathrm{~cm}^{-3}$ gas combined with very dense gas at $T_{\text {kin }} \sim 200 \mathrm{~K}$ and $n_{\text {gas }} \sim 10^{5} \mathrm{~cm}^{-3}$ (Scott et al. 2011). Another example is H-ATLAS J11460011 (Fu et al. 2012a) with $S_{500} \sim 300 \mathrm{mJy}$. The source is lensed by four galaxies in the foreground providing differential magnification factors of 17, 8 and 7 for near-IR (stars), submm (dust), and radio (gas traced by $\mathrm{CO}$ ) wavelengths. The DSFG is gas rich $\left(f_{\text {gas }} \sim 70 \%\right.$ relative to total baryons) and young with an estimated age of $20 \mathrm{Myr}$. The dusty star-burst phase is also likely to be short with a total estimated lifetime of $40 \mathrm{Myr}$ to consume the remaining gas.

While they are magnified by gravitational lensing, almost all apparently bright DSFGs selected by Herschel are also intrinsically bright or have intrinsic (i.e., magnification-corrected) luminosities that correspond to ULIRG to HyLIRG-like IR-bright galaxies. This is because the magnification factor for $S_{500}>100 \mathrm{mJy}$ samples are small with a mean value around $6_{-3}^{+5}$ (Bussmann et al. 2013). While published statistics are limited to a handful of galaxies, such a low magnification factor is in contrast to the SPT-selected lensed DSFGs at $1.4 \mathrm{~mm}$ that have magnification factors around $\sim 20$ (Hezaveh et al., 2013). The difference could easily point out biases in the sample selection with Herschel finding more of the lensed DSFGs that have large intrinsic sizes while the SPT selection is biased to intrinsically small subset of the lensed DSFGs that also happen to be primarily at higher redshifts, with latter due to the longer wavelength selection. However, this conclusion is based on limited statistics for the SPT sample. While 30 lensed Herschelselected SMGs now have well-determined magnification factors (Bussmann et al., 2013), magnification factors for SPT sample is limited to four sources (Hezaveh et al., 2013). Similarly, the intrinsic size distribution measured from lensed DSFGs could be biased low since compact sources are more likely to be highly magnified (Hezaveh et al., 2012).

On the topic of lensed DSFGs detected by SPT, Vieira et al. (2013) reported a sample of 23 spectroscopicallyconfirmed with ALMA, lensed DSFGs (of 26 targeted DSFGs). At least 10 of these DSFGs are at $z>4$ with 2 secure detections at $z>5$. Fig. 36 shows ALMA contours at $350 \mathrm{GHz}$ on HST and other near-IR imaging data for four of the lensed DSFGs studied in Hezaveh et al. (2013). These four lensed DSFGs have magnification factors that range from 5 to 22 with lens Einstein radii of 1.1 to 2.0 arcseconds. The lensing masses in the foreground range from $1-7 \times 10^{11} \mathrm{M}_{\odot}$, suggesting that SPT-selected lensed DSFGs events involve massive galaxies in the foreground. SPTS 053816-5030.8 (a.k.a. SPT0538) at $z=2.78$ involves two components, with SFR densities of $630 \pm 240 \mathrm{M}_{\odot} \mathrm{yr}^{-1}$ for the compact $(0.5 \mathrm{kpc})$ and $31 \pm 11 \mathrm{M}_{\odot} \mathrm{yr}^{-1}$ for the extended $(1.6 \mathrm{kpc})$ component, respectively (Bothwell et al. 


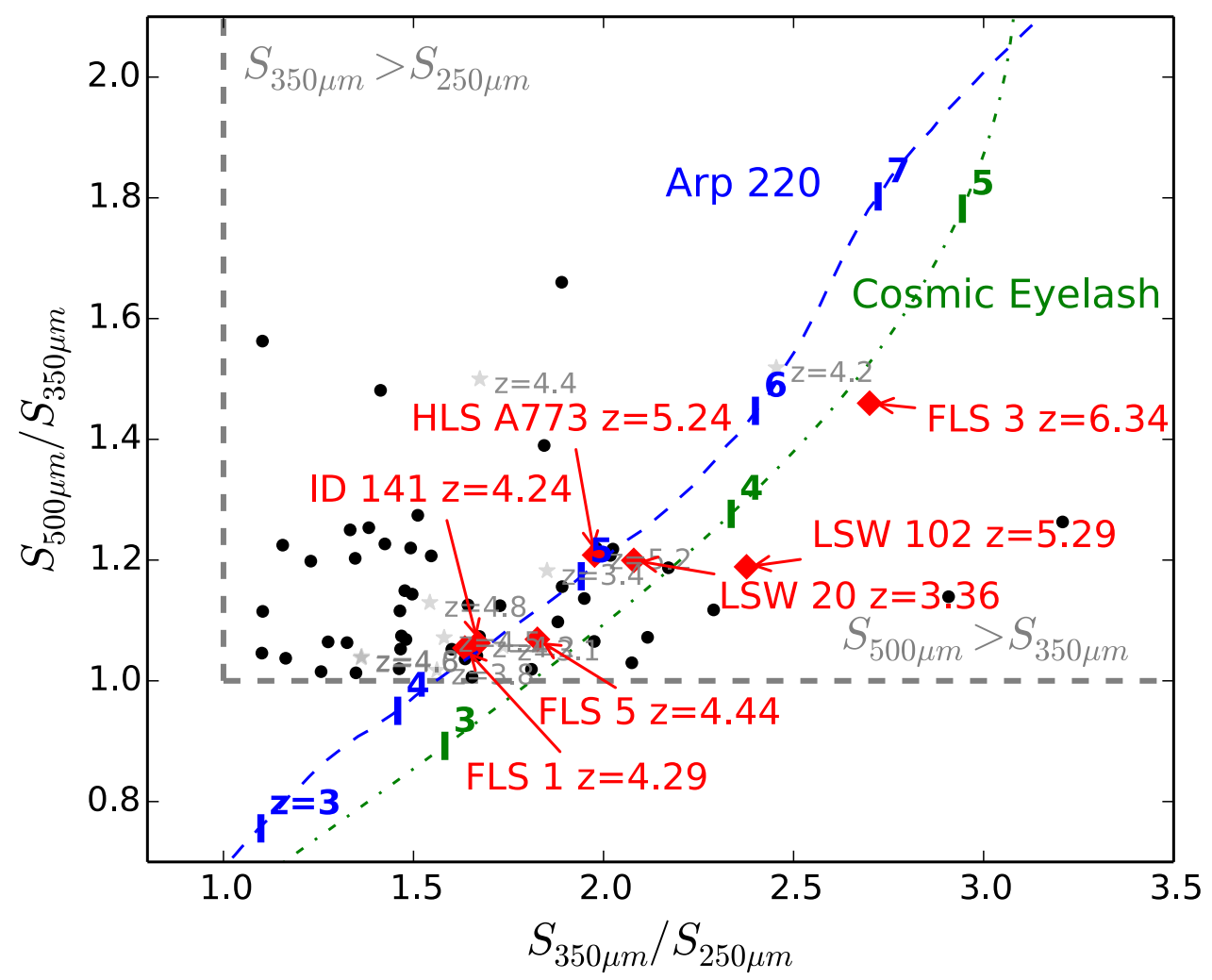

Figure 35: SPIRE color ratios for candidate $z>4$ DSFGs in Dowell et al. (2013) (black dots) with confirmed sources shown as red diamonds, including the highest redshift Herschel-selected SMG HFLS3 at $z=6.34$. SED tracks based on Arp 220 and the Cosmic Eyelash (Swinbank et al. 2010) are shown for comparison. They gray dashed lines are the selection from Dowell et al. (2013). The figure is a modified version of the same figure published in Dowell et al. (2013); its reproduction here is done with permission of the authors and AAS. 

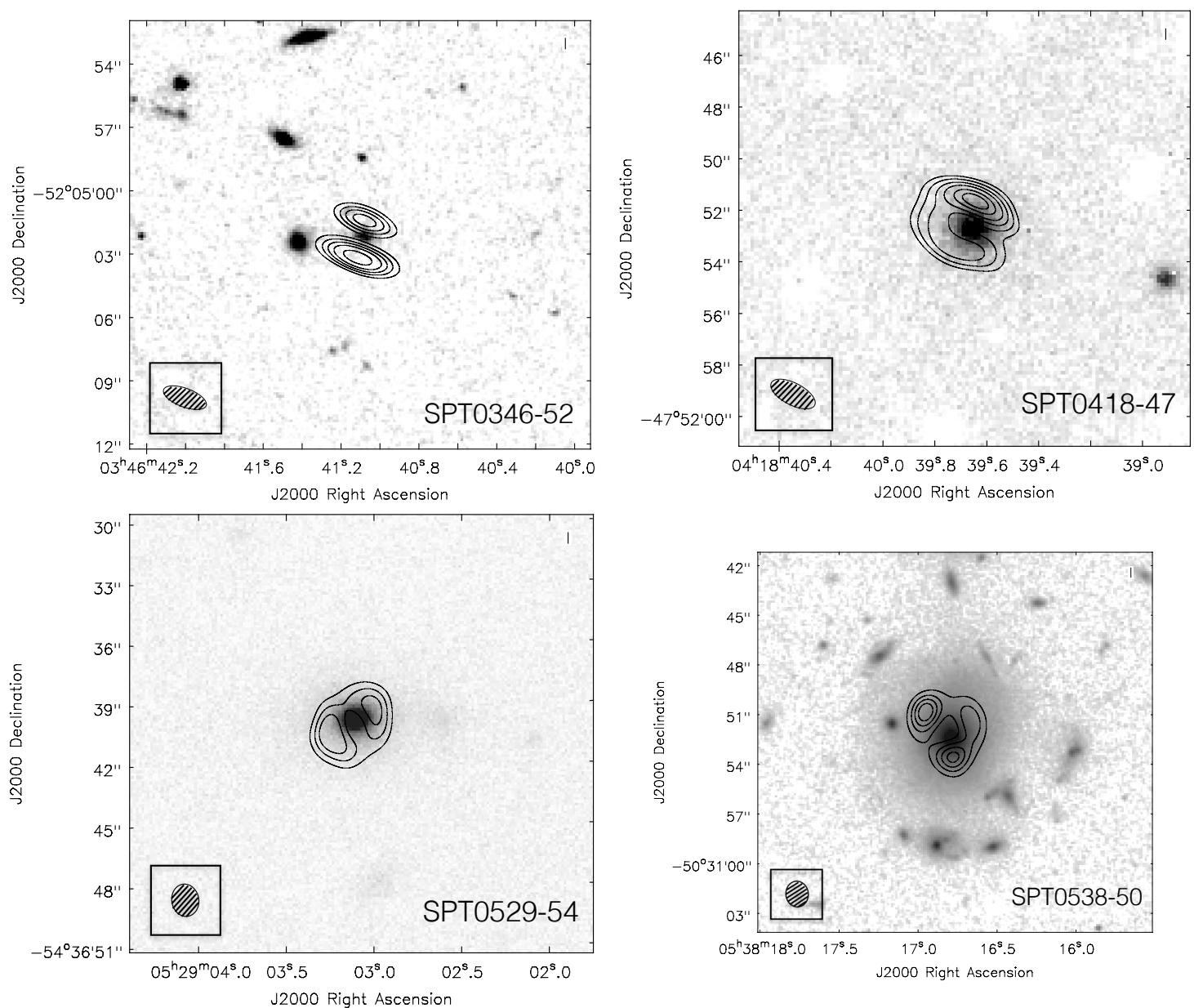

Figure 36: ALMA $350 \mathrm{GHz}$ contours of four SPT-selected lensed DSFGs on top of near-IR images of the lensing galaxy. The four targets shown here are SPT 03456-52 $(z=5.65)$, SPT 0418-47 $(z=4.22)$, SPT 0529-54 $(z=3.36)$, and SPT0538-50 $(z=2.78)$. The figure is reproduced from Hezaveh et al. (2013) with permission from the authors and AAS. 
2013b). SPT0538 has a strong $\mathrm{H}_{2} \mathrm{O}$ detection confirmed through Z-SPEc, and exhibits the highest $L_{\mathrm{H}_{2} \mathrm{O}} / L_{\mathrm{IR}}$ ratio of all high- $z$ DSFGs. With a SFR surface density reaching the levels of local ULIRGs, 70\% of the total star-formation in SPT0538-50 is in the compact region. The estimated molecular gas mass is $M_{\text {gas }} \sim 2 \times 10^{10} \mathrm{M}_{\odot}$ (Aravena et al., 2013), and the galaxy is expected to exhaust its molecular gas supply in about 20 Myr (Bothwell et al., 2013b).

\subsection{Highest redshift DSFGs}

While with the advent of wide-area submm surveys have increased the sample sizes of DSFGs at $z>2$, we are still limited to a handful of SMG-like DSFGs with $S_{850}>5 \mathrm{mJy}$ at $z>5$ (see Table 3). Most of these SMGs at $z>5$ are serendipitous discoveries.

\subsubsection{HDF850.1}

HDF850.1 dates back to the original SCUBA survey of the HDF-N by Hughes et al. (1998). The multi-wavelength counterpart identification has been challenging, despite many attempts (Downes et al., 1999, Dunlop et al., 2004, Wagg et al., 2007; Cowie et al., 2009), highlighting the challenge at identifying and studying properties of extreme DSFGs at very high redshifts. The redshift of HDF850.1 was found to be $z=5.183$, among an over-density of galaxies in the HDF-N field around the same redshift, through a combination of IRAM/PdBI CO(6-5) and CII and VLA CO(2-1) spectral line measurements (Walter et al., 2012). With the redshift determined, HDF850.1 is estimated to be forming stars at the rate of $850 \mathrm{M}_{\odot} \mathrm{yr}^{-1}$. Despite such high star-formation, gas and dust properties of HDF850.1 have been determined to be comparable to normal local star-forming galaxies, including a $L_{C[I I]} / L_{\mathrm{FIR}}$ ratio higher than extreme starbursts and AGN-hosted SMGs. Unfortunately, an infrared/optical counterpart remains undetected and the stellar properties of the galaxy has yet to be constrained.

\subsection{2. $J 1148+5251$}

$\mathrm{J} 1148+5251$ in Table 3 barely meets the canonical $850 \mu \mathrm{m}$ SMG criterion of $S_{850}>5 \mathrm{mJy}$. It was detected as a bright quasar at a redshift of 6.42 in SDSS (Fan et al., 2003). The source is bright in submm wavelengths with a far-infrared luminosity of a HyLIRG with value $\sim 2 \times 10^{13} \mathrm{~L}_{\odot}$ (Beelen et al., 2006) and a SFR in excess of 3000 $\mathrm{M}_{\odot} \mathrm{yr}^{-1}$. It is also gas rich with a total molecular gas mass of $\sim 2.5 \times 10^{10} \mathrm{M}_{\odot}$ distributed over a diameter of 5 $\mathrm{kpc}$ (Walter et al. 2003). Riechers et al. (2009) find that half of the integrated SFR is concentrated over a nuclear region of just $0.75 \mathrm{kpc}$ in radius (Riechers et al. 2009) with an SFR surface density of $1000 \mathrm{M}_{\odot} \mathrm{yr}^{-1} \mathrm{kpc}^{-2}$, close to an Eddington-limited maximal starburst (Walter et al., 2009). J1148+5251 is interesting in that the maximal SFR is spread over a kpc region while in $z \sim 2$ SMGs and local nuclear ULIRG starbursts such high SFR surface density is limited to $100 \mathrm{pc}$ areas.

\subsection{3. $A z T E C-3$}

AzTEC-3 is one of the brightest mm-wave source in the $2 \mathrm{deg} .^{2}$ COSMOS field with $S_{1.1}=11.3 \pm 1.2 \mathrm{mJy}$ (Aretxaga et al. 2011) and $S_{2 \mathrm{~mm}}=3.7 \pm 1.4 \mathrm{mJy}$ (Capak et al. 2011). With a IR luminosity of $1.7 \pm 0.8 \times 10^{13} \mathrm{~L}_{\odot}$, AzTEC-3 is starbursting with a SFR of $>1500 \mathrm{M}_{\odot} \mathrm{yr}^{-1}$. We have discussed this source with respect to its environment in Section 7.1. The molecular gas properties of the galaxy are discussed in Riechers et al. (2010b) where the $\mathrm{CO}(2-1)$, $\mathrm{CO}(5-4)$ and $\mathrm{CO}(6-5)$ observations allow an estimate on the gas mass of $\mathrm{M}_{\mathrm{gas}} \sim 5 \times 10^{10} \mathrm{M}_{\odot}$. The implied SFR is such that this gas content will be consumed in over 30 Myrs (Riechers et al., 2010a). The measured gas and stellar masses are such that the gas mass fraction is $>80 \%$ and between $30 \%$ to $80 \%$ when measured relative to the total baryon content and total dynamical mass, respectively. These fractions are comparable to $z \sim 2$ SMGs. The implications of the measured dust and stellar mass of AzTEC-3 on the formation of dust through supernovae processes are discussed in Dwek et al. (2011).

\subsubsection{HFLS3}

The Herschel-SpIRE-selected $z=6.34$ galaxy HFLS3 was found from a systematic search for $z>4$ SMGs in HerMES SPIRE maps based on the color information from 250 to $500 \mu \mathrm{m}$ (Figure 35). As the SED is redshifted, assuming typical dust temperatures at the level of 30-45 K, one expects the SED for $z>4$ DSFGs to peak at $500 \mu \mathrm{m}$ with a non-detection at $250 \mu \mathrm{m}$. The selection process of such red galaxies, using optimally filtered maps based on a color criterion, is discussed in Dowell et al. (2013). One of the reddest galaxies in this sample is HFLS3 (Riechers 
et al. 2013b) with $L_{\mathrm{FIR}} \sim 2 \times 10^{13}$ and inferred SFR of $2,900 \mathrm{M}_{\odot} \mathrm{yr}^{-1}$. It has been detected in $7 \mathrm{CO}$ lines, $7 \mathrm{H}_{2} \mathrm{O}$ lines, $\mathrm{OH}, \mathrm{OH}^{+}, \mathrm{H}_{2} \mathrm{O}^{+}, \mathrm{NH}_{3},[\mathrm{CI}]$, and [CII] lines in emission or absorption, providing a wealth of information on the conditions related to a starburst during the end of reionization epoch. The dust, molecular, and atomic gas masses of HFLS3 are $10^{9} \mathrm{M}_{\odot}, 10^{11} \mathrm{M}_{\odot}$, and $2 \times 10^{10} \mathrm{M}_{\odot}$, respectively. The gas-to-dust ratio of 80 , the gas mass fraction of $40 \%$ relative to the dynamical mass of $\sim 3 \times 10^{11} \mathrm{M}_{\odot}$, and the gas depletion time scale of $M_{\text {/rmgas }} / \mathrm{SFR} \sim 40 \mathrm{Myr}$ are comparable to $z \sim 2$ SMGs. From [CI], HFLS3 is known to contain an atomic carbon mass of $\sim 4 \times 10^{7} \mathrm{M}_{\odot}$. At the measured SFR, such a high mass of carbon could be assembled through supernovae over a timescale of 10 Myrs. The CO SLED and radiative transfer modeling show the molecular gas mass to have a kinetic temperature of about $150 \mathrm{~K}$ with a gas density around $6,000 \mathrm{~cm}^{-3}$, similar to gas densities in local ULIRGs. More interestingly, the submm spectra show $\mathrm{H}_{2} \mathrm{O}$ and $\mathrm{OH}$ lines with upper level energies $E / k_{B}>300-450 \mathrm{~K}$ and critical densities in excess of $10^{8} \mathrm{~cm}^{-3}$. These $\mathrm{H}_{2} \mathrm{O}$ line intensities and ratios are consistent with radiative pumping by infrared photons of the massive starburst in addition to collisional excitations. This scenario is different from AGN-dominated ULIRGs such as Mrk 231 where the $\mathrm{H}_{2} \mathrm{O}$ line intensities and ratios provide evidence for hard radiation associated with the luminous AGN.

\subsubsection{HLS J0918+5142}

The $z=5.2$ lensed DSFG HLS J0918+5142 was identified from SPIRE maps of galaxy cluster Abell 773 at $z=0.22$, though the DSFG is primarily lensed by a foreground galaxy at $z=0.63$ and not the galaxy cluster (Combes et al. 2012). At submm wavelengths it is magnified by a factor of $9 \pm 2$ (Rawle et al., 2013). The source is made up of multiple velocity components in the $\mathrm{CO}(1-0)$ line with one of the components at $-700 \pm 40 \mathrm{~km} \mathrm{~s}^{-1}$ having an unusually high $L_{[N I I]} / L_{[C I I]}$ ratio of $\sim 0.12$, where a ratio with typical values around 0.05 is seen in another high-Z [NII] detection at $z=4.7$ (Nagao et al., 2012). Rawle et al. (2013) identifies this velocity-resolved component as an ionized, molecular outflow. Another possibility is a less active companion galaxy with a lower density and cooler gas leading to less vigorous star-formation than in other components.

\subsubsection{SPT 0346-52}

The two SPT sources, SPT0243-49 and SPT0346-52, at $z=5.69$ and $z=5.65$ respectively, are the two $z>5$ lensed SMGs from the $1.4 \mathrm{~mm}$-selected SPT DSFGs with ALMA-determined redshifts (Vieira et al., 2013). The lens model of SPT0346-52 is described in Hezaveh et al. (2013) and is found to have a magnification in the submm of 5.6 with an intrinsic $860 \mu \mathrm{m}$ flux density of $23 \mathrm{mJy}$. This puts a intrinsic SFR surface density of $4200 \mathrm{M}_{\odot} \mathrm{yr}^{-1} \mathrm{kpc}^{-2}$, close to or well-above the Eddington limit for starbursts. 


\section{Clustering and Environment}

While most DSFGs at high- $z$ are either galaxies that appear to be in relative isolation, or an ongoing galaxy merger, a number of observations over the last five years have identified some of the most luminous DSFGs at high- $z$ as lying in potential proto-clusters of galaxies. It should be noted that the galaxies that have been identified as being potential proto-cluster members are rare-the average DSFG is not found in these environments. The DSFGs that are described in this subsection are of order the most luminous DSFGs known, and comparable to the most luminous galaxies known in the Universe.

\subsection{Environments of DSFGs}

The first DSFG to be identified as part of a proto-cluster environment was LAB1 at $z=3.09$ (Chapman et al., 2001) where the luminous $850 \mu \mathrm{m}$-detected SMG has diffuse Ly- $\alpha$ emission at a redshift with a known over-density of optical/UV-selected galaxies at $z=3.07-3.11$; the submillimeter map around the proto-cluster revealed a higher density of submm sources than in a blank-field, which led to the first studies of DSFGs' environments.

In 2009, the clustering properties of SMGs were analyzed in more detail by Chapman et al. (2009) who discovered a $z \sim 1.99$ over-density of SMGs in GOODS-N. The over-density of six SMGs and two SFRGs (so eight DSFGs in total) overlaps with an over-density of 22 optically-selected BX/BM galaxies. By comparing the stellar masses and star formation rates of both optically-selected galaxies within and outside of the over-density, Chapman et al. arrive at the conclusion that the over-density's environment is no more substantially evolved than the field. The inferred halo mass of the cluster is relatively low, so the active episode of star formation and implied high merger fraction is unexpected according to simulations.

Another prominent $850 \mu \mathrm{m}$-selected SMG thought to be in a proto-cluster was GN20, discovered in the GOODS North (hence the "GN" in GN20) field (Pope et al., 2005). Serendipitously, Daddi et al. (2009) discovered CO emission lines from the system, and placed the redshift of the SMGs at $z \sim 4$ (the physical characterization of GN20 itself was discussed in $\S 6.3$. The brightest galaxies in the area are GN20 with $S_{850}=20.3 \mathrm{mJy}$ at $z=4.055$ (potentially the brightest unlensed SMG known to date), and GN20.2a and b with a total flux density of $S_{850}=9.9 \mathrm{mJy}$ and at $z=4.051$. The concentration also contains a fourth fainter SMG at a few arcminutes away and at $z=4.0424$. Along with these SMGs, numerous Lyman break galaxies reside within 25" of GN20 (distance separation of $170 \mathrm{kpc}$ ), corresponding to an over-density of $\sim 6 \sigma$ (Daddi et al. 2009) (Fig. 32). The total estimated mass of the proto-cluster is around $\sim 10^{14} \mathrm{M}_{\odot}$. While such a mass is consistent with present-day galaxy groups or a low-mass galaxy cluster, at $z \sim 4$, such a mass corresponds to one of the highest density peaks. The evolutionary path of such a high mass halo at $z \sim 4$ is such that it will easily evolve to a massive cluster today with a total mass in excess of $\sim 5 \times 10^{15} \mathrm{M}_{\odot}$. Thus the DSFGs seen in GN20 are likely the galaxies that will evolve in to brightest cluster galaxies (BCGs).

Capak et al. (2011) examined the COSMOS data set, and found four galaxies at $z \sim 5.3$, all centered around an extreme starburst originally detected with the AzTEC camera at $1.1 \mathrm{~mm}$, COSMOS AzTEC-3 (Younger et al., 2007). The system has an inferred star formation rate of $\sim 1500 \mathrm{M}_{\odot} \mathrm{yr}^{-1}$, and within $2 \mathrm{Mpc}$ of the starburst, there are 11 objects with luminosities greater than $L_{*}$. A similar over-density of galaxies has been seen to be associated with the $z=5.2$ ScuBA SMG, HDF850.1 (Walter et al., 2012). Finding multiple SMGs undergoing a concurrent starburst, such as GN20, is likely to be rare. It will be interesting to see in the near future if currently known highest redshift SMG, HFLS3 (Riechers et al. 2013b), is associated with a proto-cluster or not.

Moving down in redshift to the peak epochs of the SMG redshift distribution at $z \sim 2.5$, Ivison et al. (2013) exploited the relationship between $L_{\mathrm{CO}}^{\prime}$ and the $\mathrm{CO}$ line width to search for luminous galaxy mergers. $L_{\mathrm{CO}}^{\prime}$ and $\mathrm{CO}$ line widths are correlated for unlensed galaxies (Bothwell et al., 2013a), while lensed galaxies show a clear departure from this relation with a flat trend (Harris et al. 2012) (Fig. 37). Searching for for the brightest galaxies in the HATLAS survey that falls on the unlensed portion of the $L_{\mathrm{CO}}^{\prime}$-CO FWHM relationship, Ivison et al. (2013) found a group of four intrinsically luminous galaxies at $z \sim 2.5$ across a $\sim 100 \mathrm{kpc}$ region. A suite of panchromatic data showed that these galaxies are extremely molecular gas rich and have star formation rates between $\sim 600-3500 \mathrm{M}_{\odot} \mathrm{yr}^{-1}$, for the individual components.

Similarly, Fu et al. (2013) identified HXMM01 from HerMES as an extremely bright SMG at $z \sim 2.3$ that resolves into two sources separated by $\sim 3^{\prime \prime}$ (roughly $25 \mathrm{kpc}$ ). The total flux density is roughly $S_{870} \sim 20 \mathrm{mJy}$, and each individual galaxy qualifies as an SMG. The individual stellar masses from the system are each $>10^{11} \mathrm{M}_{\odot}$, while the galaxy is among the brightest $\mathrm{CO}$ emitters known to date. The baryonic gas fraction of the galaxy is $\sim 50 \%$, even 


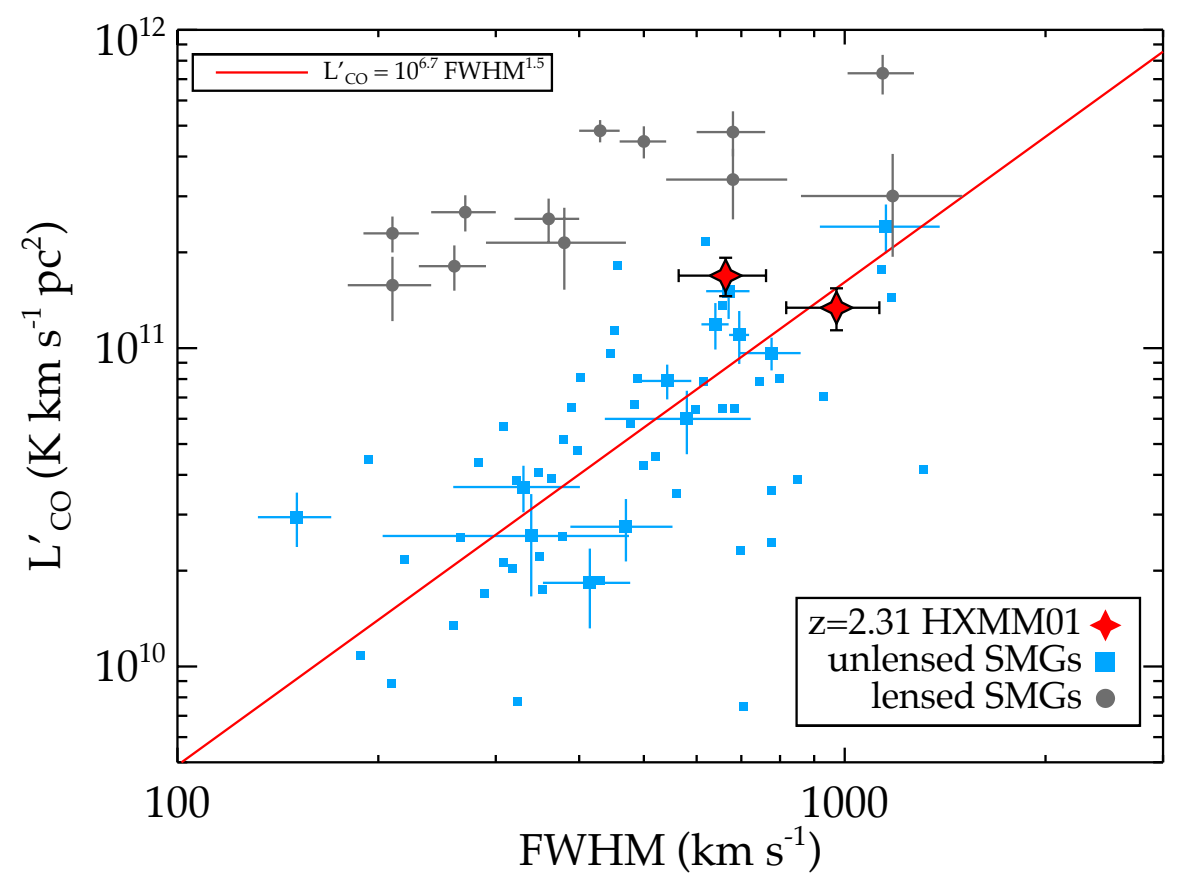

Figure 37: $L_{\mathrm{CO}}^{\prime}$ vs FWHM for $\mathrm{CO}(1-0)$ of lensed and unlensed SMGs. The two red stars show the measurements of the two components of HXMM01 The big blue squares with error bars are unlensed and lensing-corrected SMGs with CO(1-0) measurements (Riechers et al. 2011c, Ivison et al. 2011, Harris et al. 2010, Carilli et al. 2010) and the small blue squares are mostly SMGs with higher-J CO line measurements converted to $\mathrm{CO}(1-0)$ using the mean observed ratios (Bothwell et al. 2013a Carilli \& Walter 2013). The red line shows the best-fit relation for unlensed SMGs from Bothwell et al. (2013a), see also Harris et al. (2012). The grey filled circles with error bars are the GBT CO(1-0) measurements of the brightest lensed SMGs in the H-ATLAS survey (Harris et al. 2012). 

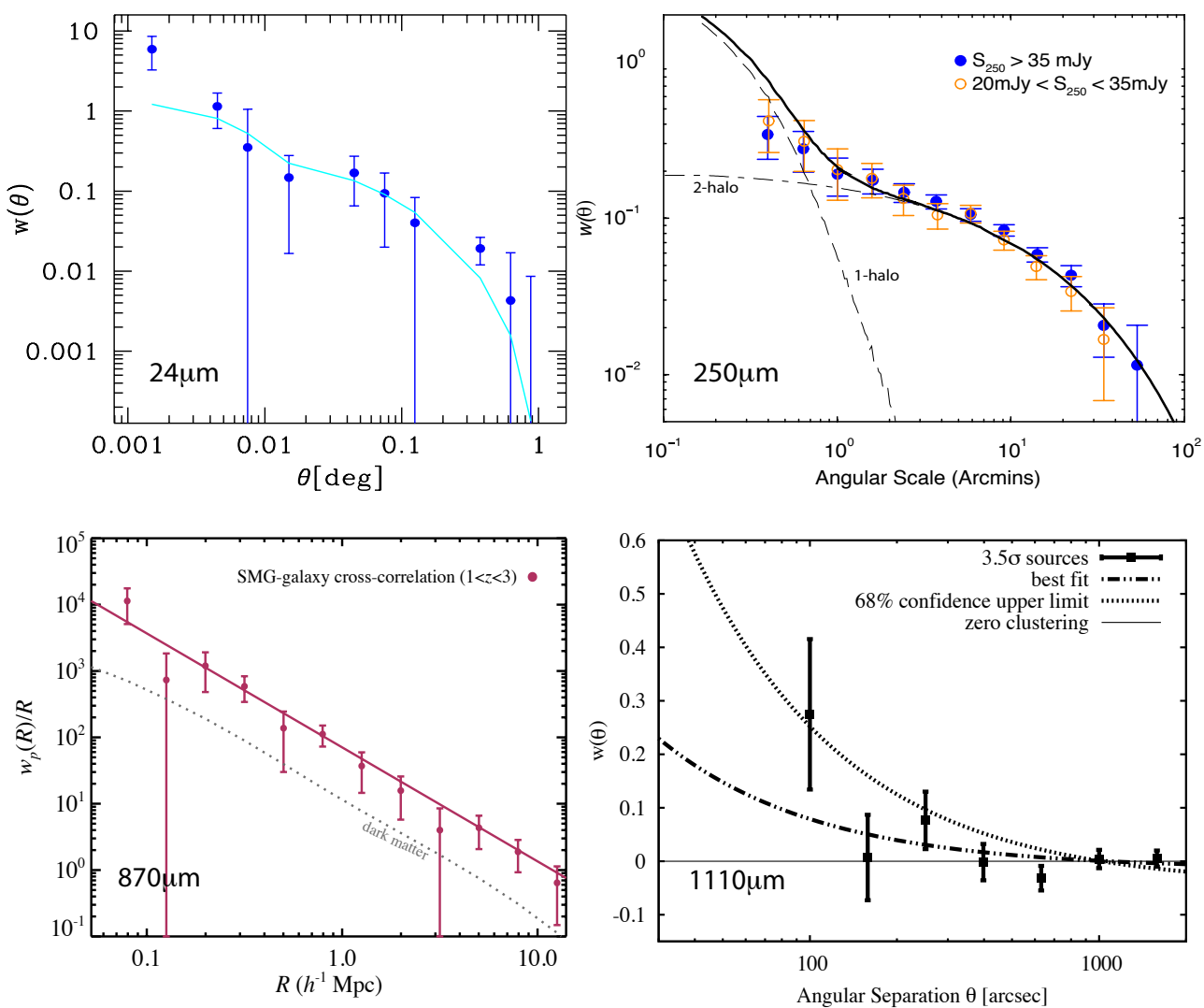

Figure 38: Clustering measurements of DSFGs at a variety of far-IR/submm wavelengths. Clockwise from top-left, we show the clustering measurements reported in the recent literature at $24 \mu \mathrm{m}$ (Magliocchetti et al. 2007) from Spitzer/MIPS, 250 $\mu \mathrm{m}$ (Cooray et al. 2010) with Herschel/SPIRE, $870 \mu \mathrm{m}$ in terms of a cross-correlation with an overlapping lyman-break galaxy distribution in ECDFS (Hickox et al. 2012) with LABOCA, and at $1.1 \mathrm{~mm}$ (Williams et al. 2011) with AzTEC on ASTE. The figure panels are reproduced with permission from the authors of each of the above references and AAS.

after accounting for $\mathrm{CO}-\mathrm{H}_{2}$ conversion factor issues (c.f. $\S 8.6$, making this one of the most gas rich systems at these masses found to date.

\subsection{Clustering of DSFGs}

The clustering of galaxies at high-redshift provides a valuable constraint on their dark matter halo masses and their occupation number, especially in the context of the halo model for large-scale structure galaxy distribution (Cooray \& Sheth, 2002). The halo mass scale of DSFGs is helpful to understand the mass scale at which starbursts are frequently found and can be compared to theoretical and numerical predictions related to efficient gas-cooling in dark matter halos (Dekel et al., 2009a b; Behroozi et al. 2013). The occupation number of DSFGs is useful to establish the satellite fraction of galaxies in the halos that host starbursts. In return the occupation number can provide information related to galaxy merging and concurrent starbursts in dark matter halos. With halo mass and occupation number in hand, one can also infer connections between seemingly different populations of galaxies at high- $z$, as well as provide strong constraints on the theory of dusty galaxy formation (as discussed in $\S 10$ ). In recent years the simple halo model has been improved with conditional luminosity functions (De Bernardis \& Cooray, 2012; Viero et al., 2012, Shang et al., 2012; Xia et al. 2012) and with functions that take into account the mass-dependent duty cycle of DSFGs and the radius-dependent efficiency for halos to convert accreted baryons into stars in dark matter halos (Béthermin et al. 2013). In this subsection, we review the results from clustering measurements of different populations of dusty galaxies. 


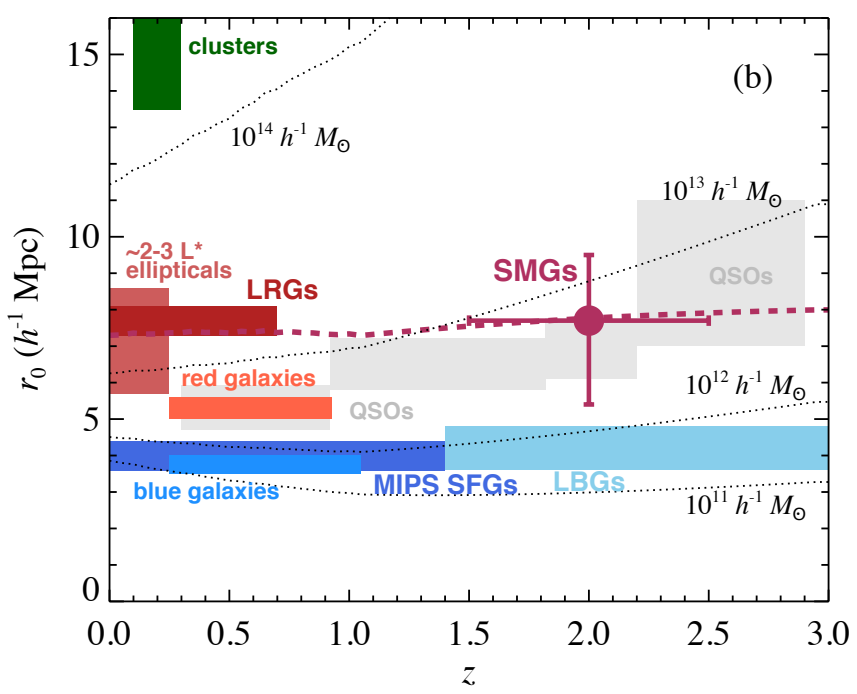

Figure 39: Auto-correlation length $r_{0}$ of DSFGs compared to a variety of galaxy populations over the redshift interval $0<z<3$. These include optically-selected SDSS QSOs at $0<z<3$ (Myers et al. 2006, Ross et al. 2009) Lyman-break galaxies (Adelberger et al. 2005), MIPS 24 $\mu$ m-selected star-forming galaxies at $0<z<1.4$ (Gilli et al. 2007), AGES and DEEP2 red and blue galaxies at $0.25<z<1$ from the AGES (Hickox et al. 2009. Coil et al. 2008) SDSS-selected luminous red galaxies (LRGs) at $0<z<0.7$ (Wake et al. 2008), and optically-selected galaxy clusters at $0.1<z<0.3$ (Estrada et al. 2009). The figure also shows the $r_{0}$ for low-redshift galaxies with r-band luminosities in the range 1.5 to $3.5 \mathrm{~L}_{\star}$, derived from the luminosity dependence of clustering (Zehavi et al. 2011). Dotted lines show $r_{0}$ versus redshift for dark matter haloes of different masses. The thick solid line shows the expected evolution in $r_{0}$ for the halo mass estimated by Hickox et al.(2012) as the best-fit halo mass for $S_{870}>$ few mJy SMGs at $z=2$. This evolution suggests that the SMGs are likely to evolve into luminous elliptical galaxies in the local Universe. The figure is reproduced with permission from Hickox et al. (2012). 
The traditional approach in clustering studies is to measure the correlation length of a population of galaxies and compare it to cosmological structure formation simulations in order to infer the halo mass of the observed galaxies. Blain et al. (2004b) provided the first measure of the clustering length of $850 \mu \mathrm{m}$ submillimeter galaxies with $S_{850}>$ $5 \mathrm{mJy}$, and provided the first evidence that SMGs reside in extremely large halo masses, with masses $\sim 10^{13} \mathrm{M}_{\odot}$ (with relatively large error bars). The measured clustering length from the Blain et al. (2004b) study was $\sim 6.9 \pm 2.1 h^{-1}$ Mpc. While the uncertainty was large, this initial measurement provided critical information that SMGs resided in the extreme tail of the halo mass function, and constrained dusty galaxy formation scenarios accordingly. Weiß et al. (2009b) performed an $870 \mu \mathrm{m}$ survey of the Extended Chandra Deep Field South (ECDFS) with the LABOCA camera on the APEX telescope, and found a spatial correlation length of $\sim 13 \pm 6 h^{-1} \mathrm{Mpc}$. While selected at a slightly longer wavelength (1.1 mm), Williams et al. (2011) presented a clustering measurement of AzTEC sources in the COSMOS field, and provided one of the first statistically significant measurements of SMGs at high- $z$. Williams et al. (2011) find upper limits on the correlation length of $\sim 6-8 h^{-1} \mathrm{Mpc}$ for $1.1 \mathrm{~mm}$ sources with $S_{1.1} \sim 3.7 \mathrm{mJy}$ (Fig. 38), which translates roughly to the $850 \mu \mathrm{m}$ selection thresholds of the earlier works.

To date, the tightest constraint on the clustering amplitude of SMGs has been performed by Hickox et al. (2012), who re-analyzed the Weiß et al. (2009a) LABOCA survey of the ECDFS, though added new redshift constraints (Wardlow et al., 2011), as well as the clustering analysis methodology of Myers et al. (2009). In this approach, instead of a direct auto-correlation of the DSFG detected at $870 \mu \mathrm{m}$, which has a low signal-to-noise ratio due to overall low source counts in even deep ground-based fields such as ECDFS in the LESS survey (Weiß et al., 2009b), the authors pursue a cross-correlation of the $870 \mu \mathrm{m}$ galaxies with optically-selected sample of galaxies in the same field with known redshifts and known clustering properties. By fixing the galaxy clustering properties, the authors can in return infer the clustering properties of the SMG sample. They infer an auto-correlation length of $7.7 \pm 2.3$ $\mathrm{h}^{-1} \mathrm{Mpc}$ at $870 \mu \mathrm{m}$ and a characteristic halo mass of $\sim 6 \times 10^{12} h^{-1} \mathrm{M}_{\odot}$ for SMGs (Hickox et al., 2012). Based on the evolution of dark matter haloes derived from simulations, Hickox et al. (2012) show that the present-day descendants of these bright $S_{870} \sim$ few mJy SMGs are typically massive elliptical galaxies equivalent to $L \sim 2$ to $3 \mathrm{~L}_{\star}$ luminousred galaxies studied in SDSS and are located in small to moderate-size galaxy groups with halo masses $\sim 10^{13} \mathrm{M}_{\odot}$ (Fig. 39).

Interestingly, Dust Obscured Galaxies (DOGs) selected for optical faintness and $24 \mu \mathrm{m}$ flux density exhibit similar clustering amplitudes as SMGs, though the amplitude is of course flux dependent. Brodwin et al. (2008) found that DOGs selected above a $24 \mu \mathrm{m}$ flux density $S_{24}>0.3 \mathrm{mJy}$ have $r_{0}=7.4 h^{-1} \mathrm{Mpc}$, quite similar to the Hickox et al. (2012) autocorrelation length of SMGs of $r_{0}=7.7 h^{-1} \mathrm{Mpc}$. The inferred halo masses for luminous SMGs and DOGs are not terribly dissimilar from constraints on optically-selected quasars (Croom et al., 2005; White et al., 2012).

Similarly, Cooray et al. (2010) utilized data from the HerMES survey to find that galaxies selected at $250 \mu \mathrm{m}$ above $30 \mathrm{mJy}$ have halo masses in the same range as observed SMGs $\left(\sim 5 \pm 4 \times 10^{12} \mathrm{M}_{\odot}\right)$ with data from the LockmanSWIRE field with close to $10^{4} 250 \mu \mathrm{m}$ galaxies. Cooray et al. (2010) also showed the excess clustering at arcminute angular scales, resulting from the 1-halo clustering term. This non-linear clustering, under the halo model ansatz, can be described as due to the correlation of multiple DSFGs in dark matter halos. The halo model fits to the clustering (Fig. 38) shows that roughly (14 \pm 8$) \%$ of DSFGs with $S_{250}>30 \mathrm{mJy}$ appear as satellites in more massive halos than $\sim 10^{12} \mathrm{M}_{\odot}$. We note that slightly different results related to galaxy clustering at SPIRE wavelengths is reported in Maddox et al. (2010). At low-redshifts with $z<0.5$, the clustering of DSFGs are similar to blue, star-forming galaxies with a correlation length $\sim 4.6 \pm 0.5 \mathrm{Mpc}$ (van Kampen et al., 2012, Guo et al., 2011) and are less-clustered than the dark matter distribution with a linear bias factor less than one $(b=0.7 \pm 0.1)$.

\subsection{Clustering of Faint, Unresolved DSFGs through the CIB Anisotropy Power Spectrum}

While the spatial distribution of individually detected galaxy counts provides information on the DSFG clustering of resolved sources, maps at far-IR/submm wavelengths can also be used to probe the clustering properties of the unresolved, fainter galaxy population. This is similar in spirit to $P(D)$ statistics that probe the faint source counts below the detection level by studying the histogram of the pixel counts associated with confusion noise.

In the case of clustering, information related to the spatial distribution of the fainter sources below the confusion noise comes from clustering studies related to the unresolved fluctuations. In particular, the angular power spectrum of the confusion noise, or the cosmic infrared background (CIB), captures the underlying spatial distribution of the fainter sources. While the fainter galaxies are individually undetected, due to gravitational growth and evolution in the large-scale structure these galaxies are expected to be clustered (Cooray et al., 2010, Hickox et al., 2012). In 

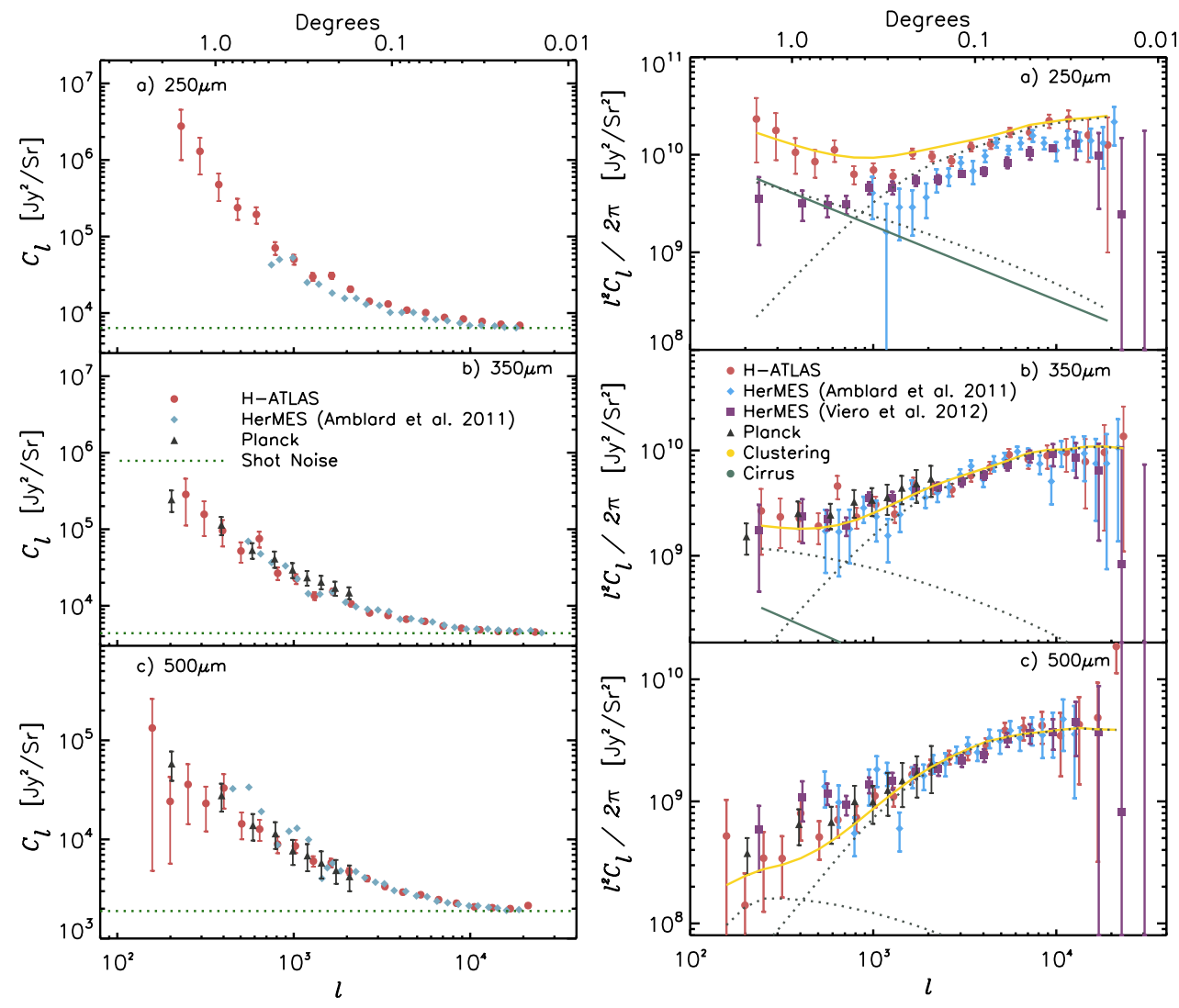

Figure 40: Angular power spectra of the CIB at $250 \mu \mathrm{m}$ (top), $350 \mu \mathrm{m}$ (middle) and $500 \mu \mathrm{m}$ (bottom). In the left panels, the power spectra are plotted as $C_{l}$ prior to the removal of the shot-noise term. In the right panels we show the power spectra as $l^{2} C_{l} / 2 \pi$ after removing the shot-noise level at each of the wave bands. The curves on the right panels show the halo models for CIB power spectrum separated into 1-halo, 2-halo, and the total signal. The solid line that scales roughly as $l^{2} C_{l} \sim l^{-0.9}$ is the best-fit Galactic cirrus fluctuation power spectrum. It is higher at $250 \mu \mathrm{m}$ relative to the amplitude at $500 \mu \mathrm{m}$ due to colder dust temperature of the Galactic ISM. The plotted measurements are from Herschel-ATLAS (Thacker et al. 2013), HerMES (Amblard et al. 2011. Viero et al. 2012 2013a), and Planck (Planck Collaboration et al. 2013b), with latter including the most recent flux calibration of the Planck/HFI channels. Within the statistical errors, these power spectra measured over multiple fields and with two different instruments agree with each other. The figure also highlights the difference between Herschel-SpIRE resolution at 18 to 36 arcseconds vs. Planck/HFI resolution at 4.5 arcminutes and above: SPIRE spectra are able to probe more accurately the 1-halo term of CIB power spectra. This figure is reproduced from Thacker et al. (2013) with permission from the authors and AAS. 
the ansatz of the halo model (Cooray \& Sheth, 2002) the faint galaxy clustering captures the statistics on how they occupy the dark matter halos in the universe. The resulting anisotropies of the CIB are then a reflection of the spatial clustering of dark matter halos that are hosted by faint DSFGs and the total CIB intensity produced by those galaxies. In practice, these CIB anisotropies, are best studied with the angular power spectrum of the background pixel intensity with or without an accounting of the bright sources that are individually detected in the maps.

Early attempts to measure the angular power spectrum of the CIB resulted in low signal-to-noise ratio measurements at $160 \mu \mathrm{m}$ with Spitzer/MIPS (Lagache et al., 2007) and at 250, 350 and 500 $\mu \mathrm{m}$ with BLAST (Viero et al., 2009). The wide area maps with Herschel-SpIRE have allowed CIB power spectrum studies to be extended to larger angular scales with improved signal-to-noise ratio measurements, while Planck/HFI have allowed these power spectrum studies to be extended to longer mm-wave wavelengths probing clustering of the CIB component arising from highest redshifts. The first clear detection of the CIB power spectrum over the angular scales between 30 arcseconds and 30 arcminute was reported in Amblard et al. (2011). Since then, additional measurements of the CIB power spectrum have come from Planck (Planck Collaboration et al., 2013b) and with additional SPIRE maps from HerMES (Viero et al., 2012) and Herschel-ATLAS (Thacker et al., 2013) (see Figure 40).

With HerMES data, Viero et al. (2012) have also presented the cross-correlations between SPIRE wavelengths, providing additional information on the faint source spatial and redshift distributions. At wavelengths corresponding to Herschel-SPIRE, the CIB power spectrum at degree angular scales, especially at $250 \mu \mathrm{m}$, is contaminated by the Galactic cirrus. The spatial resolution of SPIRE, say relative to Planck/HFI, is such that one can probe the clustered fluctuations down to 30 arcsecond angular scales. At those small non-linear scales, the clustering power spectrum is dominated by the 1-halo term associated with correlations between bright central galaxies and fainter star-forming galaxies that appear as satellites in the same dark matter halos. The halo model interpretation of the first measurements of the HerMES spectra suggest that the halo mass scale for peak star-formation activity is $\log M_{\text {peak }} / M_{\odot} \sim 13.9 \pm 0.6$ and the minimum halo mass to host dusty galaxies is $\log M_{\min } / M_{\odot} \sim 10.8 \pm 0.6$ Amblard et al. (2011). These were based on earlier predictions related to the expected Herschel and Planck CIB fluctuations Amblard \& Cooray (2007). Recent modeling, involving conditional luminosity functions (Cooray, 2006), have now improved these early conclusions (De Bernardis \& Cooray, 2012; Viero et al., 2012; Shang et al., 2012; Xia et al., 2012). The most recent models of CIB anisotropy spectra find that the effective mass scale DSFGs are at the level of $\log M_{\text {eff }} / M_{\odot} \sim 12$, consistent with the mass scale of dark matter halos where the star-formation efficiency is maximal (Viero et al., 2012, Planck Collaboration et al., 2013b).

Moving beyond statistics related to the faint DSFGs, the CIB angular power spectrum, in principle, captures the spatial distribution of the background intensity, regardless of whether the emission is from individual point sources or from smoothly varying diffuse sources, such as intra-cluster and intra-group dust. Thus the angular power spectrum is expected to be a sensitive probe of the total dust content in the Universe. The existing estimates of the dust abundance from direct emission measurements make use of the submm luminosity (Dunne et al., 2003) or dust mass (Dunne et al. 2011 functions. However, such functions are generally based out of extrapolations to fainter fluxes of the measured bright DSFG counts and the faint-end extrapolation could easily have a systematic bias. The CIB anisotropy power spectrum should capture the integrated emission from all DSFGs, including faint sources at the flux density scale that dominates the confusion noise. At shorter UV to optical wavelengths estimates of the cosmic dust abundance rely on the extinction of optical light, especially with measurements that combine magnification and extinction of quasars behind samples of foreground galaxies Ménard et al. (2010); Ménard \& Fukugita (2012). The indirect dust abundance estimates from extinction at UV and optical wavelengths should in principle be consistent with direct dust abundance measurements in the far-infrared and submm wavelengths. In Thacker et al. (2013) this comparison was made by making use of the CIB anisotropy power spectrum as a way to estimate the total dust abundance responsible for CIB fluctuations, $\Omega_{\text {dust }}(z)$, the cosmic abundance of dust relative to the critical cosmological density, as a function of redshift. Integrating over the dusty galaxy population responsible for the background anisotropies, Thacker et al. (2013) find $\Omega_{\text {dust }}=10^{-6}$ and $8 \times 10^{-6}$ in the redshift range $z \sim 0-3$. This dust abundance is consistent with estimates of the dust content in the Universe using quasar reddening and magnification measurements in the SDSS (Fig. 41 .

\subsection{Cosmic Magnification of Submm Sources}

In addition to changes to the number counts, gravitational lensing of background sources by foreground largescale structure also results in an angular cross-correlation between the background lensed source population and the foreground mass distribution. For a background population with steep number counts like DSFGs at far-IR and submm 


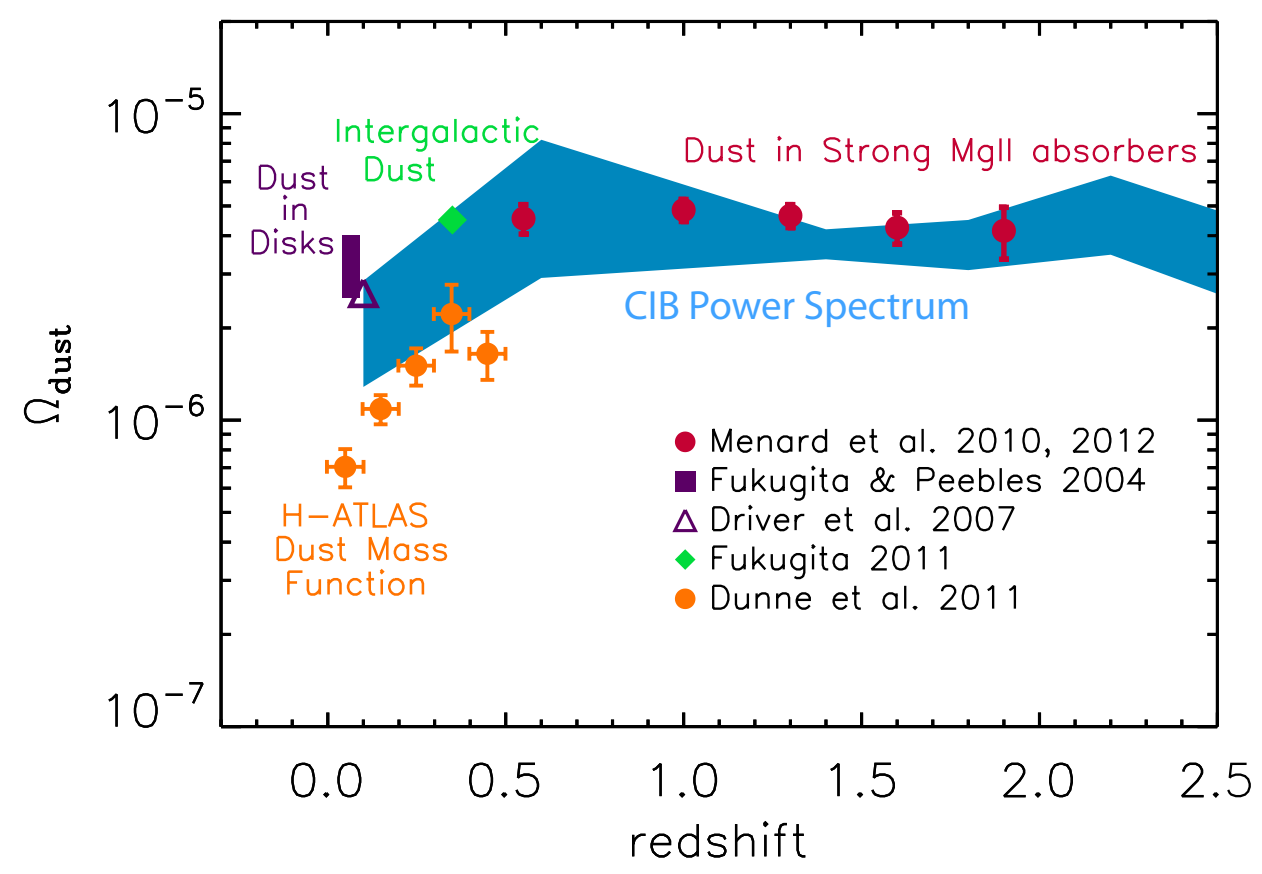

Figure 41: Cosmic abundance of dust, relative to cosmological critical density, $\Omega_{\text {dust }}$, against redshift as determined from the CIB power spectrum (Thacker et al. 2013) compared to measurements in the literature using the low-redshift dust mass function (Dunne et al. 2011), optical extinction of SDSS (Ménard et al. 2010. Ménard \& Fukugita 2012 |Fukugita 2011. Fukugita \& Peebles 2004) and 2DF (Driver et al. 2007) galaxies and quasars. This figure is reproduced from Thacker et al. (2013) with permission from the authors and AAS.

wavelengths, the resulting effect is such that near the foreground mass concentrations, where the magnification is expected to be high, there will be an overall density enhancement of DSFGs. Near low density environments, one does not expect to see an increase in the magnified DSFG counts. In terms of the large-scale structure spatial distribution this then results in a correlation between a tracer field of the foreground mass, such as foreground galaxies, and the background DSFGs. And this cross-correlation exists even if the tracer field in the foreground has no overlap in the redshift distribution with the background sources. To separate intrinsic clustering from this magnification effect, the magnification-induced cross-correlation is best measured with samples of foreground galaxies and background DSFGs that do not overlap in redshift. When combined with the number counts, such a cross-correlation study can provide constraints on cosmological parameters and galaxy bias, a key ingredient in galaxy formation and evolution models Cooray \& Sheth (2002); Jain (2002).

Previous attempts at measuring the lensing-induced cross-correlation between foreground optical galaxies and background submm sources resulted in statistically insignificant results. Almaini et al. (2005) measured the crosscorrelation between 39 submm sources detected by SCUBA and optical sources at lower redshifts with a median of about 0.5 and found some marginal evidence for a cross-correlation due to magnification. This significance was further lowered in a similar study by Blake et al. (2006). Using DSFGs detected in the early Herschel-SPIRE data of HerMES Wang et al. (2011b) found a statistically significant evidence for cosmic magnification over the angular scales of 1 to 50 arcminutes by cross-correlating DSFG samples selected to be at high redshift based on SPIRE colors against a sample of SDSS galaxies with known redshifts overlapping in the same sky area.

A separate study on cosmic magnification was presented by Hildebrandt et al. (2013). Here, instead of crosscorrelating background SPIRE sources against foreground galaxies, the authors selected a sample of low-redshift DSFGs at $250 \mu \mathrm{m}$. They were cross-correlated against a sample of $z \sim 3-5$ LBGs. The resulting cross-correlation function was interpreted as due to cosmic magnification, i.e. lensing of LBGs by dark matter halos of low-redshift DSFGs, and dust extinction. They measure the typical dark matter halo mass of bright $250 \mu \mathrm{m}$-selected DSFGs to be about $\log M_{\text {halo }} / M_{\odot}=13.2 \pm 0.08$ with a dust mass of about $6 \times 10^{-5} \times M_{\text {halo }}$ in these dark matter halos. These 

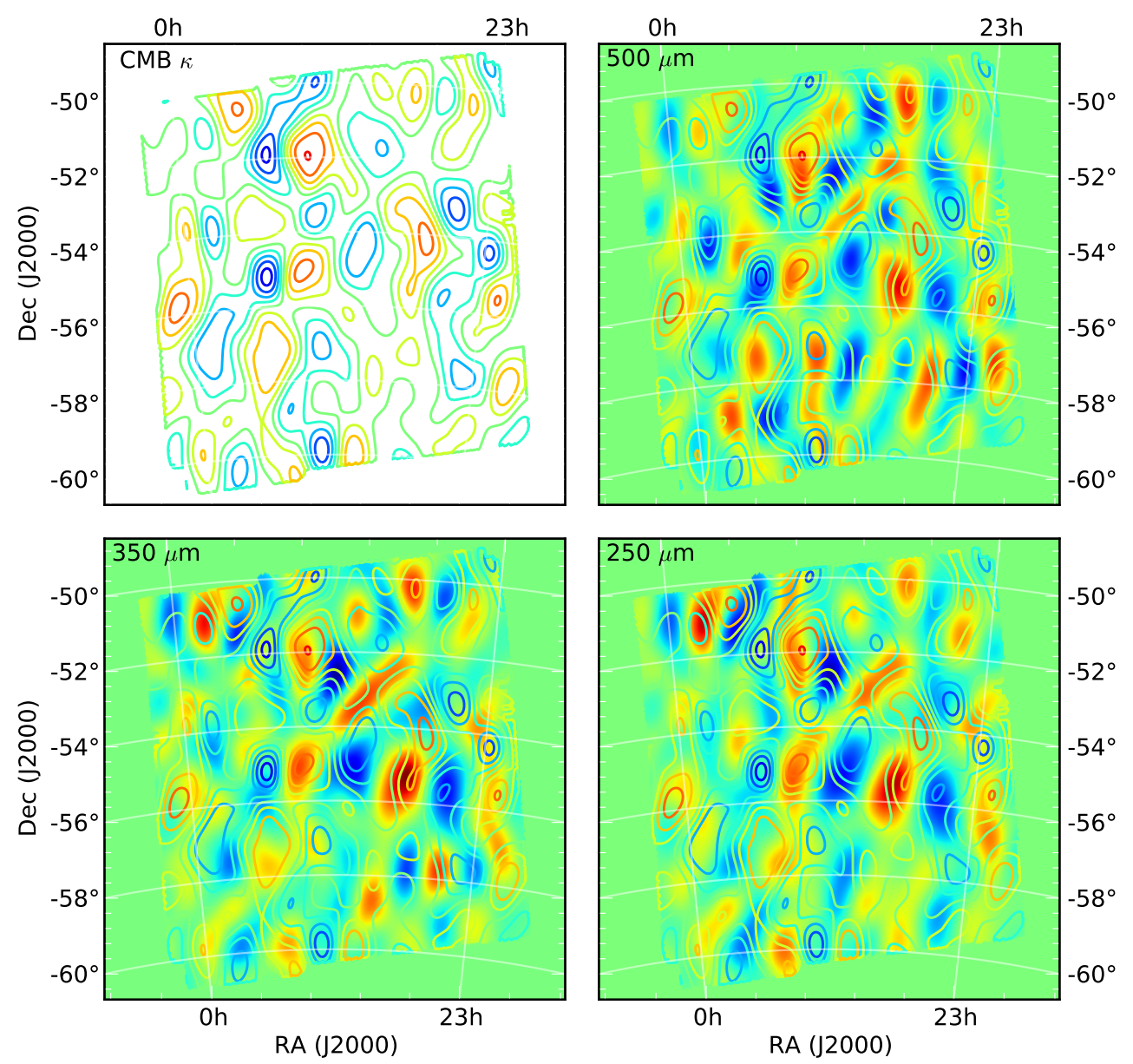

Figure 42: The South Pole Telescope (SPT) survey's lensing mass map of the CMB in its deepest 100 sq. degree area (top left) overlaid on DSFG/CIB maps from Herschel-SPIRE at $500 \mu \mathrm{m}$ (top right), $350 \mu \mathrm{m}$ (bottom left), and $250 \mu \mathrm{m}$ (bottom right). The color scale shows the CIB intensity, with red/blue showing the increased/decreased galaxy intensity, while contours represent the CMB lensing mass map. The maps and overlays show a strong cross-correlation of the two even by eye. The figure is reproduced from Holder et al. (2013) with permission from the authors and AAS.

measurements connect $250 \mu \mathrm{m}$-bright DSFGs at $z \sim 0.5$ to 1 to be hosted in massive group-sized dark matter halos instead of Milky-way or late-type like halos with total dark matter masses of around $10^{12} \mathrm{M}_{\odot}$.

\subsection{DSFGs as a tracer of the CMB lensing potential}

Gravitational lensing of the cosmic microwave background (CMB) by large-scale structure dark matter distribution has now been detected with arcminute-scale CMB experiments, such as the South Pole Telescope (van Engelen et al., 2012) and the Atacama Cosmology Telescope (Das et al., 2013) and with Planck (Planck Collaboration et al. 2013c). With the background source at the last scattering surface at a redshift of 1100, CMB is mostly lensed by large-scale structure at redshifts of $z \sim 2-3$ (Cooray \& Hu, 2000). The CIB and DSFGs that make up the background are ideal tracers of the CMB lensing potential, as was first proposed by Song et al. (2003). The cross-correlation between DSFGs and the mass map responsible for CMB lensing has now been detected with SPT (Holder et al., 2013), with a map of Herschel/SPIRE for DSFGs (Fig. 42), and separately with Planck, using Planck/HFI CIB maps (Planck Collaboration et al. 2013a). A cross-correlation of the Herschel/SPIRE map and SPT polarization maps has also been used to detect the arcminute-scale lensing signal in the CMB B-modes of polarization (Hanson et al. 2013). 


\section{Molecular Gas and Star Formation}

This section reviews the status of both observations and theory of the star-forming molecular $\left(\mathrm{H}_{2}\right)$ gas in highredshift DSFGs. When necessary, we provide some background into local observations to serve as a reference point. Other reviews in the last decade in this field can be found in Solomon \& Vanden Bout (2005), Omont (2007) and Carilli \& Walter (2013).

\subsection{Basic Definitions}

The amount of molecular line emission observed from a galaxy is typically expressed as the line luminosity. Here, we will take the example of $\mathrm{CO}$ as the emitting molecular line. $\mathrm{CO}$ could, in principle, be swapped out for other molecular species. The line luminosities are defined as either $L_{\mathrm{CO}}$, or $L_{\mathrm{CO}}^{\prime}$. These are (Solomon \& Vanden Bout, 2005):

$$
\frac{L_{\mathrm{CO}}}{\left[\mathrm{L}_{\odot}\right]}=1.04 \times 10^{-3} \times \frac{S_{\mathrm{CO}} \Delta v}{\left[\mathrm{Jy} \mathrm{km} \mathrm{s}^{-1}\right]} \times \frac{v_{\mathrm{rest}}(1+z)^{-1}}{[\mathrm{GHz}]} \frac{D_{\mathrm{L}}^{2}}{\left[\mathrm{Mpc}^{2}\right]}
$$

$S_{\mathrm{CO}} \Delta v$ is the velocity integrated CO flux (Jy $-\mathrm{km} \mathrm{s}^{-1}$ ), $v_{\text {rest }}$ is the rest frequency in $\mathrm{GHz}, D_{\mathrm{L}}$ is the luminosity distance in $\mathrm{Mpc}$, and $L_{\mathrm{CO}}$ has units of $\mathrm{L}_{\odot} . L_{\mathrm{CO}}$ is a measure of the total energy output from the $\mathrm{CO}$ line. Alternatively, the areal-integrated $\mathrm{CO}$ intensity $L_{\mathrm{CO}}^{\prime}$ is defined as:

$$
\frac{L_{\mathrm{CO}}^{\prime}}{\left[\mathrm{K} \mathrm{km} \mathrm{s}^{-1} \mathrm{pc}^{2}\right]}=3.25 \times 10^{7} \times \frac{S_{\mathrm{CO}} \Delta v}{\left[\mathrm{Jy} \mathrm{km} \mathrm{s}^{-1}\right]} \times \frac{D_{L}^{2}}{(1+z)^{3} v_{\mathrm{obs}}^{2}}\left[\frac{\mathrm{GHz}^{2}}{\mathrm{Mpc}^{2}}\right]
$$

where the pre-factor $3.25 \times 10^{7}$ is simply $c^{2} / 2 k$ (scaled by powers of 10 to make the rest of the units comply), and $k$ is Boltzmann's constant. $L_{\mathrm{CO}}^{\prime}$ is typically used to convert from a $\mathrm{CO}$ luminosity to an $\mathrm{H}_{2}$ gas mass (see the discussion in $\S 8.2$.

Exciting a molecular line is done through a combination of collisional and radiative excitation. When lines are optically thick, line photon trapping can enhance molecular excitation. A convenient metric for thinking about the conditions necessary to drive the excitation of a given molecular line is the critical density. This is the density at which the Einstein $A$ coefficients for spontaneous de-excitation out of a level is equivalent to the collisional excitation rate into a level: $n_{\text {crit }}=A / \gamma$. A summary of critical densities for a number of commonly observed molecular and atomic line transitions is given in Carilli \& Walter (2013) for $T=100 \mathrm{~K}$. Oftentimes it is assumed that if a line is observed, the densities present in the emitting gas are above the critical density for the line excitation. However, as pointed out by Evans (1999), this is too simplistic of a picture. In reality, a combination of collisional excitation and line pumping of excitation levels can be a significant enough effect that the effective density $\left(n_{\text {eff }} \approx n_{\text {crit }} / \tau_{\text {line }}\right)$ for excitation can be much lower than the critical density. The effective densities to produce a $1 \mathrm{~K}$ line for a variety of molecules and transitions are given in Evans (1999) and Reiter et al. (2011).

\subsection{Deriving $\mathrm{H}_{2}$ Gas Masses from High-Redshift Galaxies}

As one of the principle uncertainties in deriving molecular gas properties in galaxies at all redshifts is converting from the observable, carbon monoxide emission lines (hereafter, $\mathrm{CO}$ ), to the physical quantity of interest, molecular hydrogen $\left(\mathrm{H}_{2}\right)$ gas mass, we briefly review what is known about the infamous $\mathrm{CO}-\mathrm{H}_{2}$ conversion factor. We note that an excellent in depth review of the topic has recently been written by Bolatto et al. (2013).

The $\mathrm{CO}-\mathrm{H}_{2}$ conversion factor is alternatively monikered $X_{\mathrm{CO}}$ (or the "X-factor"), as well as $\alpha_{\mathrm{CO}}$. Formally, the $X_{\mathrm{CO}}$ relates the velocity-integrated $\mathrm{CO}$ intensity $\left(W_{\mathrm{CO}}\right)$ in $\mathrm{K} \mathrm{km} \mathrm{s}^{-1}$ to the gas column density via $X_{\mathrm{CO}}=N_{\mathrm{H} 2} / W_{\mathrm{CO}}$, while $\alpha_{\mathrm{CO}}$ converts from $\mathrm{CO}$ line luminosity to the total gas mass: $\alpha_{\mathrm{CO}}=M_{\mathrm{H} 2} / L_{\mathrm{CO}}^{\prime}$. The two are linearly related:

$$
\frac{X_{\mathrm{CO}}}{\left[\mathrm{cm}^{-2} / \mathrm{K} \mathrm{km} \mathrm{s}^{-1}\right]}=6.3 \times 10^{19} \times \frac{\alpha_{\mathrm{CO}}}{\left[\mathrm{M}_{\odot} \mathrm{pc}^{-2}\left(\mathrm{~K} \mathrm{~km} \mathrm{~s}^{-1}\right)^{-1}\right]}
$$

We note that this relationship does not include the contribution of Helium, and that a scale factor of $\sim 4.65 \times 10^{19}$ between $X_{\mathrm{CO}}$ and $\alpha_{\mathrm{CO}}$ is appropriate if including the contribution of Helium in the molecular gas mass. Within the 


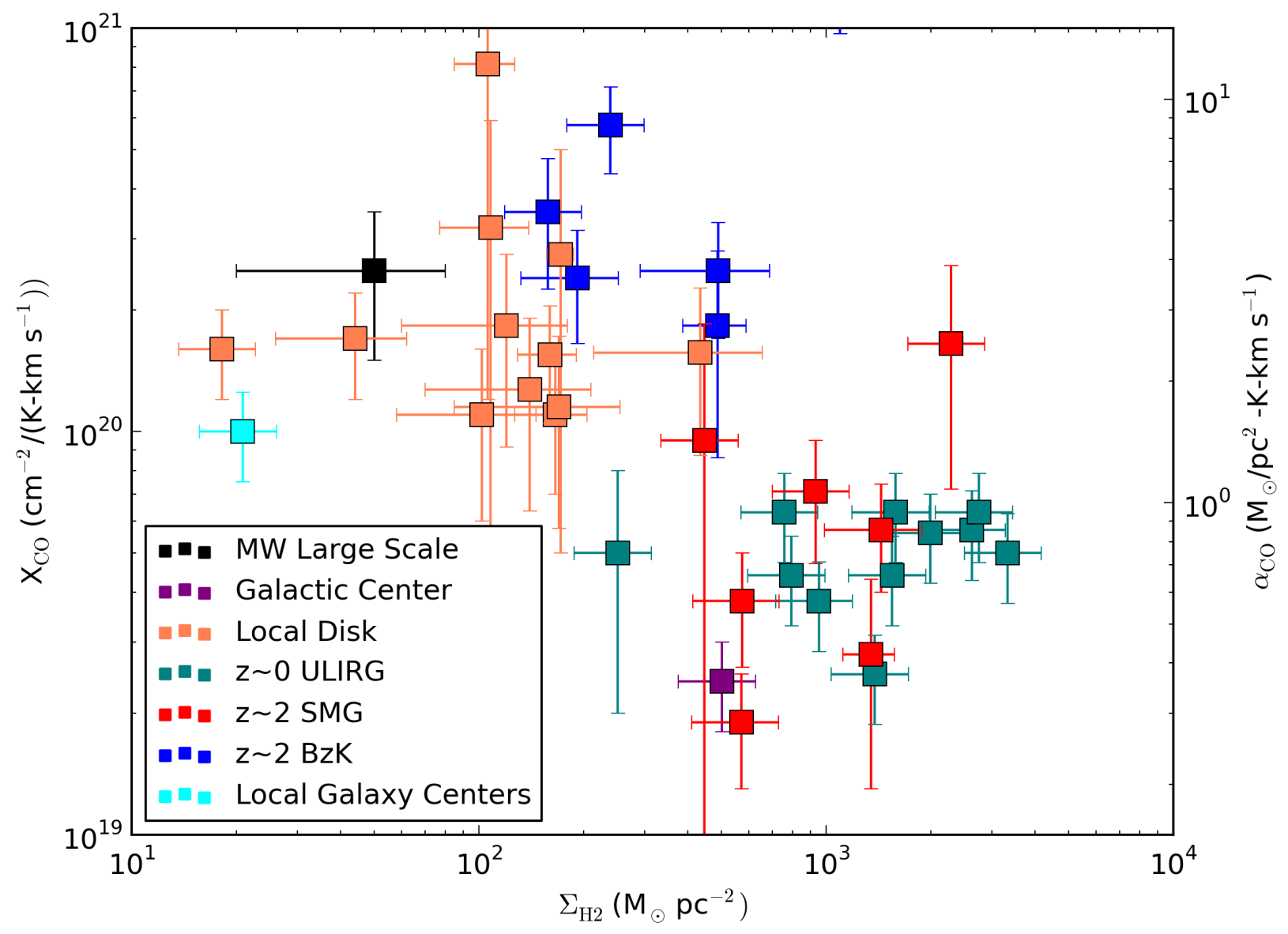

Figure 43: Observed $X_{\mathrm{CO}}$ determinations vs. galaxy surface density for the Milky Way, local galaxy disks, local galaxy disk nuclei, $z \sim 0$ ULIRGs, and $z \sim 2$ SMGs and $B z K$ galaxies. A clear inverse relationship is seen between $X_{\mathrm{CO}}$ and $\Sigma_{\mathrm{H} 2}$. Error bars are taken from uncertainties published in original papers; when no uncertainties are available, an error of 0.3 dex is assumed. Data are taken from Oka et al. (1998 MW Galactic Center), Downes \& Solomon (1998)(ULIRGs), Weiß et al. (2001); Bolatto et al. (2008); Sandstrom et al. (2013); (Donovan Meyer et al. (2012a.|2013)(Local Disks), Magdis et al. (2011); Hodge et al. (2012); Magnelli et al. (2012); Ivison et al. (2013); Fu et al. (2013)(SMGs) and Daddi et al. (2010a); Magdis et al.(2011); Magnelli et al. (2012) (BzK galaxies). The range of Milky Way values is a typical observed range of conversion factors and surface densities for Galactic clouds. We include a factor 1.36 in converting from $X_{\mathrm{CO}}$ to $\alpha_{\mathrm{CO}}$. 
Milky Way and Local Group (aside from the Small Magallenic Cloud [SMC]), the conversion factor appears to display a remarkably narrow range of $X_{\mathrm{CO}} \approx 2-4 \times 10^{20} \mathrm{~cm}^{-2} / \mathrm{K} \mathrm{km} \mathrm{s}^{-1}\left(\right.$ or $\alpha_{\mathrm{CO}} \approx 3-6 \mathrm{M}_{\odot} \mathrm{pc}^{-2}\left(\mathrm{~K} \mathrm{~km} \mathrm{~s}^{-1}\right)^{-1}$ Bloemen et al., 1986; Solomon et al., 1987; Blitz et al., 2007; Delahaye et al., 2011; Leroy et al., 2011; Donovan Meyer et al. $2012 \mathrm{~b}$ a). The independent $\mathrm{H}_{2}$ mass measurements in these observations come from virial mass measurements, dust to gas ratio assumptions (or measurements), and $\gamma$-ray observations. Given that the global physical properties of molecular clouds within the Milky Way occupy a relatively limited range (i.e. gas temperatures, GMC velocity dispersion and cloud surface density), this result is not terribly surprising (Maloney \& Black. 1988; McKee \& Ostriker, 2007; Feldmann et al. 2012a; Shetty et al., 2011b; Narayanan \& Hopkins, 2013).

Observations have noted two major points of departure from this relative constancy in the $X$-factor observed in the Local Group. First, in low-metallicity systems, the conversion factor appears to rise. Early observations of low-metallicity dwarf irregular galaxies showed a marked absence of CO emission (e.g. Tacconi \& Young, 1987). Further studies have shown that indeed there is quite a bit of "CO-dark" molecular gas in these systems and other lowmetallicity regions in galaxies, and that they are not simply molecular gas-poor (e.g. McQuinn et al., 2012; Schruba et al. 2012, Blanc et al., 2013). The theoretical basis for this is that while $\mathrm{H}_{2}$ can self-shield fairly easily, $\mathrm{CO}$ requires a column of dust approximately $A_{V} \sim 1$ to protect it from photo-dissociating radiation. At low metallicities, the relative size of the $\mathrm{CO}$ emitting region in a molecular cloud shrinks, thereby driving $X_{\mathrm{CO}}$ up (Maloney \& Wolfire, 1997; Wolfire et al., 2010, Feldmann et al., 2012a; Shetty et al., 2011b, Krumholz et al., 2011; Lagos et al., 2012, Narayanan et al., 2012b). While the effect of $\mathrm{CO}$ photodissociation may play a role in lower-metallicity galaxies at high-z (e.g. Genzel et al. 2012, Tan et al., 2013), for the typical dusty heavily star-forming systems that are the subject of this review, we can expect that this will play little part in driving any variations in $X_{\mathrm{CO}}$ (though this effect may be important in more metal-poor Lyman Break Galaxies at high-z Muñoz \& Furlanetto, 2013).

Second, and more relevant to the galaxies of interest in this review, in regions of high gas surface density (or, high star formation rate surface density), $X_{\mathrm{CO}}$ is observed to decrease from the typical Galactic value. This was seen first toward the Galactic Center (e.g. Oka et al., 1998; Strong et al., 2004), and has been noted in other nearby galactic nuclei as well (Sandstrom et al., 2013). This effect was perhaps most famously pointed out by Downes \& Solomon (1998), who showed that using a typical Milky Way $X$-factor in nearby galaxy mergers and starbursts would cause the inferred gas mass to exceed the dynamical mass. They found a range of derived $X_{\mathrm{CO}}$ values from their sample, from a factor $\sim 4-20$ less than the Galactic mean value.

Despite the dispersion seen in local ULIRGs, in the fifteen years since the original Downes \& Solomon (1998) study, the custom in the extragalactic literature has been to assume a bimodal $\mathrm{CO}-\mathrm{H}_{2}$ conversion factor. Typically, for normal star-forming systems such as the Milky Way, the community has adopted a value of $\alpha_{\mathrm{CO}} \approx 4$, similar to typical GMCs in the Local Group, and for starburst galaxies and mergers, a value of $\alpha_{\mathrm{CO}} \approx 0.8$ is typically assumed. When only considering local galaxies, there is indeed some rationale to this. Most disk galaxies that have been studied at high enough spatial resolution to resolve (at least massive) GMCs have shown that they seem to follow similar cloud scaling relations as in the Milky Way (Bolatto et al., 2008, Fukui \& Kawamura, 2010; Dobbs et al., 2013). Similarly, it is likely that the extreme pressures and high levels of turbulence typical in the ISM of nearby starbursts, that one can expect the typical cloud structure to break down. Indeed, this was the basis of the original Downes \& Solomon (1998) argument.

When considering high-redshift systems though, the case for a bimodal $X$-factor becomes quite a bit murkier. For example, disks at $z \sim 2$ can exhibit SFRs comparable to local galaxy mergers (e.g. Daddi et al. 2005). Similarly, when examining the resolved (at scales of $\sim 100 \mathrm{pc}$ ) properties of GMCs in a $z \sim 2$ disk, Swinbank et al. (2011) showed that the ISM may have a higher pressure, and hence higher molecular cloud surface densities and velocity dispersions. Either of these can severely impact $X_{\mathrm{CO}}$, and local calibrations may not apply.

As a result of this, both observational and theoretical groups have paid significant attention to constraining how $X_{\mathrm{CO}}$ varies as a function of physical environment at high-redshift in recent years. The first major observational constraints on $X_{\mathrm{CO}}$ at high- $z$ were made by Tacconi et al. (2008). These authors combined dynamical mass measurements made with high-resolution $\mathrm{CO}$ observations of $z \sim 2$ SMGs with stellar mass measurements to simultaneously constrain the stellar initial mass function (IMF), and the $\mathrm{CO}-\mathrm{H}_{2}$ conversion factor. They found a combination of a Chabrier IMF and $X$-factor comparable to what is observed in local ULIRGs to provide the best match to their data. Other constraints on $X_{\mathrm{CO}}$ in high- $z$ SMGs have come from both dust-to-gas ratio measurements (Magdis et al., 2011; Magnelli et al. 2012, Fu et al. 2013) and dynamical arguments (Tacconi et al., 2008; Daddi et al., 2010a, Hodge et al., 2012). These groups found a broad range of $X$-factors, ranging from $X_{\mathrm{CO}} \sim 2.5-6.3 \times 10^{19} \mathrm{~cm}^{-2} / \mathrm{K} \mathrm{km} \mathrm{s}^{-1}\left(\alpha_{\mathrm{CO}}\right.$ ranging 
from $\left.\sim 0.4-1 \mathrm{M}_{\odot} \mathrm{pc}^{-2}\left(\mathrm{~K} \mathrm{~km} \mathrm{~s}^{-1}\right)^{-1}\right)$. The range of $X_{\mathrm{CO}}$ values for high- $z$ SMGs ranges from lower than to higher than the typical $z \sim 0$ ULIRG value, and provides some evidence that the $X$-factor is not strictly bimodal. Moreover, Magnelli et al. (2012) find an inverse relationship between the conversion factor and and dust temperature, which is consistent with the empirical inverse powerlaw correlation between $X_{\mathrm{CO}}$ and gas surface density uncovered by Tacconi et al. (2008) and Ostriker \& Shetty (2011). Turning toward more 'normal' disk galaxies at high-z, Daddi et al. (2010b) utilize dynamical arguments to infer $\alpha_{\mathrm{CO}}=3.6 \pm 0.8 \mathrm{M}_{\odot} \mathrm{pc}^{-2}\left(\mathrm{~K} \mathrm{~km} \mathrm{~s}^{-1}\right)^{-1}$ (i.e. only slightly less than the mean Milky Way value), while Magdis et al. (2011) find a value $\alpha_{\mathrm{CO}}=4.1_{-2.7}^{+3.3} \mathrm{M}_{\odot} \mathrm{pc}^{-2}\left(\mathrm{~K} \mathrm{~km} \mathrm{~s}^{-1}\right)^{-1}$ when considering dust-to-gas ratio based arguments.

In short, at both low and high- $z$, a large range of values of $X_{\mathrm{CO}}$ is found observationally. No clear bimodality exists, nor is there any evidence for a single value that is applicable to all galaxies of a given luminosity or merger status. To be quantitative, in Figure 43 , we have compiled $X_{\mathrm{CO}}$ determinations for observed galaxies at low and highredshift as a function of inferred molecular gas surface density. These include resolved regions in local disks, galaxy nuclei, local ULIRGs, and high- $z B z K$ galaxies and SMGs. An inverse relationship between $X_{\mathrm{CO}}$ and $\Sigma_{\mathrm{H} 2}$ appears to exist, and $X_{\mathrm{CO}}$ appears to be a smoothly varying function of galaxy physical properties. Like all galaxy populations, dusty galaxies at high-z (and even individual subsets of dusty galaxies, such as SMGs) are a diverse group of galaxies, and no single conversion factor properly describes the range of physical conditions that is likely to exist in these galaxies. The large range in possible conversion factor in high- $z$ starbursts is similar to recent constraints from local ULIRGs, which suggest that local starbursts as well can have both Milky Way-like conversion factors, as well as much lower values (Papadopoulos et al. 2012).

Concomitant with the observational interest in the conversion factor in the last few years has been a flurry of theoretical activity in the field. Glover et al. (2010) and Glover \& Mac Low (2011) utilized magnetohydrodynamic models of GMC evolution combined with chemical reaction networks to show that $\mathrm{H}_{2}$ can survive in low metallicity environments, while CO can be more easily destroyed. These models were expanded upon by Shetty et al. (2011a b), who coupled these models with large velocity gradient radiative transfer simulations to explicitly predict the $\mathrm{CO}$ emission.

In the regime of large gas surface density, which is more pertinent to the galaxies at hand, Feldmann et al. (2012a) coupled the GMC models of Glover et al. (2010) to a cosmological zoom simulation of an individual galaxy at $z \sim 2$. These authors found that at high surface densities, one might expect the $X$-factor to drop, similar to the empirical findings of Ostriker \& Shetty (2011). Narayanan et al. (2011b) and Narayanan et al. (2012a) coupled 3D non-local thermodynamic equilibrium (LTE) radiative transfer calculations and dust radiative transfer simulations with smoothed particle hydrodynamic (SPH) models of disk galaxies and galaxy mergers to derive a functional form for the $\mathrm{CO}-\mathrm{H}_{2}$ conversion factor across a variety of environments. In terms of observables, they derive:

$$
X_{\mathrm{CO}}=\frac{\min \left[4,6.75 \times\left\langle W_{\mathrm{CO}}\right\rangle^{-0.32}\right] \times 10^{20}}{Z^{\prime 0.65}}
$$

where $\left\langle W_{\mathrm{CO}}\right\rangle$ is the resolved $\mathrm{CO}$ surface brightness, and $Z^{\prime}$ is the metallicity in units of solar. The idea behind this model is that higher gas velocity dispersions and temperatures in heavily star-forming environments drive up the velocity integrated intensity of the optically thick $\mathrm{CO}$ emission line, and thus decrease $X_{\mathrm{CO}}=N_{\mathrm{H} 2} / W_{\mathrm{CO}}$. Equation 21] predicts a smooth variation in $X_{\mathrm{CO}}$ based on the physical conditions within a galaxy, rather than a bimodality. Obreschkow \& Rawlings (2009) applied a Bayesian analysis to literature observational data, and recovered a similar relation between the conversion factor and CO surface brightness, while Ballantyne et al. (2013) evolved analytic models for Eddington-limited starbursts to also find an inverse relationship between $X_{\mathrm{CO}}$ and $W_{\mathrm{CO}}$. Similarly, Lagos et al. (2012) utilize a semi-analytic model coupled with a photo-dissociation region (PDR) code to investigate the relationship between $X_{\mathrm{CO}}$, metallicity, UV intensity, and gas surface density. As in other simulation methods, these authors find an inverse correlation between $X_{\mathrm{CO}}$ and $\Sigma_{\mathrm{H} 2}$.

Though the exact nature of the $\mathrm{CO}-\mathrm{H}_{2}$ conversion factor is unknown in both local and high- $z$ galaxies, significant progress has been made in the last decade. There is still a debate in the literature as to whether the $\mathrm{CO}-\mathrm{H}_{2}$ conversion factor is bimodal or continuous (e.g. Daddi et al., 2010b, Genzel et al., 2010, Narayanan et al., 2012b). This effectively boils down to assuming that the physical conditions in all starburst galaxies are exactly the same (and all normal disk galaxies are exactly the same), versus assuming that there may be dispersion and variation in different galaxies. The ramifications are severe. As we will discuss in the forthcoming subsections, whether one assumes a bimodal $X$ - 
factor or one that varies with the physical conditions in galaxies has implications for the star formation law (and hence for detailed models for star formation and ISM physics in starburst environments), and whether or not there is disagreement between the observed gas fractions of high- $z$ dusty galaxies and those that derive from theoretical models.

\subsection{Star Formation Laws and Efficiencies}

Since the original works of Schmidt (1959), and the first major surveys nearly 30 years later by Kennicutt (1989), star formation astrophysicists have sought a physical origin for an empirical form that describes the relation between the star formation rate and gas density. This is usually expressed in terms of the observable surface density terms:

$$
\Sigma_{\mathrm{SFR}}=\epsilon^{\prime} \Sigma_{\text {gas }}^{\beta}
$$

We will hereafter refer to Equation 22 interchangeably as the "Star Formation Law", the "Kennicutt-Schmidt (KS) Law", or the "KS Relation". We will principally mean the surface density version of the relation, though at times will refer to the volumetric density analog of the equation, and be explicit when doing so. We will refer repeatedly to Figure 44 in this section. In what follows, we first discuss local results, and then expand to results from high- $z$ observations. While the distinction between low and high- $z$ may seem artificial, a few issues related to both gas excitation, and murkiness related to the $X$-factor arise when considering high- $z$ observations that aren't present in low- $z$ data.

While nearly every paper that investigates molecular gas in galaxies attempts to place their galaxy on the KS relation, it can be lost in the details of comparisons to other samples why the KS law is important. In principle, there are two major components to the KS relation-the power-law exponent, $\beta$ (which is often referred to as the slope of the relation as the quantities are typically plotted in a log-log plot reflecting the large dynamic ranges involved), and the normalization, $\epsilon^{\prime}$.

The normalization of the relation, $\epsilon^{\prime}$, reflects the inverse of the gas depletion timescale ${ }^{18}$, and can be thought of as a measure of how easily stars form out of a parcel of gas. Buried in the physics that sets the efficiency are gas depletion, feedback, and turbulence-driven density distribution functions that drive the rate at which stars can form in a given parcel of gas. For example, as we will discuss later in this section, some observational claims point toward ULIRGs and SMGs having an efficiency, $\epsilon^{\prime}$ a factor $~ 10$ greater than disk galaxies. If this is correct, this suggests that a parcel of gas in a ULIRG may form stars at a rate 10 times what is seen in a disk galaxy for the same gas surface density. It is important to note that extragalactic observations typically include many GMCs in a single beam element, and thus care must be taken when the beam filling fraction of clouds may change as a function of galaxy environment.

Similarly, the exponent associated with the KS relation can reveal insight into the underlying small scale physics of star formation, even when considering globally averaged quantities such as single dish galaxy observations. The slope, $\beta$ is a critical prediction most theories of star formation, and can thus be used as a distinguishing test for different models. For example, Krumholz et al. (2009b) suggest that if the SFR in a galaxy is determined by the fraction of gas in molecular form, the cloud surface density (which occupies a narrow distribution in galaxies like the Milky Way), and turbulence-regulated star formation efficiencies $(\epsilon)$, then a linear KS relation is expected (when normalizing by the cloud free fall time, $\left.t_{\mathrm{ff}}\right)$. On the other hand, in starburst-dominated regimes, where supernova-driven turbulence dominates the velocity dispersion and gas dominates the vertical pressure, Ostriker \& Shetty (2011) and FaucherGiguère et al. (2013) find an index of $\sim 2$ should describe the KS relation. These are simply two examples, and there

\footnotetext{
${ }^{18} \epsilon^{\prime}$ is often referred to as the 'star formation efficiency', and should be distinguished from alternative definitions for the star formation efficiency that appear in the literature as well:

$$
\epsilon=\frac{M_{*}}{M_{*}+M_{\mathrm{GMC}}}
$$

where $M_{*}$ is the stellar mass formed in a cloud, and $M_{\mathrm{GMC}}$ is the mass of the parent cloud.

$$
\epsilon_{\mathrm{cosmic}}=\frac{\dot{M}_{*}}{\dot{M}_{\mathrm{grav}}}
$$

where $\dot{M}_{*}$ is the SFR within a galaxy, and $M_{\text {grav }}$ is the gas accretion rate onto the halo from the IGM. In the extragalactic literature, the star formation efficiency (SFE) most often means the inverse depletion timescale $\left(\epsilon^{\prime}\right)$, though the reader should take care as the definition from paper to paper will not always be consistent. In what follows, we will be explicit in our terminology for star formation efficiency.
} 

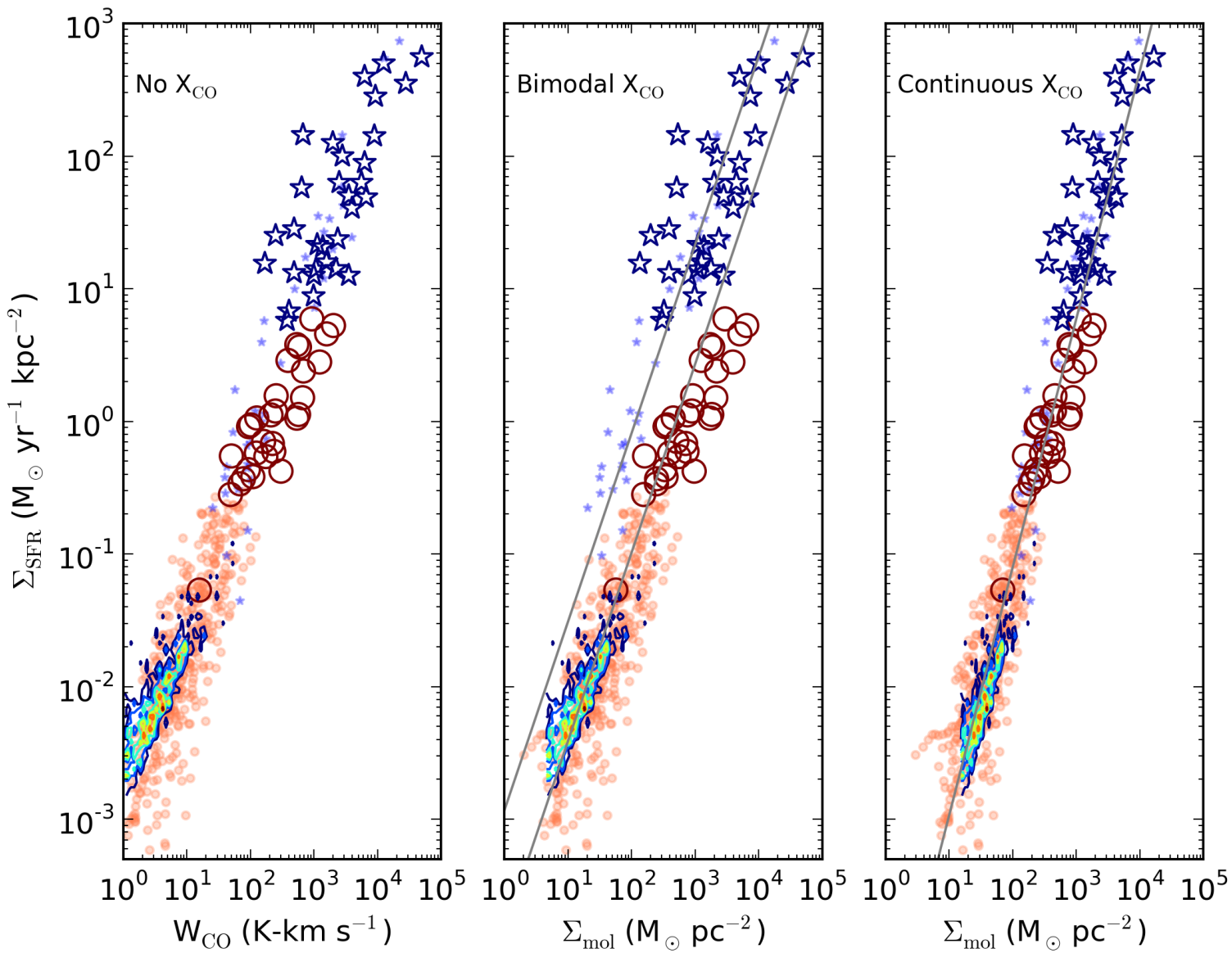

Figure 44: Dependence of Kennicutt-Schmidt (KS) star formation relation on assumed CO- $\mathrm{H}_{2}$ conversion factor $\left(X_{\mathrm{CO}}\right)$. For all plots, the circles represent quiescently star-forming galaxies (galaxies on the SFR- $M_{*}$ main sequence), and stars represent starbursts (galaxies a factor 2-3 above the main sequence). Small orange and blue symbols denote local galaxies, while large magenta and blue symbols denote galaxies at high- $z$. Contours represent resolved data from Bigiel et al. 2008). Left: KS relation plotting SFR surface density versus CO intensity (i.e. not converting to an $\mathrm{H}_{2}$ gas surface density). Middle: KS relation plotting SFR surface density vs. $\mathrm{H}_{2}$ gas surface density, assuming the traditional bimodal $X_{\mathrm{CO}}$ conversion factor. The grey lines denote the best fit sequences to the quiescent galaxies and ULIRGs. This version of the KS relation has given rise to the terminology of multiple 'modes' of star formation (a quiescent mode and a starburst mode). Right: KS relation plotting SFR surface density versus $\mathrm{H}_{2}$ gas surface density, but assuming a smoothly varying $X_{\mathrm{CO}}$ conversion factor. The functional form for $X_{\mathrm{CO}}$ is that of Equation 21 and can be arrived at either from empirical fits to observations (e.g. Ostriker \& Shetty 2011), or from the results of numerical simulations (Narayanan et al. 2012b). When assuming a smoothly varying $X_{\mathrm{CO}}$ (as opposed to a discontinuous one), the star formation relation is unimodal. 
are, of course, a wide range of theories that predict indices ranging from below unity to quadratic (see recent reviews by McKee \& Ostriker, 2007, Kennicutt \& Evans, 2012). In what follows, we will review recent progress over the last decade in this field, with a particular eye toward high- $z$ galaxies. We refer the reader to the excellent recent review by Kennicutt \& Evans (2012) for a more thorough summary of the star formation law in the Milky Way and nearby galaxies.

The seminal study of Kennicutt (1998b) compared the SFR of 36 nearby infrared-selected galaxies against the total gas mass as measured by $\mathrm{HI}$ and $\mathrm{CO}$ (as a proxy for $\mathrm{H}_{2}$ ). Kennicutt derived an index of $\beta=1.4$. Over the following decade, it became clear that the SFR is principally correlated with the $\mathrm{H}_{2}$ gas in galaxies, and not $\mathrm{HI}$ (e.g. Wong \& Blitz, 2002). Henceforth, when we refer to the star formation law, we refer to the molecular gas KS relation, and discard any contribution to the gas content from atomic gas.

Utilizing data from the THINGS, HERACLES, and BIMA SONG surveys (Walter et al., 2005; Leroy et al., 2009. Helfer et al. 2003), Bigiel et al. (2008) investigated the resolved star formation law in nearby galaxies. These authors found that the SFR is principally correlated with $\mathrm{H}_{2}$ gas (as traced by $\mathrm{CO}(\mathrm{J}=2-1)$ in this case), and unassociated with HI in nearby galaxies. This result was extended to the atomic gas-dominated outskirts of nearby galaxies by Schruba et al. (2011). A principle result from these groups is that the SFR is linearly related to the molecular gas surface density on resolved (a few hundred pc) scales, when assuming a constant line ratio from CO $(J=2-1)$ to $(J=1$ 0) (with $\Sigma_{\mathrm{SFR}}$ ), as well as a constant $X_{\mathrm{CO}}$. The results from Bigiel et al. (2008) and Leroy et al. (2008) are denoted by the colored contours in Figure 44. Utilizing a sample of 222 galaxies, Saintonge et al. (2011) found an increasing molecular depletion time scale (where the depletion time scale is the inverse of $\epsilon^{\prime}$ ) with galaxy mass, while a roughly constant atomic gas depletion time scale across their mass range of $10<\log M_{*} / \mathrm{M}_{\odot}<11.5$.

It should be noted that the claim of a linear KS law when considering normal, quiescent local galaxies has been disputed by a number of groups on varying physical grounds. For example, Liu et al. (2011) argue that when one subtracts the diffuse component from SFR maps, then a super-linear resolved KS relation emerges in NGC 3251 and NGC 5194. On the other hand, Blanc et al.(2009); Shetty et al. (2013a b) suggest that due to issues related to bisector fitting methods (which have been used in many of the resolved KS relation studies), the true KS slope should be sub-linear. Indeed, there is no consensus on the observed KS slope in galaxies, even when only considering nearby, quiescent systems.

While the numbers of high $\Sigma_{\mathrm{SFR}}$ galaxies in the local Universe are small as compared to quiescent systems, there appears to be a possible steepening of the KS relation at the transition from normal galaxies to starbursts (ULIRGs). The interpretation of this is muddied by $X$-factor assumptions, but such a steepening is potentially visible even when considering the KS law in terms of observables themselves. This is apparent in the left panel of Figure 44, where the light orange circles represent local quiescent galaxies, and the light (small) blue stars represent local starbursts. Gao \& Solomon (2004b) find a slope of $1.25-1.44$ between $L_{\mathrm{IR}}$ and $L_{\mathrm{CO}}$ (i.e. pure observables) when considering unresolved CO ( $\mathrm{J}=1-0)$ observations of both local quiescent galaxies, and nearby LIRGs and ULIRGs.

If the KS relation steepens from quiescent galaxies to ULIRGs, this may reflect a change in the physical conditions in the ISM in these galaxies, which results in a decreased star formation time scale (or increased inverse depletion time scale, $\epsilon^{\prime}$ ). Krumholz et al. (2009b) have posited that perhaps this transition may reflect a regime where GMCs are no longer regulated by internal processes, but rather the external galactic pressure exceeds the internal cloud pressure. It is plausible, in any case, that the compact, warm, and turbulent conditions at the center of a starburst drives different physical conditions in the molecular ISM, and that star formation efficiencies may be enhanced accordingly.

The dramatic increase in the number of CO detections at high- $z$ in recent years (e.g. Carilli \& Walter, 2013) have allowed for a significant expansion in the number of high $\Sigma_{\text {SFR }}$ galaxies, and thus a window into star formation in more extreme environments accordingly, though the interpretation of the results is steeply dependent on the form of the $\mathrm{CO}-\mathrm{H}_{2}$ conversion factor assumed. The first major $\mathrm{CO}$ surveys that examined the KS relation at high- $z$ were presented by Greve et al. (2005) and Bouché et al.(2007) which were limited to only the brightest sources (i.e. SMGs). Bouché et al. (2007) found that, when utilizing a local ULIRG-like $X_{\mathrm{CO}}$ conversion factor, the resulting KS relation (including local galaxies as well) was strongly super-linear, with an index of $\sim 1.7$. A super-linear index in the KS relation suggests that very high $\Sigma_{\mathrm{SFR}}$ galaxies have shorter gas depletion time scales than lower $\Sigma_{\mathrm{SFR}}$ galaxies. On the other hand, other studies by Daddi et al. (2010b) and Genzel et al. (2010) suggest that the KS relation may be linear when including high- $z$ galaxies, though these studies additionally include more quiescent galaxies on the SFR- $M_{*}$ main sequence.

The situation gets potentially murkier when considering high-z systems due to the fact that high lying lines of 
$\mathrm{CO}$ are typically observed, rather than the ground-state transition owing to $\mathrm{CO}(\mathrm{J}=1-0)$ being redshifted out of typical instrument bandpasses. Krumholz \& Thompson (2007) and Narayanan et al. (2008a c, 2011a) argue that differential excitation in observed KS relation can be strong, and that because high- $z$ galaxies are typically observed in high $\mathbf{J}$ transitions, when converting down to $\mathrm{CO}(\mathrm{J}=1-0)$, there can be systematic trends in the line ratios with $\Sigma_{\mathrm{SFR}}$ that will cause the true underlying KS relation to be steeper than what is observed. Certainly, Figure 45 highlights that no single set of line ratios can apply to high- $z$ starbursts, and there is significant dispersion. Whether or not there is a systematic trend with $\Sigma_{\mathrm{SFR}}$ is at present unclear.

Precisely determining the normalization of the KS law is comparably difficult, and is inextricably tied to a sensitive dependency on the CO-H $\mathrm{H}_{2}$ conversion factor. For example, Daddi et al. (2010b), Genzel et al. (2010) and Bothwell et al. (2010) presented relatively large samples of CO-detected galaxies at high- $z$. These included both very luminous systems such as SMGs, as well as more moderate star-forming galaxies that lie on the SFR- $M_{*}$ main sequence. When applying a Galactic $\mathrm{CO}-\mathrm{H}_{2} X_{\mathrm{CO}}$ conversion factor to local disk galaxies, and main sequence galaxies at high- $z$, and an $X_{\mathrm{CO}}$ a factor 6-8 lower for local ULIRGs and high- $z$ SMGs, a bimodal KS relation emerges. This bimodal relation is comprised of a low star formation efficiency sequence that quiescent star-forming disks at all redshifts live on, and a high star formation efficiency sequence that starbursts live on. This is shown in the middle panel of Figure 44. At face value, the interpretation of this plot is that there are two 'modes' of star formation in galaxies - a quiescent mode and starburst mode. In this physical scenario, two galaxies at a comparable set of gas surface densities may have star formation rate surface densities that vary by an order of magnitude.

A large number of theories have been posited in an attempt to understand the physical nature of the bimodal KS relation, or whether the bimodality is real. Daddi et al. (2010b) and Genzel et al. (2010) advocated a model in which the underlying star formation is driven by large scale dynamical effects, and that if one normalizes by the galaxy dynamical time (i.e. construct a relation: $\Sigma_{\mathrm{SFR}} \propto \Sigma_{\mathrm{mol}} / t_{\mathrm{dyn}}$ ), the bimodality disappears. Krumholz et al. (2012) developed a model in which the star formation rate operates on some fraction of the small-scale gas free fall time, and can be parameterized by a relation as in Equation 22. They showed that if one takes plausible values for the typical gas free fall time in galaxy disks, and luminous starbursts, the apparent bimodal relation exhibited in the Daddi et al. (2010b) and Genzel et al. (2010) data reduces to a single, unimodal relation. An alternative class of theories was presented by Ostriker \& Shetty (2011), and Narayanan et al.(2012b), who argued that the existence of a bimodal KS relation was an artifact of the usage of a bimodal $\mathrm{CO}-\mathrm{H}_{2}$ conversion factor, and that if a unimodal (either constant, or smoothly varying) $X$-factor was employed, the KS relation would no longer appear bimodal. Ostriker \& Shetty (2011) fit observed data between $X_{\mathrm{CO}}$ and $\Sigma_{\mathrm{H} 2}$ presented in Tacconi et al. (2008) to derive an empirical form for a smoothly varying $X$-factor, while Narayanan et al. (2012b) utilized numerical simulations in order to derive a theoretical model form for the conversion factor (Equation 21). After applying their models for a continuously varying $\mathrm{CO}-\mathrm{H}_{2}$ conversion factor, both Ostriker \& Shetty (2011) and Narayanan et al. (2012b) found that the bimodal KS relation reduces to a tight, unimodal relation, with index roughly 2 . This is represented in the right panel of Figure 44. A yet additional class of models exists that posits that the bimodality is in fact real, and that galaxies undergoing starbursts can go through short periods of extreme star formation efficiency. Teyssier et al. (2010) suggested that, due to extreme amounts of dense gas formed during a merger, a distributed starburst in a galaxy merger may occur with very high efficiency, driving these starbursts through a phase where they reside on the upper normalization of the bimodal KS relation.

The question is not whether or not the KS relation is bimodal, but rather how much dispersion there is in it (e.g. Feldmann et al., 2012b; Freundlich et al., 2013). The apparent bimodality is simply a statement that the inverse depletion time scale can vary by up to an order of magnitude at a given gas surface density. Characterizing how much the star formation efficiency (inverse depletion time scale) can vary, and why, is a fundamental question for both star formation and galaxy evolution astrophysics. Moving forward, detailed studies of both extreme systems at high- $z$, as well as galactic nuclei in the local Universe that can eliminate some of the uncertainties that have been present in previous surveys may provide some insight.

For example, Fu et al. (2013) utilized both gas to dust ratio constraints, as well as theoretical models in order to constrain $X_{\mathrm{CO}}$ in a detailed study of a SFR $2000 \mathrm{M}_{\odot} \mathrm{yr}^{-1} \quad \mathrm{SMG}$ caught in the act of merging at $z \sim 2$. Given their constraints on the $\mathrm{CO}-\mathrm{H}_{2}$ conversion factor for this particular object, Fu et al. (2013) concluded that the SMG will lie above an extension of the local disk KS relation (though would fall within the mix of high- $z$ galaxies [blue stars] when assuming a smoothly varying $X_{\mathrm{CO}}$; e.g. the right panel of Figure 44). A similar result was found by Ivison et al. (2013), who employed dynamical constraints on the conversion factor in a comparably luminous source to the 
Fu et al. (2013) study. Similarly, Leroy et al. (2013) employ a dust to gas ratio-dependent $X_{\mathrm{CO}}$ when studying $1 \mathrm{kpc}$ regions in nearby galaxies, and find that the star formation efficiency toward nearby galaxy centers may increase from the typical field GMC.

In summary, while significant progress has been made in understanding star formation laws as they pertain to high $z$ dusty galaxies in recent years, the field is still wide open. Even precisely determining the observed slope and dispersion in the relation is an extremely difficult task, though crucial for constraining theories of star formation.

\subsection{The Role of Dense Molecular Gas}

\subsubsection{Physics Learned from the Milky Way and Local Galaxies}

Over the last decade, a great deal of effort has been put forth in investigating the role of dense molecular gas in giant molecular clouds and galaxies. This has been motivated by Galactic studies which show a correlation between the dense molecular gas (as traced by high critical density tracers, such as $\mathrm{HCN}, \mathrm{HCO}^{+}$and $\mathrm{CS}$ ), and young stellar objects (e.g. Evans, 1999, and references therein). In contrast, owing to high optical depths in clouds, the relatively low effective density ${ }^{19}$ of $\mathrm{CO}\left(n_{\mathrm{eff}} \approx 10-100 \mathrm{~cm}^{-3}\right)$ means that it traces the bulk of the molecular mass in a cloud (modulo potential metallicity effects; see $\$ 8.2$, rather than the sites of active star formation.

In order to investigate the relationship between the star formation rate of galaxies and the dense gas mass, Solomon et al.(1992) and Gao \& Solomon (2004a b) performed the first large extragalactic surveys of HCN in nearby galaxies, targeting normal disks, LIRGs and ULIRGs between an infrared luminosity range of $\sim 7 \times 10^{9}-2 \times 10^{12} \mathrm{~L}_{\odot}$. These authors found a tight linear relationship between $\mathrm{L}_{\mathrm{IR}}$ and $L_{\mathrm{HCN}}$, suggesting that the star formation rate in galaxies is controlled by dense gas traced by HCN with effective density $n>n_{\mathrm{eff}} \approx 3 \times 10^{4} \mathrm{~cm}^{-3}$. This result was supported by observations of $\mathrm{CO}(\mathrm{J}=3-2)$ from nearby disks, LIRGs and ULIRGs that all found a roughly linear FIR- $L_{\mathrm{COJ}=3-2}$ relationship (Yao et al., 2003; Narayanan et al., 2005, Iono et al., 2009, Mao et al., 2010).

This interpretation was expanded upon by Wu et al. (2005) and Wu et al. (2010), who extended this study to dense clumps ${ }^{20}$ within the Galaxy and found a similarly linear relation between $\mathrm{L}_{\mathrm{IR}}$ and $\mathrm{HCN}$ luminosity. Complementary work utilizing $\mathrm{HCO}^{+}(\mathrm{J}=3-2)$, which has a similar effective density as $\mathrm{HCN}(\mathrm{J}=1-0)$ (Juneau et al., 2009), as well as high-visual extinction molecular gas as dense gas tracers have found roughly linear dense gas star formation laws for clumps within the Milky Way (Lada et al., 2010; Schenck et al., 2011). Mangum et al. (2008) and Mangum et al. (2013) observe formaldehyde in a sample of nearby disks and starbursts, suggesting a linear relationship between SFR and dense gas mass traced by this molecule is also possible. Wang et al. (2011a) find an slope of 0.94 between $\mathrm{L}_{\mathrm{IR}}$ and $\mathrm{CS}\left(\mathrm{J}=5-4 ; n_{\mathrm{eff}}=2 \times 10^{6}\right)$, and Graciá-Carpio et al. (2006) find a roughly linear slope with $\mathrm{HCO}^{+}(\mathrm{J}=3-2$; $n_{\mathrm{eff}}=6 \times 10^{4}$ ). These papers forward an interpretation in which there is a volume or surface density threshold for star formation within galaxies, and that dense clumps represent fundamental star formation units. In this scenario, a linear relation between SFR and the mass probed by HCN is natural. Starburst galaxies, then, simply have an increased number of dense star-forming units.

On the other hand, observations of a large number of dense gas tracers show both super-linear and sub-linear star formation laws, casting a shadow on the interpretation that $\mathrm{HCN}(\mathrm{J}=1-0)$ traces a fundamental star formation unit in galaxies. For example, Bussmann et al. (2008) observed a large subset of the Gao \& Solomon (2004a b) sample in $\mathrm{HCN}(\mathrm{J}=3-2)$ (with $n_{\mathrm{eff}}=7 \times 10^{5} \mathrm{~cm}^{-3}$, a factor of $\sim 20$ larger than the effective density of HCN $(\mathrm{J}=1-0)$ ), and found a sub-linear dense gas star formation law with index $\sim 0.7-0.8$. Similarly, Bayet et al. (2009) examined the relationship between SFR and CO emission with transitions ranging from $\mathrm{J}=1-0$ through $\mathrm{J}=12-11$, and found decreasing dense gas SFR slopes with increasing $J_{\text {upper }}$ (and, hence, increasing $\left.n_{\text {eff }}\right)$ such that the SFR-CO $(\mathrm{J}=1-0)$ relation had slope $\sim 1.4$, the SFR-CO (J=3-2) relation was roughly linear, and the SFR-CO $(\mathrm{J}=12-11)$ had slope of $\sim 0.5$. Even the results from $\mathrm{HCN}(\mathrm{J}=1-0)$ alone provide a somewhat confusing picture as some groups have found a super-linear relationship between $\mathrm{L}_{\mathrm{IR}}$ and $\mathrm{HCN}(\mathrm{J}=1-0)$ in local galaxies (García-Burillo et al., 2012). A tentative trend is evident in this series

\footnotetext{
${ }^{19}$ As a reminder, as discussed in Evans (1999), while a density of $n>n_{\text {crit }}$ is usually taken to be necessary for line emission, a variety of effects associated with radiative transfer can affect the observed line strength at a given density. Following Evans, we choose to state, rather than the critical density, the effective density $\left(n_{\text {eff }}\right.$, which is defined as the density at which a transition will have a radiation temperature of $1 \mathrm{~K}$, assuming $\log (N / \Delta v)=13.5$, and $\mathrm{T}=10 \mathrm{~K}$. A table converting $n_{\text {crit }}$ (which is typically $1-2$ orders of magnitude higher than $n_{\text {eff }}$ ) and $n_{\text {eff }}$ for a variety of molecular transitions is given in Evans (1999) and Reiter et al. (2011).

${ }^{20}$ In keeping with the standard definitions in the star formation literature, we will define "clumps" as $\sim 1$ pc entities within GMCs that may form stellar clusters, and "cores" as $\sim 0.1$ pc structures that serve as the precursors of individual or binary stars (e.g. Kennicutt \& Evans. 2012).
} 
of observations that higher critical density tracers appear to have lower SFR law slopes (Juneau et al., 2009). This trend may be evident in Milky Way clumps, though it is tentative. When Wu et al. (2010) examined the robust fits between the SFR and a variety of dense gas tracers, a number of tracers exhibited sub-linear slopes; on the other hand, the least squares fits were typically consistent with slopes of unity.

Theories on the origin of dense gas star formation laws can be broken into three camps: (1) Those that ascribe their origin to the gas density probability distribution function (PDF) in star-forming galaxies; (2) Those that connect a linear dense gas Kennicutt-Schmidt relation to a density or surface density threshold for the onset of star formation; (3) Those that appeal to chemistry models, and the influence of X-ray driven chemistry that owes either to intense starbursts or AGN input.

Models that relate the index of the SFR-line luminosity index for various dense gas tracers to the gas density PDF in galaxies were developed by Krumholz \& Thompson (2007) (utilizing analytic models for GMC structure), and Narayanan et al.(2008c) (utilizing hydrodynamic models for galaxies in evolution). In this picture, the principle driver behind the power-law index, $\beta$ in the SFR- $L_{\text {mol }}^{\beta}$ volumetric (gas mass-based) star formation law is the relationship between the gas density distribution and the effective density of the emitting dense gas tracer. A linear $\mathrm{HCN}(\mathrm{J}=1-0)$ star formation law simply reflects the relationship between the $n_{\mathrm{eff}}$ of $\mathrm{HCN}(\mathrm{J}=1-0)$, and the typical mean density in nearby galaxies. Two testable predictions arise from these models: (1) molecular lines with effective densities higher than $\mathrm{HCN}(\mathrm{J}=1-0)$ should have sub-linear star formation law slopes for local galaxies as they trace gas further out in the high-density tail of the density PDF. There may be some indication of sub-linear SFR law slopes for very high effective density tracers (e.g. Bussmann et al., 2008, Graciá-Carpio et al., 2008, Bayet et al., 2009), though larger samples are most certainly necessary. (2) Very high density systems (such as SMGs, or galactic nuclei) should have a super-linear SFR-HCN ( $\mathrm{J}=1-0)$ relation (Narayanan et al. 2008a). Indeed, observed increased HCN/CO ratios with galaxy SFR is tentative observational evidence that the gas density PDF is shifting toward higher densities in these systems (Juneau et al. 2009, Rosolowsky et al., 2011). This test will be fully realized with surveys of HCN $(\mathrm{J}=1-0)$ at high- $z$ with the VLA.

An alternative to this picture is the threshold star formation law in which meeting a volume or surface density threshold is a prerequisite to beginning the star formation process. Lada et al. (2010) find roughly linear relationships between SFR and gas above an extinction threshold of $A_{K} \approx 0.8 \mathrm{mag}$ in Galactic clumps, a result consistent with the work of Heiderman et al. (2010). These authors argue that this is comparable to a threshold surface density of $\sim 100 \mathrm{M}_{\odot} \mathrm{pc}^{-2}$, which is roughly equivalent to a volume density threshold of $\sim 10^{4-5} \mathrm{~cm}^{-3}$, depending on the cloud geometry. An attractive aspect of this picture is that the density threshold is roughly matched with the density probed by $\mathrm{HCN}(\mathrm{J}=1-0)$. This scenario suggests that $\mathrm{HCN}$ traces the dense gas that more actively forms stars better than CO, and thus predicts an SFR relation with lower dispersion. Wu et al. (2010) interpret the roughly linear relations between SFR and different dense gas tracers in their study of Galactic clumps as further evidence for this model.

A third class of models appeals to chemistry driven by X-rays in the vicinity of high star formation rate surface density environments, or an AGN (e.g. Lintott \& Viti, 2006; Meijerink et al., 2013). At least some evidence for this has been seen by Krips et al. (2008) and Graciá-Carpio et al. (2008) in nearby active systems.

\subsubsection{Dense Gas at High-Redshift}

The study of dense gas at high- $z$ is at its infancy, though it holds great promise for constraining models of star formation owing to the extreme gas physical conditions in SMGs. Gao et al. (2007) studied HCN (J=1-0) in a sample of high- $z$ SMGs and quasars, finding a relationship between the FIR luminosity in these systems and HCN luminosity, though offset from the local one (such that the high- $z$ points lie above the local linear relation). It is unclear whether this owes to higher gas density PDFs (e.g Krumholz \& Thompson, 2007; Narayanan et al., 2008c), or contribution to the FIR luminosity by AGN. Further evidence for a nonlinear FIR-HCN trend in dense, high- $z$ systems was provided by Greve et al. (2006) and Riechers et al. (2007).

Other well-studied sources include the Cloverleaf quasar (e.g. Solomon et al., 2003) and APM 0829+5255 (Wagg et al. 2005, Riechers et al. 2010b). These sources are both lensed, however, which adds the additional potential complicating factor of differential magnification. On average, a consensus finding from dense gas observations in high- $z$ SMGs is that these systems tend to have a larger fraction of their ISM in a dense phase than local field galaxies. Further evidence for this is seen in the high-excitation CO SLEDs that are typical of these sources (Carilli \& Walter. 2013; Riechers et al., 2013b). Future surveys of HCN ( $=1-0)$ from high- $z$ SMGs, and comparisons to low- $z$ relations will provide valuable insight into the physical conditions that govern star formation (e.g. Krumholz \& McKee, 2005. 
Andrews \& Thompson, 2011; Hopkins et al., 2013e). Obtaining large samples of dense gas emission lines from galaxies at high- $z$ is an important priority for the coming decade.

Alongside dense gas tracers $\mathrm{HCN}$ and $\mathrm{HCO}^{+}$, observations of water have been gaining traction in the past few years. The rotational transitions of $\mathrm{H}_{2} \mathrm{O}$ have very high critical densities $\left(\sim 10^{8} \mathrm{~cm}^{-3}\right)$, and therefore only happen in the extremely dense parts of star-forming clouds. The bulk of studies thus far have focused on low- $z$ systems (e.g. Yang et al., 2013), though at least a few have attempted to push to higher redshifts. The early studies typically detected water masers (e.g. Barvainis \& Antonucci. 2005; Impellizzeri et al., 2008), though in recent years, nonmasing sources at high- $z$ have been procured as well (Omont et al. 2011, 2013). Riechers et al. (2013b) impressively detected seven $\mathrm{H}_{2} \mathrm{O}$ lines in the $z=6.34$ galaxy HFLS3. A key result from these studies is that a clear correlation between $\mathrm{H}_{2} \mathrm{O}$ luminosity and infrared luminosity exists a range $\mathrm{L}_{\mathrm{IR}} \sim 10^{12}-10^{14} \mathrm{~L}_{\odot}$. Omont et al. (2013) find a relation $L_{\mathrm{H}_{2} \mathrm{O}} \propto \mathrm{L}_{\mathrm{IR}}{ }^{1.17}$.

\subsection{CO Excitation and Spectral Line Energy Distributions}

The CO spectral line energy distribution (CO SLED; alternatively known as the CO rotational ladder) from a galaxy provides a unique window into the bulk physical properties of the molecular gas in a given system. The SLED describes the relative strengths of $\mathrm{CO}$ emission lines, and reveals the level populations of $\mathrm{CO}$ molecules. Typically, the CO SLED of a galaxy is represented as the CO line intensity versus the rotational level of the line. The excitation of $\mathrm{CO}$ is dependent on the gas density and temperature (along with secondary effects, including the line optical depth). Generally, the relative excitation of two transitions of $\mathrm{CO}$ can be expressed as a ratio of of brightness temperatures, line luminosities or line intensities.

Typically, the warmer and denser a system, the more heavily populated the upper levels will be. For a system that is in local thermodynamic equilibrium (LTE) such that the levels can be described by Maxwell-Boltzmann statistics, the line intensity is given by the Planck function, and for warm enough temperatures (such that $E_{\text {upper }}<<k T$ ), the SLED will rise as the square of the line frequency ${ }^{21}$ Typically, these systems are referred to as 'thermalized', or 'thermal', and level populations that fall below what is expected for LTE are 'sub-thermal'. While observing high$\mathrm{J}$ CO lines in local galaxies has been difficult in the pre-Herschel and ALMA years, observations of high- $z$ SMGs have routinely been deriving well-sampled $\mathrm{CO}$ line ladders owing to the redshifting of submillimeter-wave lines into atmospherically favorable observing windows.

Constraining the CO SLED for galaxies has two main purposes. First, armed with a radiative transfer code (such as an escape probability code, or large velocity gradient (LVG) code; e.g. Krumholz, 2014), with multiple CO emission lines one can constrain the combination of temperatures and densities necessary to drive the observed CO excitation. This requires some assumption about the $\mathrm{CO}$ abundance and typical velocity gradient in emitting GMCs. Second, at high- $z$, most detections of $\mathrm{CO}$ are of high-lying transitions. In order to derive the total gas mass as traced by $\mathrm{CO}$ $(\mathrm{J}=1-0)$ (see $\$ .2$ ), one needs some knowledge regarding the $\mathrm{CO}$ excitation.

Because high- $z$ SMGs are the most extreme star-forming galaxies in the Universe, it has long been assumed that LTE is a safe assumption for the level populations. Indeed, local starburst galaxies such as M82 and NGC 253 exhibit CO SLEDs that are nearly thermal out through $\mathbf{J} \sim 5$ (Weiß et al., 2005; Hailey-Dunsheath et al., 2008). However, early detections of $\mathrm{CO}(\mathrm{J}=1-0)$ with the Green Bank Telescope (GBT) and VLA revealed that SMGs appear to exhibit a diverse range of CO SLEDs, and that at least some SMGs may indeed contain large volumes of sub-thermally excited gas (e.g. Greve et al., 2003; Hainline et al., 2006; Carilli et al., 2010, Harris et al., 2010; Ivison et al., 2011). Still, other SMGs show rather extreme conditions, and appear to have thermalized level populations through the $\mathrm{J}=6-5$ transition (e.g. HFLS3, Riechers et al., 2013b). We quantify the diversity of CO SLEDs from high- $z$ SMGS in Figure 45, where we show the CO SLEDs for all bona fide SMGs that have a CO $(\mathrm{J}=1-0)$ detection. For reference, we also show the rotational ladder for the Galaxy, M82, and what is expected for thermalized level populations so long as $E_{\text {upper }}<<k T$.

Two salient points are clear from Figure 45. First, based on the physical characterization of their ISM properties alone, SMGs appear to be a heterogeneous galaxy population. While some galaxies have CO excitation patterns consistent with very warm and dense gas, others have much weaker excitation. This may be consistent with theories that suggest that SMGs may be made up of both merger-induced starbursts caught at final coalescence (that may have

\footnotetext{
${ }^{21}$ As shown by Narayanan \& Krumholz 2014, the Rayleigh-Jeans condition is not easily met for high $\mathrm{J}$ ( $\mathrm{J} \gtrsim 6 \mathrm{CO}$ emission lines), even for extreme starbursts.
} 


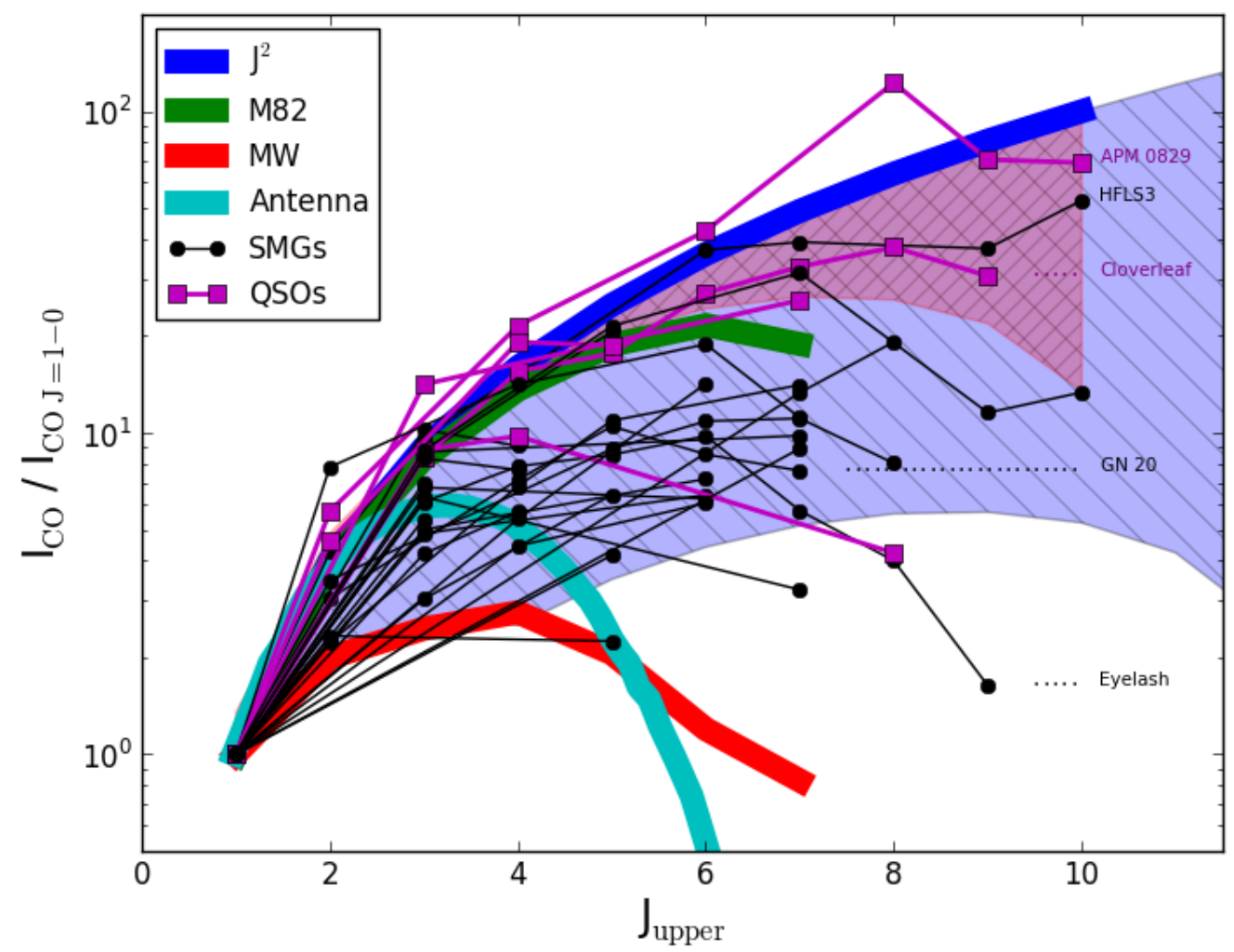

Figure 45: CO Spectral Line Energy Distribution (SLED) for high-redshift SMGs (black lines) that have a CO (J=1-0) detection. In cases of multiplicity, the SLED is for the composite system, unless the individual components also satisfy the SMG criteria $\left(S_{850}>5 \mathrm{mJy}\right)$. Galaxies are only ones that have CO 1-0. For reference, the SLED for the Milky Way is shown (red line, Fixsen et al. 1999), as well as M82 (Weiß et al. 2005. purple line,) and what is expected for LTE (blue line). As is apparent, there is a large diversity in SMG CO SLEDs, ranging from nearly thermalized through $\mathrm{J}=6$, through sub-thermal even at the $\mathrm{J}=3$-2 line. The CO SLEDs are taken from Andreani et al. (2000); Aravena et al. (2010) 2012); Baker et al. (2004); Bothwell et al. (2013a); Carilli et al. (2010); Danielson et al. (2011); Downes \& Solomon(2003); Fu et al. (2013); Greve et al. (2003) 2005); Hainline et al. (2006); Harris et al. (2010); Ivison et al. (2011| 2013); Neri et al. (2003); Papadopoulos \& Ivison (2002); Rawle et al. (2013); Riechers et al. (2011d 2013b); Scott et al. (2011); Sharon et al. (2013); Weiß et al. (2009a), and the Milky Way SLED was provided by Chris Carilli and Fabian Walter (private communication). By and large, most SMGs are thermalized through the $\mathrm{J}=2-1$ transition, and many through the $\mathrm{J}=3-2$ transition. However, assuming constant brightness temperatures at $J_{\text {upper }} \geq 3$ for high- $z$ SMGs is a poor assumption. Note, however, that the Cloverleaf and APM 0829 are lensed, and differential amplification is a major concern if different gas reservoirs have different sizes, which in turn affects their excitation. Similarly, HFLS3 has to be intrinsically hotter to be detected at $z \approx 6$, an important selection effect to consider. 
more extreme ISM conditions), as well as individual disk galaxies at high- $z$ that may have a lower-excitation ISM (e.g. Hayward et al. 2011, 2012, 2013b). Indeed, some observations of SMGs support this picture (Hodge et al., 2013b, Karim et al., 2013).

Second, it is clear that there are no 'average' line ratios for SMGs. The ladders are diverse, with line ratios at a given transition differing, at times, by an order of magnitude. This level of uncertainty can be comparable to what is present in the $\mathrm{CO}-\mathrm{H}_{2}$ conversion factor, and should be reflected in any $\mathrm{H}_{2}$ mass measurements derived from down-converting high-excitation CO lines to the 1-0 transition. In an effort to reduce this uncertainty, Narayanan \& Krumholz (2014) developed a model in which the the CO SLED is controlled by difficult-to-observe parameters such as the gas density, temperature, and line optical depths. However, these physical quantities scale well with the global galaxy SFR surface density. As a result, Narayanan \& Krumholz (2014) were able to derive a power-law parameterization for the $\mathrm{CO}$ excitation as a function of $\Sigma_{\mathrm{SFR}}$. Going forward, other theoretical or empirical calibrations for the SLED in terms of an observable proxy will be useful for interpreting high- $z$ data.

Finally, a number of recent works have pointed out that modeling high- $z$ dusty systems as single phase (single $\mathrm{T}$ and $\rho$ ) provides a poor fit to the observed SLED. For example, Harris et al. (2010), Riechers et al. (2011c) and Hodge et al. (2013a) find that some SMGs are best modeled with both a compact, high-excitation phase, as well as a more extended diffuse ISM. This is in contrast with high- $z$ quasar host galaxies, which can typically be modeled as dominated by a single high-excitation gas component (e.g. Riechers et al., 2011a; Weiß et al., 2007).

\subsection{Molecular Gas Fractions}

One of the most remarkable aspects of high- $z$ dusty galaxies is their incredibly large gas fractions as compared to present-epoch galaxies. The gas fraction, defined here as:

$$
\mathrm{f}_{\mathrm{gas}} \equiv \frac{M_{\mathrm{H} 2}}{M_{\mathrm{H} 2}+M_{*}}
$$

considers the fraction of baryons in a galaxy that is in molecular form, neglecting any contribution from HI. It is likely, however, that in the high-pressure environments typical of starburst galaxies, that the bulk of the hydrogen in the ISM is in molecular form (Blitz \& Rosolowsky, 2006, Krumholz et al., 2009a). Measurements of high- $z$ star forming galaxies (ranging from relatively quiescent $B z K$ galaxies, forming at $\sim 10-100 \mathrm{M}_{\odot} \mathrm{yr}^{-1}$, to dusty starbursts), suggest gas fractions ranging from $\mathrm{f}_{\text {gas }} \sim 0.2-0.8$ (e.g. Daddi et al., 2010a; Tacconi et al., 2010, Geach et al., 2011, Magdis et al. 2012b; Combes et al., 2013; Tacconi et al. 2013). This is to be compared to local galaxies, which show typical gas fractions $\mathrm{f}_{\text {gas }}<10 \%$ (e.g. Saintonge et al., 2011). These results have come from both from $\mathrm{CO}_{\text {inferred } \mathrm{H}_{2}}$ gas masses, as well as dust measurements (and associated dust-to-gas ratio assumptions Magdis et al., 2012a).

While no study has performed a proper mass-selected study, indications from these observations are that the average gas fraction of galaxies rises toward high-redshift. This general trend is in good agreement with cosmological galaxy formation simulations (e.g. Bouché et al., 2010, Dutton et al., 2010, Lagos et al., 2011, Davé et al., 2012). In galaxy formation theory, the baryonic gas fraction is set by a balance between gas accretion from the intergalactic medium (IGM), and the removal of gas by star formation and galactic outflows (with small perturbations from stellar mass loss and recycled gas outflows Oppenheimer et al., 2010). At higher redshifts, the baryonic inflow rate, which scales strongly with redshift (e.g. Dekel et al., 2009a, Fakhouri et al., 2010), ensures that large gas reservoirs are built up in galaxies. Geach et al. (2011) suggest that an evolution in $M_{\text {gas }} / M_{*}^{22}$ with redshift as $M_{\text {gas }} / M_{*} \propto(1+z)^{2 \pm 0.5}$ provides a reasonable fit to observed data.

On average, galaxy gas fractions decrease with increasing stellar mass. This point has been predicted in theoretical models (e.g. Davé et al., 2010, 2011; Lagos et al., 2011; Popping et al. 2012, 2013), as well as observationally confirmed (Combes et al., 2013, Saintonge et al., 2013; Tacconi et al., 2013; Santini et al., 2013). This trend is additionally seen in low-redshift galaxies, though Saintonge et al. (2011) suggest that the gas fractions of low- $z$ galaxies are more closely correlated with stellar density than stellar mass. We see this quantitatively for high-z SMGs in Figure 46, where we plot the gas fraction for all SMGs with $\mathrm{CO}(\mathrm{J}=1-0)$ detections (to minimize the relatively large uncertainty in $\mathrm{CO}$ excitation; c.f. $\S 8.5$. We use a conversion from $\mathrm{CO}$ to $\mathrm{H}_{2}$ assuming $\alpha_{\mathrm{CO}}=0.8$ for high- $z$ SMGs, and $\alpha_{\mathrm{CO}}=4$ for $B z K$ galaxies, despite the likely problems associated with assumption outlined in $\S 8.2$

\footnotetext{
${ }^{22}$ Note, this is different than our nominal definition of $\mathrm{f}_{\text {gas }}$ (Eq. 25 .
} 
This said, there is some tension between galaxy formation models and observed gas fraction in galaxies. Generally, most galaxy formation models predict galaxy gas fractions at a given mass a factor of a few lower than what is observed (e.g. Bouché et al. 2010; Dutton et al., 2010; Fu et al., 2012b; Haas et al., 2013, Davé et al., 2012). What is particularly disconcerting about this disagreement is that these models utilize a wide-range of modeling techniques: the problem is pervasive in galaxy formation theory. This is clear from Figure 46, where we show the model predictions of several groups (Benson, 2012, Lagos et al. 2012, Fu et al. 2012b, Davé et al. 2012; Popping et al., 2013) in comparison to observational determinations. This implies either a fundamental problem in our theoretical understanding of how galaxies grow over cosmic time, or an issue in our calculation of gas masses in high- $z$ galaxies.

One possible solution to this mismatch has been offered by Narayanan et al. (2012a), who suggested that the canonical "ULIRG" conversion factor $\left(\alpha_{\mathrm{CO}}=0.8\right)$ was too large for the most extreme systems at high-redshift, and that the gas temperatures and velocity dispersions were high enough to warrant even lower conversion factors. Recalling $\S 8.2$ these authors suggested that if one uses either an empirically derived form for $\alpha_{\mathrm{CO}}$ from Ostriker $\&$ Shetty (2011), or the theoretically derived continuous form for $\alpha_{\mathrm{CO}}$ from Narayanan et al. (2012b) (Equation 21), typical conversion factors for SMGs will range from $\sim 0.3-0.5$. In this case, there can be reconciliation between the gas fractions of many observed SMGs, and the gamut of theories that predict lower gas fractions. Interestingly, there is at least one observed case of an massive $\left(M_{*} \approx 2 \times 10^{11} \mathrm{M}_{\odot}\right)$ SMG that exhibits a high gas fraction $f_{\text {gas }} \sim 50 \%$, even when considering the aforementioned models for $\alpha_{\mathrm{CO}}$ (Fu et al., 2013).

An alternative theoretical solution has been suggested by Gabor \& Bournaud (2013), who utilized a combination of analytic arguments and high-resolution numerical simulations to show that star formation in galaxies undergoing heavy accretion from the IGM may be delayed owing to energy input into the disk from the accreted gas. The increase in turbulence driven by the accreted gas reduces the star formation efficiency, and allows gas fractions to rise accordingly (though see Hopkins et al., 2013b, for counter arguments).

Finally, Tacconi et al. (2013) suggest that the tension between galaxy gas fractions measured in observations and simulated galaxies may owe to incompleteness in the observations. In particular, when correcting for incomplete sampling of galaxies in the SFR- $M_{*}$ main sequence (owing to SFR cuts), the observed gas fractions can come down in better agreement with cosmological simulations.

Going forward, a key advance will be to construct a large enough sample of galaxies at high-redshift within narrow stellar mass bins in order to derive reliable measurements of the evolution of galaxy gas fractions (at a given stellar mass) with redshift.

\subsection{Molecular Gas Morphology and Dynamics}

Over the past decade, the advent of (sub)mm-wave interferometers, and increased capabilities of radio-wave interferometers have allowed for meaningful samples of CO, FIR, and radio morphologies of high- $z$ dusty galaxies. Here we discuss morphology and dynamics from $\mathrm{CO}$, in contrast to the earlier discussions provided in $\S 5$. The $\mathrm{CO}$ spatial extent can constrain the $\mathrm{H}_{2}$ gas surface density, which aids in placing a galaxy on the Kennicutt-Schmidt star formation relation (c.f. $\S 8.3$. Molecular and atomic line dynamics can provide information regarding the physical origin of a galaxy (i.e. if it is a dynamically hot, as one might expect from a merger, or dynamically cold), and in the cases of multiple $\mathrm{CO}$ lines, can even provide a map of the thermal and density structure in high- $z$ galaxies.

High-resolution observations with the PdBI, VLA and ALMA interferometers have afforded detailed studies of the molecular gas morphology as traced by various CO transitions in high- $z$ SMGs. Molecular line morphologies give both a measure of the emitting-region size for gas surface density measurements, as well as an idea of the dynamics of the gas. As discussed in $\S 8.5$, the excitation of $\mathrm{CO}$ in high- $z$ starburst galaxies is relatively diverse, meaning that different transitions can, at times, trace different spatial extents. In Figure 47, we plot the ratio of the sizes of the FWHM CO line widths of the $\mathrm{CO}(\mathrm{J}=1-0)$ and $\mathrm{CO}(\mathrm{J}=3-2)$ emission lines for all high- $z$ dusty galaxies where both measurements are available. While the line width is not a measure of the emission size directly ${ }^{23}$, it does reflect a combination of the mass enclosed in the emitting region and the spatial extent of the gas. Though the sample sizes are small, some features are clear. The majority of sources have similar line widths in the CO $(\mathrm{J}=1-0)$ transition,

\footnotetext{
${ }^{23}$ We choose to use the line width as a proxy for the emitting region size for two reasons. First, it removes any ambiguity as to how a size is defined from one study to another. Second, the sample size of galaxies that have direct morphology measures of multiple CO transitions is incredibly small.
} 


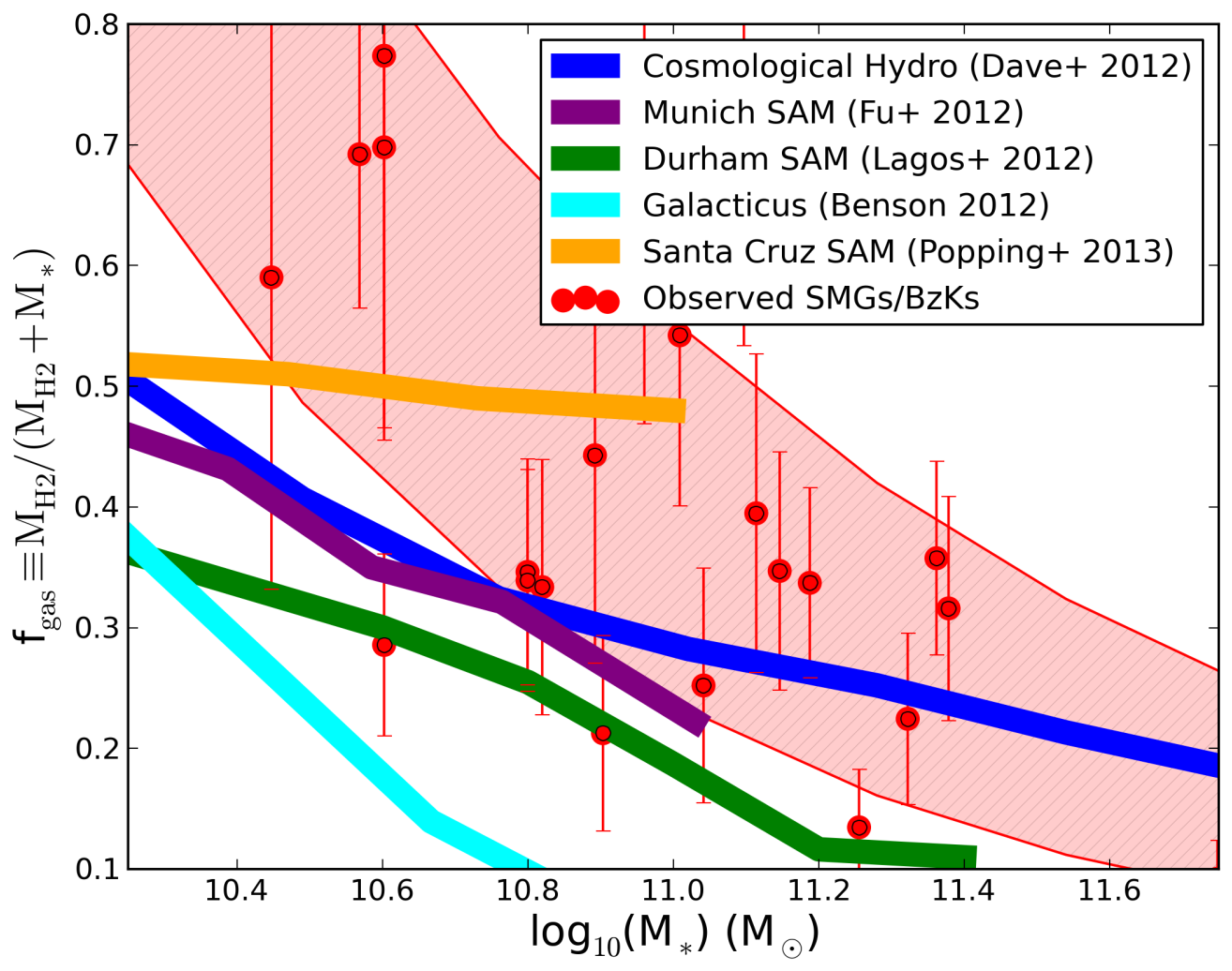

Figure 46: Molecular gas fraction as a function of stellar mass for SMGs (red points). These are compared to theoretical models by Benson (2012); Lagos et al. (2012); Fu et al. (2012b); Popping et al. (2013) and Davé et al. (2012). The red shaded region shows the approximate region spanned by the observations. The models span a range of methods, from bona fide cosmological hydrodynamic simulations to four different Semi-Analytic prescriptions. By and large, inferred gas fractions of observed high- $z$ galaxies are all a factor of a few larger than any theoretical model predicts. The observed gas fractions are computed assuming a CO- $\mathrm{H}_{2}$ conversion factor of $\alpha_{\mathrm{CO}}=0.8$ for SMGs, and $\alpha_{\mathrm{CO}}=4$ for $B z K$ galaxies, which, for better or worse, is the canonical assumption in the literature. Given the large spread in potential CO excitation (c.f. $\$ 8.5$, we only consider galaxies with $\mathrm{CO}(\mathrm{J}=1-0)$ detections to remove the uncertainty in down-converting high-excitation lines to the ground transition. The $\mathrm{CO}(\mathrm{J}=1-0)$ measurements were reported by Aravena et al. (2010 2012); Baker et al. (2004); Bothwell et al. (2010) 2013a); Carilli et al. (2010); Daddi et al. (2010a); Fu et al. (2013); Greve et al. (2003); Hainline et al. (2006); Ivison et al. (2011).2013); Scott et al. (2011); Sharon et al. (2013); Swinbank et al. (2011); Riechers et al. (2011c |2013b). 


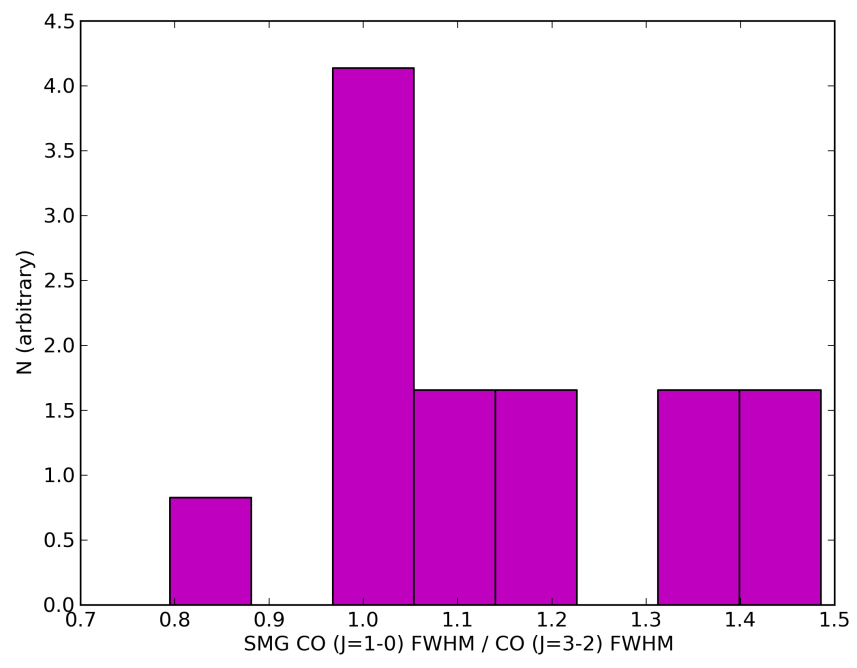

Figure 47: $\mathrm{CO} \mathrm{J}=1-0 / \mathrm{CO} \mathrm{J}=3-2$ line width ratios. The power toward high ratios suggests stratification in the gas density, temperature or volume filling factor, and hence, $\mathrm{CO}$ emitting region for high-J lines. The data are compiled from the Carilli \& Walter (2013) compendium.

and the denser gas tracer, $\mathrm{CO}(\mathrm{J}=3-2)$, though there is a clear high ratio tail where the $\mathrm{CO}(\mathrm{J}=1-0)$ line width is roughly $10-50 \%$ larger than the $\mathrm{CO}(\mathrm{J}=3-2)$ emitting region. This effect is expected to be more dramatic when considering even higher-lying transitions (which are often probed in high-redshift galaxies due to a combination of telescope instrumentation and the desire for the highest spatial resolution studies possible). Care must be taken when interpreting results that depend on $\mathrm{CO}$ dynamics that derive from high-lying lines.

The molecular gas that has been mapped in normal disk-like galaxies at high- $z$ tends to be extended, compared to local starbursts. This is notable because the global SFRs of high- $z$ disks can oftentimes be comparable to those of compact nuclear starbursts in the local Universe (Daddi et al., 2005). Aravena et al. (2010) and Daddi et al. (2010a) find that $B z K$ galaxies are extended on scales of $\sim 6-10 \mathrm{kpc}(\mathrm{FWHM})$ in the $\mathrm{CO}(\mathrm{J}=1-0)$ and $(\mathrm{J}=2-1)$ transitions. Tacconi et al. (2013) find CO (J=3-2) sizes $\left(R_{1 / 2}\right)$ for a large sample of $z \sim 1-2$ disks ranging from $4-10 \mathrm{kpc}$, and a reasonable match between the $\mathrm{CO}$ size and the optical/UV size.

Turning to starbursts, the number of bona fide $\mathrm{CO}(\mathrm{J}=1-0)$ detections of high- $z$ dusty starbursts are few, owing to the lack of sensitive radio interferometers preceding the VLA. Utilizing the VLA, Ivison et al. (2011) found CO (J=1-0) FWHM sizes of $\sim 16 \mathrm{kpc}$ from a sample of SMGs, distinctly more extended than the $\sim 1-3 \mathrm{kpc}$ halflight radii derived from higher- $J$ lines by Tacconi et al. (2008). Similarly, Riechers et al. (2011c) find source radii of $\sim 3-15 \mathrm{kpc}$ in a sample of 3 SMGs detected in $\mathrm{CO}(\mathrm{J}=1-0)$, and note again that these sizes are notably more extended than the $\mathrm{CO}(\mathrm{J}=4-3)$ and $(\mathrm{J}=6-5)$ sizes measured by Engel et al. (2010) for the same galaxies. Interestingly, Riechers et al. (2011a) inferred, based on CO line ratios, that the $\mathrm{J}=1-0$ emitting region from a sample of quasar host galaxies at similar redshifts to the aforementioned SMG studies $(z \sim 2)$ is relatively compact. One could use this data to argue that QSOs and SMGs derive from distinctly different galaxy populations (though would have to reconcile the similar clustering measurements; Hickox et al., 2012), or that quasars derive from SMGs after undergoing a size transformation (as might be expected from a galaxy merger). We emphasize that the radii for many SMGs that have extended CO ( $\mathrm{J}=1-0)$ sizes are $\lesssim 4 \mathrm{kpc}$ when probing higher lying transitions (Downes \& Solomon, 2003; Genzel et al., 2003, Neri et al. 2003; Tacconi et al., 2006, 2008). Note also that these differential sizes for high-excitation gas reservoirs can also exist in quasars, a problem which could be exacerbated in lensed sources due to differential amplification. The differences in source radii likely derive in part from a density or excitation stratification in the gas, but also in part from a genuine diversity in the source population.

One of the principle results of high-resolution CO mapping of starbursts at high- $z$ has been for studies of the dynamics of the gas. Maps of exquisite resolution of $z \sim 1-2$ disks have provided the first clear evidence for ordered 
rotation and galaxy disks at high- $z$ (Genzel et al., 2003, Daddi et al., 2010a, Tacconi et al., 2010, 2013). A large step forward was made by Tacconi et al. (2010), who presented a rotation curve out to $\sim 8 \mathrm{kpc}$ from the galaxy's center of a $z \sim 1$ disk galaxy, then from Swinbank et al. (2011) who presented the disk-like kinematics in the Cosmic Eyelash at $z=2.3$ (see $\$ 6.2$ ).

For higher-luminosity (starburst) systems at high-z, the main thrust for obtaining CO-based dynamics has been with the motivation of understanding whether or not these systems owe their extreme luminosities and star formation rates to galaxy mergers, or whether they may result from secular processes within a galaxy disk. The results are varied. Some observations have found clear evidence for rotating molecular disks in even extremely bright SMGs and quasars (e.g Tacconi et al., 2008, Bothwell et al., 2010, Carilli et al., 2010; Hodge et al., 2012, Deane et al., 2013)), while other studies have found more seemingly disrupted systems when examining the velocity contours (Tacconi et al., 2008; Engel et al., 2010, Bothwell et al., 2013a, Riechers et al., 2011b, 2013b). Still other groups have shown potentially extremely convincing evidence for ongoing mergers at high- $z$ by showing multiple counterparts that are potentially two galaxies caught in the act of merging, prior to final coalescence (e.g. Engel et al., 2010, Yan et al., 2010; Riechers et al. 2011a; Ivison et al., 2013, Fu et al., 2013). This said, there are reasonable counter-arguments to each of these examples. For example, numerical simulations by Springel \& Hernquist (2005) and Hopkins et al. (2009) have shown that very gas-rich mergers at high-redshift can quickly re-form a gaseous disk soon after the merger, due to the dissipational nature of gas.

Narayanan et al. (2009) showed that for the specific example of galaxy mergers massive enough to form luminous systems comparable to observed SMGs, the synthetic CO velocity contours would show signs of ordered rotation some fraction of the time. Thus, observed rotational signatures in high- $z$ systems do not rule out galaxy mergers. Similarly, Davé et al. (2010) showed that SMGs that are fueled primarily from accretion from the IGM (rather than via major mergers) could still show somewhat disrupted disks, due to the sporadic nature of gas accretion.

Finally, even the seemingly clear-cut case of seeing a Also, the impact of the galaxy merger in driving the observed luminosity is potentially minimal (Narayanan et al. 2009, 2010a, Lanz et al., 2013). As an anecdotal example, a distant observer may consider the Milky Way and Andromeda as a pair of galaxies undergoing a merger (depending on the line of sight), yet neither is a "merger-induced starburst galaxy". The ramifications of these sorts of studies are indeed important. Theoretical models aiming to quantify what fraction of galaxies at a given luminosity are galaxy mergers versus disks produce a wide range of answers (e.g. Baugh et al., 2005, Davé et al. 2010, Dekel et al., 2009a, Narayanan et al., 2010a; Hopkins et al., 2010, González et al., 2011; Hayward et al., 2013b), and any observational constraints in this area are quite valuable (we discuss this issue in more detail in $\$ 10$ ).

What is clear, at present, is that SMGs appear to be a diverse population with respect to their gas kinematics. Clear examples for ordered rotation, non-Keplerian dynamics, and multiple counterparts potentially prior to merging exist. From the dynamical information alone, the submillimeter selection appears to cull a diverse set of systems from high- $z$ galaxies.

Going forward, perhaps one of the most exciting avenues in CO morphology studies of high- $z$ systems will be detailed studies of the ISM in gravitationally lensed systems, comparable to the exquisitely imaged Cosmic Eyelash (see $\$$ 6.2) galaxy by Swinbank et al. (2010) and Swinbank et al. (2011). High-redshift galaxies allow for a unique opportunity to study star formation and ISM structure in a much higher-pressure environment than our own galaxy, and may give clues as to how star formation proceeds in galaxies ranging from local ULIRGs through the most extreme starbursts at high-redshift. Swinbank et al. (2010, 2011) found that in the high-pressure environment of the Eyelash, GMCs lie off of local cloud scaling relations (i.e. "Larson's Laws"), and that at a given cloud radius, the GMCs in the Eyelash has higher velocity dispersion. Interestingly, this is also true for massive, dense clumps in our galaxy (Shirley et al., 2003, and references therein).

These sorts of constraints on the basic physical properties of the ISM in these extreme environments can have significant impact in both the astrophysics of star formation and galaxy evolution. For example, models that invoke a variety of physical mechanisms, from accretion and star formation (Goldbaum et al. 2011) to stellar feedback (Hopkins et al. 2012c, Krumholz et al. 2014) in setting the basic properties of molecular clouds will be impacted by our understanding of the structure of the ISM in these test cases. Similarly, nearly every model for the origin and potential variations in the IMF depend on the physical properties of the molecular ISM on small scales (e.g. Krumholz, 2011b, Hopkins, 2013, Padoan \& Nordlund, 2002, Narayanan \& Davé, 2012, 2013). Because the exact form of the IMF impacts stellar mass estimates, star formation histories, the generation of stellar winds, and interstellar, circum and intergalactic metal enrichment (e.g. Arrigoni et al., 2010; Weidner et al. 2013), these sorts of studies can be 
impactful on a large range of scales.

\subsection{Synthesis}

This is a particularly exciting time for high-redshift star formation and molecular gas studies. With the advent of ALMA, as well as substantial upgrades to other ground based facilities (such as the JVLA and PdBI), we are able to detect molecular lines at high- $z$ in a fraction of the time that was previously required. Similarly, numerical simulations are reaching a point where they can resolve giant molecular clouds on galaxy-wide scales.

Going forward, it will be critical to place strict constraints on the major uncertainties in molecular line observations. These include a comprehensive picture (either a theoretical model, or observed empirical relations) for the $\mathrm{CO}-\mathrm{H}_{2}$ conversion factor, as well as the conversion from high-J $\mathrm{CO}$ states to low $\mathrm{J}$ states.

At the same time, we are lacking a comprehensive understanding of the relationship between the physical state of the ISM, and how star formation subsequently proceeds. How do the density, temperature, and velocity dispersion PDFs affect molecular cloud star formation rates and the initial mass function of stars formed? What is the physical structure of the ISM in high- $z$ galaxies, and how does this impact ongoing star formation? Observations of lensed high-redshift galaxies at high resolution will help elucidate some of these issues. Similarly, observations of both molecular clouds in the Milky Way, and more extreme regions in the local Universe hold great promise for resolving outstanding issues in high-redshift star formation. 


\section{Atomic Lines}

The launch of the Herschel Space Observatory and advent of increasingly sensitive submm spectrometers has allowed for the characterization of FIR fine structure lines at low and high-redshift respectively. The predominant lines studied are those of neutral and ionized carbon, nitrogen and oxygen, with a particular emphasis on the [CII] ionized fine structure line. These lines are the dominant cooling lines for diffuse ISM, and can provide a diagnostic into both the cold neutral medium, HII regions, and photodissociation regions (PDRs) in galaxies. Accordingly, we review here what is known about $[\mathrm{CII}]$ at high-redshift, though we note results from other lines when appropriate.

The [CII] $158 \mu \mathrm{m}$ line (in reality $157.7 \mu \mathrm{m}$ ) is a fine structure line which is thought to be excited by collisions with neutral hydrogen, or with free electrons and protons when the electron density is sufficiently high. Important sources of free electrons are from dust grains and PAHs via photo-electric heating (Draine, 1978; Helou et al., 2001) from UV photons.

The first detection of [CII] at high- $z$ was presented by Maiolino et al. (2005), who detected the line in the luminous $z=6.4$ quasar host galaxy J1148+5251. Since then, the number of detections of the $158 \mu \mathrm{m}[\mathrm{CII}]$ emission line at highredshift have grown rapidly. Luminous quasar host galaxies (e.g. Maiolino et al., 2005; Iono et al., 2006; Maiolino et al. 2009, Walter et al., 2009; Wagg et al. 2010, Stacey et al. 2010, Gallerani et al., 2012, Carniani et al., 2013), SMGs (e.g. Ivison et al. 2010d, Stacey et al. 2010; Cox et al. 2011; De Breuck et al., 2011; Carilli et al., 2013, Swinbank et al., 2012; Wagg et al. 2012; George et al., 2013; Huynh et al., 2013) and normal star-forming galaxies (e.g. Graciá-Carpio et al. 2011) at high-redshift have all been detected in [CII]. The line is thought to be an important coolant in the ISM, and can make up as much as $1 \%$ of the far infrared luminosity of a galaxy (Nikola et al., 1998, Malhotra et al. 2001; Stacey et al. 2010). However, the sites of origin of [CII] emission can be diverse, and thus its power as a diagnostic for the physical conditions in the ISM of early Universe galaxies is still debated. Because it has a relatively low ionization potential $(11.3 \mathrm{eV}$, compared to the $13.6 \mathrm{eV}$ characteristic of $\mathrm{HI})$, [CII] can arise from both neutral gas, as well as ionized regions. Beyond this, the critical densities for [CII] emission can range from $\sim 5-50 \mathrm{~cm}^{-3}$ for collisions with electrons, to $\sim 1-8 \times 10^{3} \mathrm{~cm}^{-3}$ for collisions with neutral atomic or molecular hydrogen (Goldsmith et al., 2012). Hence, the line is relatively easy to excite.

At the same time, other lines provide complementary information to $[\mathrm{CII}]$ in high- $z$ galaxies. For example, $[\mathrm{NII}]$ at $205 \mu \mathrm{m}$ has an ionization potential of $14.5 \mathrm{eV}$, and thus traces ionized ISM. Because the [NII] transition has a critical density and second ionization potential very similar to that of [CII], the ratio of the two lines can serve as a diagnostic for the amount of [CII] arising from the ionized medium (e.g. Decarli et al. 2014).

\subsection{The [CII]-FIR deficit in Galaxies}

The Milky Way has a [CII]/FIR luminosity ratio of roughly 0.003 , as do other nearby disk galaxies with some scatter. In the early days of [CII] observations of low-redshift galaxies with the Long Wavelength Spectrometer (LWS) on the Infrared Space Observatory (ISO), it was recognized that very luminous infrared galaxies such as ULIRGs appear to have a deficit in [CII] emission compared to their FIR luminosities. However, present-epoch ULIRGs emit roughly 10\% of the expected [CII] flux, given their far infrared luminosities (e.g. Malhotra et al., 1997, 2001; Luhman et al., 1998, 2003). A variety of possible explanations have been posited for the apparent deficit. These include optically thick [CII] emission in the presence of large dust columns. Alternatively, if the UV radiation fields are softer owing either to dust extinction in compact starbursts, or a varying IMF, there may be less heating of hydrogen and electrons, and hence less collisions with ionized carbon. Other explanations for the [CII]/FIR deficit include high ionization parameters in the vicinity of an AGN, and saturated [CII] emission in extremely dense environments. (e.g. Luhman et al., 1998, Abel et al., 2009; Papadopoulos et al., 2010; Sargsyan et al., 2012). Based on the existence of a deficit in [NII] (among other lines, such as [OI], Farrah et al. (2013) proposed a combination of a harsher interstellar radiation field in HII regions with increased dust grain charging in the ISM as a potential origin of the line deficits. In Figure 48, we plot the current state of CII-FIR measurements for low and high- $z$ galaxies. The grey points denote low- $z$ galaxies, and highlight the deficit of $L_{[\mathrm{CII}]}$ at high FIR luminosities.

The results from high- $z$ systems have been more mixed. Stacey et al. (2010) compiled a number of new detections with literature measurements to suggest that the [CII]-FIR deficit persists when including high- $z$ galaxies. The high- $z$ points that show a deficit in this study tended to host an AGN. Wang et al. (2013a) found that the [CII]/FIR deficit also persisted in their sample of five $z \sim 6$ quasars detected by ALMA, and Rawle et al. (2013) found a deficit for an extreme starburst SMG at $z \sim 5$. Similarly, Iono et al. (2006) found [CII]/FIR ratios comparable to local ULIRGs in a 
$z \sim 5$ quasar. This deficit is potentially not confined to [CII] emission. Graciá-Carpio et al. (2011) examined the [CII] emission properties (along with a host of other FIR lines, including [OI], [OIII], [NII] and [NIII]), and found evidence for a (line flux)/FIR deficit for all of these lines. Similarly, Farrah et al. (2013) found deficits in [OI] 63, [OI] 145, [NII] and [CII] at high $L_{\mathrm{IR}}$. Graciá-Carpio et al. (2011) found that when plotting the deficit against $L_{\mathrm{FIR}} / M_{\mathrm{H} 2}$ instead of $L_{\mathrm{FIR}}$, the deficit began uniformly around $L_{\mathrm{FIR}} / M_{\mathrm{H} 2} \approx 80 \mathrm{~L}_{\odot} / \mathrm{M}_{\odot}$ for each of the emission lines. This result was confirmed for [NII] by Decarli et al. (2012). Interestingly, Pope et al. (2013) find a deficit in $L_{\mathrm{PAH} 6.2} / \mathrm{L}_{\mathrm{IR}}$ with $\mathrm{L}_{\mathrm{IR}}$ for both local and high- $z$ DSFGs, with a similar offset as is possibly seen in the [CII]/FIR deficit in high- $z$ galaxies.

On the other hand, some studies have found no deficit when examining [CII], as well as other nebular lines (e.g. Hailey-Dunsheath et al., 2010; Wagg et al., 2010, De Breuck et al., 2011; Ferkinhoff et al., 2011; Swinbank et al., 2012; Coppin et al., 2012). These studies have found that at typical ULIRG luminosities, where local galaxies exhibit a clear [CII]/FIR deficit, some high- $z$ SMGs appear to show a [CII] excess.

The issue may be that comparing galaxies at low and high- $z$ via simple luminosity bins is comparing apples and oranges. At a given stellar mass, galaxies at high- $z$ have a higher SFR (and hence, $L_{\mathrm{FIR}}$ ) than a $z=0$ analog. Similarly, at a fixed $L_{\mathrm{FIR}}$, galaxies at high- $z$ tend to be more spatially extended than present epoch counterparts, which may mitigate whatever physical mechanism produces a deficit in local galaxies. Indeed, further examination of Figure 48 suggests that perhaps the [CII]-FIR deficit continues to persist at high- $z$, though the relation is simply shifted in FIR luminosity. Hence, one reasonable approach for future studies may involve comparing the [CII]/FIR ratio against luminosity surface density, as in Díaz-Santos et al. (2013).

\section{2. [CII] as a Star Formation Rate Indicator}

The role of [CII] as a SFR tracer in galaxies is under debate, though it is a topic of great interest in the community. If a reasonable calibration between [CII] and SFR can be determined, the line would serve as a powerful probe of high- $z$ galaxies. At the least, [CII] does not suffer from extinction as heavily as more traditional shorter-wavelength SFR tracers.

Leech et al. (1999) presented an examination of [CII] emission from 19 Virgo cluster spirals, and found a positive correlation between the line luminosity and FIR luminosity, with less dispersion than is seen in local ULIRGs. Boselli et al. (2002) found a relation between [CII] and $\mathrm{H} \alpha$ luminosity in galaxies.

de Looze et al. (2011) presented an analysis of [CII] emission from 24 nearby star-forming galaxies, and compared the line luminosity to star formation rates determined from UV data (taken from GALEX), and MIPS $24 \mu \mathrm{m}$ fluxes. These authors find a relatively tight correlation between the SFR of galaxies and [CII] luminosity between $\log$ (SFR) $\approx[-1,2]$. de Looze et al. (2011) attribute the positive correlation to two possible explanations. The first is that the [CII] flux from PDRs comes from the regions extremely close to star-forming regions, near the border of HII regions and neutral gas. The second is that the $[\mathrm{CII}]$ emission principally arises from cold neutral medium, and the relation between SFR and [CII] emission is simply a manifestation of the global Kennicutt-Schmidt law. Similarly, Sargsyan et al. (2012) found that, when excluding AGN selected by the $6.2 \mu \mathrm{m}$ PAH equivalent width, [CII] correlates well with the $L_{\mathrm{IR}}$ of nearby galaxies, and is thus a reasonable SFR tracer in these environments. These authors further suggest that the [CII]-FIR deficit therefore owes to increased contribution to the infrared luminosity by embedded AGN. Farrah et al. (2013) report an empirical calibration of the [CII]-SFR relation as well.

On the other hand, the existence of a deficit of [CII] emission at high infrared luminosities (with the deficits beginning at seemingly increasing IR luminosity at increasing redshifts; c.f. Figure 48) suggests that [CII] may not be a robust tracer of a galaxy's SFR, at least in the high luminosity regime. Beyond this, as is apparent from Figure 48, at a given infrared luminosity, there is approximately an order of magnitude scatter in the [CII]/FIR ratio. Some of this scatter may owe to the fact that [CII] can arise from both neutral and ionized gas. Sargsyan et al. (2012) and Farrah et al. (2013) show a $\sim 1$ dex scatter in their SFR-[CII] relations.

\section{3. [CII] Morphologies and Dynamics}

At high-redshift, utilizing $\mathrm{CO}$ as a tracer of the morphologies and dynamics of neutral gas can become problematic. First, low-metallicity galaxies will suffer from a decreased abundance of $\mathrm{CO}$, making the molecule harder to detect (c.f. $\S 8.2$. Second, low $\Sigma_{\text {SFR }}$ galaxies will typically have lower $\mathrm{CO}$ excitation. At increasing redshifts, a given receiver detects increasingly high excitation lines, which may be faint in low $\Sigma_{\text {SFR }}$ galaxies. Both of these have motivated the exploration of alternative gas dynamical tracers in high- $z$ galaxies, such as [CII]. 


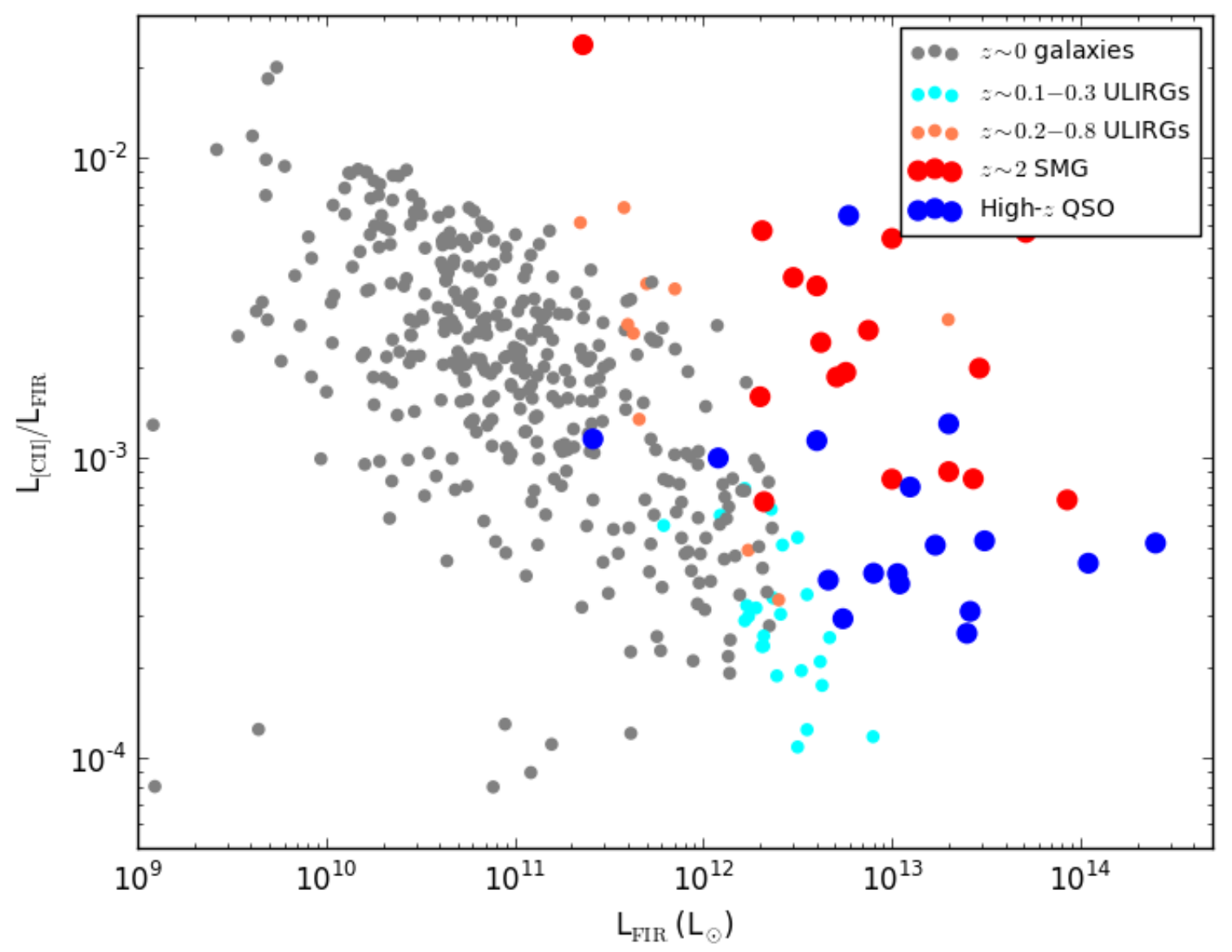

Figure 48: Relationship between $L_{\mathrm{CII}} / L_{\mathrm{FIR}}$ ratio and $L_{\mathrm{FIR}}$ for both local galaxies and galaxies at mid $(z \sim 0.1-0.3)$ and high- $z$. At a fixed redshift, there appears to be a deficit in the [CII]/FIR ratio at high FIR luminosities. This said, the high- $z$ points appear to be offset from the low- $z$ data. The local data is compiled by Brauher et al. (2008), with new data by Díaz-Santos et al. (2013) from the GOALS survey included as well. The data at moderate redshifts is from Farrah et al.|2013) and Rigopoulou et al. (2014). The high- $z$ data is subdivided into quasars and SMGs, and comes from Cox et al. (2011); De Breuck et al. (2011); George et al. (2013); Graciá-Carpio et al. (2011); Ivison et al. (2010d); Maiolino et al. (2005); Rawle et al. (2013); Stacey et al. (2010); Swinbank et al.(2012); Valtchanov et al. (2011); Venemans et al. (2012); Wagg et al. (2012); Wang et al. (2013a); Willott et al. (2013) 
A number of studies have used extremely high resolution [CII] observations to constrain the size of the emitting region of high- $z$ quasar host galaxies. Walter et al. (2009) derived a source size of $\sim 1.5 \mathrm{kpc}$, and suggested that the galaxy was undergoing an Eddington-limited starburst event. Similar compact sizes were derived for two $z \sim 4$ quasar host galaxies by Gallerani et al. (2012) and Carniani et al. (2013), who also detected companion galaxies potentially due to merge with the quasar host. Carniani et al.(2013) and Wang et al.(2013a) displayed the power of using ALMA to examine the dynamics of [CII]-emitting gas in quasar host galaxies, and showed that a number of galaxies in their sample potentially exhibited rotating gas disks.

This all said, there have been a number of notable non-detections of [CII] at high- $z$ that have called into question the reliability of [CII] as a gas tracer in high- $z$ galaxies. For example, Kanekar et al. (2013) searched for [CII] emission from a lensed Lyman- $\alpha$ emitter at $z \sim 6.5$, and showed a non detection. Similarly, Ouchi et al. (2013) utilized ALMA to search for [CII] from Himiko, a luminous galaxy at $z \sim 6.5$ that forms stars at $\sim 100 \mathrm{M}_{\odot} \mathrm{yr}^{-1}$. These authors showed a non detection to limits $L_{\mathrm{CII}}<5.4 \times 10^{7} \mathrm{~L} \odot$. If these results are confirmed by followup deep observations of Himiko and other similar galaxies, they may suggest that [CII] emission may not be ubiquitous in early-Universe galaxies, even those forming stars at a relatively prodigious rate. Whether the lower metallicity of Himiko drives the [CII] non-detection is an open question. Further investigation into the origin of [CII] emission, and its value both as an SFR tracer, as well as gas dynamics probe are clearly warranted.

Going forward, increased observational constraints on the origin of [CII] emission in star-forming galaxies will be critical. Recently, Decarli et al. (2014) examined the unique $z \sim 4.7$ interacting system BR1202-0725 in [NII] and [CII]. BR1202 consists of a quasar, SMG and two Lyman- $\alpha$ emitters. These authors found large [CII]/[NII] ratios for the QSO and SMG, though ratios closer to unity for one Lyman- $\alpha$ emitter, suggesting ionized gas as the principle source of $[\mathrm{CII}]$ emission in the latter source.

Finally, we conclude this section by briefly noting that observations of neutral atomic carbon, CI, are gaining traction in recent years. CI is a simple three level system that is typically optically thin-detection of multiple lines allows for observed constraints on the excitation temperature and column density (of carbon), and serves as a crosscheck against values derived from CO (Walter et al., 2011; Alaghband-Zadeh et al., 2013). 


\section{The Theory of forming Dusty Galaxies}

The theory of forming dusty galaxies at high-redshift has been a confounding, frustrating, and difficult subject under the general umbrella topic of astrophysical galaxy formation. As we will present in this section, while theorists have thrown every tool in their toolbox at the problem, and the methods that aim to model the origin and evolution of high- $z$ starbursts are extremely diverse, nearly all models struggle with (generally different) aspects of matching observations of high- $z$ dusty galaxies.

By and large, as with observations, the principle focus of theorists modeling DSFGs has been in modeling the high$z$ Submillimeter Galaxy population. This said, surveys of other dusty galaxy populations with Spitzer and Herschel have motivated some targeted modeling efforts (e.g. Lacey et al. 2008, 2010; Narayanan et al., 2010a, Niemi et al., 2012), and have at the least provided a strong constraint on general galaxy formation models (e.g. Somerville et al., 2012).

Our principle aims in this section are to overview the general methods employed in modeling high- $z$ dusty galaxies, and to describe the main differences in them, along with some of their strengths and weaknesses. We will then summarize the major results in the theoretical literature of dusty galaxy formation over the last decade, and conclude the section with an outline of key differences and observable tests of the models. Finally, we note that this section is a review solely of the formation of dusty galaxies. For a more comprehensive recent review of galaxy formation theory, please see Benson (2010).

\subsection{Overview of Dusty Galaxy Modeling Methods}

In this section, we will first overview the principle methods that have been used in modeling high- $z$ dusty systems, paying particular attention to how they model the details of the FIR/submm emission. The modeling methods fall into three broad classes: Semi-Analytic Models (SAMs), Cosmological Hydrodynamic Simulations, and Hybrid Models. We will briefly discuss Empirical (also known as "Backwards Evolution") methods in the models section as they are often described as "models" and compared to observations. This said, as we will point out in this section, while empirical methods are useful in predicting the evolution of observed source counts, they are not bona fide models. Rather, they are purely based on extrapolation of observations, and thus not predictive theories of galaxy formation. Hence, our discussion of these types of methods in this review will be limited. We summarize the salient points of galaxy formation modeling methods in Table 4 as they pertain to DSFG modeling.

The main purpose in this section is to highlight both the complementarity between methods, as well as the basic level at which astrophysical processes are no longer directly simulated, but rather implemented in the simulations via an analytic prescription. In principle, every simulation requires an analytic prescription at some level. The difference in scale where these prescriptions are imposed vary dramatically for galaxy formation models that aim to simulate dusty galaxies, and can range from prescriptions on the parsec scale, to prescriptions on dark matter halo-scales. In Table 5. we summarize the main points made in this section, and in particular, outline key testable predictions made by many of the major SMG modeling groups.

\subsubsection{Semi-Analytic Models}

The term "Semi-Analytic Modeling" (SAMs) typically refers to modeling methods that draw on a combination of both numerical simulations (to model the cosmic evolution of dark matter halos), and analytic approximations (to model the structure of galaxies, and physics associated with the baryonic/luminous component of galaxies). The original framework itself goes back to seminal papers by White \& Frenk (1991), Cole (1991) and Lacey \& Silk (1991), and was advanced substantially by a number of groups (Baugh et al. 1996, 1998, Kauffmann et al., 1993, Kauffmann, 1996; Kauffmann \& Charlot, 1998, Kauffmann et al. 1999: Somerville \& Primack, 1999). Because SAMs are typically a combination of large-scale dark matter only simulation ${ }^{24}$ with fairly detailed analytic prescriptions of galaxy formation and evolution, particular SAMs that drive a large number of papers are typically referred to in the literature by their host institution/city, such as the "Durham SAM", the "Santa Cruz SAM", or the "Munich SAM". One exciting aspect of SAMs is that they are relatively inexpensive to run. Because of this, it can be quite inexpensive

\footnotetext{
${ }^{24}$ It should be noted that some subset of SAMs build upon analytic prescriptions that have been calibrated from large cosmological dark-matter only simulations, rather than via direct numerical simulation.
} 
to run a large range of model parameter choices (and variations on prescriptions for physical processes) with the SAM methodology (e.g. the new publicly available code, Galacticus Benson, 2012). Because of this, SAMs are readily used for isolating the effects of an individual astrophysical process (see Croton et al., 2006, for an example of this.). One promising way forward with SAMs is the construction of large grids of models that aim to constrain galaxy formation parameter spaces via comparison to observations through Markov Chain Monte Carlo models (e.g. Lu et al., 2011, 2012).

For the majority of SAMs, the only directly simulated quantity is the dark matter-only structure formation models. With the assumption of a given cosmology and initial perturbation spectrum, these simulations are evolved forward to capture the gravitational collapse of dark matter halos, and their mergers with cosmic time. The analytic prescriptions by which the baryonic physics is treated is usually what sets the principle distinction between different SAMs. A small number of SAMs include additional layers of simulated quantities (e.g. Lagos et al., 2013), though to our knowledge no SAM numerically solves the Euler equations, and typically treat the geometries of galaxies as highly simplified. In Table 2 of his review article, Benson (2010) summarizes some of the key physical processes that are (or are not) captured by five major semi-analytic codes, though the detailed treatment of an individual physical process (e.g. star formation) can vary from model to model, and even individual models (e.g. the Durham Model) can have a number of sub-branches that differ in detail. Generally, the analytic prescriptions for baryonic physics that are incorporated into SAMs include gas cooling, feedback from stellar evolution and AGN, star formation in molecular gas, and chemical enrichment of the ISM and IGM. The structure of galaxies is necessarily derived via simplified prescriptions (e.g. exponential disks; disks with some bulge component; ellipticals).

The models of galaxies are related to observations in a semi-analytic framework typically via one of two methodologies. The first is to directly apply dust radiative transfer simulations to the galaxy model outputs. A grid of dust distribution and stellar sources is set up in a simplified geometry (e.g. an axisymmetric disk with a bulge component) corresponding to the assumption of the source structure in the SAM. The emission is then calculated from this analytic geometry self-consistently with a code such as GRASIL (e.g. Silva et al., 1998). Because the source geometry is analytically defined, it is nontrivial to describe the resolution of a SAM, other than the typical force resolution of the base dark matter-only simulation. The model galaxies are typically broken up into some number of grid elements for the GRASIL calculations, though within that, sub-resolution molecular clouds are included making the definition of 'resolution' even more complicated to define in the SAM framework. A key advantage of this formalism is that the computation time is relatively quick, allowing for parameter-space surveys in (e.g.) the assumed IMF or dust content. This methodology has been employed by (as an example) Baugh et al. (2005); Lacey et al. (2008); González et al. (2011) and Fontanot \& Somerville (2011).

The other common means for prescribing the SEDs to galaxies assigned to halos in SAMs is via SED templates derived for observed galaxies (e.g. Dale \& Helou, 2002; Chary \& Elbaz, 2001; Rieke et al., 2009). These templates, calibrated for $z \sim 0$ LIRGs and ULIRGs, can usually be parameterized in terms of the bolometric luminosity of the galaxy, which is a readily available quantity from SAMs. Because these methods are rooted in observations, the SEDs are not predictive. This method is advantageous in that it is even faster than the aforementioned coupling of simplified geometries with dust radiative transfer codes, is not dependent on the particular geometry assumed for a given galaxy, not dependent on the resolution of a gridded model, and is grounded in observational data. The uncertainty, of course, is whether locally-calibrated observational templates are applicable to galaxies at the same luminosity at high- $z$. A recent example of this sort of application of observed SED templates to SAMs is presented in Somerville et al. (2012); Kim et al. (2014).

\subsubsection{Cosmological Hydrodynamic Simulations}

Cosmological hydrodynamic simulations build on the dark matter structure formation models that are the base of SAMs, and hydrodynamically simulate the evolution of the baryonic components of galaxies. This is the major difference between SAMs and cosmological hydrodynamic simulations: the former assign the physical properties of galaxies via analytic models, while the latter derive them from bona fide hydrodynamic simulations. Each has distinct advantages, and the methods are complementary. SAMs provide the only feasible possibility for surveying parameter spaces, and describing a reasonable landscape within which to pursue more costly simulations. Hydrodynamic simulations offer the advantage of potentially providing a more realistic model for the formation and evolution of galaxies, but are so costly that they typically can only explore a few parameter choices. When a set of simulations do not match 
an aspect of observations (as they are bound to do), it can be very difficult in cosmological hydrodynamic simulations to identify the source of the mismatch.

A number of publicly available codes exist for the purposes of cosmological simulations that are commonly employed 25 , such as AMIGA, GADGET, ENZo, Flash, and RAMSEs (Knebe et al., 2001; Springel, 2005; The Enzo Collaboration et al. 2013, Fryxell et al., 2000; Teyssier, 2002), as well as other well-known and tested codes that are not necessarily public (e.g. GASOLINE, ART and AREPo; Wadsley et al., 2004, Kravtsov, 1999, Springel, 2010, ${ }^{26}$ The physics included in hydrodynamic cosmological simulations is varied even among a common set of codes, and is certainly extremely diverse when considering simulations run with different codes. The hydrodynamic methods employed vary from code to code as well, ranging from particle based methods (i.e. smoothed particle hydrodynamics; SPH), to grid-based codes (most principally adaptive mesh refinement; AMR), to hybrid-based methods. In many ways, the different methods agree between one another, though in others there can be stark differences (see, e.g., Nelson et al., 2013, for a recent example.). A number of code comparison projects have been performed, and are currently underway (e.g. Scannapieco et al., 2012, Kim et al., 2014).

\subsubsection{Idealized and Hybrid Models}

As a complementary method to cosmological hydrodynamic simulations, a number of groups have explored the formation of dusty galaxies via idealized hydrodynamic galaxy simulations. These typically involve evolving forward the hydrodynamic properties of baryons in galaxies that have been initialized with idealized conditions. These are generally disk galaxies, or mergers between two disks. These have an advantage of allowing for relatively high resolution (a few-100 pc). The disadvantage, of course, is that there is no knowledge of the cosmological context of the galaxies. So, perturbations in the galaxy structure due to environment are lost, as well as the ability to simulate surveys of galaxies.

These simulations are typically coupled with dust radiative transfer to calculate their observed SED properties. This is their main advantage when comparing to SAMs or cosmological hydrodynamic techniques. Idealized simulations offer high enough resolution that the distribution of luminous sources and dust are well-known for the calculation of the emergent SED. Similarly, idealized simulations typically offer high temporal resolution, which allows for studies in the color evolution of galaxies.

In order to infer cosmological statistics from idealized simulations, a number of groups (e.g. Hopkins et al., 2010, Hayward et al. 2013b) have developed techniques where they combine duty cycles of particular events with galaxy merger rates and mass functions derived from cosmological dark matter-only simulations. As an illustrative example, Hayward et al. (2013b) inferred the number counts of $850 \mu \mathrm{m}$-selected SMGs by running a large suite of idealized disk galaxies and mergers over a large range of galaxy masses and merger mass ratios through dust radiative transfer calculations. This generated the typical submm duty cycle as a function of galaxy mass and merger mass ratio. These authors then combined these duty cycles with observed stellar mass functions from $z \sim 2-4$ (in order to derive the contribution to the submm number counts from non-interacting galaxies), as well as theoretical galaxy-galaxy merger rates derived from cosmological simulations (in order to add in the contribution from galaxy mergers). This technique has the advantage of effectively keeping $\sim 1-100$ pc resolution over large volumes (e.g. Hopkins et al., 2013c a d), which is entirely unfeasible with a bona fide cosmological simulation. The downside is that the impact of events such as gas accretion from the intergalactic medium, or galaxy harassment in dense environments may not be captured in this technique (though see Moster et al., 2014, for a novel methodology that can include some of these effects).

These sorts of hybrid models are unlike cosmological simulations or SAMs in that they cannot be evolved forward to infer the properties of the descendents of high- $z$ DSFGs. However, as we will discuss, there are a large number of observations that can constrain the models. These range from the mean physical properties of the galaxies of interest (e.g. SFRs, gas fractions, stellar masses and halo masses) to statistical inferences such as the breakdown of galaxy morphologies, and overlap with other galaxy populations.

\subsubsection{Empirical Methods}

A number of empirical methods exist in the literature to predict the evolution of number counts at different wavelengths. These methods are not 'models' as the SAMs, cosmological simulations, or hybrid methods discussed pre-

\footnotetext{
${ }^{25}$ Throughout, we refer to successors of a given code [e.g. GADGET-2, GADGET-3 by the base code name, and leave off the version number.

${ }^{26}$ A relatively up-to-date wiki describing both these codes, as well as a number of astrophysical codes is available at http://astrosim.net/code/.
} 
viously in that they provide no physical information about the observed galaxies, and almost solely utilize observed parametric forms for luminosity functions and galaxy SEDs in order to make their predictions. In other words, rather than being ab initio models of galaxy formation and evolution, the empirical methods are phenomenological models with little to no physics that are constrained to fit the existing observations when making predictions for future surveys. This said, they provide strong constraints both on the SED shapes and potential evolution of galaxy luminosity functions, and are well-used in the literature as a consequence.

While the details are varied, the basic principles underlying empirical methods are similar. At their heart, empirical methods rely on combining observed SED templates of galaxies with observed luminosity functions. The free parameters in the luminosity function are then tuned such that the model matches the known number counts of existing populations in order to make predictions for surveys at as yet unobserved wavelengths and/or redshifts. This is, of course, a simplistic description of empirical methods, and indeed many groups have added significant complexities to this basic idea. For example, Negrello et al. (2007) combined phenomenological models of starburst galaxy SED evolution with the Granato et al. (2004) SAM in order to make specific predictions regarding the counts of SMGs when considering lensed populations.

\subsection{Main Results from Theories of Dusty Galaxies}

As alluded to in $\S 10.1$, forming dusty galaxies at high- $z$ has been a confounding problem for theorists across the board. In this section, we summarize the main results over the past decade, divided by the general class of methods that have attempted to understand luminous sources at high- $z$. As the reader will notice, coming up with galaxies in simulations that can reproduce some observed and inferred physical attributes of high- $z$ dusty galaxies is fairly straight forward. Doing so while also matching constraints from other low or high- $z$ galaxy populations simultaneously is significantly harder. To our knowledge, no model to date has simultaneously matched the observed number counts of SMGs while also matching their inferred physical properties, as well as the $z \sim 0$ stellar mass function in an $a b$ initio manner. A comparison of some of the outlined methods may also be found in van Kampen et al. (2005). As a reference for the forthcoming section, in Figure 49 , we show the observed $850 \mu \mathrm{m}$ differential number counts with a variety of theoretical models overlaid.

\subsubsection{Semi-Analytic Methods}

A number of SAMs are contemporaneous with the first submm/IR surveys at high- $z$, and are summarized in full in the last major review on SMGs by Blain et al. (2002). Of note, Guiderdoni et al. (1998), Blain et al. (1999), and Devriendt \& Guiderdoni (2000) provided predictions for and comparisons with these nascent deep submm surveys. These, and other predictions at the time tended to under-predict the number of SMGs, a problem that, as we will see, will prove to be pervasive in SAMs. In particular, these papers provided early indications that extreme assumptions were necessary to produce SMGs.

Once deep submillimeter number counts were maturing in the literature, the difficulty in producing enough SMGs in cosmological models was pointed out in a seminal paper by Baugh et al. (2005). These authors updated the thencurrent version of the Durham GALForm SAM (Cole et al. 2000, Granato et al., 2000) to investigate the physical properties of high- $z$ SMGs. By coupling their model with GRAsIL dust radiative transfer calculations, Baugh et al. (2005) found that they under-predicted the observed number counts of SMGs by a factor of $\sim 30-50$. Constraining the model was a goal of simultaneously matching the observed LBG distribution at high- $z$, as well as the $z=0 K$-band luminosity function and IRAS $60 \mu \mathrm{m}$ luminosity function. Beyond this, the model had a goal of not allowing the dust temperature to be a free parameter, which was a key update to empirical models that pre-dated this one.

As a solution to matching the observed SMG number counts while retaining a match to the observed LBG and $z=0$ $K$-band luminosity function, Baugh et al. (2005) modified three major aspects of the original Granato et al. (2000) model. First, the star formation time scale in disks is modified to be dependent on the circular velocity, rather than the dynamical time scale. This has the effect of making galaxies more gas rich. The second modification was to allow starbursts in galaxy mergers that involved minor mergers (down to mass ratios of 1:20), rather than just in major mergers, if the minor mergers were sufficiently gas rich $\left(f_{\text {gas }}>0.75\right)$. The third modification, which is likely the most dramatic change, was to the stellar initial mass function in starburst galaxies. For quiescently star-forming galaxies, the model imposed a Kennicutt (1983) model IMF, with $d n / d \ln (m) \propto m^{-x}$, with $x=0.4$ for $M<1 \mathrm{M}_{\odot}$, and $x=1.5$ for $M>1 \mathrm{M}_{\odot}$. For starbursts that are triggered by galaxy mergers, the IMF was modified to being 


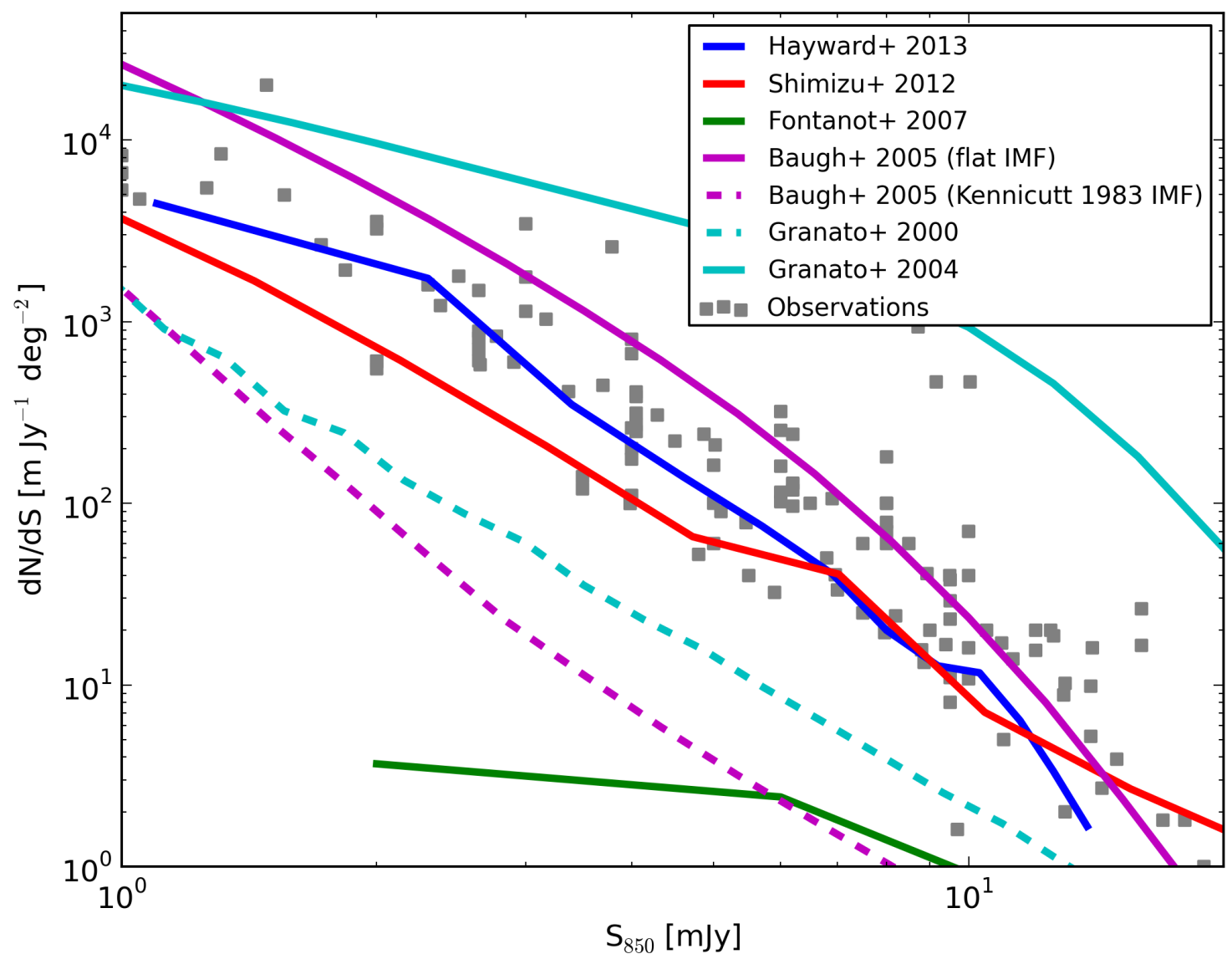

Figure 49: Observed $850 \mu \mathrm{m}$ differential number counts (grey squares), with theoretical curves from various models over-plotted as colored lines

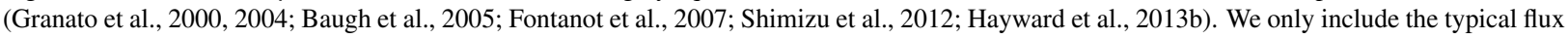
density range of observed points that are considered by theoretical models that aim to match the SMG number counts. Note, we do not include the Davé et al. (2010) hydrodynamic model, or empirical models, as the theoretical counts in these cases are forced to match the observed counts by construction. 
extremely top heavy (in fact, flat), with $x=0$ for all stellar masses. While the transition from a Kennicutt (1983) IMF to a flat IMF does not have a physical basis, there are a few attractive aspects to a top-heavy IMF in starbursts (beyond those already outlined in $\S 5.4$. First, the majority of stars formed in galaxy mergers are done during early quiescent phases, rather than bursts (e.g. Cox et al. 2008). Hence, modifying the IMF in bursts alone does not severely impact the present-epoch stellar mass function. Second, increasing the fraction of massive stars formed in a generation results in both more UV luminosity per stellar mass formed, as well as more dust. Hence, while the bolometric luminosity of the galaxy increases, the spectrum is allowed to remain cold, fostering the formation of submillimeter-detectable galaxies. In this model $>99 \%$ of SMGs are triggered by galaxy mergers, and hence the IMF is predicted to be flat in essentially all SMGs. The difference in the Baugh et al. (2005) predictions for a Kennicutt (1983) and flat IMF is shown in Figure 49 .

These models were examined further by González et al. (2011), who examined the physical properties of the SMGs formed in the Baugh et al. model in detail. González et al. (2011) find that $~ 75 \%$ of the SMGs formed in the Durham model do so in minor (mass ratio $>1: 20$ ) mergers, with baryonic gas fractions $>75 \%$. Roughly $22 \%$ of the SMGs are formed in major mergers, and $0.7 \%$ in quiescent galaxies. This is in stark contrast to some of the results from cosmological hydrodynamic and hybrid models, as we will discuss later. The resulting morphology of these galaxies is a disk plus bulge, with most $(>2 / 3)$ of the descendants having a bulge to total $(B / T)$ mass ratio $>0.5$.

Swinbank et al. (2008) showed that the Baugh et al. (2005) model for SMG formation matched the observed SMG number counts, FIR and mm-wave colors, and potentially redshift distribution (though this is, of course, an area of hot debate; c.f. § 4). Similarly, a number of works have expanded this model to investigate other galaxy populations and shown reasonable correspondence with observations. Le Delliou et al. (2005, 2006) examine the properties of Ly $\alpha$ emitters in the Baugh et al. (2005) GALForm model, and find good correspondence with the observed counts, magnitudes and equivalent widths (modulo assumptions regarding a fixed escape fraction). Similarly, Lacey et al. (2008) compared the galaxies formed in the Baugh et al. (2005) model to existing Spitzer data to show that the model matches a number of IR constraints, including the strong evolution in the galaxy mid-IR luminosity function. In particular, these authors find that the modified IMF (i.e. flat IMF in starbursts) is necessary to match the observed mid-IR luminosity function evolution, and that a normal IMF in starbursts may be excluded as it predicts too little evolution in the Durham SAM.

However, Swinbank et al. (2008) show that the the synthetic $K$-band and mid-IR $(3-8 \mu \mathrm{m})$ colors of the model galaxies in the Baugh et al. (2005) model are too low by up to a factor $\sim 10$ as compared to observed SMGs. This owes to a typical stellar mass of $\sim 10^{10} \mathrm{M}_{\odot}$ for SMGs in the Durham model. Qualitatively, this can be explained by the idea that a flat IMF (in merger-driven starbursts) was necessary to reproduce the observed cold dust colors of SMGs in the model. The IMF is only flat in merger-driven bursts in this model, though there are not enough mergers between massive galaxies to account for the full SMG number counts. Hence, the model required lower mass galaxies to serve as SMGs, which shows tension with observed $K$-band and mid-IR colors. In a similar vein, Bothwell et al. (2013a) showed that at $z>3$, observed SMGs and those made in the GALForm model are discrepant in their predicted and observed molecular gas fractions by a factor of 2, with the model gas fractions approaching unity by $z \sim 4$. While gas fractions in SMGs are observed to be high (modulo potential $X$-factor effects; c.f. $\S 8.6$, a gas fraction $\left(f_{\text {gas }}=M_{\text {gas }} /\left(M_{\text {gas }}+M_{*}\right)>0.9\right.$ as predicted by the GALForm model for $z \sim 4$ galaxies is not currently supported by observations. Finally, the flat IMF that GALFORM requires in order to match the observed SMG number counts and evolution in the galaxy mid-IR luminosity function has not been observed in any nearby environment (see the recent review by Bastian et al. 2010). While some circumstantial evidence may point toward more bottom-light IMFs in heavily star-forming environments (c.f. § 5.4), nothing approaching an IMF as extreme as a flat one has ever been observed. Beyond this, joint constraints on the $X$-factor, stellar mass, dynamical mass and IMF of observed high- $z$ SMGs exclude a flat IMF, in the case where the gas reservoir's size is relatively compact at low- $J$ (Tacconi et al. 2008). In summary, the flat IMF GALFORM model put forth by the Durham group has shown success in matching a number of observations of high- $z$ galaxies. On the other hand, there are a few notable mismatches when confronted with observations that suggest that the GALFORm model, at least as far as dusty galaxies at high- $z$ go, will lead to further physical insight as it is further tuned in an effort to fully match basic observational constraints of SMGs.

In complementary work, Fontanot et al. (2007) used the Model for the Rise of Galaxies and Active Galactic Nuclei (MORGANA) to investigate whether the observed $850 \mu \mathrm{m}$ counts could be accounted for in hierarchical galaxy formation SAMs without any extreme assumptions regarding the IMF. Similar to Baugh et al. (2005), Fontanot et al. (2007) combined their SAM with GRASIL radiative transfer in order to predict the synthetic colors of their model galaxies. 
Utilizing a Salpeter IMF, and an updated model for gas cooling onto halos, this group was able to match the observed $850 \mu \mathrm{m}$ number counts. The physical form of the SMGs formed in the MORGANA simulations are notably different from those formed in GALFORM, however. While the latter suggested that SMGs were almost exclusively mergers, Fontanot et al. (2007) found that mergers only dominated galaxies with the highest star formation rates, SFR $>200 \mathrm{M}_{\odot} \mathrm{yr}^{-1}$. As we will discuss, the idea of SMGs owing to both heavily star-forming (non-merging) galaxies, as well as mergers will be prevalent in both the cosmological hydrodynamic simulations, as well as the hybrid models for SMG formation. In principle, the difference in the models is that the Fontanot et al. (2007) model is able to form heavily star-forming galaxies without mergers owing to more efficient gas cooling from the IGM. It is noted, however, that this model over produces stars in massive galaxies, and therefore is incompatible with observations of local galaxies.

Somerville et al. (2012) presented an updated form of the Somerville \& Primack (1999) SAM (i.e. the "Santa Cruz SAM") that was combined with estimates for dust attenuation by both diffuse dust and stellar birth clouds surrounding young clusters. The energy absorbed by dust was assumed to be re-radiated in the infrared, and combined these with both theoretical SED templates by Devriendt et al. (1999), as well as the locally-calibrated observed SED templates by Rieke et al. (2009). These authors found that in order to reproduce the (rest) UV and optical luminosity functions at high-z, an evolving dust-to-metals ratio was necessary. In other words, galaxies at a comparable luminosity at highredshift compared to a local analog needed less dust obscuration. This made reproducing the observed properties of dusty galaxies at high- $z$ more difficult, however, and as a consequence the Somerville et al. (2012) model was unable to match the observed $250 \mu \mathrm{m}$ SPIRE number counts, as well as the $850 \mu \mathrm{m}$ ScuBA counts. As shown by Niemi et al. (2012), the Somerville et al. (2012) model works rather well at lower redshifts (i.e. $z<2$ ), though shows increasing tension with observations at $z>2$. The discrepancy in the Santa Cruz SAM in matching IR and submm counts becomes progressively worse at increasing wavelength. Interestingly, these models show good agreement between the physical properties of their identified Herschel-detected galaxies, and those inferred from observations (including stellar and gas masses, star formation rate, and disk sizes). The Somerville et al. (2012) and Niemi et al. (2012) models predicted that galaxies of increasing infrared luminosity were more likely to owe their origin to mergers, though only roughly half of Herschel-detected galaxies were formed in mergers: the other 50\% arose from heavily star-forming galaxies fueled by the gas-rich environment characteristic of the $z \sim 2-4$ Universe.

\subsubsection{Cosmological Hydrodynamic Simulations}

One of the first cosmological hydrodynamic simulations to address the origin of high- $z$ SMGs were by Fardal et al. 2001 ${ }^{27}$ utilizing the parallel SPH code, TREESPH. This work combined cosmological SPH galaxy formation models with $7 h^{-1} \mathrm{kpc}$ spatial resolution (equivalent Plummer softening length) with an empirical recipe for converting the SFR of a galaxy to $850 \mu \mathrm{m}$ flux density (assuming a dust temperature and SED slope $\beta$ ) to calculate the synthetic emission properties of high- $z$ galaxies. These authors were amongst the first to advocate a scenario in which nearly all SMGs at high- $z$ formed from massive, heavily star-forming galaxies that are not undergoing a merger. This will be a common feature of cosmological hydrodynamic models. A key feature of this model is that SMGs are typically not undergoing starbursts, but rather typically have much lower SFRs than observations suggest of the population, with a median simulated SFR of $\sim 100 \mathrm{M}_{\odot} \mathrm{yr}^{-1}$ with long duration SFR timescales.

Finlator et al. (2006) discussed a similar scenario for the formation of SMGs. When exploring the physical properties of $z \sim 4$ galaxies in a cosmological hydrodynamic simulation run with GADGET, these authors noted a number of galaxies in their simulated volume with SFRs exceeding $100 \mathrm{M}_{\odot} \mathrm{yr}^{-1}$, with two exceeding $1000 \mathrm{M}_{\odot} \mathrm{yr}^{-1}$. Subsequently, Dekel et al. (2009a) showed that these large SFRs could owe to the growth of galaxies via the accretion of cold gas from the IGM, at a time when the Universe was denser, and hence cooling times were shorter. Dekel et al. (2009a) suggest that approximately half of SMGs with flux densities $S_{850}>5 \mathrm{mJy}$ form in mergers with mass ratio $>M_{1} / M_{2}>0.1$, while the other half owe their large SFRs to smoother gas accretion from the IGM.

Building on these studies, Davé et al. (2010) performed the most extensive study of the physical properties of SMGs in cosmological hydrodynamic simulations to date. These authors ran a $100 \mathrm{Mpc} h^{-1} \operatorname{cosmological}$ simulation with the $N$-body/SPH code GADGET, with an effective resolution of $3.75 \mathrm{kpc} h^{-1}$. The simulations, which included physical prescriptions for momentum-driven winds and chemical enrichment, have previously shown good agreement

\footnotetext{
${ }^{27}$ While the Fardal et al. (2001) work was never formally published, it appears on the arXiv, and is still referred to heavily in the theoretical SMG literature. Hence, we include it in our review.

${ }^{28}$ Note, that the term "major merger" is typically attributed to galaxies with mass ratio $M_{1} / M_{2}>1 / 3$, rather than 0.1 .
} 
with the galaxy mass-metallicity relation (Finlator \& Davé, 2008), observed IGM enrichment (Oppenheimer \& Davé, 2006, 2008, 2009), and the cosmological evolution of the UV-luminosity function. Davé et al. (2010) identified SMGs as the most heavily star-forming galaxies in their simulations, and chose all SMGs above a given SFR threshold such that their abundance matched the observed number counts of SMGs. Hence, these simulations took the ansatz of matching the number counts by construction, and asked what the physical properties of these galaxies in cosmological hydrodynamic simulations were. This threshold SFR for SMG identification was $180 \mathrm{M}_{\odot} \mathrm{yr}^{-1}$.

As in previous cosmological hydrodynamic simulations, the bulk of the SMGs modeled by Davé et al. (2010) were massive star-forming galaxies that were not undergoing a merger-driven starburst. As such, these galaxies, for the most part, live on an extension of the galaxy SFR- $M_{*}$ main sequence defined by lower-mass galaxies. The model galaxies had typical $\log \left(M_{*}\right)=[11,11.8]$, baryonic gas fractions $\sim 20-40 \%$, and SFRs ranging from $180-570 \mathrm{M}_{\odot} \mathrm{yr}^{-1}$. While the many of the physical properties of the simulated SMGs in the Davé et al. (2010) model match those inferred from observations, the SFRs tend to be a factor $\sim 2-5$ lower than what is observed. This dilemma originates in the fact that there are not enough mergers in the simulations for all SMGs to be accounted for by mergers, at least for galaxies as massive as $M_{*} \sim 10^{11} \mathrm{M}_{\odot}$ (e.g. Guo \& White, 2008; Hopkins et al. 2010). Without bursts, the SFR is, to first order, regulated by the gas accretion rate from the IGM, which is dictated by the ever-growing gravitational potential in the halo (e.g. Dekel et al., 2009b). Similar issues plague most cosmological simulations, which fail to match the observed normalization of the SFR- $M_{*}$ relation at $z \sim 2$ (e.g. Davé, 2008; Weinmann et al., 2011). Suggestions of a bottom-light IMF have been put forth to ameliorate the issue (e.g. Narayanan \& Davé, 2012, 2013), though this may come at the cost of introducing a tension with stellar absorption line measurements in local massive galaxies, the presumed descendants of high- $z$ starbursts (c.f. § 5.4). In the Davé et al. (2010) model, 1 galaxy out of the 41 in their simulated sample was undergoing a major $\left(M_{1} / M_{2}>1 / 3\right)$ merger.

Finally, Shimizu et al. (2012) performed cosmological hydrodynamic simulations with GADGET as well, though including a simplified dust absorption model and grey-body emission for the SED. These authors find that they are able to reproduce the number counts and clustering amplitude of observed SMGs, and predict a stellar mass of $>10^{11} \mathrm{M}_{\odot}$ for all $S_{850}>1 \mathrm{mJy}$ SMGs (note that this is different from the canonical definition used by the other theoretical models described here, of $S_{850}>5 \mathrm{mJy}$ ). Shimizu et al. (2012) find many more galaxies with SFR $>500 \mathrm{M}_{\odot} \mathrm{yr}^{-1}$ than Davé et al. (2010), though also simulate many more galaxies such that it is not clear how the percentages compare. The stellar masses found in this study are amongst the largest compared to the other models discussed thus far, with $\log \left(M_{*}\right)=[11,12.4]$.

\subsubsection{Idealized and Hybrid Simulations}

High-resolution galaxy merger and isolated disk simulations have long been examined to study the detailed star formation properties of star-forming galaxies. Chakrabarti et al. (2008) coupled a series of eight 1:1 idealized major merger GADGET SPH simulations with RADISHE, a 3D Monte Carlo radiative equilibrium dust radiative transfer code to calculate the synthetic SED properties of these galaxies. Chakrabarti et al.(2008) showed, as has been previously seen by a number of groups, that galaxies of increasing mass have increasing star formation rates, and that coplanar galaxy mergers tend to undergo larger bursts than those merging orthogonally. These authors examined the time evolution of the $850 \mu \mathrm{m}$ flux density for galaxy mergers, as well as the black hole growth rates, and synthetic Spitzer and Herschel colors.

The models of Chakrabarti et al. (2008) found peak SFRs of 3000-4000 $\mathrm{M}_{\odot} \mathrm{yr}^{-1}$, comparable to some inferred estimates of observed galaxies. This said, they were only marginally able to produce a galaxy that was as bright as $\mathrm{S}_{850} \approx 4 \mathrm{mJy}$ for a timescale of 10-20 Myr with even their most massive galaxies. The brightest galaxy in this study $(4 \mathrm{mJy}$ at $850 \mu \mathrm{m})$ had a stellar mass $M_{*}=9.4 \times 10^{11} \mathrm{M}_{\odot}$, suggesting that the majority of $S_{850}>5$ mJy SMGs in this model would require $M_{*}>10^{12} \mathrm{M}_{\odot}$. Referring to $\S 5.3$, this includes only the most massive galaxies utilizing the Michałowski et al. (2012) estimates, which are at the upper end of SMG mass estimates and potentially not even valid since they exceed constraints from dynamical mass estimates.

Narayanan et al. (2010c) examined the properties of a series of galaxy mergers over a range of galaxy masses and merger mass ratios, as well as idealized isolated disk galaxies utilizing GADGET as the $N$-body/hydrodynamics solver. These authors coupled these simulations with sunRISE, a 3D Monte Carlo dust radiative transfer code in order to produce the simulated SED and nebular line properties of their simulated galaxies (Jonsson, 2006; Jonsson et al., 2010, Jonsson \& Primack, 2010). A key difference between SUNRISE and RADISHE is the inclusion of photodissociation region (PDR) modeling. The UV absorption and subsequent long-wavelength re-emission from the cold PDRs surrounding 
Table 4: Summary of Galaxy Formation Model Methods Described Here

\begin{tabular}{|l|l|l|l|}
\hline \hline Model Type & Directly Simulated & Scale of Analytic Approximations & How Radiative Transfer is Done \\
\hline \hline SAM & Cosmic Dark Matter & Galaxy Scales & $\begin{array}{l}\text { Axisymmetric analytic galaxy } \\
\text { models coupled with 3D dust } \\
\text { radiative transfer }\end{array}$ \\
\hline $\begin{array}{l}\text { Cosmological } \\
\text { Hydro }\end{array}$ & $\begin{array}{l}\text { Cosmic Dark Matter } \\
\text { Baryons in Galaxies and IGM }\end{array}$ & $\lesssim 5 \mathrm{kpc}$ & $\begin{array}{l}\text { Either N/A or } \\
\text { analytic approximations }\end{array}$ \\
\hline Idealized & $\begin{array}{l}\text { Baryons in Galaxies } \\
\text { (decoupled from environment) }\end{array}$ & Molecular Cloud $(\lesssim 100 \mathrm{pc})$ & 3D dust radiative transfer \\
\hline Hybrid & $\begin{array}{l}\text { Cosmic Dark Matter } \\
\text { Baryons in Galaxies } \\
\text { (decoupled from environment) }\end{array}$ & Molecular Cloud $(\lesssim 100 \mathrm{pc})$ & 3D dust radiative transfer \\
\hline
\end{tabular}

$†$ This column refers to what component of the galaxy formation models is directly simulated via numerical modeling (as opposed to via analytic approximation)

young stellar clusters formed in the galaxy merger is captured in SUNRISE via the inclusion of MAPPINGS photoionzation calculations (Groves et al. 2008). Narayanan et al. (2010c) suggested that the bulk of the $850 \mu \mathrm{m}$ flux density to arise during a galaxy merger was due to the obscuration by stellar birth clouds, though neglecting these, but treating the molecular ISM as having a volume filling fraction of unity could achieve the same effect. These galaxies produced SMGs with a range of peak flux densities, from the common $S_{850}=5 \mathrm{mJy}$ to the extreme $15 \mathrm{mJy}$ (comparable to, e.g. GN20). The stellar masses ranged from $\sim 3-8 \times 10^{11} \mathrm{M}_{\odot}$, and the galaxies lived in haloes ranging from $\sim 7-20 \times 10^{12} \mathrm{M}_{\odot}$.

Follow-up work by Narayanan et al. (2009) investigated the simulated CO properties of the Narayanan et al. (2010c) model SMG sample by combining the GADGET simulations with TURTLEBEAch (Narayanan et al. 2006a b. 2008 b d) 3D non-LTE Monte Carlo molecular line radiative transfer calculations in post-processing. These authors found that SMGs that formed in galaxy mergers show broad CO line widths, varied CO morphologies, and typically subthermal CO excitation, comparable to previous observations (c.f. $\$ 8.5$ ).

Similarly, Narayanan et al. (2010b) examined the synthetic optical and Spitzer colors from these simulations with a goal of understanding the physical properties of DOGs. In this model, DOGs may owe to a variety of origins, with lower-luminosity DOGs generally being ascribed to isolated disk galaxies, and higher luminosity DOGs arising almost exclusively from mergers. The most luminous DOGs with a power-law mid-IR SED may represent an transition stage between a starburst-dominated merger-driven SMG, and an optical quasar phase. During this phase, the black holes grow rapidly, and rise from lying below the $M_{\mathrm{BH}}-\sigma$ relation to on it. Moreover, because of the diversity of the DOG population, there is a large overlap between the SMG and DOG populations in this picture, a result that is confirmed by at least some observations (Pope et al., 2008a).

The Narayanan et al. (2009, 2010c) series of models were expanded upon substantially in a series of papers by Hayward et al. (2011, 2012, 2013a b) and Hayward (2013). In summary, these papers comprise a nuanced picture of SMGs in which SMGs do not owe specifically to either isolated disk galaxies, or galaxy mergers, but a combination of the two. Along with these, blended pairs of galaxies (sometimes physically associated, other times, not) make up the observed SMG number counts.

Hayward et al. (2011) showed that during the coalescence of a galaxy merger, galaxies become more compact, and drop in dust mass driving an increased dust temperature. This has the effect of starbursts being relatively inefficient at boosting submm flux density. These authors showed that the relationship between the SFR of a galaxy and 850 flux density is relatively weak. A major finding from this work, as well as Hayward et al. (2012), was that it is unlikely that starbursts alone can make up the full SMG galaxy number counts, and that pairs of galaxies in-spiralling in toward a final merger coalescence may contribute substantially.

While idealized simulations such as those performed by Chakrabarti et al. (2008) and Narayanan et al. (2009. $2010 \mathrm{a}$ c) have the advantage of having high-enough spatial resolution that their physical geometry, and consequent radiative transfer solutions are well-characterized, they offer no information about the general population as they are 
not cosmological calculations. The hybrid methodology to extrapolate the properties of idealized simulations to a cosmological context was first developed by Hopkins et al. (2010). The basic construct behind these models was to utilize theoretical halo mass functions, and assign galaxies to them utilizing an abundance matching technique (e.g. Conroy \& Wechsler, 2009, Behroozi et al., 2010). These would then be combined with the results of high-resolution merger calculations in order to determine the typical burst luminosity from a galaxy merger, or steady-state SFR from an isolated disk galaxy of a given mass. By convolving the two, Hopkins et al. (2010) measured the contribution of mergers, AGN and isolated-disk galaxies to observed infrared luminosity functions.

This methodology was improved upon by Hayward et al. (2013b), and directly applied to observations of high- $z$ dusty galaxies. By running a large suite of idealized disk galaxy simulations, as well as galaxy mergers over a range of galaxy masses, merger mass ratios, and merger orbits, these authors derived a mean submm duty cycle for galaxies as a function of these galaxy physical properties. In order to simulate the results from deep submm surveys, Hayward et al. (2013b) then combined these results with simulated galaxy merger rates, as well as observed stellar mass functions (in order to quantify the abundance of non-interacting galaxies). By combining this multi-scale methodology, these authors found they were able to match the observed SMG number counts when accounting for the full contribution of merger-induced starbursts, non-interacting galaxies, and galaxy pairs that were physically associated. Hayward et al. (2013a) showed that physically unassociated galaxies (i.e. galaxies at substantially different redshifts) may also contribute to the observed SMG number counts.

These sorts of hybrid methods have both distinct advantages and disadvantages. The most positive aspect of this multi-scale methodology is that it it allows for an extremely large dynamic range of physical processes to be modeled. For example, the Hayward et al. (2013b) model effectively has $\sim 50-100$ pc resolution (i.e. resolving the surfaces of large giant molecular clouds) over cosmological volumes. This sort of treatment is important when modeling both the detailed processes that occur in a chaotic system, such as a galaxy merger, as well as resolving the spatial distribution of luminous sources and dust. Because the simulations are anchored in high-resolution hydrodynamic models, few assumptions regarding the spatial geometry of the system have to be made going into the radiative transfer calculations. This is something that cosmological simulations, at least in their current state, are unable to do given resolution limitations. On the down side, this approach is not truly ab initio as a bona fide cosmological simulation. For example, the hybrid approach does not allow for a direct investigation as to the role of continuous gas-replenishment of galaxies from the IGM.

\subsection{Testable Predictions and Key Differences between Models}

In Table 5, we summarize the key testable predictions of some of the major models for SMG formation in the literature. We provide literature references for each model, as well as list the major codes and methodologies used. While the exact same physical quantities are not always available for each model, Table 5 should provide a relatively direct way of comparing the direct predictions from different SMG formation models. While some of the predictions were available from the literature, others had to be obtained via private communication. Oftentimes, the simulations were no longer available owing to computer crashes and retirements; hence, some predictions may not be available. 
Table 5: Summary of SMG Theoretical Models and Key Predictions ${ }^{\dagger}$

\begin{tabular}{|c|c|c|c|}
\hline "Model Reference & Code & Methodology & $\begin{array}{l}\text { Distinguishing Predictions } \\
\text { for } z=2 \text { SMGs }\end{array}$ \\
\hline Granato et al. (2004) & GALFORM & SAM & No Predictions Available \\
\hline Baugh et al. $(\overline{005})$ & GALFORM & SAM & $M_{*}=2.1 \times 10^{10 \ddagger} \mathrm{M}_{\odot}$ \\
\hline González et al. (2011) & GRASIL & Dust Radiative Transfer & $\begin{array}{l}M_{\text {halo }}=2.2 \times 10^{12 \ddagger} \mathrm{M}_{\odot} \\
22 \% \text { major }\left(M_{1} / M_{2}>1 / 3\right) \text { mergers } \\
77 \% \text { minor }\left(M_{1} / M_{2}<1 / 3\right) \text { mergers } \\
\mathrm{f}_{\text {gas }}>0.75 \text { (for minor mergers) } \\
S_{850}>5 \text { mJy duty cycle }{ }^{\ddagger} 0.1 \mathrm{Gyr} \\
\text { Flat stellar IMF in starbursts }\end{array}$ \\
\hline Chakrabarti et al. (2008) & $\begin{array}{l}\text { GADGET } \\
\text { RADISHE }\end{array}$ & $\begin{array}{c}\text { Idealized Hydro } \\
\text { Dust Radiative Transfer }\end{array}$ & $\begin{array}{l}M_{*}>9.4 \times 10^{11} \mathrm{M}_{\odot} \\
\text { necessary for } S_{850}>5 \mathrm{mJy}\end{array}$ \\
\hline Fontanot et al. (2007) & MORGANA & SAM & $\begin{array}{l}M_{*}=3.5 \times 10^{11} \mathrm{M}_{\odot}{ }^{* \ddagger} \\
M_{\text {halo }}=7 \times 10^{13} \mathrm{M}_{\odot}^{\ddagger} \\
f_{\text {gas }}=0.33^{\ddagger} \\
\text { SFR }=183 \mathrm{M}_{\odot} \mathrm{yr}^{-1 \ddagger}\end{array}$ \\
\hline Dekel et al. (2009a) & RAMSES & Cosmological Hydro (AMR) & $\begin{array}{l}\sim 1 / 2 \text { of SMGs with } S_{850}>5 \mathrm{mJy} \\
\text { will be mergers with } M_{1} / M_{2}>0.1\end{array}$ \\
\hline Davé et al. (2010) & GADGET & Cosmological Hydro (SPH) & $\begin{array}{l}M_{*} \approx 10^{11}-5 \times 10^{11} \mathrm{M}_{\odot} \\
M_{\text {halo }} \approx 6 \times 10^{12}-4 \times 10^{13} \mathrm{M}_{\odot} \\
2 \% \text { major }\left(M_{1} / M_{2}>1 / 3\right) \text { mergers } \\
f_{\text {gas }}=0.2-0.4 \\
\mathrm{SFR} \approx 180-570 \mathrm{M}_{\odot} \mathrm{yr}^{-1}\end{array}$ \\
\hline Somerville et al. (2012) & SANTA CRUZ & SAM & No Predictions Available* \\
\hline Shimizu et al. (2012) & GADGET & Cosmological Hydro (SPH) & $\begin{array}{l}M_{*}=5-35 \times 10^{11} \mathrm{M}_{\odot}{ }^{*} \\
M_{\text {halo }}=1.4-10 \times 10^{13} \mathrm{M}_{\odot} \\
f_{\text {gas }}=0.7-0.8 \\
\text { SFR }=250-1950\end{array}$ \\
\hline \begin{tabular}{|l|} 
Hayward et al. $(2013 b)$ \\
Narayanan et al. $(2010 \mathrm{c})$
\end{tabular} & $\begin{array}{l}\text { GADGET } \\
\text { SUNRISE }\end{array}$ & $\begin{array}{c}\text { Hybrid } \\
\text { Dust Radiative Transfer }\end{array}$ & $\begin{array}{l}\text { Physically Associated Galaxies: } \\
M_{*}=6-10 \times 10^{10} \mathrm{M}_{\odot} \\
M_{\text {halo }}=3-5 \times 10^{12} \mathrm{M}_{\odot} \\
\text { SFR }>160 \mathrm{M}_{\odot} \mathrm{yr}^{-1}\end{array}$ \\
\hline Hayward et al. (2013a) & ART & SAM & $\begin{array}{l}\text { Physically Unassociated Galaxies (blends)*: } \\
\text { Median } M_{*}=9 \times 10^{10} \mathrm{M}_{\odot} \\
\text { Median } M_{\text {halo }}=5 \times 10^{12} \mathrm{M}_{\odot} \\
\text { Median SFR }=190 \mathrm{M}_{\odot} \mathrm{yr}^{-1}\end{array}$ \\
\hline
\end{tabular}

$\lceil$ We only include SAMs, hydrodynamic and hybrid models as they make bona fide predictions for physical quantities.

$\$$ Median quantities for $S_{850}>5$ mJy SMGs

* Numbers obtained from authors via private communication. 


\section{Future directions}

The study of dusty star-forming galaxies (DSFGs) at high redshifts has passed through major milestones over the last decade. At the time of the Blain et al. (2002) review, the principle DSFGs were identified at $850 \mu \mathrm{m}$ as submillimeter galaxies (SMGs) with flux densities in excess of a few mJy scattered over a few square degrees. The rate of discovery of SMGs with the initial Scuba instrument on JCMT was at the level of one per 10 hours of observations on the sky. Since Scuba, new instruments on single-dish ground-based experiments, especially Laboca, AzTEC, Scuba-2, and SPT have expanded the submm surveys with an order of magnitude increase in the discovery rate. Moving from single-dish observations, interferometers such as VLA, SMA, CARMA and IRAM/PdBI allowed detailed follow-up studies on the nature of these galaxies at high resolution, especially on the molecular gas content and distribution.

Over the last five years, another order of magnitude improvement in our ability to discover DSFGs at high redshifts came from the space-based observations with the Herschel Space Observatory. The SPIRE instrument could image a square degree on the sky down below the source confusion noise of 6 to $8 \mathrm{mJy}$ at 250,350 and $500 \mu \mathrm{m}$, simultaneously, in less than two hours. A square degree imaged with SPIRE generally contains about 1000 sources individually detected, while the source confusion itself contains significant information on the counts and spatial distribution of the fainter sources below the individual detection threshold. With over 1200 square degrees mapped and existing in the data archive, Herschel has now left a lasting legacy for follow-up observations over the coming decades with new facilities.

Among the most prominent new facilities which will see countless advances in this area over the next decade is the Atacama Large Millimeter Array (ALMA). At its full capability, ALMA will be able to detect the ionized gas emission as traced by the $158 \mu \mathrm{m}$ [CII] line from a Milky Way-like galaxy at $z \sim 3$ in less than 24 hours of observations. Reaching these depths will be critical in understanding fundamental differences between luminous DSFGs and normal galaxies at high redshift.

Moving forward, during the remainder of this decade and early next decade, we expect significant advances in our understanding of DSFGs at high redshifts from planned facilities like CCAT, GLT (Greenland $12 \mathrm{~m}$ Telescope), JWST, SPICA, and thirty meter-class telescopes with adaptive optics over arcminute areas on the sky in infrared wavelengths. We end our review with a list of scientific questions and goals for future science programs relating to DSFGs. Over the coming years we hope to:

1. Resolve $100 \%$ of the cosmic infrared background at submm wavelengths into individual galaxies. For reference we note that deep images with Herschel-SPIRE have only resolved $10 \%$ of the cosmic infrared background at 350 and $500 \mu \mathrm{m}$ to individual galaxies, while indirect techniques like stacking on known galaxy populations or using gravitational lensing and high-resolution instruments like ScuBA- 2 can account for $\sim 80 \%$ of the background. It will become necessary to resolve the background directly in submm wavelength imaging data to address if the galaxies that make up the remainder of the background are very high redshift star-forming galaxies or a fainter galaxy population at low redshifts, and to what extent the background is comprised of those two populations.

2. Build large, complete, luminosity limited samples of DSFGs which do not suffer from the selection biases plaguing present-day samples, which are defined by their selection at single-submillimeter bolometer band wavelengths. This will enable a proper accounting for the integrated contribution of dust-enshrouded star formation to the cosmic star-formation rate density (SFRD) and relative importance of DSFGs relative to the much more numerous and well-studied optically-selected galaxy populations.

3. Find statistically significant samples of $z>4$ DSFGs and SMGs to be able to measure the LFs at $z=4$ to the epoch of reionization at $z>6$ to establish the proportion of the early cosmic SFRD which is enshrouded by dust. This will shed light on dust obscuration and dust production mechanisms shortly after the epoch of reionization. Currently only a handful of SMGs are known at $z>5$ and most are gravitationally lensed with the accuracy of lensing models limiting our ability to use them for a cosmological measurement of the SFRD. Separately, measurements of the escape fraction of the Ly- $\alpha$ photons $f_{\mathrm{esc}}$ of $z>6$ SMGs (like HFLS3) will be necessary to establish if such sources with vigorous star-formation, are an important contributor to the UV ionizing photon background responsible for reionization at the highest redshifts of the Universe. If the SMGs are an important contributor they could easily dominate the UV photon budget and alleviate the current need for a reionization model dominated by UV photons from faint, small galaxies.

4. Identify and follow-up lensed DSFGs in resolved detail. While lensed DSFGs cause some issues with interpretation, they are also useful in many other ways in the pre- $30 \mathrm{~m}$ class telescope era. Through spatial enhancement 
provided by extreme lensing magnification events, it will become necessary to study the internal structure of $L=10^{10}-10^{13} \mathrm{~L}_{\odot}$ sub-LIRGs to HyLIRGs at $z \sim 2-4$ to study how their internal physical processes, on the scale of several 10-100 pc, might differ or be similar to star-forming molecular clouds in nearby galaxies.

5. Re-calibrate star formation rate indicators for dusty galaxies. The existing calibrations related to SFR and luminosity are limited to near-by galaxies, a handful of calibrators, or subsamples of distinctly optically-selected galaxy samples. In the future it will become necessary to re-calibrate extinction estimates at optical wavelengths, particularly relevant to studies during the epoch of reionization to establish the abundance of dust in the early Universe. In general, it is also crucial that we re-calibrate all of the star-formation indicators with new dust and gas information currently in hand at high redshifts as the existing relations may have significant evolutionary trends that are currently ignored.

6. Reveal the physical mechanisms driving the incredible luminosities in high- $z$ DSFGs. While some evidence has pointed to their obvious merger-dominated histories, other work has argued strongly that the gas depletion timescales are long enough to be steady-state, fed through bombardment of gas from filaments in the early Universe. A clean computation of the fractional contribution of merger-driven activity towards cosmic star formation, particularly amongst DSFG populations, is needed. Likewise, we need to acquire an enhanced understanding of DSFGs' place in the context of the galaxy main sequence by disentangling uncertainties in stellar mass and SFR estimates. Future observations that will more precisely determine these quantities will be extremely valuable.

7. Gain a better understanding of the interplay and coevolution of star-forming galaxies and their active galactic nuclei (AGN). Probing the formation and evolution supermassive black holes at galaxy centers in tandem with their host galaxy's star formation history is critical to understanding how relevant different suggested evolutionary trajectories are to galaxy formation and evolution. Both observational and theoretical work in this area should improve drastically in the coming years with statistically larger data samples becoming available and enhanced models which will shed light on AGN feedback.

8. Map out $[\mathrm{CII}]$ and $\mathrm{CO}(1-0)$ gas in all $z>6$ galaxies and combine these observations with low-frequency $21-\mathrm{cm}$ radio interferometers studying the epoch of reionization. Measuring the ionizing bubble sizes of star-forming galaxies and the growth of ionized bubble size during reionization as a function of the star-formation rate and gas mass will shed light on the physical processes responsible for reionization. These studies are could be done in the context of intensity mapping where galaxies are not individually resolved with either [CII] or $\mathrm{CO}(1-0)$ line, but instead could be pursued with statisical analysis like intensity power spectra and cross power spectral analysis.

9. Understand the physical origins of [CII] emission, when it is a good SFR tracer, and where the [CII]-IR luminosity deficit originates. Also, we should aim to understand how [CII] line intensity varies with the metallicity. Similarly, we should aim at contrasting high and low-excitation tracers in order to observationally probe coeval AGN and starburst tracers. There are still large uncertainties on our understanding of molecular and ionized gas processes at submm and mm wavelengths.

10. Develop a comprehensive theoretical/simulated model that accounts for various DSFG observables: the submm number counts, DSFG clustering and environments, and the redshift distribution of various DSFG populations, while not violating other cosmic constraints gathered from other observation data (e.g. luminosity functions and stellar mass functions).

This list is by no means exhaustive, but is representative of some of the major goals of this burgeoning field in the coming years. Many of the future developments will depend on specific capabilities of forthcoming observatories, but the underlying theme is very clear: while we have made significant advances over the last ten years, in our ability to find and understand DSFGs, we are far from fully understanding the physical processes that govern high redshift dust-obscured star-formation and the assembly and evolution of the first galaxies. 


\section{Acknowledgements}

During the preparation of this manuscript, many colleagues and collaborators in the community contributed data to this work and/or provided helpful feedback on its content. We would like to thank the COSMOS collaboration for permitting the public use of their data, which was used to make Figure 10. We also thank Justin Spilker for sharing the composite millimeter spectrum of SPT DSFGs from his upcoming paper (plotted here in Figure 16), and Scott Chapman for sharing the composite rest-frame ultraviolet spectrum of $850 \mu \mathrm{m}$-selected SMGs from his upcoming paper (plotted here in Figure 26). We are extremely grateful to the many other members of the community that willingly shared their published data or simulation results for the purposes of plots in this review, including Andrew Baker, Manda Banerji, Carlton Baugh, Andrew Benson, Matt Bothwell, Chris Carilli, Anna Danielson, Romeel Davé, Tanio Diaz-Santos, Duncan Farrah, Fabio Fontanot, Hai Fu, Jian Fu, Javier Gracia-Carpio, Steve Hailey-Dunsheath, Chris Hayward, Jacqueline Hodge, Mark Krumholz, Cedric Lacey, Claudia Lagos, Jen Donovan Meyer, Gergo Popping, Dominik Riechers, Dimitra Rigopoulou, Karin Sandstrom, Kim Scott, Chelsea Sharon, Ikko Shimizu, Rachel Somerville, Mark Swinbank, and Fabian Walter. We also wish to thank the many colleagues who gave us permission to reprint figures from their previous published papers here, including Phil Hopkins (Figure 2), Sam Kim (Figure 9), Laura Mocanu (Figure 15), David Alexander (Figure 27), Karin Menéndez-Delmestre (Figure 28), Susannah AlaghbandZadeh (Figure 29), Scott Chapman and Jeyhan Kartaltepe (Figure 30), Mark Swinbank (Figure 31), Jacqueline Hodge (Figure 32), Mattia Negrello (Figure 33), Shane Bussmann (Figure 34), Darren Dowell and Alex Conley (Figure 35), Yashar Hezaveh (Figure 36), Manuela Magliocchetti, Ryan Hickox and Christina Williams (Figure 38), Cameron Thacker (Figures 40 and 41), and Gil Holder (Figure 42).

Furthermore, we would like to thank the many expert members of our community who took time to read and offer comments on a preliminary draft of this review; the manuscript was significantly improved with their feedback: Susannah Alaghband-Zadeh, Andrew Benson, Matthieu Béthermin, R. Shane Bussmann, Alexander Conley, Edward Chapin, Romeel Davé, Aaron Evans, Neal Evans, Duncan Farrah, Chris Hayward, Jacqueline Hodge, Rob Ivison, Claudia Lagos, Dan Marrone, Alexandra Pope, David Sanders, Ian Smail, Joaquin Vieira, and Marco Viero. We would also like to thank our anonymous reviewer who provided many excellent and insightful suggestions for improving the manuscript. Also, we would like to acknowledge the contributions of many collaborators, especially, members of the Herschel/SPIRE Instrument Science Team, HerMES, H-ATLAS, and COSMOS.

In addition, we would like to thank the participants of the Aspen Center for Physics Summer 2013 Workshop "The Obscured Universe: Dust and Gas in Distant Starburst Galaxies" for spurring the discussions which motivated the writing of this review. We thank Marc Kamionkowski for inviting us to submit a review article on the dusty star-forming galaxies and for his help during the writing and editorial process. CMC would like to acknowledge generous support from a McCue Fellowship through the University of California, Irvine's Center for Cosmology and support from a Hubble Fellowship, grant HST-HF-51268.01-A from Space Telescope Science Institute for support during the preparation of this review. DN is supported by the NSF via grant AST-1009452. AC is supported by a combination of NSF AST-1313319 and NASA/JPL funding for US guaranteed time and open time programs with the Herschel Space Observatory. 


\section{Glossary of Dusty Star-Forming Galaxy Acronyms}

\section{- DOG: Dust-Obscured Galaxy}

DOGs are extremely red galaxies selected by their $R$ - [24] color to be redder than most ultraluminous infrared galaxies at all redshifts. The formal selection is defined in Dey et al. (2008) as galaxies which satisfy the following two criteria: (1) $F_{24 \mu m} \geq 0.3 \mathrm{mJy}$, and (2) $(R-[24]) \geq 14$ (Vega) mag. This corresponds to flux density ratios $F_{24 \mu \mathrm{m}} / F_{\mathrm{R}} \geq 982$. Pope et al. (2008b) later loosened the first criteria to accept galaxies of lower flux densities, i.e. $F_{24 \mu \mathrm{m}} \geq 100 \mu \mathrm{Jy}$.

- DSFG: Dusty Star-Forming Galaxy

DSFG is a generic term for star-forming galaxies which contain substantial amounts of dust or whose rest-frame optical/ultraviolet light might be significantly obscured. There is no strict observational definition for DSFGs, although the term has been used to refer to both extreme starbursts and more moderate star-forming galaxies.

- HSG: Herschel-selected Galaxy

Herschel-selected galaxies can refer to any galaxy selected in the Herschel PACS $(100 \mu \mathrm{m}, 160 \mu \mathrm{m})$ or SPIRE $(250 \mu \mathrm{m}, 350 \mu \mathrm{m}$, or $500 \mu \mathrm{m})$ bands. Casey et al. (2012a b) define HSGs as galaxies detected at $>3 \sigma$ significance in at least one of the three SPIRE bands, where $\sigma$ represents the instrumental and confusion noise uncertainty, so the detection criteria for HSGs are approximately $S_{250}>12 \mathrm{mJy}, S_{350}>14 \mathrm{mJy}$, and $S_{500}>15 \mathrm{mJy}$.

- HyLIRG/HLIRG: Hyperluminous Infrared Galaxy HyLIRGs (or HLIRGs) are defined as having $8-1000 \mu \mathrm{m}$ luminosities between $10^{13} \mathrm{~L} \odot$ and $10^{14} \mathrm{~L} \odot$.

- LIRG: Luminous Infrared Galaxy

LIRGs are defined as having $8-1000 \mu \mathrm{m}$ luminosities between $10^{11} \mathrm{~L} \odot$ and $10^{12} \mathrm{~L} \odot$.

- MMG: MilliMeter-selected Galaxy

Galaxies detected at millimeter wavelengths; this is a broad term which refers to SMG-like galaxies which are detected around $1 \mathrm{~mm}$ (ranging from $\sim 850 \mu \mathrm{m}-1.2 \mathrm{~mm}$ ), as opposed to galaxies detected from $250-500 \mu \mathrm{m}$. MMG is also an alternate term for 'SMG.'

- OFRG: Optically Faint Radio Galaxy

OFRGs are optically-faint $(i \lesssim 23) \mu$ Jy radio galaxies which are not $850 \mu$ m-detected $\left(S_{850} \lesssim 2-5\right.$ mJy Chapman et al. 2004a). OFRGs were originally proposed as an alternate class of dusty star-forming galaxy which are not luminous at $850 \mu \mathrm{m}$ due to a temperature bias selection effect. The radio emission is thought to be dominated by star-formation and not AGN.

- SFG: Star-Forming Galaxy

$\mathrm{SFG}$ is a generic term for normal star-forming galaxies which might include star-forming $B z K$ galaxies, BX/BM galaxies, LBGs. SFGs exclude ULIRGs, SMGs, or other extreme starburst populations summarized in this review. Although the definition of SFG is not strictly defined in observational terms, several works have claimed that SFGs can be broadly described as sitting on the normal galaxy 'main sequence' (Noeske et al., 2007b) and are mostly not made up of secularly evolving, disk galaxies (Shapiro et al., 2008, Förster Schreiber et al., 2009. Tacconi et al., 2010; Daddi et al., 2010a; Genzel et al., 2010).

- SFRG: Submillimeter Faint, Star-Forming Radio Galaxy

SFRGs are submillimeter-faint $\mu$ Jy radio galaxies like OFRGs, although unlike OFRGs, SFRGs need not be optically faint. SFRGs were described in Casey et al. (2009a) as an updated classification to OFRGs; since many SMGs are not optically faint $(i>23)$, removing the optically-faint classification from submillimeterfaint galaxies was necessary to estimate how many dusty galaxies were missing from ScubA surveys in the pre-Herschel era. 


\section{- SMG: Submillimeter Galaxy}

SMGs were initially defined as galaxies detected at $850 \mu \mathrm{m}$ with the Scuba instrument at the James Clerk Maxwell Telescope (JCMT); they have flux densities $S_{850} \gtrsim 2-5 \mathrm{mJy}$. More recently, the term 'SMG' has been used in a more broad sense to apply not only to galaxies luminous at $850 \mu \mathrm{m}$, but galaxies with continuum detections $\gtrsim 1 \mathrm{mJy}$ anywhere from $\sim 250 \mu \mathrm{m}-2 \mathrm{~mm}$.

- ULIRG: Ultraluminous Infrared Galaxy

ULIRGs are defined as having $8-1000 \mu \mathrm{m}$ luminosities between $10^{12} \mathrm{~L}_{\odot}$ and $10^{13} \mathrm{~L}_{\odot}$.

\section{References}

Abel, N. P., Dudley, C., Fischer, J., Satyapal, S., \& van Hoof, P. A. M. 2009, ApJ, 701, 1147

Abel, T. \& Wandelt, B. D. 2002, MNRAS, 330, L53

Adelberger, K. L. \& Steidel, C. C. 2000, ApJ, 544, 218

Adelberger, K. L., Steidel, C. C., Pettini, M., Shapley, A. E., Reddy, N. A., \& Erb, D. K. 2005, ApJ, 619, 697

Alaghband-Zadeh, S., Chapman, S. C., Swinbank, A. M., Smail, I., Danielson, A. L. R., Decarli, R., Ivison, R. J., Meijerink, R., Weiss, A., \& van der Werf, P. P. 2013, MNRAS, 435, 1493

Alaghband-Zadeh, S., Chapman, S. C., Swinbank, A. M., Smail, I., Harrison, C. M., Alexander, D. M., Casey, C. M., Davé, R., Narayanan, D., Tamura, Y., \& Umehata, H. 2012, MNRAS, 3329

Alberts, S., Wilson, G. W., Lu, Y., Johnson, S., et al. 2013, MNRAS, 431, 194

Alexander, D. M., Bauer, F. E., Brandt, W. N., Daddi, E., Hickox, R. C., Lehmer, B. D., Luo, B., Xue, Y. Q., Young, M., Comastri, A., Del Moro, A., Fabian, A. C., Gilli, R., Goulding, A. D., Mainieri, V., Mullaney, J. R., Paolillo, M., Rafferty, D. A., Schneider, D. P., Shemmer, O., \& Vignali, C. 2011, ApJ, 738, 44

Alexander, D. M., Bauer, F. E., Chapman, S. C., Smail, I., Blain, A. W., Brandt, W. N., \& Ivison, R. J. 2005a, ApJ, 632 , 736

Alexander, D. M., Brandt, W. N., Smail, I., Swinbank, A. M., Bauer, F. E., Blain, A. W., Chapman, S. C., Coppin, K. E. K., Ivison, R. J., \& Menéndez-Delmestre, K. 2008, AJ, 135, 1968

Alexander, D. M., Smail, I., Bauer, F. E., Chapman, S. C., Blain, A. W., Brandt, W. N., \& Ivison, R. J. 2005b, Nature, 434, 738

Allamandola, L. J., Tielens, A. G. G. M., \& Barker, J. R. 1985, ApJ, 290, L25

Almaini, O., Dunlop, J. S., Willott, C. J., Alexander, D. M., Bauer, F. E., \& Liu, C. T. 2005, MNRAS, 358,875

Almaini, O., Scott, S. E., Dunlop, J. S., Manners, J. C., Willott, C. J., Lawrence, A., Ivison, R. J., Johnson, O., Blain, A. W., Peacock, J. A., Oliver, S. J., Fox, M. J., Mann, R. G., Pérez-Fournon, I., González-Solares, E., Rowan-Robinson, M., Serjeant, S., Cabrera-Guerra, F., \& Hughes, D. H. 2003, MNRAS, 338, 303

Amblard, A. \& Cooray, A. 2007, ApJ, 670, 903

Amblard, A., Cooray, A., Serra, P., Altieri, B., Arumugam, V., Aussel, H., Blain, A., Bock, J., Boselli, A., Buat, V., Castro-Rodríguez, N., Cava, A., Chanial, P., Chapin, E., Clements, D. L., Conley, A., Conversi, L., Dowell, C. D., Dwek, E., Eales, S., Elbaz, D., Farrah, D., Franceschini, A., Gear, W., Glenn, J., Griffin, M., Halpern, M., Hatziminaoglou, E., Ibar, E., Isaak, K., Ivison, R. J., Khostovan, A. A., Lagache, G., Levenson, L., Lu, N., Madden, S., Maffei, B., Mainetti, G., Marchetti, L., Marsden, G., Mitchell-Wynne, K., Nguyen, H. T., O’Halloran, B., Oliver, S. J., Omont, A., Page, M. J., Panuzzo, P., Papageorgiou, A., Pearson, C. P., Pérez-Fournon, I., Pohlen, M., Rangwala, N., Roseboom, I. G., Rowan-Robinson, M., Portal, M. S., Schulz, B., Scott, D., Seymour, N., Shupe, D. L., Smith, A. J., Stevens, J. A., Symeonidis, M., Trichas, M., Tugwell, K., Vaccari, M., Valiante, E., Valtchanov, I., Vieira, J. D., Vigroux, L., Wang, L., Ward, R., Wright, G., Xu, C. K., \& Zemcov, M. 2011, Nature, 470, 510

Amblard, A., Cooray, A., Serra, P., Temi, P., Barton, E., Negrello, M., Auld, R., Baes, M., Baldry, I. K., Bamford, S., Blain, A., Bock, J., Bonfield, D., Burgarella, D., Buttiglione, S., Cameron, E., Cava, A., Clements, D., Croom, S., Dariush, A., de Zotti, G., Driver, S., Dunlop, J., Dunne, L., Dye, S., Eales, S., Frayer, D., Fritz, J., Gardner, J. P., Gonzalez-Nuevo, J., Herranz, D., Hill, D., Hopkins, A., Hughes, D. H., Ibar, E., Ivison, R. J., Jarvis, M., Jones, D. H., Kelvin, L., Lagache, G., Leeuw, L., Liske, J., Lopez-Caniego, M., Loveday, J., Maddox, S., Michałowski, M., Norberg, P., Parkinson, H., Peacock, J. A., Pearson, C., Pascale, E., Pohlen, M., Popescu, C., Prescott, M., Robotham, A., Rigby, E., Rodighiero, G., Samui, S., Sansom, A., Scott, D., Serjeant, S., Sharp, R., Sibthorpe, B., Smith, D. J. B., Thompson, M. A., Tuffs, R., Valtchanov, I., van Kampen, E., van der Werf, P., Verma, A., Vieira, J., \& Vlahakis, C. 2010, A\&A, 518, L9+

Andreani, P., Cimatti, A., Loinard, L., \& Röttgering, H. 2000, A\&A, 354, L1

Andrews, B. H. \& Thompson, T. A. 2011, ApJ, 727, 97

Aravena, M., Carilli, C., Daddi, E., Wagg, J., Walter, F., Riechers, D., Dannerbauer, H., Morrison, G. E., Stern, D., \& Krips, M. 2010, ApJ, 718, 177

Aravena, M., Carilli, C. L., Salvato, M., Tanaka, M., Lentati, L., Schinnerer, E., Walter, F., Riechers, D., Smolcić, V., Capak, P., Aussel, H., Bertoldi, F., Chapman, S. C., Farrah, D., Finoguenov, A., Le Floc'h, E., Lutz, D., Magdis, G., Oliver, S., Riguccini, L., Berta, S., Magnelli, B., \& Pozzi, F. 2012, MNRAS, 426, 258

Aravena, M., Murphy, E. J., Aguirre, J. E., Ashby, M. L. N., Benson, B. A., Bothwell, M., Brodwin, M., Carlstrom, J. E., Chapman, S. C., Crawford, T. M., de Breuck, C., Fassnacht, C. D., Gonzalez, A. H., Greve, T. R., Gullberg, B., Hezaveh, Y., Holder, G. P., Holzapfel, W. L., Keisler, R., Malkan, M., Marrone, D. P., McIntyre, V., Reichardt, C. L., Sharon, K., Spilker, J. S., Stalder, B., Stark, A. A., Vieira, J. D., \& Weiß, A. 2013, MNRAS, 433, 498

Aretxaga, I., Hughes, D. H., Coppin, K., Mortier, A. M. J., Wagg, J., Dunlop, J. S., Chapin, E. L., Eales, S. A., Gaztañaga, E., Halpern, M., Ivison, R. J., van Kampen, E., Scott, D., Serjeant, S., Smail, I., Babbedge, T., Benson, A. J., Chapman, S., Clements, D. L., Dunne, L., Dye, S., Farrah, D., Jarvis, M. J., Mann, R. G., Pope, A., Priddey, R., Rawlings, S., Seigar, M., Silva, L., Simpson, C., \& Vaccari, M. 2007, MNRAS, 379, 1571 
Aretxaga, I., Wilson, G. W., Aguilar, E., Alberts, S., Scott, K. S., Scoville, N., Yun, M. S., Austermann, J., Downes, T. P., Ezawa, H., Hatsukade, B., Hughes, D. H., Kawabe, R., Kohno, K., Oshima, T., Perera, T. A., Tamura, Y., \& Zeballos, M. 2011, MNRAS, 415,3831

Arrigoni, M., Trager, S. C., Somerville, R. S., \& Gibson, B. K. 2010, MNRAS, 402, 173

Auger, M. W., Treu, T., Gavazzi, R., Bolton, A. S., Koopmans, L. V. E., \& Marshall, P. J. 2010, ApJ, 721, L163

Austermann, J. E., Dunlop, J. S., Perera, T. A., Scott, K. S., Wilson, G. W., Aretxaga, I., Hughes, D. H., Almaini, O., Chapin, E. L., Chapman,

S. C., Cirasuolo, M., Clements, D. L., Coppin, K. E. K., Dunne, L., Dye, S., Eales, S. A., Egami, E., Farrah, D., Ferrusca, D., Flynn, S., Haig,

D., Halpern, M., Ibar, E., Ivison, R. J., van Kampen, E., Kang, Y., Kim, S., Lacey, C., Lowenthal, J. D., Mauskopf, P. D., McLure, R. J., Mortier,

A. M. J., Negrello, M., Oliver, S., Peacock, J. A., Pope, A., Rawlings, S., Rieke, G., Roseboom, I., Rowan-Robinson, M., Scott, D., Serjeant, S.,

Smail, I., Swinbank, A. M., Stevens, J. A., Velazquez, M., Wagg, J., \& Yun, M. S. 2010, MNRAS, 401, 160

Baker, A. J., Tacconi, L. J., Genzel, R., Lehnert, M. D., \& Lutz, D. 2004, ApJ, 604, 125

Ballantyne, D. R., Armour, J. N., \& Indergaard, J. 2013, ApJ, 765, 138

Banerji, M., Chapman, S. C., Smail, I., Alaghband-Zadeh, S., Swinbank, A. M., Dunlop, J. S., Ivison, R. J., \& Blain, A. W. 2011, MNRAS, 418, 1071

Barger, A. J., Cowie, L. L., \& Richards, E. A. 2000, AJ, 119, 2092

Barger, A. J., Cowie, L. L., Sanders, D. B., Fulton, E., Taniguchi, Y., Sato, Y., Kawara, K., \& Okuda, H. 1998, Nature, 394,248

Barger, A. J., Cowie, L. L., Smail, I., Ivison, R. J., Blain, A. W., \& Kneib, J. 1999, AJ, 117, 2656

Barger, A. J., Cowie, L. L., \& Wang, W. 2007, ApJ, 654, 764

Barger, A. J., Wang, W.-H., Cowie, L. L., Owen, F. N., Chen, C.-C., \& Williams, J. P. 2012, ApJ, 761, 89

Barnard, V. E., Vielva, P., Pierce-Price, D. P. I., Blain, A. W., Barreiro, R. B., Richer, J. S., \& Qualtrough, C. 2004, MNRAS, 352, 961

Barvainis, R. \& Antonucci, R. 2005, ApJ, 628, L89

Bastian, N., Covey, K. R., \& Meyer, M. R. 2010, ARA\&A, 48, 339

Baugh, C. M., Cole, S., \& Frenk, C. S. 1996, MNRAS, 283, 1361

Baugh, C. M., Cole, S., Frenk, C. S., \& Lacey, C. G. 1998, ApJ, 498, 504

Baugh, C. M., Lacey, C. G., Frenk, C. S., Granato, G. L., Silva, L., Bressan, A., Benson, A. J., \& Cole, S. 2005, MNRAS, 356,1191

Bautz, M. W., Malm, M. R., Baganoff, F. K., Ricker, G. R., Canizares, C. R., Brandt, W. N., Hornschemeier, A. E., \& Garmire, G. P. 2000, ApJ, 543, L119

Bayet, E., Gerin, M., Phillips, T. G., \& Contursi, A. 2009, MNRAS, 399, 264

Beelen, A., Cox, P., Benford, D. J., Dowell, C. D., Kovács, A., Bertoldi, F., Omont, A., \& Carilli, C. L. 2006, ApJ, 642, 694

Beelen, A., Omont, A., Bavouzet, N., Kovács, A., Lagache, G., De Breuck, C., Weiss, A., Menten, K. M., Colbert, J. W., Dole, H., Siringo, G., \& Kreysa, E. 2008, A\&A, 485, 645

Behroozi, P. S., Conroy, C., \& Wechsler, R. H. 2010, ApJ, 717, 379

Behroozi, P. S., Wechsler, R. H., \& Conroy, C. 2013, ApJ, 770, 57

Bell, E. F. 2003, ApJ, 586, 794

Benson, A. J. 2010, Phys. Rep., 495, 33

-. 2012, New Astronomy, 17, 175

Berta, S., Lonsdale, C. J., Polletta, M., Savage, R. S., Franceschini, A., Buttery, H., Cimatti, A., Dias, J., Feruglio, C., Fiore, F., Held, E. V., La Franca, F., Maiolino, R., Marconi, A., Matute, I., Oliver, S. J., Ricciardelli, E., Rubele, S., Sacchi, N., Shupe, D., \& Surace, J. 2007, A\&A, 476, 151

Berta, S., Magnelli, B., Nordon, R., Lutz, D., Wuyts, S., Altieri, B., Andreani, P., Aussel, H., Castañeda, H., Cepa, J., Cimatti, A., Daddi, E., Elbaz, D., Förster Schreiber, N. M., Genzel, R., Le Floc'h, E., Maiolino, R., Pérez-Fournon, I., Poglitsch, A., Popesso, P., Pozzi, F., Riguccini, L., Rodighiero, G., Sanchez-Portal, M., Sturm, E., Tacconi, L. J., \& Valtchanov, I. 2011, A\&A, 532, A49

Bertoldi, F., Carilli, C., Aravena, M., Schinnerer, E., Voss, H., Smolcic, V., Jahnke, K., Scoville, N., Blain, A., Menten, K. M., Lutz, D., Brusa, M., Taniguchi, Y., Capak, P., Mobasher, B., Lilly, S., Thompson, D., Aussel, H., Kreysa, E., Hasinger, G., Aguirre, J., Schlaerth, J., \& Koekemoer, A. 2007, ApJS, 172, 132

Béthermin, M., Daddi, E., Magdis, G., Sargent, M. T., Hezaveh, Y., Elbaz, D., Le Borgne, D., Mullaney, J., Pannella, M., Buat, V., Charmandaris, V., Lagache, G., \& Scott, D. 2012a, ApJ, 757, L23

Béthermin, M., Dole, H., Beelen, A., \& Aussel, H. 2010a, A\&A, 512, A78

Béthermin, M., Dole, H., Cousin, M., \& Bavouzet, N. 2010b, A\&A, 516, A43

Béthermin, M., Dole, H., Lagache, G., Le Borgne, D., \& Penin, A. 2011, A\&A, 529, A4

Béthermin, M., Wang, L., Doré, O., Lagache, G., Sargent, M., Daddi, E., Cousin, M., \& Aussel, H. 2013, A\&A, 557, A66

Béthermin, M. et al. 2012b, A\&A, 542

Bielby, R. M., Hill, M. D., Metcalfe, N., \& Shanks, T. 2012, MNRAS, 419, 1315

Biggs, A. D. \& Ivison, R. J. 2008, MNRAS, 385, 893

Biggs, A. D., Ivison, R. J., Ibar, E., Wardlow, J. L., Dannerbauer, H., Smail, I., Walter, F., Weiß, A., Chapman, S. C., Coppin, K. E. K., De Breuck,

C., Dickinson, M., Knudsen, K. K., Mainieri, V., Menten, K., \& Papovich, C. 2011, MNRAS, 413, 2314

Biggs, A. D., Younger, J. D., \& Ivison, R. J. 2010, MNRAS, 408, 342

Bigiel, F., Leroy, A., Walter, F., Brinks, E., de Blok, W. J. G., Madore, B., \& Thornley, M. D. 2008, AJ, 136, 2846

Blain, A. W. 1996, MNRAS, 283, 1340

Blain, A. W., Assef, R., Stern, D., Tsai, C.-W., et al. 2013, ApJ, 778, 113

Blain, A. W., Barnard, V. E., \& Chapman, S. C. 2003, MNRAS, 338, 733

Blain, A. W., Chapman, S. C., Smail, I., \& Ivison, R. 2004a, ApJ, 611, 52

-. 2004b, ApJ, 611, 725

Blain, A. W., Jameson, A., Smail, I., Longair, M. S., Kneib, J.-P., \& Ivison, R. J. 1999, MNRAS, 309, 715

Blain, A. W., Smail, I., Ivison, R. J., Kneib, J., \& Frayer, D. T. 2002, Phys. Rep., 369, 111

Blake, C., Pope, A., Scott, D., \& Mobasher, B. 2006, MNRAS, 368, 732 
Blanc, G. A., Heiderman, A., Gebhardt, K., Evans, II, N. J., \& Adams, J. 2009, ApJ, 704, 842

Blanc, G. A., Schruba, A., Evans, II, N. J., Jogee, S., Bolatto, A., Leroy, A. K., Song, M., van den Bosch, R. C. E., Drory, N., Fabricius, M., Fisher, D., Gebhardt, K., Heiderman, A., Marinova, I., Vogel, S., \& Weinzirl, T. 2013, ApJ, 764, 117

Blitz, L., Fukui, Y., Kawamura, A., Leroy, A., Mizuno, N., \& Rosolowsky, E. 2007, in Protostars and Planets V, ed. B. Reipurth, D. Jewitt, \& K. Keil, 81-96

Blitz, L. \& Rosolowsky, E. 2006, ApJ, 650, 933

Bloemen, J. B. G. M., Strong, A. W., Mayer-Hasselwander, H. A., Blitz, L., Cohen, R. S., Dame, T. M., Grabelsky, D. A., Thaddeus, P., Hermsen, W., \& Lebrun, F. 1986, A\&A, 154, 25

Bolatto, A. D., Leroy, A. K., Rosolowsky, E., Walter, F., \& Blitz, L. 2008, ApJ, 686, 948

Bolatto, A. D., Wolfire, M., \& Leroy, A. K. 2013, ARA\&A, 51, 207

Bolzonella, M., Miralles, J., \& Pelló, R. 2000, A\&A, 363, 476

Bond, N. A., Benford, D. J., Gardner, J. P., Amblard, A., Fleuren, S., Blain, A. W., Dunne, L., Smith, D. J. B., Maddox, S. J., Hoyos, C., Baes, M., Bonfield, D., Bourne, N., Bridge, C., Buttiglione, S., Cava, A., Clements, D., Cooray, A., Dariush, A., de Zotti, G., Driver, S., Dye, S., Eales, S., Eisenhardt, P., Hopwood, R., Ibar, E., Ivison, R. J., Jarvis, M. J., Kelvin, L., Robotham, A. S. G., Temi, P., Thompson, M., Tsai, C.-W., van der Werf, P., Wright, E. L., Wu, J., \& Yan, L. 2012, ApJ, 750, L18

Bondi, M., Ciliegi, P., Venturi, T., Dallacasa, D., Bardelli, S., Zucca, E., Athreya, R. M., Gregorini, L., Zanichelli, A., Le Fèvre, O., Contini, T., Garilli, B., Iovino, A., Temporin, S., \& Vergani, D. 2007, A\&A, 463, 519

Borys, C., Chapman, S., Halpern, M., \& Scott, D. 2003, MNRAS, 344, 385

Borys, C., Smail, I., Chapman, S. C., Blain, A. W., Alexander, D. M., \& Ivison, R. J. 2005, ApJ, 635, 853

Boselli, A., Gavazzi, G., Lequeux, J., \& Pierini, D. 2002, A\&A, 385, 454

Bothwell, M. S., Chapman, S. C., Tacconi, L., Smail, I., Ivison, R. J., Casey, C. M., Bertoldi, F., Beswick, R., Biggs, A., Blain, A. W., Cox, P., Genzel, R., Greve, T. R., Kennicutt, R., Muxlow, T., Neri, R., \& Omont, A. 2010, MNRAS, 405, 219

Bothwell, M. S., Smail, I., Chapman, S. C., Genzel, R., Ivison, R. J., Tacconi, L. J., Alaghband-Zadeh, S., Bertoldi, F., Blain, A. W., Casey, C. M., Cox, P., Greve, T. R., Lutz, D., Neri, R., Omont, A., \& Swinbank, A. M. 2013a, MNRAS, 429, 3047

Bothwell, M. S. et al. 2013b, ArXiv e-prints

Bouché, N., Cresci, G., Davies, R., Eisenhauer, F., Förster Schreiber, N. M., Genzel, R., Gillessen, S., Lehnert, M., Lutz, D., Nesvadba, N., Shapiro, K. L., Sternberg, A., Tacconi, L. J., Verma, A., Cimatti, A., Daddi, E., Renzini, A., Erb, D. K., Shapley, A., \& Steidel, C. C. 2007, ApJ, 671, 303

Bouché, N., Dekel, A., Genzel, R., Genel, S., Cresci, G., Förster Schreiber, N. M., Shapiro, K. L., Davies, R. I., \& Tacconi, L. 2010, ApJ, 718, 1001

Bouwens, R. J., Illingworth, G. D., Blakeslee, J. P., \& Franx, M. 2006, ApJ, 653, 53

Bracco, A., Cooray, A., Veneziani, M., Amblard, A., Serra, P., Wardlow, J., Thompson, M. A., White, G., Auld, R., Baes, M., Bertoldi, F., Buttiglione, S., Cava, A., Clements, D. L., Dariush, A., de Zotti, G., Dunne, L., Dye, S., Eales, S., Fritz, J., Gomez, H., Hopwood, R., Ibar, I., Ivison, R. J., Jarvis, M., Lagache, G., Lee, M. G., Leeuw, L., Maddox, S., Michałowski, M., Pearson, C., Pohlen, M., Rigby, E., Rodighiero, G., Smith, D. J. B., Temi, P., Vaccari, M., \& van der Werf, P. 2011, MNRAS, 412, 1151

Bradford, C. M., Aguirre, J. E., Aikin, R., Bock, J. J., Earle, L., Glenn, J., Inami, H., Maloney, P. R., Matsuhara, H., Naylor, B. J., Nguyen, H. T., \& Zmuidzinas, J. 2009, ApJ, 705, 112

Brand, K., Brown, M. J. I., Dey, A., Jannuzi, B. T., Kochanek, C. S., Kenter, A. T., Fabricant, D., Fazio, G. G., Forman, W. R., Green, P. J., Jones, C. J., McNamara, B. R., Murray, S. S., Najita, J. R., Rieke, M., Shields, J. C., \& Vikhlinin, A. 2006, ApJ, 641, 140

Brandl, B. R., Bernard-Salas, J., Spoon, H. W. W., Devost, D., Sloan, G. C., Guilles, S., Wu, Y., Houck, J. R., Weedman, D. W., Armus, L., Appleton, P. N., Soifer, B. T., Charmandaris, V., Hao, L., Higdon, J. A., Marshall, S. J., \& Herter, T. L. 2006, ApJ, 653, 1129

Brauher, J. R., Dale, D. A., \& Helou, G. 2008, ApJS, 178, 280

Brewer, B. J., Dutton, A. A., Treu, T., Auger, M. W., Marshall, P. J., Barnabè, M., Bolton, A. S., Koo, D. C., \& Koopmans, L. V. E. 2012, MNRAS, 422,3574

Bridge, C. R., Blain, A., Borys, C. J. K., Petty, S., Benford, D., Eisenhardt, P., Farrah, D., Griffith, R. L., Jarrett, T., Lonsdale, C., Stanford, S. A., Stern, D., Tsai, C.-W., Wright, E. L., \& Wu, J. 2013, ApJ, 769, 91

Brodwin, M., Dey, A., Brown, M. J. I., Pope, A., Armus, L., Bussmann, S., Desai, V., Jannuzi, B. T., \& Le Floc'h, E. 2008, ApJ, 687 , L65

Brusa, M., Zamorani, G., Comastri, A., Hasinger, G., Cappelluti, N., Civano, F., Finoguenov, A., Mainieri, V., Salvato, M., Vignali, C., Elvis, M., Fiore, F., Gilli, R., Impey, C. D., Lilly, S. J., Mignoli, M., Silverman, J., Trump, J., Urry, C. M., Bender, R., Capak, P., Huchra, J. P., Kneib, J. P., Koekemoer, A., Leauthaud, A., Lehmann, I., Massey, R., Matute, I., McCarthy, P. J., McCracken, H. J., Rhodes, J., Scoville, N. Z., Taniguchi, Y., \& Thompson, D. 2007, ApJS, 172, 353

Bruzual, G. \& Charlot, S. 2003, MNRAS, 344, 1000

Buat, V., Noll, S., Burgarella, D., Giovannoli, E., Charmandaris, V., Pannella, M., Hwang, H. S., Elbaz, D., Dickinson, M., Magdis, G., Reddy, N., \& Murphy, E. J. 2012, A\&A, 545, A141

Budavári, T. \& Szalay, A. S. 2008, ApJ, 679, 301

Burgarella, D., Buat, V., \& Iglesias-Páramo, J. 2005, MNRAS, 360, 1413

Bussmann, R. S., Dey, A., Armus, L., Brown, M. J. I., Desai, V., Gonzalez, A. H., Jannuzi, B. T., Melbourne, J., \& Soifer, B. T. 2012, ApJ, 744, 150

Bussmann, R. S., Dey, A., Lotz, J., Armus, L., Brand, K., Brown, M. J. I., Desai, V., Eisenhardt, P., Higdon, J., Higdon, S., Jannuzi, B. T., Le Floc'h, E., Melbourne, J., Soifer, B. T., \& Weedman, D. 2009, ApJ, 693, 750

Bussmann, R. S., Dey, A., Lotz, J., Armus, L., Brown, M. J. I., Desai, V., Eisenhardt, P., Higdon, J., Higdon, S., Jannuzi, B. T., Le Floc'h, E., Melbourne, J., Soifer, B. T., \& Weedman, D. 2011, ApJ, 733, 21

Bussmann, R. S., Narayanan, D., Shirley, Y. L., Juneau, S., Wu, J., Solomon, P. M., Vanden Bout, P. A., Moustakas, J., \& Walker, C. K. 2008, ApJ, $681, \mathrm{~L} 73$

Bussmann, R. S., Perez-Fournon, I., Amber, S., Calanog, J., Gurwell, M. A., Dannerbauer, H., De Bernardis, F., Fu, H., Harris, A. I., Krips, M., 
Lapi, A., Maiolino, R., Omont, A., Riechers, D., Wardlow, J., Baker, A. J., Birkinshaw, M., Bock, J., Bourne, N., Clements, D. L., Cooray, A., De Zotti, G., Dunne, L., Dye, S., Eales, S., Farrah, D., Gavazzi, R., Gonzalez Nuevo, J., Hopwood, R., Ibar, E., Ivison, R. J., Laporte, N., Maddox, S., Martinez-Navajas, P., Michalowski, M., Negrello, M., Oliver, S. J., Roseboom, I. G., Scott, D., Serjeant, S., Smith, A. J., Smith, M., Streblyanska, A., Valiante, E., van der Werf, P., Verma, A., Vieira, J. D., Wang, L., \& Wilner, D. 2013, ArXiv e-prints

Cai, Z.-Y., Lapi, A., Xia, J.-Q., De Zotti, G., Negrello, M., Gruppioni, C., Rigby, E., Castex, G., Delabrouille, J., \& Danese, L. 2013 , ApJ, 768, 21 Calzetti, D. 2001, PASP, 113, 1449

Calzetti, D., Kinney, A. L., \& Storchi-Bergmann, T. 1994, ApJ, 429, 582

Capak, P., Carilli, C. L., Lee, N., Aldcroft, T., Aussel, H., Schinnerer, E., Wilson, G. W., Yun, M. S., Blain, A., Giavalisco, M., Ilbert, O., Kartaltepe, J., Lee, K., McCracken, H., Mobasher, B., Salvato, M., Sasaki, S., Scott, K. S., Sheth, K., Shioya, Y., Thompson, D., Elvis, M., Sanders, D. B., Scoville, N. Z., \& Tanaguchi, Y. 2008, ApJ, 681, L53

Capak, P. L., Riechers, D., Scoville, N. Z., Carilli, C., Cox, P., Neri, R., Robertson, B., Salvato, M., Schinnerer, E., Yan, L., Wilson, G. W., Yun, M., Civano, F., Elvis, M., Karim, A., Mobasher, B., \& Staguhn, J. G. 2011, Nature, 470, 233

Cappellari, M., McDermid, R. M., Alatalo, K., Blitz, L., Bois, M., Bournaud, F., Bureau, M., Crocker, A. F., Davies, R. L., Davis, T. A., de Zeeuw, P. T., Duc, P.-A., Emsellem, E., Khochfar, S., Krajnović, D., Kuntschner, H., Morganti, R., Naab, T., Oosterloo, T., Sarzi, M., Scott, N., Serra, P., Weijmans, A.-M., \& Young, L. M. 2013, MNRAS, 432, 1862

Cappellari, M. et al. 2012, Nature, 484, 485

Caputi, K. I., Lagache, G., Yan, L., Dole, H., Bavouzet, N., Le Floc'h, E., Choi, P. I., Helou, G., \& Reddy, N. 2007, ApJ, 660, 97

Carilli, C. L., Daddi, E., Riechers, D., Walter, F., Weiss, A., Dannerbauer, H., Morrison, G. E., Wagg, J., Davé, R., Elbaz, D., Stern, D., Dickinson, M., Krips, M., \& Aravena, M. 2010, ApJ, 714, 1407

Carilli, C. L., Hodge, J., Walter, F., Riechers, D., Daddi, E., Dannerbauer, H., \& Morrison, G. E. 2011, ApJ, 739, L33

Carilli, C. L., Riechers, D., Walter, F., Maiolino, R., Wagg, J., Lentati, L., McMahon, R., \& Wolfe, A. 2013, ApJ, 763, 120

Carilli, C. L. \& Walter, F. 2013, ARA\&A, 51, 105

Carilli, C. L. \& Yun, M. S. 1999, ApJ, 513, L13

Carlstrom, J. E. et al. 2011, PASP, 123, 568

Carniani, S., Marconi, A., Biggs, A., Cresci, G., Cupani, G., D’Odorico, V., Humphreys, E., Maiolino, R., Mannucci, F., Molaro, P., Nagao, T., Testi, L., \& Zwaan, M. A. 2013, A\&A, 559, A29

Casey, C. M. 2012, MNRAS, 425, 3094

Casey, C. M., Berta, S., Béthermin, M., Bock, J., Bridge, C., Budynkiewicz, J., Burgarella, D., Chapin, E., Chapman, S. C., Clements, D. L., Conley, A., Conselice, C. J., Cooray, A., Farrah, D., Hatziminaoglou, E., Ivison, R. J., le Floc'h, E., Lutz, D., Magdis, G., Magnelli, B., Oliver, S. J., Page, M. J., Pozzi, F., Rigopoulou, D., Riguccini, L., Roseboom, I. G., Sanders, D. B., Scott, D., Seymour, N., Valtchanov, I., Vieira, J. D., Viero, M., \& Wardlow, J. 2012a, ApJ, 761, 140

Casey, C. M., Berta, S., Béthermin, M., Bock, J., Bridge, C., Burgarella, D., Chapin, E., Chapman, S. C., Clements, D. L., Conley, A., Conselice, C. J., Cooray, A., Farrah, D., Hatziminaoglou, E., Ivison, R. J., le Floc'h, E., Lutz, D., Magdis, G., Magnelli, B., Oliver, S. J., Page, M. J., Pozzi, F., Rigopoulou, D., Riguccini, L., Roseboom, I. G., Sanders, D. B., Scott, D., Seymour, N., Valtchanov, I., Vieira, J. D., Viero, M., \& Wardlow, J. 2012b, ApJ, 761, 139

Casey, C. M., Chapman, S. C., Beswick, R. J., Biggs, A. D., Blain, A. W., Hainline, L. J., Ivison, R. J., Muxlow, T. W. B., \& Smail, I. 2009a, MNRAS, 399, 121

Casey, C. M., Chapman, S. C., Daddi, E., Dannerbauer, H., Pope, A., Scott, D., Bertoldi, F., Beswick, R. J., Blain, A. W., Cox, P., Genzel, R., Greve, T. R., Ivison, R. J., Muxlow, T. W. B., Neri, R., Omont, A., Smail, I., \& Tacconi, L. J. 2009b, MNRAS, 400,670

Casey, C. M., Chapman, S. C., Muxlow, T. W. B., Beswick, R. J., Alexander, D. M., \& Conselice, C. J. 2009c, MNRAS, 395, 1249

Casey, C. M., Chapman, S. C., Neri, R., Bertoldi, F., Smail, I., Coppin, K., Greve, T. R., Bothwell, M. S., Beswick, R. J., Blain, A. W., Cox, P., Genzel, R., Muxlow, T. W. B., Omont, A., \& Swinbank, A. M. 2011a, MNRAS, 415, 2723

Casey, C. M., Chapman, S. C., Smail, I., Alaghband-Zadeh, S., Bothwell, M. S., \& Swinbank, A. M. 2011b, MNRAS, 411,2739

Casey, C. M., Chen, C.-C., Cowie, L., Barger, A., Capak, P., Ilbert, O., Koss, M., Lee, N., Le Floc'h, E., Sanders, D. B., \& Williams, J. P. 2013, ArXiv e-prints

Chabrier, G. 2003, ApJ, 586, L133

Chakrabarti, S., Fenner, Y., Cox, T. J., Hernquist, L., \& Whitney, B. A. 2008, ApJ, 688, 972

Chapin, E. L., Chapman, S. C., Coppin, K. E., Devlin, M. J., Dunlop, J. S., Greve, T. R., Halpern, M., Hasselfield, M. F., Hughes, D. H., Ivison, R. J., Marsden, G., Moncelsi, L., Netterfield, C. B., Pascale, E., Scott, D., Smail, I., Viero, M., Walter, F., Weiss, A., \& van der Werf, P. 2010, MNRAS, 1682

- 2011, MNRAS, 411, 505

Chapin, E. L., Pope, A., Scott, D., Aretxaga, I., Austermann, J. E., Chary, R.-R., Coppin, K., Halpern, M., Hughes, D. H., Lowenthal, J. D., Morrison, G. E., Perera, T. A., Scott, K. S., Wilson, G. W., \& Yun, M. S. 2009, MNRAS, 398, 1793

Chapman, S. C., Barger, A. J., Cowie, L. L., Scott, D., Borys, C., Capak, P., Fomalont, E. B., Lewis, G. F., Richards, E. A., Steffen, A. T., Wilson, G., \& Yun, M. 2003a, ApJ, 585, 57

Chapman, S. C., Blain, A., Ibata, R., Ivison, R. J., Smail, I., \& Morrison, G. 2009, ApJ, 691, 560

Chapman, S. C., Blain, A. W., Ivison, R. J., \& Smail, I. R. 2003b, Nature, 422, 695

-. 2003c, Nature, 422, 695

Chapman, S. C., Blain, A. W., Smail, I., \& Ivison, R. J. 2005, ApJ, 622, 772

Chapman, S. C., Ivison, R. J., Roseboom, I. G., Auld, R., Bock, J., Brisbin, D., Burgarella, D., Chanial, P., Clements, D. L., Cooray, A., Eales, S., Franceschini, A., Giovannoli, E., Glenn, J., Griffin, M., Mortier, A. M. J., Oliver, S. J., Omont, A., Page, M. J., Papageorgiou, A., Pearson, C. P., Pérez-Fournon, I., Pohlen, M., Rawlings, J. I., Raymond, G., Rodighiero, G., Rowan-Robinson, M., Scott, D., Seymour, N., Smith, A. J., Symeonidis, M., Tugwell, K. E., Vaccari, M., Vieira, J. D., Vigroux, L., Wang, L., \& Wright, G. 2010, MNRAS, 409, L13

Chapman, S. C., Lewis, G. F., Scott, D., Borys, C., \& Richards, E. 2002, ApJ, 570, 557

Chapman, S. C., Richards, E. A., Lewis, G. F., Wilson, G., \& Barger, A. J. 2001, ApJ, 548, L147 
Chapman, S. C., Smail, I., Blain, A. W., \& Ivison, R. J. 2004a, ApJ, 614, 671

Chapman, S. C., Smail, I., Windhorst, R., Muxlow, T., \& Ivison, R. J. 2004b, ApJ, 611, 732

Charlot, S. \& Fall, S. M. 2000, ApJ, 539, 718

Charmandaris, V., Mirabel, I. F., Tran, D., Laurent, O., Cesarsky, C. J., Gallais, P., Sauvage, M., \& Vigroux, L. 1997, in Extragalactic Astronomy in the Infrared, ed. G. A. Mamon, T. X. Thuan, \& J. Tran Thanh Van, 283

Chary, R. \& Elbaz, D. 2001, ApJ, 556, 562

Chen, C.-C., Cowie, L. L., Barger, A. J., Casey, C. M., Lee, N., Sanders, D. B., Wang, W.-H., \& Williams, J. P. 2013a, ApJ, 762, 81

Chen, C.-C. et al. 2013b, ApJ

Chomiuk, L. \& Povich, M. S. 2011, AJ, 142, 197

Ciliegi, P., Zamorani, G., Hasinger, G., Lehmann, I., Szokoly, G., \& Wilson, G. 2003, A\&A, 398, 901

Clauset, A., Rohilla Shalizi, C., \& Newman, M. E. J. 2007, ArXiv e-prints

Clements, D. L., Bendo, G., Pearson, C., Khan, S. A., Matsuura, S., \& Shirahata, M. 2011, MNRAS, 411, 373

Clements, D. L., Rigby, E., Maddox, S., Dunne, L., Mortier, A., Pearson, C., Amblard, A., Auld, R., Baes, M., Bonfield, D., Burgarella, D., Buttiglione, S., Cava, A., Cooray, A., Dariush, A., de Zotti, G., Dye, S., Eales, S., Frayer, D., Fritz, J., Gardner, J. P., Gonzalez-Nuevo, J., Herranz, D., Ibar, E., Ivison, R., Jarvis, M. J., Lagache, G., Leeuw, L., Lopez-Caniego, M., Negrello, M., Pascale, E., Pohlen, M., Rodighiero, G., Samui, S., Serjeant, S., Sibthorpe, B., Scott, D., Smith, D. J. B., Temi, P., Thompson, M., Valtchanov, I., van der Werf, P., \& Verma, A. 2010, A\&A, 518, L8

Coil, A. L., Newman, J. A., Croton, D., Cooper, M. C., Davis, M., Faber, S. M., Gerke, B. F., Koo, D. C., Padmanabhan, N., Wechsler, R. H., \& Weiner, B. J. 2008, ApJ, 672, 153

Cole, S. 1991, ApJ, 367, 45

Cole, S., Lacey, C. G., Baugh, C. M., \& Frenk, C. S. 2000, MNRAS, 319, 168

Combes, F., García-Burillo, S., Braine, J., Schinnerer, E., Walter, F., \& Colina, L. 2013, A\&A, 550, A41

Combes, F., Rex, M., Rawle, T. D., Egami, E., Boone, F., Smail, I., Richard, J., Ivison, R. J., Gurwell, M., Casey, C. M., Omont, A., Berciano Alba, A., Dessauges-Zavadsky, M., Edge, A. C., Fazio, G. G., Kneib, J.-P., Okabe, N., Pelló, R., Pérez-González, P. G., Schaerer, D., Smith, G. P., Swinbank, A. M., \& van der Werf, P. 2012, A\&A, 538, L4

Condon, J. J. 1992, ARA\&A, 30, 575

Conley, A. et al. 2011, ApJ, 732, L35

Connolly, A. J., Szalay, A. S., Dickinson, M., Subbarao, M. U., \& Brunner, R. J. 1997, ApJ, 486, L11

Conroy, C. 2013, ARA\&A, 51, 393

Conroy, C. \& van Dokkum, P. 2012a, ApJ, 747, 69

Conroy, C. \& van Dokkum, P. G. 2012b, ApJ, 760, 71

Conroy, C. \& Wechsler, R. H. 2009, ApJ, 696, 620

Cooray, A. 2006, MNRAS, 365, 842

Cooray, A., Amblard, A., Wang, L., Arumugam, V., Auld, R., Aussel, H., Babbedge, T., Blain, A., Bock, J., Boselli, A., Buat, V., Burgarella, D., Castro-Rodriguez, N., Cava, A., Chanial, P., Clements, D. L., Conley, A., Conversi, L., Dowell, C. D., Dwek, E., Eales, S., Elbaz, D., Farrah, D., Fox, M., Franceschini, A., Gear, W., Glenn, J., Griffin, M., Halpern, M., Hatziminaoglou, E., Ibar, E., Isaak, K., Ivison, R. J., Khostovan, A. A., Lagache, G., Levenson, L., Lu, N., Madden, S., Maffei, B., Mainetti, G., Marchetti, L., Marsden, G., Mitchell-Wynne, K., Mortier, A. M. J., Nguyen, H. T., O’Halloran, B., Oliver, S. J., Omont, A., Page, M. J., Panuzzo, P., Papageorgiou, A., Pearson, C. P., Perez Fournon, I., Pohlen, M., Rawlings, J. I., Raymond, G., Rigopoulou, D., Rizzo, D., Roseboom, I. G., Rowan-Robinson, M., Schulz, B., Scott, D., Serra, P., Seymour, N., Shupe, D. L., Smith, A. J., Stevens, J. A., Symeonidis, M., Trichas, M., Tugwell, K. E., Vaccari, M., Valtchanov, I., Vieira, J. D., Vigroux, L., Ward, R., Wright, G., Xu, C. K., \& Zemcov, M. 2010, A\&A, 518, L22

Cooray, A. \& Hu, W. 2000, ApJ, 534, 533

Cooray, A. \& Sheth, R. 2002, Phys. Rep., 372,

Coppin, K., Chapin, E. L., Mortier, A. M. J., Scott, S. E., Borys, C., Dunlop, J. S., Halpern, M., Hughes, D. H., Pope, A., Scott, D., Serjeant, S., Wagg, J., Alexander, D. M., Almaini, O., Aretxaga, I., Babbedge, T., Best, P. N., Blain, A., Chapman, S., Clements, D. L., Crawford, M., Dunne, L., Eales, S. A., Edge, A. C., Farrah, D., Gaztañaga, E., Gear, W. K., Granato, G. L., Greve, T. R., Fox, M., Ivison, R. J., Jarvis, M. J., Jenness, T., Lacey, C., Lepage, K., Mann, R. G., Marsden, G., Martinez-Sansigre, A., Oliver, S., Page, M. J., Peacock, J. A., Pearson, C. P., Percival, W. J., Priddey, R. S., Rawlings, S., Rowan-Robinson, M., Savage, R. S., Seigar, M., Sekiguchi, K., Silva, L., Simpson, C., Smail, I., Stevens, J. A., Takagi, T., Vaccari, M., van Kampen, E., \& Willott, C. J. 2006, MNRAS, 372, 1621

Coppin, K., Halpern, M., Scott, D., Borys, C., \& Chapman, S. 2005, MNRAS, 357, 1022

Coppin, K., Halpern, M., Scott, D., Borys, C., Dunlop, J., Dunne, L., Ivison, R., Wagg, J., Aretxaga, I., Battistelli, E., Benson, A., Blain, A., Chapman, S., Clements, D., Dye, S., Farrah, D., Hughes, D., Jenness, T., van Kampen, E., Lacey, C., Mortier, A., Pope, A., Priddey, R., Serjeant, S., Smail, I., Stevens, J., \& Vaccari, M. 2008a, MNRAS, 384, 1597

Coppin, K., Pope, A., Menéndez-Delmestre, K., Alexander, D. M., Dunlop, J. S., Egami, E., Gabor, J., Ibar, E., Ivison, R. J., Austermann, J. E., Blain, A. W., Chapman, S. C., Clements, D. L., Dunne, L., Dye, S., Farrah, D., Hughes, D. H., Mortier, A. M. J., Page, M. J., Rowan-Robinson, M., Scott, D., Simpson, C., Smail, I., Swinbank, A. M., Vaccari, M., \& Yun, M. S. 2010, ApJ, 713, 503

Coppin, K. E. K., Danielson, A. L. R., Geach, J. E., Hodge, J. A., Swinbank, A. M., Wardlow, J. L., Bertoldi, F., Biggs, A., Brandt, W. N., Caselli, P., Chapman, S. C., Dannerbauer, H., Dunlop, J. S., Greve, T. R., Hamann, F., Ivison, R. J., Karim, A., Knudsen, K. K., Menten, K. M., Schinnerer, E., Smail, I., Spaans, M., Walter, F., Webb, T. M. A., \& van der Werf, P. P. 2012, MNRAS, 427, 520

Coppin, K. E. K., Smail, I., Alexander, D. M., Weiss, A., Walter, F., Swinbank, A. M., Greve, T. R., Kovacs, A., De Breuck, C., Dickinson, M., Ibar, E., Ivison, R. J., Reddy, N., Spinrad, H., Stern, D., Brandt, W. N., Chapman, S. C., Dannerbauer, H., van Dokkum, P., Dunlop, J. S., Frayer, D., Gawiser, E., Geach, J. E., Huynh, M., Knudsen, K. K., Koekemoer, A. M., Lehmer, B. D., Menten, K. M., Papovich, C., Rix, H., Schinnerer, E., Wardlow, J. L., \& van der Werf, P. P. 2009, MNRAS, 395, 1905

Coppin, K. E. K., Swinbank, A. M., Neri, R., Cox, P., Alexander, D. M., Smail, I., Page, M. J., Stevens, J. A., Knudsen, K. K., Ivison, R. J., Beelen, A., Bertoldi, F., \& Omont, A. 2008b, MNRAS, 389, 45 
Cowie, L. L., Barger, A. J., \& Kneib, J.-P. 2002, AJ, 123, 2197

Cowie, L. L., Barger, A. J., Wang, W.-H., \& Williams, J. P. 2009, ApJ, 697, L122

Cowie, L. L., Songaila, A., Hu, E. M., \& Cohen, J. G. 1996, AJ, 112, 839

Cox, P., Krips, M., Neri, R., Omont, A., Güsten, R., Menten, K. M., Wyrowski, F., Weiß, A., Beelen, A., Gurwell, M. A., Dannerbauer, H., Ivison, R. J., Negrello, M., Aretxaga, I., Hughes, D. H., Auld, R., Baes, M., Blundell, R., Buttiglione, S., Cava, A., Cooray, A., Dariush, A., Dunne, L., Dye, S., Eales, S. A., Frayer, D., Fritz, J., Gavazzi, R., Hopwood, R., Ibar, E., Jarvis, M., Maddox, S., Michałowski, M., Pascale, E., Pohlen, M., Rigby, E., Smith, D. J. B., Swinbank, A. M., Temi, P., Valtchanov, I., van der Werf, P., \& de Zotti, G. 2011, ApJ, 740, 63

Cox, T. J., Jonsson, P., Somerville, R. S., Primack, J. R., \& Dekel, A. 2008, MNRAS, 384, 386

Crawford, T. M., Switzer, E. R., Holzapfel, W. L., Reichardt, C. L., Marrone, D. P., \& Vieira, J. D. 2010, ApJ, 718, 513

Croom, S. M., Boyle, B. J., Shanks, T., Smith, R. J., Miller, L., Outram, P. J., Loaring, N. S., Hoyle, F., \& da Ângela, J. 2005 , MNRAS, 356, 415

Croton, D. J., Springel, V., White, S. D. M., De Lucia, G., Frenk, C. S., Gao, L., Jenkins, A., Kauffmann, G., Navarro, J. F., \& Yoshida, N. 2006, MNRAS, 365, 11

da Cunha, E., Charlot, S., \& Elbaz, D. 2008, MNRAS, 388, 1595

Daddi, E., Alexander, D. M., Dickinson, M., Gilli, R., Renzini, A., Elbaz, D., Cimatti, A., Chary, R., Frayer, D., Bauer, F. E., Brandt, W. N., Giavalisco, M., Grogin, N. A., Huynh, M., Kurk, J., Mignoli, M., Morrison, G., Pope, A., \& Ravindranath, S. 2007a, ApJ, 670, 173

Daddi, E., Bournaud, F., Walter, F., Dannerbauer, H., Carilli, C. L., Dickinson, M., Elbaz, D., Morrison, G. E., Riechers, D., Onodera, M., Salmi, F., Krips, M., \& Stern, D. 2010a, ApJ, 713, 686

Daddi, E., Dannerbauer, H., Stern, D., Dickinson, M., Morrison, G., Elbaz, D., Giavalisco, M., Mancini, C., Pope, A., \& Spinrad, H. 2009, ApJ, 694,1517

Daddi, E., Dickinson, M., Morrison, G., Chary, R., Cimatti, A., Elbaz, D., Frayer, D., Renzini, A., Pope, A., Alexander, D. M., Bauer, F. E., Giavalisco, M., Huynh, M., Kurk, J., \& Mignoli, M. 2007b, ApJ, 670, 156

Daddi, E., Elbaz, D., Walter, F., Bournaud, F., Salmi, F., Carilli, C., Dannerbauer, H., Dickinson, M., Monaco, P., \& Riechers, D. 2010b, ApJ, 714, L118

Daddi, E. et al. 2005, ApJ, 631, L13

Dale, D. A. \& Helou, G. 2002, ApJ, 576, 159

Dale, D. A., Helou, G., Contursi, A., Silbermann, N. A., \& Kolhatkar, S. 2001, ApJ, 549, 215

Danielson, A. L. R., Swinbank, A. M., Smail, I., Bayet, E., van der Werf, P. P., Cox, P., Edge, A. C., Henkel, C., \& Ivison, R. J. 2013, MNRAS

Danielson, A. L. R., Swinbank, A. M., Smail, I., Cox, P., Edge, A. C., Weiss, A., Harris, A. I., Baker, A. J., De Breuck, C., Geach, J. E., Ivison, R. J., Krips, M., Lundgren, A., Longmore, S., Neri, R., \& Flaquer, B. O. 2011, MNRAS, 410, 1687

Das, S., Louis, T., Nolta, M. R., Addison, G. E., Battistelli, E. S., Bond, J., Calabrese, E., Devlin, D. C. M. J., Dicker, S., Dunkley, J., Dünner, R., Fowler, J. W., Gralla, M., Hajian, A., Halpern, M., Hasselfield, M., Hilton, M., Hincks, A. D., Hlozek, R., Huffenberger, K. M., Hughes, J. P., Irwin, K. D., Kosowsky, A., Lupton, R. H., Marriage, T. A., Marsden, D., Menanteau, F., Moodley, K., Niemack, M. D., Page, L. A., Partridge, B., Reese, E. D., Schmitt, B. L., Sehgal, N., Sherwin, B. D., Sievers, J. L., Spergel, D. N., Staggs, S. T., Swetz, D. S., Switzer, E. R., Thornton, R., Trac, H., \& Wollack, E. 2013, ArXiv e-prints

Davé, R. 2008, MNRAS, 385, 147

Davé, R., Finlator, K., \& Oppenheimer, B. D. 2011, MNRAS, 416, 1354

-. 2012, MNRAS, 421, 98

Davé, R., Finlator, K., Oppenheimer, B. D., Fardal, M., Katz, N., Kereš, D., \& Weinberg, D. H. 2010, MNRAS, 404, 1355

De Bernardis, F. \& Cooray, A. 2012, ApJ, 760, 14

De Breuck, C., Maiolino, R., Caselli, P., Coppin, K., Hailey-Dunsheath, S., \& Nagao, T. 2011, A\&A, 530, L8

de Jong, T., Clegg, P. E., Rowan-Robinson, M., Soifer, B. T., Habing, H. J., Houck, J. R., Aumann, H. H., \& Raimond, E. 1984, ApJ, 278 , L67

de Jong, T., Klein, U., Wielebinski, R., \& Wunderlich, E. 1985, A\&A, 147, 6

de Looze, I., Baes, M., Bendo, G. J., Cortese, L., \& Fritz, J. 2011, MNRAS, 416, 2712

Deane, R. P., Heywood, I., Rawlings, S., \& Marshall, P. J. 2013, MNRAS, 434, 23

Decarli, R., Walter, F., Carilli, C., Bertoldi, F., Cox, P., Ferkinhoff, C., Groves, B., Maiolino, R., Neri, R., Riechers, D., \& Weiss, A. 2014, ApJ, 782, L17

Decarli, R., Walter, F., Neri, R., Bertoldi, F., Carilli, C., Cox, P., Kneib, J. P., Lestrade, J. F., Maiolino, R., Omont, A., Richard, J., Riechers, D., Thanjavur, K., \& Weiss, A. 2012, ApJ, 752, 2

Dekel, A., Birnboim, Y., Engel, G., Freundlich, J., Goerdt, T., Mumcuoglu, M., Neistein, E., Pichon, C., Teyssier, R., \& Zinger, E. 2009a, Nature, 457,451

Dekel, A., Sari, R., \& Ceverino, D. 2009b, ApJ, 703, 785

Delahaye, T., Fiasson, A., Pohl, M., \& Salati, P. 2011, A\&A, 531, A37+

Desai, V., Soifer, B. T., Dey, A., Jannuzi, B. T., Le Floc'h, E., Bian, C., Brand, K., Brown, M. J. I., Armus, L., Weedman, D. W., Cool, R., Stern, D., \& Brodwin, M. 2008, ApJ, 679, 1204

Desai, V., Soifer, B. T., Dey, A., Le Floc'h, E., Armus, L., Brand, K., Brown, M. J. I., Brodwin, M., Jannuzi, B. T., Houck, J. R., Weedman, D. W., Ashby, M. L. N., Gonzalez, A., Huang, J., Smith, H. A., Teplitz, H., Willner, S. P., \& Melbourne, J. 2009, ApJ, 700, 1190

Devlin, M. J., Ade, P. A. R., Aretxaga, I., Bock, J. J., Chapin, E. L., Griffin, M., Gundersen, J. O., Halpern, M., Hargrave, P. C., Hughes, D. H., Klein, J., Marsden, G., Martin, P. G., Mauskopf, P., Moncelsi, L., Netterfield, C. B., Ngo, H., Olmi, L., Pascale, E., Patanchon, G., Rex, M., Scott, D., Semisch, C., Thomas, N., Truch, M. D. P., Tucker, C., Tucker, G. S., Viero, M. P., \& Wiebe, D. V. 2009, Nature, 458, 737

Devriendt, J. E. G. \& Guiderdoni, B. 2000, A\&A, 363, 851

Devriendt, J. E. G., Guiderdoni, B., \& Sadat, R. 1999, A\&A, 350, 381

Dey, A., Soifer, B. T., Desai, V., Brand, K., Le Floc'h, E., Brown, M. J. I., Jannuzi, B. T., Armus, L., Bussmann, S., Brodwin, M., Bian, C., Eisenhardt, P., Higdon, S. J., Weedman, D., \& Willner, S. P. 2008, ApJ, 677, 943

Díaz-Santos, T., Armus, L., Charmandaris, V., Stierwalt, S., Murphy, E. J., Haan, S., Inami, H., Malhotra, S., Meijerink, R., Stacey, G., Petric, A. O., Evans, A. S., Veilleux, S., van der Werf, P. P., Lord, S., Lu, N., Howell, J. H., Appleton, P., Mazzarella, J. M., Surace, J. A., Xu, C. K., 
Schulz, B., Sanders, D. B., Bridge, C., Chan, B. H. P., Frayer, D. T., Iwasawa, K., Melbourne, J., \& Sturm, E. 2013, ApJ, 774, 68

Díaz-Santos, T., Charmandaris, V., Armus, L., Petric, A. O., Howell, J. H., Murphy, E. J., Mazzarella, J. M., Veilleux, S., Bothun, G., Inami, H., Appleton, P. N., Evans, A. S., Haan, S., Marshall, J. A., Sanders, D. B., Stierwalt, S., \& Surace, J. A. 2010, ApJ, 723, 993

Dickinson, M., Giavalisco, M., \& GOODS Team. 2003, in The Mass of Galaxies at Low and High Redshift, ed. R. Bender \& A. Renzini, 324

Dobbs, C. L., Krumholz, M. R., Ballesteros-Paredes, J., Bolatto, A. D., Fukui, Y., Heyer, M., Mac Low, M.-M., Ostriker, E. C., \& VázquezSemadeni, E. 2013, arXiv/1312.3223

Dole, H., Le Floc'h, E., Pérez-González, P. G., Papovich, C., Egami, E., Lagache, G., Alonso-Herrero, A., Engelbracht, C. W., Gordon, K. D., Hines, D. C., Krause, O., Misselt, K. A., Morrison, J. E., Rieke, G. H., Rieke, M. J., Rigby, J. R., Young, E. T., Bai, L., Blaylock, M., Neugebauer, G., Beichman, C. A., Frayer, D. T., Mould, J. R., \& Richards, P. L. 2004, ApJS, 154, 87

Donley, J. L., Koekemoer, A. M., Brusa, M., Capak, P., Cardamone, C. N., Civano, F., Ilbert, O., Impey, C. D., Kartaltepe, J. S., Miyaji, T., Salvato, M., Sanders, D. B., Trump, J. R., \& Zamorani, G. 2012, ApJ, 748, 142

Donley, J. L., Rieke, G. H., Alexander, D. M., Egami, E., \& Pérez-González, P. G. 2010, ApJ, 719, 1393

Donovan Meyer, J., Koda, J., Momose, R., Fukuhara, M., Mooney, T., Towers, S., Egusa, F., Kennicutt, R., Kuno, N., Carty, M., Sawada, T., \& Scoville, N. 2012a, ApJ, 744, 42

-. 2012b, ApJ, 744, 42

Donovan Meyer, J., Koda, J., Momose, R., Mooney, T., Egusa, F., Carty, M., Kennicutt, R., Kuno, N., Rebolledo, D., Sawada, T., Scoville, N., \& Wong, T. 2013, ApJ, 772, 107

Dopita, M. A., Groves, B. A., Fischera, J., Sutherland, R. S., Tuffs, R. J., Popescu, C. C., Kewley, L. J., Reuland, M., \& Leitherer, C. 2005, ApJ, 619,755

Dowell, C. D., Allen, C. A., Babu, R. S., Freund, M. M., Gardner, M., Groseth, J., Jhabvala, M. D., Kovacs, A., Lis, D. C., Moseley, Jr., S. H., Phillips, T. G., Silverberg, R. F., Voellmer, G. M., \& Yoshida, H. 2003, in Society of Photo-Optical Instrumentation Engineers (SPIE) Conference Series, Vol. 4855, Society of Photo-Optical Instrumentation Engineers (SPIE) Conference Series, ed. T. G. Phillips \& J. Zmuidzinas, $73-87$

Dowell, C. D., Conley, A., Glenn, J., Arumugam, V., Asboth, V., Aussel, H., Bertoldi, F., Bethermin, M., Bock, J., Boselli, A., Bridge, C., Buat, V., Burgarella, D., Cabrera-Lavers, A., Casey, C. M., Chapman, S. C., Clements, D. L., Conversi, L., Cooray, A., Dannerbauer, H., De Bernardis, F., Ellsworth-Bowers, T. P., Farrah, D., Franceschini, A., Griffin, M., Gurwell, M. A., Halpern, M., Hatziminaoglou, E., Heinis, S., Ibar, E., Ivison, R. J., Laporte, N., Marchetti, L., Martinez-Navajas, P., Marsden, G., Morrison, G. E., Nguyen, H. T., O’Halloran, B., Oliver, S. J., Omont, A., Page, M. J., Papageorgiou, A., Pearson, C. P., Petitpas, G., Perez-Fournon, I., Pohlen, M., Riechers, D., Rigopoulou, D., Roseboom, I. G., Rowan-Robinson, M., Sayers, J., Schulz, B., Scott, D., Seymour, N., Shupe, D. L., Smith, A. J., Streblyanska, A., Symeonidis, M., Vaccari, M., Valtchanov, I., Vieira, J. D., Viero, M., Wang, L., Wardlow, J., Xu, C. K., \& Zemcov, M. 2013, ArXiv e-prints

Downes, A. J. B., Peacock, J. A., Savage, A., \& Carrie, D. R. 1986, MNRAS, 218, 31

Downes, D., Neri, R., Greve, A., Guilloteau, S., Casoli, F., Hughes, D., Lutz, D., Menten, K. M., Wilner, D. J., Andreani, P., Bertoldi, F., Carilli, C. L., Dunlop, J., Genzel, R., Gueth, F., Ivison, R. J., Mann, R. G., Mellier, Y., Oliver, S., Peacock, J., Rigopoulou, D., Rowan-Robinson, M., Schilke, P., Serjeant, S., Tacconi, L. J., \& Wright, M. 1999, A\&A, 347, 809

Downes, D. \& Solomon, P. M. 1998, ApJ, 507, 615

-. 2003, ApJ, 582, 37

Draine, B. T. 1978, ApJS, 36, 595

-. 2006, ApJ, 636, 1114

Draine, B. T. \& Li, A. 2007, ApJ, 657, 810

Driver, S. P., Popescu, C. C., Tuffs, R. J., Liske, J., Graham, A. W., Allen, P. D., \& de Propris, R. 2007, MNRAS, 379, 1022

Dullemond, C. P. \& van Bemmel, I. M. 2005, A\&A, 436, 47

Dumas, G., Schinnerer, E., Tabatabaei, F. S., Beck, R., Velusamy, T., \& Murphy, E. 2011, AJ, 141, 41

Dunlop, J. et al. 2007, Spitzer Proposal, 40021

Dunlop, J. S. 2011, in Astronomical Society of the Pacific Conference Series, Vol. 446, Galaxy Evolution: Infrared to Millimeter Wavelength Perspective, ed. W. Wang, J. Lu, Z. Luo, Z. Yang, H. Hua, \& Z. Chen, 209

Dunlop, J. S., Ade, P. A. R., Bock, J. J., Chapin, E. L., Cirasuolo, M., Coppin, K. E. K., Devlin, M. J., Griffin, M., Greve, T. R., Gundersen, J. O., Halpern, M., Hargrave, P. C., Hughes, D. H., Ivison, R. J., Klein, J., Kovacs, A., Marsden, G., Mauskopf, P., Netterfield, C. B., Olmi, L., Pascale, E., Patanchon, G., Rex, M., Scott, D., Semisch, C., Smail, I., Targett, T. A., Thomas, N., Truch, M. D. P., Tucker, C., Tucker, G. S., Viero, M. P., Walter, F., Wardlow, J. L., Weiss, A., \& Wiebe, D. V. 2010, MNRAS, 1354

Dunlop, J. S., McLure, R. J., Yamada, T., Kajisawa, M., Peacock, J. A., Mann, R. G., Hughes, D. H., Aretxaga, I., Muxlow, T. W. B., Richards, A. M. S., Dickinson, M., Ivison, R. J., Smith, G. P., Smail, I., Serjeant, S., Almaini, O., \& Lawrence, A. 2004, MNRAS, 350,769

Dunne, L., Eales, S., Ivison, R., Morgan, H., \& Edmunds, M. 2003, Nature, 424, 285

Dunne, L. \& Eales, S. A. 2001, MNRAS, 327, 697

Dunne, L., Gomez, H. L., da Cunha, E., Charlot, S., Dye, S., Eales, S., Maddox, S. J., Rowlands, K., Smith, D. J. B., Auld, R., Baes, M., Bonfield, D. G., Bourne, N., Buttiglione, S., Cava, A., Clements, D. L., Coppin, K. E. K., Cooray, A., Dariush, A., de Zotti, G., Driver, S., Fritz, J., Geach, J., Hopwood, R., Ibar, E., Ivison, R. J., Jarvis, M. J., Kelvin, L., Pascale, E., Pohlen, M., Popescu, C., Rigby, E. E., Robotham, A., Rodighiero, G., Sansom, A. E., Serjeant, S., Temi, P., Thompson, M., Tuffs, R., van der Werf, P., \& Vlahakis, C. 2011, MNRAS, 417, 1510

Dupac, X., Bernard, J.-P., Boudet, N., Giard, M., Lamarre, J.-M., Mény, C., Pajot, F., Ristorcelli, I., Serra, G., Stepnik, B., \& Torre, J.-P. 2003, A\&A, 404, L11

Dutton, A. A., Treu, T., Brewer, B. J., Marshall, P. J., Auger, M. W., Barnabè, M., Koo, D. C., Bolton, A. S., \& Koopmans, L. V. E. 2013, MNRAS, 428,3183

Dutton, A. A., van den Bosch, F. C., \& Dekel, A. 2010, MNRAS, 405, 1690

Dwek, E., Arendt, R. G., Hauser, M. G., Fixsen, D., Kelsall, T., Leisawitz, D., Pei, Y. C., Wright, E. L., Mather, J. C., Moseley, S. H., Odegard, N., Shafer, R., Silverberg, R. F., \& Weiland, J. L. 1998, ApJ, 508, 106

Dwek, E., Staguhn, J. G., Arendt, R. G., Capak, P. L., Kovacs, A., Benford, D. J., Fixsen, D., Karim, A., Leclercq, S., Maher, S. F., Moseley, S. H., Schinnerer, E., \& Sharp, E. H. 2011, ApJ, 738, 36 
Dye, S., Eales, S. A., Aretxaga, I., Serjeant, S., Dunlop, J. S., Babbedge, T. S. R., Chapman, S. C., Cirasuolo, M., Clements, D. L., Coppin, K. E. K., Dunne, L., Egami, E., Farrah, D., Ivison, R. J., van Kampen, E., Pope, A., Priddey, R., Rieke, G. H., Schael, A. M., Scott, D., Simpson, C., Takagi, T., Takata, T., \& Vaccari, M. 2008, MNRAS, 386, 1107

Dye, S., Negrello, M., Hopwood, R., Nightingale, J. W., Bussmann, R. S., Amber, S., Bourne, N., Cooray, A., Dariush, A., Dunne, L., Eales, S. A., Gonzalez-Nuevo, J., Ibar, E., Ivison, R. J., Maddox, S., Valiante, E., \& Smith, M. 2013, ArXiv e-prints

Eales, S., Dunne, L., Clements, D., Cooray, A., de Zotti, G., Dye, S., Ivison, R., Jarvis, M., Lagache, G., Maddox, S., Negrello, M., Serjeant, S., Thompson, M. A., van Kampen, E., Amblard, A., Andreani, P., Baes, M., Beelen, A., Bendo, G. J., Benford, D., Bertoldi, F., Bock, J., Bonfield, D., Boselli, A., Bridge, C., Buat, V., Burgarella, D., Carlberg, R., Cava, A., Chanial, P., Charlot, S., Christopher, N., Coles, P., Cortese, L., Dariush, A., da Cunha, E., Dalton, G., Danese, L., Dannerbauer, H., Driver, S., Dunlop, J., Fan, L., Farrah, D., Frayer, D., Frenk, C., Geach, J., Gardner, J., Gomez, H., González-Nuevo, J., González-Solares, E., Griffin, M., Hardcastle, M., Hatziminaoglou, E., Herranz, D., Hughes, D., Ibar, E., Jeong, W.-S., Lacey, C., Lapi, A., Lawrence, A., Lee, M., Leeuw, L., Liske, J., López-Caniego, M., Müller, T., Nandra, K., Panuzzo, P., Papageorgiou, A., Patanchon, G., Peacock, J., Pearson, C., Phillipps, S., Pohlen, M., Popescu, C., Rawlings, S., Rigby, E., Rigopoulou, M., Robotham, A., Rodighiero, G., Sansom, A., Schulz, B., Scott, D., Smith, D. J. B., Sibthorpe, B., Smail, I., Stevens, J., Sutherland, W., Takeuchi, T., Tedds, J., Temi, P., Tuffs, R., Trichas, M., Vaccari, M., Valtchanov, I., van der Werf, P., Verma, A., Vieria, J., Vlahakis, C., \& White, G. J. 2010, PASP, 122, 499

Eales, S., Lilly, S., Webb, T., Dunne, L., Gear, W., Clements, D., \& Yun, M. 2000, AJ, 120, 2244

Eales, S., Smith, M. W. L., Auld, R., Baes, M., Bendo, G. J., Bianchi, S., Boselli, A., Ciesla, L., Clements, D., Cooray, A., Cortese, L., Davies, J., De Looze, I., Galametz, M., Gear, W., Gentile, G., Gomez, H., Fritz, J., Hughes, T., Madden, S., Magrini, L., Pohlen, M., Spinoglio, L., Verstappen, J., Vlahakis, C., \& Wilson, C. D. 2012, ApJ, 761, 168

Eddington, A. S. 1913, MNRAS, 73, 359

Efstathiou, A. \& Rowan-Robinson, M. 1995, MNRAS, 273, 649

-. 2003, MNRAS, 343, 322

Efstathiou, A., Rowan-Robinson, M., \& Siebenmorgen, R. 2000, MNRAS, 313, 734

Efstathiou, A. \& Siebenmorgen, R. 2009, A\&A, 502, 541

Egami, E. et al. 2004, ApJS, 154, 130

Elbaz, D., Dickinson, M., Hwang, H. S., Díaz-Santos, T., Magdis, G., Magnelli, B., Le Borgne, D., Galliano, F., Pannella, M., Chanial, P., Armus, L., Charmandaris, V., Daddi, E., Aussel, H., Popesso, P., Kartaltepe, J., Altieri, B., Valtchanov, I., Coia, D., Dannerbauer, H., Dasyra, K., Leiton, R., Mazzarella, J., Alexander, D. M., Buat, V., Burgarella, D., Chary, R.-R., Gilli, R., Ivison, R. J., Juneau, S., Le Floc'h, E., Lutz, D., Morrison, G. E., Mullaney, J. R., Murphy, E., Pope, A., Scott, D., Brodwin, M., Calzetti, D., Cesarsky, C., Charlot, S., Dole, H., Eisenhardt, P., Ferguson, H. C., Förster Schreiber, N., Frayer, D., Giavalisco, M., Huynh, M., Koekemoer, A. M., Papovich, C., Reddy, N., Surace, C., Teplitz, H., Yun, M. S., \& Wilson, G. 2011, A\&A, 533, A119+

Elsner, F., Feulner, G., \& Hopp, U. 2008, A\&A, 477, 503

Engel, H., Tacconi, L. J., Davies, R. I., Neri, R., Smail, I., Chapman, S. C., Genzel, R., Cox, P., Greve, T. R., Ivison, R. J., Blain, A., Bertoldi, F., \& Omont, A. 2010, ApJ, 724, 233

Estrada, J., Sefusatti, E., \& Frieman, J. A. 2009, ApJ, 692, 265

Evans, II, N. J. 1999, ARA\&A, 37, 311

Faber, S. M., Phillips, A. C., Kibrick, R. I., Alcott, B., Allen, S. L., Burrous, J., Cantrall, T., Clarke, D., Coil, A. L., Cowley, D. J., Davis, M., Deich, W. T. S., Dietsch, K., Gilmore, D. K., Harper, C. A., Hilyard, D. F., Lewis, J. P., McVeigh, M., Newman, J., Osborne, J., Schiavon, R., Stover, R. J., Tucker, D., Wallace, V., Wei, M., Wirth, G., \& Wright, C. A. 2003, in Society of Photo-Optical Instrumentation Engineers (SPIE) Conference Series, Vol. 4841, Society of Photo-Optical Instrumentation Engineers (SPIE) Conference Series, ed. M. Iye \& A. F. M. Moorwood, 1657-1669

Fabian, A. C. et al. 2000, MNRAS, 315, L8

Fadda, D., Yan, L., Lagache, G., Sajina, A., Lutz, D., Wuyts, S., Frayer, D. T., Marcillac, D., Le Floc'h, E., Caputi, K., Spoon, H. W. W., Veilleux, S., Blain, A., \& Helou, G. 2010, ApJ, 719, 425

Fakhouri, O., Ma, C.-P., \& Boylan-Kolchin, M. 2010, MNRAS, 406, 2267

Fan, X., Strauss, M. A., Schneider, D. P., Becker, R. H., White, R. L., Haiman, Z., Gregg, M., Pentericci, L., Grebel, E. K., Narayanan, V. K., Loh, Y.-S., Richards, G. T., Gunn, J. E., Lupton, R. H., Knapp, G. R., Ivezić, Ž., Brandt, W. N., Collinge, M., Hao, L., Harbeck, D., Prada, F., Schaye, J., Strateva, I., Zakamska, N., Anderson, S., Brinkmann, J., Bahcall, N. A., Lamb, D. Q., Okamura, S., Szalay, A., \& York, D. G. 2003, AJ, 125, 1649

Fardal, M. A., Katz, N., Weinberg, D. H., \& Davé, R. 2007, MNRAS, 379, 985

Fardal, M. A., Katz, N., Weinberg, D. H., Davé, R., \& Hernquist, L. 2001, arXiv/0107290

Farrah, D., Afonso, J., Efstathiou, A., Rowan-Robinson, M., Fox, M., \& Clements, D. 2003, MNRAS, 343,585

Farrah, D., Bernard-Salas, J., Spoon, H. W. W., Soifer, B. T., Armus, L., Brandl, B., Charmandaris, V., Desai, V., Higdon, S., Devost, D., \& Houck, J. 2007, ApJ, 667, 149

Farrah, D., Lebouteiller, V., Spoon, H. W. W., Bernard-Salas, J., Pearson, C., Rigopoulou, D., Smith, H. A., González-Alfonso, E., Clements, D. L., Efstathiou, A., Cormier, D., Afonso, J., Petty, S. M., Harris, K., Hurley, P., Borys, C., Verma, A., Cooray, A., \& Salvatelli, V. 2013, ApJ, 776, 38

Farrah, D., Lonsdale, C. J., Weedman, D. W., Spoon, H. W. W., Rowan-Robinson, M., Polletta, M., Oliver, S., Houck, J. R., \& Smith, H. E. 2008, ApJ, 677, 957

Faucher-Giguère, C.-A., Quataert, E., \& Hopkins, P. F. 2013, MNRAS, 433, 1970

Feldmann, R., Gnedin, N. Y., \& Kravtsov, A. V. 2012a, ApJ, 747, 124

-. 2012b, ApJ, 758, 127

Ferkinhoff, C., Brisbin, D., Nikola, T., Parshley, S. C., Stacey, G. J., Phillips, T. G., Falgarone, E., Benford, D. J., Staguhn, J. G., \& Tucker, C. E. 2011, ApJ, 740, L29

Ferkinhoff, C., Hailey-Dunsheath, S., Nikola, T., Parshley, S. C., Stacey, G. J., Benford, D. J., \& Staguhn, J. G. 2010, ApJ, 714 , L147 
Ferreras, I., La Barbera, F., de la Rosa, I. G., Vazdekis, A., de Carvalho, R. R., Falcón-Barroso, J., \& Ricciardelli, E. 2013, MNRAS, 429, L15 Finlator, K. \& Davé, R. 2008, MNRAS, 385, 2181

Finlator, K., Davé, R., Papovich, C., \& Hernquist, L. 2006, ApJ, 639, 672

Fiolet, N., Omont, A., Lagache, G., Bertincourt, B., Fadda, D., Baker, A. J., Beelen, A., Berta, S., Boulanger, F., Farrah, D., Kovács, A., Lonsdale, C., Owen, F., Polletta, M., Shupe, D., \& Yan, L. 2010, A\&A, 524, A33

Fiore, F., Grazian, A., Santini, P., Puccetti, S., Brusa, M., Feruglio, C., Fontana, A., Giallongo, E., Comastri, A., Gruppioni, C., Pozzi, F., Zamorani, G., \& Vignali, C. 2008, ApJ, 672, 94

Fixsen, D. J., Bennett, C. L., \& Mather, J. C. 1999, ApJ, 526, 207

Fixsen, D. J., Dwek, E., Mather, J. C., Bennett, C. L., \& Shafer, R. A. 1998, ApJ, 508, 123

Fleuren, S., Sutherland, W., Dunne, L., Smith, D. J. B., Maddox, S. J., González-Nuevo, J., Findlay, J., Auld, R., Baes, M., Bond, N. A., Bonfield, D. G., Bourne, N., Cooray, A., Buttiglione, S., Cava, A., Rigby, E. E., Robotham, A., Scott, D., Temi, P., Thompson, M. A., Valiante, E., \& Werf, P. v. d. 2012, MNRAS, 423, 2407

Fontanot, F., Monaco, P., Silva, L., \& Grazian, A. 2007, MNRAS, 382, 903

Fontanot, F. \& Somerville, R. S. 2011, MNRAS, 416, 2962

Förster Schreiber, N. M., Genzel, R., Bouché, N., Cresci, G., Davies, R., Buschkamp, P., Shapiro, K., Tacconi, L. J., Hicks, E. K. S., Genel, S., Shapley, A. E., Erb, D. K., Steidel, C. C., Lutz, D., Eisenhauer, F., Gillessen, S., Sternberg, A., Renzini, A., Cimatti, A., Daddi, E., Kurk, J., Lilly, S., Kong, X., Lehnert, M. D., Nesvadba, N., Verma, A., McCracken, H., Arimoto, N., Mignoli, M., \& Onodera, M. 2009, ApJ, 706, 1364 Förster Schreiber, N. M., Genzel, R., Lutz, D., Kunze, D., \& Sternberg, A. 2001, ApJ, 552, 544

Förster Schreiber, N. M., Genzel, R., Lutz, D., \& Sternberg, A. 2003, ApJ, 599, 193

Fox, M. J., Efstathiou, A., Rowan-Robinson, M., Dunlop, J. S., Scott, S., Serjeant, S., Mann, R. G., Oliver, S., Ivison, R. J., Blain, A., Almaini, O., Hughes, D., Willott, C. J., Longair, M., Lawrence, A., \& Peacock, J. A. 2002, MNRAS, 331, 839

Frayer, D. T., Ivison, R. J., Scoville, N. Z., Yun, M., Evans, A. S., Smail, I., Blain, A. W., \& Kneib, J.-P. 1998, ApJ, 506, L7

Freundlich, J., Combes, F., Tacconi, L. J., Cooper, M. C., Genzel, R., Neri, R., Bolatto, A., Bournaud, F., Burkert, A., Cox, P., Davis, M., Förster Schreiber, N. M., Garcia-Burillo, S., Gracia-Carpio, J., Lutz, D., Naab, T., Newman, S., Sternberg, A., \& Weiner, B. 2013, A\&A, 553, A130

Fritz, J., Franceschini, A., \& Hatziminaoglou, E. 2006, MNRAS, 366, 767

Fryxell, B., Olson, K., Ricker, P., Timmes, F. X., Zingale, M., Lamb, D. Q., MacNeice, P., Rosner, R., Truran, J. W., \& Tufo, H. 2000, ApJS, 131, 273

Fu, H., Cooray, A., Feruglio, C., Ivison, R. J., Riechers, D. A., Gurwell, M., Bussmann, R. S., Harris, A. I., Altieri, B., Aussel, H., Baker, A. J., Bock, J., Boylan-Kolchin, M., Bridge, C., Calanog, J. A., Casey, C. M., Cava, A., Chapman, S. C., Clements, D. L., Conley, A., Cox, P., Farrah, D., Frayer, D., Hopwood, R., Jia, J., Magdis, G., Marsden, G., Martínez-Navajas, P., Negrello, M., Neri, R., Oliver, S. J., Omont, A., Page, M. J., Pérez-Fournon, I., Schulz, B., Scott, D., Smith, A., Vaccari, M., Valtchanov, I., Vieira, J. D., Viero, M., Wang, L., Wardlow, J. L., \& Zemcov, M. 2013, Nature, 498, 338

Fu, H., Jullo, E., Cooray, A., Bussmann, R. S., Ivison, R. J., Pérez-Fournon, I., Djorgovski, S. G., Scoville, N., Yan, L., Riechers, D. A., Aguirre, J., Auld, R., Baes, M., Baker, A. J., Bradford, M., Cava, A., Clements, D. L., Dannerbauer, H., Dariush, A., De Zotti, G., Dole, H., Dunne, L., Dye, S., Eales, S., Frayer, D., Gavazzi, R., Gurwell, M., Harris, A. I., Herranz, D., Hopwood, R., Hoyos, C., Ibar, E., Jarvis, M. J., Kim, S., Leeuw, L., Lupu, R., Maddox, S., Martínez-Navajas, P., Michałowski, M. J., Negrello, M., Omont, A., Rosenman, M., Scott, D., Serjeant, S., Smail, I., Swinbank, A. M., Valiante, E., Verma, A., Vieira, J., Wardlow, J. L., \& van der Werf, P. 2012a, ApJ, 753, 134

Fu, J., Kauffmann, G., Li, C., \& Guo, Q. 2012b, MNRAS, 424, 2701

Fukugita, M. 2011, ArXiv e-prints

Fukugita, M. \& Peebles, P. J. E. 2004, ApJ, 616, 643

Fukui, Y. \& Kawamura, A. 2010, ARA\&A, 48, 547

Gabor, J. M. \& Bournaud, F. 2013, MNRAS

Galametz, M., Kennicutt, R. C., Albrecht, M., Aniano, G., Armus, L., Bertoldi, F., Calzetti, D., Crocker, A. F., Croxall, K. V., Dale, D. A., Donovan Meyer, J., Draine, B. T., Engelbracht, C. W., Hinz, J. L., Roussel, H., Skibba, R. A., Tabatabaei, F. S., Walter, F., Weiss, A., Wilson, C. D., \& Wolfire, M. G. 2012, MNRAS, 425, 763

Gallerani, S., Neri, R., Maiolino, R., Martín, S., De Breuck, C., Walter, F., Caselli, P., Krips, M., Meneghetti, M., Nagao, T., Wagg, J., \& Walmsley, M. 2012, A\&A, 543, A114

Gao, Y., Carilli, C. L., Solomon, P. M., \& Vanden Bout, P. A. 2007, ApJ, 660, L93

Gao, Y. \& Solomon, P. M. 2004a, ApJS, 152, 63

-. 2004b, ApJ, 606, 271

García-Burillo, S., Usero, A., Alonso-Herrero, A., Graciá-Carpio, J., Pereira-Santaella, M., Colina, L., Planesas, P., \& Arribas, S. 2012, A\&A, 539, A8

Garn, T. \& Alexander, P. 2008, MNRAS, 391, 1000

Gavazzi, R., Cooray, A., Conley, A., Aguirre, J. E., Amblard, A., Auld, R., Beelen, A., Blain, A., Blundell, R., Bock, J., Bradford, C. M., Bridge, C., Brisbin, D., Burgarella, D., Chanial, P., Chapin, E., Christopher, N., Clements, D. L., Cox, P., Djorgovski, S. G., Dowell, C. D., Eales, S., Earle, L., Ellsworth-Bowers, T. P., Farrah, D., Franceschini, A., Fu, H., Glenn, J., González Solares, E. A., Griffin, M., Gurwell, M. A., Halpern, M., Ibar, E., Ivison, R. J., Jarvis, M., Kamenetzky, J., Kim, S., Krips, M., Levenson, L., Lupu, R., Mahabal, A., Maloney, P. D., Maraston, C., Marchetti, L., Marsden, G., Matsuhara, H., Mortier, A. M. J., Murphy, E., Naylor, B. J., Neri, R., Nguyen, H. T., Oliver, S. J., Omont, A., Page, M. J., Papageorgiou, A., Pearson, C. P., Pérez-Fournon, I., Pohlen, M., Rangwala, N., Rawlings, J. I., Raymond, G., Riechers, D., Rodighiero, G., Roseboom, I. G., Rowan-Robinson, M., Schulz, B., Scott, D., Scott, K. S., Serra, P., Seymour, N., Shupe, D. L., Smith, A. J., Symeonidis, M., Tugwell, K. E., Vaccari, M., Valiante, E., Valtchanov, I., Verma, A., Vieira, J. D., Vigroux, L., Wang, L., Wardlow, J., Wiebe, D., Wright, G., Xu, C. K., Zeimann, G., Zemcov, M., \& Zmuidzinas, J. 2011, ApJ, 738, 125

Geach, J. E., Chapin, E. L., Coppin, K. E. K., Dunlop, J. S., Halpern, M., Smail, I., van der Werf, P., Serjeant, S., Farrah, D., Roseboom, I., Targett, T., Arumugam, V., Asboth, V., Blain, A., Chrysostomou, A., Clarke, C., Ivison, R. J., Jones, S. L., Karim, A., Mackenzie, T., Meijerink, R., Michalowski, M. J., Scott, D., Simpson, J., Swinbank, A. M., Alexander, D., Almaini, O., Aretxaga, I., Best, P., Chapman, S., Clements, 
D. L., Conselice, C., Danielson, A. L. R., Eales, S., Edge, A. C., Gibb, A., Hughes, D., Jenness, T., Knudsen, K. K., Lacey, C., Marsden, G., McMahon, R., Oliver, S., Page, M. J., Peacock, J. A., Rigopoulou, D., Robson, E. I., Spaans, M., Stevens, J., Webb, T. M. A., Willott, C., Wilson, C. D., \& Zemcov, M. 2013, MNRAS, 432, 53

Geach, J. E., Smail, I., Moran, S. M., MacArthur, L. A., Lagos, C. d. P., \& Edge, A. C. 2011, ApJ, 730, L19

Genzel, R., Baker, A. J., Tacconi, L. J., Lutz, D., Cox, P., Guilloteau, S., \& Omont, A. 2003, ApJ, 584, 633

Genzel, R., Tacconi, L. J., Combes, F., Bolatto, A., Neri, R., Sternberg, A., Cooper, M. C., Bouché, N., Bournaud, F., Burkert, A., Comerford, J., Cox, P., Davis, M., Förster Schreiber, N. M., Garcia-Burillo, S., Gracia-Carpio, J., Lutz, D., Naab, T., Newman, S., Saintonge, A., Shapiro, K., Shapley, A., \& Weiner, B. 2012, ApJ, 746, 69

Genzel, R., Tacconi, L. J., Gracia-Carpio, J., Sternberg, A., Cooper, M. C., Shapiro, K., Bolatto, A., Bouché, N., Bournaud, F., Burkert, A., Combes, F., Comerford, J., Cox, P., Davis, M., Schreiber, N. M. F., Garcia-Burillo, S., Lutz, D., Naab, T., Neri, R., Omont, A., Shapley, A., \& Weiner, B. 2010, MNRAS, 407, 2091

Georgantopoulos, I., Rovilos, E., \& Comastri, A. 2011, A\&A, 526, A46

George, R. D., Ivison, R. J., Hopwood, R., Riechers, D. A., Bussmann, R. S., Cox, P., Dye, S., Krips, M., Negrello, M., Neri, R., Serjeant, S., Valtchanov, I., Baes, M., Bourne, N., Clements, D. L., De Zotti, G., Dunne, L., Eales, S. A., Ibar, E., Maddox, S., Smith, M. W. L., Valiante, E., \& van der Werf, P. 2013, MNRAS, 436, L99

Gilli, R., Daddi, E., Chary, R., Dickinson, M., Elbaz, D., Giavalisco, M., Kitzbichler, M., Stern, D., \& Vanzella, E. 2007, A\&A, 475, 83

Gilli, R., Su, J., Norman, C., Vignali, C., Comastri, A., Tozzi, P., Rosati, P., Stiavelli, M., Brandt, W. N., Xue, Y. Q., Luo, B., Castellano, M., Fontana, A., Fiore, F., Mainieri, V., \& Ptak, A. 2011, ApJ, 730, L28

Glenn, J., Conley, A., Béthermin, M., Altieri, B., Amblard, A., Arumugam, V., Aussel, H., Babbedge, T., Blain, A., Bock, J., Boselli, A., Buat, V., Wright, G., Xu, C. K., \& Zemcov, M. 2010, MNRAS, 409, 109

Glover, S. C. O., Federrath, C., Mac Low, M.-M., \& Klessen, R. S. 2010, MNRAS, 404, 2

Glover, S. C. O. \& Mac Low, M.-M. 2011, MNRAS, 412, 337

Goldbaum, N. J., Krumholz, M. R., Matzner, C. D., \& McKee, C. F. 2011, ApJ, 738, 101

Goldsmith, P. F., Langer, W. D., Pineda, J. L., \& Velusamy, T. 2012, ApJS, 203, 13

González, J. E., Lacey, C. G., Baugh, C. M., \& Frenk, C. S. 2011, MNRAS, 413, 749

Gorjian, V., Brodwin, M., Kochanek, C. S., Murray, S., Stern, D., Brand, K., Eisenhardt, P. R., Ashby, M. L. N., Barmby, P., Brown, M. J. I., Dey, A., Forman, W., Jannuzi, B. T., Jones, C., Kenter, A. T., Pahre, M. A., Shields, J. C., Werner, M. W., \& Willner, S. P. 2008, ApJ, 679, 1040

Goto, T., Takagi, T., Matsuhara, H., Takeuchi, T. T., Pearson, C., Wada, T., Nakagawa, T., Ilbert, O., Le Floc'h, E., Oyabu, S., Ohyama, Y., Malkan, M., Lee, H. M., Lee, M. G., Inami, H., Hwang, N., Hanami, H., Im, M., Imai, K., Ishigaki, T., Serjeant, S., \& Shim, H. 2010, A\&A, 514, A6+ Graciá-Carpio, J., García-Burillo, S., \& Planesas, P. 2008, ApSS, 313, 331

Graciá-Carpio, J., García-Burillo, S., Planesas, P., \& Colina, L. 2006, ApJ, 640, L135

Graciá-Carpio, J., Sturm, E., Hailey-Dunsheath, S., Fischer, J., Contursi, A., Poglitsch, A., Genzel, R., González-Alfonso, E., Sternberg, A., Verma, A., Christopher, N., Davies, R., Feuchtgruber, H., de Jong, J. A., Lutz, D., \& Tacconi, L. J. 2011, ApJ, 728, L7

Granato, G. L., De Zotti, G., Silva, L., Bressan, A., \& Danese, L. 2004, ApJ, 600, 580

Granato, G. L., Lacey, C. G., Silva, L., Bressan, A., Baugh, C. M., Cole, S., \& Frenk, C. S. 2000, ApJ, 542, 710

Greve, T. R., Hainline, L. J., Blain, A. W., Smail, I., Ivison, R. J., \& Papadopoulos, P. P. 2006, AJ, 132, 1938

Greve, T. R., Ivison, R. J., Bertoldi, F., Stevens, J. A., Dunlop, J. S., Lutz, D., \& Carilli, C. L. 2004, MNRAS, 354, 779

Greve, T. R., Ivison, R. J., \& Papadopoulos, P. P. 2003, ApJ, 599, 839

Greve, T. R., Pope, A., Scott, D., Ivison, R. J., Borys, C., Conselice, C. J., \& Bertoldi, F. 2008, MNRAS, 389,1489

Greve, T. R., Vieira, J. D., Weiß, A., Aguirre, J. E., Aird, K. A., Ashby, M. L. N., Benson, B. A., Bleem, L. E., Bradford, C. M., Brodwin, M., Carlstrom, J. E., Chang, C. L., Chapman, S. C., Crawford, T. M., de Breuck, C., de Haan, T., Dobbs, M. A., Downes, T., Fassnacht, C. D., Fazio, G., George, E. M., Gladders, M., Gonzalez, A. H., Halverson, N. W., Hezaveh, Y., High, F. W., Holder, G. P., Holzapfel, W. L., Hoover, S., Hrubes, J. D., Johnson, M., Keisler, R., Knox, L., Lee, A. T., Leitch, E. M., Lueker, M., Luong-Van, D., Malkan, M., Marrone, D. P., McIntyre, V., McMahon, J. J., Mehl, J., Menten, K. M., Meyer, S. S., Montroy, T., Murphy, E. J., Natoli, T., Padin, S., Plagge, T., Pryke, C., Reichardt, C. L., Rest, A., Rosenman, M., Ruel, J., Ruhl, J. E., Schaffer, K. K., Sharon, K., Shaw, L., Shirokoff, E., Stalder, B., Stanford, S. A., Staniszewski, Z., Stark, A. A., Story, K., Vanderlinde, K., Walsh, W., Welikala, N., \& Williamson, R. 2012, ApJ, 756, 101

Greve, T. R. et al. 2005, MNRAS, 359, 1165

Griffin et al. 2010, A\&A, 518, L3

Groves, B., Dopita, M. A., Sutherland, R. S., Kewley, L. J., Fischera, J., Leitherer, C., Brandl, B., \& van Breugel, W. 2008, ApJS, 176, 438 Gruppioni, C. et al. 2013, MNRAS, 432, 23

Guiderdoni, B., Hivon, E., Bouchet, F. R., \& Maffei, B. 1998, MNRAS, 295, 877

Guo, Q., Cole, S., Lacey, C. G., Baugh, C. M., Frenk, C. S., Norberg, P., Auld, R., Baldry, I. K., Bamford, S. P., Bourne, N., Buttiglione, E. S., Cava, A., Cooray, A., Croom, S., Dariush, A., de Zotti, G., Driver, S., Dunne, L., Dye, S., Eales, S., Fritz, J., Hopkins, A., Hopwood, R., Ibar, E., Ivison, R. J., Jarvis, M., Jones, D. H., Kelvin, L., Liske, J., Loveday, J., Maddox, S. J., Parkinson, H., Pascale, E., Peacock, J. A., Pohlen, M., Prescott, M., Rigby, E. E., Robotham, A., Rodighiero, G., Sharp, R., Smith, D. J. B., Temi, P., \& van Kampen, E. 2011, MNRAS, 412, 2277 Guo, Q. \& White, S. D. M. 2008, MNRAS, 384, 2

Haas, M. R., Schaye, J., Booth, C. M., Dalla Vecchia, C., Springel, V., Theuns, T., \& Wiersma, R. P. C. 2013, MNRAS, 435, 2931

Hailey-Dunsheath, S., Nikola, T., Stacey, G. J., Oberst, T. E., Parshley, S. C., Benford, D. J., Staguhn, J. G., \& Tucker, C. E. 2010 , ApJ, 714, L162 Hailey-Dunsheath, S., Nikola, T., Stacey, G. J., Oberst, T. E., Parshley, S. C., Bradford, C. M., Ade, P. A. R., \& Tucker, C. E. 2008 , ApJ, 689, L109 Hainline, L. J., Blain, A. W., Greve, T. R., Chapman, S. C., Smail, I., \& Ivison, R. J. 2006, ApJ, 650, 614

Hainline, L. J., Blain, A. W., Smail, I., Alexander, D. M., Armus, L., Chapman, S. C., \& Ivison, R. J. 2011, ApJ, 740, 96

Hanson, D., Hoover, S., Crites, A., Ade, P. A. R., Aird, K. A., Austermann, J. E., Beall, J. A., Bender, A. N., Benson, B. A., Bleem, L. E., Bock, J. J., Carlstrom, J. E., Chang, C. L., Chiang, H. C., Cho, H.-M., Conley, A., Crawford, T. M., de Haan, T., Dobbs, M. A., Everett, W., Gallicchio, J., Gao, J., George, E. M., Halverson, N. W., Harrington, N., Henning, J. W., Hilton, G. C., Holder, G. P., Holzapfel, W. L., Hrubes, J. D., Huang, N., Hubmayr, J., Irwin, K. D., Keisler, R., Knox, L., Lee, A. T., Leitch, E., Li, D., Liang, C., Luong-Van, D., Marsden, G., McMahon, 
J. J., Mehl, J., Meyer, S. S., Mocanu, L., Montroy, T. E., Natoli, T., Nibarger, J. P., Novosad, V., Padin, S., Pryke, C., Reichardt, C. L., Ruhl, J. E., Saliwanchik, B. R., Sayre, J. T., Schaffer, K. K., Schulz, B., Smecher, G., Stark, A. A., Story, K. T., Tucker, C., Vanderlinde, K., Vieira, J. D., Viero, M. P., Wang, G., Yefremenko, V., Zahn, O., \& Zemcov, M. 2013, Physical Review Letters, 111, 141301

Häring, N. \& Rix, H.-W. 2004, ApJ, 604, L89

Harris, A. I., Baker, A. J., Frayer, D. T., Smail, I., Swinbank, A. M., Riechers, D. A., van der Werf, P. P., Auld, R., Baes, M., Bussmann, R. S., Buttiglione, S., Cava, A., Clements, D. L., Cooray, A., Dannerbauer, H., Dariush, A., De Zotti, G., Dunne, L., Dye, S., Eales, S., Fritz, J., González-Nuevo, J., Hopwood, R., Ibar, E., Ivison, R. J., Jarvis, M. J., Maddox, S., Negrello, M., Rigby, E., Smith, D. J. B., Temi, P., \& Wardlow, J. 2012, ApJ, 752, 152

Harris, A. I., Baker, A. J., Jewell, P. R., Rauch, K. P., Zonak, S. G., O’Neil, K., Shelton, A. L., Norrod, R. D., Ray, J., \& Watts, G. 2007, in Astronomical Society of the Pacific Conference Series, Vol. 375, From Z-Machines to ALMA: (Sub)Millimeter Spectroscopy of Galaxies, ed. A. J. Baker, J. Glenn, A. I. Harris, J. G. Mangum, \& M. S. Yun, 82

Harris, A. I., Baker, A. J., Zonak, S. G., Sharon, C. E., Genzel, R., Rauch, K., Watts, G., \& Creager, R. 2010, ApJ, 723, 1139

Harwit, M. \& Pacini, F. 1975, ApJ, 200, L127

Hatsukade, B., Ohta, K., Seko, A., Yabe, K., \& Akiyama, M. 2013, ApJ, 769, L27

Hatsukade, B. et al. 2011, MNRAS, 411, 102

Hayward, C. C. 2013, MNRAS, 432, L85

Hayward, C. C., Behroozi, P. S., Somerville, R. S., Primack, J. R., Moreno, J., \& Wechsler, R. H. 2013a, MNRAS

Hayward, C. C., Jonsson, P., Kereš, D., Magnelli, B., Hernquist, L., \& Cox, T. J. 2012, MNRAS, 424, 951

Hayward, C. C., Kereš, D., Jonsson, P., Narayanan, D., Cox, T. J., \& Hernquist, L. 2011, ApJ, 743, 159

Hayward, C. C., Narayanan, D., Kereš, D., Jonsson, P., Hopkins, P. F., Cox, T. J., \& Hernquist, L. 2013b, MNRAS, 428,2529

Hayward, C. C., Torrey, P., Springel, V., Hernquist, L., \& Vogelsberger, M. 2013c, arXiv/1309.2942

Heiderman, A., Evans, II, N. J., Allen, L. E., Huard, T., \& Heyer, M. 2010, ApJ, 723, 1019

Helfer, T. T., Thornley, M. D., Regan, M. W., Wong, T., Sheth, K., Vogel, S. N., Blitz, L., \& Bock, D. C.-J. 2003, ApJS, 145, 259

Helou, G., Khan, I. R., Malek, L., \& Boehmer, L. 1988, ApJS, 68, 151

Helou, G., Malhotra, S., Hollenbach, D. J., Dale, D. A., \& Contursi, A. 2001, ApJ, 548, L73

Helou, G., Soifer, B. T., \& Rowan-Robinson, M. 1985, ApJ, 298, L7

Hennebelle, P. \& Chabrier, G. 2008, ApJ, 684, 395

Héraudeau, P., Oliver, S., del Burgo, C., Kiss, C., Stickel, M., Mueller, T., Rowan-Robinson, M., Efstathiou, A., Surace, C., Tóth, L. V., Serjeant, S., Alexander, D. M., Franceschini, A., Lemke, D., Morel, T., Pérez-Fournon, I., Puget, J.-L., Rigopoulou, D., Rocca-Volmerange, B., \& Verma, A. 2004, MNRAS, 354, 924

Hezaveh, Y. D. \& Holder, G. P. 2011, ApJ, 734, 52

Hezaveh, Y. D., Marrone, D. P., Fassnacht, C. D., Spilker, J. S., Vieira, J. D., Aguirre, J. E., Aird, K. A., Aravena, M., Ashby, M. L. N., Bayliss, M., Benson, B. A., Bleem, L. E., Bothwell, M., Brodwin, M., Carlstrom, J. E., Chang, C. L., Chapman, S. C., Crawford, T. M., Crites, A. T., De Breuck, C., de Haan, T., Dobbs, M. A., Fomalont, E. B., George, E. M., Gladders, M. D., Gonzalez, A. H., Greve, T. R., Halverson, N. W., High, F. W., Holder, G. P., Holzapfel, W. L., Hoover, S., Hrubes, J. D., Husband, K., Hunter, T. R., Keisler, R., Lee, A. T., Leitch, E. M., Lueker, M., Luong-Van, D., Malkan, M., McIntyre, V., McMahon, J. J., Mehl, J., Menten, K. M., Meyer, S. S., Mocanu, L. M., Murphy, E. J., Natoli, T., Padin, S., Plagge, T., Reichardt, C. L., Rest, A., Ruel, J., Ruhl, J. E., Sharon, K., Schaffer, K. K., Shaw, L., Shirokoff, E., Stalder, B., Staniszewski, Z., Stark, A. A., Story, K., Vanderlinde, K., Weiß, A., Welikala, N., \& Williamson, R. 2013, ApJ, 767, 132

Hezaveh, Y. D., Marrone, D. P., \& Holder, G. P. 2012, ApJ, 761, 20

Hickox, R. C., Jones, C., Forman, W. R., Murray, S. S., Kochanek, C. S., Eisenstein, D., Jannuzi, B. T., Dey, A., Brown, M. J. I., Stern, D., Eisenhardt, P. R., Gorjian, V., Brodwin, M., Narayan, R., Cool, R. J., Kenter, A., Caldwell, N., \& Anderson, M. E. 2009, ApJ, 696, 891

Hickox, R. C., Wardlow, J. L., Smail, I., Myers, A. D., Alexander, D. M., Swinbank, A. M., Danielson, A. L. R., Stott, J. P., Chapman, S. C., Coppin, K. E. K., Dunlop, J. S., Gawiser, E., Lutz, D., van der Werf, P., \& Weiß, A. 2012, MNRAS, 421, 284

Hildebrand, R. H. 1983, QJRAS, 24, 267

Hildebrandt, H., van Waerbeke, L., Scott, D., Béthermin, M., Bock, J., Clements, D., Conley, A., Cooray, A., Dunlop, J. S., Eales, S., Erben, T., Farrah, D., Franceschini, A., Glenn, J., Halpern, M., Heinis, S., Ivison, R. J., Marsden, G., Oliver, S. J., Page, M. J., Pérez-Fournon, I., Smith, A. J., Rowan-Robinson, M., Valtchanov, I., van der Burg, R. F. J., Vieira, J. D., Viero, M., \& Wang, L. 2013, MNRAS, 429, 3230

Hill, M. D. \& Shanks, T. 2011, MNRAS, 410, 762

Hodge, J. A., Carilli, C. L., Walter, F., Daddi, E., \& Riechers, D. 2013a, ApJ, 776, 22

Hodge, J. A., Carilli, C. L., Walter, F., de Blok, W. J. G., Riechers, D., Daddi, E., \& Lentati, L. 2012, ApJ, 760, 11

Hodge, J. A., Karim, A., Smail, I., Swinbank, A. M., Walter, F., Biggs, A. D., Ivison, R. J., Weiss, A., Alexander, D. M., Bertoldi, F., Brandt, W. N., Chapman, S. C., Coppin, K. E. K., Cox, P., Danielson, A. L. R., Dannerbauer, H., De Breuck, C., Decarli, R., Edge, A. C., Greve, T. R., Knudsen, K. K., Menten, K. M., Rix, H.-W., Schinnerer, E., Simpson, J. M., Wardlow, J. L., \& van der Werf, P. 2013b, ApJ, 768, 91

Holder, G. P., Viero, M. P., Zahn, O., Aird, K. A., Benson, B. A., Bhattacharya, S., Bleem, L. E., Bock, J., Brodwin, M., Carlstrom, J. E., Chang, C. L., Cho, H.-M., Conley, A., Crawford, T. M., Crites, A. T., de Haan, T., Dobbs, M. A., Dudley, J., George, E. M., Halverson, N. W., Holzapfel, W. L., Hoover, S., Hou, Z., Hrubes, J. D., Keisler, R., Knox, L., Lee, A. T., Leitch, E. M., Lueker, M., Luong-Van, D., Marsden, G., Marrone, D. P., McMahon, J. J., Mehl, J., Meyer, S. S., Millea, M., Mohr, J. J., Montroy, T. E., Padin, S., Plagge, T., Pryke, C., Reichardt, C. L., Ruhl, J. E., Sayre, J. T., Schaffer, K. K., Schulz, B., Shaw, L., Shirokoff, E., Spieler, H. G., Staniszewski, Z., Stark, A. A., Story, K. T., van Engelen, A., Vanderlinde, K., Vieira, J. D., Williamson, R., \& Zemcov, M. 2013, ApJ, 771, L16

Holland, W. S., Robson, E. I., Gear, W. K., Cunningham, C. R., Lightfoot, J. F., Jenness, T., Ivison, R. J., Stevens, J. A., Ade, P. A. R., Griffin, M. J., Duncan, W. D., Murphy, J. A., \& Naylor, D. A. 1999, MNRAS, 303, 659

Holland, W. S. et al. 2013, MNRAS, 430, 2513

Hönig, S. F., Beckert, T., Ohnaka, K., \& Weigelt, G. 2006, A\&A, 452, 459

Hopkins, A. M. \& Beacom, J. F. 2006, ApJ, 651, 142

Hopkins, P. F. 2012, MNRAS, 423, 2037 
-. 2013, MNRAS, 433, 170

Hopkins, P. F., Cox, T. J., Hernquist, L., Narayanan, D., Hayward, C. C., \& Murray, N. 2013a, MNRAS, 430, 1901

Hopkins, P. F., Cox, T. J., Younger, J. D., \& Hernquist, L. 2009, ApJ, 691, 1168

Hopkins, P. F., Hayward, C. C., Narayanan, D., \& Hernquist, L. 2012a, MNRAS, 420, 320

Hopkins, P. F., Hernquist, L., Cox, T. J., Di Matteo, T., Martini, P., Robertson, B., \& Springel, V. 2005a, ApJ, 630, 705

Hopkins, P. F., Hernquist, L., Cox, T. J., Di Matteo, T., Robertson, B., \& Springel, V. 2006, ApJS, 163, 1

Hopkins, P. F., Hernquist, L., Cox, T. J., \& Kereš, D. 2008, ApJS, 175, 356

Hopkins, P. F., Hernquist, L., Hayward, C. C., \& Narayanan, D. 2012b, MNRAS, 425, 1121

Hopkins, P. F., Hernquist, L., Martini, P., Cox, T. J., Robertson, B., Di Matteo, T., \& Springel, V. 2005b, ApJ, 625, L71

Hopkins, P. F., Kereš, D., \& Murray, N. 2013b, MNRAS, 432, 2639

Hopkins, P. F., Kereš, D., Murray, N., Hernquist, L., Narayanan, D., \& Hayward, C. C. 2013c, MNRAS, 433, 78

Hopkins, P. F., Narayanan, D., \& Murray, N. 2013d, MNRAS, 432, 2647

Hopkins, P. F., Narayanan, D., Murray, N., \& Quataert, E. 2013e, MNRAS

Hopkins, P. F., Quataert, E., \& Murray, N. 2012c, MNRAS, 421, 3488

Hopkins, P. F., Richards, G. T., \& Hernquist, L. 2007, ApJ, 654, 731

Hopkins, P. F., Younger, J. D., Hayward, C. C., Narayanan, D., \& Hernquist, L. 2010, MNRAS, 402, 1693

Hopwood, R., Wardlow, J., Cooray, A., Khostovan, A. A., Kim, S., Negrello, M., da Cunha, E., Burgarella, D., Aretxaga, I., Auld, R., Baes, M., Barton, E., Bertoldi, F., Bonfield, D. G., Blundell, R., Buttiglione, S., Cava, A., Clements, D. L., Cooke, J., Dannerbauer, H., Dariush, A., de Zotti, G., Dunlop, J., Dunne, L., Dye, S., Eales, S., Fritz, J., Frayer, D., Gurwell, M. A., Hughes, D. H., Ibar, E., Ivison, R. J., Jarvis, M. J., Lagache, G., Leeuw, L., Maddox, S., Michałowski, M. J., Omont, A., Pascale, E., Pohlen, M., Rigby, E., Rodighiero, G., Scott, D., Serjeant, S., Smail, I., Smith, D. J. B., Temi, P., Thompson, M. A., Valtchanov, I., van der Werf, P., Verma, A., \& Vieira, J. D. 2011, ApJ, 728, L4

Houck, J. R., Soifer, B. T., Weedman, D., Higdon, S. J. U., Higdon, J. L., Herter, T., Brown, M. J. I., Dey, A., Jannuzi, B. T., Le Floc'h, E., Rieke, M., Armus, L., Charmandaris, V., Brandl, B. R., \& Teplitz, H. I. 2005, ApJ, 622, L105

Hughes, D. H., Aretxaga, I., Chapin, E. L., Gaztañaga, E., Dunlop, J. S., Devlin, M. J., Halpern, M., Gundersen, J., Klein, J., Netterfield, C. B., Olmi, L., Scott, D., \& Tucker, G. 2002, MNRAS, 335, 871

Hughes, D. H., Dunlop, J. S., \& Rawlings, S. 1997, MNRAS, 289, 766

Hughes, D. H., Serjeant, S., Dunlop, J., Rowan-Robinson, M., Blain, A., Mann, R. G., Ivison, R., Peacock, J., Efstathiou, A., Gear, W., Oliver, S., Lawrence, A., Longair, M., Goldschmidt, P., \& Jenness, T. 1998, Nature, 394, 241

Hung, C.-L., Sanders, D. B., Casey, C. M., Lee, N., Barnes, J. E., Capak, P., Kartaltepe, J. S., Koss, M., Larson, K. L., Le Floc'h, E., Lockhart, K., Man, A. W. S., Mann, A. W., Riguccini, L., Scoville, N., \& Symeonidis, M. 2013, ApJ, 778, 129

Huynh, M. T., Norris, R. P., Coppin, K. E. K., Emonts, B. H. C., Ivison, R. J., Seymour, N., Smail, I., Smolčić, V., Swinbank, A. M., Brandt, W. N., Chapman, S. C., Dannerbauer, H., De Breuck, C., Greve, T. R., Hodge, J. A., Karim, A., Knudsen, K. K., Menten, K. M., van der Werf, P. P., Walter, F., \& Weiss, A. 2013, MNRAS, 431, L88

Ibar, E., Ivison, R. J., Biggs, A. D., Lal, D. V., Best, P. N., \& Green, D. A. 2009, MNRAS, 397, 281

Ilbert, O., Capak, P., Salvato, M., Aussel, H., McCracken, H. J., Sanders, D. B., Scoville, N., Kartaltepe, J., Arnouts, S., Le Floc'h, E., Mobasher, B., Taniguchi, Y., Lamareille, F., Leauthaud, A., Sasaki, S., Thompson, D., Zamojski, M., Zamorani, G., Bardelli, S., Bolzonella, M., Bongiorno, A., Brusa, M., Caputi, K. I., Carollo, C. M., Contini, T., Cook, R., Coppa, G., Cucciati, O., de la Torre, S., de Ravel, L., Franzetti, P., Garilli, B., Hasinger, G., Iovino, A., Kampczyk, P., Kneib, J.-P., Knobel, C., Kovac, K., Le Borgne, J. F., Le Brun, V., Fèvre, O. L., Lilly, S., Looper, D., Maier, C., Mainieri, V., Mellier, Y., Mignoli, M., Murayama, T., Pellò, R., Peng, Y., Pérez-Montero, E., Renzini, A., Ricciardelli, E., Schiminovich, D., Scodeggio, M., Shioya, Y., Silverman, J., Surace, J., Tanaka, M., Tasca, L., Tresse, L., Vergani, D., \& Zucca, E. 2009, ApJ, 690,1236

Impellizzeri, C. M. V., McKean, J. P., Castangia, P., Roy, A. L., Henkel, C., Brunthaler, A., \& Wucknitz, O. 2008, Nature, 456, 927

Iono, D., Wilson, C. D., Yun, M. S., Baker, A. J., Petitpas, G. R., Peck, A. B., Krips, M., Cox, T. J., Matsushita, S., Mihos, J. C., \& Pihlstrom, Y. 2009, ApJ, 695, 1537

Iono, D., Yun, M. S., Elvis, M., Peck, A. B., Ho, P. T. P., Wilner, D. J., Hunter, T. R., Matsushita, S., \& Muller, S. 2006, ApJ, 645, L97

Irwin, M. J., Ibata, R. A., Lewis, G. F., \& Totten, E. J. 1998, ApJ, 505, 529

Ivison, R. J., Alexander, D. M., Biggs, A. D., Brandt, W. N., Chapin, E. L., Coppin, K. E. K., Devlin, M. J., Dickinson, M., Dunlop, J., Dye, S., Eales, S. A., Frayer, D. T., Halpern, M., Hughes, D. H., Ibar, E., Kovács, A., Marsden, G., Moncelsi, L., Netterfield, C. B., Pascale, E., Patanchon, G., Rafferty, D. A., Rex, M., Schinnerer, E., Scott, D., Semisch, C., Smail, I., Swinbank, A. M., Truch, M. D. P., Tucker, G. S., Viero, M. P., Walter, F., Weiß, A., Wiebe, D. V., \& Xue, Y. Q. 2010a, MNRAS, 402, 245

Ivison, R. J., Greve, T. R., Dunlop, J. S., Peacock, J. A., Egami, E., Smail, I., Ibar, E., van Kampen, E., Aretxaga, I., Babbedge, T., Biggs, A. D., Blain, A. W., Chapman, S. C., Clements, D. L., Coppin, K., Farrah, D., Halpern, M., Hughes, D. H., Jarvis, M. J., Jenness, T., Jones, J. R., Mortier, A. M. J., Oliver, S., Papovich, C., Pérez-González, P. G., Pope, A., Rawlings, S., Rieke, G. H., Rowan-Robinson, M., Savage, R. S., Scott, D., Seigar, M., Serjeant, S., Simpson, C., Stevens, J. A., Vaccari, M., Wagg, J., \& Willott, C. J. 2007, MNRAS, 380,199

Ivison, R. J., Greve, T. R., Smail, I., Dunlop, J. S., Roche, N. D., Scott, S. E., Page, M. J., Stevens, J. A., Almaini, O., Blain, A. W., Willott, C. J., Fox, M. J., Gilbank, D. G., Serjeant, S., \& Hughes, D. H. 2002, MNRAS, 337,

Ivison, R. J., Magnelli, B., Ibar, E., Andreani, P., Elbaz, D., Altieri, B., Amblard, A., Arumugam, V., Auld, R., Aussel, H., Babbedge, T., Berta, S., Blain, A., Bock, J., Bongiovanni, A., Boselli, A., Buat, V., Burgarella, D., Castro-Rodríguez, N., Cava, A., Cepa, J., Chanial, P., Cimatti, A., Cirasuolo, M., Clements, D. L., Conley, A., Conversi, L., Cooray, A., Daddi, E., Dominguez, H., Dowell, C. D., Dwek, E., Eales, S., Farrah, D., Förster Schreiber, N., Fox, M., Franceschini, A., Gear, W., Genzel, R., Glenn, J., Griffin, M., Gruppioni, C., Halpern, M., Hatziminaoglou, E., Isaak, K., Lagache, G., Levenson, L., Lu, N., Lutz, D., Madden, S., Maffei, B., Magdis, G., Mainetti, G., Maiolino, R., Marchetti, L., Morrison, G. E., Mortier, A. M. J., Nguyen, H. T., Nordon, R., O’Halloran, B., Oliver, S. J., Omont, A., Owen, F. N., Page, M. J., Panuzzo, P., Papageorgiou, A., Pearson, C. P., Pérez-Fournon, I., Pérez García, A. M., Poglitsch, A., Pohlen, M., Popesso, P., Pozzi, F., Rawlings, J. I., Raymond, G., Rigopoulou, D., Riguccini, L., Rizzo, D., Rodighiero, G., Roseboom, I. G., Rowan-Robinson, M., Saintonge, A., Sanchez Portal, M., Santini, P., Schulz, B., Scott, D., Seymour, N., Shao, L., Shupe, D. L., Smith, A. J., Stevens, J. A., Sturm, E., Symeonidis, M., Tacconi, 
L., Trichas, M., Tugwell, K. E., Vaccari, M., Valtchanov, I., Vieira, J., Vigroux, L., Wang, L., Ward, R., Wright, G., Xu, C. K., \& Zemcov, M. 2010b, A\&A, 518, L31+

Ivison, R. J., Papadopoulos, P. P., Smail, I., Greve, T. R., Thomson, A. P., Xilouris, E. M., \& Chapman, S. C. 2011, MNRAS, 412, 1913

Ivison, R. J., Smail, I., Barger, A. J., Kneib, J.-P., Blain, A. W., Owen, F. N., Kerr, T. H., \& Cowie, L. L. 2000, MNRAS, 315, 209

Ivison, R. J., Smail, I., Le Borgne, J.-F., Blain, A. W., Kneib, J.-P., Bezecourt, J., Kerr, T. H., \& Davies, J. K. 1998, MNRAS, 298, 583

Ivison, R. J., Smail, I., Papadopoulos, P. P., Wold, I., Richard, J., Swinbank, A. M., Kneib, J.-P., \& Owen, F. N. 2010c, MNRAS, 404, 198

Ivison, R. J., Swinbank, A. M., Smail, I., Harris, A. I., Bussmann, R. S., Cooray, A., Cox, P., Fu, H., Kovács, A., Krips, M., Narayanan, D.,

Negrello, M., Neri, R., Peñarrubia, J., Richard, J., Riechers, D. A., Rowlands, K., Staguhn, J. G., Targett, T. A., Amber, S., Baker, A. J., Bourne,

N., Bertoldi, F., Bremer, M., Calanog, J. A., Clements, D. L., Dannerbauer, H., Dariush, A., De Zotti, G., Dunne, L., Eales, S. A., Farrah, D.,

Fleuren, S., Franceschini, A., Geach, J. E., George, R. D., Helly, J. C., Hopwood, R., Ibar, E., Jarvis, M. J., Kneib, J.-P., Maddox, S., Omont,

A., Scott, D., Serjeant, S., Smith, M. W. L., Thompson, M. A., Valiante, E., Valtchanov, I., Vieira, J., \& van der Werf, P. 2013, ApJ, 772,137

Ivison, R. J., Swinbank, A. M., Swinyard, B., Smail, I., Pearson, C. P., Rigopoulou, D., Polehampton, E., Baluteau, J.-P., Barlow, M. J., Blain,

A. W., Bock, J., Clements, D. L., Coppin, K., Cooray, A., Danielson, A., Dwek, E., Edge, A. C., Franceschini, A., Fulton, T., Glenn, J., Griffin,

M., Isaak, K., Leeks, S., Lim, T., Naylor, D., Oliver, S. J., Page, M. J., Pérez Fournon, I., Rowan-Robinson, M., Savini, G., Scott, D., Spencer,

L., Valtchanov, I., Vigroux, L., \& Wright, G. S. 2010d, A\&A, 518, L35

Jain, B. 2002, ApJ, 580, L3

John, T. L. 1988, A\&A, 193, 189

Johnson, S. P., Wilson, G. W., Wang, Q. D., Williams, C. C., Scott, K. S., Yun, M. S., Pope, A., Lowenthal, J., Aretxaga, I., Hughes, D., Kim, M. J.,

Kim, S., Tamura, Y., Kohno, K., Ezawa, H., Kawabe, R., \& Oshima, T. 2013, MNRAS, 431, 662

Jonsson, P. 2006, MNRAS, 372, 2

Jonsson, P., Groves, B. A., \& Cox, T. J. 2010, MNRAS, 403, 17

Jonsson, P. \& Primack, J. R. 2010, New Astronomy, 15, 509

Joseph, R. D. \& Wright, G. S. 1985, MNRAS, 214, 87

Juneau, S., Dickinson, M., Alexander, D. M., \& Salim, S. 2011, ApJ, 736, 104

Juneau, S., Narayanan, D. T., Moustakas, J., Shirley, Y. L., Bussmann, R. S., Kennicutt, R. C., \& Vanden Bout, P. A. 2009, ApJ, 707, 1217

Kanekar, N., Wagg, J., Ram Chary, R., \& Carilli, C. L. 2013, ApJ, 771, L20

Karim, A., Swinbank, A. M., Hodge, J. A., Smail, I. R., Walter, F., Biggs, A. D., Simpson, J. M., Danielson, A. L. R., Alexander, D. M., Bertoldi, F., de Breuck, C., Chapman, S. C., Coppin, K. E. K., Dannerbauer, H., Edge, A. C., Greve, T. R., Ivison, R. J., Knudsen, K. K., Menten, K. M., Schinnerer, E., Wardlow, J. L., Weiß, A., \& van der Werf, P. 2013, MNRAS, 432, 2

Kartaltepe, J. S., Dickinson, M., Alexander, D. M., Bell, E. F., Dahlen, T., Elbaz, D., Faber, S. M., Lotz, J., McIntosh, D. H., Wiklind, T., Altieri, B., Aussel, H., Bethermin, M., Bournaud, F., Charmandaris, V., Conselice, C. J., Cooray, A., Dannerbauer, H., Davé, R., Dunlop, J., Dekel, A., Ferguson, H. C., Grogin, N. A., Hwang, H. S., Ivison, R., Kocevski, D., Koekemoer, A., Koo, D. C., Lai, K., Leiton, R., Lucas, R. A., Lutz, D., Magdis, G., Magnelli, B., Morrison, G., Mozena, M., Mullaney, J., Newman, J. A., Pope, A., Popesso, P., van der Wel, A., Weiner, B., \& Wuyts, S. 2012, ApJ, 757, 23

Kartaltepe, J. S., Sanders, D. B., Le Floc'h, E., Frayer, D. T., Aussel, H., Arnouts, S., Ilbert, O., Salvato, M., Scoville, N. Z., Surace, J., Yan, L., Capak, P., Caputi, K., Carollo, C. M., Cassata, P., Civano, F., Hasinger, G., Koekemoer, A. M., Le Fèvre, O., Lilly, S., Liu, C. T., McCracken, H. J., Schinnerer, E., Smolčić, V., Taniguchi, Y., Thompson, D. J., Trump, J., Baldassare, V. F., \& Fiorenza, S. L. 2010, ApJ, 721, 98

Kartaltepe, J. S., Sanders, D. B., Scoville, N. Z., Calzetti, D., Capak, P., Koekemoer, A., Mobasher, B., Murayama, T., Salvato, M., Sasaki, S. S., \& Taniguchi, Y. 2007, ApJS, 172, 320

Kauffmann, G. 1996, MNRAS, 281, 487

Kauffmann, G. \& Charlot, S. 1998, MNRAS, 294, 705

Kauffmann, G., Colberg, J. M., Diaferio, A., \& White, S. D. M. 1999, MNRAS, 303, 188

Kauffmann, G., White, S. D. M., \& Guiderdoni, B. 1993, MNRAS, 264, 201

Kawada, M., Baba, H., Barthel, P. D., Clements, D., Cohen, M., Doi, Y., Figueredo, E., Fujiwara, M., Goto, T., Hasegawa, S., Hibi, Y., Hirao, T., Hiromoto, N., Jeong, W.-S., Kaneda, H., Kawai, T., Kawamura, A., Kester, D., Kii, T., Kobayashi, H., Kwon, S. M., Lee, H. M., Makiuti, S., Matsuo, H., Matsuura, S., Müller, T. G., Murakami, N., Nagata, H., Nakagawa, T., Narita, M., Noda, M., Oh, S. H., Okada, Y., Okuda, H., Oliver, S., Ootsubo, T., Pak, S., Park, Y.-S., Pearson, C. P., Rowan-Robinson, M., Saito, T., Salama, A., Sato, S., Savage, R. S., Serjeant, S., Shibai, H., Shirahata, M., Sohn, J., Suzuki, T., Takagi, T., Takahashi, H., Thomson, M., Usui, F., Verdugo, E., Watabe, T., White, G. J., Wang, L., Yamamura, I., Yamauchi, C., \& Yasuda, A. 2007, PASJ, 59, 389

Kawara, K., Matsuhara, H., Okuda, H., Taniguchi, Y., Sato, Y., Sofue, Y., Wakamatsu, K., Oyabu, S., Sanders, D. B., \& Cowie, L. L. 2004, A\&A, 413,843

Kelly, B. C., Shetty, R., Stutz, A. M., Kauffmann, J., Goodman, A. A., \& Launhardt, R. 2012, ApJ, 752, 55

Kennicutt, R. C. \& Evans, N. J. 2012, ARA\&A, 50, 531

Kennicutt, Jr., R. C. 1983, ApJ, 272, 54

-. 1989, ApJ, 344, 685

-. 1998a, ARA\&A, 36, 189

-. 1998b, ApJ, 498, 541

Kewley, L. J., Groves, B., Kauffmann, G., \& Heckman, T. 2006, MNRAS, 372, 961

Kim, J.-h., Abel, T., Agertz, O., Bryan, G. L., Ceverino, D., Christensen, C., Conroy, C., Dekel, A., Gnedin, N. Y., Goldbaum, N. J., Guedes, J., Hahn, O., Hobbs, A., Hopkins, P. F., Hummels, C. B., Iannuzzi, F., Keres, D., Klypin, A., Kravtsov, A. V., Krumholz, M. R., Kuhlen, M., Leitner, S. N., Madau, P., Mayer, L., Moody, C. E., Nagamine, K., Norman, M. L., Onorbe, J., O’Shea, B. W., Pillepich, A., Primack, J. R., Quinn, T., Read, J. I., Robertson, B. E., Rocha, M., Rudd, D. H., Shen, S., Smith, B. D., Szalay, A. S., Teyssier, R., Thompson, R., Todoroki, K., Turk, M. J., Wadsley, J. W., Wise, J. H., Zolotov, A., \& AGORA Collaboration29, f. t. 2014, ApJS, 210, 14

Kim, J.-h., Wise, J. H., \& Abel, T. 2009, ApJ, 694, L123

Kim, S., Wardlow, J. L., Cooray, A., Fleuren, S., Sutherland, W., Khostovan, A. A., Auld, R., Baes, M., Bussmann, R. S., Buttiglione, S., Cava, 
A., Clements, D., Dariush, A., De Zotti, G., Dunne, L., Dye, S., Eales, S., Fritz, J., Hopwood, R., Ibar, E., Ivison, R., Jarvis, M., Maddox, S., Michałowski, M. J., Pascale, E., Pohlen, M., Rigby, E., Scott, D., Smith, D. J. B., Temi, P., \& van der Werf, P. 2012, ApJ, 756, 28

Kirkpatrick, A., Pope, A., Alexander, D. M., Charmandaris, V., Daddi, E., Dickinson, M., Elbaz, D., Gabor, J., Hwang, H. S., Ivison, R., Mullaney, J., Pannella, M., Scott, D., Altieri, B., Aussel, H., Bournaud, F., Buat, V., Coia, D., Dannerbauer, H., Dasyra, K., Kartaltepe, J., Leiton, R., Lin, L., Magdis, G., Magnelli, B., Morrison, G., Popesso, P., \& Valtchanov, I. 2012, ApJ, 759, 139

Kirkpatrick, A., Pope, A., Charmandaris, V., Daddi, E., Elbaz, D., Hwang, H. S., Pannella, M., Scott, D., Altieri, B., Aussel, H., Coia, D., Dannerbauer, H., Dasyra, K., Dickinson, M., Kartaltepe, J., Leiton, R., Magdis, G., Magnelli, B., Popesso, P., \& Valtchanov, I. 2013, ApJ, 763, 123

Knebe, A., Green, A., \& Binney, J. 2001, MNRAS, 325, 845

Knudsen, K. K., van der Werf, P. P., \& Kneib, J.-P. 2008, MNRAS, 384, 1611

Koprowski, M. P., Dunlop, J. S., Michalowski, M. J., Cirasuolo, M., \& Bowler, R. A. A. 2013, ArXiv e-prints

Koss, M., Mushotzky, R., Baumgartner, W., Veilleux, S., Tueller, J., Markwardt, C., \& Casey, C. M. 2013, ApJ, 765, L26

Kovács, A., Chapman, S. C., Dowell, C. D., Blain, A. W., Ivison, R. J., Smail, I., \& Phillips, T. G. 2006, ApJ, 650, 592

Kovács, A., Omont, A., Beelen, A., Lonsdale, C., Polletta, M., Fiolet, N., Greve, T. R., Borys, C., Cox, P., De Breuck, C., Dole, H., Dowell, C. D., Farrah, D., Lagache, G., Menten, K. M., Bell, T. A., \& Owen, F. 2010, ApJ, 717, 29

Kravtsov, A. V. 1999, PhD thesis, NEW MEXICO STATE UNIVERSITY

Kreysa, E., Gemünd, H.-P., Gromke, J., Haslam, C. G. T., Reichertz, L., Haller, E. E., Beeman, J. W., Hansen, V., Sievers, A., \& Zylka, R. 1999, Infrared Physics and Technology, 40, 191

Kriek, M. \& Conroy, C. 2013, ApJ, 775, L16

Krips, M., Neri, R., García-Burillo, S., Martín, S., Combes, F., Graciá-Carpio, J., \& Eckart, A. 2008, ApJ, 677, 262

Kroupa, P. 2002, Science, 295, 82

Kruijssen, J. M. D. \& Longmore, S. N. 2013, MNRAS

Krumholz, M. R. 2011a, ApJ, 743, 110

-. 2011b, ApJ, 743, 110

-. 2014, MNRAS, 437, 1662

Krumholz, M. R., Bate, M. R., Arce, H. G., Dale, J. E., Gutermuth, R., Klein, R. I., Li, Z.-Y., Nakamura, F., \& Zhang, Q. 2014, arXiv/1401.2473

Krumholz, M. R., Dekel, A., \& McKee, C. F. 2012, ApJ, 745, 69

Krumholz, M. R., Leroy, A. K., \& McKee, C. F. 2011, ApJ, 731, 25

Krumholz, M. R. \& McKee, C. F. 2005, ApJ, 630, 250

Krumholz, M. R., McKee, C. F., \& Tumlinson, J. 2009a, ApJ, 693, 216

-. 2009b, ApJ, 699, 850

Krumholz, M. R. \& Thompson, T. A. 2007, ApJ, 669, 289

Lacey, C. \& Silk, J. 1991, ApJ, 381, 14

Lacey, C. G., Baugh, C. M., Frenk, C. S., Benson, A. J., Orsi, A., Silva, L., Granato, G. L., \& Bressan, A. 2010, MNRAS, 405, 2

Lacey, C. G., Baugh, C. M., Frenk, C. S., Silva, L., Granato, G. L., \& Bressan, A. 2008, MNRAS, 385, 1155

Lacki, B. C. \& Thompson, T. A. 2010, ApJ, 717, 196

Lacki, B. C., Thompson, T. A., \& Quataert, E. 2010, ApJ, 717, 1

Lacy, M., Storrie-Lombardi, L. J., Sajina, A., Appleton, P. N., Armus, L., Chapman, S. C., Choi, P. I., Fadda, D., Fang, F., Frayer, D. T., Heinrichsen, I., Helou, G., Im, M., Marleau, F. R., Masci, F., Shupe, D. L., Soifer, B. T., Surace, J., Teplitz, H. I., Wilson, G., \& Yan, L. 2004, ApJS, 154, 166 Lada, C. J., Lombardi, M., \& Alves, J. F. 2010, ApJ, 724, 687

Lagache, G., Bavouzet, N., Fernandez-Conde, N., Ponthieu, N., Rodet, T., Dole, H., Miville-Deschênes, M.-A., \& Puget, J.-L. 2007, ApJ, 665, L89

Lagache, G., Dole, H., Puget, J.-L., Pérez-González, P. G., Le Floc'h, E., Rieke, G. H., Papovich, C., Egami, E., Alonso-Herrero, A., Engelbracht, C. W., Gordon, K. D., Misselt, K. A., \& Morrison, J. E. 2004, ApJS, 154, 112

Lagache, G., Puget, J.-L., \& Dole, H. 2005, ARA\&A, 43, 727

Lagos, C. D. P., Baugh, C. M., Lacey, C. G., Benson, A. J., Kim, H.-S., \& Power, C. 2011, MNRAS, 418,1649

Lagos, C. d. P., Bayet, E., Baugh, C. M., Lacey, C. G., Bell, T. A., Fanidakis, N., \& Geach, J. E. 2012, MNRAS, 426, 2142

Lagos, C. d. P., Lacey, C. G., \& Baugh, C. M. 2013, MNRAS, 436, 1787

Laird, E. S., Nandra, K., Pope, A., \& Scott, D. 2010, MNRAS, 401, 2763

Lanz, L., Zezas, A., Brassington, N., Smith, H. A., Ashby, M. L. N., da Cunha, E., Fazio, G. G., Hayward, C. C., Hernquist, L., \& Jonsson, P. 2013, ApJ, 768, 90

Lapi, A., González-Nuevo, J., Fan, L., Bressan, A., De Zotti, G., Danese, L., Negrello, M., Dunne, L., Eales, S., Maddox, S., Auld, R., Baes, M., Bonfield, D. G., Buttiglione, S., Cava, A., Clements, D. L., Cooray, A., Dariush, A., Dye, S., Fritz, J., Herranz, D., Hopwood, R., Ibar, E., Ivison, R., Jarvis, M. J., Kaviraj, S., López-Caniego, M., Massardi, M., Michałowski, M. J., Pascale, E., Pohlen, M., Rigby, E., Rodighiero, G., Serjeant, S., Smith, D. J. B., Temi, P., Wardlow, J., \& van der Werf, P. 2011, ApJ, 742, 24

Larson, R. B. 1981, MNRAS, 194, 809

Laurent, G. T., Aguirre, J. E., Glenn, J., Ade, P. A. R., Bock, J. J., Edgington, S. F., Goldin, A., Golwala, S. R., Haig, D., Lange, A. E., Maloney, P. R., Mauskopf, P. D., Nguyen, H., Rossinot, P., Sayers, J., \& Stover, P. 2005, ApJ, 623, 742

Laurent, O., Mirabel, I. F., Charmandaris, V., Le Floc'h, E., Lutz, D., \& Genzel, R. 2000, in ESA Special Publication, Vol. 456, ISO Beyond the Peaks: The 2nd ISO Workshop on Analytical Spectroscopy, ed. A. Salama, M. F. Kessler, K. Leech, \& B. Schulz, 249

Le Delliou, M., Lacey, C., Baugh, C. M., Guiderdoni, B., Bacon, R., Courtois, H., Sousbie, T., \& Morris, S. L. 2005, MNRAS, 357, L11

Le Delliou, M., Lacey, C. G., Baugh, C. M., \& Morris, S. L. 2006, MNRAS, 365, 712

Le Floc'h, E., Papovich, C., Dole, H., Bell, E. F., Lagache, G., Rieke, G. H., Egami, E., Pérez-González, P. G., Alonso-Herrero, A., Rieke, M. J., Blaylock, M., Engelbracht, C. W., Gordon, K. D., Hines, D. C., Misselt, K. A., Morrison, J. E., \& Mould, J. 2005, ApJ, 632, 169

Le Floc'h, E. et al. 2004, ApJS, 154, 170

Lee, N., Sanders, D. B., Casey, C. M., Scoville, N. Z., Hung, C.-L., Le Floc'h, E., Ilbert, O., Aussel, H., Capak, P., Kartaltepe, J. S., Roseboom, I., 
Salvato, M., Aravena, M., Bock, J., Oliver, S. J., Riguccini, L., \& Symeonidis, M. 2013, ArXiv e-prints

Leech, K. J., Völk, H. J., Heinrichsen, I., Hippelein, H., Metcalfe, L., Pierini, D., Popescu, C. C., Tuffs, R. J., \& Xu, C. 1999, MNRAS, 310,317

Leger, R. F. \& Puget, J.-L. 1984, A\&A, 137, 5

Leitherer, C. \& Heckman, T. M. 1995, ApJS, 96, 9

Lemke, D. et al. 1996, A\&A, 315, 64

Leroy, A. K., Bolatto, A., Gordon, K., Sandstrom, K., Gratier, P., Rosolowsky, E., Engelbracht, C. W., Mizuno, N., Corbelli, E., Fukui, Y., \& Kawamura, A. 2011, ApJ, 737, 12

Leroy, A. K., Walter, F., Bigiel, F., Usero, A., Weiss, A., Brinks, E., de Blok, W. J. G., Kennicutt, R. C., Schuster, K.-F., Kramer, C., Wiesemeyer, H. W., \& Roussel, H. 2009, AJ, 137, 4670

Leroy, A. K., Walter, F., Brinks, E., Bigiel, F., de Blok, W. J. G., Madore, B., \& Thornley, M. D. 2008, AJ, 136, 2782

Leroy, A. K., Walter, F., Sandstrom, K., Schruba, A., Munoz-Mateos, J.-C., Bigiel, F., Bolatto, A., Brinks, E., de Blok, W. J. G., Meidt, S., Rix, H.-W., Rosolowsky, E., Schinnerer, E., Schuster, K.-F., \& Usero, A. 2013, AJ, 146, 19

Lewis, G. F., Chapman, S. C., \& Helou, G. 2005, ApJ, 621, 32

Lilly, S. J., Tresse, L., Hammer, F., Crampton, D., \& Le Fevre, O. 1995, ApJ, 455, 108

Lindner, R. R., Baker, A. J., Omont, A., Beelen, A., Owen, F. N., Bertoldi, F., Dole, H., Fiolet, N., Harris, A. I., Ivison, R. J., Lonsdale, C. J., Lutz, D., \& Polletta, M. 2011, ApJ, 737, 83

Lintott, C. \& Viti, S. 2006, ApJ, 646, L37

Lisenfeld, U., Isaak, K. G., \& Hills, R. 2000, MNRAS, 312, 433

Lisenfeld, U., Volk, H., \& Xu, C. 1996, A\&A, 306, 677

Liu, G., Koda, J., Calzetti, D., Fukuhara, M., \& Momose, R. 2011, ApJ, 735, 63

Lonsdale, C. J., Persson, S. E., \& Matthews, K. 1984, ApJ, 287, 95

Lonsdale, C. J., Polletta, M. d. C., Omont, A., Shupe, D., Berta, S., Zylka, R., Siana, B., Lutz, D., Farrah, D., Smith, H. E., Lagache, G., DeBreuck, C., Owen, F., Beelen, A., Weedman, D., Franceschini, A., Clements, D., Tacconi, L., Afonso-Luis, A., Pérez-Fournon, I., Cox, P., \& Bertoldi, F. 2009, ApJ, 692, 422

Lonsdale, C. J. et al. 2003, PASP, 115, 897

Lotz, J. M., Davis, M., Faber, S. M., Guhathakurta, P., Gwyn, S., Huang, J., Koo, D. C., Le Floc'h, E., Lin, L., Newman, J., Noeske, K., Papovich, C., Willmer, C. N. A., Coil, A., Conselice, C. J., Cooper, M., Hopkins, A. M., Metevier, A., Primack, J., Rieke, G., \& Weiner, B. J. 2008, ApJ, 672,177

Lu, Y., Mo, H. J., Katz, N., \& Weinberg, M. D. 2012, MNRAS, 421, 1779

Lu, Y., Mo, H. J., Weinberg, M. D., \& Katz, N. 2011, MNRAS, 416, 1949

Luhman, M. L., Satyapal, S., Fischer, J., Wolfire, M. G., Cox, P., Lord, S. D., Smith, H. A., Stacey, G. J., \& Unger, S. J. 1998, ApJ, 504, L11

Luhman, M. L., Satyapal, S., Fischer, J., Wolfire, M. G., Sturm, E., Dudley, C. C., Lutz, D., \& Genzel, R. 2003, ApJ, 594, 758

Lutz, D., Dunlop, J. S., Almaini, O., Andreani, P., Blain, A., Efstathiou, A., Fox, M., Genzel, R., Hasinger, G., Hughes, D., Ivison, R. J., Lawrence,

A., Mann, R. G., Oliver, S., Peacock, J. A., Rigopoulou, D., Rowan-Robinson, M., Scott, S., Serjeant, S., \& Tacconi, L. 2001, A\&A, 378, 70

Lutz, D., Mainieri, V., Rafferty, D., Shao, L., Hasinger, G., Weiß, A., Walter, F., Smail, I., Alexander, D. M., Brandt, W. N., Chapman, S., Coppin, K., Förster Schreiber, N. M., Gawiser, E., Genzel, R., Greve, T. R., Ivison, R. J., Koekemoer, A. M., Kurczynski, P., Menten, K. M., Nordon, R., Popesso, P., Schinnerer, E., Silverman, J. D., Wardlow, J., \& Xue, Y. Q. 2010, ApJ, 712, 1287

Lutz, D. et al. 2011, A\&A, 532

Madau, P., Ferguson, H. C., Dickinson, M. E., Giavalisco, M., Steidel, C. C., \& Fruchter, A. 1996, MNRAS, 283,1388

Maddox, S. J., Dunne, L., Rigby, E., Eales, S., Cooray, A., Scott, D., Peacock, J. A., Negrello, M., Smith, D. J. B., Benford, D., Amblard, A., Auld, R., Baes, M., Bonfield, D., Burgarella, D., Buttiglione, S., Cava, A., Clements, D., Dariush, A., de Zotti, G., Dye, S., Frayer, D., Fritz, J., Gonzalez-Nuevo, J., Herranz, D., Ibar, E., Ivison, R., Jarvis, M. J., Lagache, G., Leeuw, L., Lopez-Caniego, M., Pascale, E., Pohlen, M., Rodighiero, G., Samui, S., Serjeant, S., Temi, P., Thompson, M., \& Verma, A. 2010, A\&A, 518, L11

Magdis, G. E., Daddi, E., Béthermin, M., Sargent, M., Elbaz, D., Pannella, M., Dickinson, M., Dannerbauer, H., da Cunha, E., Walter, F., Rigopoulou, D., Charmandaris, V., Hwang, H. S., \& Kartaltepe, J. 2012a, ApJ, 760, 6

Magdis, G. E., Daddi, E., Elbaz, D., Sargent, M., Dickinson, M., Dannerbauer, H., Aussel, H., Walter, F., Hwang, H. S., Charmandaris, V., Hodge, J., Riechers, D., Rigopoulou, D., Carilli, C., Pannella, M., Mullaney, J., Leiton, R., \& Scott, D. 2011, ApJ, 740, L15

Magdis, G. E., Daddi, E., Sargent, M., Elbaz, D., Gobat, R., Dannerbauer, H., Feruglio, C., Tan, Q., Rigopoulou, D., Charmandaris, V., Dickinson, M., Reddy, N., \& Aussel, H. 2012b, ApJ, 758, L9

Magdis, G. E., Elbaz, D., Hwang, H. S., Amblard, A., Arumugam, V., Aussel, H., Blain, A., Bock, J., Boselli, A., Buat, V., Castro-Rodríguez, N., Cava, A., Chanial, P., Clements, D. L., Conley, A., Conversi, L., Cooray, A., Dowell, C. D., Dwek, E., Eales, S., Farrah, D., Franceschini, A., Glenn, J., Griffin, M., Halpern, M., Hatziminaoglou, E., Huang, J., Ibar, E., Isaak, K., Le Floc'h, E., Lagache, G., Levenson, L., Lonsdale, C. J., Lu, N., Madden, S., Maffei, B., Mainetti, G., Marchetti, L., Morrison, G. E., Nguyen, H. T., O’Halloran, B., Oliver, S. J., Omont, A., Owen, F. N., Page, M. J., Pannella, M., Panuzzo, P., Papageorgiou, A., Pearson, C. P., Pérez-Fournon, I., Pohlen, M., Rigopoulou, D., Rizzo, D., Roseboom, I. G., Rowan-Robinson, M., Schulz, B., Scott, D., Seymour, N., Shupe, D. L., Smith, A. J., Stevens, J. A., Strazzullo, V., Symeonidis, M., Trichas, M., Tugwell, K. E., Vaccari, M., Valtchanov, I., Vigroux, L., Wang, L., Wright, G., Xu, C. K., \& Zemcov, M. 2010, MNRAS, 409, 22

Magliocchetti, M., Silva, L., Lapi, A., de Zotti, G., Granato, G. L., Fadda, D., \& Danese, L. 2007, MNRAS, 375, 1121

Magnelli, B., Elbaz, D., Chary, R. R., Dickinson, M., Le Borgne, D., Frayer, D. T., \& Willmer, C. N. A. 2009, A\&A, 496, 57

-. 2011, A\&A, 528, A35

Magnelli, B., Lutz, D., Berta, S., Altieri, B., Andreani, P., Aussel, H., Castañeda, H., Cava, A., Cepa, J., Cimatti, A., Daddi, E., Dannerbauer, H., Dominguez, H., Elbaz, D., Förster Schreiber, N., Genzel, R., Grazian, A., Gruppioni, C., Magdis, G., Maiolino, R., Nordon, R., Pérez Fournon, I., Pérez García, I., Poglitsch, A., Popesso, P., Pozzi, F., Riguccini, L., Rodighiero, G., Saintonge, A., Santini, P., Sanchez-Portal, M., Shao, L., Sturm, E., Tacconi, L., Valtchanov, I., Wieprecht, E., \& Wiezorrek, E. 2010, A\&A, 518, L28

Magnelli, B., Lutz, D., Saintonge, A., Berta, S., Santini, P., Symeonidis, M., Altieri, B., Andreani, P., Aussel, H., Béthermin, M., Bock, J., 
Bongiovanni, A., Cepa, J., Cimatti, A., Conley, A., Daddi, E., Elbaz, D., Förster Schreiber, N. M., Genzel, R., Ivison, R. J., Le Floc'h, E., Magdis, G., Maiolino, R., Nordon, R., Oliver, S. J., Page, M., Pérez García, A., Poglitsch, A., Popesso, P., Pozzi, F., Riguccini, L., Rodighiero, G., Rosario, D., Roseboom, I., Sanchez-Portal, M., Scott, D., Sturm, E., Tacconi, L. J., Valtchanov, I., Wang, L., \& Wuyts, S. 2013a, ArXiv e-prints

Magnelli, B., Popesso, P., Berta, S., Pozzi, F., Elbaz, D., Lutz, D., Dickinson, M., Altieri, B., Andreani, P., Aussel, H., Béthermin, M., Bongiovanni, A., Cepa, J., Charmandaris, V., Chary, R.-R., Cimatti, A., Daddi, E., Förster Schreiber, N. M., Genzel, R., Gruppioni, C., Harwit, M., Hwang, H. S., Ivison, R. J., Magdis, G., Maiolino, R., Murphy, E., Nordon, R., Pannella, M., Pérez García, A., Poglitsch, A., Rosario, D., Sanchez-Portal, M., Santini, P., Scott, D., Sturm, E., Tacconi, L. J., \& Valtchanov, I. 2013b, A\&A, 553, A132

Magnelli, B., Saintonge, A., Lutz, D., Tacconi, L. J., Berta, S., Bournaud, F., Charmandaris, V., Dannerbauer, H., Elbaz, D., Förster-Schreiber, N. M., Graciá-Carpio, J., Ivison, R., Maiolino, R., Nordon, R., Popesso, P., Rodighiero, G., Santini, P., \& Wuyts, S. 2012, A\&A, 548, A22

Maiolino, R., Caselli, P., Nagao, T., Walmsley, M., De Breuck, C., \& Meneghetti, M. 2009, A\&A, 500, L1

Maiolino, R., Cox, P., Caselli, P., Beelen, A., Bertoldi, F., Carilli, C. L., Kaufman, M. J., Menten, K. M., Nagao, T., Omont, A., Weiß, A., Walmsley, C. M., \& Walter, F. 2005, A\&A, 440, L51

Malhotra, S., Helou, G., Stacey, G., Hollenbach, D., Lord, S., Beichman, C. A., Dinerstein, H., Hunter, D. A., Lo, K. Y., Lu, N. Y., Rubin, R. H., Silbermann, N., Thronson, Jr., H. A., \& Werner, M. W. 1997, ApJ, 491, L27

Malhotra, S., Kaufman, M. J., Hollenbach, D., Helou, G., Rubin, R. H., Brauher, J., Dale, D., Lu, N. Y., Lord, S., Stacey, G., Contursi, A., Hunter, D. A., \& Dinerstein, H. 2001, ApJ, 561, 766

Maloney, P. \& Black, J. H. 1988, ApJ, 325, 389

Maloney, P. R. \& Wolfire, M. G. 1997, in IAU Symposium, Vol. 170, IAU Symposium, ed. W. B. Latter, S. J. E. Radford, P. R. Jewell, J. G. Mangum, \& J. Bally, 299-301

Mangum, J. G., Darling, J., Henkel, C., \& Menten, K. M. 2013, ApJ, 766, 108

Mangum, J. G., Darling, J., Menten, K. M., \& Henkel, C. 2008, ApJ, 673, 832

Mao, R.-Q., Schulz, A., Henkel, C., Mauersberger, R., Muders, D., \& Dinh-V-Trung. 2010, ApJ, 724, 1336

Maraston, C. 2005, MNRAS, 362, 799

Marsden, D., Gralla, M., Marriage, T. A., Switzer, E. R., Partridge, B., Massardi, M., Morales, G., Addison, G., Bond, J., Crichton, D., Das, S., Devlin, M., Dunner, R., Hajian, A., Hilton, M., Hincks, A., Hughes, J. P., Irwin, K., Kosowsky, A., Menanteau, F., Moodley, K., Niemack, M., Page, L., Reese, E. D., Schmitt, B., Sehgal, N., Sievers, J., Staggs, S., Swetz, D., Thornton, R., \& Wollack, E. 2013, ArXiv e-prints

Marsden, G. et al. 2009, ApJ, 707, 1729

Martin, C. L. 2005, ApJ, 621, 227

McDermid, R. M., Alatalo, K., Blitz, L., Bois, M., Bournaud, F., Bureau, M., Cappellari, M., Crocker, A. F., Davies, R. L., Davis, T. A., de Zeeuw, P. T., Duc, P.-A., Emsellem, E., Khochfar, S., Krajnović, D., Kuntschner, H., Lablanche, P.-Y., Morganti, R., Naab, T., Oosterloo, T., Sarzi, M., Scott, N., Serra, P., Weijmans, A.-M., \& Young, L. M. 2012, in IAU Symposium, Vol. 284, IAU Symposium, ed. R. J. Tuffs \& C. C. Popescu, 244-247

McKee, C. F. \& Ostriker, E. C. 2007, ARA\&A, 45, 565

McLure, R. J., Jarvis, M. J., Targett, T. A., Dunlop, J. S., \& Best, P. N. 2006, MNRAS, 368, 1395

McQuinn, K. B. W., Skillman, E. D., Dalcanton, J. J., Dolphin, A. E., Cannon, J. M., Holtzman, J., Weisz, D. R., \& Williams, B. F. 2012, ApJ, 751, 127

Meijerink, R., Kristensen, L. E., Weiß, A., van der Werf, P. P., Walter, F., Spaans, M., Loenen, A. F., Fischer, J., Israel, F. P., Isaak, K., Papadopoulos, P. P., Aalto, S., Armus, L., Charmandaris, V., Dasyra, K. M., Diaz-Santos, T., Evans, A., Gao, Y., González-Alfonso, E., Güsten, R., Henkel, C., Kramer, C., Lord, S., Martín-Pintado, J., Naylor, D., Sanders, D. B., Smith, H., Spinoglio, L., Stacey, G., Veilleux, S., \& Wiedner, M. C. 2013, ApJ, 762, L16

Melbourne, J., Peng, C. Y., Soifer, B. T., Urrutia, T., Desai, V., Armus, L., Bussmann, R. S., Dey, A., \& Matthews, K. 2011, AJ, 141, 141

Ménard, B. \& Fukugita, M. 2012, ApJ, 754, 116

Ménard, B., Scranton, R., Fukugita, M., \& Richards, G. 2010, MNRAS, 405, 1025

Menéndez-Delmestre, K., Blain, A. W., Alexander, D. M., Smail, I., Armus, L., Chapman, S. C., Frayer, D. T., Ivison, R. J., \& Teplitz, H. I. 2007, ApJ, 655, L65

Menéndez-Delmestre, K., Blain, A. W., Smail, I., Alexander, D. M., Chapman, S. C., Armus, L., Frayer, D., Ivison, R. J., \& Teplitz, H. 2009, ApJ, 699,667

Menéndez-Delmestre, K., Blain, A. W., Swinbank, M., Smail, I., Ivison, R. J., Chapman, S. C., \& Gonçalves, T. S. 2013, ApJ, 767, 151

Merloni, A. 2004, MNRAS, 353, 1035

Meurer, G. R., Heckman, T. M., \& Calzetti, D. 1999, ApJ, 521, 64

Meurer, G. R., Heckman, T. M., Lehnert, M. D., Leitherer, C., \& Lowenthal, J. 1997, AJ, 114, 54

Michałowski, M., Hjorth, J., \& Watson, D. 2010, A\&A, 514, A67

Michałowski, M. J., Dunlop, J. S., Cirasuolo, M., Hjorth, J., Hayward, C. C., \& Watson, D. 2012, A\&A, 541, A85

Miller, G. E. \& Scalo, J. M. 1979, ApJS, 41, 513

Mocanu, L. M., Crawford, T. M., Vieira, J. D., Aird, K. A., Aravena, M., Austermann, J. E., Benson, B. A., Béthermin, M., Bleem, L. E., Bothwell, M., Carlstrom, J. E., Chang, C. L., Chapman, S., Cho, H., Crites, A. T., de Haan, T., Dobbs, M. A., Everett, W. B., George, E. M., Halverson, N. W., Harrington, N., Hezaveh, Y., Holder, G. P., Holzapfel, W. L., Hoover, S., Hrubes, J. D., Keisler, R., Knox, L., Lee, A. T., Leitch, E. M., Lueker, M., Luong-Van, D., Marrone, D. P., McMahon, J. J., Mehl, J., Meyer, S. S., Mohr, J. J., Montroy, T. E., Natoli, T., Padin, S., Plagge, T., Pryke, C., Rest, A., Reichardt, C. L., Ruhl, J. E., Sayre, J. T., Schaffer, K. K., Shirokoff, E., Spieler, H. G., Spilker, J. S., Stalder, B., Staniszewski, Z., Stark, A. A., Story, K. T., Switzer, E. R., Vanderlinde, K., \& Williamson, R. 2013, ArXiv e-prints

Moster, B. P., Macciò, A. V., \& Somerville, R. S. 2014, MNRAS, 437, 1027

Muñoz, J. A. \& Furlanetto, S. R. 2013, MNRAS, 435, 2676

Murakami, H., Baba, H., Barthel, P., Clements, D. L., Cohen, M., Doi, Y., Enya, K., Figueredo, E., Fujishiro, N., Fujiwara, H., Fujiwara, M., Garcia-Lario, P., Goto, T., Hasegawa, S., Hibi, Y., Hirao, T., Hiromoto, N., Hong, S. S., Imai, K., Ishigaki, M., Ishiguro, M., Ishihara, D., Ita, 
Y., Jeong, W.-S., Jeong, K. S., Kaneda, H., Kataza, H., Kawada, M., Kawai, T., Kawamura, A., Kessler, M. F., Kester, D., Kii, T., Kim, D. C., Kim, W., Kobayashi, H., Koo, B. C., Kwon, S. M., Lee, H. M., Lorente, R., Makiuti, S., Matsuhara, H., Matsumoto, T., Matsuo, H., Matsuura, S., Müller, T. G., Murakami, N., Nagata, H., Nakagawa, T., Naoi, T., Narita, M., Noda, M., Oh, S. H., Ohnishi, A., Ohyama, Y., Okada, Y., Okuda, H., Oliver, S., Onaka, T., Ootsubo, T., Oyabu, S., Pak, S., Park, Y.-S., Pearson, C. P., Rowan-Robinson, M., Saito, T., Sakon, I., Salama, A., Sato, S., Savage, R. S., Serjeant, S., Shibai, H., Shirahata, M., Sohn, J., Suzuki, T., Takagi, T., Takahashi, H., Tanabé, T., Takeuchi, T. T., Takita, S., Thomson, M., Uemizu, K., Ueno, M., Usui, F., Verdugo, E., Wada, T., Wang, L., Watabe, T., Watarai, H., White, G. J., Yamamura, I., Yamauchi, C., \& Yasuda, A. 2007, PASJ, 59, 369

Murgia, M., Helfer, T. T., Ekers, R., Blitz, L., Moscadelli, L., Wong, T., \& Paladino, R. 2005, A\&A, 437, 389

Murphy, E. J. 2009, ApJ, 706, 482

Murphy, E. J., Chary, R.-R., Dickinson, M., Pope, A., Frayer, D. T., \& Lin, L. 2011, ApJ, 732, 126

Murphy, E. J., Helou, G., Kenney, J. D. P., Armus, L., \& Braun, R. 2008, ApJ, 678, 828

Myers, A. D., Brunner, R. J., Richards, G. T., Nichol, R. C., Schneider, D. P., Vanden Berk, D. E., Scranton, R., Gray, A. G., \& Brinkmann, J. 2006, ApJ, 638, 622

Myers, A. D., White, M., \& Ball, N. M. 2009, MNRAS, 399, 2279

Nagao, T., Maiolino, R., De Breuck, C., Caselli, P., Hatsukade, B., \& Saigo, K. 2012, A\&A, 542, L34

Narayanan, D., Bothwell, M., \& Davé, R. 2012a, MNRAS, 426, 1178

Narayanan, D., Cox, T. J., Hayward, C. C., \& Hernquist, L. 2011a, MNRAS, 412, 287

Narayanan, D., Cox, T. J., Hayward, C. C., Younger, J. D., \& Hernquist, L. 2009, MNRAS, 400, 1919

Narayanan, D., Cox, T. J., \& Hernquist, L. 2008a, ApJ, 681, L77

Narayanan, D., Cox, T. J., Kelly, B., Davé, R., Hernquist, L., Di Matteo, T., Hopkins, P. F., Kulesa, C., Robertson, B., \& Walker, C. K. 2008b, ApJS, 176, 331

Narayanan, D., Cox, T. J., Robertson, B., Davé, R., Di Matteo, T., Hernquist, L., Hopkins, P., Kulesa, C., \& Walker, C. K. 2006a, ApJ, 642, L107 Narayanan, D., Cox, T. J., Shirley, Y., Davé, R., Hernquist, L., \& Walker, C. K. 2008c, ApJ, 684, 996

Narayanan, D. \& Davé, R. 2012, MNRAS, 423, 3601

-. 2013, MNRAS, 436, 2892

Narayanan, D., Dey, A., Hayward, C. C., Cox, T. J., Bussmann, R. S., Brodwin, M., Jonsson, P., Hopkins, P. F., Groves, B., Younger, J. D., \& Hernquist, L. 2010a, MNRAS, 407, 1701

-. 2010b, MNRAS, 407, 1701

Narayanan, D., Groppi, C. E., Kulesa, C. A., \& Walker, C. K. 2005, ApJ, 630, 269

Narayanan, D., Hayward, C. C., Cox, T. J., Hernquist, L., Jonsson, P., Younger, J. D., \& Groves, B. 2010c, MNRAS, 401 , 1613

Narayanan, D. \& Hopkins, P. F. 2013, MNRAS

Narayanan, D. \& Krumholz, M. 2014, arXiv/1401.2998

Narayanan, D., Krumholz, M., Ostriker, E. C., \& Hernquist, L. 2011b, MNRAS, 418, 664

Narayanan, D., Krumholz, M. R., Ostriker, E. C., \& Hernquist, L. 2012b, MNRAS, 421, 3127

Narayanan, D., Kulesa, C. A., Boss, A., \& Walker, C. K. 2006b, ApJ, 647, 1426

Narayanan, D., Li, Y., Cox, T. J., Hernquist, L., Hopkins, P., Chakrabarti, S., Davé, R., Di Matteo, T., Gao, L., Kulesa, C., Robertson, B., \& Walker, C. K. 2008d, ApJS, 174, 13

Nayakshin, S. \& Sunyaev, R. 2005, MNRAS, 364, L23

Negrello, M., Hopwood, R., De Zotti, G., Cooray, A., Verma, A., Bock, J., Frayer, D. T., Gurwell, M. A., Omont, A., Neri, R., Dannerbauer, H., Leeuw, L. L., Barton, E., Cooke, J., Kim, S., da Cunha, E., Rodighiero, G., Cox, P., Bonfield, D. G., Jarvis, M. J., Serjeant, S., Ivison, R. J., Dye, S., Aretxaga, I., Hughes, D. H., Ibar, E., Bertoldi, F., Valtchanov, I., Eales, S., Dunne, L., Driver, S. P., Auld, R., Buttiglione, S., Cava, A., Grady, C. A., Clements, D. L., Dariush, A., Fritz, J., Hill, D., Hornbeck, J. B., Kelvin, L., Lagache, G., Lopez-Caniego, M., Gonzalez-Nuevo, J., Maddox, S., Pascale, E., Pohlen, M., Rigby, E. E., Robotham, A., Simpson, C., Smith, D. J. B., Temi, P., Thompson, M. A., Woodgate, B. E., York, D. G., Aguirre, J. E., Beelen, A., Blain, A., Baker, A. J., Birkinshaw, M., Blundell, R., Bradford, C. M., Burgarella, D., Danese, L., Dunlop, J. S., Fleuren, S., Glenn, J., Harris, A. I., Kamenetzky, J., Lupu, R. E., Maddalena, R. J., Madore, B. F., Maloney, P. R., Matsuhara, H., Michaowski, M. J., Murphy, E. J., Naylor, B. J., Nguyen, H., Popescu, C., Rawlings, S., Rigopoulou, D., Scott, D., Scott, K. S., Seibert, M., Smail, I., Tuffs, R. J., Vieira, J. D., van der Werf, P. P., \& Zmuidzinas, J. 2010, Science, 330, 800

Negrello, M., Hopwood, R., Dye, S., da Cunha, E., Serjeant, S., Fleuren, S., Bussmann, R. S., Cooray, A., Dannerbauer, H., Gonzalez-Nuevo, J., Lapi, A., Omont, A., Amber, S., Auld, R., Baes, M., Buttiglione, S., Cava, A., Danese, L., Dariush, A., De Zotti, G., Dunne, L., Eales, S., Fritz, J., Ibar, E., Ivison, R., Kim, S., Maddox, S., Michalowski, M. J., Pascale, E., Pohlen, M., Rigby, E., Rowlands, K., Smith, D. J. B., Sutherland, W., Temi, P., \& Wardlow, J. 2013, ArXiv e-prints

Negrello, M., Perrotta, F., González-Nuevo, J., Silva, L., de Zotti, G., Granato, G. L., Baccigalupi, C., \& Danese, L. 2007 , MNRAS, 377,1557

Nelson, D., Vogelsberger, M., Genel, S., Sijacki, D., Kereš, D., Springel, V., \& Hernquist, L. 2013, MNRAS, 429, 3353

Nenkova, M., Ivezić, Ž., \& Elitzur, M. 2002, ApJ, 570, L9

Nenkova, M., Sirocky, M. M., Ivezić, Ž., \& Elitzur, M. 2008, ApJ, 685, 147

Neri, R., Genzel, R., Ivison, R. J., Bertoldi, F., Blain, A. W., Chapman, S. C., Cox, P., Greve, T. R., Omont, A., \& Frayer, D. T. 2003, ApJ, 597, L113

Neugebauer, G., Habing, H. J., van Duinen, R., Aumann, H. H., Baud, B., Beichman, C. A., Beintema, D. A., Boggess, N., Clegg, P. E., de Jong, T., Emerson, J. P., Gautier, T. N., Gillett, F. C., Harris, S., Hauser, M. G., Houck, J. R., Jennings, R. E., Low, F. J., Marsden, P. L., Miley, G., Olnon, F. M., Pottasch, S. R., Raimond, E., Rowan-Robinson, M., Soifer, B. T., Walker, R. G., Wesselius, P. R., \& Young, E. 1984, ApJ, 278, L1

Niemi, S.-M., Somerville, R. S., Ferguson, H. C., Huang, K.-H., Lotz, J., \& Koekemoer, A. M. 2012, MNRAS, 421, 1539

Nikola, T., Genzel, R., Herrmann, F., Madden, S. C., Poglitsch, A., Geis, N., Townes, C. H., \& Stacey, G. J. 1998, ApJ, 504, 749

Nikola, T., Hailey-Dunsheath, S., Stacey, G. J., Benford, D. J., Moseley, Jr., S. H., \& Staguhn, J. G. 2003, in Society of Photo-Optical Instrumentation Engineers (SPIE) Conference Series, Vol. 4855, Millimeter and Submillimeter Detectors for Astronomy, ed. T. G. Phillips \& J. Zmuidzinas, 
88-99

Noeske, K. G., Faber, S. M., Weiner, B. J., Koo, D. C., Primack, J. R., Dekel, A., Papovich, C., Conselice, C. J., Le Floc'h, E., Rieke, G. H., Coil, A. L., Lotz, J. M., Somerville, R. S., \& Bundy, K. 2007a, ApJ, 660, L47

Noeske, K. G., Weiner, B. J., Faber, S. M., Papovich, C., Koo, D. C., Somerville, R. S., Bundy, K., Conselice, C. J., Newman, J. A., Schiminovich, D., Le Floc'h, E., Coil, A. L., Rieke, G. H., Lotz, J. M., Primack, J. R., Barmby, P., Cooper, M. C., Davis, M., Ellis, R. S., Fazio, G. G., Guhathakurta, P., Huang, J., Kassin, S. A., Martin, D. C., Phillips, A. C., Rich, R. M., Small, T. A., Willmer, C. N. A., \& Wilson, G. 2007b, ApJ, 660, L43

Noll, S., Burgarella, D., Giovannoli, E., Buat, V., Marcillac, D., \& Muñoz-Mateos, J. C. 2009, A\&A, 507, 1793

Nordon, R., Lutz, D., Genzel, R., Berta, S., Wuyts, S., Magnelli, B., Altieri, B., Andreani, P., Aussel, H., Bongiovanni, A., Cepa, J., Cimatti, A., Daddi, E., Fadda, D., Förster Schreiber, N. M., Lagache, G., Maiolino, R., Pérez García, A. M., Poglitsch, A., Popesso, P., Pozzi, F., Rodighiero, G., Rosario, D., Saintonge, A., Sanchez-Portal, M., Santini, P., Sturm, E., Tacconi, L. J., Valtchanov, I., \& Yan, L. 2012, ApJ, 745, 182

Nordon, R., Lutz, D., Saintonge, A., Berta, S., Wuyts, S., Förster Schreiber, N. M., Genzel, R., Magnelli, B., Poglitsch, A., Popesso, P., Rosario, D., Sturm, E., \& Tacconi, L. J. 2013, ApJ, 762, 125

Obreschkow, D. \& Rawlings, S. 2009, MNRAS, 394, 1857

Oesch, P. A., Bouwens, R. J., Illingworth, G. D., Carollo, C. M., Franx, M., Labbé, I., Magee, D., Stiavelli, M., Trenti, M., \& van Dokkum, P. G. 2010, ApJ, 709, L16

Oka, T., Hasegawa, T., Hayashi, M., Handa, T., \& Sakamoto, S. 1998, ApJ, 493, 730

Oke, J. B., Cohen, J. G., Carr, M., Cromer, J., Dingizian, A., Harris, F. H., Labrecque, S., Lucinio, R., Schaal, W., Epps, H., \& Miller, J. 1995, PASP, 107,375

Oliver, S., Frost, M., Farrah, D., Gonzalez-Solares, E., Shupe, D. L., Henriques, B., Roseboom, I., Alfonso-Luis, A., Babbedge, T. S. R., Frayer, D., Lencz, C., Lonsdale, C. J., Masci, F., Padgett, D., Polletta, M., Rowan-Robinson, M., Siana, B., Smith, H. E., Surace, J. A., \& Vaccari, M. 2010, MNRAS, 405, 2279

Oliver, S. J., Bock, J., Altieri, B., Amblard, A., Arumugam, V., Aussel, H., Babbedge, T., Beelen, A., Béthermin, M., Blain, A., Boselli, A., Bridge, C., Brisbin, D., Buat, V., Burgarella, D., Castro-Rodríguez, N., Cava, A., Chanial, P., Cirasuolo, M., Clements, D. L., Conley, A., Conversi, L., Cooray, A., Dowell, C. D., Dubois, E. N., Dwek, E., Dye, S., Eales, S., Elbaz, D., Farrah, D., Feltre, A., Ferrero, P., Fiolet, N., Fox, M., Franceschini, A., Gear, W., Giovannoli, E., Glenn, J., Gong, Y., González Solares, E. A., Griffin, M., Halpern, M., Harwit, M., Hatziminaoglou, E., Heinis, S., Hurley, P., Hwang, H. S., Hyde, A., Ibar, E., Ilbert, O., Isaak, K., Ivison, R. J., Lagache, G., Le Floc'h, E., Levenson, L., Faro, B. L., Lu, N., Madden, S., Maffei, B., Magdis, G., Mainetti, G., Marchetti, L., Marsden, G., Marshall, J., Mortier, A. M. J., Nguyen, H. T., O’Halloran, B., Omont, A., Page, M. J., Panuzzo, P., Papageorgiou, A., Patel, H., Pearson, C. P., Pérez-Fournon, I., Pohlen, M., Rawlings, J. I., Raymond, G., Rigopoulou, D., Riguccini, L., Rizzo, D., Rodighiero, G., Roseboom, I. G., Rowan-Robinson, M., Sánchez Portal, M., Schulz, B., Scott, D., Seymour, N., Shupe, D. L., Smith, A. J., Stevens, J. A., Symeonidis, M., Trichas, M., Tugwell, K. E., Vaccari, M., Valtchanov, I., Vieira, J. D., Viero, M., Vigroux, L., Wang, L., Ward, R., Wardlow, J., Wright, G., Xu, C. K., \& Zemcov, M. 2012, MNRAS, 424, 1614

Omont, A. 2007, Reports on Progress in Physics, 70, 1099

Omont, A., Neri, R., Cox, P., Lupu, R., Guélin, M., van der Werf, P., Weiß, A., Ivison, R., Negrello, M., Leeuw, L., Lehnert, M., Smail, I., Verma, A., Baker, A. J., Beelen, A., Aguirre, J. E., Baes, M., Bertoldi, F., Clements, D. L., Cooray, A., Coppin, K., Dannerbauer, H., de Zotti, G., Dye, S., Fiolet, N., Frayer, D., Gavazzi, R., Hughes, D., Jarvis, M., Krips, M., Michałowski, M. J., Murphy, E. J., Riechers, D., Serjeant, S., Swinbank, A. M., Temi, P., Vaccari, M., Vieira, J. D., Auld, R., Buttiglione, B., Cava, A., Dariush, A., Dunne, L., Eales, S. A., Fritz, J., Gomez, H., Ibar, E., Maddox, S., Pascale, E., Pohlen, M., Rigby, E., Smith, D. J. B., Bock, J., Bradford, C. M., Glenn, J., Scott, K. S., \& Zmuidzinas, J. 2011, A\&A, 530, L3

Omont, A., Yang, C., Cox, P., Neri, R., Beelen, A., Bussmann, R. S., Gavazzi, R., van der Werf, P., Riechers, D., Downes, D., Krips, M., Dye, S., Ivison, R., Vieira, J. D., Weiß, A., Aguirre, J. E., Baes, M., Baker, A. J., Bertoldi, F., Cooray, A., Dannerbauer, H., De Zotti, G., Eales, S. A., Fu, H., Gao, Y., Guélin, M., Harris, A. I., Jarvis, M., Lehnert, M., Leeuw, L., Lupu, R., Menten, K., Michałowski, M. J., Negrello, M., Serjeant, S., Temi, P., Auld, R., Dariush, A., Dunne, L., Fritz, J., Hopwood, R., Hoyos, C., Ibar, E., Maddox, S., Smith, M. W. L., Valiante, E., Bock, J., Bradford, C. M., Glenn, J., \& Scott, K. S. 2013, A\&A, 551, A115

Onaka, T., Matsuhara, H., Wada, T., Fujishiro, N., Fujiwara, H., Ishigaki, M., Ishihara, D., Ita, Y., Kataza, H., Kim, W., Matsumoto, T., Murakami, H., Ohyama, Y., Oyabu, S., Sakon, I., Tanabé, T., Takagi, T., Uemizu, K., Ueno, M., Usui, F., Watarai, H., Cohen, M., Enya, K., Ootsubo, T., Pearson, C. P., Takeyama, N., Yamamuro, T., \& Ikeda, Y. 2007, PASJ, 59, 401

Oppenheimer, B. D. \& Davé, R. 2006, MNRAS, 373, 1265

-. 2008, MNRAS, 387, 577

-. 2009, MNRAS, 395, 1875

Oppenheimer, B. D., Davé, R., Kereš, D., Fardal, M., Katz, N., Kollmeier, J. A., \& Weinberg, D. H. 2010, MNRAS, 406, 2325

Ostriker, E. C. \& Shetty, R. 2011, ApJ, 731, 41

Ouchi, M., Ellis, R., Ono, Y., Nakanishi, K., Kohno, K., Momose, R., Kurono, Y., Ashby, M. L. N., Shimasaku, K., Willner, S. P., Fazio, G. G., Tamura, Y., \& Iono, D. 2013, ApJ, 778, 102

Pacifici, C., Kassin, S. A., Weiner, B., Charlot, S., \& Gardner, J. P. 2013, ApJ, 762, L15

Padoan, P. \& Nordlund, A. 2002, ApJ, 576, 870

Papadopoulos, P. P., Isaak, K., \& van der Werf, P. 2010, ApJ, 711, 757

Papadopoulos, P. P. \& Ivison, R. J. 2002, ApJ, 564, L9

Papadopoulos, P. P., van der Werf, P., Xilouris, E., Isaak, K. G., \& Gao, Y. 2012, ApJ, 751, 10

Papovich, C., Finkelstein, S. L., Ferguson, H. C., Lotz, J. M., \& Giavalisco, M. 2011, MNRAS, 412, 1123

Papovich, C., Rudnick, G., Le Floc'h, E., van Dokkum, P. G., Rieke, G. H., Taylor, E. N., Armus, L., Gawiser, E., Huang, J., Marcillac, D., \& Franx, M. 2007, ApJ, 668, 45

Paradis, D., Bernard, J.-P., \& Mény, C. 2009, A\&A, 506, 745

Pascale, E. et al. 2009, ApJ, 707, 1740

Patanchon, G., Ade, P. A. R., Bock, J. J., Chapin, E. L., Devlin, M. J., Dicker, S. R., Griffin, M., Gundersen, J. O., Halpern, M., Hargrave, P. C., 
Hughes, D. H., Klein, J., Marsden, G., Mauskopf, P., Moncelsi, L., Netterfield, C. B., Olmi, L., Pascale, E., Rex, M., Scott, D., Semisch, C., Thomas, N., Truch, M. D. P., Tucker, C., Tucker, G. S., Viero, M. P., \& Wiebe, D. V. 2009, ApJ, 707, 1750

Peng, C. Y., Impey, C. D., Rix, H., Kochanek, C. S., Keeton, C. R., Falco, E. E., Lehár, J., \& McLeod, B. A. 2006, ApJ, 649, 616

Perera, T. A., Chapin, E. L., Austermann, J. E., Scott, K. S., Wilson, G. W., Halpern, M., Pope, A., Scott, D., Yun, M. S., Lowenthal, J. D., Morrison, G., Aretxaga, I., Bock, J. J., Coppin, K., Crowe, M., Frey, L., Hughes, D. H., Kang, Y., Kim, S., \& Mauskopf, P. D. 2008, MNRAS, 391,1227

Pérez-González, P. G., Rieke, G. H., Egami, E., Alonso-Herrero, A., Dole, H., Papovich, C., Blaylock, M., Jones, J., Rieke, M., Rigby, J., Barmby, P., Fazio, G. G., Huang, J., \& Martin, C. 2005, ApJ, 630, 82

Pérez-González, P. G., Rieke, G. H., Villar, V., Barro, G., Blaylock, M., Egami, E., Gallego, J., Gil de Paz, A., Pascual, S., Zamorano, J., \& Donley, J. L. 2008, ApJ, 675, 234

Perrotta, F., Baccigalupi, C., Bartelmann, M., De Zotti, G., \& Granato, G. L. 2002, MNRAS, 329, 445

Piovan, L., Tantalo, R., \& Chiosi, C. 2006, MNRAS, 366, 923

Planck Collaboration, Ade, P. A. R., Aghanim, N., Armitage-Caplan, C., Arnaud, M., Ashdown, M., Atrio-Barandela, F., Aumont, J., Baccigalupi, C., Banday, A. J., \& et al. 2013a, ArXiv e-prints

-. 2013b, ArXiv e-prints

Planck Collaboration, Ade, P. A. R., Aghanim, N., Armitage-Caplan, C., Arnaud, M., Ashdown, M., Banday, A. J., \& et al. 2013c, ArXiv e-prints Poglitsch, A., Waelkens, C., Geis, N., Feuchtgruber, H., Vandenbussche, B., Rodriguez, L., Krause, O., Renotte, E., van Hoof, C., Saraceno, P., Cepa, J., Kerschbaum, F., Agnèse, P., Ali, B., Altieri, B., Andreani, P., Augueres, J.-L., Balog, Z., Barl, L., Bauer, O. H., Belbachir, N., Benedettini, M., Billot, N., Boulade, O., Bischof, H., Blommaert, J., Callut, E., Cara, C., Cerulli, R., Cesarsky, D., Contursi, A., Creten, Y., De Meester, W., Doublier, V., Doumayrou, E., Duband, L., Exter, K., Genzel, R., Gillis, J.-M., Grözinger, U., Henning, T., Herreros, J., Huygen, R., Inguscio, M., Jakob, G., Jamar, C., Jean, C., de Jong, J., Katterloher, R., Kiss, C., Klaas, U., Lemke, D., Lutz, D., Madden, S., Marquet, B., Martignac, J., Mazy, A., Merken, P., Montfort, F., Morbidelli, L., Müller, T., Nielbock, M., Okumura, K., Orfei, R., Ottensamer, R., Pezzuto, S., Popesso, P., Putzeys, J., Regibo, S., Reveret, V., Royer, P., Sauvage, M., Schreiber, J., Stegmaier, J., Schmitt, D., Schubert, J., Sturm, E., Thiel, M., Tofani, G., Vavrek, R., Wetzstein, M., Wieprecht, E., \& Wiezorrek, E. 2010, A\&A, 518, L2

Polletta, M., Tajer, M., Maraschi, L., Trinchieri, G., Lonsdale, C. J., Chiappetti, L., Andreon, S., Pierre, M., Le Fèvre, O., Zamorani, G., Maccagni, D., Garcet, O., Surdej, J., Franceschini, A., Alloin, D., Shupe, D. L., Surace, J. A., Fang, F., Rowan-Robinson, M., Smith, H. E., \& Tresse, L. 2007, ApJ, 663, 81

Pope, A., Borys, C., Scott, D., Conselice, C., Dickinson, M., \& Mobasher, B. 2005, MNRAS, 358, 149

Pope, A., Bussmann, R. S., Dey, A., Meger, N., Alexander, D. M., Brodwin, M., Chary, R.-R., Dickinson, M. E., Frayer, D. T., Greve, T. R., Huynh, M., Lin, L., Morrison, G., Scott, D., \& Yan, C.-H. 2008a, ApJ, 689, 127

Pope, A., Chary, R.-R., Alexander, D. M., Armus, L., Dickinson, M., Elbaz, D., Frayer, D., Scott, D., \& Teplitz, H. 2008b, ApJ, 675, 1171

Pope, A., Scott, D., Dickinson, M., Chary, R., Morrison, G., Borys, C., Sajina, A., Alexander, D. M., Daddi, E., Frayer, D., MacDonald, E., \& Stern, D. 2006, MNRAS, 370, 1185

Pope, A., Wagg, J., Frayer, D., Armus, L., Chary, R.-R., Daddi, E., Desai, V., Dickinson, M. E., Elbaz, D., Gabor, J., \& Kirkpatrick, A. 2013, ApJ, 772,92

Popping, G., Caputi, K. I., Somerville, R. S., \& Trager, S. C. 2012, MNRAS, 425, 2386

Popping, G., Somerville, R. S., \& Trager, S. C. 2013, arXiv/1308.6764

Puget, J.-L., Abergel, A., Bernard, J.-P., Boulanger, F., Burton, W. B., Desert, F.-X., \& Hartmann, D. 1996, A\&A, 308, L5

Rangwala, N., Maloney, P. R., Glenn, J., Wilson, C. D., Rykala, A., Isaak, K., Baes, M., Bendo, G. J., Boselli, A., Bradford, C. M., Clements, D. L., Cooray, A., Fulton, T., Imhof, P., Kamenetzky, J., Madden, S. C., Mentuch, E., Sacchi, N., Sauvage, M., Schirm, M. R. P., Smith, M. W. L., Spinoglio, L., \& Wolfire, M. 2011, ApJ, 743, 94

Rawle, T. D., Egami, E., Bussmann, R. S., Gurwell, M., Ivison, R. J., Boone, F., Combes, F., Danielson, A. L. R., Rex, M., Richard, J., Smail, I., Swinbank, A. M., Blain, A. W., Clement, B., Dessauges-Zavadsky, M., Edge, A. C., Fazio, G. G., Jones, T., Kneib, J.-P., Omont, A., Perez-Gonzalez, P. G., Schaerer, D., Valtchanov, I., van der Werf, P. P., Walth, G., Zamojski, M., \& Zemcov, M. 2013, arXiv/1310.4090

Reddy, N., Dickinson, M., Elbaz, D., Morrison, G., Giavalisco, M., Ivison, R., Papovich, C., Scott, D., Buat, V., Burgarella, D., Charmandaris, V., Daddi, E., Magdis, G., Murphy, E., Altieri, B., Aussel, H., Dannerbauer, H., Dasyra, K., Hwang, H. S., Kartaltepe, J., Leiton, R., Magnelli, B., \& Popesso, P. 2012, ApJ, 744, 154

Reddy, N. A. \& Steidel, C. C. 2009, ApJ, 692, 778

Reddy, N. A., Steidel, C. C., Pettini, M., Adelberger, K. L., Shapley, A. E., Erb, D. K., \& Dickinson, M. 2008, ApJS, 175,48

Refregier, A. \& Loeb, A. 1997, ApJ, 478, 476

Reiter, M., Shirley, Y. L., Wu, J., Brogan, C., Wootten, A., \& Tatematsu, K. 2011, ApJS, 195, 1

Riechers, D. A., Capak, P. L., Carilli, C. L., Cox, P., Neri, R., Scoville, N. Z., Schinnerer, E., Bertoldi, F., \& Yan, L. 2010a, ApJ, 720, L131

Riechers, D. A., Carilli, C. L., Maddalena, R. J., Hodge, J., Harris, A. I., Baker, A. J., Walter, F., Wagg, J., Vanden Bout, P. A., Weiß, A., \& Sharon, C. E. 2011a, ApJ, 739, L32

Riechers, D. A., Cooray, A., Omont, A., Neri, R., Harris, A. I., Baker, A. J., Cox, P., Frayer, D. T., Carpenter, J. M., Auld, R., Aussel, H., Beelen, A., Blundell, R., Bock, J., Brisbin, D., Burgarella, D., Chanial, P., Chapman, S. C., Clements, D. L., Conley, A., Dowell, C. D., Eales, S., Farrah, D., Franceschini, A., Gavazzi, R., Glenn, J., Griffin, M., Gurwell, M., Ivison, R. J., Kim, S., Krips, M., Mortier, A. M. J., Oliver, S. J., Page, M. J., Papageorgiou, A., Pearson, C. P., Pérez-Fournon, I., Pohlen, M., Rawlings, J. I., Raymond, G., Rodighiero, G., Roseboom, I. G., Rowan-Robinson, M., Scott, K. S., Seymour, N., Smith, A. J., Symeonidis, M., Tugwell, K. E., Vaccari, M., Vieira, J. D., Vigroux, L., Wang, L., Wardlow, J., \& Wright, G. 2011b, ApJ, 733, L12

Riechers, D. A., Hodge, J., Walter, F., Carilli, C. L., \& Bertoldi, F. 2011c, ApJ, 739, L31

Riechers, D. A., Pope, A., Daddi, E., Armus, L., Carilli, C. L., Walter, F., Hodge, J., Chary, R.-R., Morrison, G. E., Dickinson, M., Dannerbauer, H., \& Elbaz, D. 2013a, ArXiv e-prints

Riechers, D. A., Walter, F., Bertoldi, F., Carilli, C. L., Aravena, M., Neri, R., Cox, P., Weiß, A., \& Menten, K. M. 2009, ApJ, 703 , 1338

Riechers, D. A., Walter, F., Carilli, C. L., \& Bertoldi, F. 2007, ApJ, 671, L13 
Riechers, D. A., Walter, F., Carilli, C. L., Cox, P., Weiss, A., Bertoldi, F., \& Menten, K. M. 2011d, ApJ, 726, 50

Riechers, D. A., Weiß, A., Walter, F., \& Wagg, J. 2010b, ApJ, 725, 1032

Riechers, D. A. et al. 2013b, Nature, 496, 329

Rieke, G. H., Alonso-Herrero, A., Weiner, B. J., Pérez-González, P. G., Blaylock, M., Donley, J. L., \& Marcillac, D. 2009, ApJ, 692, 556

Rieke, G. H., Loken, K., Rieke, M. J., \& Tamblyn, P. 1993, ApJ, 412, 99

Rieke, G. H., Young, E. T., Engelbracht, C. W., Kelly, D. M., Low, F. J., Haller, E. E., Beeman, J. W., Gordon, K. D., Stansberry, J. A., Misselt, K. A., Cadien, J., Morrison, J. E., Rivlis, G., Latter, W. B., Noriega-Crespo, A., Padgett, D. L., Stapelfeldt, K. R., Hines, D. C., Egami, E., Muzerolle, J., Alonso-Herrero, A., Blaylock, M., Dole, H., Hinz, J. L., Le Floc'h, E., Papovich, C., Pérez-González, P. G., Smith, P. S., Su, K. Y. L., Bennett, L., Frayer, D. T., Henderson, D., Lu, N., Masci, F., Pesenson, M., Rebull, L., Rho, J., Keene, J., Stolovy, S., Wachter, S., Wheaton, W., Werner, M. W., \& Richards, P. L. 2004, ApJS, 154, 25

Rigby, J. R., Marcillac, D., Egami, E., Rieke, G. H., Richard, J., Kneib, J.-P., Fadda, D., Willmer, C. N. A., Borys, C., van der Werf, P. P., Pérez-González, P. G., Knudsen, K. K., \& Papovich, C. 2008, ApJ, 675, 262

Rigopoulou, D., Hopwood, R., Magdis, G. E., Thatte, N., Swinyard, B. M., Farrah, D., Huang, J.-S., Alonso-Herrero, A., Bock, J. J., Clements, D., Cooray, A., Griffin, M. J., Oliver, S., Pearson, C., Riechers, D., Scott, D., Smith, A., Vaccari, M., Valtchanov, I., \& Wang, L. 2014, ApJ, 781, L15

Robitaille, T. P. \& Whitney, B. A. 2010, ApJ, 710, L11

Rodighiero, G., Daddi, E., Baronchelli, I., Cimatti, A., Renzini, A., Aussel, H., Popesso, P., Lutz, D., Andreani, P., Berta, S., Cava, A., Elbaz, D., Feltre, A., Fontana, A., Förster Schreiber, N. M., Franceschini, A., Genzel, R., Grazian, A., Gruppioni, C., Ilbert, O., Le Floch, E., Magdis, G., Magliocchetti, M., Magnelli, B., Maiolino, R., McCracken, H., Nordon, R., Poglitsch, A., Santini, P., Pozzi, F., Riguccini, L., Tacconi, L. J., Wuyts, S., \& Zamorani, G. 2011, ApJ, 739, L40

Rodighiero, G. \& Franceschini, A. 2004, A\&A, 419, L55

Rodighiero, G., Vaccari, M., Franceschini, A., Tresse, L., Le Fevre, O., Le Brun, V., Mancini, C., Matute, I., Cimatti, A., Marchetti, L., Ilbert, O., Arnouts, S., Bolzonella, M., Zucca, E., Bardelli, S., Lonsdale, C. J., Shupe, D., Surace, J., Rowan-Robinson, M., Garilli, B., Zamorani, G., Pozzetti, L., Bondi, M., de la Torre, S., Vergani, D., Santini, P., Grazian, A., \& Fontana, A. 2010, A\&A, 515, A8+

Roseboom, I. G., Dunlop, J. S., Cirasuolo, M., Geach, J. E., Smail, I., Halpern, M., van der Werf, P., Almaini, O., Arumugam, V., Asboth, V., Auld, R., Blain, A., Bremer, M. N., Bock, J., Bowler, R., Buitrago, F., Chapin, E., Chapman, S., Chrysostomou, A., Clarke, C., Conley, A., Coppin, K. E. K., Danielson, A. L. R., Farrah, D., Glenn, J., Hatziminaoglou, E., Ibar, E., Ivison, R. J., Jenness, T., van Kampen, E., Karim, A., Mackenzie, T., Marsden, G., Meijerink, R., Michałowski, M. J., Oliver, S. J., Page, M. J., Pearson, E., Scott, D., Simpson, J. M., Smith, D. J. B., Spaans, M., Swinbank, A. M., Symeonidis, M., Targett, T., Valiante, E., Viero, M., Wang, L., Willott, C. J., \& Zemcov, M. 2013, ArXiv e-prints

Roseboom, I. G., Ivison, R. J., Greve, T. R., Amblard, A., Arumugam, V., Auld, R., Aussel, H., Bethermin, M., Blain, A., Bock, J., Boselli, A., Brisbin, D., Buat, V., Burgarella, D., Castro-Rodríguez, N., Cava, A., Chanial, P., Chapin, E., Chapman, S., Clements, D. L., Conley, A., Conversi, L., Cooray, A., Dowell, C. D., Dunlop, J. S., Dwek, E., Eales, S., Elbaz, D., Farrah, D., Franceschini, A., Glenn, J., Griffin, M., Halpern, M., Hatziminaoglou, E., Ibar, E., Isaak, K., Lagache, G., Levenson, L., Lu, N., Madden, S., Maffei, B., Mainetti, G., Marchetti, L., Marsden, G., Morrison, G., Mortier, A. M. J., Nguyen, H. T., O’Halloran, B., Oliver, S. J., Omont, A., Page, M. J., Panuzzo, P., Papageorgiou, A., Pearson, C. P., Pérez-Fournon, I., Pohlen, M., Rawlings, J. I., Raymond, G., Rigopoulou, D., Rizzo, D., Rodighiero, G., Rowan-Robinson, M., Schulz, B., Scott, D., Seymour, N., Shupe, D. L., Smith, A. J., Stevens, J. A., Symeonidis, M., Trichas, M., Tugwell, K. E., Vaccari, M., Valtchanov, I., Vieira, J. D., Viero, M. P., Vigroux, L., Wardlow, J., Wang, L., Wright, G., Xu, C. K., \& Zemcov, M. 2012, MNRAS, 419, 2758

Roseboom, I. G., Oliver, S. J., Kunz, M., Altieri, B., Amblard, A., Arumugam, V., Auld, R., Aussel, H., Babbedge, T., Béthermin, M., Blain, A., Bock, J., Boselli, A., Brisbin, D., Buat, V., Burgarella, D., Castro-Rodríguez, N., Cava, A., Chanial, P., Chapin, E., Clements, D. L., Conley, A., Conversi, L., Cooray, A., Dowell, C. D., Dwek, E., Dye, S., Eales, S., Elbaz, D., Farrah, D., Fox, M., Franceschini, A., Gear, W., Glenn, J., Solares, E. A. G., Griffin, M., Halpern, M., Harwit, M., Hatziminaoglou, E., Huang, J., Ibar, E., Isaak, K., Ivison, R. J., Lagache, G., Levenson, L., Lu, N., Madden, S., Maffei, B., Mainetti, G., Marchetti, L., Marsden, G., Mortier, A. M. J., Nguyen, H. T., O’Halloran, B., Omont, A., Page, M. J., Panuzzo, P., Papageorgiou, A., Patel, H., Pearson, C. P., Pérez-Fournon, I., Pohlen, M., Rawlings, J. I., Raymond, G., Rigopoulou, D., Rizzo, D., Rowan-Robinson, M., Portal, M. S., Schulz, B., Scott, D., Seymour, N., Shupe, D. L., Smith, A. J., Stevens, J. A., Symeonidis, M., Trichas, M., Tugwell, K. E., Vaccari, M., Valtchanov, I., Vieira, J. D., Vigroux, L., Wang, L., Ward, R., Wright, G., Xu, C. K., \& Zemcov, M. 2010, MNRAS, 409, 48

Rosolowsky, E., Pineda, J. E., \& Gao, Y. 2011, MNRAS, 415, 1977

Ross, N. P., Shen, Y., Strauss, M. A., Vanden Berk, D. E., Connolly, A. J., Richards, G. T., Schneider, D. P., Weinberg, D. H., Hall, P. B., Bahcall, N. A., \& Brunner, R. J. 2009, ApJ, 697, 1634

Roussel, H., Sauvage, M., Vigroux, L., \& Bosma, A. 2001, A\&A, 372, 427

Rowan-Robinson, M., Broadhurst, T., Oliver, S. J., Taylor, A. N., Lawrence, A., McMahon, R. G., Lonsdale, C. J., Hacking, P. B., Conrow, T., Saunders, W., Ellis, R. S., Efstathiou, G. P., \& Condon, J. J. 1991, Nature, 351, 719

Rujopakarn, W., Rieke, G. H., Eisenstein, D. J., \& Juneau, S. 2011, ApJ, 726, 93

Saintonge, A., Kauffmann, G., Wang, J., Kramer, C., Tacconi, L. J., Buchbender, C., Catinella, B., Graciá-Carpio, J., Cortese, L., Fabello, S., Fu, J., Genzel, R., Giovanelli, R., Guo, Q., Haynes, M. P., Heckman, T. M., Krumholz, M. R., Lemonias, J., Li, C., Moran, S., Rodriguez-Fernandez, N., Schiminovich, D., Schuster, K., \& Sievers, A. 2011, MNRAS, 415, 61

Saintonge, A., Lutz, D., Genzel, R., Magnelli, B., Nordon, R., Tacconi, L. J., Baker, A. J., Bandara, K., Berta, S., Förster Schreiber, N. M., Poglitsch, A., Sturm, E., Wuyts, E., \& Wuyts, S. 2013, ApJ, 778, 2

Sajina, A., Yan, L., Fadda, D., Dasyra, K., \& Huynh, M. 2012, ApJ, 757, 13

Sajina, A., Yan, L., Lutz, D., Steffen, A., Helou, G., Huynh, M., Frayer, D., Choi, P., Tacconi, L., \& Dasyra, K. 2008, ApJ, 683, 659

Salpeter, E. E. 1955, ApJ, 121, 161

Sanders, D. B., Mazzarella, J. M., Kim, D.-C., Surace, J. A., \& Soifer, B. T. 2003, AJ, 126, 1607

Sanders, D. B. \& Mirabel, I. F. 1985, ApJ, 298, L31

-. 1996, ARA\&A, 34, 749

Sanders, D. B., Scoville, N. Z., Young, J. S., Soifer, B. T., Schloerb, F. P., Rice, W. L., \& Danielson, G. E. 1986, ApJ, 305, L45 
Sanders, D. B., Soifer, B. T., Elias, J. H., Madore, B. F., Matthews, K., Neugebauer, G., \& Scoville, N. Z. 1988, ApJ, 325,74

Sanders, D. B. et al. 2007, ApJS, 172, 86

Sandstrom, K. M., Leroy, A. K., Walter, F., Bolatto, A. D., Croxall, K. V., Draine, B. T., Wilson, C. D., Wolfire, M., Calzetti, D., Kennicutt, R. C., Aniano, G., Donovan Meyer, J., Usero, A., Bigiel, F., Brinks, E., de Blok, W. J. G., Crocker, A., Dale, D., Engelbracht, C. W., Galametz, M., Groves, B., Hunt, L. K., Koda, J., Kreckel, K., Linz, H., Meidt, S., Pellegrini, E., Rix, H.-W., Roussel, H., Schinnerer, E., Schruba, A., Schuster, K.-F., Skibba, R., van der Laan, T., Appleton, P., Armus, L., Brandl, B., Gordon, K., Hinz, J., Krause, O., Montiel, E., Sauvage, M., Schmiedeke, A., Smith, J. D. T., \& Vigroux, L. 2013, ApJ, 777, 5

Santini, P., Maiolino, R., Magnelli, B., Lutz, D., Lamastra, A., Li Causi, G., Eales, S., Andreani, P., Berta, S., Buat, V., Cooray, A., Cresci, G., Daddi, E., Farrah, D., Fontana, A., Franceschini, A., Genzel, R., Granato, G., Grazian, A., Le Floc'h, E., Magdis, G., Magliocchetti, M., Mannucci, F., Menci, N., Nordon, R., Oliver, S., Popesso, P., Pozzi, F., Riguccini, L., Rodighiero, G., Rosario, D. J., Salvato, M., Scott, D., Silva, L., Tacconi, L., Viero, M., Wang, L., Wuyts, S., \& Xu, K. 2013, ArXiv e-prints

Sargent, M. T., Béthermin, M., Daddi, E., \& Elbaz, D. 2012, ApJ, 747, L31

Sargent, M. T., Schinnerer, E., Murphy, E., Carilli, C. L., Helou, G., Aussel, H., Le Floc'h, E., Frayer, D. T., Ilbert, O., Oesch, P., Salvato, M., Smolčić, V., Kartaltepe, J., \& Sanders, D. B. 2010, ApJ, 714, L190

Sargsyan, L., Lebouteiller, V., Weedman, D., Spoon, H., Bernard-Salas, J., Engels, D., Stacey, G., Houck, J., Barry, D., Miles, J., \& Samsonyan, A. 2012, ApJ, 755, 171

Saunders, W., Rowan-Robinson, M., Lawrence, A., Efstathiou, G., Kaiser, N., Ellis, R. S., \& Frenk, C. S. 1990, MNRAS, 242, 318

Scannapieco, C., Wadepuhl, M., Parry, O. H., Navarro, J. F., Jenkins, A., Springel, V., Teyssier, R., Carlson, E., Couchman, H. M. P., Crain, R. A., Dalla Vecchia, C., Frenk, C. S., Kobayashi, C., Monaco, P., Murante, G., Okamoto, T., Quinn, T., Schaye, J., Stinson, G. S., Theuns, T., Wadsley, J., White, S. D. M., \& Woods, R. 2012, MNRAS, 423, 1726

Schartmann, M., Meisenheimer, K., Camenzind, M., Wolf, S., Tristram, K. R. W., \& Henning, T. 2008, A\&A, 482, 67

Schenck, D. E., Shirley, Y. L., Reiter, M., \& Juneau, S. 2011, AJ, 142, 94

Schleicher, D. R. G. \& Beck, R. 2013, A\&A, 556, A142

Schmidt, M. 1959, ApJ, 129, 243

Schruba, A., Leroy, A. K., Walter, F., Bigiel, F., Brinks, E., de Blok, W. J. G., Dumas, G., Kramer, C., Rosolowsky, E., Sandstrom, K., Schuster, K., Usero, A., Weiss, A., \& Wiesemeyer, H. 2011, AJ, 142, 37

Schruba, A., Leroy, A. K., Walter, F., Bigiel, F., Brinks, E., de Blok, W. J. G., Kramer, C., Rosolowsky, E., Sandstrom, K., Schuster, K., Usero, A., Weiss, A., \& Wiesemeyer, H. 2012, AJ, 143, 138

Scott, K. S., Austermann, J. E., Perera, T. A., Wilson, G. W., Aretxaga, I., Bock, J. J., Hughes, D. H., Kang, Y., Kim, S., Mauskopf, P. D., Sanders, D. B., Scoville, N., \& Yun, M. S. 2008, MNRAS, 385, 2225

Scott, K. S., Lupu, R. E., Aguirre, J. E., Auld, R., Aussel, H., Baker, A. J., Beelen, A., Bock, J., Bradford, C. M., Brisbin, D., Burgarella, D., Carpenter, J. M., Chanial, P., Chapman, S. C., Clements, D. L., Conley, A., Cooray, A., Cox, P., Dowell, C. D., Eales, S., Farrah, D., Franceschini, A., Frayer, D. T., Gavazzi, R., Glenn, J., Griffin, M., Harris, A. I., Ibar, E., Ivison, R. J., Kamenetzky, J., Kim, S., Krips, M., Maloney, P. R., Matsuhara, H., Mortier, A. M. J., Murphy, E. J., Naylor, B. J., Neri, R., Nguyen, H. T., Oliver, S. J., Omont, A., Page, M. J., Papageorgiou, A., Pearson, C. P., Pérez-Fournon, I., Pohlen, M., Rawlings, J. I., Raymond, G., Riechers, D., Rodighiero, G., Roseboom, I. G., Rowan-Robinson, M., Scott, D., Seymour, N., Smith, A. J., Symeonidis, M., Tugwell, K. E., Vaccari, M., Vieira, J. D., Vigroux, L., Wang, L., Wright, G., \& Zmuidzinas, J. 2011, ApJ, 733, 29

Scott, K. S., Wilson, G. W., Aretxaga, I., Austermann, J. E., Chapin, E. L., Dunlop, J. S., Ezawa, H., Halpern, M., Hatsukade, B., Hughes, D. H., Kawabe, R., Kim, S., Kohno, K., Lowenthal, J. D., Montaña, A., Nakanishi, K., Oshima, T., Sanders, D., Scott, D., Scoville, N., Tamura, Y., Welch, D., Yun, M. S., \& Zeballos, M. 2012, MNRAS, 423, 575

Scott, K. S., Yun, M. S., Wilson, G. W., Austermann, J. E., Aguilar, E., Aretxaga, I., Ezawa, H., Ferrusca, D., Hatsukade, B., Hughes, D. H., Iono, D., Giavalisco, M., Kawabe, R., Kohno, K., Mauskopf, P. D., Oshima, T., Perera, T. A., Rand, J., Tamura, Y., Tosaki, T., Velazquez, M., Williams, C. C., \& Zeballos, M. 2010, MNRAS, 405, 2260

Scott, S. E., Dunlop, J. S., \& Serjeant, S. 2006, MNRAS, 370, 1057

Scott, S. E., Fox, M. J., Dunlop, J. S., Serjeant, S., Peacock, J. A., Ivison, R. J., Oliver, S., Mann, R. G., Lawrence, A., Efstathiou, A., RowanRobinson, M., Hughes, D. H., Archibald, E. N., Blain, A., \& Longair, M. 2002, MNRAS, 331, 817

Scoville, N., Aussel, H., Brusa, M., Capak, P., Carollo, C. M., Elvis, M., Giavalisco, M., Guzzo, L., Hasinger, G., Impey, C., Kneib, J., LeFevre, O., Lilly, S. J., Mobasher, B., Renzini, A., Rich, R. M., Sanders, D. B., Schinnerer, E., Schminovich, D., Shopbell, P., Taniguchi, Y., \& Tyson, N. D. 2007, ApJS, 172, 1

Scoville, N. Z. 2012, ArXiv e-prints

Scoville, N. Z. \& Kwan, J. 1976, ApJ, 206, 718

Scoville, N. Z. et al. 2013, ArXiv e-prints

Sedgwick, C., Serjeant, S., Pearson, C., Matsuura, S., Shirahata, M., Oyabu, S., Goto, T., Matsuhara, H., Clements, D. L., Negrello, M., \& White, G. J. 2011, MNRAS, 416, 1862

Serjeant, S. 2012, MNRAS, 424, 2429

Serjeant, S., Dye, S., Mortier, A., Peacock, J., Egami, E., Cirasuolo, M., Rieke, G., Borys, C., Chapman, S., Clements, D., Coppin, K., Dunlop, J., Eales, S., Farrah, D., Halpern, M., Mauskopf, P., Pope, A., Rowan-Robinson, M., Scott, D., Smail, I., \& Vaccari, M. 2008, MNRAS, 386,1907 Shang, C., Haiman, Z., Knox, L., \& Oh, S. P. 2012, MNRAS, 421, 2832

Shapiro, K. L., Genzel, R., Förster Schreiber, N. M., Tacconi, L. J., Bouché, N., Cresci, G., Davies, R., Eisenhauer, F., Johansson, P. H., Krajnović, D., Lutz, D., Naab, T., Arimoto, N., Arribas, S., Cimatti, A., Colina, L., Daddi, E., Daigle, O., Erb, D., Hernandez, O., Kong, X., Mignoli, M., Onodera, M., Renzini, A., Shapley, A., \& Steidel, C. 2008, ApJ, 682, 231

Shapley, A. E., Steidel, C. C., Pettini, M., \& Adelberger, K. L. 2003, ApJ, 588, 65

Sharon, C. E., Baker, A. J., Harris, A. I., \& Thomson, A. P. 2013, ApJ, 765, 6

Shetty, R., Glover, S. C., Dullemond, C. P., \& Klessen, R. S. 2011a, MNRAS, 412, 1686

Shetty, R., Kauffmann, J., Schnee, S., \& Goodman, A. A. 2009a, ApJ, 696, 676 
Shetty, R., Kauffmann, J., Schnee, S., Goodman, A. A., \& Ercolano, B. 2009b, ApJ, 696, 2234

Shetty, R., Kelly, B. C., \& Bigiel, F. 2013a, MNRAS, 430, 288

Shetty, R., Kelly, B. C., Rahman, N., Bigiel, F., Bolatto, A. D., Clark, P. C., Klessen, R. S., \& Konstandin, L. K. 2013b, MNRAS

Shetty, R. et al. 2011b, MNRAS, 415, 3253

Shimizu, I., Yoshida, N., \& Okamoto, T. 2012, MNRAS, 427, 2866

Shirley, Y. L., Evans, II, N. J., Young, K. E., Knez, C., \& Jaffe, D. T. 2003, ApJS, 149, 375

Siebenmorgen, R. \& Krügel, E. 2007, A\&A, 461, 445

Silva, L., Granato, G. L., Bressan, A., \& Danese, L. 1998, ApJ, 509, 103

Simpson, C. \& Eisenhardt, P. 1999, PASP, 111, 691

Simpson, J., Swinbank, M., Smail, I., Alexander, D., Brandt, N., Bertoldi, F., de Breuck, C., Chapman, S., Coppin, K., da Cunha, E., Danielson, A., Dannerbauer, H., Greve, T., Hodge, J., Ivison, R., Karim, A., Knudsen, K., Poggianti, B., Schinnerer, E., Thomson, A., Walter, F., Wardlow, J., Weiss, A., \& van der Werf, P. 2013, ArXiv e-prints

Siringo, G., Kreysa, E., De Breuck, C., Kovacs, A., Lundgren, A., Schuller, F., Stanke, T., Weiss, A., Guesten, R., Jethava, N., May, T., Menten, K. M., Meyer, H.-G., Starkloff, M., \& Zakosarenko, V. 2010, The Messenger, 139, 20

Siringo, G., Kreysa, E., Kovács, A., Schuller, F., Weiß, A., Esch, W., Gemünd, H.-P., Jethava, N., Lundershausen, G., Colin, A., Güsten, R., Menten, K. M., Beelen, A., Bertoldi, F., Beeman, J. W., \& Haller, E. E. 2009, A\&A, 497, 945

Smail, I., Chapman, S. C., Blain, A. W., \& Ivison, R. J. 2004, ApJ, 616, 71

Smail, I., Ivison, R. J., \& Blain, A. W. 1997, ApJ, 490, L5+

Smail, I., Ivison, R. J., Blain, A. W., \& Kneib, J. 2002, MNRAS, 331, 495

Smail, I., Ivison, R. J., Blain, A. W., \& Kneib, J.-P. 1998, ApJ, 507, L21

Smail, I., Ivison, R. J., Owen, F. N., Blain, A. W., \& Kneib, J.-P. 2000, ApJ, 528, 612

Smail, I., Swinbank, A. M., Ivison, R. J., \& Ibar, E. 2011, MNRAS, 414, L95

Smith, A. J., Wang, L., Oliver, S. J., Auld, R., Bock, J., Brisbin, D., Burgarella, D., Chanial, P., Chapin, E., Clements, D. L., Conversi, L., Cooray, A., Dowell, C. D., Eales, S., Farrah, D., Franceschini, A., Glenn, J., Griffin, M., Ivison, R. J., Mortier, A. M. J., Page, M. J., Papageorgiou, A., Pearson, C. P., Pérez-Fournon, I., Pohlen, M., Rawlings, J. I., Raymond, G., Rodighiero, G., Roseboom, I. G., Rowan-Robinson, M., Savage, R., Scott, D., Seymour, N., Symeonidis, M., Tugwell, K. E., Vaccari, M., Valtchanov, I., Vigroux, L., Ward, R., Wright, G., \& Zemcov, M. 2012, MNRAS, 419, 377

Smith, C. H., Aitken, D. K., \& Roche, P. F. 1989, MNRAS, 241, 425

Smith, D. J. B., Dunne, L., Maddox, S. J., Eales, S., Bonfield, D. G., Jarvis, M. J., Sutherland, W., Fleuren, S., Rigby, E. E., Thompson, M. A., Baldry, I. K., Bamford, S., Buttiglione, S., Cava, A., Clements, D., Cooray, A., Croom, S., Dariush, A., de Zotti, G., Driver, S. P., Dunlop, J. S., Fritz, J., Hill, D. T., Hopkins, A., Hopwood, R., Ibar, E., Ivison, R. J., Jones, D. H., Kelvin, L., Leeuw, L., Liske, J., Loveday, J., Madore, B. F., Norberg, P., Panuzzo, P., Pascale, E., Pohlen, M., Popescu, C. C., Prescott, M., Robotham, A., Rodighiero, G., Scott, D., Seibert, M., Sharp, R., Temi, P., Tuffs, R. J., van der Werf, P., \& van Kampen, E. 2011, MNRAS, 416, 857

Smolčić, V., Aravena, M., Navarrete, F., Schinnerer, E., Riechers, D. A., Bertoldi, F., Feruglio, C., Finoguenov, A., Salvato, M., Sargent, M., McCracken, H. J., Albrecht, M., Karim, A., Capak, P., Carilli, C. L., Cappelluti, N., Elvis, M., Ilbert, O., Kartaltepe, J., Lilly, S., Sanders, D., Sheth, K., Scoville, N. Z., \& Taniguchi, Y. 2012, A\&A, 548, A4

Snyder, G. F., Hayward, C. C., Sajina, A., Jonsson, P., Cox, T. J., Hernquist, L., Hopkins, P. F., \& Yan, L. 2013, ApJ, 768,168

Soifer, B. T., Rowan-Robinson, M., Houck, J. R., de Jong, T., Neugebauer, G., Aumann, H. H., Beichman, C. A., Boggess, N., Clegg, P. E., Emerson, J. P., Gillett, F. C., Habing, H. J., Hauser, M. G., Low, F. J., Miley, G., \& Young, E. 1984, ApJ, 278, L71

Solomon, P., Vanden Bout, P., Carilli, C., \& Guelin, M. 2003, Nature, 426, 636

Solomon, P. M., Downes, D., \& Radford, S. J. E. 1992, ApJ, 387, L55

Solomon, P. M., Rivolo, A. R., Barrett, J., \& Yahil, A. 1987, ApJ, 319, 730

Solomon, P. M. \& Sage, L. J. 1988, ApJ, 334, 613

Solomon, P. M. \& Vanden Bout, P. A. 2005, ARA\&A, 43, 677

Somerville, R. S., Gilmore, R. C., Primack, J. R., \& Domínguez, A. 2012, MNRAS, 423, 1992

Somerville, R. S. \& Primack, J. R. 1999, MNRAS, 310, 1087

Song, Y.-S., Cooray, A., Knox, L., \& Zaldarriaga, M. 2003, ApJ, 590, 664

Spiniello, C., Koopmans, L. V. E., Trager, S. C., Czoske, O., \& Treu, T. 2011, MNRAS, 417, 3000

Spiniello, C., Trager, S. C., Koopmans, L. V. E., \& Chen, Y. P. 2012, ApJ, 753, L32

Springel, V. 2005, MNRAS, 364, 1105

- 2010, MNRAS, 401, 791

Springel, V. \& Hernquist, L. 2005, ApJ, 622, L9

Stacey, G. J., Hailey-Dunsheath, S., Ferkinhoff, C., Nikola, T., Parshley, S. C., Benford, D. J., Staguhn, J. G., \& Fiolet, N. 2010, ApJ, 724,957

Staguhn, J. G., Benford, D. J., Allen, C. A., Moseley, S. H., Sharp, E. H., Ames, T. J., Brunswig, W., Chuss, D. T., Dwek, E., Maher, S. F., Marx, C. T., Miller, T. M., Navarro, S., \& Wollack, E. J. 2006, in Society of Photo-Optical Instrumentation Engineers (SPIE) Conference Series, Vol. 6275, Society of Photo-Optical Instrumentation Engineers (SPIE) Conference Series

Staguhn, J. G., Benford, D. J., Fixsen, D. J., Hilton, G., Irwin, K. D., Jhabvala, C. A., Kovacs, A., Leclercq, S., Maher, S. F., Miller, T. M., Moseley, S. H., Sharp, E. H., \& Wollack, E. J. 2012, in Society of Photo-Optical Instrumentation Engineers (SPIE) Conference Series, Vol. 8452, Society of Photo-Optical Instrumentation Engineers (SPIE) Conference Series

Staguhn, J. G. et al. 2013, ArXiv e-prints

Staniszewski, Z. et al. 2009, ApJ, 701, 32

Stark, D. P., Ellis, R. S., Bunker, A., Bundy, K., Targett, T., Benson, A., \& Lacy, M. 2009, ApJ, 697, 1493

Stark, D. P., Schenker, M. A., Ellis, R., Robertson, B., McLure, R., \& Dunlop, J. 2013, ApJ, 763, 129

Stern, D., Eisenhardt, P., Gorjian, V., Kochanek, C. S., Caldwell, N., Eisenstein, D., Brodwin, M., Brown, M. J. I., Cool, R., Dey, A., Green, P., Jannuzi, B. T., Murray, S. S., Pahre, M. A., \& Willner, S. P. 2005, ApJ, 631, 163 
Stolte, A., Brandner, W., Grebel, E. K., Lenzen, R., \& Lagrange, A.-M. 2005, ApJ, 628, L113

Strong, A. W., Moskalenko, I. V., Reimer, O., Digel, S., \& Diehl, R. 2004, A\&A, 422, L47

Sutherland, W. \& Saunders, W. 1992, MNRAS, 259, 413

Swetz, D. S., Ade, P. A. R., Amiri, M., Appel, J. W., Battistelli, E. S., Burger, B., Chervenak, J., Devlin, M. J., Dicker, S. R., Doriese, W. B., Dünner, R., Essinger-Hileman, T., Fisher, R. P., Fowler, J. W., Halpern, M., Hasselfield, M., Hilton, G. C., Hincks, A. D., Irwin, K. D., Jarosik, N., Kaul, M., Klein, J., Lau, J. M., Limon, M., Marriage, T. A., Marsden, D., Martocci, K., Mauskopf, P., Moseley, H., Netterfield, C. B., Niemack, M. D., Nolta, M. R., Page, L. A., Parker, L., Staggs, S. T., Stryzak, O., Switzer, E. R., Thornton, R., Tucker, C., Wollack, E., \& Zhao, Y. 2011, ApJS, 194, 41

Swinbank, A. M., Chapman, S. C., Smail, I., Lindner, C., Borys, C., Blain, A. W., Ivison, R. J., \& Lewis, G. F. 2006, MNRAS, 371 , 465

Swinbank, A. M., Karim, A., Smail, I., Hodge, J., Walter, F., Bertoldi, F., Biggs, A. D., de Breuck, C., Chapman, S. C., Coppin, K. E. K., Cox, P., Danielson, A. L. R., Dannerbauer, H., Ivison, R. J., Greve, T. R., Knudsen, K. K., Menten, K. M., Simpson, J. M., Schinnerer, E., Wardlow, J. L., Weiß, A., \& van der Werf, P. 2012, MNRAS, 427, 1066

Swinbank, A. M., Lacey, C. G., Smail, I., Baugh, C. M., Frenk, C. S., Blain, A. W., Chapman, S. C., Coppin, K. E. K., Ivison, R. J., Gonzalez, J. E., \& Hainline, L. J. 2008, MNRAS, 391, 420

Swinbank, A. M., Papadopoulos, P. P., Cox, P., Krips, M., Ivison, R. J., Smail, I., Thomson, A. P., Neri, R., Richard, J., \& Ebeling, H. 2011, ApJ, 742,11

Swinbank, A. M., Smail, I., Chapman, S. C., Blain, A. W., Ivison, R. J., \& Keel, W. C. 2004, ApJ, 617, 64

Swinbank, A. M., Smail, I., Longmore, S., Harris, A. I., Baker, A. J., De Breuck, C., Richard, J., Edge, A. C., Ivison, R. J., Blundell, R., Coppin, K. E. K., Cox, P., Gurwell, M., Hainline, L. J., Krips, M., Lundgren, A., Neri, R., Siana, B., Siringo, G., Stark, D. P., Wilner, D., \& Younger, J. D. 2010, Nature, 464, 733

Swinbank, M., Simpson, J., Smail, I., Harrison, C., Hodge, J., Karim, A., Walter, F., Alexander, D., Brandt, N., de Breuck, C., da Cunha, E., Chapman, S., Coppin, K., Danielson, A., Dannerbauer, H., Decarli, R., Greve, T., Ivison, R., Knudsen, K., Lagos, C., Schinnerer, E., Thomson, A., Wardlow, J., Weiss, A., \& van der Werf, P. 2013, ArXiv e-prints

Symeonidis, M. et al. 2013, MNRAS, 431, 2317

Tabatabaei, F., Krause, M., \& Beck, R. 2005, Astronomische Nachrichten, 326, 532

Tabatabaei, F. S., Beck, R., Krause, M., Berkhuijsen, E. M., Gehrz, R., Gordon, K. D., Hinz, J. L., Humphreys, R., McQuinn, K., Polomski, E., Rieke, G. H., \& Woodward, C. E. 2007, A\&A, 466, 509

Tabatabaei, F. S., Braine, J., Xilouris, E. M., Kramer, C., Boquien, M., Combes, F., Henkel, C., Relano, M., Verley, S., Gratier, P., Israel, F., Wiedner, M. C., Roellig, M., Schuster, K. F., \& van derWerf, P. 2013, ArXiv e-prints

Tacconi, L. J., Genzel, R., Neri, R., Cox, P., Cooper, M. C., Shapiro, K., Bolatto, A., Bouché, N., Bournaud, F., Burkert, A., Combes, F., Comerford, J., Davis, M., Schreiber, N. M. F., Garcia-Burillo, S., Gracia-Carpio, J., Lutz, D., Naab, T., Omont, A., Shapley, A., Sternberg, A., \& Weiner, B. 2010, Nature, 463, 781

Tacconi, L. J., Neri, R., Chapman, S. C., Genzel, R., Smail, I., Ivison, R. J., Bertoldi, F., Blain, A., Cox, P., Greve, T., \& Omont, A. 2006, ApJ, 640, 228

Tacconi, L. J., Neri, R., Genzel, R., Combes, F., Bolatto, A., Cooper, M. C., Wuyts, S., Bournaud, F., Burkert, A., Comerford, J., Cox, P., Davis, M., Förster Schreiber, N. M., García-Burillo, S., Gracia-Carpio, J., Lutz, D., Naab, T., Newman, S., Omont, A., Saintonge, A., Shapiro Griffin, K., Shapley, A., Sternberg, A., \& Weiner, B. 2013, ApJ, 768, 74

Tacconi, L. J. \& Young, J. S. 1987, ApJ, 322, 681

Tacconi, L. J. et al. 2008, ApJ, 680, 246

Takagi, T., Ohyama, Y., Goto, T., Matsuhara, H., Oyabu, S., Wada, T., Pearson, C. P., Lee, H. M., Im, M., Lee, M. G., Shim, H., Hanami, H., Ishigaki, T., Imai, K., White, G. J., Serjeant, S., \& Malkan, M. 2010, A\&A, 514, A5

Takagi, T., Vansevicius, V., \& Arimoto, N. 2003, PASJ, 55, 385

Tan, Q., Daddi, E., Sargent, M., Magdis, G., Hodge, J., Béthermin, M., Bournaud, F., Carilli, C., Dannerbauer, H., Dickinson, M., Elbaz, D., Gao, Y., Morrison, G., Owen, F., Pannella, M., Riechers, D., \& Walter, F. 2013, ApJ, 776, L24

Targett, T. A., Dunlop, J. S., Cirasuolo, M., McLure, R. J., Bruce, V. A., Fontana, A., Galametz, A., Paris, D., Davé, R., Dekel, A., Faber, S. M., Ferguson, H. C., Grogin, N. A., Kartaltepe, J. S., Kocevski, D. D., Koekemoer, A. M., Kurczynski, P., Lai, K., \& Lotz, J. 2013, MNRAS, 432, 2012

Teyssier, R. 2002, A\&A, 385, 337

Teyssier, R., Chapon, D., \& Bournaud, F. 2010, ApJ, 720, L149

Thacker, C., Cooray, A., Smidt, J., De Bernardis, F., Mitchell-Wynne, K., Amblard, A., Auld, R., Baes, M., Clements, D. L., Dariush, A., De Zotti, G., Dunne, L., Eales, S., Hopwood, R., Hoyos, C., Ibar, E., Jarvis, M., Maddox, S., Michałowski, M. J., Pascale, E., Scott, D., Serjeant, S., Smith, M. W. L., Valiante, E., \& van der Werf, P. 2013, ApJ, 768, 58

The Enzo Collaboration, Bryan, G. L., Norman, M. L., O’Shea, B. W., Abel, T., Wise, J. H., Turk, M. J., Reynolds, D. R., Collins, D. C., Wang, P., Skillman, S. W., Smith, B., Harkness, R. P., Bordner, J., Kim, J.-h., Kuhlen, M., Xu, H., Goldbaum, N., Hummels, C., Kritsuk, A. G., Tasker, E., Skory, S., Simpson, C. M., Hahn, O., Oishi, J. S., So, G. C., Zhao, F., Cen, R., \& Li, Y. 2013, arXiv/1307.2265

Thomas, D., Maraston, C., Bender, R., \& Mendes de Oliveira, C. 2005, ApJ, 621, 673

Thompson, T. A., Quataert, E., \& Murray, N. 2005, ApJ, 630, 167

Thompson, T. A., Quataert, E., \& Waxman, E. 2007, ApJ, 654, 219

Thomson, A. P., Ivison, R. J., Smail, I., Swinbank, A. M., Weiss, A., Kneib, J.-P., Papadopoulos, P. P., Baker, A. J., Sharon, C. E., \& van Moorsel, G. A. 2012, MNRAS, 425, 2203

Tortora, C., Romanowsky, A. J., \& Napolitano, N. R. 2013, ApJ, 765, 8

Treister, E., Urry, C. M., Schawinski, K., Cardamone, C. N., \& Sanders, D. B. 2010, ApJ, 722, L238

Treister, E., Urry, C. M., \& Virani, S. 2009, ApJ, 696, 110

Treu, T., Auger, M. W., Koopmans, L. V. E., Gavazzi, R., Marshall, P. J., \& Bolton, A. S. 2010, ApJ, 709, 1195

Tsai, C.-W., Eisenhardt, P., Wu, J., Bridge, C., et al. 2013, in Astronomical Society of the Pacific Conference Series, Vol. 477, Astronomical Society 
of the Pacific Conference Series, ed. W.-H. Sun, C. K. Xu, N. Z. Scoville, \& D. B. Sanders

U, V., Sanders, D. B., Mazzarella, J. M., Evans, A. S., Howell, J. H., Surace, J. A., Armus, L., Iwasawa, K., Kim, D.-C., Casey, C. M., Vavilkin, T., Dufault, M., Larson, K. L., Barnes, J. E., Chan, B. H. P., Frayer, D. T., Haan, S., Inami, H., Ishida, C. M., Kartaltepe, J. S., Melbourne, J. L., \& Petric, A. O. 2012, ApJS, 203, 9

Valiante, E., Lutz, D., Sturm, E., Genzel, R., Tacconi, L. J., Lehnert, M. D., \& Baker, A. J. 2007, ApJ, 660, 1060

Valtchanov, I., Virdee, J., Ivison, R. J., Swinyard, B., van der Werf, P., Rigopoulou, D., da Cunha, E., Lupu, R., Benford, D. J., Riechers, D., Smail, I., Jarvis, M., Pearson, C., Gomez, H., Hopwood, R., Altieri, B., Birkinshaw, M., Coia, D., Conversi, L., Cooray, A., de Zotti, G., Dunne, L., Frayer, D., Leeuw, L., Marston, A., Negrello, M., Portal, M. S., Scott, D., Thompson, M. A., Vaccari, M., Baes, M., Clements, D., Michałowski, M. J., Dannerbauer, H., Serjeant, S., Auld, R., Buttiglione, S., Cava, A., Dariush, A., Dye, S., Eales, S., Fritz, J., Ibar, E., Maddox, S., Pascale, E., Pohlen, M., Rigby, E., Rodighiero, G., Smith, D. J. B., Temi, P., Carpenter, J., Bolatto, A., Gurwell, M., \& Vieira, J. D. 2011, MNRAS, 415, 3473

van der Kruit, P.C. 1971, A\&A, 15, 110

-. 1973, A\&A, 29, 263

van der Werf, P. P. et al. 2010, A\&A, 518, L42

van Dokkum, P. G. 2008, ApJ, 674, 29

van Dokkum, P. G. \& Conroy, C. 2010, Nature, 468, 940

-. 2012, ApJ, 760, 70

van Engelen, A., Keisler, R., Zahn, O., Aird, K. A., Benson, B. A., Bleem, L. E., Carlstrom, J. E., Chang, C. L., Cho, H. M., Crawford, T. M., Crites, A. T., de Haan, T., Dobbs, M. A., Dudley, J., George, E. M., Halverson, N. W., Holder, G. P., Holzapfel, W. L., Hoover, S., Hou, Z., Hrubes, J. D., Joy, M., Knox, L., Lee, A. T., Leitch, E. M., Lueker, M., Luong-Van, D., McMahon, J. J., Mehl, J., Meyer, S. S., Millea, M., Mohr, J. J., Montroy, T. E., Natoli, T., Padin, S., Plagge, T., Pryke, C., Reichardt, C. L., Ruhl, J. E., Sayre, J. T., Schaffer, K. K., Shaw, L., Shirokoff, E., Spieler, H. G., Staniszewski, Z., Stark, A. A., Story, K., Vanderlinde, K., Vieira, J. D., \& Williamson, R. 2012, ApJ, 756, 142

van Kampen, E., Percival, W. J., Crawford, M., Dunlop, J. S., Scott, S. E., Bevis, N., Oliver, S., Pearce, F., Kay, S. T., Gaztañaga, E., Hughes, D. H., \& Aretxaga, I. 2005, MNRAS, 359, 469

van Kampen, E., Smith, D. J. B., Maddox, S., Hopkins, A. M., Valtchanov, I., Peacock, J. A., Michałowski, M. J., Norberg, P., Eales, S., Dunne, L., Liske, J., Baes, M., Scott, D., Rigby, E., Robotham, A., van der Werf, P., Ibar, E., Jarvis, M. J., Loveday, J., Auld, R., Baldry, I. K., Bamford, S., Cameron, E., Croom, S., Buttiglione, S., Cava, A., Cooray, A., Driver, S., Dunlop, J. S., Dariush, A., Fritz, J., Ivison, R. J., Pascale, E., Pohlen, M., Rodighiero, G., Temi, P., Bonfield, D. G., Hill, D., Jones, D. H., Kelvin, L., Parkinson, H., Prescott, M., Sharp, R., de Zotti, G., Serjeant, S., Popescu, C. C., \& Tuffs, R. J. 2012, MNRAS, 426, 3455

Veilleux, S., Kim, D.-C., \& Sanders, D. B. 2002, ApJS, 143, 315

Veilleux, S., Sanders, D. B., \& Kim, D.-C. 1997, ApJ, 484, 92

-. 1999, ApJ, 522, 139

Venemans, B. P., McMahon, R. G., Walter, F., Decarli, R., Cox, P., Neri, R., Hewett, P., Mortlock, D. J., Simpson, C., \& Warren, S. J. 2012, ApJ, 751, L25

Veneziani, M., Ade, P. A. R., Bock, J. J., Boscaleri, A., Crill, B. P., de Bernardis, P., De Gasperis, G., de Oliveira-Costa, A., De Troia, G., Di Stefano, G., Ganga, K. M., Jones, W. C., Kisner, T. S., Lange, A. E., MacTavish, C. J., Masi, S., Mauskopf, P. D., Montroy, T. E., Natoli, P., Netterfield, C. B., Pascale, E., Piacentini, F., Pietrobon, D., Polenta, G., Ricciardi, S., Romeo, G., \& Ruhl, J. E. 2010, ApJ, 713, 959

Vernet, J. \& Cimatti, A. 2001, A\&A, 380, 409

Vieira, J. D., Crawford, T. M., Switzer, E. R., Ade, P. A. R., Aird, K. A., Ashby, M. L. N., Benson, B. A., Bleem, L. E., Brodwin, M., Carlstrom, J. E., Chang, C. L., Cho, H.-M., Crites, A. T., de Haan, T., Dobbs, M. A., Everett, W., George, E. M., Gladders, M., Hall, N. R., Halverson, N. W., High, F. W., Holder, G. P., Holzapfel, W. L., Hrubes, J. D., Joy, M., Keisler, R., Knox, L., Lee, A. T., Leitch, E. M., Lueker, M., Marrone, D. P., McIntyre, V., McMahon, J. J., Mehl, J., Meyer, S. S., Mohr, J. J., Montroy, T. E., Padin, S., Plagge, T., Pryke, C., Reichardt, C. L., Ruhl, J. E., Schaffer, K. K., Shaw, L., Shirokoff, E., Spieler, H. G., Stalder, B., Staniszewski, Z., Stark, A. A., Vanderlinde, K., Walsh, W., Williamson, R., Yang, Y., Zahn, O., \& Zenteno, A. 2010, ApJ, 719, 763

Vieira, J. D., Marrone, D. P., Chapman, S. C., De Breuck, C., Hezaveh, Y. D., Wei $\beta$, A., Aguirre, J. E., Aird, K. A., Aravena, M., Ashby, M. L. N., Bayliss, M., Benson, B. A., Biggs, A. D., Bleem, L. E., Bock, J. J., Bothwell, M., Bradford, C. M., Brodwin, M., Carlstrom, J. E., Chang, C. L., Crawford, T. M., Crites, A. T., de Haan, T., Dobbs, M. A., Fomalont, E. B., Fassnacht, C. D., George, E. M., Gladders, M. D., Gonzalez, A. H., Greve, T. R., Gullberg, B., Halverson, N. W., High, F. W., Holder, G. P., Holzapfel, W. L., Hoover, S., Hrubes, J. D., Hunter, T. R., Keisler, R., Lee, A. T., Leitch, E. M., Lueker, M., Luong-van, D., Malkan, M., McIntyre, V., McMahon, J. J., Mehl, J., Menten, K. M., Meyer, S. S., Mocanu, L. M., Murphy, E. J., Natoli, T., Padin, S., Plagge, T., Reichardt, C. L., Rest, A., Ruel, J., Ruhl, J. E., Sharon, K., Schaffer, K. K., Shaw, L., Shirokoff, E., Spilker, J. S., Stalder, B., Staniszewski, Z., Stark, A. A., Story, K., Vanderlinde, K., Welikala, N., \& Williamson, R. 2013, Nature, 495, 344

Viero, M. P., Ade, P. A. R., Bock, J. J., Chapin, E. L., Devlin, M. J., Griffin, M., Gundersen, J. O., Halpern, M., Hargrave, P. C., Hughes, D. H., Klein, J., MacTavish, C. J., Marsden, G., Martin, P. G., Mauskopf, P., Moncelsi, L., Negrello, M., Netterfield, C. B., Olmi, L., Pascale, E., Patanchon, G., Rex, M., Scott, D., Semisch, C., Thomas, N., Truch, M. D. P., Tucker, C., Tucker, G. S., \& Wiebe, D. V. 2009 , ApJ, 707,1766

Viero, M. P., Moncelsi, L., Mentuch, E., Buitrago, F., Bauer, A. E., Chapin, E. L., Conselice, C. J., Devlin, M. J., Halpern, M., Marsden, G., Netterfield, C. B., Pascale, E., Pérez-González, P. G., Rex, M., Scott, D., Smith, M. W. L., Truch, M. D. P., Trujillo, I., \& Wiebe, D. V. 2012, MNRAS, 421, 2161

Viero, M. P., Wang, L., Zemcov, M., Addison, G., Amblard, A., Arumugam, V., Aussel, H., Béthermin, M., Bock, J., Boselli, A., Buat, V., Burgarella, D., Casey, C. M., Clements, D. L., Conley, A., Conversi, L., Cooray, A., De Zotti, G., Dowell, C. D., Farrah, D., Franceschini, A., Glenn, J., Griffin, M., Hatziminaoglou, E., Heinis, S., Ibar, E., Ivison, R. J., Lagache, G., Levenson, L., Marchetti, L., Marsden, G., Nguyen, H. T., O’Halloran, B., Oliver, S. J., Omont, A., Page, M. J., Papageorgiou, A., Pearson, C. P., Pérez-Fournon, I., Pohlen, M., Rigopoulou, D., Roseboom, I. G., Rowan-Robinson, M., Schulz, B., Scott, D., Seymour, N., Shupe, D. L., Smith, A. J., Symeonidis, M., Vaccari, M., Valtchanov, I., Vieira, J. D., Wardlow, J., \& Xu, C. K. 2013a, ApJ, 772, 77

Viero, M. P. et al. 2013b, ApJ, 779, 32 
Volk, H. 1989, A\&A, 218, 67

Wadsley, J. W., Stadel, J., \& Quinn, T. 2004, New Astronomy, 9, 137

Wagg, J., Carilli, C. L., Wilner, D. J., Cox, P., De Breuck, C., Menten, K., Riechers, D. A., \& Walter, F. 2010, A\&A, 519, L1

Wagg, J., Hughes, D. H., Aretxaga, I., Chapin, E. L., Dunlop, J. S., Gaztañaga, E., \& Devlin, M. 2007, MNRAS, 375, 745

Wagg, J., Wiklind, T., Carilli, C. L., Espada, D., Peck, A., Riechers, D., Walter, F., Wootten, A., Aravena, M., Barkats, D., Cortes, J. R., Hills, R., Hodge, J., Impellizzeri, C. M. V., Iono, D., Leroy, A., Martín, S., Rawlings, M. G., Maiolino, R., McMahon, R. G., Scott, K. S., Villard, E., \& Vlahakis, C. 2012, ApJ, 752, L30

Wagg, J., Wilner, D. J., Neri, R., Downes, D., \& Wiklind, T. 2005, ApJ, 634, L13

Wake, D. A., Sheth, R. K., Nichol, R. C., Baugh, C. M., Bland-Hawthorn, J., Colless, M., Couch, W. J., Croom, S. M., de Propris, R., Drinkwater, M. J., Edge, A. C., Loveday, J., Lam, T. Y., Pimbblet, K. A., Roseboom, I. G., Ross, N. P., Schneider, D. P., Shanks, T., \& Sharp, R. G. 2008, MNRAS, 387, 1045

Wall, J. V., Pope, A., \& Scott, D. 2008, MNRAS, 383, 435

Walter, F., Bertoldi, F., Carilli, C., Cox, P., Lo, K. Y., Neri, R., Fan, X., Omont, A., Strauss, M. A., \& Menten, K. M. 2003 , Nature, 424,406

Walter, F., Brinks, E., de Blok, W. J. G., Thornley, M. D., \& Kennicutt, R. C. 2005, in Astronomical Society of the Pacific Conference Series, Vol. 331, Extra-Planar Gas, ed. R. Braun, 269

Walter, F., Decarli, R., Carilli, C., Bertoldi, F., Cox, P., da Cunha, E., Daddi, E., Dickinson, M., Downes, D., Elbaz, D., Ellis, R., Hodge, J., Neri, R., Riechers, D. A., Weiss, A., Bell, E., Dannerbauer, H., Krips, M., Krumholz, M., Lentati, L., Maiolino, R., Menten, K., Rix, H.-W., Robertson, B., Spinrad, H., Stark, D. P., \& Stern, D. 2012, Nature, 486, 233

Walter, F., Riechers, D., Cox, P., Neri, R., Carilli, C., Bertoldi, F., Weiss, A., \& Maiolino, R. 2009, Nature, 457, 699

Walter, F., Weiß, A., Downes, D., Decarli, R., \& Henkel, C. 2011, ApJ, 730, 18

Wang, J., Zhang, Z., \& Shi, Y. 2011a, MNRAS, 416, L21

Wang, L., Cooray, A., Farrah, D., Amblard, A., Auld, R., Bock, J., Brisbin, D., Burgarella, D., Chanial, P., Clements, D. L., Eales, S., Franceschini, A., Glenn, J., Gong, Y., Griffin, M., Heinis, S., Ibar, E., Ivison, R. J., Mortier, A. M. J., Oliver, S. J., Page, M. J., Papageorgiou, A., Pearson, C. P., Pérez-Fournon, I., Pohlen, M., Rawlings, J. I., Raymond, G., Rodighiero, G., Roseboom, I. G., Rowan-Robinson, M., Scott, D., Serra, P., Seymour, N., Smith, A. J., Symeonidis, M., Tugwell, K. E., Vaccari, M., Vieira, J. D., Vigroux, L., \& Wright, G. 2011 b, MNRAS, 414,596

Wang, R., Carilli, C. L., Beelen, A., Bertoldi, F., Fan, X., Walter, F., Menten, K. M., Omont, A., Cox, P., Strauss, M. A., \& Jiang, L. 2007, AJ, 134, 617

Wang, R., Wagg, J., Carilli, C. L., Walter, F., Lentati, L., Fan, X., Riechers, D. A., Bertoldi, F., Narayanan, D., Strauss, M. A., Cox, P., Omont, A., Menten, K. M., Knudsen, K. K., Neri, R., \& Jiang, L. 2013a, ApJ, 773, 44

Wang, S. X., Brandt, W. N., Luo, B., Smail, I., Alexander, D. M., Danielson, A. L. R., Hodge, J. A., Karim, A., Lehmer, B. D., Simpson, J. M., Swinbank, A. M., Walter, F., Wardlow, J. L., Xue, Y. Q., Chapman, S. C., Coppin, K. E. K., Dannerbauer, H., De Breuck, C., Menten, K. M., \& van der Werf, P. 2013b, ApJ, 778, 179

Wang, W.-H., Cowie, L. L., \& Barger, A. J. 2006, ApJ, 647, 74

Wang, W.-H., Cowie, L. L., Barger, A. J., \& Williams, J. P. 2011c, ApJ, 726, L18

Wardlow, J. L., Cooray, A., De Bernardis, F., Amblard, A., Arumugam, V., Aussel, H., Baker, A. J., Béthermin, M., Blundell, R., Bock, J., Boselli, A., Bridge, C., Buat, V., Burgarella, D., Bussmann, R. S., Cabrera-Lavers, A., Calanog, J., Carpenter, J. M., Casey, C. M., Castro-Rodríguez, N., Cava, A., Chanial, P., Chapin, E., Chapman, S. C., Clements, D. L., Conley, A., Cox, P., Dowell, C. D., Dye, S., Eales, S., Farrah, D., Ferrero, P., Franceschini, A., Frayer, D. T., Frazer, C., Fu, H., Gavazzi, R., Glenn, J., González Solares, E. A., Griffin, M., Gurwell, M. A., Harris, A. I., Hatziminaoglou, E., Hopwood, R., Hyde, A., Ibar, E., Ivison, R. J., Kim, S., Lagache, G., Levenson, L., Marchetti, L., Marsden, G., Martinez-Navajas, P., Negrello, M., Neri, R., Nguyen, H. T., O'Halloran, B., Oliver, S. J., Omont, A., Page, M. J., Panuzzo, P., Papageorgiou, A., Pearson, C. P., Pérez-Fournon, I., Pohlen, M., Riechers, D., Rigopoulou, D., Roseboom, I. G., Rowan-Robinson, M., Schulz, B., Scott, D., Scoville, N., Seymour, N., Shupe, D. L., Smith, A. J., Streblyanska, A., Strom, A., Symeonidis, M., Trichas, M., Vaccari, M., Vieira, J. D., Viero, M., Wang, L., Xu, C. K., Yan, L., \& Zemcov, M. 2013, ApJ, 762, 59

Wardlow, J. L., Smail, I., Coppin, K. E. K., Alexander, D. M., Brandt, W. N., Danielson, A. L. R., Luo, B., Swinbank, A. M., Walter, F., Weiß, A., Xue, Y. Q., Zibetti, S., Bertoldi, F., Biggs, A. D., Chapman, S. C., Dannerbauer, H., Dunlop, J. S., Gawiser, E., Ivison, R. J., Knudsen, K. K., Kovács, A., Lacey, C. G., Menten, K. M., Padilla, N., Rix, H.-W., \& van der Werf, P. P. 2011, MNRAS, 415,1479

Webb, T. M., Eales, S., Foucaud, S., Lilly, S. J., McCracken, H., Adelberger, K., Steidel, C., Shapley, A., Clements, D. L., Dunne, L., Le Fèvre, O., Brodwin, M., \& Gear, W. 2003, ApJ, 582, 6

Weidner, C., Ferreras, I., Vazdekis, A., \& La Barbera, F. 2013, MNRAS, 435, 2274

Weingartner, J. C. \& Draine, B. T. 2001, ApJ, 548, 296

Weinmann, S. M., Neistein, E., \& Dekel, A. 2011, MNRAS, 417, 2737

Weiß, A., De Breuck, C., Marrone, D. P., Vieira, J. D., Aguirre, J. E., Aird, K. A., Aravena, M., Ashby, M. L. N., Bayliss, M., Benson, B. A., Béthermin, M., Biggs, A. D., Bleem, L. E., Bock, J. J., Bothwell, M., Bradford, C. M., Brodwin, M., Carlstrom, J. E., Chang, C. L., Chapman, S. C., Crawford, T. M., Crites, A. T., de Haan, T., Dobbs, M. A., Downes, T. P., Fassnacht, C. D., George, E. M., Gladders, M. D., Gonzalez, A. H., Greve, T. R., Halverson, N. W., Hezaveh, Y. D., High, F. W., Holder, G. P., Holzapfel, W. L., Hoover, S., Hrubes, J. D., Husband, K., Keisler, R., Lee, A. T., Leitch, E. M., Lueker, M., Luong-Van, D., Malkan, M., McIntyre, V., McMahon, J. J., Mehl, J., Menten, K. M., Meyer, S. S., Murphy, E. J., Padin, S., Plagge, T., Reichardt, C. L., Rest, A., Rosenman, M., Ruel, J., Ruhl, J. E., Schaffer, K. K., Shirokoff, E., Spilker, J. S., Stalder, B., Staniszewski, Z., Stark, A. A., Story, K., Vanderlinde, K., Welikala, N., \& Williamson, R. 2013, ApJ, 767, 88

Weiß, A., Downes, D., Neri, R., Walter, F., Henkel, C., Wilner, D. J., Wagg, J., \& Wiklind, T. 2007, A\&A, 467, 955

Weiß, A., Ivison, R. J., Downes, D., Walter, F., Cirasuolo, M., \& Menten, K. M. 2009a, ApJ, 705, L45

Weiß, A., Kovács, A., Coppin, K., Greve, T. R., Walter, F., Smail, I., Dunlop, J. S., Knudsen, K. K., Alexander, D. M., Bertoldi, F., Brandt, W. N., Chapman, S. C., Cox, P., Dannerbauer, H., De Breuck, C., Gawiser, E., Ivison, R. J., Lutz, D., Menten, K. M., Koekemoer, A. M., Kreysa, E., Kurczynski, P., Rix, H., Schinnerer, E., \& van der Werf, P. P. 2009b, ApJ, 707, 1201

Weiß, A., Neininger, N., Hüttemeister, S., \& Klein, U. 2001, A\&A, 365, 571

Weiß, A., Walter, F., \& Scoville, N. Z. 2005, A\&A, 438, 533 
White, M., Myers, A. D., Ross, N. P., Schlegel, D. J., Hennawi, J. F., Shen, Y., McGreer, I., Strauss, M. A., Bolton, A. S., Bovy, J., Fan, X., Miralda-Escude, J., Palanque-Delabrouille, N., Paris, I., Petitjean, P., Schneider, D. P., Viel, M., Weinberg, D. H., Yeche, C., Zehavi, I., Pan, K., Snedden, S., Bizyaev, D., Brewington, H., Brinkmann, J., Malanushenko, V., Malanushenko, E., Oravetz, D., Simmons, A., Sheldon, A., \& Weaver, B. A. 2012, MNRAS, 424, 933

White, S. D. M. \& Frenk, C. S. 1991, ApJ, 379, 52

Wilkins, S. M., Trentham, N., \& Hopkins, A. M. 2008, MNRAS, 385, 687

Williams, C. C., Giavalisco, M., Porciani, C., Yun, M. S., Pope, A., Scott, K. S., Austermann, J. E., Aretxaga, I., Hatsukade, B., Lee, K.-S., Wilson, G. W., Cybulski, R., Hughes, D. H., Kawabe, R., Kohno, K., Perera, T., \& Schloerb, F. P. 2011, ApJ, 733, 92

Willott, C. J., Omont, A., \& Bergeron, J. 2013, ApJ, 770, 13

Wilson, G. W., Austermann, J. E., Perera, T. A., Scott, K. S., Ade, P. A. R., Bock, J. J., Glenn, J., Golwala, S. R., Kim, S., Kang, Y., Lydon, D., Predmore, C. R., Roberts, C. M., Souccar, K., \& Yun, M. S. 2008, MNRAS, 386, 807

Wolfire, M. G., Hollenbach, D., \& McKee, C. F. 2010, ApJ, 716, 1191

Wong, T. \& Blitz, L. 2002, ApJ, 569, 157

Wright, E. L., Eisenhardt, P. R. M., Mainzer, A. K., Ressler, M. E., Cutri, R. M., Jarrett, T., Kirkpatrick, J. D., Padgett, D., McMillan, R. S., Skrutskie, M., Stanford, S. A., Cohen, M., Walker, R. G., Mather, J. C., Leisawitz, D., Gautier, III, T. N., McLean, I., Benford, D., Lonsdale, C. J., Blain, A., Mendez, B., Irace, W. R., Duval, V., Liu, F., Royer, D., Heinrichsen, I., Howard, J., Shannon, M., Kendall, M., Walsh, A. L., Larsen, M., Cardon, J. G., Schick, S., Schwalm, M., Abid, M., Fabinsky, B., Naes, L., \& Tsai, C.-W. 2010, AJ, 140, 1868

Wu, J., Evans, II, N. J., Gao, Y., Solomon, P. M., Shirley, Y. L., \& Vanden Bout, P. A. 2005, ApJ, 635, L173

Wu, J., Evans, II, N. J., Shirley, Y. L., \& Knez, C. 2010, ApJS, 188, 313

Wu, J., Vanden Bout, P. A., Evans, II, N. J., \& Dunham, M. M. 2009, ApJ, 707, 988

Xia, J.-Q., Negrello, M., Lapi, A., De Zotti, G., Danese, L., \& Viel, M. 2012, MNRAS, 422, 1324

Yamamura, I., Makiuti, S., Ikeda, N., Fukuda, Y., Oyabu, S., Koga, T., \& White, G. J. 2010, VizieR Online Data Catalog, 2298,0

Yan, L., Choi, P. I., Fadda, D., Marleau, F. R., Soifer, B. T., Im, M., Armus, L., Frayer, D. T., Storrie-Lombardi, L. J., Thompson, D. J., Teplitz, H. I., Helou, G., Appleton, P. N., Chapman, S., Fan, F., Heinrichsen, I., Lacy, M., Shupe, D. L., Squires, G. K., Surace, J., \& Wilson, G. 2004a, ApJS, 154, 75

Yan, L., Helou, G., Fadda, D., Marleau, F. R., Lacy, M., Wilson, G., Soifer, B. T., Drozdovsky, I., Masci, F., Armus, L., Teplitz, H. I., Frayer, D. T., Surace, J., Storrie-Lombardi, L. J., Appleton, P. N., Chapman, S., Choi, P., Fan, F., Heinrichsen, I., Im, M., Schmitz, M., Shupe, D. L., \& Squires, G. K. 2004b, ApJS, 154, 60

Yan, L., Tacconi, L. J., Fiolet, N., Sajina, A., Omont, A., Lutz, D., Zamojski, M., Neri, R., Cox, P., \& Dasyra, K. M. 2010, ApJ, 714, 100

Yang, C., Gao, Y., Omont, A., Liu, D., Isaak, K. G., Downes, D., van der Werf, P. P., \& Lu, N. 2013, ApJ, 771, L24

Yao, L., Seaquist, E. R., Kuno, N., \& Dunne, L. 2003, ApJ, 588, 771

Young, J. S., Schloerb, F. P., Kenney, J. D., \& Lord, S. D. 1986, ApJ, 304, 443

Younger, J. D., Fazio, G. G., Huang, J.-S., Yun, M. S., Wilson, G. W., Ashby, M. L. N., Gurwell, M. A., Lai, K., Peck, A. B., Petitpas, G. R., Wilner, D. J., Iono, D., Kohno, K., Kawabe, R., Hughes, D. H., Aretxaga, I., Webb, T., Martínez-Sansigre, A., Kim, S., Scott, K. S., Austermann, J., Perera, T., Lowenthal, J. D., Schinnerer, E., \& Smolčić, V. 2007, ApJ, 671, 1531

Younger, J. D., Fazio, G. G., Wilner, D. J., Ashby, M. L. N., Blundell, R., Gurwell, M. A., Huang, J.-S., Iono, D., Peck, A. B., Petitpas, G. R., Scott, K. S., Wilson, G. W., \& Yun, M. S. 2008, ApJ, 688, 59

Younger, J. D., Hayward, C. C., Narayanan, D., Cox, T. J., Hernquist, L., \& Jonsson, P. 2009a, MNRAS, 396, L66

Younger, J. D., Omont, A., Fiolet, N., Huang, J., Fazio, G. G., Lai, K., Polletta, M., Rigopoulou, D., \& Zylka, R. 2009b, MNRAS, 394,1685

Yun, M. S. \& Carilli, C. L. 2002, ApJ, 568, 88

Yun, M. S., Reddy, N. A., \& Condon, J. J. 2001, ApJ, 554, 803

Yun, M. S., Scott, K. S., Guo, Y., Aretxaga, I., Giavalisco, M., Austermann, J. E., Capak, P., Chen, Y., Ezawa, H., Hatsukade, B., Hughes, D. H., Iono, D., Johnson, S., Kawabe, R., Kohno, K., Lowenthal, J., Miller, N., Morrison, G., Oshima, T., Perera, T. A., Salvato, M., Silverman, J., Tamura, Y., Williams, C. C., \& Wilson, G. W. 2012, MNRAS, 420, 957

Zamojski, M., Yan, L., Dasyra, K., Sajina, A., Surace, J., Heckman, T., \& Helou, G. 2011, ApJ, 730, 125

Zehavi, I., Zheng, Z., Weinberg, D. H., Blanton, M. R., Bahcall, N. A., Berlind, A. A., Brinkmann, J., Frieman, J. A., Gunn, J. E., Lupton, R. H., Nichol, R. C., Percival, W. J., Schneider, D. P., Skibba, R. A., Strauss, M. A., Tegmark, M., \& York, D. G. 2011, ApJ, 736, 59

Zemcov, M., Blain, A., Cooray, A., Béthermin, M., Bock, J., Clements, D. L., Conley, A., Conversi, L., Dowell, C. D., Farrah, D., Glenn, J., Griffin, M., Halpern, M., Jullo, E., Kneib, J.-P., Marsden, G., Nguyen, H. T., Oliver, S. J., Richard, J., Roseboom, I. G., Schulz, B., Scott, D., Shupe, D. L., Smith, A. J., Valtchanov, I., Viero, M., Wang, L., \& Wardlow, J. 2013, ApJ, 769, L31

Zweibel, E. 2013, Nature, 502, 453 